Franz Kneer, Klaus G. Puschmann, and Axel D. Wittmann (Ed.)

\title{
Modern Solar Facilities - Advanced Solar Science
}

Proceedings of a Workshop held at Göttingen September 27-29, 2006

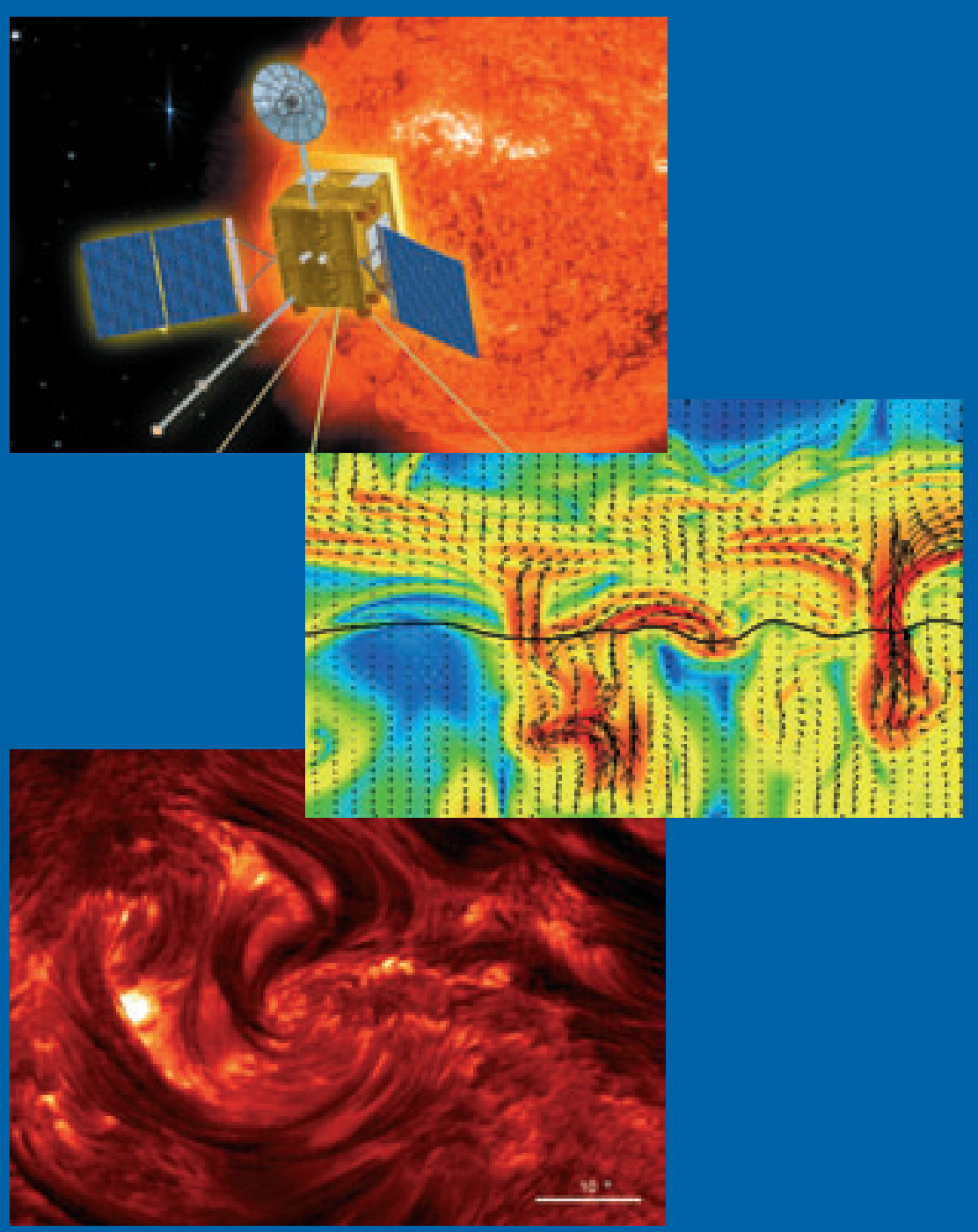

Universitätsverlag Göttingen 

Franz Kneer, Klaus G. Puschmann, and Axel D. Wittmann (Ed.)

Modern Solar Facilities

Except where otherwise noted, this work is licensed under a Creative Commons License

(c)

SORTERIGHIS RESERVED 
erschienen im Universitätsverlag Göttingen 2007 
Franz Kneer, Klaus G. Puschmann, Axel D. Wittmann (Ed.)

Modern Solar Facilities Advanced Solar Science

Proceedings of a Workshop held at Göttingen September 27-29, 2006

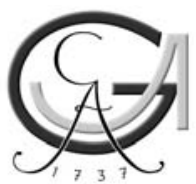

Universitätsverlag Göttingen 2007 


\title{
Bibliographische Information der Deutschen Nationalbibliothek
}

Die Deutsche Nationalbibliothek verzeichnet diese Publikation in der Deutschen Nationalbibliographie; detaillierte bibliographische Daten sind im Internet über $<$ http://dnb.ddb.de> abrufbar

\author{
Address of the Editor in Chief \\ Prof. Dr. Franz Kneer \\ Institut für Astrophysik der Georg-August-Universität Göttingen \\ Friedrich-Hund-Platz 1, 37077 Göttingen \\ kneer@,Astro.Physik.Uni-Goettingen.DE \\ http://www.astro.physik.uni-goettingen.de/ kneer
}

This work is protected by German Intellectual Property Right Law. It is also available as an Open Access version through the publisher's homepage and the Online Catalogue of the State and University Library of Goettingen

(http://www.sub.uni-goettingen.de). Users of the free online version are invited to read, download and distribute it under the licence agreement shown in the online version. Users may also print a small number for educational or private use. However they may not sell print versions of the online book.

Umschlaggestaltung: Margo Bargheer

Cover images: Top: Artist's view of the Solar Orbiter spacecraft near the Sun (from Fleck, p. 23). Middle: Logarithm of the (color-coded) electric current density in a vertical cross section of sub-photospheric and photospheric layers, according to a numerical MHD simulation. Arrows show strength and direction of the magnetic field (from Steiner, p. 332). Bottom: Flaring active region observed on April 26, 2006, with the German VTT at Izaña/Tenerife in the line centre of $\mathrm{H} \alpha$ (from Sánchez-Andrade Nuño et al., p. 274)

(C) 2007 Universitätsverlag Göttingen

http://univerlag.uni-goettingen.de

ISBN: 978-3-938616-84-0 


\section{Contents}

Summary of the workshop

F. Kneer, K. G. Puschmann, and A. D. Wittmann

\section{Telescopes and instrumentation}

Future solar space missions (invited)

B. Fleck

LYRA - a solar UV radiometer using diamond detectors

A. Theissen, A. BenMoussa, U. Schühle, J.-F. Hochedez, and W. Schmutz

Ground-based solar facilities in the U.S.A. (invited)

C. Denker, D. E. Gary, and T. R. Rimmele

New high resolution solar telescope GREGOR (invited)

R. Volkmer, O. von der Lühe, F. Kneer, J. Staude, H. Balthasar, T. Berkefeld,

P. Caligari, M. Collados, C. Halbgewachs, F. Heidecke, A. Hofmann, M. Klvaña,

M. Sobotka, H. Nicklas, E. Popow, K. G. Puschmann, W. Schmidt, D. Soltau,

K. Strassmeier, and A. D. Wittmann

From the "Göttingen" Fabry-Perot Interferometer to the GREGOR FPI

K. G. Puschmann, F. Kneer, H. Nicklas, and A. D. Wittmann

Gregor@Night. A concept for a night-time spectrograph for the $1.5 \mathrm{~m}$ solar telescope GREGOR

K. G. Strassmeier, M. Woche, T. Granzer, M. I. Andersen, W. Schmidt, and P. Koubsky

Multi-wavelength observations at the German VTT on Tenerife -

Possible combinations of post-focus instruments

C. Beck, K. Mikurda, L. R. Bellot Rubio, T. Kentischer, and M. Collados

Instrumental developments at the Gregory Coudé Telescope (GCT) at IRSOL

M. Bianda, R. Ramelli, A. Feller, J. O. Stenflo, and G. Küveler

Imaging polarimetry with a tunable narrow-band filter

A. Feller, M. Bianda, and J. O. Stenflo

Methods in high-resolution solar spectroscopy

F. Kneer 
SUNRISE: High resolution UV/VIS observations of the Sun

from the stratosphere (invited)

A. M. Gandorfer, S. K. Solanki, P. Barthol, V. Martínez Pillet, W. Schmidt,

A. M. Title, M. Knölker, and the SUNRISE team

ZERODUR mirror substrates for solar telescopes

T. Döhring, R. Jedamzik, and P. Hartmann

\section{High-resolution and high-precision observations}

Future missions for helioseismology (invited)

M. Roth

Helioseismology at MPS

L. Gizon, R. Cameron, J. Jackiewicz, M. Roth, H. Schunker, and T. Stahn

Structure and evolution of supergranulation from local helioseismology

J. Hirzberger, L. Gizon, S. K. Solanki, and T. L. Duvall, Jr.

Solar adaptive optics (invited)

T. Berkefeld

Anisoplanatic optical transfer functions for solar adaptive optics from simulation using wave front sensor data

M. Sailer and $O$. von der Lühe

Solar image restoration (invited)

M. G. Löfdahl, M. J. van Noort, and C. Denker

Probabilistic suppression of astronomical image degradations

M. Haindl and S. Šimberová

Advances, challenges and limitations of speckle reconstruction and deconvolution

K. Mikurda, O. von der Lühe, F. Wöger, and W. Schmidt

Reversed granulation in Fe I $7090.4 \AA$

K. Janssen and G. Cauzzi

The height dependence of temperature - velocity correlation in the solar photosphere

J. Koza, A. Kučera, J. Rybák, and H. Wöhl

Polarimetry in the visible and near infrared (invited)

M. Collados

Dynamics of the quiet photosphere and its magnetic field under high spatial resolution

K. G. Puschmann, F. Kneer, and I. Domínguez Cerdeña

Internetwork magnetic fields

M. J. Martínez González, M. Collados, and B. Ruiz Cobo

High-resolution CN spectroscopy of small-scale solar magnetic features

V. V. Zakharov, A. Gandorfer, and S. K. Solanki 
Magnetic properties of G-band bright points

C. Beck, K. Mikurda, L. R. Bellot Rubio, R. Schlichenmaier, and P. Sütterlin

Photospheric magnetic field and chromospheric emission

R. Rezaei, R. Schlichenmaier, C. Beck, and W. Schmidt

Velocity distribution of chromospheric downflows

R. Aznar Cuadrado, S. K. Solanki, and A. Lagg

Off-limb spectroscopy of the He I $10830 \AA$ A multiplet: observations vs. modelling B. Sánchez-Andrade Nuño, R. Centeno, K. G. Puschmann, J. Trujillo Bueno, and F. Kneer

Study of polar faculae with north pole coverage of the Sun

J. Blanco Rodríguez, O. Okunev, K. G. Puschmann, and F. Kneer

Effects of the "false" magnetic-field imbalance in solar active regions

I. V. Oreshina and B. V. Somov

Magnetic source of the solar cycle variation of the Mn I $539.4 \mathrm{~nm}$ line

S. Danilovic, S. K. Solanki, W. Livingston, N. Krivova, and I. Vince

Reconstruction of irradiance from 2D magnetograms and modification of the $1 / \mu$-correction paradigm

A. Reiners, G. Basri, K. Soto, F. Ramos Stierle, and T. Lewis

Variations in solar indices during the $23^{\text {rd }}$ solar cycle

N. Oklay

Structure and flattening of the solar corona

M. T. Özkan, A. Ökten, N. Oklay, A. Gültekin, M. Kara, Z. F. Bostancı, M. Başal, and N. Al Erdoğan

Fine structure in a dark umbra

M. Sobotka and K. G. Puschmann

Dynamics of a solar pore with light bridge

S. Giordano, F. Berrilli, D. Del Moro, and V. Penza

$\mathrm{Ca}$ II $\mathrm{H}$ line wing images of sunspot penumbrae

recorded with the Swedish 1-m Solar Telescope

G. Narayan, M. J. van Noort, and G. Scharmer

Temporal evolution of intensity, velocity and magnetic field of sunspots at high spatial resolution

N. Bello González, F. Kneer, and K. G. Puschmann

Magnetic vector field above a sunspot

P. Gömöry and H. Balthasar 
The observational counterpart of the rising flux tube model?

J. Jurčák and M. Sobotka

Simultaneous polarimetric observations with VTT and THEMIS

H. Balthasar and V. Bommier

On the inhomogeneities of the sunspot penumbra

R. Schlichenmaier, D. A. N. Müller, and C. Beck

Modified $p$-modes in penumbral filaments

D. S. Bloomfield, A. Lagg, S. K. Solanki, and J. M. Borrero

Wave propagation and shock formation in diverse magnetic structures

R. Centeno, M. Collados, and J. Trujillo Bueno

Evolution of coronal hole boundaries seen in EIT $195 \AA$ and TRACE $171 \AA$ images M. S. Madjarska and T. Wiegelmann

Magnetic field structure and dynamics in coronal hole - active region interface zone I. A. Bilenko

Evolution of the photospheric magnetic field in the source regions

of coronal mass ejections

V. Bothmer and D. Tripathi

Two-dimensional imaging of the $\mathrm{He}_{3} / \mathrm{H} \beta$ emission ratio

in quiescent solar prominences

E. Wiehr, G. Stellmacher, and J. Hirzberger

Wavelet-based method for coronal loop oscillations analysis

D. Tothova, D. E. Innes, and S. K. Solanki

Magnetic stereoscopy of coronal loops in NOAA 8891

L. Feng, T. Wiegelmann, and B. Inhester

Observations of a flaring active region in $\mathrm{H} \alpha$

B. Sánchez-Andrade Nuño, K. G. Puschmann, and F. Kneer

On the thermal and non-thermal excitation effects as studied in the $\mathrm{H} \alpha, \mathrm{H} \beta$

and Ca II $8542 \AA$ line profiles in a solar flare

P. Kotrč and L. K. Kashapova

Solar HXR- and $\gamma$-ray emission measurements in 2005 by SONG/CORONAS-F

near minimum of the last activity cycle

I. N. Myagkova, S. N. Kuznetsov, E. A. Muravieva, and L. I. Starostin

\section{Theory and interpretation}

Inversion techniques: From observations to atmospheres (invited)

B. Ruiz Cobo

Stokes profile inversion in Meso-Structured Magnetic Atmospheres

T. A. Carroll 
Line ratio method applied to inter-network magnetic fields

E. Khomenko and M. Collados

The Ba II $\lambda 4554$ resonance line and solar granulation

V. L. Olshevsky and N. G. Shchukina

Magnetic mappers of the quiet solar atmosphere

J. Trujillo Bueno

Molecular Hanle effect in the Paschen-Back regime: theory and application

A. I. Shapiro, S. V. Berdyugina, D. M. Fluri, and J. O. Stenflo

Recent progresses in the simulation of small-scale magnetic fields (invited)

O. Steiner

Solar mesogranulation as a cellular automaton effect

L. Matloch, R. Cameron, D. Schmitt, and M. Schüssler

Sunspot models with bright rings

L. L. Kitchatinov and G. Rüdiger

Numerical modeling of MHD wave propagation in sunspots: a 3D case

V. Olshevsky, E. Khomenko, and M. Collados

Seismology of sunspot atmosphere: from chromospheric resonance

to nonlinear antireflection

Y. D. Zhugzhda

Ray tracing of ion-cyclotron waves in a coronal funnel

R. Mecheri and E. Marsch

Nanoflare model of emission line radiance distributions in active region coronae

H. Safari, D. E. Innes, S. K. Solanki, and A. Pauluhn

Modeling large-scale coronal structures

P. Ruan, T. Wiegelmann, and B. Inhester

Magnetic flux transport and the lifetimes of spots on active cool stars

E. Işık, M. Schüssler, and S. K. Solanki

Mass loss rates and wind ram pressures of cool stars

V. Holzwarth and M. Jardine

List of participants 



\section{Summary of the workshop}

An international workshop entitled: 'Modern Solar Facilities - Advanced Solar Science' was held in Göttingen from 27 to 29 September, 2006. It took place in the new physics building located in the northern campus of the Georg-August-Universität. The workshop was attended by 88 participants from 24 different countries (see the list of participants, pp. 375 381 , containing the institutional addresses at the time of the workshop). With 11 invited talks, 28 contributed talks, and 44 poster contributions the workshop gathered leading researchers in the field and gave a broad overview of the current state of solar research.

The following three main topics were covered by the invited talks, contributed talks, and poster presentations during the workshop:

1. Solar telescopes, instruments and techniques

This included space missions and ground-based facilities in operation and under development, as well as new post-focus instrumentation.

2. High-resolution observations and results

This included high-resolution and high-precision solar observations, methods and results, including adaptive optics, image processing, spectroscopy and polarimetry, as well as helioseismology.

3. Theoretical models, simulations, and interpretations

This included model calculations, inversion techniques, numerical simulations including line formation, diagnostics with the Zeeman and the Hanle effects, and MHD simulations.

Solar physics is developing at a fast pace. During the recent years we have witnessed, and will witness in the years to come, observational facilities on ground and in space of unprecedented potential. Large telescopes of the 1-meter class and larger are in operation or will soon be commissioned (e.g. GREGOR, $1.5 \mathrm{~m}$ ). Most of the existing and newlyplanned ground-based solar telescopes are equipped with adaptive optics (AO). The design and the planning for the Advanced Technology Solar Telescope (ATST) have reached a state that it can possibly be brought into operation within the next five years. In 2006 the space missions Solar-B (HINODE) and STEREO have been launched successfully. SUNRISE, in its first stage a balloon experiment, shall follow within a few years. Likewise, on the side of detectors and of data acquisition in general, we notice immense improvements. And last but not least, new fast computing facilities including very efficient numerical codes allow realistic simulations of processes of the Sun. All these developments permit an enormous progress in solar science. Especially the understanding of small-scale dynamics on the Sun, in the solar atmosphere, and in sub-photospheric layers, is rapidly growing. 
According to subject across the sessions, the workshop covered the scientific topics:

1. Solar interior and helioseismology

2. Solar photosphere and chromosphere, and their dynamics in quiet and active regions

3. Solar corona and solar wind, including flares, CMEs, and few stellar topics.

The invited keynotes gave a broad overview of various fields of research: future space missions (Fleck), new solar facilities in the US (Denker), the construction of GREGOR (Volkmer) and SUNRISE (Gandorfer), helioseismology (Roth), various new and important techniques, viz. adaptive optics (Berkefeld), image restoration (Löfdahl), polarimetry (Collados), research into faculae at the poles of the Sun (Okunev, not reprinted here), and more theoretical aspects as line-formation inversion techniques (Ruiz Cobo) and solar MHD numerical simulations (Steiner).

During the workshop as well as in these proceedings, poster and oral contributions were treated on equal rights. There was ample time for a brief oral presentation of each poster and for extended poster sessions during the workshop. Details for the workshop were made available on the conference website, of which a trailer version may still be visited (for a while): http://www.astro.physik.uni-goettingen.de/ solconf

The contributed talks and posters covered a wide variety of subjects. There were presentations on requirements for high-quality optics and spectrometers, instrumental improvements, and intelligent data analysis. The attendants witnessed the increasing scientific potential of (local) helioseismology to probe the interior of the Sun. Presentations on the solar granulation, its interaction with waves and magnetic fields showed that this topic has remained exciting and contains much physics not yet fully understood.

The magnetism of the Sun plays an important role in the dynamics of the solar interior and atmosphere, as well as of stars in general. Thus, many contributions dealt with magnetic phenomena on the Sun, with few contributions on stellar magnetism. The presented research ranges from magnetic cycle variations to large-scale magnetic activity, from global properties of the corona to magnetic fields in the corona, from their rootings in the photosphere to prominences and coronal mass ejections (CMEs). The success of space research was further underlined by works on coronal loop oscillations and stereoscopy of coronal loops.

Magnetic fields in the network and internetwork regions of the quiet Sun were addressed as well as faculae on the disk, near the limb, and at the poles of the Sun. Quiet Sun observations also dealt with chromospheric flows and spicules.

Certainly, many new, exciting, and high-quality results on pores and sunspots were presented. These included research into sunspot umbrae, flows and waves, magnetic fields, and magnetodynamic processes in pores, sunspots, light bridges, penumbrae, and sunspot surroundings. Observations of flares, representing violent magnetic processes, observed in chromospheric lines, such as $\mathrm{H} \alpha$, and in hard $\mathrm{X}$ rays and $\gamma$ rays, were also discussed.

With regard to the interpretation of spectroscopic and polarimetric data, fresh ideas of radiative transfer have arisen. It was shown how small-scale, even sub-resolution, fluctuations in temperature, velocity, and magnetic field can be treated and which diagnostic spectroscopic features would be appropriate to conclude, via inversion techniques, on the physical properties of the solar atmosphere. Beyond Zeeman diagnostics, the Hanle effect allows to scrutinize sub-resolution, fully entangled, weak magnetic fields. 
Last but not least, theoretical analyses and numerical modeling are contributing to the rapid growth of understanding of solar dynamics. We learned about (magneto)convection in granulation and mesogranulation, about the structure of umbral dots and the evolution of pores, about the deep structure of sunspots, and waves in sunspots. And again, initiated by observations from space, cyclotron waves in coronal funnels, magnetic processes in nanoflares, and magnetic loop structures in general, are on the way of being understood.

The meeting went smoothly and, in the opinion of all we spoke, proceeded in a relaxed atmosphere inviting for scientific discussions. Not all of the attendees could find the time and ease to prepare a written version of their contribution. Some of the presentations were combined by groups of authors to just one extended contribution. These are the reasons why the present volume contains only 71 of the 83 papers presented during the workshop.

This workshop will, foreseeably, have been the last solar meeting under the present structure of our institute, which originated from the Universitäts-Sternwarte (1816-2005): There, the discipline of Solar Physics was once founded by eminent scientists like Karl Schwarzschild $(\approx 1910$ : Solar atmophere, solar corona, solar ballooning), Hans Kienle $(\approx$ 1930: Solar radiation measurements, spectroheliographic techniques), Karl-Otto Kiepenheuer $(\approx 1940$ : Solar activity, solar observations, UV spectroscopy), Paul ten Bruggencate $(\approx 1950)$, and Egon-Horst Schröter $(\approx 1970)$, with solar telescopes at the Hainberg Observatory (1944-2005), at IRSL/Locarno (1961-1983), and at Tenerife (1984-...).

We, the undersigned organizers and editors, have tried to compose these proceedings in a reasonable sequence of topics, which was not always possible in view of the wide research fields addressed by the various contributions. We have put efforts into careful refereeing and correction, to our best knowledge.

The organizers acknowledge financial, technical, and logistic support by the following institutions and company:

Deutsche Forschungsgemeinschaft,

Land Niedersachsen,

Georg-August-Universität Göttingen,

Fakultät für Physik, Universität Göttingen,

Firma SCHOTT AG/Mainz,

Institut für Astrophysik, Universität Göttingen.

It is a pleasure to thank all those who have, by preparing the meeting, by attending the meeting, and by sending written versions of their papers, contributed to the enjoyable, scientifically valuable workshop. We appreciate the encouragement by all participants and contributors, during the workshop and during the preparation of these proceedings. Special thanks are due to Dieter Schmitt for his invaluable help in realising this book. And finally we wish to thank the Universitätsverlag Göttingen for publishing this book in its Scientific Proceedings series.

Göttingen, 1 March 2007

F. Kneer, K. G. Puschmann, A. D. Wittmann 

Part 1

\section{Telescopes and instrumentation}



Modern Solar Facilities - Advanced Solar Science, 17-26

F. Kneer, K. G. Puschmann, A. D. Wittmann (eds.)

(C) Universitätsverlag Göttingen 2007

\title{
Future solar space missions
}

\author{
B. Fleck \\ ESA Research and Scientific Support Department, c/o NASA/GSFG, Greenbelt, MD, USA \\ Email: bernhard.fleck@esa.int
}

\begin{abstract}
The coming years promise to be the golden era of solar and heliospheric physics, with STEREO, Hinode (Solar-B) and SDO enhancing the current fleet of solar space missions and affording new opportunities for improved understanding of the Sun-heliosphere system. Looking beyond that, however, there is a significant gap until Solar Orbiter will be launched in 2015 (nearly 20 years after the launch of SOHO). This paper provides an overview of the next generation of solar space missions.
\end{abstract}

\section{Introduction}

Space-borne observations from Yohkoh, SOHO, TRACE, and RHESSI have produced stunning results that have invigorated solar research and challenged existing models of the Sun. The next generation of solar space missions that includes Hinode (known as Solar-B before its launch on 21 September 2006), STEREO (launched on 25 October 2006), SDO, Solar Orbiter, Solar Sentinels, and other smaller, focused missions promises to continue this "solar renaissance" by providing the ability to investigate solar processes on their fundamental scales, whether sub-arcsecond or global in nature, and by adding new viewing angles - high latitude, far-side, and out of Sun-Earth line viewing as well as close encounters.

\section{Hinode}

Hinode ("sunrise", formerly known as Solar-B; Shimizu et al. 2002) is an ISAS mission with US, UK and ESA participation as follow-on to the highly successful Yohkoh mission. It was launched on 21 September 2006. The key scientific objectives of Hinode are to study the generation and transport of magnetic fields and their role in heating and structuring the chromosphere and corona, and in eruptive events and flares. The 3-axis stabilized satellite (total mass $\approx 900 \mathrm{~kg}$ ) was launched into a polar sun-synchronous orbit with inclination $97.9^{\circ}$ and an altitude of approximately $600 \mathrm{~km}$. The nominal mission lifetime is three years.

Hinode's scientific payload comprises three instruments. At the heart of Hinode is a diffraction limited 50-cm aperture Solar Optical Telescope (SOT), which feeds a Focal Plane Package (FPP) designed for high resolution photospheric and chromospheric imaging and spectro-polarimetry. In addition there are two coronal instruments, the X-Ray Telescope (XRT) and the Extreme-ultraviolet Imaging Spectrometer (EIS).

The SOT is the largest solar telescope launched into space. It provides diffraction limited resolution from $3880-6600 \AA$ and feeds the FPP which consists of the following three main components: 
(i) a broad-band interference filter imager (BFI),

(ii) a narrow-band tunable birefringent filter imager (NFI),

(iii) a spectro-polarimeter (SP), essentially a space version of the HAO Advanced Stokes Polarimeter.

The image is stabilized to better than 0.02 arcsec over a range from 0.02 to $20 \mathrm{~Hz}$ by a correlation tracker and active tilt mirror. The broad-band filter system makes diffraction-limited images with 0.05 arcsec pixels in the $\mathrm{Ca}$ II $\mathrm{H}$ line, $\mathrm{CN}$ and $\mathrm{G}$ bandheads, and continuum bands. Its maximum field-of-view (FOV) is $216 \times 108 \mathrm{arcsec}^{2}$. It has a common focal plane with the narrow-band filter system, which makes filtergrams, (vector) magnetograms, Dopplergrams, and Stokes images in several photospheric lines, $\mathrm{Mg}$ b, and $\mathrm{H} \alpha$. It has 0.08 arcsec pixels and a FOV up to $165 \times 328 \operatorname{arcsec}^{2}$. The spectro-polarimeter makes vector magnetic measurements from Stokes spectra of the Fe I lines 6301 and $6302 \AA$, with 0.16 arcsec pixels and a FOV same as that of the narrow-band filter. The SP and filter imagers can be used to observe simultaneously on the same target region.

XRT is an enhanced version of the SXT instrument on Yohkoh, providing coronal images at wavelengths from 2 to $60 \AA$. The image scale is 1 arcsec/pixel which is a factor 2.5 better than that of SXT, and it responds to a broader range of plasma temperatures. XRT is a grazing-incidence (GI) modified Wolter I X-ray telescope, of $35 \mathrm{~cm}$ inner diameter and $2.7 \mathrm{~m}$ focal length. The $2048 \times 2048$ back-illuminated CCD has $13.5 \mu \mathrm{m}$ pixels, corresponding to 1.0 arcsec and giving full Sun field of view. A small optical telescope using the same CCD provides visible light images for coalignment with the SOT.

EIS consists of a multilayer-coated off-axis telescope mirror (150 mm aperture) and a multilayer-coated toroidal grating spectrometer. It uses two back-thinned CCDs with $13.5 \mu \mathrm{m}$ pixels as detectors. The instrument includes thin-film aluminum filters to reject longer wavelength radiation, a slit mechanism and two back-thinned CCD detectors at the focal plane. Monochromatic images are formed either by rastering the solar image across a narrow entrance slit or by using a very wide slit. The mirror and spectrometer combined have a spatial resolution capability of 2 arcsec while the plate scale is $1^{\prime \prime} /$ pixel. The spectral resolution is good enough to allow the measurement of velocity to $\pm 3 \mathrm{~km} / \mathrm{s}$. Half of each optic is coated to optimize reflectance at $170-210 \AA$, and the other half to optimize reflectance at $250-290 \AA$. Each wavelength range is imaged onto a separate back-thinned CCD detector of high quantum efficieny $(\approx 80 \%$ ) for the chosen wavelengths. The two wavelength bands include emission lines formed over a temperature range from roughly 0.1 to $20 \mathrm{MK}$.

\section{STEREO}

The principal scientific objective of NASA's Solar-TErrestrial RElations Observatory (STEREO; Kaiser 2005) is to understand the origin and consequences of coronal mass ejections (CMEs). By using two identically instrumented spacecraft, one drifting ahead of Earth and one behind, it will provide new perspectives on the structure of the solar corona and CMEs. It will obtain simultaneous images of the Sun from the two spacecraft and build a 3-D picture of CMEs and the complex structures around them. It will also study the propagation of disturbances through the heliosphere and their effects at Earth orbit. STEREO is a multilateral international collaboration involving participants from the United States, France, 


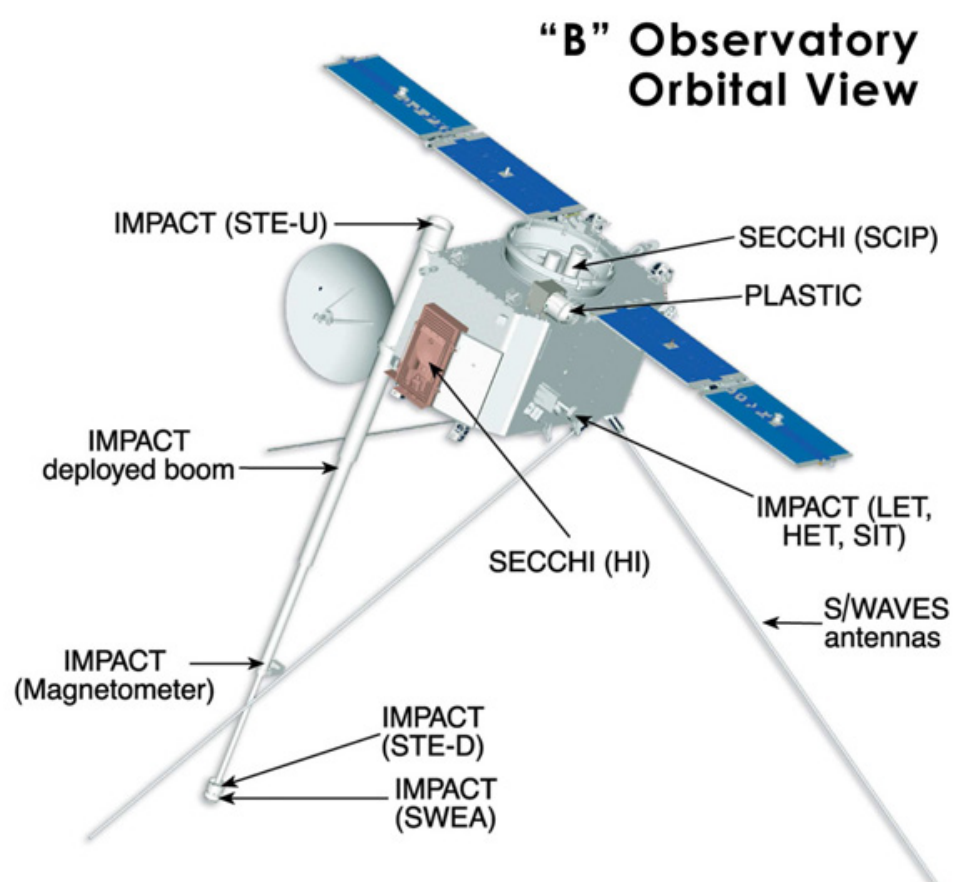

Figure 1. Schematic view of one of the twin STEREO spacecraft. Each carries four instrument suites: SECCHI, SWAVES, IMPACT, and PLASTIC.

Germany, and the United Kingdom. It was launched on a Delta II 7925-10L on 25 October 2006, and science operations started on 22 January 2007.

Each of the two spacecraft weighs approximately $620 \mathrm{~kg}$ and provides $540 \mathrm{~W}$ power. They are separating from Earth at the average rate of 22.5 degrees per year ( $45^{\circ}$ per year between the two spacecraft). The nomincal mission lifetime is two years.

The two spacecraft carry four identical instrument suites (Fig. 1):

(i) SECCHI, the Sun Earth Connection Coronal and Heliospheric Investigation, which includes four instruments: two white-light coronagraphs (covering the range 1.25$4 \mathrm{R}_{\odot}$, and 2-15 $\mathrm{R}_{\odot}$ ), an EUV imager (full-disk, $1^{\prime \prime}$ pixels) and a heliospheric imager (HI, an externally occulted coronagraph that can image the heliosphere from $12 \mathrm{R}_{\odot}$ to beyond Earth's orbit)

(ii) STEREO/WAVES (SWAVES), an interplanetary radio burst tracker that traces the generation and evolution of traveling radio disturbances from the Sun to the orbit of Earth.

(iii) IMPACT, the In situ Measurements of Particles and CME Transients investigation, which measures the 3-D distribution and plasma characteristics of solar energetic particles and the local vector magnetic field.

(iv) PLASTIC, the PLAsma and SupraThermal Ion and Composition experiment, which provides plasma characteristics of protons, alpha particles and heavy ions. 


\section{Proba-2}

Proba-2, currently under development and due for launch in May 2008, is the second in ESA's series of small, low-cost satellites that are being used to validate new spacecraft technologies while also carrying scientific instruments. Proba-2's dimensions are similar to a washing maschine $(80 \times 70 \times 60 \mathrm{~cm})$. It will weigh about $120 \mathrm{~kg}$ of which $35 \mathrm{~kg}$ will be for the payload.

The Proba satellites are part of ESA's In-Orbit Technology Demonstration Programme. Among the new technologies that will be demonstrated on Proba-2 are a new type of lithiumion battery, an advanced data and power management system, combined carbon-fibre and aluminium structural panels, new models of reaction wheels, star trackers and GPS receivers, an upgraded telecommand system with a decoder largely implemented in software, a digital Sun-sensor, a fibre-sensor system for monitoring temperatures and pressures around the spacecraft, a very high precision flux-gate magnetometer, an experimental solar panel with a solar flux concentrator, a xenon gas propulsion system using resistojet thrusters and a solidstate nitrogen gas generator to pressurise the propellant tanks, and an exploration microcamera (X-CAM) with panoramic optics.

Proba-2 carries also four scientific experiments, two of which are for solar observations, the other two for space weather measurements. The first one is SWAP (Sun Watcher with APS detectors and image Processing), a small EUV imager based on an off-axis RitcheyChrétien telescope design, that will image a FOV of 54 arcmin of the 1 million degree solar corona. A spectral band around $17.5 \mathrm{~nm}$ will be selected by aluminium filters and multilayer coatings on the primary and secondary mirrors. A $1024 \times 1024$ pixel CMOS APS detector (instead of a more classical CCD camera), will be coated with a luminescent phosphor coating to boost the EUV sensitivity. SWAP will be capable of high-cadence $(1 \mathrm{~min})$ imaging and will complement SOHO/EIT and STEREO/SECCHI observations.

The other solar instrument is LYRA (Lyman Alpha RAdiometer), which will measure solar irradiance in four passpands relevant to solar physics, space weather and aeronomy with a cadence of up to $100 \mathrm{~Hz}$, using novel, solar-blind diamond detectors. The four channels are i) Lyman-alpha, ii) the 200-220 nm Herzberg continuum range, iii) Aluminum channel including $\mathrm{He}$ II at $30.4 \mathrm{~nm}$, and iv) the hotter Zirconium channel.

\section{SDO}

The Solar Dynamics Observatory (SDO, Hathaway et al. 2001) is the first cornerstone mission in NASA's Living With a Star (LWS) programme, the goals of which are "to develop the scientific understanding necessary to effectively address those aspects of the coupled Sun-Earth system that directly affect life and society". SDO's objective in this programme is to understand the nature and source of solar variability that affects life and society. Key questions to be addressed by SDO are: What mechanisms drive the quasi-periodic 11-year cycle of solar activity? How is active region magnetic flux synthesized, concentrated, and dispersed across the solar surface? Where do the observed variations in the Sun's total and spectral irradiance arise, and how do they relate to the magnetic activity cycle? Is it possible to make accurate and reliable forecasts of space weather and climate?

SDO (Fig. 2) will carry three instrument suites: 


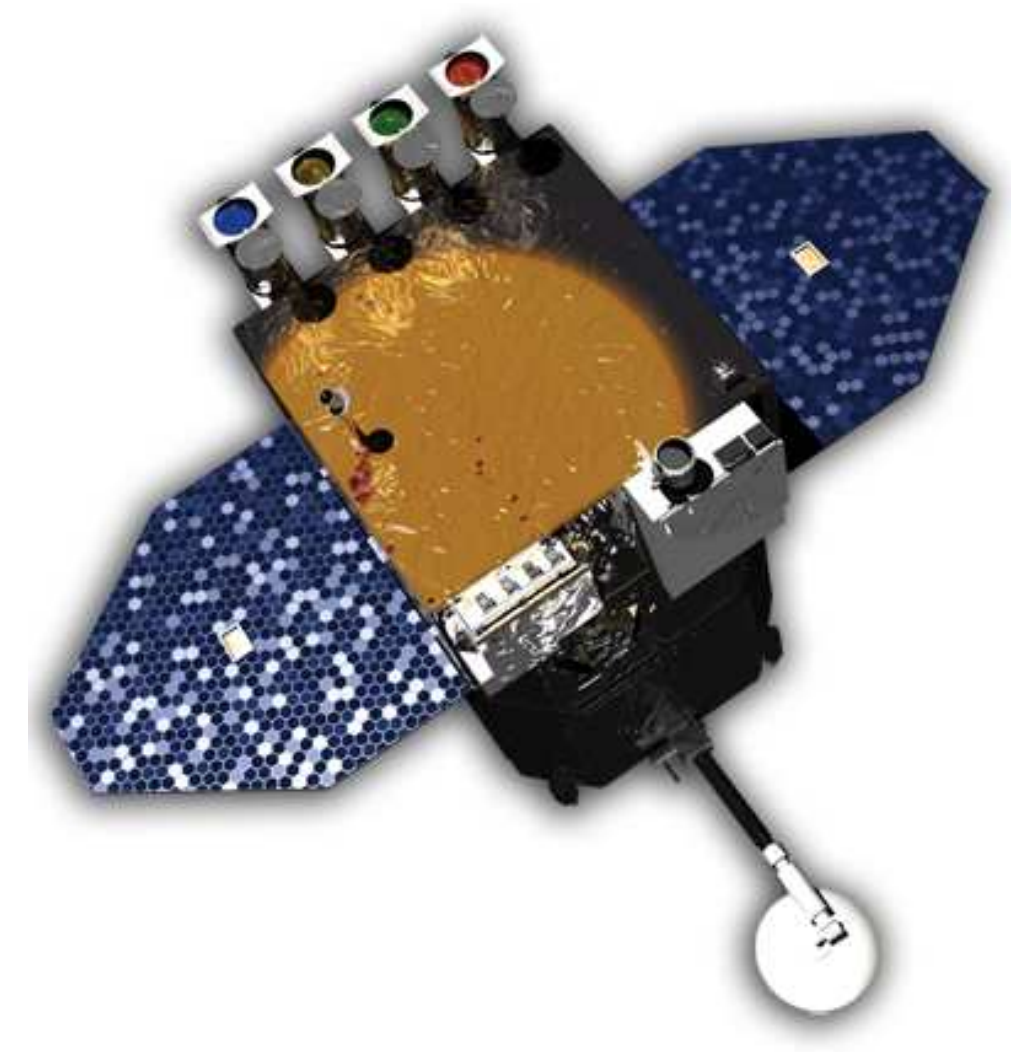

Figure 2. Schematic view of the Solar Dynamics Observatory (SDO), which carries three scientific instruments (AIA, HMI, EVE - see text).

- The Helioseismic and Magnetic Imager (HMI), a high resolution helioseismograph/vectormagnetograph that will provide stabilized 1"-resolution full-disk Doppler velocity and line-of-sight magnetic flux images every 45 seconds, and vector-magnetic field maps every 90 seconds. HMI has significant heritage from the SOHO MDI instrument with enhancements to provide higher resolution (e.g. $4 \mathrm{k} \times 4 \mathrm{k}$ detectors), higher cadence, and the addition of a second channel to provide full Stokes polarization measurements.

- The Atmospheric Imaging Assembly (AIA) will provide coverage of the entire visible solar hemisphere, observed from photospheric to coronal temperatures, at 1 arcsec resolution, with a characteristic cadence of 10 seconds for each channel. AIA comprises four dual normal-incidence telescopes with multi-layer optics that enable it to cycle through a set of seven EUV channels centered on strong emission lines of iron (ranging from Fe IX through Fe XXIII) and Helium II $304 \AA$, plus two UV channels near $1600 \AA$ and a broad-band visible channel. Each telescope contains a 4096×4096 CCD camera system and has a 41 arcmin field of view. 
- The EUV Variability Experiment (EVE) will measure the solar EUV irradiance with unprecedented spectral resolution, temporal cadence, accuracy, and precision. EVE consists of three subsytems: The Multiple EUV Grating Spectrograph (MEGS) measures the 40-1200 $\AA$ spectral irradiance with $1 \AA$ spectral resolution and with 10 second cadence. The Optics Free Spectrometer (OFS), being ionization cells, provides daily in-flight calibrations for the MEGS channels. The EUV Spectrophotometer (ESP) completes the spectral coverage at 1-50 ̊ and 1190-1250 A. Collectively, EVE will measure EUV irradiance from 1 to $1250 \AA$ with $7 \%$ accuracy and $4 \%$ long-term precision.

Target launch date for SDO is August 2008. It will fly in an inclined geosynchrononous orbit, which satisfies the requirements for a high scientific data rate well in excess of $100 \mathrm{Mbps}$ and nearly continuous observations with a single dedicated ground station.

\section{PICARD}

PICARD (Thuillier et al. 2006) is a CNES microsatellite mission to measure the solar diameter, solar shape, solar differential rotation, and the solar constant over three years to investigate the nature of their relations and their variabilities. A key objective is to provide a reliable measurement of the ratio $W=\frac{d \ln (R)}{d \ln (L)}$. The main instrument on PICARD is a $110 \mathrm{~mm}$ telescope that will provide full disk images of the Sun on a $2 \mathrm{k} \times 2 \mathrm{k}$ CCD detector in 4 wavelengths bands: $230 \mathrm{~nm}, 548 \mathrm{~nm}, 160 \mathrm{~nm}$, and Ly $\alpha$. In addition, it carries two instruments to measure total solar irradiance and solar spectral irradiance in 5 channels, including two UV channels corresponding to the spectral domains important for ozone photochemistry. Launch of PICARD is planned for early 2009.

\section{Solar Orbiter}

\subsection{Science goals}

The top-level scientific goals of the Solar Orbiter mission (Fig. 3, Marsch et al. 2001, 2005; Marsden \& Fleck 2003) are to:

- Determine the properties, dynamics and interactions of plasma, fields and particles in the near-Sun heliosphere;

- Investigate the links between the solar surface, corona and inner heliosphere;

- Explore, at all latitudes, the energetics, dynamics and fine-scale structure of the Suns magnetized atmosphere;

- Probe the solar dynamo by observing the Suns high-latitude field, flows and seismic waves.

To achieve these goals, Solar Orbiter will incorporate both a near-Sun and a high-latitude phase. The near-Sun phase of the mission will enable the Orbiter spacecraft to approach the Sun as close as 48 solar radii $(\approx 0.22 \mathrm{AU})$ during part of its orbit, thereby permitting observations from a quasi-helio-synchronous vantage point (so-called co-rotation.). At these distances, the angular speed of a spacecraft near its perihelion approximately matches the 


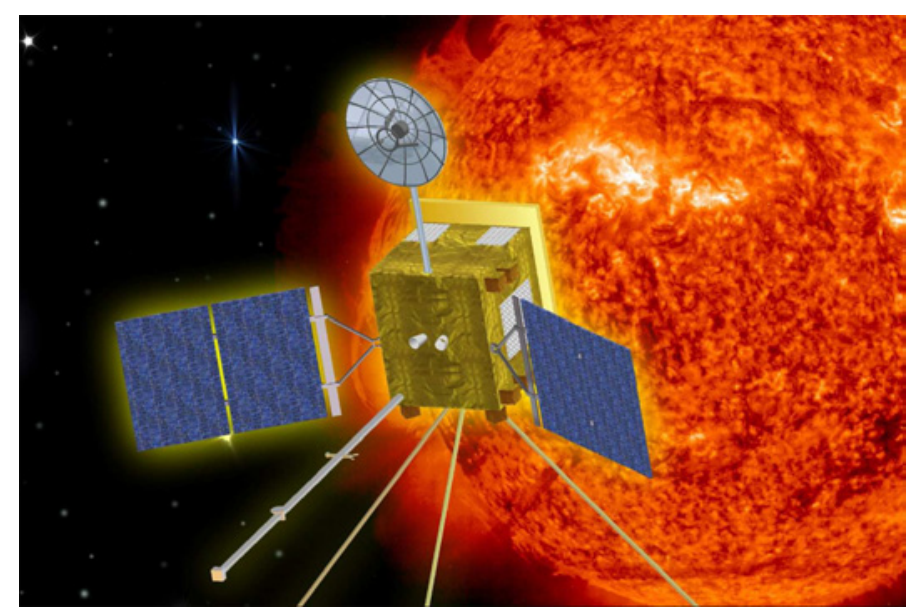

Figure 3. Artist's concept of the Solar Orbiter.

rotation rate of the Sun, enabling instruments to track a given point on the Sun surface for several days. During the out-of-ecliptic phase of the mission, the Orbiter will reach intermediate solar latitudes (up to $35^{\circ}$ in the extended phase), making possible detailed studies of the Suns polar caps by the remote-sensing instruments.

Figure 4 shows the ecliptic projection of the spacecraft trajectory in a coordinate system that is fixed with respect to the Sun-Earth line. It can be seen that the full range of solar longitudes is covered during the mission, including a number of "far-side" perihelion passages. This will provide excellent opportunities for coordinated observations, in particular with near-Earth observing platforms. It is also envisaged to have close coordination between Solar Orbiter and the NASA Living With a Star Sentinels programme whose Inner Heliospheric Sentinel spacecraft will have trajectories that are similar to the near-ecliptic orbits of Solar Orbiter.

\subsection{Reference payload}

A summary of the Solar Orbiter reference payload is provided in Table 1. The reference payload can be grouped into three main categories: a) In-Situ instruments; b) Remote-Sensing instruments, constrained to follow a 1 arcsec, 1 meter philosophy (representing the spatial resolution and maximum allowed envelope for the largest units); c) Payload Support Elements (e.g. boom, doors and windows, etc.). The table refers to the core payload complement, reflecting the science prioritization given by the Science Definition Team. All figures reported in the table include design maturity margins (depending on heritage). Detailed descriptions of the reference instruments are given in the Payload Definition Document (ESAESTEC, SCI-A/2004/175/AO). The scientific requirements for the payload are given in the Science Requirements Document (Marsden \& Marsch 2005).

Currently, discussions are ongoing concerning the possibility of joining Solar Orbiter and NASA's Solar Sentinels to form a single collaborative programme. This would have potential benefits for both missions, not only from a cost point of view, but also scientifically, as there 


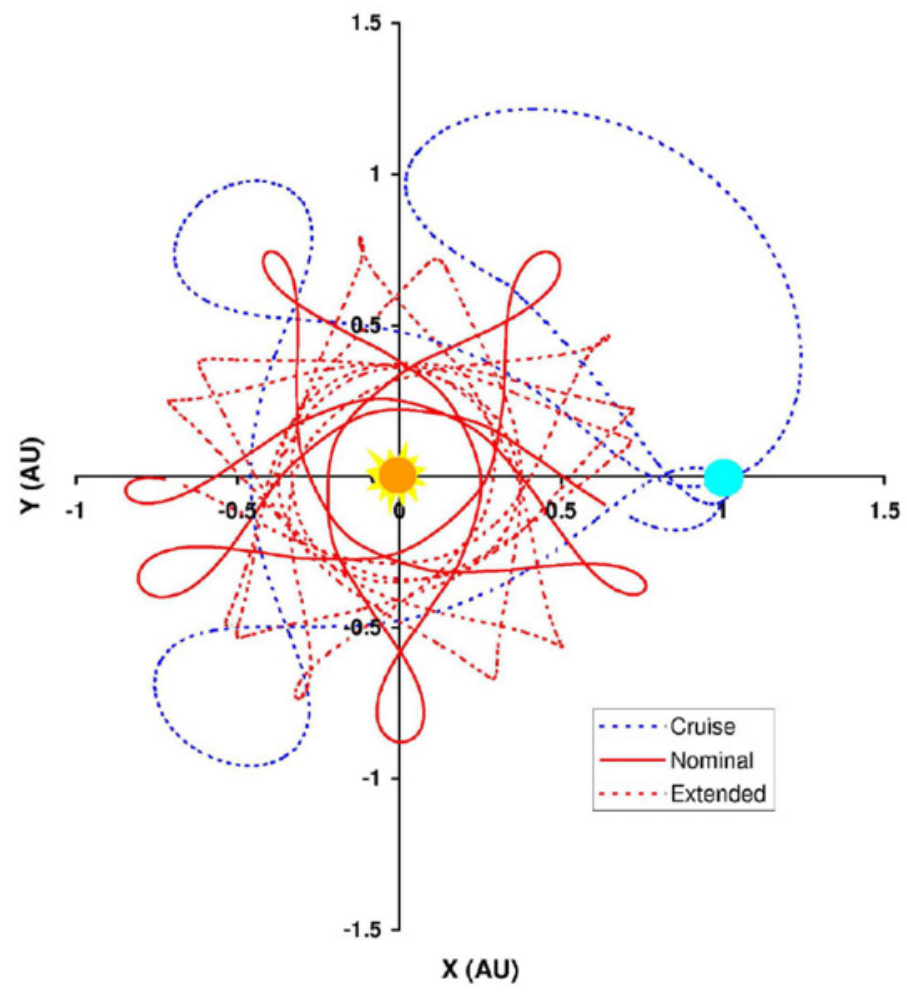

Figure 4. Spacecraft trajectory (cruise, nominal and extended phases) plotted in a coordinate system with the Sun-Earth line fixed (2015 launch opportunity).

\begin{tabular}{lcc}
\hline \hline Instrument & Mass [kg] & Power [W] \\
\hline a) In-Situ instruments & & \\
Solar Wind Plasma Analyzer (SWA) & 16.5 & 15.5 \\
Radio and Plasma Wave Analyzer (RPW) & 13.0 & 7.0 \\
Magnetometer (MAG) & 2.1 & 1.5 \\
Energetic Particle Detector (EPD) & 9.0 & 8.5 \\
Dust Particle Detector (DPD) & 1.8 & 6 \\
Neutron Gamma ray Detector (NGD) & 5.5 & 5.5 \\
Remote-Sensing instruments & & \\
Visible Imager \& Magnetograph (VIM) & 30.4 & 35 \\
EUV Spectrometer (EUS) & 18.0 & 25 \\
EUV Imager (EUI) & 20.4 & 28 \\
Coronagraph (COR) & 18.3 & 30 \\
Spectrometer Telescope Imaging X-rays (STIX) & 4.4 & 4 \\
c) Payload Support Elements (PSE) & $\mathbf{2 8 . 4}$ & $\mathbf{4}$ \\
\hline TOTAL & $\mathbf{1 6 7 . 8}$ & $\mathbf{1 7 0 . 0}$ \\
\hline \hline
\end{tabular}

Table 1. The Solar Orbiter Reference Payload as given in the Payload Definition Document. 
are clear synergies between the two projects. An element of the proposed collaboration is a joint launch provided by NASA. A detailed technical evaluation has yet to be made, however, and until this has been done, no firm decision can be taken.

\section{Solar Sentinels}

NASAs LWS Sentinels mission (Lin et al. 2006) is a multispacecraft mission that will study (1) the acceleration and transport of solar energetic particles (SEPs) and (2) the initiation and evolution of coronal mass ejections (CMEs) and interplanetary shocks in the inner heliosphere. The full Sentinels program as described in the Sentinels Science and Technology Definition Team Report (Lin et al. 2006) comprises (1) a constellation of four identically instrumented Inner Heliospheric Sentinels to make in-situ measurements of the plasma, energetic particle, and fields environment as close to the Sun as $0.25 \mathrm{AU}$ as well as multipoint remote-sensing observations of solar X-ray, radio, gamma-ray, and neutron emissions; (2) a Near-Earth Sentinel in Sun-synchronous orbit for ultraviolet and white-light observations of the corona; and (3) a Farside Sentinel in heliocentric orbit at 1 AU to measure the photospheric magnetic field from positions $60^{\circ}$ to $120^{\circ}$ ahead of the Earth. Launch is envisaged for the first half of the next decade (cf. Section 7.2).

\section{KuaFu}

$\mathrm{KuaFu}$ (Xia et al. 2007) is a Chinese space weather mission currently under study. It would consist of three satellites: KuaFu-A, KuaFu-B1 and B2. KuaFu-A is the solar component and would be operated in the Sun-Earth Lagrangian point L1, while B1 and B2 fly in polar orbits around Earth. The mission is designed to explore solar disturbances and their effects on the near-Earth space. Launch of KuaFu is planned for the first half of the next decade. The model payload of KuaFu-A includes a full-disk EUV and Ly- $\alpha$ imager, a white-light and Ly- $\alpha$ coronagraph, a hard X-ray and $\gamma$-ray spectrometer, a total solar irradiance monitor, and a particles and fields package.

\section{Other solar missions in NASA's $S^{3} \mathrm{C} 2005$ roadmap}

NASA's Sun-Solar-System Connection Science and Technology Roadmap lists several other solar missions:

- Near-term missions (launch planned by 2015)

- Solar Probe - a mission to understand the processes that heat the solar corona and produce the solar wind. It will carry a comprehensive suite of in-situ instruments as well as remote sensing instruments into the corona (perihelion at 4 solar radii).

- Intermediate-term missions (launch planned between 2015 and 2025)

- Doppler - a mission to study the physical mechanisms of mass flow and energy release in the solar atmosphere. The model payload includes UV and EUV spectrometers and images and a filter magnetograph for surface magnetic field measurements. 
- Solar Polar Imager - a mission to measure the Sun's polar magnetic field and polar flows, and to image global effects of CMEs and the evolution of the full $3 \mathrm{D}$ corona. It will require solar sail technology to get into a $60^{\circ}$ inclined orbit at $0.48 \mathrm{AU}$.

- Telemachus - another polar mission, but on a ballistic trajectory with Jupiter gravity assist. The final orbit would be a $0.2 \times 2.5 \mathrm{AU}$ orbit at $90^{\circ}$ inclination with a period of 1.5 years.

- Future missions (launch planned after 2025)

- Reconnection and Microscale (RAM) - a mission for ultra-high (0.02 arcsec/pixel) EUV coronal imaging and high resolution (0.1 $\operatorname{arcsec})$ EUV/UV spectroscopy.

- Magnetic Transition Region Probe (MTRAP) - a "super-Hinode" with a $6 \mathrm{~m}$ optical telescope and high resolution UV/EUV imagers and spectrometers.

\section{Conclusions}

The vast domain from the solar interior to the heliopause is a highly complex physical system, linked to the Sun by its magnetic field and expanding atmosphere, the solar wind. To understand the complex structure and dynamics of the Sun-Heliosphere system requires a multi-spacecraft approach that combines high resolution observations, both remote sensing and in situ, with observations from multiple viewing angles and from out-of-ecliptic vantage points. The missions described above represent an exciting fleet of solar space missions. The multiple technique, multiple vantage point approach will open up new areas of research, expanding considerably from our near-Earth/ecliptic view, and will provide crucial new information for solving some of the long-standing scientific questions about the nature of our daylight star and its influence on Earth.

\section{References}

Hathaway, D. et al. 2001, Solar Dynamics Observatory - Report of the Science Definition Team. http://lws.gsfc.nasa.gov/lws_mission_ sdo.htm

Kaiser, M. L. 2005, Adv. Space Res., 36, 1483

Marsch, E., Martínez Pillet, V., Fleck, B., \& Marsden, R. G. (eds.) 2001, ”Solar Encounter”, Proceedings of the First Solar Orbiter Workshop, ESA SP-493

Marsch, E., Marsden, R. G., Harrison, R. A., Wimmer-Schweingruber, R., \& Fleck, B. 2005, Adv. Space Res., 36, 1360

Marsden, R. G. \& Fleck, B. 2003, Adv. Space Res., 32, 2699

Marsden, R. G. \& Marsch, E. 2005, Solar Orbiter Science Requirements Document, ESA-ESTEC, SCI-SH/2005/100/RGM

Lin, R. P., et al. 2006, Solar Sentinels: Report of the Science and Technology Definition Team, NASA/TM 2006214137

Shimizu, T., \& the Solar-B Team 2002, Adv. Space Res., 29, 2009

Thuillier, G., Dewitte, S., Schmutz, W., \& the PICARD Team 2006, Adv. Space Res., 38, 1792

Xia, L.-D., Tu, C.-Y., Schwenn, R., Donovan, E., Wang, J.-S., \& Zhang, Y.-W. 2007, Adv. Space Res., in press 


\title{
LYRA - a solar UV radiometer using diamond detectors
}

\author{
A. Theissen ${ }^{1, *}$, A. BenMoussa ${ }^{2}$, U. Schühle ${ }^{1}$, J.-F. Hochedez ${ }^{2}$, and W. Schmutz ${ }^{3}$ \\ ${ }^{1}$ Max-Planck-Institut für Sonnensystemforschung, Katlenburg-Lindau, Germany \\ ${ }^{2}$ Royal Observatory of Belgium, Brussels, Belgium \\ ${ }^{3}$ Physikalisch-Meteorologisches Observatorium Davos and World Radiation Center \\ (PMOD/WRC), Switzerland \\ *Email: theissen@mps.mpg.de
}

\begin{abstract}
LYRA, the Lyman- $\alpha$ radiometer, is a high-cadence $(100 \mathrm{~Hz})$ solar VUV radiometer which will measure disk-integrated irradiances in 4 wavelength channels. Special emphasis is given on novel detectors based on diamond which will be tested for the first time in space. Two kinds of detectors are employed: MSM- and PiN-type detectors. Their particular advantage compared to silicon detectors lies in their solar blindness with a UV/visible reduction ratio of at least four orders of magnitude, which simplifies the design of UV instruments.
\end{abstract}

\section{Introduction}

Three areas of research are particularly dependent on monitoring the UV solar irradiance: since the solar UV irradiance is the main source of energy deposited in the Earth's atmosphere and governs its thermal and chemical structure, it provides valuable data for atmospheric studies, e.g. in investigating its ozone balance (Egorova et al. 2004). It is also a main indicator for the radiation and particle environment in space ('Space Weather'), impacting Earth orbiting satellites, ground-based telecommunication, and other technologies. Last but not least, it is a main source of information about physical processes on the Sun itself. Past, present and future missions dedicated to solar irradiance monitoring all show a particular strength in terms of wavelength coverage, spectral resolution, cadence, time coverage or mission lifetime (for an overview, see Table 1 in Hochedez et al. 2006). LYRA will be unique in adding the capability of very high cadence measurements, aiming at a highest possible cadence of $100 \mathrm{~Hz}$, i.e. providing a set of measurements every $10 \mathrm{~ms}$. As LYRA will regularly monitor solar eclipses, it will also scan the vertical absorption profile of the Earth's atmosphere, where high cadence translates into a high spatial resolution.

A main driver of the mission is the first time use of novel diamond photoconducting and semiconducting technologies in space. Together with other instruments, most notably SWAP ('Sun Watcher with APS detectors and image Processing', Berghmans et al. 2006) which will provide EUV images, LYRA is part of the Proba2 (project for on-board autonomy) spacecraft of the European Space Agency (ESA), a mission aimed at demonstrating novel space technologies and due for launch in late 2007.

This proceedings paper gives a short description of the mission and diamond detectors. 


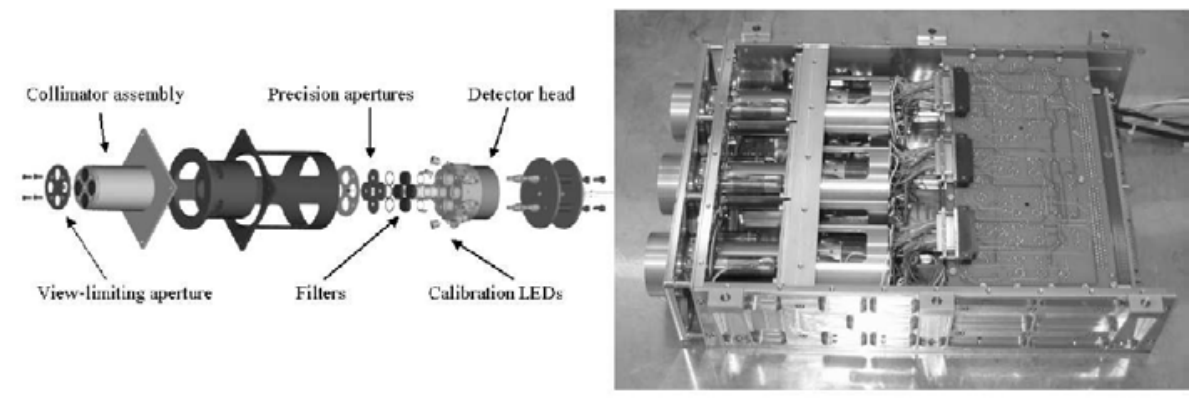

Figure 1. Sketch of a LYRA unit with 4 wavelength channels (left) and photograph of the LYRA instrument and its 3 radiometer units (right).

More detailed information and a complete list of publications can be found on the web-site at http://lyra.oma.be.

\section{Instrument description}

LYRA will measure the disk-integrated solar irradiance in four wavelength bands: $1-20 \mathrm{~nm}$ (Zirconium filter channel), 17-70 nm (Aluminium filter channel), 115-125 nm (Ly- $\alpha$ line), and 200-220 nm (Herzberg continuum). The spectral filter transmittances are plotted in Fig. 2. LYRA consists of three almost identical units (they only have minor differences in detectors, see Sect. 3) of which any two can operate simultaneously. This allows an intercomparison of the performance of each unit for calibration purposes, and monitoring of eventual degradation with time. UV LEDs in each unit provide additional calibration. Figure 1 shows a sketch of one unit, consisting of collimator, 3- $\mathrm{mm}$ precision apertures, filters, detectors and calibration UV LEDs, together with a photograph of the assembled instrument with all three units. Detectors are designed and fabricated in Belgium by IMOMEC together with the National Institute for Materials Science (NIMS) in Tsukuba, Japan. The optical, electronical and mechanical design is provided by the Physikalisch-Meteorologisches Observatorium Davos (PMOD) in Switzerland. The unobstructed field of view is $2.06 \mathrm{deg}$ on a circular detector sensitive area with $4.2 \mathrm{~mm}$ diameter.

\section{Diamond detectors}

Key elements of the LYRA design are novel solar-blind detectors based on diamond. Solar blindness stems from the fact that diamond is a wide bandgap semiconductor with a gap energy of $\approx 5.5 \mathrm{eV}$ at room temperature. This translates into a cutoff-wavelength in sensitivity of $\approx 225 \mathrm{~nm}$. The detector response has a UV/visible ratio around that cutoff-wavelength of more than 4 orders of magnitude, resulting in a high radiation hardness - see Fig. 2 for typical spectral detector response curves. This is an advantage for the instrument design, as filters for the sole use of blocking the unwanted visible radiation are no longer necessary. Furthermore, diamond detectors do not need to be cooled to reduce noise. Next to a few silicon detectors for comparison purposes, LYRA is equipped with two kinds of diamond 


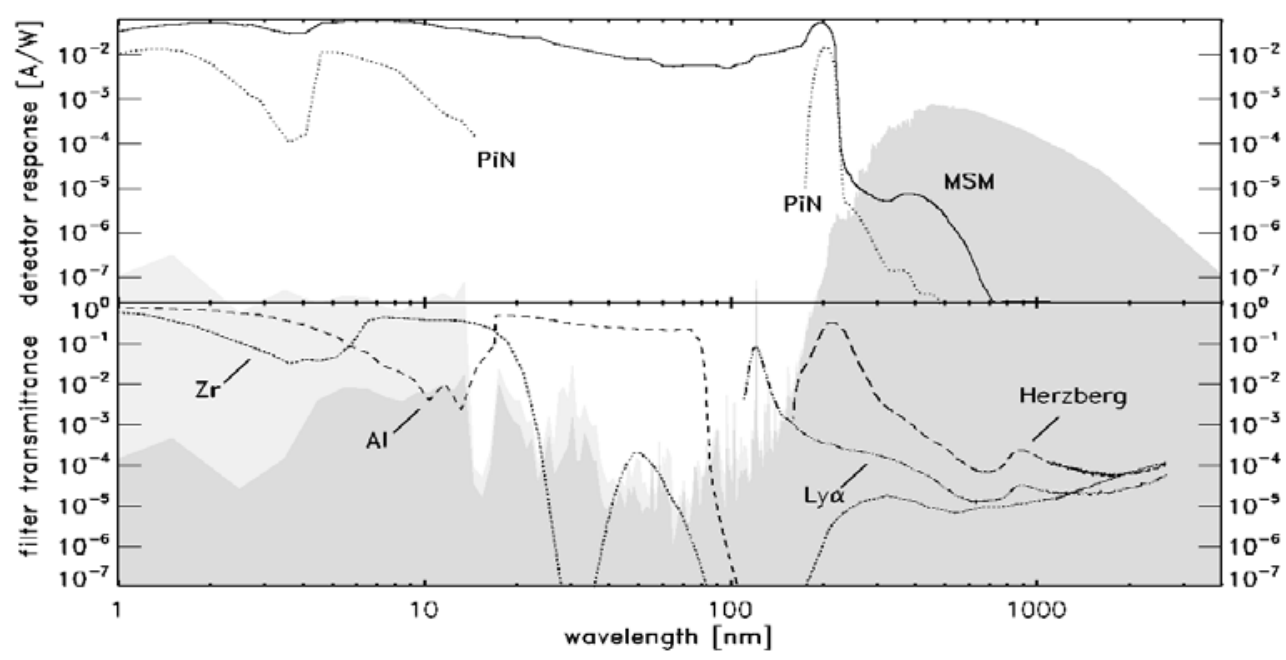

Figure 2. Spectral response of a typical MSM and PiN diamond detector (upper plot) and spectral characteristics of the filters used in each LYRA unit (lower plot). Shaded areas: UV spectra for solar minimum and solar maximum for comparison (no scale, source: TIMED-SEE experiment).
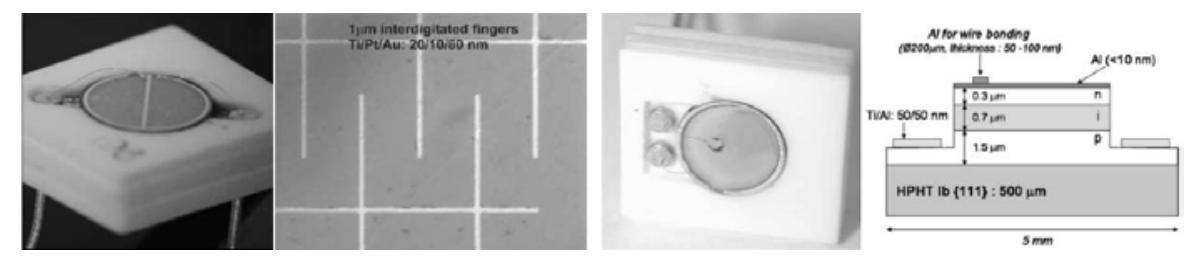

Figure 3. From left to right: photograph of an MSM detector; closeup showing the Ti/Pt/Au contacts which are on a $5 \mathrm{~V}$ potential difference causing a photocurrent due to electron-hole pairs created by incident UV light; photograph of a PiN detector; schematic representation showing the $\mathrm{p}, \mathrm{i}$ and $\mathrm{n}$ layers.

detectors: photoconductor (metal-semiconductor-metal or MSM) and photodiode (PiN) detectors. Figure 3 shows photographs and sketches for both types of detectors.

The signal stability, linearity and spectral responsivity of both kinds of detectors have extensively been studied by BenMoussa et al. (2006a,b, and references therein). All detectors show a good signal stability over extended periods of time (several $1000 \mathrm{~s}$ in the experiments undertaken) and an instantaneous response to changes in photon flux with the exception of a few individual MSM detectors where the detector signal gradually builds up after switching on a UV source (Fig. 4). This is probably due to chemical impurities or structural defects and is under further investigation. Detectors for LYRA are thus selected individually in order to guarantee the best possible response and stability. Both detector types deviate slightly from linearity (Fig. 5) which is physically explained in BenMoussa et al. (2006a,b). These papers also provide measurements of the spectral responses (typical responses are given in Fig. 2) 

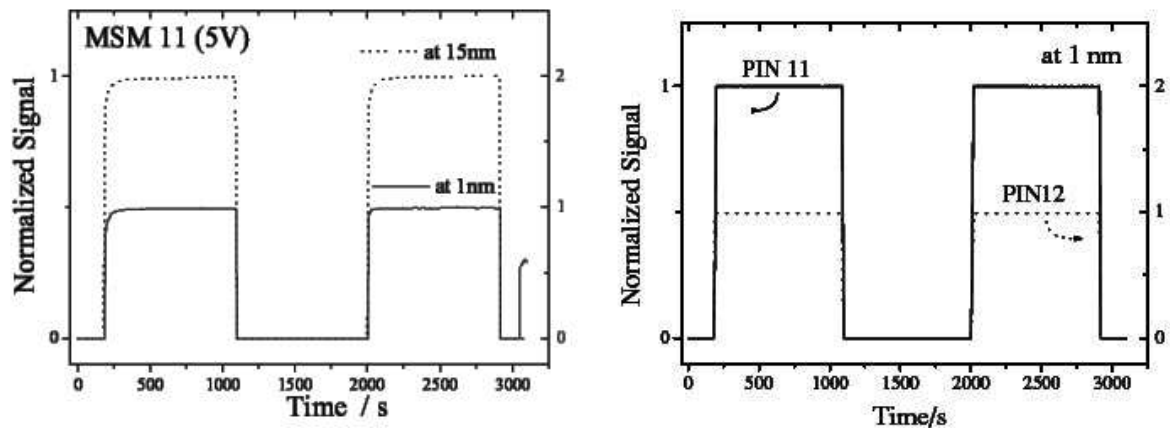

Figure 4. Normalized signal vs. time for some individual MSM and PiN detectors. The input beam was switched on and off approx. every $1000 \mathrm{~s}$. The plot demonstrates the necessity of individual testing: while both PiN detectors shown here (\#11 and \#12) operate perfectly, the particular MSM detector in this example (\#11) exhibits an inert response to changes in photon flux.
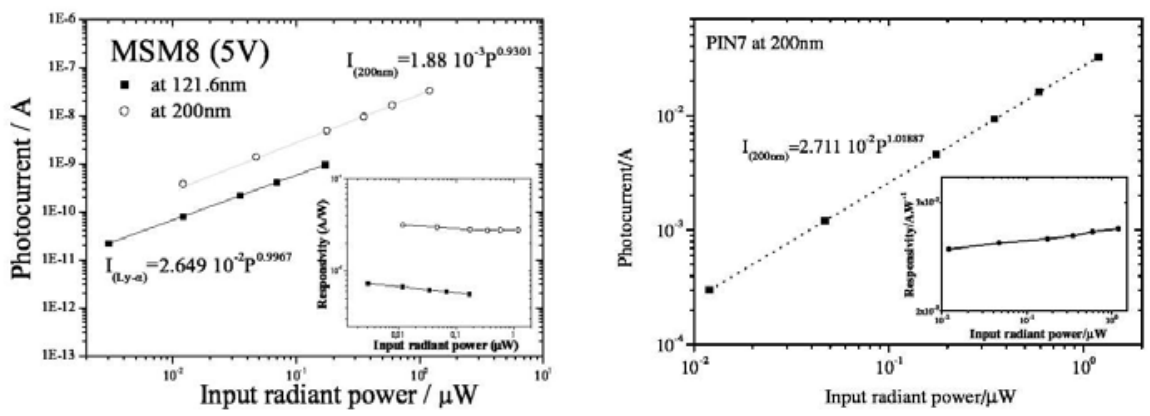

Figure 5. Measured photocurrent vs. incident radiant power at $121.6 \mathrm{~nm}$ (MSM, left) and at 200nm (both MSM, left, and PiN, right). MSM behaves slightly sublinear, and PiN behaves slightly superlinear. Insets: responsivities, slightly dependent on incident power (sub- and superlinearity).

along with fits to theoretical models, and detector flatfields.

\section{References}

BenMoussa, A., Theissen, A., Scholze, F., et al. 2006a, Nuclear Instruments and Methods in Phys. Res. A, 568, 398

BenMoussa, A., Schühle, U., Scholze, F., et al. 2006b, Meas. Sci. Technol., 17, 913

Berghmans, D., Hochedez, J.-F., Defise, J. M., et al. 2006, Advances in Space Res., 38, 1807

Egorova, T., Rozanov, E., Manzini, E., et al. 2004, Geophys. Res. Lett., 31, 6119

Hochedez, J.-F., Schmutz, W., Nesladek, M., et al. 2006, Advances in Space Res., 37, 303 
Modern Solar Facilities - Advanced Solar Science, 31-38

F. Kneer, K. G. Puschmann, A. D. Wittmann (eds.)

(C) Universitätsverlag Göttingen 2007

\title{
Ground-based solar facilities in the U.S.A.
}

\author{
C. Denker ${ }^{1,2, *}$, D. E. Gary ${ }^{1}$, and T. R. Rimmele ${ }^{3}$ \\ ${ }^{1}$ New Jersey Institute of Technology, Newark, NJ, U.S.A. \\ ${ }^{2}$ Astrophysikalisches Institut Potsdam, Potsdam, Germany \\ ${ }^{3}$ National Solar Observatory/Sacramento Peak, Sunspot, NM, U.S.A. \\ *Email: cdenker@adm.njit.edu
}

\begin{abstract}
In this review, we present the status of new ground-based facilities for optical and radio observations of the Sun in the United States. The 4-meter aperture Advanced Technology Solar Telescope (ATST) under the stewardship of the National Solar Observatory (NSO) has successfully completed its design phase and awaits funding approval. The 1.6-meter aperture New Solar Telescope (NST) at Big Bear Solar Observatory (BBSO) is currently under construction. Complementing these optical telescopes is the Frequency Agile Solar Radiotelescope (FASR) - an instrument for dynamic broadband imaging spectroscopy covering a multitude of radio frequencies from $50 \mathrm{MHz}$ to $20 \mathrm{GHz}$. Imaging spectroscopy and polarimetry are common features of these telescopes, which will provide new insight regarding the evolution and nature of solar magnetic fields. High-resolution observations of solar activity, bridging the solar atmosphere from the photosphere to the corona, will be obtained with a dedicated suite of instruments. Special emphasis of this review will be put on the interplay between instrumentation and scientific discovery.
\end{abstract}

\section{Introduction}

Astronomy, astrophysics and solar physics in the United States are periodically evaluated by panels of the National Research Council (Parker 1998; McKee \& Taylor 2001; Lanzerotti 2003). In the latest review, the Panel on the Sun and Heliospheric Physics as part of the Solar and Space Physics Decadal Survey (Lanzerotti 2003) identified and prioritized four science questions for new research initiatives in solar and heliospheric physics: (1) What physical processes are responsible for coronal heating and solar wind acceleration, and what controls the development and evolution of the solar wind in the innermost heliosphere. (2) What determines the magnetic structure of the Sun and its evolution in time, and what physical processes determine how and where magnetic flux emerges from beneath the photosphere? (3) What is the physics of explosive energy release in the solar atmosphere, and how do the resulting heliospheric disturbances evolve in space and time? (4) What is the physical nature of the outer heliosphere, and how does the heliosphere interact with the galaxy? The unique combination and research thrusts of the three U.S. ground-based initiatives FASR (Gary 2003; Bastian 2003), NST (Denker et al. 2006), and ATST (Rimmele et al. 2003; Keil et al. 2004a; Oschmann et al. 2004; Rimmele et al. 2005; Wagner et al. 2006) in collaboration with many other national and international programs will undoubtedly advance solar and heliospheric physics across this broad theme in the years to come. These major initiatives are also well-aligned with current and future space missions. 
Before discussing these major projects in detail, some of the other U.S. ground-based projects should briefly be mentioned. The Global Oscillation Network Group (GONG, Harvey et al. 1996) is a world-wide network of six observing stations to study the internal structure and dynamics of the Sun using helioseismology. In particular, the Sun's "five minute" oscillations are determined from line shifts due to the Doppler effect, which are measured in the Ni I line at $676.8 \mathrm{~nm}$ with a polarizing Michelson interferometer. Synoptic Optical Longterm Investigations of the Sun (SOLIS, Keller et al. 2003) is a synoptic facility to understand the solar activity cycle and monitor solar irradiance changes. The SOLIS instrument suite consists of a Vector Spectromagnetograph (VSM), a Full Disk Patrol (FDP) instrument and an Integrated Sunlight Spectrometer (ISS). Another instrument for synoptic studies of the Sun is the Optical Solar Patrol Network (OSPAN), formerly known as the Improved Solar Observing Optical Network (ISOON, Neidig et al. 1998). OSPAN data products include $\mathrm{H} \alpha$, He I $1083.0 \mathrm{~nm}$ and red continuum full-disk images as well as line-of-sight magnetograms in the Ca I line at $612.2 \mathrm{~nm}$. Future instruments include the Coronal Solar Magnetism Observatory (COSMO), a meter-class coronagraph, which has been proposed by the High Altitude Observatory. COSMO would replace the existing Mauna Loa Solar Observatory operated by the National Center for Atmospheric Research.

\section{Frequency Agile Solar Radiotelescope}

FASR (Figure 1) is an instrument dedicated to solar observations with a field-of-view encompassing the entire solar disk. Broadband imaging spectro-polarimetry will be used to study the solar atmosphere (White et al. 2003) from the middle chromosphere to the corona (about one solar radius above the surface). The FASR array is a three-armed logarithmic spiral with self-similar scaling containing a total of 200 individual antennas. The array consists of 100 antennas with dish sizes of $2 \mathrm{~m}, 60$ antennas with dish sizes of $6 \mathrm{~m}$ and $40 \mathrm{log}$-periodic dipoles.

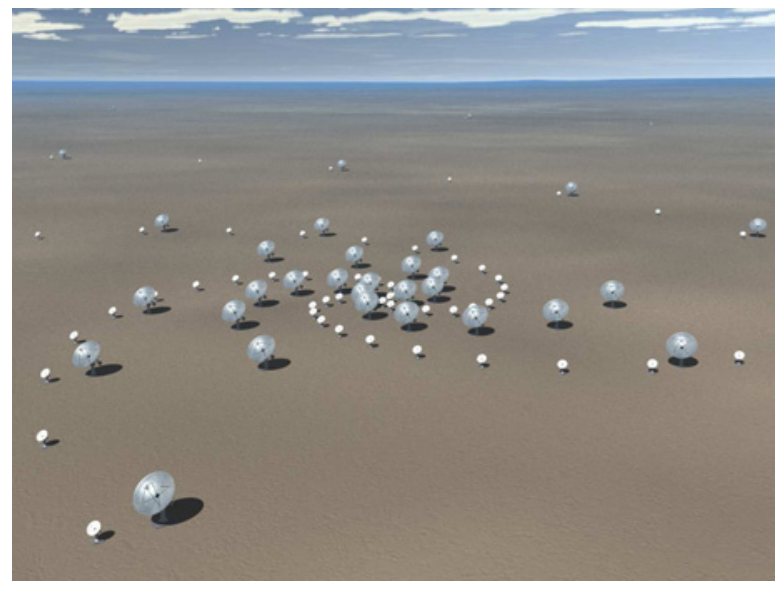

Figure 1. Frequency Agile Solar Radiotelescope.

The longest baseline is about $5 \mathrm{~km}$. Thus, a roughly circular area with same diameter is required for the future FASR site. Other site characteristics include low levels of radio frequency interference (RFI) over the entire band from $50 \mathrm{MHz}$ to $20 \mathrm{GHz}$ and a benign environment to minimize corrosion and ease maintenance (Gary \& Keller 2003).

The array will observe low frequency radio emission from $50 \mathrm{MHz}$ to $20 \mathrm{GHz}$, which originates predominantly in the solar corona. Bremsstrahlung or free-free radiation, gyrosynchroton radiation and plasma radiation are the three most important emission mechanisms in this frequency range. The lower frequency boundary is close to the electron plasma 
frequency of the ionosphere above an observer. Thus, it defines the observing window for ground-based radio observations.

The Sun's radio signal is highly variable. The brightness can change by orders of magnitude on extremely short time-scales. Therefore, FASR has to combine a large dynamic range with high temporal resolution. The time resolution is about $10 \mathrm{~ms}$ from $100 \mathrm{MHz}$ to $3 \mathrm{GHz}$ and about $100 \mathrm{~ms}$ for frequencies larger than $3 \mathrm{GHz}$. The spectral resolution $\Delta \lambda / \lambda$ ranges from $0.1 \%$ in the low frequency part to $1 \%$ in the high frequency part of the spectrum.

The instrument has the ability to measure the full Stokes vector. However, the Stokes $I$ and $V$ components are more important, since strong differential Faraday rotation in the corona will obscure any intrinsic linear polarization measured in Stokes $Q$ and $U$. True coronal magnetograms are the end product of imaging spectro-polarimetry with FASR. Another access to coronal magnetic fields is given by optical observations in the near-infrared, which is one of the ATST's main objectives.

One of FASR's science goals is to obtain a more detailed understanding of the elusive nature of solar radio bursts (Bastian 2004). The character of the bursts strongly depends on frequency. The duration of radio bursts is relatively short lasting only about 5 min to $15 \mathrm{~min}$. Bursts below about $3 \mathrm{GHz}$ are characterized by a frequency shift of the emission with time, usually from high to low frequencies. For example, the frequency drift is much faster for Type III than for Type II bursts. The cause of Type III radio bursts are beams of non-thermal electrons with energies in the range from a few to $100 \mathrm{keV}$. The propagation speed of electrons in the beams can reach a few tenths of the speed of light. In contrast to these emissions, the dominant emission mechanisms for Type IV radio bursts are plasma and gyrosynchroton radiation. The various forms of broad-band emissions are typically associated with flares. Type II and IV radio bursts and CMEs are closely associated. Fast CME-driven or flareassociated ejecta and blast waves such as Moreton or EIT waves produce shocks, which in turn are responsible for Type II radio bursts.

The physical conditions near the energy release site can be accessed by radio observations (see e.g., Gary \& Keller 2004, for a detailed introduction to solar radiophysics), since they can accurately measure the plasma and gyrosynchroton radiation produced by non-thermal electrons. The interaction of electron beams with a fixed electron number density at a given location can be studied with imaging spectroscopy. Images sampled at different frequencies contain the information on the electron beam trajectory. In the case of decimetric Type III radio bursts, it allows to discern between upward and downward beam trajectories. Thus, energy release sites can be connected to beam trajectories and to coronal magnetic field topology, which yields a comprehensive picture of eruptive phenomena in the chromosphere and corona.

Advances in broadband devices, digital circuitry and computing power have made FASR feasible. These technological innovations have played the same critical role in solar radiophysics as advances in adaptive optics (AO) for optical telescopes. Several key FASR components are currently being developed, which include some prototyping efforts to mitigate risks. These studies are funded as PI-type grants to individual members of the FASR project. The overall design and development (D\&D) is funded by the Advanced Instrumentation and Technology program of the Astronomical Sciences Division (AST) within the National Science Foundation (NSF). Several strategies are investigated on how to implement FASR, once the D\&D studies are successfully completed. One option is to build a scaled-down prototype with about $10 \%$ of the antennas, which could be funded within NSF AST. 


\section{New Solar Telescope at Big Bear Solar Observatory}

The proximity of the Sun allows us to study surface structure in fine detail. Even if observed with the largest telescopes, almost all of the other stars remain point sources. The recent quest for solar telescopes with apertures larger than one meter can be traced to two technological breakthroughs: solar AO (Rimmele 2000) and an open telescope design and its first successful implementation, the Dutch Open Telescope (DOT, Rutten et al. 2004). These advances in technology help to overcome the limitations posed

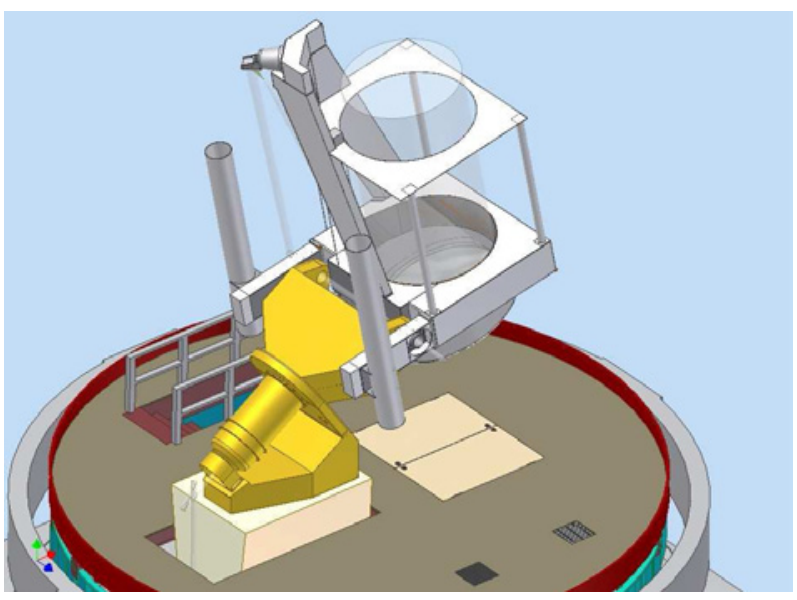

Figure 2. New Solar Telescope at Big Bear Solar Observatory. by Earth's turbulent atmosphere. Observations close to the diffraction limit of this new generation of telescopes are now feasible and will provide the first glimpse at the intrinsic scales of magneto-convection, which are only $100 \mathrm{~km}$ or less on the solar surface.

NST (Figure 2) is a 1.6-meter, off-axis Gregory-type telescope replacing a number of older solar telescopes at BBSO. The project is a collaboration between BBSO, the Korean Astronomical Observatory (KAO) and Institute for Astronomy at the University of Hawai' $i$. All major contracts for design and fabrication of NST are in place and construction of components is well underway. BBSO is ideally suited for campaign-style solar observations. NST will provide unprecedented time series with high spatial and high temporal resolution. Both are required to follow the dynamics of active regions and eruptive phenomena on the Sun. Synoptic observations and space weather-related studies have historically been one of the research focusses at BBSO (Gallagher et al. 2002).

The old metal dome at BBSO was replaced in March 2006 with a larger one of $10 \mathrm{~m}$ diameter, 5/8 fiberglass sphere manufactured by MFG Ratech. The design is based on the dome for the Southern Astrophysical Research (SOAR) telescope (Teran et al. 2000). Fourteen vent gates with automated, proportional positioned dampers are spaced around the equator of the dome to control the air flow across the open structure telescope. DFM Engineering supplies the equatorial mount, telescope tube, primary mirror (M1) cell, positioning actuators and M1 supports, the optical support structure, pointing control system, mounts and mirrors for M3 through M5, and M1 handling equipment. An unbalanced system was developed to meet the overall mass requirement imposed by the existing pier. Unbalanced moments are taken up by the declination (DEC) drive system. The center-of-gravity about the right ascension (RA) axis will vary with RA position. This will be compensated by a pair of moving 1/2 ton counter-weights located in tubes near the DEC axis (see Figure 2).

M1 has a $1.6 \mathrm{~m}$ clear aperture $(1.7 \mathrm{~m}$ Zerodur blank with $10 \mathrm{~cm}$ thickness). The telescope optics are based on a Gregory-type design with two additional flat mirrors to direct the light into the declination and coudé axes, respectively. The M1 $f$-ratio is $f / 2.4$ corresponding 
to a focal length of $4.1 \mathrm{~m}$. The final $f$-ratio in the Gregory focus is $f / 52$ and the effective focal length of the telescope is $83.2 \mathrm{~m}$. The Steward Observatory at the University of Arizona is polishing M1 and is developing computer-generated hologram techniques required to produce 8.4-meter aperture, off-axis mirrors. M1 is a 1/5 scale test project for the Giant Magellan Telescope (Martin et al. 2004).

In addition to a large-aperture telescope, dedicated post-focus instruments have to be developed to exploit the capabilities of the new telescope. The NST post-focus instruments were tailored towards space weather and solar activity studies, i.e., preference was given to obtain high spatial and high temporal resolution data. Two imaging vector magnetographs for the visible and near-infrared wavelengths regions are currently being developed (Denker et al. 2003a,b) and have produced first science results. A scanning grating spectrograph with higher spectral resolution will be built by KAO. These instruments benefit from a high-order AO system, which was developed in a collaboration between NSO and BBSO (Rimmele 2000; Didkovsky et al. 2003; Ren et al. 2003; Rimmele et al. 2004a). According to the ATST site survey (Hill et al. 2004, 2006), the median Fried parameter $r_{0}$ at BBSO is about $6.0 \mathrm{~cm}$, with about 1000 hours per year when the Fried parameter $r_{0}$ exceeds $7.0 \mathrm{~cm}$. This indicates that AO-corrected data will be available for substantial periods of time. The special location of BBSO inside Big Bear Lake results in an almost flat profile of the Fried parameter $r_{0}$ with time. Thus, AO-corrected observations can be obtained from sunrise to sunset. As far as imaging is concerned, even higher correction can be achieved by combining AO and post-facto image correction (Denker et al. 2005). Considering that image restoration is no longer limited by computing resources (Denker et al. 2001), high-resolution observations can be routinely obtained, which is a necessity for space weather monitoring and forecast.

\section{Advanced Technology Solar Telescope}

The ATST (Figure 3) is a 4-meter aperture, all-reflecting, off-axis Gregory-type telescope with integrated AO. In 13 reflections, the light is directed to a $16.4 \mathrm{~m}$ diameter, co-rotating coudé optical laboratory. The light path includes an upgrade option for multi-conjugate AO (Berkefeld et al. 2006; Rimmele et al. 2006). The telescope delivers a $300^{\prime \prime}$ FOV to the Nasmyth observing station, which is unvignetted over $240^{\prime \prime}$. The FOV at the coudé observing station will be reduced to $120^{\prime \prime} \times 120^{\prime \prime}$ to ease the optical design of the post-focus instrumentation and to achieve a Strehl ratio of 0.8 across the entire field. The polarization and calibration optics are integral parts of the ATST located at the Gregory observing station and serve all post-focus instruments. The coudé optical laboratory provides ample space for multi-instrument set-ups and flexibility to integrate user instruments. The ATST project includes four facility-class, first light instruments (Rimmele et al. 2004b).

The Visible-Light Broadband Imager (VBI, Uitenbroek et al. 2006) combines high-order AO with image restoration. Two channels, covering wavelength regions in the blue from $350 \mathrm{~nm}$ to $400 \mathrm{~nm}$ and in the red from $630 \mathrm{~nm}$ to $900 \mathrm{~nm}$, are outfitted with fast, large-format CCD detectors for phase diversity and speckle imaging. The Visible Spectro-Polarimeter (ViSP, Elmore et al. 2005) is a medium dispersion spectrograph based on the horizontal spectrograph currently in operation at NSO's Dunn Solar Telescope. ViSP will carry out precision measurements of full state of polarization simultaneously at diverse wavelengths from $380 \mathrm{~nm}$ to $900 \mathrm{~nm}$. Fully resolved line profiles will provide quantitative diagnostics of 
the magnetic field vector as a function of height in the solar atmosphere. The Visible Tunable Filter (VTF, Gary et al. 2003) is an imaging spectro-polarimeter (triple etalon Fabry-Perot) covering the visible wavelength range from $450 \mathrm{~nm}$ to $750 \mathrm{~nm}$. Its design was guided by the successful Telecentric Etalon Solar Spectrometer (TESOS, Kentischer et al. 1998; Tritschler et al. 2002) of the Kiepenheuer-Institut für Sonnenphysik in Freiburg, Germany. VTF will deliver Stokes spectro-polarimetry, accurate surface photometry, line-of-sight velocity measurements and transverse flows from feature tracking. High-cadence and the possibility of post-facto image restoration make VTF an ideal instrument to study the dynamics of eruptive events and evolutionary changes of solar activity. The first light coronal instrument will be mounted at Nasmyth station and provide measurements of the coronal magnetic field. The operational mode of ATST will be quite different from current solar facilities. Instead of observer-defined instruments, the aforementioned suite of fixed facility instruments will provide standard data products and enable batch observing (Rimmele et al. 2004b).

The primary ATST mission is to perform high-resolution studies of the Sun's magnetic fields from the photosphere to the corona (Rimmele et al. 2003; Keil et al. 2004b). The term "high-resolution" in this context refers to the spatial, temporal and spectral (including polarization) domains in their entirety. The intrinsic spatial scales of solar magnetic fields can be as small as just a few tens of kilometers. Starting with the dynamics of small-scale flux tubes and their amplifications by

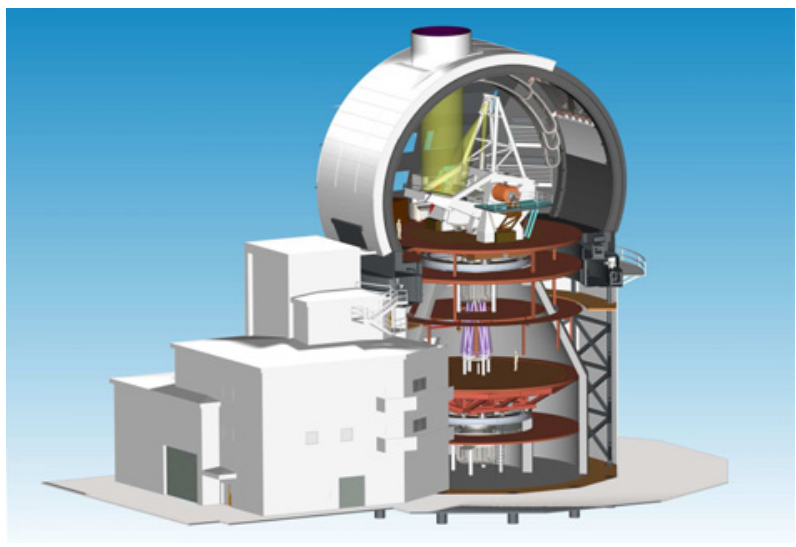

Figure 3. Advanced Technology Solar Telescope (ATST). plasma flows, sunspots and active regions are the next steps in merging and concentrating magnetic flux. The dynamics of sunspots occur on a time-scale of just a few minutes. Magnetic reconnection above active regions can lead to violent eruptions in form of flares, filament/promincence eruption and coronal mass ejections (CMEs). Magnetic reconnection on smaller scales and/or wave phenomena are linked to coronal heating. On longer time-scales the 11-year sunspot cycle is only one of the many signatures of the 22-year magnetic cycle of the Sun. In addition, to the more traditional spectro-polarimetric methods in the visible wavelength regime, technological advances have provided access to the near- and thermalinfrared. Site selection and instrument design with a special emphasis on scattered light performance give ATST infrared capabilities to directly measure coronal magnetic fields.

The ATST site survey has been a community effort to identify a location, which combines the best seeing and environmental conditions to fulfill the science goals of the ATST (Hill et al. 2004, 2006). This site survey has been the most extensive study of daytime seeing conditions so far. Initially, 72 candidate sites were selected and ranked according to feasibility, environmental conditions, and climate. Six sites were initially tested with an identical set of instruments (Beckers et al. 2003; Lin \& Penn 2004; Socas-Navarro et al. 2005). These sites represented a large sample of geographical features: mountain sites, mountain-lake sites, and 
mountain-island sites. After an interim comparison of the seeing conditions, the selection was narrowed to three sites (Big Bear Solar Observatory in Southern California, Observatorio Roque de los Muchachos on La Palma, Spain, and Mees Solar Observatory (MSO) on Haleakalā, Maui, Hawai'i). All finalist sites had about the same number of annual hours of seeing conditions with $r_{0}>7 \mathrm{~cm}$ (about 1000 hours), which confirmed their status as premier sites for solar observations. However, Haleakalā had the most annual hours (about 400 hours) of excellent seeing ( $r_{0}>12.0 \mathrm{~cm}$ at a height of $28 \mathrm{~m}$ above ground). Haleakalā had also the most annual hours of low sky brightness required for coronal observations. Thus, MSO was chosen as the future ATST site.

The NSF-funded ATST D\&D efforts started in late 2001. In September 2005, ATST reached the readiness stage within NSF's Major Research Equipment and Facilities Construction program. Once the site decision was made, geotechnical studies were carried out at Haleakalā in early 2005. In parallel, an Environmental Impact Study (EIS) was initiated in 2005, which included several public hearings. The EIS process will be concluded by the end of 2006. As a result, the exterior shape and overall footprint of the ATST facility has advanced to the point, where it is now largely frozen. Since 2004, several NSF cost and design reviews were held and the preliminary design review was passed successfully in October 2006. The ATST is expected to be considered for a new start in spring of 2007 by the National Science Board, which is the U.S. science policy adviser to the President and the Congress. A positive recommendation could lead to an inclusion in the President's budget for fiscal year 2009. This would also signal the beginning of the ATST construction. In this scenario, ATST would see first light in 2012 and telescope and first-light instrumentation would be fully commissioned by 2014. Current efforts focus on preparation for contracting of major ATST sub-systems to keep the project on target.

\section{Conclusions}

ATST, NST and FASR will obtain comprehensive observations of the Sun from its surface to the outer atmosphere. A systems approach is required, which integrates data of the photosphere, chromosphere and corona, to study the nature and evolution of magnetic fields, which are the cause of many energetic phenomena such as flares, prominence/filament eruptions and CMEs. Acceleration of energetic particles, propagation of energetic and eruptive phenomena into the interplanetary medium and formation of coronal and interplanetary shocks are all topics, which rely on a detailed understanding of the near-Sun evolution of magnetic fields, thus, establishing a close connection between ATST, NST and FASR and space-based instruments studying the heliosphere and interplanetary medium. The synergies among these ground-based U.S. solar facilities will break new ground in solar physics and space science and bring us closer to answer some of the fundamental questions (Keil et al. 2004b): (1) What is the nature of solar magnetism? (2) How does magnetism control our star? (3) How can we model and predict the Sun's changing outputs that affect the Earth?

Acknowledgements. This work was supported by NSF under grants ATM 00-86999, ATM 0236945, IIS ITR 03-24816, AST 03-52915 and AST MRI 00-79482 and by NASA under grant NAG 5-12782. The National Solar Observatory is operated by the Association of Universities for Research in Astronomy under a cooperative agreement with the National Science Foundation, for the benefit of the astronomical community. 


\section{References}

Bastian, T. S. 2004, P\&SS, 52, 1381

Bastian, T. S. 2003, Proc. SPIE, 4853, 98

Beckers, J. M., Liu, Z., \& Jin, Z. 2003, Proc. SPIE, 4853, 273

Berkefeld, T., Soltau, D., \& von der Lühe, O. 2006, Proc. SPIE, 6272,4

Denker, C., Yang, G., \& Wang, H. 2001, Solar Phys., 202, 63

Denker, C., Didkovsky, L., Ma, J. \& 5 co-authors 2003a, Astron. Nachr./Astron. Not., 324, 332

Denker, C., Ma, J., Wang, H. \& 4 co-authors 2003b, Proc. SPIE, 4853, 223

Denker, C., Mascarinas, D., Xu, Y. \& 5 co-authors 2005, Solar Phys., 227, 217

Denker, C., Goode, P. R., Ren, D. \& 17 co-authors 2006, Proc. SPIE, 6267, 62670A

Didkovsky, L. V., Dolgushyn, A., Marquette, W. H. \& 10 co-authors 2003, Proc. SPIE, 4853, 630

Elmore, D. F., Socas-Navarro, H., Card, G. L., \& Streander, K. V. 2005, Proc. SPIE, 5901, 60

Gallagher, P. T., Denker, C., Yurchyshyn, V. \& 4 co-authors 2002, Ann. Geophys., 20, 1105

Gary, D. E. 2003, J. Korean Astron. Soc., 36, 135

Gary, D. E. \& Keller, C. U. 2003, Proc. SPIE, 4853, 523

Gary, D. E. \& Keller, C. U. 2004, Solar and Space Weather Radiophysics - Current Status and Future

Developments (Kluwer Academic Publishers, Dordrecht, The Netherlands)

Gary, G. A., Balasubramaniam, K. S., \& Sigwarth, M. 2003, Proc. SPIE, 4853, 252

Harvey, J. W., Hill, F., \& Hubbard, R. \& 14 co-authors 1996, Science, 533, 163

Hill, F., Beckers, J., Brandt, P. \& 17 co-authors 2004, Proc. SPIE, 5489, 122

Hill, F., Beckers, J., Brandt, P. \& 18 co-authors 2006, Proc. SPIE, 6267, 62671T

Keil, S. L., Oschmann, J. M., Rimmele, T. R. \& 8 co-authors 2004a, Proc. SPIE, 5489, 625

Keil, S. L., Rimmele, T. R., Oschmann, J. M. \& 4 co-authors 2004b, IAU Symp., 223, 581

Keller, C. U., Harvey, J. W., \& Giampapa, M. S. 2003, Proc. SPIE, 4853, 194

Kentischer, T. J., Schmidt, W., Sigwarth, M., \& von Uexküll, M. 1998, A\&A, 340, 569

Lanzerotti, L. 2003, The Sun to the Earth - and Beyond: A Decadal Research Strategy in Solar and Space Physics (National Academy Press, Washington, DC)

Lin, H. \& Penn, M. J. 2004, PASP, 116, 652

Martin, H. M., Burge, J. H., Cuerden, B. \& 3 co-authors 2004, Proc. SPIE, 5494, 62

McKee, C. \& Taylor, J. 2001, Astronomy and Astrophysics in the New Millenium. (National Academy Press, Washington, DC)

Neidig, D., Wiborg, P., Confer, M. \& 17 co-authors 1998, ASP Conf. Ser., 140, 519

Oschmann, J., Dalrymple, N., Warner, M. \& 6 co-authors 2004, Proc. SPIE, 5171, 160

Parker, E. N. 1998, Ground-Based Solar Research: An Assessment and Strategy for the Future (National Academy Press, Washington, DC)

Ren, D., Hegwer, S. L., Rimmele, T. R., Didkovsky, L. V., \& Goode, P. R. 2003, Proc. SPIE, 4853, 630

Rimmele, T. R. 2000, Proc. SPIE, 4007, 218

Rimmele, T. R., Keil, S. L., Keller, C. U. \& 10 co-authors 2003, ASP Conf. Ser., 286, 3

Rimmele, T. R., Richards, K., Hegwer, S. \& 10 co-authors 2004a, Proc. SPIE, 5171, 179

Rimmele, T., Hubbard, R. P., Balasubramaniam,K. S. \& 12 co-authors 2004b, Proc. SPIE, 5492, 944

Rimmele, T. R., Keil, S. L., Wagner, J. \& 8 co-authors 2005, Proc. SPIE, 5901, 41

Rimmele, T., Richards, K., Roche,J., Hegwer,S., \& Tritschler,A. 2006, Proc. SPIE, 6272, 5

Rutten, R. J., Hammerschlag, R. H., Bettonvil, F C. M. \& 2 co-authors 2004, A\&A, 413, 1183

Socas-Navarro, H., Beckers, J., Brandt, P. \& 17 co-authors 2005, Publ. Astron. Soc. Pac., 117, 1296

Teran, J. U., Porter, D. S., Hileman, E. A. \& Neff, D. H. 2000, Proc. SPIE, 4004, 155

Tritschler, A., Schmidt, W., Langhans, K., \& Kentischer, T. J. 2002, Solar Phys., 211, 17

Uitenbroek, H., Tritschler, A., An, H. K., \& Berger, T. 2006, Proc. SPIE, 6269, 193

Wagner, J., Rimmele, T. R., Keil, S. L. \& 12 co-authors 2006, Proc. SPIE, 6267, 626709

White, S., Lee, J., Aschwanden, M. A., \& Bastian, T. S. 2003, Proc. SPIE, 4853, 531 


\title{
New high resolution solar telescope GREGOR
}

\author{
R. Volkmer ${ }^{1, *}$, O. von der Lühe ${ }^{1}$, F. Kneer ${ }^{2}$, J. Staude $^{3}$, H. Balthasar ${ }^{3}$, T. Berkefeld ${ }^{1}$, \\ P. Caligari ${ }^{1}$, M. Collados ${ }^{4}$, C. Halbgewachs ${ }^{1}$, F. Heidecke ${ }^{1}$, A. Hofmann $^{3}$, \\ M. KIvaňa ${ }^{5}$, M. Sobotka ${ }^{5}$, H. Nicklas ${ }^{2}$, E. Popow ${ }^{3}$, K. G. Puschmann ${ }^{2}$, W. Schmidt ${ }^{1}$, \\ D. Soltau ${ }^{1}$, K. Strassmeier ${ }^{3}$, and A. D. Wittmann ${ }^{2}$ \\ ${ }^{1}$ Kiepenheuer-Institut für Sonnenphysik, Freiburg, Germany \\ ${ }^{2}$ Institut für Astrophysik, Göttingen, Germany \\ ${ }^{3}$ Astrophysikalisches Institut Potsdam, Germany \\ ${ }^{4}$ Instituto de Astrofísica de Canarias, La Laguna, Spain \\ ${ }^{5}$ Astronomical Institute, AS CR, Ondřejov, Czech Republic \\ *Email: volkmer@kis.uni-freiburg.de
}

\begin{abstract}
The $1.5 \mathrm{~m}$ solar telescope GREGOR is being constructed at Tenerife, Spain. Its purpose is to observe with high spatial and spectral resolution small-scale dynamic magnetic features on the Sun. The telescope is completely open with retractable dome and actively cooled primary mirror made of silicon carbide to minimize thermal effects on the image quality. After completion it will be one of the most powerful solar telescopes. This paper presents a general overview of the telescope characteristics and the current status.
\end{abstract}

\section{Introduction}

The magnetic activity of the Sun plays a dominant role in virtually all processes in the solar atmosphere. It is responsible for the energy balance of the outer atmosphere, it causes the activity cycle and the concomitant variability of the solar luminosity and it produces most of the sometimes spectacular phenomena like sunspots, prominences, flares and coronal mass ejections. Theoretical studies and numerical simulations suggest that much of the interaction between the solar plasma and the magnetic field occurs on very small spatial scales corresponding to an angle less than 0.1 arcsec. It is therefore important to have a large enough telescope which can resolve small details. In addition, a large aperture is needed to achieve the photometric accuracy and sensitivity needed for a quantitative physical understanding of the solar magnetic field.

GREGOR will be a new solar telescope with large aperture and modern scientific instruments. It is designed to observe solar magnetic structure below $70 \mathrm{~km}$ size on the Sun. The polarimetric capabilities will allow high precision measurements of magnetic features in the solar photosphere and chromosphere. The large size of the aperture will provide enough light and spatial resolution to allow studies of highly dynamic magnetic and velocity fields on the Sun.

Key features of the telescope are a Gregory coudé optical configuration, an open structure, an alt-azimuth mount and a completely retractable dome to make the entire system as 


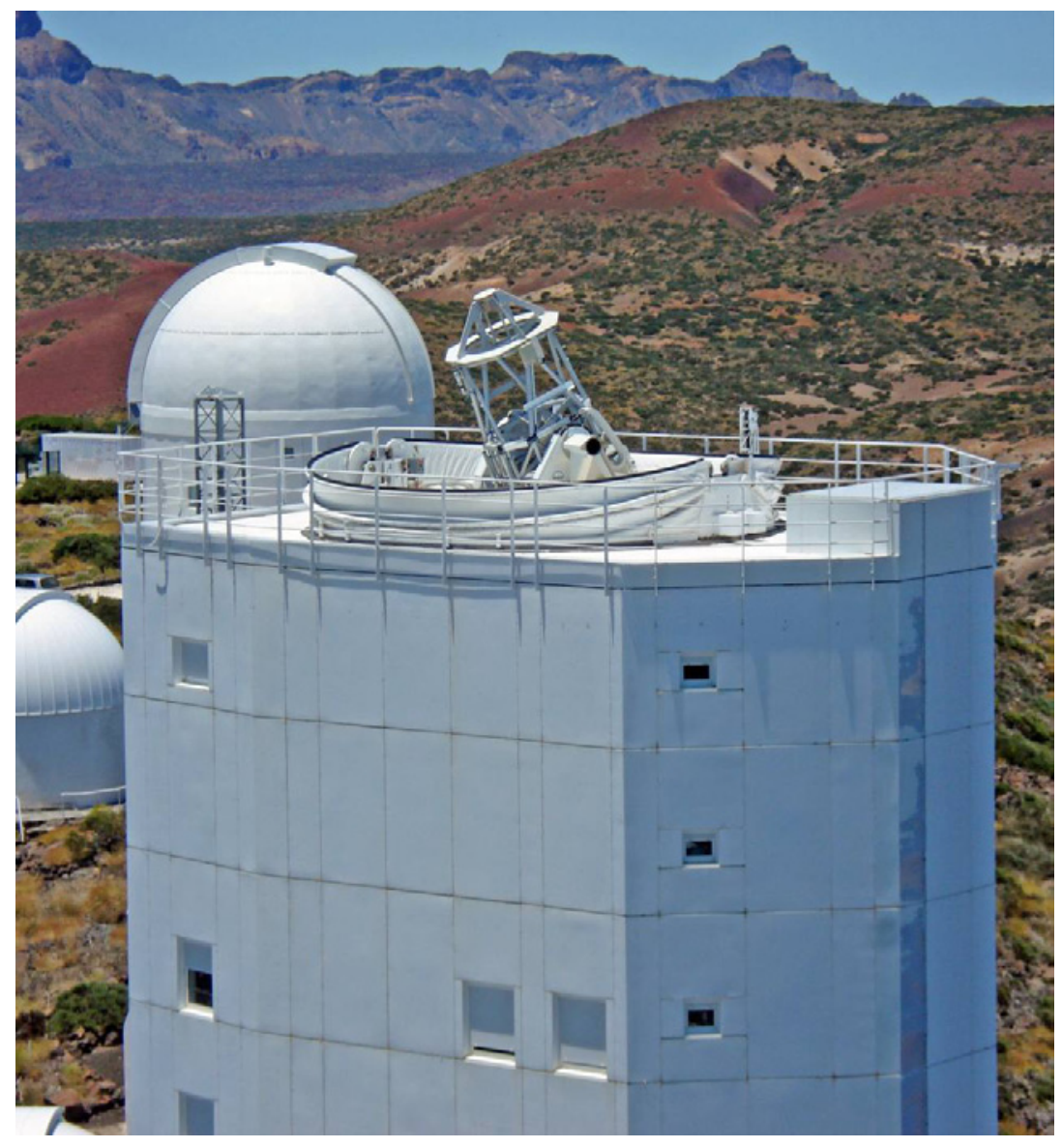

Figure 1. GREGOR telescope with open dome at Obervatorio del Teide, Izaña/Tenerife.

permeable as possible to wind. The first three mirrors are made of light-weighted carbon reinforced silicon carbide, primarily for reasons of thermal control of the optics. An adaptive optics system feeds a compensated image to several post-focus instruments.

The instrument is currently under construction at the Observatorio del Teide on Tenerife, Spain. It is build by a consortium of three German institutes, the Kiepenheuer-Institut für Sonnenphysik (KIS), the Astrophysikalisches Institut Potsdam (AIP), the Institut für Astrophysik Göttingen (IAG), and additional European partners.

\section{Optical configuration}

\subsection{Optical path}

The main mirrors of solar telescopes are heated by solar radiation. The transfer of this heat to the ambient air results in substantial image disturbances. Therefore the main mirrors of 
large, open telescopes must be controlled thermally.

The first three mirrors (M1 to M3 in Figure 2) of the GREGOR telescope are made from silicon carbide (Cesic). Cesic has a very high stiffness, a good lightweight capability and, compared with Zerodur or other glass ceramics, a very high thermal conductivity. This allows removing the absorbed heat from the backside of the main mirrors. The GREGOR primary mirror is cooled by an active temperature controlled air stream. Secondary and tertiary mirrors are passively cooled by radiation and wind flow.

The optical configuration is a Gregory coudé which consists of three powered mirrors. A water cooled field stop at F1 at the primary focus rejects most of the sunlight (97\%) and only the required field of view of a diameter of 3 arcmin will pass through. A polarimetry package is located near the secondary focal plane at the intersection of the azimuth and elevation axis before the first oblique reflection. The third mirror provides the final focus through the coude optics and image de-rotator in the area below the telescope.

Since GREGOR is an alt-azimuth telescope, image rotation needs to be compensated during observations. The image de-rotator consists of three mirrors mounted in a rotating cage with its axis in line with the azimuth axis of the telescope.

A flat mirror redirects the beam horizontally into the laboratory feeding the adaptive optics (AO) system, which delivers a stabilized science image to several post-focus instruments. The effective focal length is about $56 \mathrm{~m}$ and observations are possible at two final focal planes in two laboratories.

Both image de-rotator and adaptive optics can be removed from the light path to use the telescope e.g. for night time observations.

Most of the optical components have already been manufactured. During 2006 the optical elements from M3 to M16 were preliminary integrated and aligned (Soltau et al. 2006). Only the primary and secondary silicon carbide mirrors are missing. The secondary mirror is expected to be integrated in 2007 and the primary in 2008.

\section{Adaptive optics}

A "first-light" adaptive optics system will be used from the beginning (Berkefeld et al. 2003). A tip-tilt mirror and a deformable mirror with 80 actuators compensate atmospheric and fixed optical aberrations. The tip-tilt mirror will also be used for spatial scanning purposes, e.g. for the long-slit spectrograph. Each post-focus instrument will have its separate ShackHartmann-type wave front sensor with 78 illuminated sub-apertures and a 12 arcsec field of view. There are plans to develop a high-order adaptive optics system using one of the coudé mirrors for a ground-layer deformable mirror. The development of multi-conjugate adaptive optics (MCAO) for compensating image disturbances in a larger field of view is under way.

\section{Structure}

The telescope tube is a completely open Serrurier structure. This allows free access of the wind to cool optics and mechanics heated by solar radiation. The telescope shall have full performance in pointing and tracking up to $20 \mathrm{~m} / \mathrm{sec}$ wind speed.

The telescope design is very stiff. The calculated eigenfrequency is about $13 \mathrm{~Hz}$. The telescope is driven by standard gear motors and uses cross roller bearings in azimuth and 


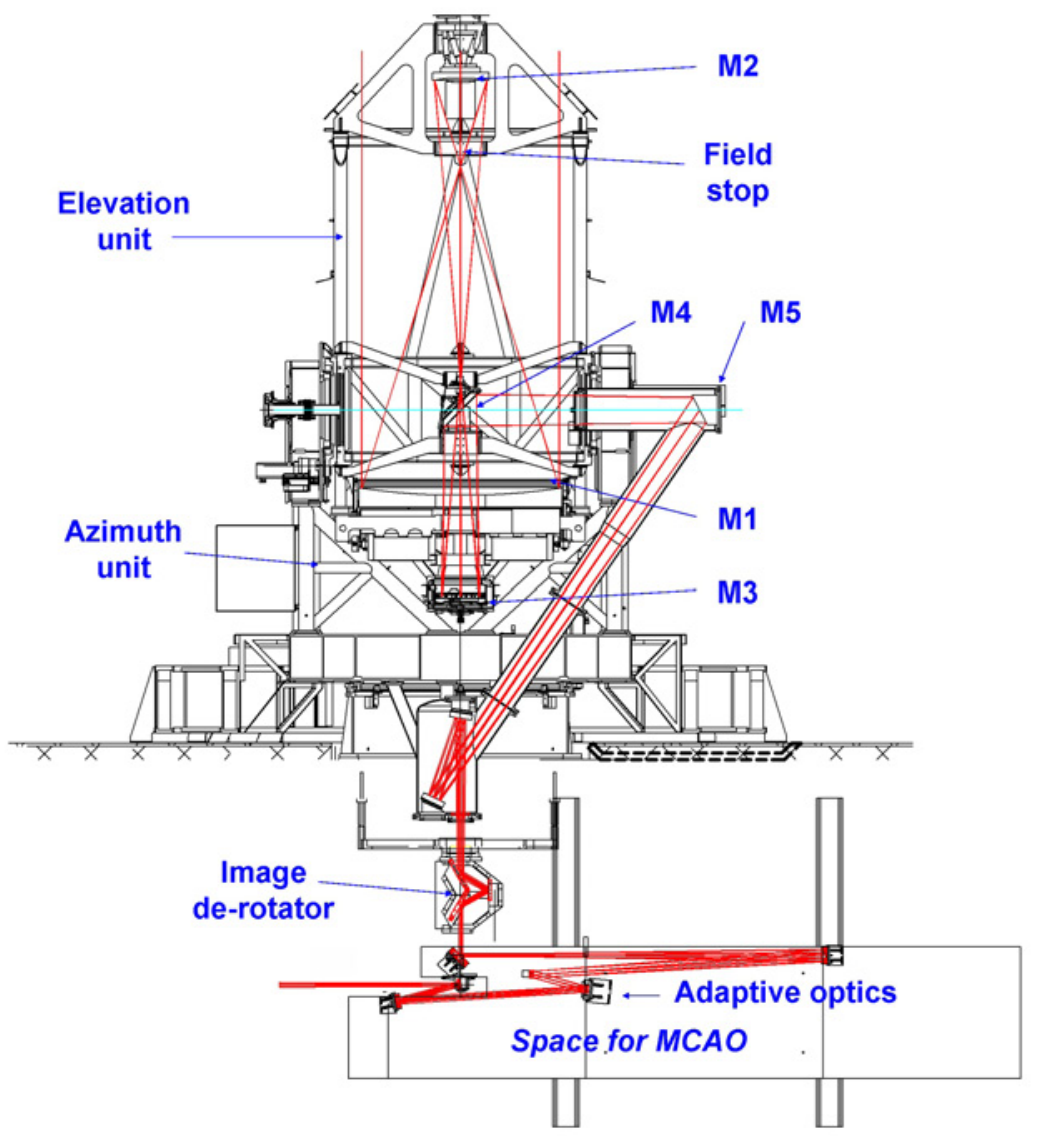

Figure 2. Structure and optical light path of the GREGOR telescope.

elevation axis.

The telescope structure and its control system were successfully tested at the telescope site. Preliminary tests showed that the tracking accuracy is better than 1 arcsec (Volkmer et al. 2005). The measured first eigenfrequency is $12 \mathrm{~Hz}$ which is in a good accordance with the calculated value.

\section{Building and dome}

Before erection of the GREGOR telescope the building housed during the past 17 years a $45 \mathrm{~cm}$ free aperture Gregory coudé telescope. This telescope was removed in 2002 together with its dome.

The complete ceiling of the highest level was exchanged to get a better stiffness. During the reconstruction of the building the complete electrical cabling and network was renewed. 
The new retractable dome was mounted in 2004. The design is similar to the Dutch Open Telescope (DOT) on La Palma.

Two optical laboratories offer space for scientific instruments. The optical lab on the 5th floor will host most of the post-focus instruments. The slit spectrograph will be located in the optical lab of the 4 th floor.

The observer can handle the complete telescope from the control room in the 3rd floor. This separation reduces interferences with the scientific instruments.

\section{Control system}

The GREGOR control system consists of different modules. Each sub-system, such as e.g. post-focus instruments, telescope control system, adaptive optics and dome uses its dedicated program module with graphical user interface.

The modules are communicating via a newly developed communication protocol (DCP). The telescope and the instruments could be controlled remotely from a separate control room in the observatory building. The data will be stored on a large central data storage server.

The field bus systems CoSM (communication of short messages) and a trigger bus system were developed to communicate with peripheral devices such as motors and temperature sensors and to synchronize cameras of different instruments. Both systems are finished and partially integrated.

\section{Post-focus devices}

Already from the beginning GREGOR will have two first-light post-focus instruments.

The first is a two-dimensional spectro-polarimeter using two Fabry-Perot interferometers in a collimated beam as the wavelength selectors. The image acquisition and control systems as well as one of the two interferometers were tested very successfully at the VTT already in April 2005 (Puschmann et al. 2006). The instrument demonstrated an acquisition rate of about 10 frames/s with a 1.2 million pixels field of view and a 3 pm FWHM spectral resolution at $550 \mathrm{~nm}$.

The second post-focus instrument is a long-slit echelle spectrograph which observes mainly in the near-infrared (Collados et al. 2007). The grating of the original spectrograph will be reused. The camera system is similar to the TIP II camera (Tenerife Infrared Polarimeter) which is currently used at the VTT.

Additional cameras for speckle reconstruction will also be installed in the optical laboratory from the beginning.

The POLIS spectro-polarimeter (Schmidt et al. 2003) at the GREGOR telescope will be installed when the commissioning phase is concluded.

A high-resolution, fibre-fed, double-echelle stellar spectrograph has been designed for using the telescope at night. This spectrograph will be remotely controlled and integration will start not earlier than 2009. 


\section{Conclusions}

The $1.5 \mathrm{~m}$ solar telescope GREGOR is about to complete its erection phase. The major parts of the structure are integrated, the dome is mounted and the control system is ready. The post-focus instruments are proceeding well and the plane optic has been installed. The manufacturing of the main optics, despite the early start in the project, has proven to be significantly more difficult than expected. The optics will be delivered not before the beginning of 2008 and commissioning is planned during summer 2008.

\section{References}

Berkefeld, T., Soltau, D., \& von der Lühe, O. 2003, Astron. Nachr., 324, 296

Collados, M., Díaz, J. J., Hernández, E., López, R., \& Páez, E. 2007, in Proc. Sociedad Española de Astronomía, Barcelona, September 2006, in press

Puschmann, K. G., Kneer, F., Seelemann, T., \& Wittmann, A. D. 2006, A\&A, 451, 1151

Schmidt, W., Beck, C., Kentischer, T., Elmore, D., \& Lites, B. 2003, Astron. Nachr., 324, 300

Soltau, D., Berkefeld, T., \& Volkmer, R. 2006, in Proceedings Ground-based and Airborne Telescopes, ed. Stepp, Larry M., Proceedings of the SPIE, Volume 6267

Volkmer, R., von der Lühe, O., Kneer, F., Staude, J., Berkefeld, T., Caligari, P., Halbgewachs, C., Schmidt, W., Soltau, D., Nicklas, H., Wittmann, A., Balthasar, H., Hofmann, A., Strassmeier, K., Sobotka, M., Klvaňa, M., \& Collados, M. 2006, in Proceedings Optics for EUV, X-Ray, and Gamma-Ray Astronomy II, ed. O. Citterio \& S. L. O'Dell, Proceedings of the SPIE, Volume 5901, 75. 


\title{
From the "Göttingen" Fabry-Perot Interferometer to the GREGOR FPI
}

\author{
K. G. Puschmann*, F. Kneer, H. Nicklas, and A. D. Wittmann \\ Institut für Astrophysik, Göttingen, Germany \\ *Email: kgp@astro.physik.uni-goettingen.de
}

\begin{abstract}
Fabry-Perot Interferometers (FPIs) have advantages over slit spectrographs, allowing fast two-dimensional, narrowband imaging and post factum image reconstruction of the spectropolarimetric data obtained. The resulting intensity, velocity and magnetic field maps are a fundamental base for the understanding of the dynamics of the solar atmosphere and its magnetic fields at smallest spatial scales. Efforts are undertaken to provide, with the "Göttingen" Fabry-Perot interferometer, an up-todate post-focus instrument for the German $1.5 \mathrm{~m}$ GREGOR solar telescope. Therefore a renewal of the spectrometer has been achieved during the first half of 2005. First observations at the German Vacuum Tower Telescope (VTT) reveal new scientific aspects and a very promising outlook for the future at GREGOR. In this contribution a general description of the upgraded spectrometer is given. Its final optical design at GREGOR is described and an optical analysis of the GREGOR FPI is outlined. Latest results with the new instrument obtained at the VTT are presented.
\end{abstract}

\section{Introduction}

For the understanding of the dynamics of the fine-structure of the solar atmosphere and of its magnetic field two-dimensional spectroscopic and polarimetric observations at high spatial, spectral and temporal resolution are required. Fabry-Perot Interferometers (FPIs) allow efficient narrowband imaging in two dimensions. The good performance of the "Göttingen" Fabry-Perot interferometer (Bendlin et al. 1992) has been demonstrated in several publications during the last years. In combination with post factum image reconstruction many new insights in different topics of solar physics have been gained (Wunnenberg et al. 2002; Domínguez Cerdeña et al. 2003a,b; Okunev \& Kneer 2004; Bello González et al. 2005; Hirzberger et al. 2005). The "Göttingen" Fabry-Perot interferometer (later on GREGOR FPI) will be one of the post-focus instruments at the German $1.5 \mathrm{~m}$ GREGOR solar telescope. Therefore a renewal of the spectrometer has been achieved during the first half of 2005 (Puschmann et al. 2006). In this contribution we summarise in Section 1 the new features of the upgraded instrument presently mounted at the German Vacuum Tower Telescope (VTT), present in Section 2 its final optical design for GREGOR and show in Section 3 results of an optics analysis on this optical setup for the two main beams of the instrument. As an outlook on what we can expect from the spectrometer once it will be mounted at GREGOR, latest observational results at the VTT obtained with the new instrument are presented in the final section. 


\section{Main components of the new instrument}

The upgrade consisted mainly in the integration of the new features described below.

New detectors: Two Imager QE CCDs from LaVision GmbH (Göttingen) with the Sony chip ICX285AL with a full well capacity of $18000 \mathrm{e}^{-}$and a read-out noise of $4.5 \mathrm{e}^{-}$. The AD conversion is done with 12 bit resolution. The detectors have a spectral range from $3200 \AA$ up to $9000 \AA$ with a maximum quantum efficiency of $60 \%$ at $5500 \AA$. The chip has a size of $8.8 \times 6.7 \mathrm{~mm}^{2}$ which corresponds to $1376 \times 1040$ pixels with a pixel size of $6.45 \times 6.45 \mu \mathrm{m}^{2}$.

A new etalon from IC Optical Systems (diameter $\oslash=70 \mathrm{~mm}$, finesse $\mathcal{F} \sim 46$, spacing $\|=1.1 \mathrm{~mm})$. The other FPI from IC Optical Systems with $\oslash=50 \mathrm{~mm}, \mathcal{F} \sim 36$, and $\|=$ $0.125 \mathrm{~mm}$ has yet remained. It will be replaced during the first half of 2007 by a second narrowband etalon from IC Optical Systems $(\oslash=70 \mathrm{~mm}, \mathcal{F} \sim 46, \|=1.4 \mathrm{~mm})$. Both etalons will then have a high reflectivity coating (reflectivity $R \sim 0.95$ ) in the wavelength range $5300-8600 \AA$. The combination of two narrowband etalons will strongly suppress both side bands and extended wings of the Airy transmission function. The narrower transmission with FWHM $=20-75 \mathrm{~m} \AA$ will further improve the spectral purity and resolution of the spectrometer. All etalons are controlled now by CS100 three-channel controllers of IC Optical Systems, with a three-axis capacitance bridge stabilisation system which enables the parallelism and cavity spacing of the etalon to be servo-stabilised. The cavity spacing during observation is controlled digitally via PC (RS-232 port) for scanning in wavelength.

Together with new PC hardware the Software Package Davis 7 from LaVision has been purchased and has been adapted to the spectrometer, new software with a Graphical User Interface (GUI) for camera read-out and FPI control has been developed and implemented.

The integration of a filter wheel, housing up to 5 different narrowband interference filters, is nearly finished and will allow to observe quasi-simultaneously several spectral regions. Efforts are undertaken to replace in 2007 the Stokes $V$ polarimeter by ferro-electric liquid crystals to improve the polarimetric accuracy with beam exchange. For further details and a comparison between the former and upgraded system see Puschmann et al. (2006).

\section{Optical design for GREGOR}

In Fig. 1 we present the final optical design of the spectrometer for its use at GREGOR. The instrument will be transferred to the new telescope as soon the latter will be operative. It will be mounted on three optical benches in the $5^{\text {th }}$ floor of the telescope building. Together with the main components, the yet purchased optical rails and additional optical elements (e.g. lenses, folding mirrors) are illustrated.

A beam splitter (BS1) in front of the science focus (F4) feeds part of the light into the Wave Front Sensor (WFS) of the Adaptive Optics system (AO). Two achromatic lenses (TL1, TL2) transfer the science focus to F5. A beam splitting cube (BS2) feeds $5 \%$ of the incoming light into the broadband channel, where images are taken by the CCD1 detector using a broadband (FWHM 100 ̊) interference filter (BIF). The achromatic lenses (TL3, TL4) are chosen

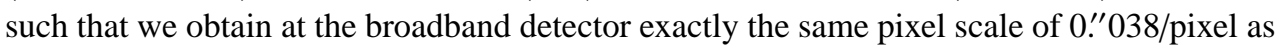
at the narrowband one, yielding a total field of view (FOV) of 52 ." $2 \times 39$." 5 .

$95 \%$ of the incoming light is transmitted into the narrowband channel, where the light beam passes a filter wheel, housing up to 5 narrowband interference filters $(\mathrm{FWHM}=6-$ 


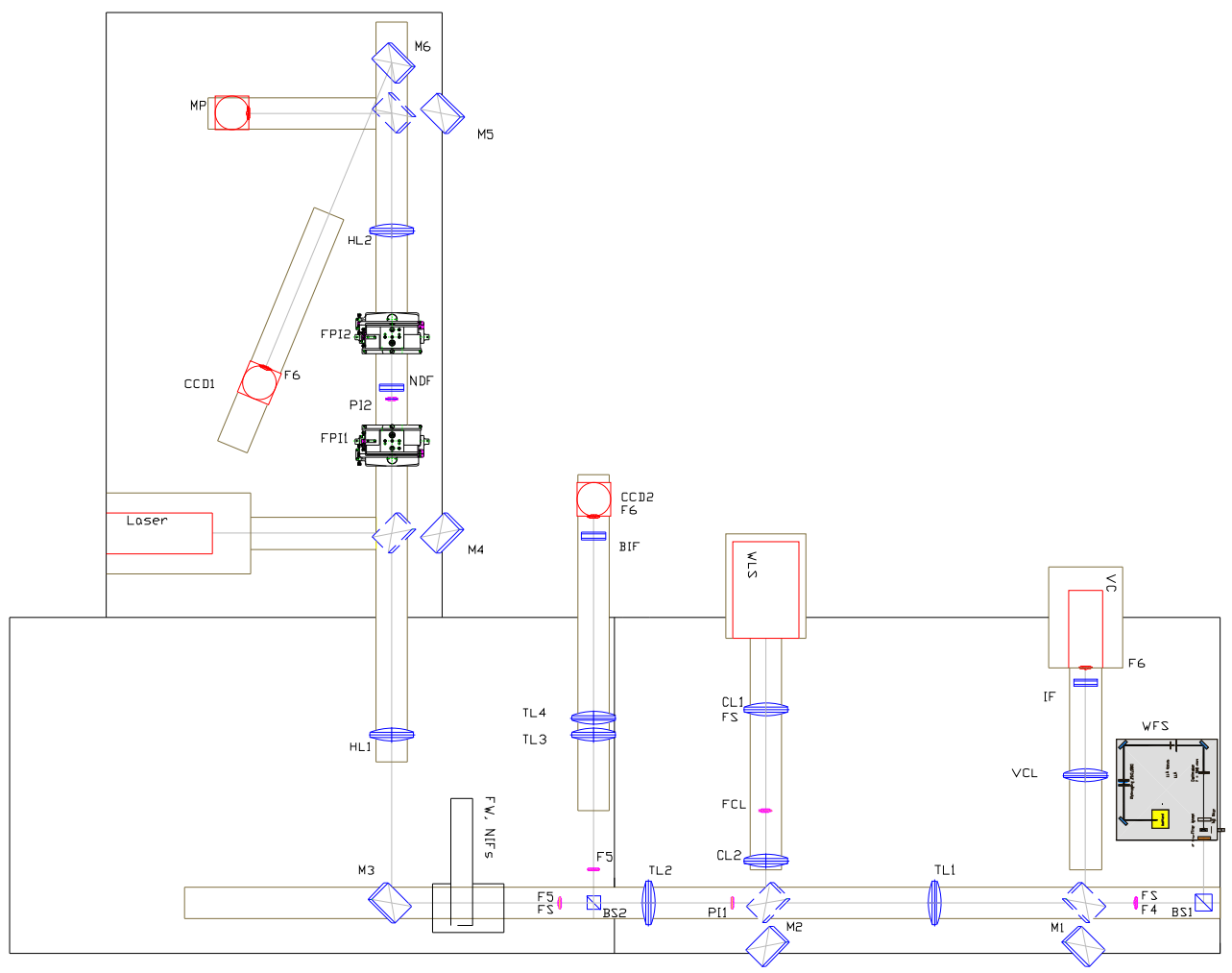

Figure 1. Final optical design of the spectrometer at GREGOR. WFS: Wavefront sensor; VC: Video camera; WLS: White light source (projector); CCD1, CCD2: CCD detectors; FPI1, FPI2: Narrowband and broadband etalon; MP: Photomultiplier; FW: Filter wheel; NIFs: Narrowband interference filters; BIF: Broadband interference filter; IF: Interference filter; NDF: Neutral density filter (NG11, $2 \mathrm{~mm}, 63 \%$ transmission); achromatic lenses: TL1 $(f=600 \mathrm{~mm})$, TL2 $(f=250 \mathrm{~mm})$, TL3 $(f=400 \mathrm{~mm})$, TL4 (f $=600 \mathrm{~mm}), \mathrm{HL} 1(\mathrm{f}=1000 \mathrm{~mm}), \mathrm{HL} 2(\mathrm{f}=1500 \mathrm{~mm}), \mathrm{VCL}(\mathrm{f}=200 \mathrm{~mm})$; Plano-convex lenses: CL1 $(\mathrm{f}=300 \mathrm{~mm})$, CL2 ( $\mathrm{f}=150 \mathrm{~mm})$; M1, M2, M4, M5: Removable folding mirrors; M3, M6: Fixed folding mirrors; F4, F5, F6, FCL: Foci; PI1, PI2: Pupil images; BS1, BS2: Beam-splitter; FS: Field stop. See the text for a detailed description.

$10 \AA$ ). The achromatic collimator lens HL1 produces an image of the telescope pupil (PB2) in whose vicinity the two etalons (FPI1, FPI2) are mounted. A neutral density filter (NDF) in between the two etalons will remove inter-etalon reflexes. The camera lens HL2 produces a focus (F6) at the CCD2 detector where images are taken simultaneously with the broadband ones. A polarimeter can be placed in front of CCD2. In the narrowband channel the light beam is folded twice by fixed folding mirrors (M3, M6) to keep the space required by the instrument as small as possible.

The field stop at F1 limits the total amount of light entering the spectrometer and is larger than the FOV. The stop at F2 can be adjusted to the final FOV on the CCD2 detector and is used to avoid an overlapping of the images separated by the polarimeter. 

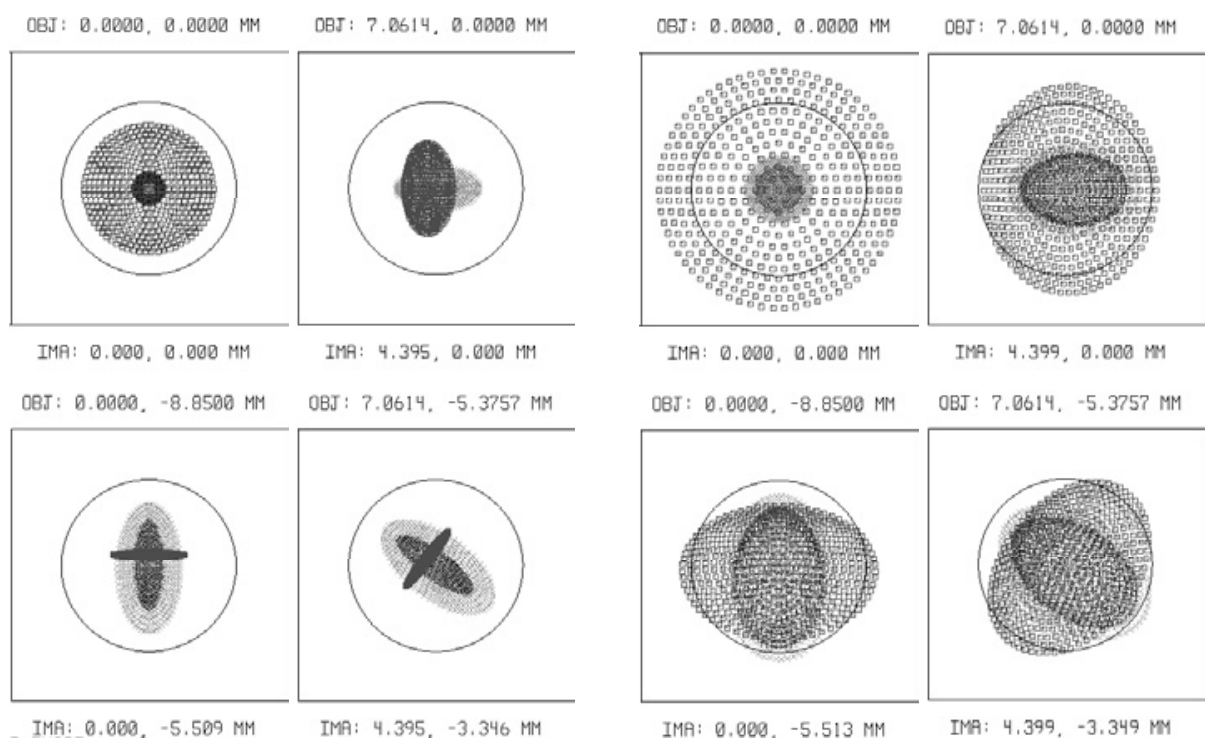

Figure 2. Spot diagrams at the foci of the broadband (left four panels) and narrowband beam (right four panels), respectively. Shown are the spot diagrams for the total field of view (FOV) at optical axis (upper left), at the middle of the edge (upper right), at the corner (lower right) and for the maximum radial distance (lower left). The rms diameters at the three wavelengths $530 \mathrm{~nm}$ (crosses), $580 \mathrm{~nm}$ (diagonal crosses) and $630 \mathrm{~nm}$ (squares) are typically $11 \mu \mathrm{m}$ and $21 \mu \mathrm{m}$ for the broadband and narrowband channel, respectively, compared to an Airy disk diameter (circle) of $33 \mu \mathrm{m}$ for both channels. The focusing is done for the field edge with equal weight of wavelengths.

In addition, we have provided a video channel, a white-light source for spectral calibration purposes as well as a laser and photomultiplier channel for finesse adjustment of the two etalons. Most of the optics has been already delivered to Tenerife. Lens and mirror mounts are being manufactured presently at our workshop in Göttingen.

\section{Optical design analysis}

The Zemax optical design program was used to ray trace the different beams which have been set up using solely commercial achromatic lenses of Linos Photonics. Special care was taken in positioning the achromat doublets and matching the glass melts that were commercially available for the required size of up to $100 \mathrm{~mm}$ diameter. In Fig. 2 we present the ray trace results as spot diagrams for the two main optical channels, the narrowband channel, including the Fabry-Perot etalons, and the broadband channel, considering for both an imaging onto $1376 \times 1040$ pixel CCD detectors. The two etalons have been modelled as plane-parallel plates without any inclination relative to the beam. Within the spectral range $530 \mathrm{~nm}-630 \mathrm{~nm}$, the spot diagrams (presented for the lower right quadrant of both CCD detectors) with rms diameters of $11 \mu \mathrm{m}$ and $21 \mu \mathrm{m}$, respectively, are well within the Airy disk of $33 \mu \mathrm{m}$. The results are very encouraging with respect to the diffraction limit. The broadband channel shows a two times better performance due to its smaller beam diameter. 


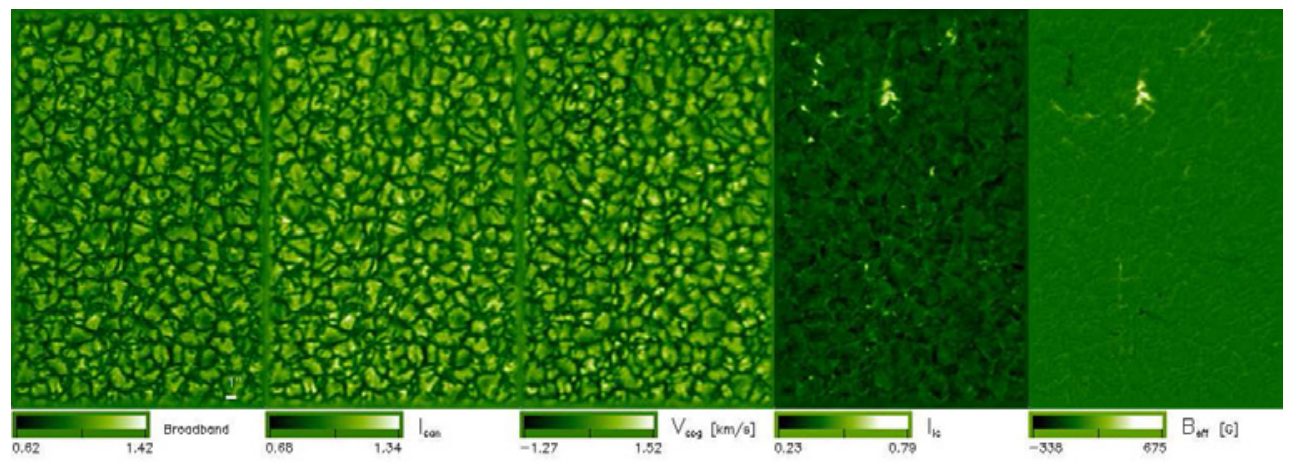

Figure 3. Quiet Sun near disk centre. From left to right: Broadband and narrowband continuum intensity, velocity, line centre intensity and effective magnetic field with a spatial resolution $<0$. .' $^{\prime}$. FOV: $29^{\prime \prime} \times 43^{\prime \prime}$. The broadband images possess a $\Delta \mathrm{I}_{\mathrm{rms}}$ of $11 \%$ after image reconstruction. The measured magnetic flux densities amount up to $800 \mathrm{G}$ in areas of network boundary and up to $180 \mathrm{G}$ in the internetwork. A good correlation between intergranular bright points and magnetic field is revealed when comparing the continuum and line centre images with the magnetic field maps.

\section{Outlook to Gregor - latest results with the upgraded FPI at the VTT}

The scientific output of the spectrometer at GREGOR can be extrapolated from two results recently obtained with the new instrument at the VTT in combination with adaptive optics.

Firstly, we comment on an excellent $1 \mathrm{~h} 10 \mathrm{~min}$ time series of a quiet solar region near disk centre, acquired on $4^{\text {th }}$ of May 2006 revealing the dynamics of the quiet photosphere and its magnetic field at smallest scales. Spectropolarimetric images were obtained in the magnetically sensitive Fe I $5247 \AA$ and Fe I $5250 \AA$ lines with Landé factors of 2.0 and 3.0, respectively. The lines have been scanned quasi simultaneously with a temporal cadence of 17 seconds each. The spectral resolution was $\sim 27 \mathrm{~m} \AA$. Thanks to speckle reconstruction methods (Puschmann \& Sailer 2006; Krieg et al. 1998) the achieved spatial resolution of the obtained intensity, velocity and magnetic field maps is below 0." 3 (see Fig. 3). First analyses reveal strong dynamics of small-scale magnetic fields in the quiet Sun. Field structures, elongated along intergranular lanes, combine and split, and leave strong fingerprints at high photospheric layers. The polarisation signal strengthens and weakens in reaction to granular convection processes, indicating advection and diffusion of magnetic flux at smallest scales. Indications of flux annihilation, flux tube evacuation or signs of upwards propagating shock fronts are illustrated. Recent numerical MHD simulations come close to the observations presented here. Our findings lend support to the efforts to apply the Zeeman effect to very high angular resolution observation with large solar telescopes, in addition to Hanle diagnostics. For further details see Puschmann et al. (2007).

Secondly, we mention a 45 minute time series of the flaring solar active region NOAA AR 0875, obtained on April 26 ${ }^{\text {th }}$, 2006. 2D spectrograms with high spatial resolution, containing 21 different wavelength positions along the $\mathrm{H} \alpha$ line, have been obtained, scanning with a cadence of $\sim 20 \mathrm{~s}$. After applying the speckle reconstruction techniques mentioned above the spatial resolution turns out to be nearly diffraction-limited. Figure 4 (left panel) 

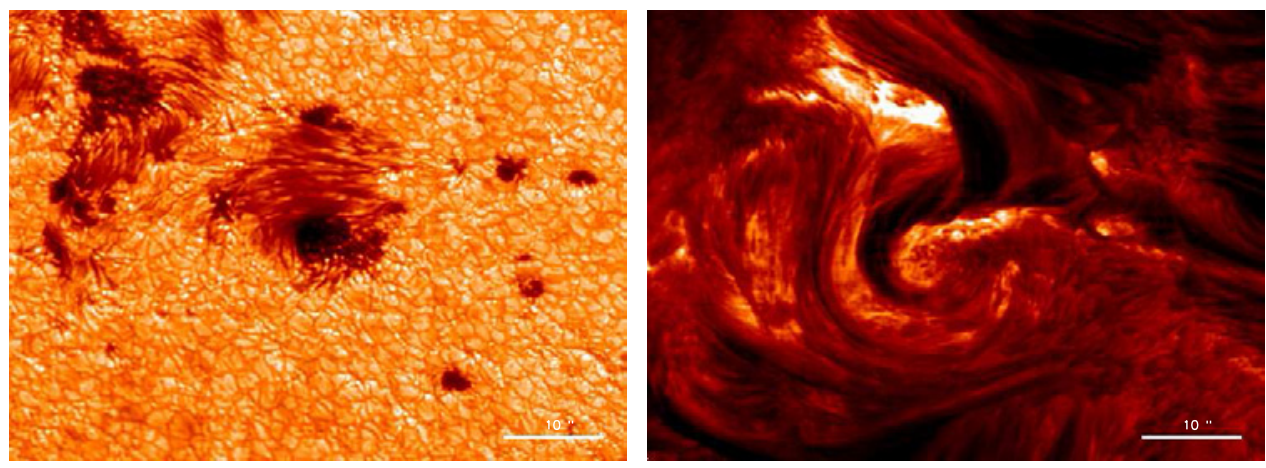

Figure 4. Active region NOAA AR 10875 at $\mu=0.59$ observed with the new Göttingen Fabry-Perot interferometer, VTT, April $26^{\text {th }}, 2006$. FOV: $63^{\prime \prime} \times 47^{\prime \prime}$. Left: Speckle reconstructed broadband image at $6300 \AA$ A. Right: Reconstructed narrowband line centre image at $6563 \AA$. The spatial resolution in both images is $<0$. .' 3 . Intensities have been rescaled for better visualisation of fainter features.

gives an example of one speckle reconstructed broadband image, showing the lower part of a large sunspot with many bright umbral dots and several pores, two of them with a complex penumbral connection. A wide range of different chromospheric features like fibrils, filaments and a sigmoid-like structure are found in the line core image presented in Fig. 4 (right panel). The data reveal a unique possibility to study the dynamics of different chromospheric features at the smallest scales and to analyse their connection to the structures rooted more deep in the photosphere. Besides, the several solar flare events observed as well as the search for Alfvén waves will be an interesting topic of future research. For further details see Sánchez-Andrade Nuño et al. (2007).

Acknowledgements. KGP thanks the Deutsche Forschungsgemeinschaft for support through grant KN 152/29-1,2. The Vacuum Tower Telescope is operated by the Kiepenheuer-Institut für Sonnenphysik, Freiburg, at the Spanish Observatorio del Teide of the Instituto de Astrofísica de Canarias.

\section{References}

Bello González, N., Okunev, O. V., Domínguez Cerdeña, I., Kneer, F., \& Puschmann, K. G. 2005, A\&A, 434, 317

Bendlin, C., Volkmer, R., \& Kneer, F. 1992, A\&A, 257, 817

Domínguez Cerdeña, I., Sánchez Almeida, J., \& Kneer, F. 2003a, A\&A, 407, 741

Domínguez Cerdeña, I., Kneer, F., \& Sánchez Almeida, J. 2003b, ApJ, 582, L55

Hirzberger, J., Stangl, S., Gersin, K., Jurçák, J., Puschmann, K. G., et al. 2005, A\&A, 442, 1079

Krieg, J., Kneer, F., Koschinsky, M., Ritter, C., \& Stark, J. L. 1998, in "A Crossroads for European

Solar and Heliospheric Physics", ed. R. H. Harris, F. Moreno Insertis \& E. Priest, ESA SP-417, 317

Okunev, O. V. \& Kneer F. 2004, A\&A, 425, 321

Puschmann, K. G. \& Sailer, M. J. 2006, A\&A, 454, 1011

Puschmann, K. G., Kneer, F., Seelemann, T., \& Wittmann, A. D. 2006, A\&A, 451, 1151

Puschmann, K. G., Kneer, F., \& Domínguez Cerdeña, I. 2007, (this volume)

Sánchez-Andrade Nuño, B., Puschmann, K. G., \& Kneer, F. 2007, (this volume)

Wunnenberg, M., Kneer, F., \& Hirzberger, J. 2002, A\&A, 395, L5 
Modern Solar Facilities - Advanced Solar Science, 51-54

F. Kneer, K. G. Puschmann, A. D. Wittmann (eds.)

(C) Universitätsverlag Göttingen 2007

\title{
Gregor@Night. \\ A concept for a night-time spectrograph for the $1.5 \mathrm{~m}$ solar telescope GREGOR
}

\author{
K. G. Strassmeier ${ }^{1, *}$, M. Woche ${ }^{1}$, T. Granzer ${ }^{1}$, M. I. Andersen ${ }^{1}$, W. Schmidt ${ }^{2}$, and \\ P. Koubsky ${ }^{3}$ \\ ${ }^{1}$ Astrophysikalisches Institut Potsdam (AIP), Potsdam, Germany \\ ${ }^{2}$ Kiepenheuer-Institut für Sonnenphysik (KIS), Freiburg, Germany \\ ${ }^{3}$ Astronomical Institute, Academy of Sciences, Prague, Czech Republic \\ *Email: kstrassmeier@aip.de
}

\begin{abstract}
Gregor@Night is the concept for a fiber-fed double echelle spectrograph for the night-time use of GREGOR. Its design specifications are driven by a science case based on the solar-stellar connection, ranging from the search and characterization of solar analogs, the characterization of late-type stars with exoplanets, asteroseismology with high time resolution and cadence, stellar cycle analogs and many other related topics. The spectrographs are based on a white-pupil design with a $110 \mathrm{~mm}$ beam and are optimized for the wavelength ranges 360-490 nm and 510-870 nm, respectively and achieve a two-pixel resolution of 100,000 for an entrance aperture of 3 " at $20 \%$ throughput. The instrument would be fully automated and no on-site night observer required.
\end{abstract}

\section{The new GREGOR solar telescope}

GREGOR is an open telescope on an alt-azimuthal mount with an aperture of $1.5 \mathrm{~m}$ (Figure 1, cf. Volkmer et al. 2007, this volume). It will be equipped with an adaptive optics system in order to compensate for the deformation of the wavefront of the incoming light caused by atmospheric turbulence. It is the only way to reconcile the conflicting requirements of high spatial, spectral, and temporal resolutions and of spectro-polarimetric precision.

The optical design includes an axial-symmetric 3-mirror configuration where the first two mirrors form a classical Gregory telescope. The first three mirrors (M1, M2 and M3) are curved to provide imaging. The effective focal length is about $55 \mathrm{~m}$, the entrance pupil diameter is $150 \mathrm{~cm}$, and therefore the effective focal ratio is $\mathrm{f} / 36.5$ and the image scale becomes $3.75^{\prime \prime} / \mathrm{mm}$. A cooled field stop at the prime focus (F1) reflects most of the sunlight out of the telescope and transmits a field of view of 300". The solar science focus can be fed into either of the two topmost floors of the building. Here, different high performance focal instruments will be added. These are, e.g., a high-resolution Fabry-Perot filter spectrometer, different polarimeters, a Czerny-Turner spectrograph, and instrumentation for infrared spectroscopy and polarimetry. 


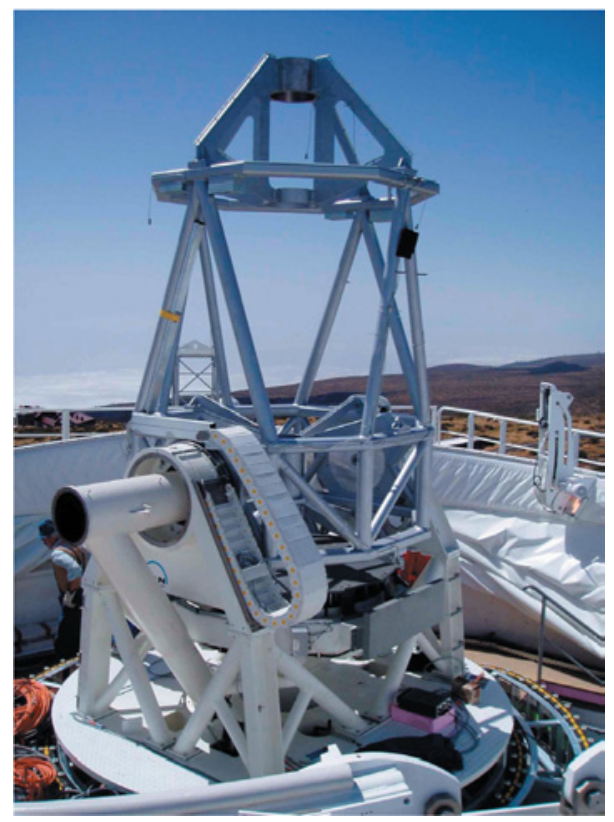

Figure 1. The GREGOR telescope as of summer 2006. The fiber pickup for Gregor@Night would be in a bent Gregorian focus delivered by the F2/M4 unit (partially seen in the center of the telescope tube and in detail in Fig. 4).

\section{Science case: a search for solar twins}

Extrapolated number counts based on various sample directions suggest a total of up to 400 billion stars in our galaxy. Detailed extrapolation from the local neighborhood hints towards a total of $\approx 26$ billion $\mathrm{G}$ dwarfs in our galaxy of which roughly one billion is expected to be of spectral classification G2. Therefore, there should be plenty of 'Suns' around us, but apparently there are not. Of the many attempts to find them, only two stars were classified as "nearly solar analogs" (18 Sco and HD98618). Is the fact that we have such troubles finding solar twins a sign of a solar-stellar disconnection? Or in other words, what do we learn from the fact that stars with solar parameters are so rare. Or aren't they rare at all and we just haven't looked deeply enough?

Therefore, we propose a large survey to search for solar twins. High-resolution spectroscopy with Gregor@Night shall be obtained for the entire lifetime of the GREGOR telescope. On the observing menu would be all known early G-stars brighter than, say, 12th magnitude. The criteria are similarity of spectral appearance due to effective temperature and gravity expressed in terms of excitation equilibrium of many iron lines, detailed chemical abundances, and dynamic parameters (e.g. stellar rotation). Also, extra-solar planets are an important part of the solar-stellar connection. Did they provide extra rotational braking in the early years? Is angular-momentum dissipation due to planet formation the mechanism to bring the planet host star's magnetic activity to a halt and then enable "normal" biological surface evolution in a habitable zone? 


\section{Spectrograph design}

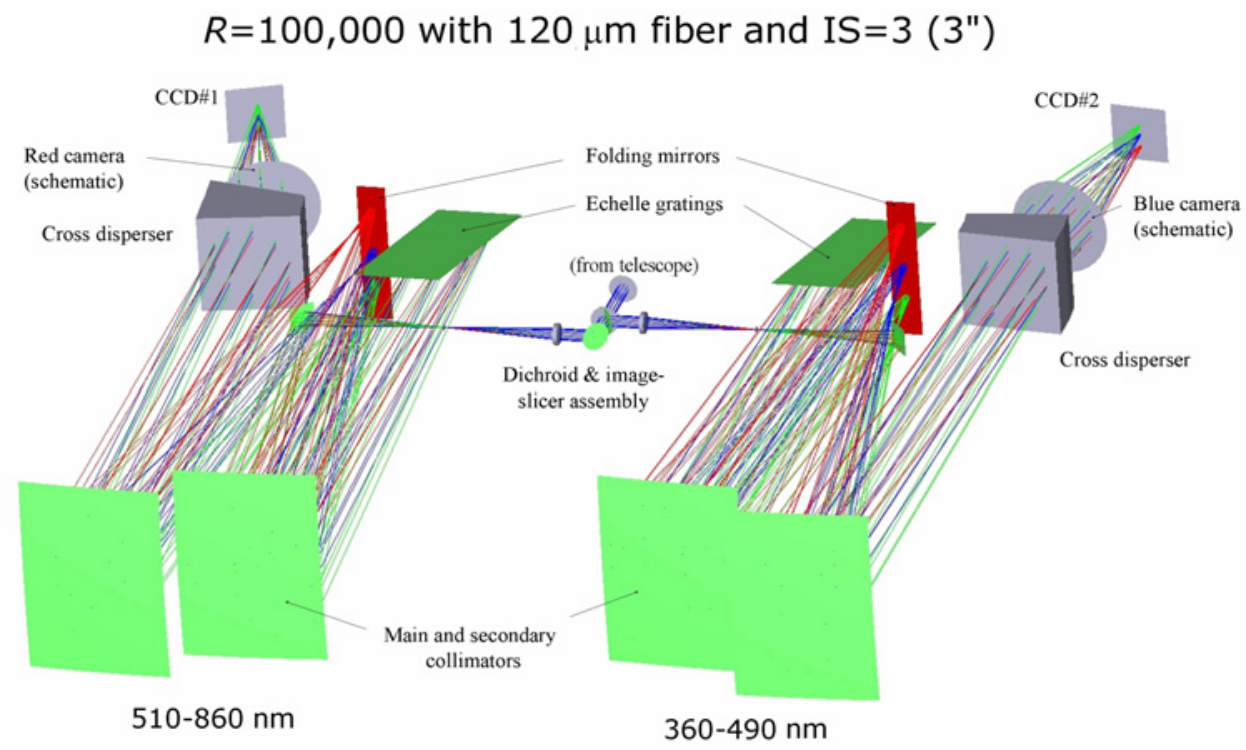

Figure 2. Optical design of Gregor@Night. It consists of two white-pupil spectrographs following the VLT/UVES design but with a fiber feed and the immersed three-slice waveguide image slicer (IS) developed for PEPSI/LBT. Two 4k-CCD cameras would cover the spectrum from $360 \mathrm{~nm}$ to $870 \mathrm{~nm}$ in a single exposure at $R=100,000$.

Figure 2 is a preliminary optical design of the spectrograph. Each arm is based on an R4 echelle grating in Littrow configuration of dimension $110 \times 420 \mathrm{~mm}$ with 41.6 and 31.6 lines/mm for the blue and the red grating, respectively (Figure 3). Both collimators are offaxis parabolas with size roughly $300 \mathrm{~mm}$ by $120 \mathrm{~mm}$ and $160 \mathrm{~mm}$, respectively, and are f/10 at a total length of $1.6 \mathrm{~m}$. The main collimator is used in double reflection. The wavelength coverage is $360-490 \mathrm{~nm}$ and $510-870 \mathrm{~nm}$ for the blue and the red arm, respectively. The cross dispersion required is relatively large and two coupled prisms enclosing a volume-phaseholographic grating are envisioned. One paraxial f/2.6 camera per arm with 6 , possibly 7 lenses images the light then on the $61.4 \times 61.4 \mathrm{~mm}$ CCD. The detectors themselves are the current STA $4 \mathrm{k} \times 4 \mathrm{k} 15-\mu \mathrm{m}$ thinned devices foreseen for first light of the PEPSI spectrograph at the LBT.

To increase the overall efficiency of the instrument we intend to employ a "waveguide" image slicer, as for PEPSI (www.aip.de/pepsi). With a 3-slice slicer and a $120 \mu \mathrm{m}$ core fiber we would reach a $3^{\prime \prime}$ aperture on the sky at a spectral resolving power of 100,000. With the $1.5 \mathrm{~m}$ entrance aperture of GREGOR and an estimated $10 \%$ overall efficiency, a $\mathrm{S} / \mathrm{N}$ ratio of 200:1 could be reached in 1 hour integration time for a star of 10th magnitude. The practical limiting magnitude is set by the accumulated guiding errors for coadding individual CCD frames (together with the overall system efficiency) and is expected to be near 13-14th magnitude in $V$. 

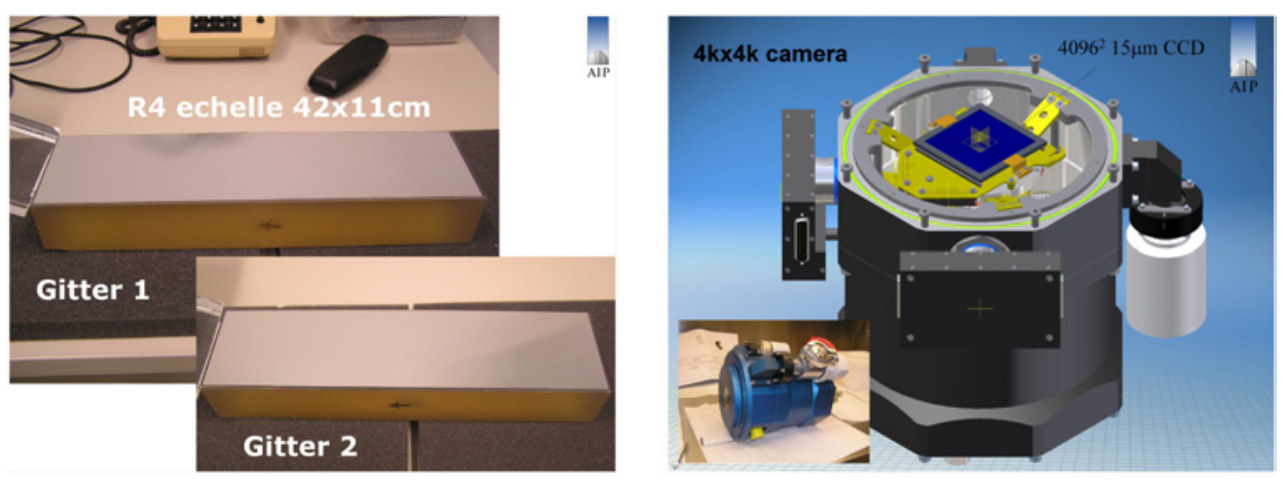

Figure 3. Left: The two R4 echelle gratings for Gregor@Night from Richardson Grating Lab. Right: Design of the $4 \mathrm{k}-\mathrm{CCD}$ camera.
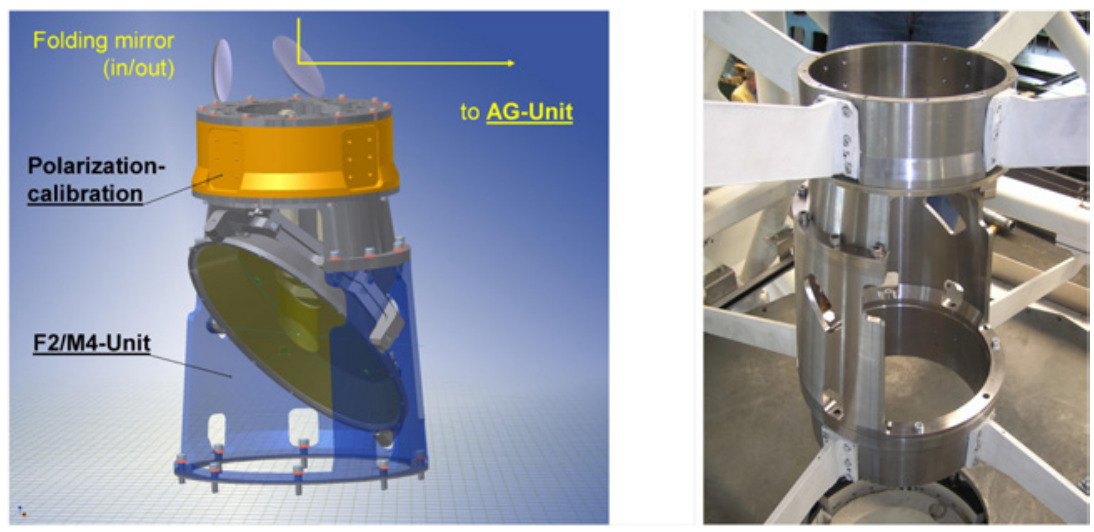

Figure 4. Left: Concept of the light pick-up system. A folding mirror must be inserted into the Gregorian beam to divert the light to the night-time acquisition and guiding (AG) unit. Right: It is foreseen to be mounted on top of the solar-light polarimetric calibration unit, itself mounted on the F2/M4 unit shown.

\section{The day-night interface}

The robotic acquisition and guiding (AG) unit is based on the STELLA design (see www.aip.de/stella). In this case acquisition and guiding is performed on a frame-transfer CCD with a grey beam splitter of approximately $3 \%$ of the light of the target. An aluminized glass plate with an aperture stop also protects the fiber micro lens. Light injection into the STSU fiber would be through the GREGOR F2-focus at $\mathrm{f} / 6$.

After a manual switch to night-time operation by the day-time telescope operator, a weather station takes over the night-time control as a master in a slave/master environment. The target scheduling, acquisition, guiding and data management is envisioned in full automatic mode, no night-time personnel is required to run the spectrograph. Data would be transported to Germany via the Internet and automatically run through an analysis pipeline. 


\title{
Multi-wavelength observations at the German VTT on Tenerife - Possible combinations of post-focus instruments
}

\author{
C. Beck ${ }^{1, *}$, K. Mikurda ${ }^{1}$, L. R. Bellot Rubio ${ }^{2}$, T. Kentischer ${ }^{1}$, and M. Collados ${ }^{3}$ \\ ${ }^{1}$ Kiepenheuer-Institut für Sonnenphysik, Freiburg, Germany \\ ${ }^{2}$ Instituto de Astrofísica de Andalucía (CSIC), Granada, Spain \\ ${ }^{3}$ Instituto de Astrofísica de Canarias, La Laguna, Spain \\ *Email: cbeck@kis.uni-freiburg.de
}

\begin{abstract}
To study the small-scale dynamic processes of magneto-convection in the solar photosphere in more detail than currently achieveable, not only the spatial resolution has to be increased, but also the information content of observations. In order to do so, several wavelengths and spectral lines must be observed simultaneously. This is often achieved by coordinated campaigns at several telescopes with different post-focus instrumentation. The German Vacuum Tower Telescope (VTT) on Tenerife offers the possibility to operate several dedicated instruments - spectrometers, polarimeters, imaging systems - at the same time. We describe some of the possible combinations of post-focus instruments, and present examples of multi-wavelength data obtained recently.
\end{abstract}

\section{Introduction}

Progress in solar physics has been achieved recently on both the observational and the theoretical side. Simulations of the solar atmosphere now extend from photospheric layers up to the chromosphere (e.g. Wedemeyer et al. 2004). The new Swedish 1-m telescope on La Palma has led to the discovery of several peculiarities that went unnoticed before, e.g., the dark cores of penumbral filaments seen by Scharmer et al. (2002) and Sütterlin et al. (2004). The same amount of surprises is to be expected with the advent of the next-generation solar telescopes, like the 1.5-m GREGOR telescope on Tenerife (von der Lühe et al. 2001) or the 4-m ATST in Hawaii (Keil et al. 2003). However, the increase of spatial resolution does not make life much easier for observers. To understand the physical processes on the Sun not only the spatial resolution - which determines the spatial scales that are visible - is critical, but also the information content of the observations - that allows to understand what is happening. In the excitement over the new observational findings, the equally important issue of information content seems to be undervalued. In this contribution, we show how the German Vacuum Tower Telescope on Tenerife can be used to maximize the information content of solar observations at a moderate spatial resolution of around 1" by operating several post-focus instruments simultaneously. 

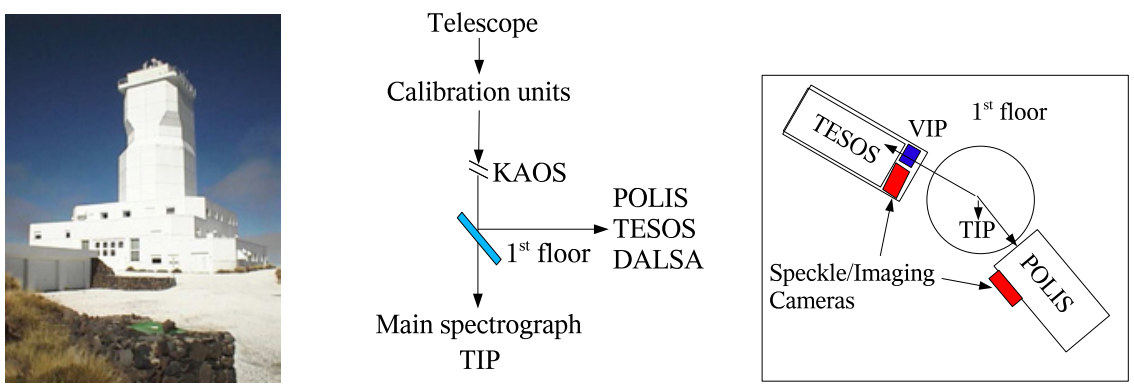

Figure 1. The building of the German VTT (left) with a sketch of the optical layout (middle) and the location of the post-focus instruments in the first floor (right). The light can be divided between the main spectrograph in the basement and the optical lab in the first floor.

\section{The German Vacuum Tower Telescope}

The German Vacuum Tower Telescope consists of a coelostat system on top of the elevenfloor building, and a 70-cm primary mirror of $46 \mathrm{~m}$ focal length. The light is fed in downwards by the coelostat, and the focal plane is finally located close to the first floor of the building. Before reaching the focus, the light passes through the Kiepenheuer-Institute adaptive optics system (KAOS, von der Lühe et al. 2003) that improves the spatial resolution by a real-time correction of wavefront aberrations. With good seeing conditions, the diffraction limit of around 0.' 6 in infrared wavelengths can be reached. Calibration units for infrared and visible wavelengths can be inserted in front of the adaptive optics system for the derivation of the polarimetric properties of each instrument (cf. middle panel of Fig. 1). In the first floor, the light can be divided between the main spectrograph in the basement and the post-focus instruments located in the first floor. As dichroic beamsplitters are available, this can be done without any loss of light.

\section{Post-focus instrumentation and possible combinations}

The POlarimetric LIttrow Spectrograph (POLIS, Beck et al. 2005) is a stand-alone slitspectrograph vector polarimeter with two channels. The first channel observes the full Stokes vector of the two photospheric Fe I lines at $630 \mathrm{~nm}$, while the second channel observes the intensity profile of the chromospheric Ca II H line. Two slits of 0 .' $^{\prime} 18$ and 0.'48 width are available. The spatial sampling along the $45^{\prime \prime}$-slit $\left(90^{\prime \prime}\right.$ with polarizing beamsplitter rotated) is 0.'15@630 nm. The Tenerife Infrared Polarimeter (TIP, Collados et al. 2007) is a slit-spectrograph vector polarimeter for infrared wavelengths between $1 \mu \mathrm{m}$ and $2 \mu \mathrm{m}$. The instrument is integrated into the main spectrograph. The slit width can be varied between 0.37 and 0!'90. The 70" -slit is sampled with 0.'17 per pixel. The TElecentric SOlar Spectrometer (TESOS, Kentischer et al. 1998) is a 2-D spectrometer that uses three etalons in telecentric mounting for wavelength scanning. It can be used in a wavelength range from 400 to $800 \mathrm{~nm}$. With its prefilter wheel up to 4 different spectral ranges can be observed

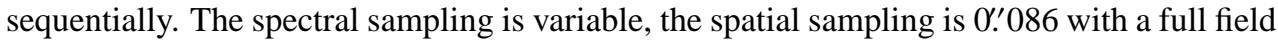
of view (FOV) of around $40^{\prime \prime} \times 40^{\prime \prime}$. The KIS-IAA Visible Imaging Polarimeter (VIP, Bellot 


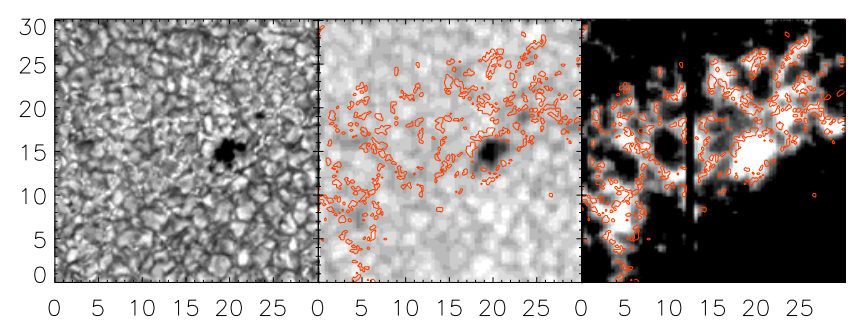

Figure 2. Left to right: G-band intensity, continuum intensity in IR, magnetic flux. Contours outline G-band bright points. Scales are in arcsecs.

Rubio et al. 2006) is not a stand-alone instrument, but a modulator package with nematic liquid crystals that can be used to upgrade TESOS (or the main spectrograph) to a full vector or Stokes-V polarimeter. It can be used for wavelengths of 400 to $800 \mathrm{~nm}$ like TESOS. Due to its dual beam design, the FOV for VIP@TESOS is $20^{\prime \prime} \times 40^{\prime \prime}$. The DALSA cameras are fast $1 \mathrm{k} \times 1 \mathrm{k}$ cameras that can be used to obtain the image bursts necessary for speckle reconstruction. Spatial sampling and FOV depend on the image scale used; a sampling of $0{ }^{\prime} 05$ in a FOV of $60^{\prime \prime} \times 60^{\prime \prime}$ is usual. The DALSA cameras can be used in any wavelength for which suitable prefilters are available, e.g., in the $\mathrm{G}$ band. Additional low-noise cameras (AT, XEDAR, PCO) are also available for imaging in spectral lines with very low light level (H $\alpha$, Ca II K). TIP can be used simultaneously with either TESOS (or VIP@TESOS) and any imaging/speckle channel in front of TESOS, or with POLIS and an imaging/speckle channel @ $500 \mathrm{~nm}$ at the side of POLIS (cf. rightmost panel of Fig. 1).

\section{Examples of multi-wavelength data}

We have chosen two data sets from 2005 and 2006 as examples of multi-wavelength data. In 2005, we used the combination of TIP and TESOS together with a speckle channel in the G band in front of TESOS. We observed a pore and the surrounding area with network fields and quiet Sun at a heliocentric angle of $12^{\circ}$ on October, 11, 2005. Figure 2 shows a section of the FOV in the $\mathrm{G}$ band and the continuum intensity at $1.5 \mu \mathrm{m}$ after alignment of the data. The G-band images were speckle-reconstructed to improve the spatial resolution up to the diffraction limit. The spectra of TIP have been inverted with the SIR code (Ruiz Cobo \& del Toro Iniesta 1992) to obtain the magnetic field vector and the magnetic flux (rightmost image). With this combination, one can study the structure and evolution of magnetic fields and their signature in the $\mathrm{G}$ band. The intensity profiles of the FeI line at $557.6 \mathrm{~nm}$ from TESOS are not included yet, but will give additional information on temperature stratification and flow velocities at higher spatial resolution than the TIP data.

The second example (Fig. 3) shows data obtained in July 2006, with the combination of TIP and POLIS. A DALSA speckle camera was placed at the side of POLIS, observing at a wavelength of $500 \mathrm{~nm}$. This combination of instruments yields information on the photospheric magnetic fields from four different magnetic sensitive spectral lines, covering a large height range in the solar photosphere. The second channel of POLIS with the chromospheric $\mathrm{Ca}$ II $\mathrm{H}$ line yields information on the chromospheric signature of areas where fields are present or absent in the field of view. This allows to address the question of the chromospheric heating mechanism in different structures, like in the magnetic network and 

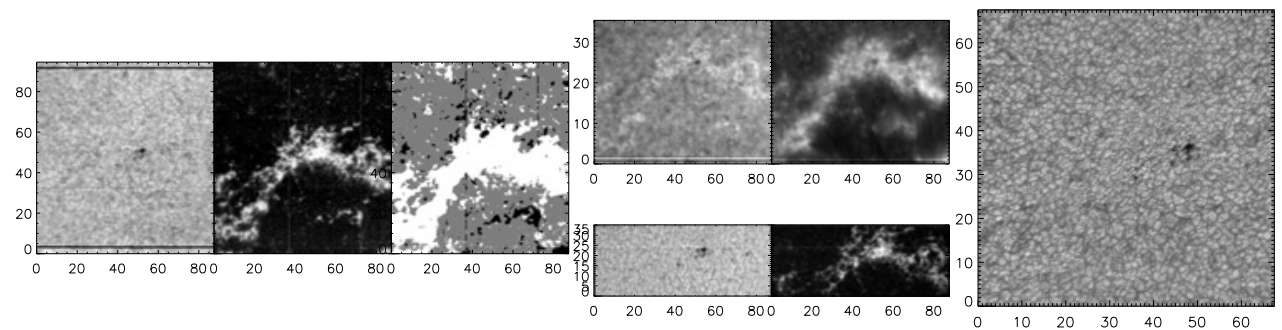

Figure 3. Left: POLIS@630nm, Stokes I, Stokes V, polarity of fields. Middle, top: POLIS@396nm, wing intensity, line core intensity. Middle, bottom: TIP@1565 nm, Stokes I, Stokes V. Right: specklereconstructed DALSA image @ $500 \mathrm{~nm}$. The DALSA image has been taken from the middle of the series of about 80 speckle bursts, which were taken during the scan across the area by TIP and POLIS.

in the internetwork, which is devoid of strong field concentrations.

\section{Conclusions}

The German Vacuum Tower Telescope allows to combine several post-focus instruments for simultaneous multi-wavelength observations. Spectropolarimetry in the photosphere can be combined with high-resolution imaging in photospheric or chromospheric lines. The Ca II $\mathrm{H}$ channel of POLIS allows to obtain chromospheric spectra. The possible combinations of the instruments TIP, TESOS, POLIS, VIP, and speckle (or imaging) channels open a variety of options to address current topics in solar physics, where multi-wavelength information is crucial.

Acknowledgements. This work was supported by the DFG under grant SCHL 514/2-1 and SCHM 1168/3, and by the Spanish MEC under Programa Ramón y Cajal and project ESP2003-07735-C0403. The VTT is operated by the Kiepenheuer-Institut für Sonnenphysik at the Spanish Observatorio del Teide of the Instituto de Astrofísica de Canarias (IAC).

\section{References}

Beck, C., Schmidt, W., Kentischer, T., \& Elmore, D. 2005, A\&A, 437, 1159

Bellot Rubio, L. R., Tritschler, A., Kentischer, T., Beck, C., \& Del Toro Iniesta, J. C. 2006, Solar Active Regions and 3D Magnetic Structure, 26th meeting of the IAU, Joint Discussion 3, 16-17 August, 2006, Prague, Czech Republic, JD03, \#58, 3

Collados, M., Lagg, A., Díaz García, J. J., et al. 2007, in ASP Conf. Ser., in prep.

Keil, S., Rimmele, T., Keller, C., \& The ATST Team. 2003, Astron. Nachr., 324, 303

Kentischer, T. J., Schmidt, W., Sigwarth, M., \& Uexkuell, M. V. 1998, A\&A, 340, 569

Ruiz Cobo, B. \& del Toro Iniesta, J. C. 1992, ApJ, 398, 375

Sütterlin, P., Bellot Rubio, L. R., \& Schlichenmaier, R. 2004, A\&A, 424, 1049

Scharmer, G. B., Gudiksen, B. V., Kiselman, D., Löfdahl, M. G., \& Rouppe van der Voort, L. H. M. 2002, Nature, 420, 151

von der Lühe, O., Schmidt, W., Soltau, D., et al. 2001, Astron. Nachr., 322, 353

von der Lühe, O., Soltau, D., Berkefeld, T., \& Schelenz, T. 2003, in Proceedings of the SPIE, Volume 4853,187

Wedemeyer, S., Freytag, B., Steffen, M., Ludwig, H.-G., \& Holweger, H. 2004, A\&A, 414, 1121 


\title{
Instrumental developments at the Gregory Coudé Telescope (GCT) at IRSOL
}

\author{
M. Bianda ${ }^{1,2, *}$, R. Ramelli ${ }^{1}$, A. Feller ${ }^{2}$, J. O. Stenflo ${ }^{2,3}$, and G. Küveler ${ }^{4}$ \\ ${ }^{1}$ Istituto Ricerche Solari Locarno, Locarno, Switzerland \\ ${ }^{2}$ Institute of Astronomy, ETH Zentrum, Zurich, Switzerland \\ ${ }^{3}$ Faculty of Mathematics \& Science, University of Zurich, Zurich, Switzerland \\ ${ }^{4}$ Fachhochschule Wiesbaden, Rüsselsheim, Germany \\ *Email: mbianda@irsol.ch
}

\begin{abstract}
The research projects carried out with the Gregory Coudé Telescope (GCT) at Istituto Ricerche Solari Locarno (IRSOL) are mainly focused on high precision polarimetry. The Zurich IMaging POLarimeter (ZIMPOL) developed at ETH Zurich and installed permanently at the GCT at IRSOL allows a polarimetric precision down to $10^{-5}$ to be reached. This makes it possible to perform several accurate spectro-polarimetric measurements of scattering polarization and to investigate solar magnetic fields through the signatures of the Hanle and Zeeman effects. The research programs are currently being extended to monochromatic imaging of the Stokes vector with a recently installed Fabry-Perot rapidly tunable filter system with a narrow pass band of about $30 \mathrm{~m} \AA$. The spatial resolution is being improved by the installation of an adaptive optics (AO) system.
\end{abstract}

\section{Introduction}

The great advances in high precision polarimetry that have been achieved with the introduction of the Zurich IMaging POLarimeter (ZIMPOL) a decade ago opened a new window in solar physics. Polarimetry is in fact a very powerful tool that can be used to study solar magnetic fields as well as the physical processes behind the generation of polarization in atomic and molecular spectral lines. Magnetic field measurements through Zeeman effect signatures, which appear in the presence of strong and oriented magnetic fields, have long been performed at many observatories. With the high polarimetric precision of ZIMPOL it has become possible to extend the magnetic field diagnostics to weak fields and to fields which are tangled on scales below the spatial resolution, which are invisible to the Zeeman effect but get revealed by the Hanle effect (Hanle 1924).

Spectro-polarimetry is currently the main field of research at the Istituto Ricerche Solari Locarno (IRSOL). Advantage is taken from the circumstance that a ZIMPOL system is permanently installed at IRSOL. In addition, the GCT is very well suited for polarimetric measurements, since the amount of instrumental polarization is low and stays practically constant during the observing day, since it is a function of declination only. Therefore it can easily be accounted for. With a Fabry-Perot filter system and an adaptive optics system recently installed at IRSOL we plan to start several new interesting projects. 


\section{IRSOL - The institute}

The observatory at the Istituto Ricerche Solari Locarno (IRSOL), located in southern Switzerland, was constructed in 1958/59 by the Universitäts-Sternwarte Göttingen (USG), Germany. In 1984, after USG moved its observing activity to the facilities at Observatorio del Teide on Tenerife, a local foundation (FIRSOL) acquired the observatory in Locarno. The partially dismantled instrumentation was rebuilt and improved, in collaboration with USG (now IAG), Technical Highschool (FH) of Applied Sciences of Wiesbaden (Germany), and the Institute of Astronomy at ETH Zurich. The scientific collaboration with ETH Zurich allowed the implementation at IRSOL of an important polarimetry observing program, first with a beam exchange polarimeter and then with ZIMPOL.

\section{Instrumentation at IRSOL}

The IRSOL telescope is a $45 \mathrm{~cm}$ aperture Gregory coudé type instrument with $25 \mathrm{~m}$ effective focal length. A field stop with a pinhole of about $2.5 \mathrm{~mm}$ diameter in the prime focus reduces the field of view to a 200 arcsec diameter circular image. The rest of the solar image is reflected away from the main light beam. This reduces heating and scattered light and is of particular advantage when observing low intensity structures like sunspots, spicules, or prominences. The relative orientation of the two folding mirrors M3 and M4 (coudé) changes only with declination and is orthogonal at the time of the equinoxes. As a consequence the instrumental polarization, originating through oblique reflections, is almost constant during the day and virtually vanishes during the equinoxes (Sánchez Almeida et al. 1991). A Gregory coudé type telescope is thus very well suited for polarimetric measurements.

An automatic guiding system developed by the FH Wiesbaden (Küveler et al. 1998) is also available. Its operation is based on the solar image obtained from the light reflected by the field stop in the primary focus.

The Czerny-Turner spectrograph with $10 \mathrm{~m}$ focal length is based on a $180 \times 360 \mathrm{~mm}$ grating with 316 lines per $\mathrm{mm}$ and $63^{\circ}$ blaze angle. A prism based predisperser allows to select the spectral band entering in the spectrograph without overlap of the grating orders.

Monochromatic imaging observations of the solar surface can be performed using the recently installed Fabry-Perot filter system in collimated configuration (Feller et al. 2006). The system uses two temperature controlled lithium niobate etalons with an aperture of $70 \mathrm{~mm}$. The transmitted wavelength can be selected by electrically tuning the refractive index of the etalon medium, by varying the temperature, or by tilting the etalon. The bandwidth is about $30 \mathrm{mÅ}$.

An adaptive optics (AO) system based on a tip-tilt mirror and a 37 actuator deformable mirror is currently being installed and tested in collaboration with the University of Applied Sciences of Southern Switzerland, SUPSI, and with ETH Zurich. The system follows the design of the infrared AO system installed at the McMath-Pierce Solar Telescope at Kitt Peak (Keller et al. 2003). The first tests made with the tip-tilt mirror have already given good results (see Figure 1).

Two polarimeters are available. The oldest one operating at IRSOL is the dual beam exchange device based on a Savart plate and rotating quarter and half wave retarder plates (Bianda et al. 1998). A polarimetric precision of a few $10^{-4}$ can be reached, but it can be affected by seeing-induced cross-talk, because the technique requires two exposures taken 


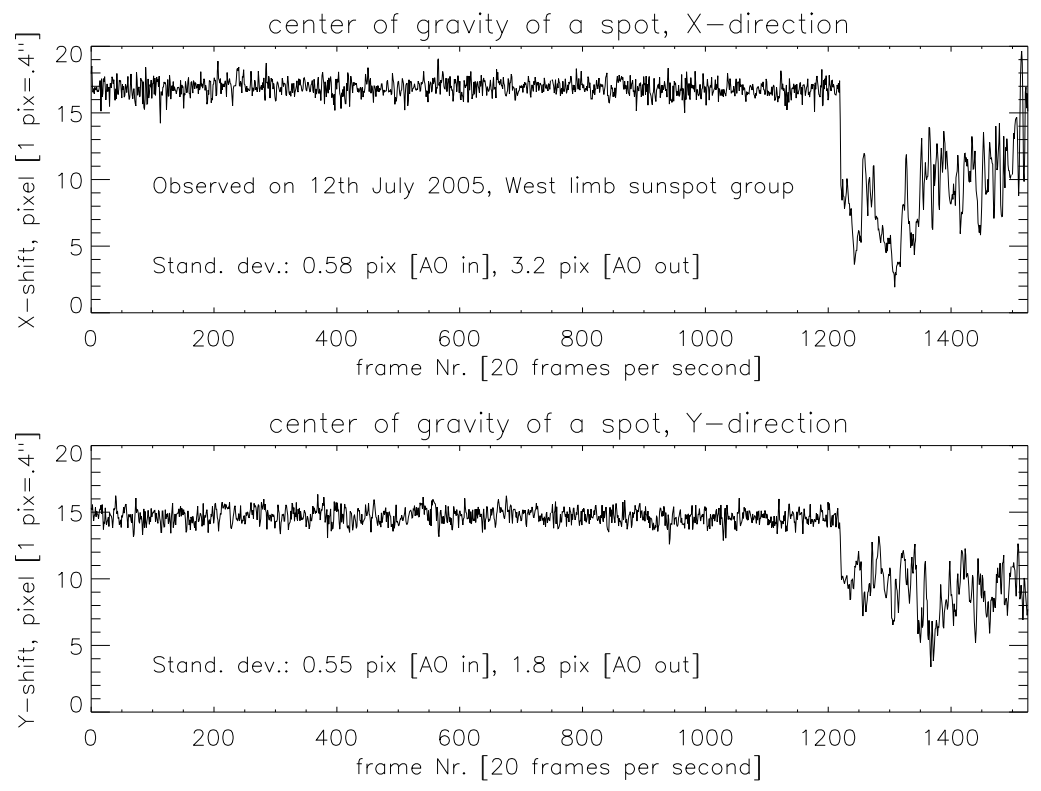

Figure 1. First tests with the tip-tilt correction of the AO system. The two plots show the displacement of the barycentre of a sunspot in the $x$ resp. $y$ direction with and without tip-tilt correction. The tip-tilt mirror was switched off around frame number 1200 .

at different times. The second polarimeter is ZIMPOL (Povel 1995; Gandorfer et al. 2004), which is installed permanently at IRSOL since 1998. Its main advantage is that it is free from seeing-induced effects thanks to its high modulation rate: $42 \mathrm{kHz}$ (obtained with a piezoelastic modulator), or $1 \mathrm{kHz}$ (obtained with ferro-electric liquid crystal modulators). Another advantage is that the same pixels of the CCD ZIMPOL sensor are used to measure all Stokes parameters. Therefore the Stokes $Q / I, U / I$ and $V / I$ images are not influenced by different pixel efficiencies. The ZIMPOL polarimetric accuracy depends mainly on the photon statistics. With long exposure times it has already been possible at IRSOL to reach an accuracy of about $10^{-5}$.

\section{Scientific research projects at IRSOL}

The research projects at IRSOL take advantage of the very good polarimetric and spectral accuracy of the instrumentation. A large amount of observing time is available to carry out monitoring measurements or for projects requiring long observing times (which cannot easily be done at large telescope facilities, where the observing time is shared by different research groups according to a predefined program). In addition it is possible to be very flexible with the programs to allow fast reaction to particular solar events. The modular layout of the instrumentation inside the observing room is very convenient for installation and testing of new instrumentation.

Examples of the scientific results obtained at IRSOL in recent years are: 
- Investigations of the Hanle effect in the quiet chromosphere (Bianda et al. 1999).

- Publication of the first two volumes of the "Atlas of the Second Solar Spectrum" (Gandorfer 2000, 2002).

- Discovery of vast amounts of hidden magnetism in the solar photosphere (Trujillo Bueno et al. 2004; Stenflo 2004).

- Determination of novel constraints on impact polarization in solar flares (Bianda et al. 2005).

- Measurements of full Stokes profiles in prominences in $\mathrm{H} \alpha, \mathrm{He} \mathrm{D}_{3}, \mathrm{H} \beta$, and in spicules in $\mathrm{He}_{3}$ (Ramelli et al. 2005a,b).

- First polarimetric measurements of the Paschen-Back effect in $\mathrm{CaH}$ transitions (Berdyugina et al. 2006).

Different other observing programs are also foreseen in the future. They will focus on solar magnetism and polarimetry with the Fabry-Perot filter system or with the spectrograph. They also include synoptic type programs (e.g. variations of the Hanle-effect signatures with heliographic latitude and solar cycle). Furthermore IRSOL is open to coordinated type programs with other observatories: simultaneous observations of solar features with complementary sets of instruments or supporting type observations that complement the science of another project.

Acknowledgements. We are grateful for the financial support provided by the canton of Ticino, the city of Locarno, ETH Zurich and the Fondazione Aldo e Cele Daccò.

\section{References}

Berdyugina, S. V., Fluri, D. M., Ramelli, R., Bianda, M., Gisler, D., \& Stenflo, J. O. 2006, ApJ, 649, L49

Bianda, M., Solanki, S. K., \& Stenflo, J. O. 1998, A\&A, 331, 760

Bianda, M., Stenflo, J. O., \& Solanki, S. K. 1999, A\&A, 350, 1060

Bianda, M., Benz, A. O., Stenflo, J. O., Küveler, G., \& Ramelli, R. 2005, A\&A, 434, 1183

Feller, A., et al. 2006, Proceedings of the Fourth Solar Polarization Workshop, Boulder 2005

Gandorfer, A. 2000, VdF, ISBN 3-7281-2764-7

Gandorfer, A. 2002, VdF, ISBN 3-7281-2844-4

Gandorfer, A., et al. 2004, A\&A, 422, 703

Hanle, W. 1924, Z. Phys., 30, 93

Keller, C. U., Plymate, C. \& Ammons, S. M. 2003, Proceedings of the SPIE, 4853, 35

Küveler, G., et al. 1998, Solar Phys., 182, 247

Povel, H. P. 1995, Opt. Eng. 34, 1870

Ramelli, R., \& Bianda, M. 2005a, Astron. Astrophys. Space Science Library, 320, 215

Ramelli, R., Bianda, M., Trujillo Bueno, J., Merenda, L., \& Stenflo, J. O. 2005b, in D. E. Innes, A.

Lagg, \& S. K. Solanki (eds.), ESA SP-596: Chromospheric and Coronal Magnetic Fields

Sánchez Almeida, J., Martínez Pillet, V., \& Wittmann, A. D. 1991, Solar Phys., 134, 1

Stenflo, J. O. 2004, Nature, 430, 304

Trujillo Bueno, J., Shchukina, N., \& Asensio Ramos, A. 2004, Nature, 430, 326 
Modern Solar Facilities - Advanced Solar Science, 63-66

F. Kneer, K. G. Puschmann, A. D. Wittmann (eds.)

(C) Universitätsverlag Göttingen 2007

\title{
Imaging polarimetry with a tunable narrow-band filter
}

\author{
A. Feller ${ }^{1, *}$, M. Bianda ${ }^{2}$, and J. O. Stenflo ${ }^{1,3}$ \\ ${ }^{1}$ Institute of Astronomy, ETH Zurich, Switzerland \\ ${ }^{2}$ Istituto Ricerche Solari Locarno, Switzerland \\ ${ }^{3}$ Faculty of Mathematics \& Science, University of Zurich, Switzerland \\ *Email: feller@astro.phys.ethz.ch
}

\begin{abstract}
We have developed a fully tunable narrow-band filter system to be used in combination with ZIMPOL for monochromatic imaging vector polarimetry. It is designed for the whole visible spectrum, from the UV at about $395 \mathrm{~nm}$, to the red at about $660 \mathrm{~nm}$, with a band width of 30-50 mA. The main components are two lithium niobate Fabry-Pérot etalons made of Y-cut crystals, which means that the transmission spectra and tuning parameters are different for the two orthogonal states of polarisation. This allows to enhance the finesse with a double-pass configuration where the polarisation is rotated by 90 degrees between the passes, a possibility that is not available to other Fabry-Pérot systems. The filter system is available in two different optical configurations: telecentric, for high spatial resolution, and collimated, with a large field of view and high throughput. The telecentric configuration is compact and can be easily used at foreign telescopes like the SST, whereas the collimated configuration is permanently installed at IRSOL. We present the instrument and its operation.
\end{abstract}

\section{Introduction}

During the last decade our institute has developed the powerful CCD-based imaging polarimeter system ZIMPOL (Zurich IMaging POLarimeter), described in detail by Povel (1995) and Gandorfer et al. (2004). Until now ZIMPOL has mainly been used in combination with a spectrograph (cf. Stenflo 2004). The new narrow-band filter system will allow us to combine high-precision polarimetry with quasi-monochromatic imaging, thus providing a new scientific dimension to our work with ZIMPOL. An exploratory attempt in this direction with the UBF at Sacramento Peak Observatory (New Mexico/U.S.A.) has given a first glimpse of the type of data that may be obtained (Stenflo et al. 2002).

\section{Instrument}

Our filter system is based on two Fabry-Pérot (FP) etalons, made of Y-cut lithium niobate crystals. They are birefringent and each optical axis has a different transmission spectrum and different tuning parameters.

The system is currently available in two optical setups (cf. Figure 1), where the etalons are placed in series (tandem configuration). The collimated setup is permanently installed at the Istituto Ricerche Solari Locarno (IRSOL) and provides a large field of view and high 

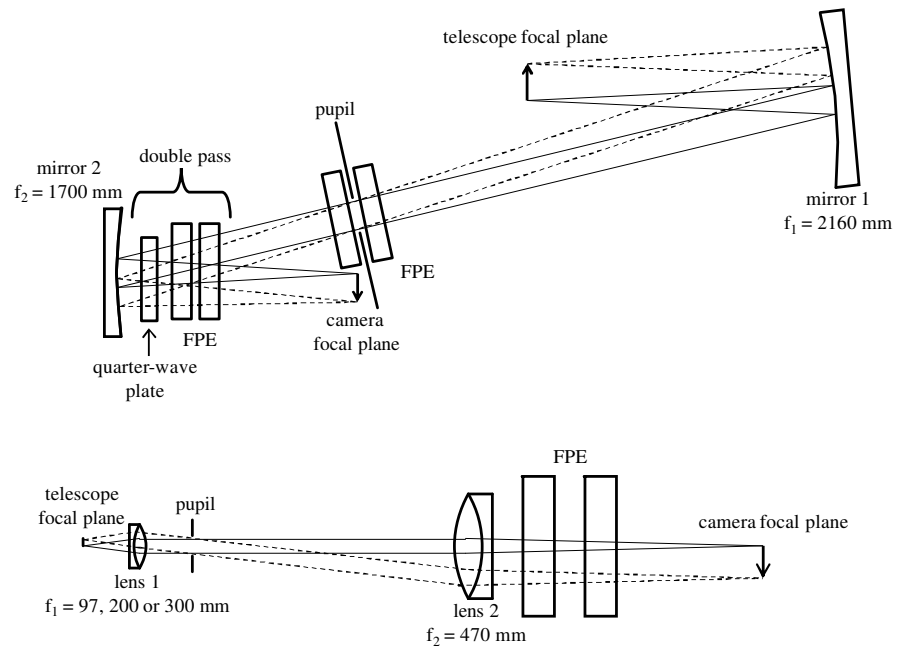

Figure 1. Schematic drawing of the two available optical setups (not to scale). Top: Collimated setup. This setup is designed for low spatial but high spectral resolution. It also permits to experiment with a double-pass configuration. Bottom: Telecentric setup. At the highest magnification ZIMPOL works close to the diffraction limit of the Swedish Solar Telescope. The compact design aims at portability.

Table 1. Properties of the optical setups

\begin{tabular}{lll}
\hline & Collimated (IRSOL) & Telecentric (SST) \\
\hline Camera illumination & $\mathrm{F} / 44$ & $\mathrm{~F} / 223, \mathrm{~F} / 108, \mathrm{~F} / 72$ \\
Field of view & $181 \times 132 \operatorname{arcsec}^{2}$ & $29 \times 21-91 \times 66 \mathrm{arcsec}^{2}$ \\
Max. spatial resolution & $0.5 \operatorname{arcsec}(\mathrm{sampling} \mathrm{limited})$ & $0.1 \operatorname{arcsec}($ diffraction limited) \\
Effect on filter passband & field-dependent blue-shift of $22 \mathrm{~m} \AA$ & broadening of $1 \mathrm{~m} \AA$ \\
Used FP aperture & $40-60 \mathrm{~mm}$ & $24-27 \mathrm{~mm}$ \\
Imaging optics & spherical mirrors & achromatic doublet lenses (F2 / N-BK7) \\
Unfolded setup length & $9.3 \mathrm{~m}$ & $1.4-1.8 \mathrm{~m}$ \\
\hline
\end{tabular}

throughput. This setup will also be used later on to experiment with a double-pass configuration, where the polarisation is rotated by 90 degrees between the passes, as described by Netterfield et al. (1997). The telecentric setup works at higher magnifications. Although it can also be used at IRSOL, it is mainly designed for observations with the Swedish Solar Telescope (SST). The main properties of both setups are summarised in Table 1.

\section{Operation}

The transmission spectrum of one FP etalon can be altered by varying the optical thickness of the cavity. With our $\mathrm{LiNbO}_{3}$ etalons this can be achieved in three ways: tilting broadens 

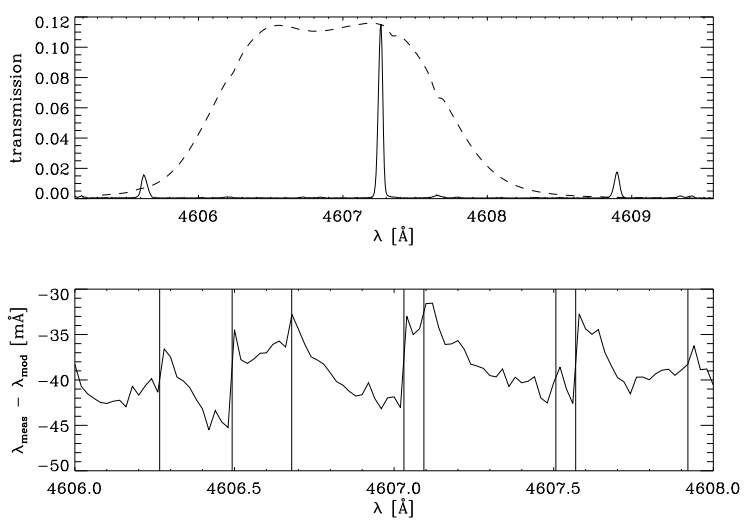

Figure 2. Top: Example of the tandem transmission spectrum (solid curve) in the range of the $\mathrm{Sr}$ I prefilter (dashed curve). The prefilter curve is scaled to the tandem peak transmission. Bottom: Difference $\lambda_{\text {meas }}-\lambda_{\text {mod }}$ between measured and modelled peak positions during a tandem scan through the prefilter. Apart from the $40 \mathrm{~m} \AA$ offset, which can be easily calibrated, the residuals are in the order of $5 \mathrm{~mA}$ or about $10 \%$ of the FWHM. At the positions marked by the vertical lines the combination of the individual etalon transmission peaks is switched.

the transmission peaks and is mainly used for ghost suppression (cf. Feller et al. 2007).

Temperature tuning is slow ( $\sim 10 \mathrm{~m} \AA /$ minute) and is used to set the etalons at an optimum home position within a spectral region of interest. Tuning by voltage is much faster ( $\sim 80 \mathrm{~m} \AA / \mathrm{s})$ and is used for spectral scans or to cycle between several discrete spectral positions, which is required for differential Zeeman and Hanle diagnostics. Under computer control one has the possibility to access these three tuning parameters within the framework of the ZIMPOL II software, which allows to script different measuring procedures and to synchronise the etalons for tandem operation.

As opposed to air-spaced etalons it is difficult to model the wavelength dependence of the optical cavity thickness with sufficient accuracy at once for the whole spectral working range. We therefore calibrate our etalons for each prefilter separately. With the help of the high-resolution spectrograph available at IRSOL we record the transmission spectra of the individual etalons at different temperatures $T$ and voltages $V$ and derive from them $j$ model parameters $p_{i, j}(T, V)$ for each etalon and optical axis combination $i$. The so calibrated etalon models allow us to predict the spectral position of any transmission peak within the prefilter passband with an accuracy of a few $m \AA$, which is required to keep the etalons in phase (cf. Figure 2).

\section{Conclusions and outlook}

The test measurements at IRSOL (cf. example in Figure 3) have shown that the tandem configuration is performing well in both optical setups. The telecentric setup will have its debut at the SST in October this year for observations of limb-scattering polarisation at high spatial resolution, namely in Ca I (4227 ̊) and Sr I (4607 ̊). 
A. Feller et al.: Imaging polarimetry with a tunable narrow-band filter
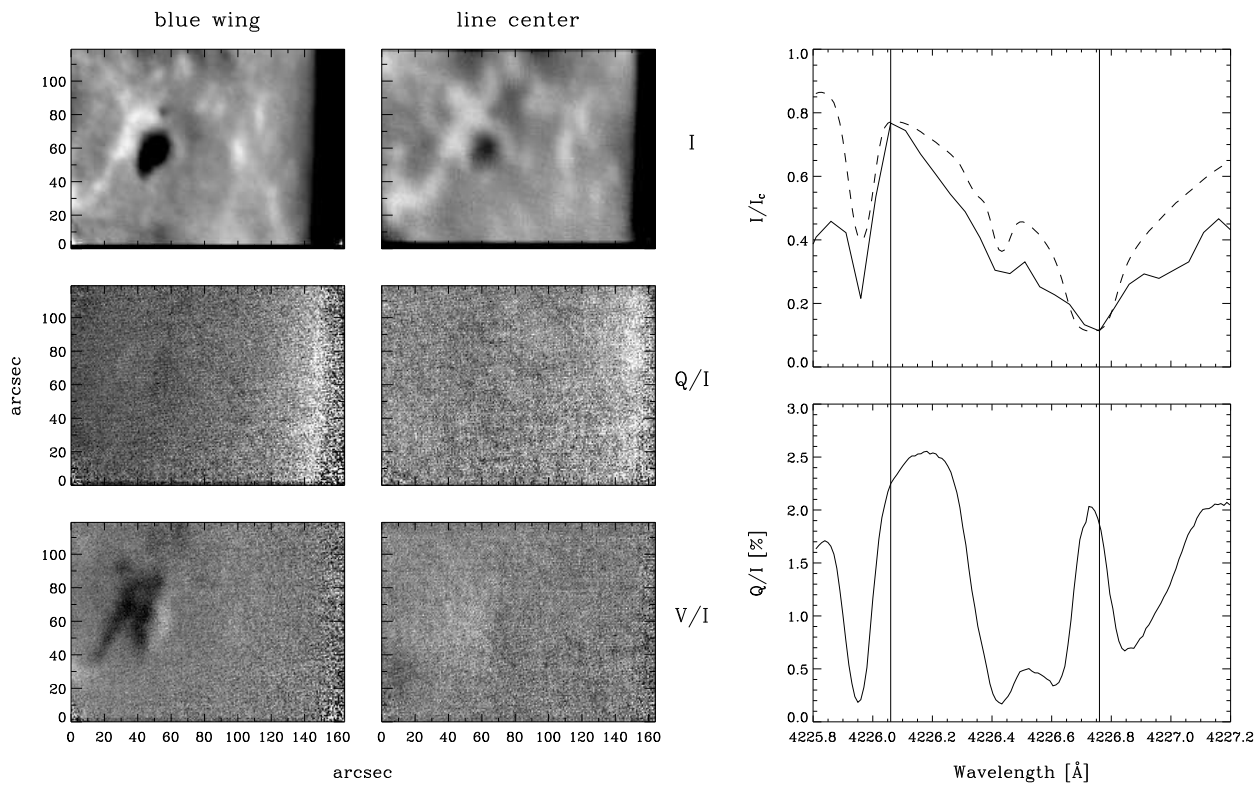

Figure 3. First light observation in the Ca I prefilter of the active region NOAA 10905, close to the west limb on August 31, 2006. Upper right: scan with the FP filter (solid line). As comparison, the corresponding spectrum from Gandorfer (2002) is overplotted (dashed line). Lower right: $Q / I$ from Gandorfer (2002) for a limb distance of $\mu=0.1$. Left: Series of Stokes images recorded with ZIMPOL at the two spectral positions indicated by the vertical lines in the scan. The amplitude of the greyscale for Stokes $Q / I$ and $V / I$ is $2 \%$. To enhance the contrast, the limb darkening has been removed in the Stokes $I$ images.

In parallel to scientific work with the current setups, instrumental development will go on at IRSOL in terms of the described double-pass configuration and a hybrid system, based on the filter and the IRSOL spectrograph.

Acknowledgements. Support for this work has been obtained through grants Nos. 200020-101603 and 2160-64533.01 from the Swiss Nationalfonds and grant No. 0-43066-01 from ETH Zurich.

\section{References}

Feller, A., Boller, A., Stenflo, J. O. 2007, in Proc. 4th International Workshop of Solar Polarization, ed. R. Casini \& B. W. Lites, ASP Conf. Series, in press

Gandorfer, A. M. 2002, The Second Solar Spectrum. Volume 2: $3910 \AA$ to 4630 A. (vdf, ETH Zurich)

Gandorfer, A. M., Steiner, H. P., Povel, P., et al. 2004, A\&A, 422, 703

Netterfield, R. P., Freund, C. H., Seckold, J. A., \& Walsh, C. J. 1997, Appl. Opt., 36, 4556

Povel, H. 1995, Opt. Eng., 34, 1870

Stenflo, J. O. 2004, Rev. Mod. Astron., 17, 269

Stenflo, J. O., Gandorfer, A., Holzreuter, R., Gisler, D., Keller, C. U., \& Bianda, M. 2002, A\&A, 389, 314 
Modern Solar Facilities - Advanced Solar Science, 67-68

F. Kneer, K. G. Puschmann, A. D. Wittmann (eds.)

(C) Universitätsverlag Göttingen 2007

\title{
Methods in high-resolution solar spectroscopy
}

\author{
F. Kneer \\ Institut für Astrophysik, Göttingen, Germany
}

\begin{abstract}
After few historical remarks, the needs in high-resolution spectroscopy are outlined. They are concerned with temporal, spatial, and wavelength resolution for a reasonable field of view of supergranulation cell or larger. At 0 .' $^{\prime} 1$ spatial resolution $(\hat{\approx} 72.5 \mathrm{~km}$ on the Sun) the measurement for a snapshot should be finished within the transit time $t_{\mathrm{tr}} \approx 10 \mathrm{~s}$ (size of resolution element/sound speed). Finally, the performances of grating spectrometers and Fabry-Perot interferometers are compared. The latter are far superior to grating spectrographs due to their high resolution-luminosity product.
\end{abstract}

\section{Introduction}

Joseph von Fraunhofer (re-)discovered in 1819 the principle of diffraction gratings (Born \& Wolf 1970). His first gratings were made by winding fine wires around two parallel screws. About 1846, Friedrich Adolph Nobert ruled, first as test objects for microscopes, grooves with $1 / 4.000 \mathrm{~mm}$ distance into glass which he also used as gratings for spectroscopy (Hentschel 1998). Henry Augustus Rowland revolutionized spectroscopy with the development of his accurate ruling engine for large gratings in 1882 (Hentschel 1998). A further method for spectroscopy, with eventually extremely high spectral resolution, was invented by M. P. A. Charles Fabry and J.-B. Alfred Perot in the year 1897 (Hernandez 1988). Two parallel, flat and semi-transparent mirrors, called an etalon, cause by multi-reflection interference of many rays and lead to a channel spectrum. Since then, from the end of the 19 th century till today at the beginning of the 21 st century, both grating spectrographs and Fabry-Perot interferometers (FPIs) have experienced enormous developments with regard to precision, reflectivity, size and many other requirements. Both are widely in used in astrophysical observations, but have different properties and especially FPIs are more difficult to handle (see, e.g., Kneer \& Hirzberger 2001).

\section{Grating spectrometers vs. FPI spectrometers in high-resolution work}

Let us adopt the following needs to obtain data relevant for future studies into small-scale processes in the solar atmosphere. We wish to observe a reasonable field of view (FOV) of $60^{\prime \prime} \times 60^{\prime \prime}$ with an angular resolution of 0 . $^{\prime \prime}(\hat{\approx} 72.5 \mathrm{~km}$ on the Sun). The spectral resolution should be $\mathcal{R}=\lambda / \delta \lambda=\nabla \times \infty \prime^{\nabla}$ and the temporal resolution should admit to cover the FOV with a cadence of $10 \mathrm{~s}$, which is the transit time $t_{\mathrm{tr}}=72.5 \mathrm{~km} / c_{\mathrm{s}}$ with $c_{\mathrm{s}}=$ soundspeed.

The performance of a spectrometer can be quantified by the resolution-luminosity product

$$
\mathcal{R} \times L=\lambda / \delta \lambda \times S \times \Omega \times \tau,
$$


where $S=$ cross-section of light beam, $\Omega=$ solid angle of beam through $S$, and $\tau=$ transmission of the device, e.g., grating or FPI. Table 1 then compares the properties of grating spectrometers with spectrometers based on FPIs.

Table 1. Comparison of grating spectrometers (GS) vs. Fabry-Perot based spectrometers (FPI).

\begin{tabular}{|c|c|c|c|}
\hline & GS & FPI & ratio FPI/GS \\
\hline $\mathcal{R}$ & $5 \times 10^{5}$ & $5 \times 10^{5}$ & 1.0 \\
\hline$S(\hat{=})$ & $60^{\prime \prime} \times 00^{\prime \prime} 05$ & $60^{\prime \prime} \times 60^{\prime \prime}$ & 1,200 \\
\hline$\Omega$ & $\propto\left(\oslash_{\mathrm{Tel}} / \mathrm{f}_{\mathrm{eff}}\right)^{2}=1 / N^{2}$ & $1 / N^{2}$ & 1.0 \\
\hline$\tau$ & $\approx 0.1$ & $\approx 0.5$ & 5.0 \\
\hline $\begin{array}{l}\text { spectral coverage } \\
(S C)\end{array}$ & $\begin{array}{c}9 \times 0.5 \AA \\
\text { (9 CCDs, coverage } \\
\text { by each: } 0.5 \AA \text { ) }\end{array}$ & $\begin{array}{c}3 \times 0.005 \AA \\
(3 \text { FPIs, coverage } \\
\text { by scanning: } 0.5 \AA \text { ) }\end{array}$ & $3.3 \times 10^{-3}$ \\
\hline number of steps $(N S)$ & $60^{\prime \prime} / 0^{\prime \prime} 05=1.2 \times 10^{3}$ & $\begin{array}{c}3 \times(0.5 \AA) /(0.005 \AA)=300 \\
(3 \text { lines per FPI and cadence })\end{array}$ & 0.25 \\
\hline $\bar{R} \cdot S \cdot \Omega \cdot \tau$ & & & 6,000 \\
\hline $\mathcal{R} \cdot S \cdot \Omega \cdot \tau \cdot S C / N S$ & & & 80 \\
\hline
\end{tabular}

It is obvious that, for the above needs, FPI spectrometers are far superior to grating spectrometers. In fact, the needs can not be met with today's grating spectrometers. Besides, for observations from ground, image reconstruction is needed to arrive at the required spatial resolution, which can "easily" be performed with imaging spectrometers as FPIs. Our experience shows that they allow short exposure times in the 5-10 ms range, even for polarimetry. So speckle techniques can be applied.

In conclusion, due to their excellent performance, FPI spectrometers have become powerful post-focus instruments and are used with increasing success for two-dimensional highresolution solar spectroscopy (Puschmann et al. 2006).

\section{References}

Born, M. \& Wolf, E. 1970, Principles of Optics, 4th ed., Pergamon Press, Oxford

Hentschel, K. 1998, Zum Zusammenspiel von Experiment und Theorie, I, Verlag Dr. Kovač, Hamburg Hernandez, G. 1988, Fabry-Perot Interferometers, Cambridge Studies in Modern Optics, 3, Cambridge University Press, Cambridge

Kneer, F. \& Hirzberger, J. 2001, Astron. Nachr./Astron. Notes, 322, 375

Puschmann, K. G., Kneer, F., Seelemann, T., \& Wittmann, A. D. 2006, A\&A, 451, 1151 


\title{
SUNRISE: High resolution UV/VIS observations of the Sun from the stratosphere
}

\author{
A. M. Gandorfer ${ }^{1, *}$, S. K. Solanki ${ }^{1}$, P. Barthol ${ }^{1}$, V. Martínez Pillet $^{2}$, W. Schmidt ${ }^{3}$ \\ A. M. Title ${ }^{4}$, M. Knölker ${ }^{5}$, and the SUNRISE team \\ ${ }^{1}$ Max-Planck-Institut für Sonnensystemforschung, Katlenburg-Lindau, Germany \\ ${ }^{2}$ Instituto de Astrofísica de Canarias, La Laguna, Tenerife, Spain \\ ${ }^{3}$ Kiepenheuer-Institut für Sonnenphysik, Freiburg, Germany \\ ${ }^{4}$ Lockheed-Martin Solar and Astrophysics Lab., Palo Alto, CA, USA \\ ${ }^{5}$ High Altitude Observatory, Boulder, CO, USA \\ *Email: gandorfer@mps.mpg.de
}

\begin{abstract}
SUNRISE is an international project for the development, construction, and operation of a balloon-borne solar telescope with an aperture of $1 \mathrm{~m}$, working in the UV/VIS spectral domain. The main scientific goal of SUNRISE is to understand the structure and dynamics of the magnetic field in the atmosphere of the Sun. SUNRISE will provide near diffraction-limited images of the photosphere and chromosphere with an unpredecented resolution down to $35 \mathrm{~km}$ on the solar surface at wavelengths around $220 \mathrm{~nm}$. The focal-plane instrumentation consists of a polarization sensitive spectrograph, a Fabry-Perot filter magnetograph, and a phase-diverse filter imager working in the near UV. The first stratospheric long-duration balloon flight of SUNRISE is planned in summer 2009 from the Swedish ESRANGE station. SUNRISE is a joint project of the German Max-Planck-Institut für Sonnensystemforschung (MPS), Katlenburg-Lindau, with the Kiepenheuer-Institut für Sonnenphysik (KIS), Freiburg, Germany, the High-Altitude Observatory (HAO), Boulder, USA, the Lockheed-Martin Solar and Astrophysics Lab. (LMSAL), Palo Alto, USA, and the Spanish IMaX consortium.

In this paper we will present a brief description of the scientific and technological aspects of SUNRISE.
\end{abstract}

\section{Introduction: Science with SUNRISE}

The solar atmosphere is pervaded by magnetic fields which are at the root of the many fascinating phenomena grouped together under the name solar activity. The magnetic processes that govern solar activity locally determine 'space weather' as well as being potentially significant drivers of terrestrial climate variability on a time scale of decades to centuries. If we are to understand these fundamental processes, we must learn how the magnetic field interacts with the solar plasma and must uncover the conversion of energy between its mechanical, magnetic, radiative, and thermal forms. The solar photosphere represents the key interaction region: Thermal, kinetic and magnetic energy all are of the same order of magnitude and transform easily from one form into another. The interaction between convection, radiation and magnetic field in the electrically conducting solar plasma leads to the creation of a rich variety of magnetic structure, from huge sunspots down to intense magnetic field concentrations on length scales down to a few tens of km, as illustrated in Fig. 1. 
In order to best address these major scientific questions the SUNRISE instruments should provide images of the magnetic structure and measurements of the magnetic field, the flow velocity, and thermodynamic properties of the plasma ...

- ... with a spatial resolution down to $\simeq 35 \mathrm{~km}$ on the Sun,

- ... on a sufficiently large field of view to cover the magnetic connectivity in the solar atmosphere $(\simeq 30 \mathrm{Mm})$,

- ... over a sufficiently long time to follow the evolution of magnetically active regions (i.e., several days), and

- ... simultaneously in different heights of the solar atmosphere.

This leads to the concept of a diffraction-limited operation of a telescope of $1 \mathrm{~m}$ aperture in the visible and UV spectral ranges (down to $\simeq 220 \mathrm{~nm}$ ), with in-flight alignment capability, equipped with a filter imager, a polarimetric spectrograph, and an imaging magnetograph, on long-duration stratospheric balloon flights in the framework of NASA's LDB program.

\section{SUNRISE mission concept}

Ground based observations of the Sun are suffering from several limitations. The Earth's atmosphere does not allow access to the interesting solar UV radiation between $220 \mathrm{~nm}$ and $370 \mathrm{~nm}$. In addition, atmospheric turbulence usually is creating image blur, so that high resolution imaging is possible only occasionally. Long term and high resolution observations especially in the UV are therefore conducted from space-borne instruments. Avoiding the high costs associated with space missions, but taking the advantages of being above $99 \%$ of the Earth's atmosphere, SUNRISE shall be flown as a balloon-borne stratospheric solar

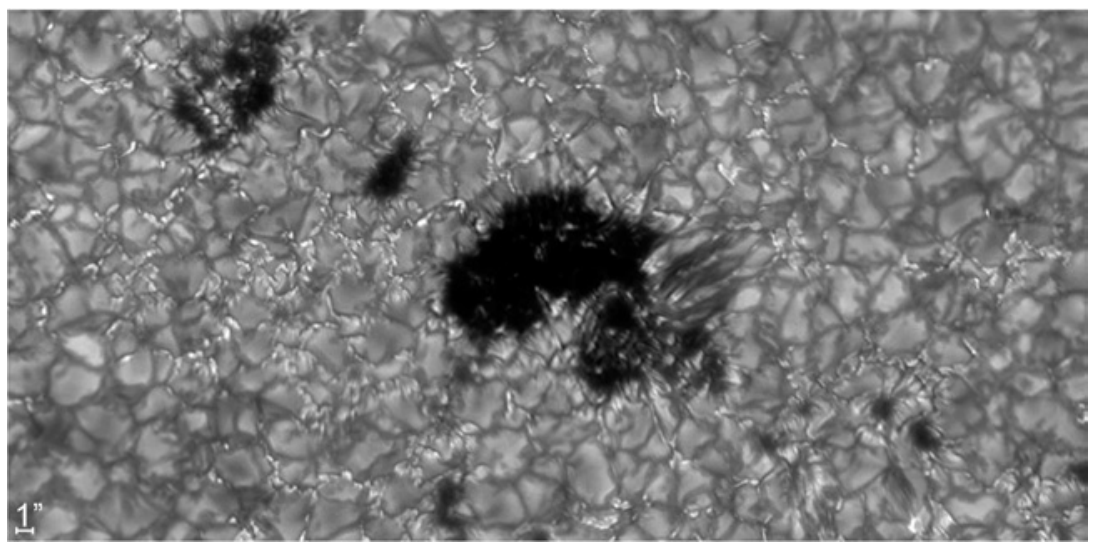

Figure 1. High resolution image of a magnetic solar region observed in the light of the so-called G band, an absorption band of the $\mathrm{CH}$ molecule at $430.5 \mathrm{~nm}$. In this wavelength band the small scale manifestations of solar magnetism can be seen as bright features with high intensity contrast (image taken by V. Zakharov at the Swedish Solar Telescope on La Palma, Spain). 
observatory in the framework of NASA's LDB (Long Duration Balloon) program. A zeropressure helium balloon with an inflated volume of $835.000 \mathrm{~m}^{3}$ and $130 \mathrm{~m}$ diameter will lift the $\approx 1800 \mathrm{~kg}$ science payload to float altitudes of $35-40 \mathrm{~km}$. NASA LDB missions recently have been launched mainly from Williams Field near McMurdo, Antarctica $\left(77.86^{\circ} \mathrm{S}\right.$, $167.13^{\circ} \mathrm{E}$ ), with a launch window from December to January. NASA recently expanded their launch capabilities cooperating with ESRANGE $\left(67.89^{\circ} \mathrm{N}, 21.10^{\circ} \mathrm{E}\right)$ near Kiruna, Sweden, allowing missions to be launched during the northern summer period in addition. Relatively stable wind systems at float altitude take the balloon and instrument on circum-polar trajectories with flight durations of 9-12 days per revolution. Launching balloons during solstice conditions close to the polar circle offers uninterrupted solar observations without day/night cycles at stratospheric altitudes. Permanent sunlight and only small elevation changes of the Sun form ideal conditions, so that undisturbed observation and power generation for the instruments are guaranteed. Furthermore, thermal conditions do not vary significantly and the balloon floats at nearly constant altitude. The preferred launch site for the first SUNRISE mission is ESRANGE, although the flight duration currently is limited to 4-5 days due to the lack of Russian overflight permissions for NASA balloons. ESRANGE provides excellent infrastructure and is accessible with much lower logistical effort compared to McMurdo. SUNRISE is designed for about two weeks autonomous operation. However, during commissioning and the initial phase of operation direct contact to the instrument is highly desirable. A high speed communication system (E-Link, developed by ESRANGE) will be used by SUNRISE on rental basis. It acts as transparent ethernet connection with up to $2 \mathrm{Mbit} / \mathrm{s}$ up- and downlink over a distance of up to $500 \mathrm{~km}$. Additional mobile ground stations along the trajectory would even allow extended line-of-sight communication.

\section{Observational philosophy}

The SUNRISE postfocus instrumentation consists of 5 units, three out of which are science instruments, the other two are system units for image stabilization and light distribution. The SUNRISE science requirements demand simultaneous observations of all three science instruments. This is ensured by ISLID, the Image Stabilization and Light Distribution system of SUNRISE, which will be described in detail below. ISLID contains a fast tip-tilt mirror, which is controlled by a correlating wavefront sensor (CWS), which will be described below. ISLID is based on dichroic beam-splitters, which guide the different wavelength bands to the individual science branches in the most efficient way. Part of the light, which is not used for scientific analysis is fed to the CWS. In this way, simultaneous observations are possible with maximum photon flux in each channel. While the technical realisation of the individual science instruments will be described in more detail below, we will list their specific roles here:

a) SUFI

The Sunrise Filter Imager (SUFI) samples the photosphere and chromosphere in distinct wavelength bands. The channel at $225 \mathrm{~nm}$ allows studies of the upper photosphere and lower chromosphere at a spatial resolution of $0.05 \operatorname{arcsec}(35 \mathrm{~km}$ on the Sun). At the same time, this wavelength is important for the stratospheric ozone household. The $\mathrm{OH}$ band at $313 \mathrm{~nm}$ and the $\mathrm{CN}$ band at $388 \mathrm{~nm}$ provide high contrast, and thus sensitivity to thermal inhomogeneities in the photosphere. The Mg II k line (singly 
ionized magnesium) at $279.6 \mathrm{~nm}$ is an excellent thermometer for the chromospheric temperature structure.

b) SUPOS

The achievement of the main science goals of SUNRISE depends on quantitative and accurate measurements of the strength and orientation of the magnetic field with appropriate spatial, spectral, and temporal resolution. The Sunrise Polarimetric Spectrograph (SUPOS) allows high-resolution vector-polarimetry, simultaneously providing photospheric and chromospheric magnetic field measurements.

c) $\mathrm{IMaX}$

The Imaging Magnetograph EXperiment for Sunrise (IMaX) is an imaging vector magnetograph based upon a tunable narrow-band filter. The instrument will provide fast-cadence two-dimensional maps of the complete magnetic vector, the line-of-sight velocity, and continuum frames with high spatial resolution.

IMaX images will be taken in two to four narrow wavelength bands in either wing of the photospheric spectral line of Fe I (neutral iron) at $525.06 \mathrm{~nm}$.

\section{Instrument description}

\subsection{Gondola}

High resolution imaging requires a stable platform. This is one of the main tasks for the gondola, situated about $100 \mathrm{~m}$ below the balloon at the end of the flight train. The gondola core is designed as an aluminum/steel framework structure (see Fig. 2), being relatively lightweight but providing the required stiffness and protection to the sensitive optical instrument. The telescope and instrumentation need to be precision pointed toward the Sun. Azimuthal control of the whole payload is performed via a momentum transfer unit at the top of the gondola, the elevation of the telescope is adjusted by a two stage linear drive in a range of $0^{\circ}$ to $45^{\circ}$. Control loops, fed by signals from a high precision Sun sensor, keep the pointing constant within a few arcsec. Electrical power is provided to the instruments and electronics via large solar panels, attached left and right at the front of the gondola framework structure. They benefit from the precision pointing toward the Sun, assuring nearly constant output of more than $1.5 \mathrm{~kW}$. On the rear side of the gondola, shaded by the solar panels from direct Sun illumination, the instrument control elecronics is located on two racks. The racks are inclined with respect to the structure due to thermal reasons. This orientation minimizes radiative input from the Earth and the hot solar panels onto the electronics, and maximizes the dissipation of generated heat to the cold sky above the instrument. A commanding and communication package provided by CSBF (Columbia Science Ballooning Facility, a branch of NASA) is located underneath the gondola structure. It has separate solar panels at all four sides of the gondola to stay operational even in case of pointing loss. The package allows commanding and housekeeping downlink via TDRS and Inmarsat satellites along the complete trajectory. Shock absorbing crash pads at the very lower end of the gondola shall reduce mechanical loads during touch down and landing, thus enabling SUNRISE to be reflown with hopefully minimal refurbishment effort. The complete payload has dimensions of 5.5 meters in width and length and is about 6.6 meters high. The gondola 
structure, the power and pointing systems are provided by the High Altitude Observatory (HAO), NCAR, Boulder, USA.

\subsection{SUNRISE Telescope}

The SUNRISE telescope is a light weight Gregory-type reflector with $1 \mathrm{~m}$ clear aperture and $25 \mathrm{~m}$ effective focal length. The main mirror with a parabolic surface and focal length of $2.5 \mathrm{~m}$ is an extremely light-weight Zerodur mirror currently being manufactured under responsibility of SAGEM, France, under contract with the German Kayser-Threde company.

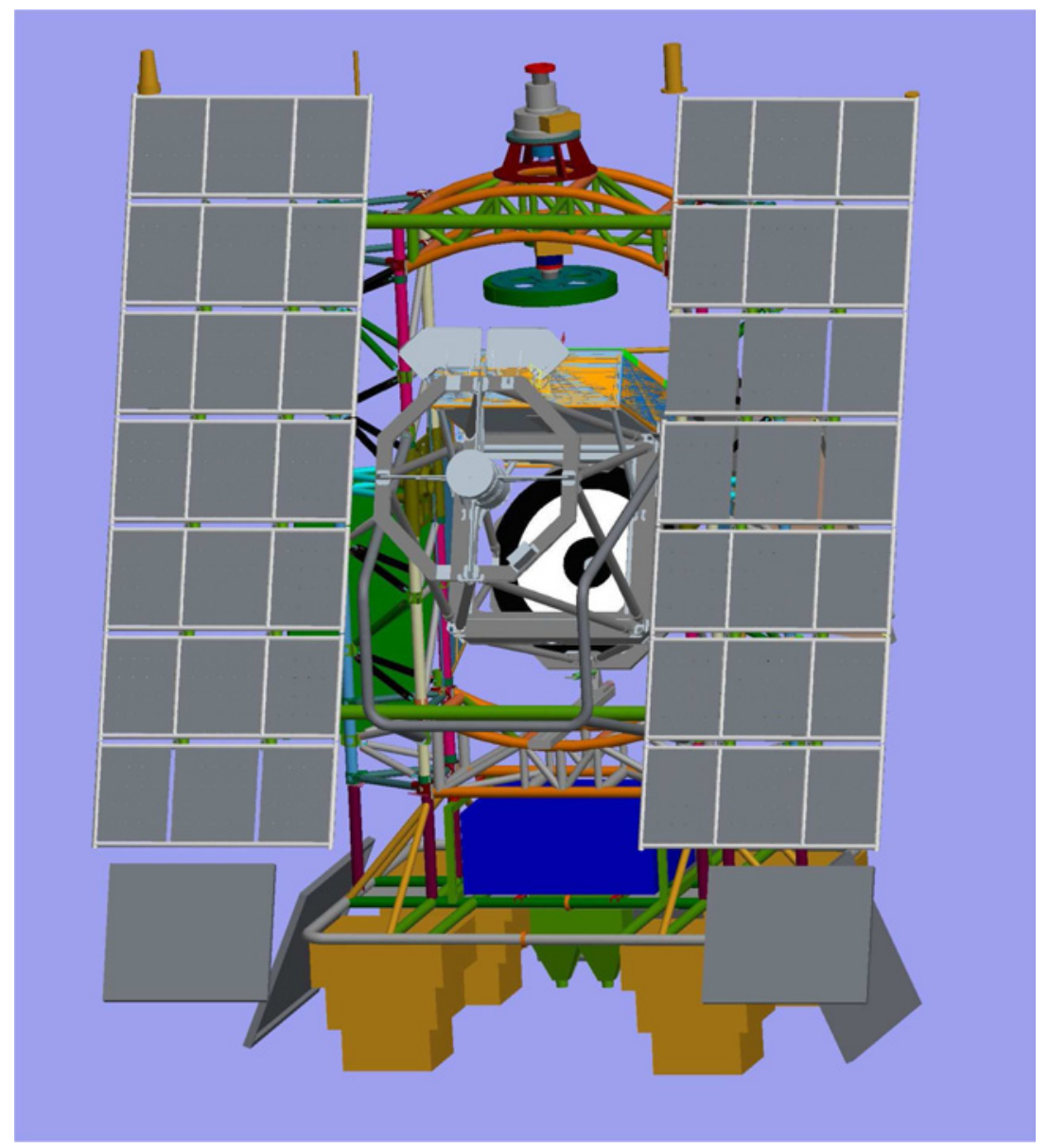

Figure 2. Sketch of the telescope in the gondola with solar panels, landing shock absorbers, and attachment ring to the balloon. The telesope is mounted in an altitude-azimuthal mount. The instrument platform carrying the science instruments is attached to the telescope central frame on top of the telescope. The instrument platform houses all three science instruments (SUPOS, SUFI, and IMaX) as well as the Image Stabilization and Light Distribution system (ISLID) and the Correlating Wavefront Sensor (CWS). 
In the primary focus a field stop is placed, a heat rejection wedge with a hole that defines the useable field of view, corresponding to $148000 \mathrm{~km}$ on the solar surface. The field stop reflects $99 \%$ of the incoming light out of the telescope. This reduces the heat load on the focal-plane instrumentation to about $10 \mathrm{~W}$. The light passing through the field stop is reflected off M2 and folded back by two flat mirrors M3 and M4 to feed the focal-plane package. The latter is mounted piggy-back on the telescope structure and consists of the science instruments and the wavefront sensor/correlation tracker unit. A tip-tilt steering mirror is controlled by the correlation tracker and provides precise pointing and guiding. Stray light is minimized by the field stop in F1, by covering the telescope structure with MLI from both inside and outside, by a set of baffle rings, and by an inner primary-mirror-bore baffle.

The optical system of the SUNRISE telescope is semi-active: relative alignment of M2 to M1 is controlled by low order wavefront sensing in the science focus and passing control signals to the M2 mount. This technique is also used for focusing. The telescope will be built by Kayser-Threde (Munich) under supervision of the Max-Planck-Institut für Sonnensystemforschung, Katlenburg-Lindau, Germany.

\subsection{Image Stabilization and Light Distribution System: ISLID}

ISLID performs two main tasks: First, it forms a real image of the telescope aperture on a fast tip-tilt mirror, which is used to stabilize the image and compensate for residual image motion due to gondola shake and vibrations within the system. This is done with a field lens in the telescope's secondary focus. In order to allow for the UV part of the solar spectrum to be transmitted the lens is made from fused silica and is uncoated. Reimaging is achieved with a two mirror arrangement (for SUFI) and additional refractive optics for SUPOS, IMaX, and CWS.

The second task of ISLID is the light distribution to the different post-focus instruments in a most photon efficient manner by guiding only the dedicated wavelength bands of the instruments by the use of dichroic beam splitters.

\subsection{Correlating Wavefront Sensor: CWS}

The CWS is used in two ways, for precision image stabilization and guiding, and to control proper alignment of the telescope.

Guiding is performed in a closed-loop servo system that consists of a correlation tracker (CT) to provide the error signal and a tip-tilt mirror performing the correction.

A wavefront sensor measures the actual state of the optical alignment and generates an appropriate error signal. A control system converts this error signal into actuation signals which are used to drive and align the secondary mirror, M2.

The detection principle is based on a correlation tracker generating tip and tilt error signals. However, instead of sensing the position of a single image derived from the entire pupil of the telescope, 7 subapertures sense the local wavefront tilt in two zones of the pupil. The information derived from the 7 independently analysed images of the same solar scene suffices to determine the coefficients of a Zernike function decomposition of the wavefront error up to the third radial degree. The coefficients for tip and tilt, defocus, and Seidel coma are used as error signals for the control system. The system is able to measure and 
compensate axial and lateral displacement of the secondary mirror, M2, as well as dynamic image displacement errors.

The correlation tracker/wavefront sensor unit including the tip-tilt mirror and the control software is developed by the Kiepenheuer-Institut für Sonnenphysik, Freiburg, Germany.

\subsection{SUNRISE Polarimetric Spectrograph: SUPOS}

The spectropolarimeter SUPOS has undergone a considerable design change and is now designed as a single spectral line high resolution grating spectrograph working at a wavelength around $854 \mathrm{~nm}$. Here a chromospheric line of singly ionized calcium allows for simultaneous magnetic field diagnostics due to its Zeeman splitting. The line is formed in different layers, spanning from the photosphere to the chromosphere. While the interpretation of the data is more demanding as compared to purely photospheric lines, the advantage of having access to chromospheric magnetism more than compensates for this. The full polarisation state of the spectral line will be detected using 2 nematic liquid crystal variable retarders followed by a polarizing beam splitter. The CCD camera is read in synchronism with the electrooptical modulation in order to demodulate the polarization signal.

\subsection{SUNRISE Filtergraph: SUFI}

The SUNRISE filter imager (SUFI) is the instrument allowing for the highest angular resolution of down to 0.05 arcsec. This corresponds to the diffraction limit of the $1 \mathrm{~m}$ mirror for a wavelength of $225 \mathrm{~nm}$. In order to achieve near diffraction limited imaging, a phasediverse imaging technique is used by splitting the image in front of the CCD detector: half of the CCD area collects the focused image, while a special optical arrangement forms a second image of the same scene on the second half of the CCD, now with a defocus of one wave. Postfacto restoration of the image free from static aberrations of the optical path can be achieved. The optical arrangement is a Schwarzschild system to magnify the telescope secondary focus scale by a factor of 5 onto the CCD. SUFI works in distinct wavelength bands in the near UV between $220 \mathrm{~nm}$ and $390 \mathrm{~nm}$, which are selected by IAD coated interference filters sitting in a double filter wheel to ensure sufficient blocking against the strong visible and near infrared parts of the solar irradiance.

\subsection{Imaging Magnetograph eXperiment: IMaX}

IMaX is a polarisation sensitive filtergraph developed for observations of Doppler shifts and polarisation in a Zeeman sensitive spectral line of neutral iron at $525.06 \mathrm{~nm}$.

A tunable $\mathrm{LiNbO}_{3}$ solid state Fabry-Perot etalon is used in double pass. This configuration significantly saves mass and power and relaxes the demanding requirements on passband stability. Since the free spectral range of such a system is quite small, a narrowband interference filter (FWHM $0.1 \mathrm{~nm}$ ) must be used. Both, prefilter and etalon must be thermally stabilized. Imaging is done with two synchronised CCD cameras for phase diversity reconstruction.

Polarimetry is done with two nematic liquid crystal modulators. Four switching states are needed for full Stokes vector polarimetry. A two-state observing mode is foreseen for longitudinal magnetometry (only circular polarisation) at high cadence. 
IMaX is being developed by a Spanish consortium led by the Instituto de Astrofísica de Canarias, La Laguna (Tenerife), in cooperation with the Instituto de Astrofísica de Andalucía, Granada, the Instituto Nacional de Tecnicas Aeroespaciales, Madrid, and the Grupo de Astronomía y Ciencias del Espacio, Valencia.

\section{Schedule}

SUNRISE plans to conduct a continental U.S. test flight with the gondola, main parts of the electronics and a dummy telescope in October 2007. The telescope delivery to MPS is expected early 2008. Assembly, alignment and calibration of telescope and postfocus instrumentation, the system integration and testing with the refurbished gondola are planned until end of 2008. Mission preparation and shipping to ESRANGE will be beginning 2009, aiming at a launch during the May/June 2009 launch window.

Acknowledgements. The German contribution to SUNRISE is funded by the Bundesministerium für Wirtschaft und Technologie through Deutsches Zentrum für Luft- und Raumfahrt e.V. (DLR), grant no. 50 OU 0401. 
Modern Solar Facilities - Advanced Solar Science, 77-82

F. Kneer, K. G. Puschmann, A. D. Wittmann (eds.)

(C) Universitätsverlag Göttingen 2007

\title{
ZERODUR mirror substrates for solar telescopes
}

\author{
T. Döhring", R. Jedamzik, and P. Hartmann \\ SCHOTT AG, Mainz, Germany \\ *Email: thorsten.doehring@schott.com
}

\begin{abstract}
The zero-expansion glass ceramic material, ZERODUR, is well known for nighttime telescope mirror substrates. Also for solar telescopes, ZERODUR is often selected as mirror blank material. Examples are the Swedish $1 \mathrm{~m}$ Solar Telescope (SST), the balloon-born telescope SUNRISE, and the New Solar Telescope (NST) of the Big Bear Solar Observatory. The properties of ZERODUR are discussed with respect to the special technical requirements of solar observatories, resulting in the conclusion that mirrors made of this glass ceramic material are an excellent choice for solar telescopes.
\end{abstract}

\section{Introduction}

In 2003, SCHOTT celebrated the anniversary event of 100 years of delivery of astronomical mirror blanks (Hartmann \& Morian 2004). Thereby the successful history of the zero expansion glass ceramic material ZERODUR now lasts more than 35 years. This glass ceramic from SCHOTT has been the material of choice in astronomy for decades, thanks to its special properties such as extremely high thermal and mechanical stability. The most important and significant properties of the glass ceramic material ZERODUR are the extremely low coefficient of linear thermal expansion (CTE) as well as the homogeneity of this coefficient throughout the entire piece. SCHOTT has already delivered more than 250 large astronomy mirror blanks with dimensions up to $8.2 \mathrm{~m}$. Today most of the major modern nighttime optical telescopes of the $4 \mathrm{~m}$ class and the $8-10 \mathrm{~m}$ class are equipped with ZERODUR. These include KECK I, KECK II, HET, GTC, LAMOST, and VLT.

The Sun is our nearest star and too bright to be observed with naked unprotected eyes. Hence, it is surprising that also for solar observations there is a strong trend towards in creasing telescope diameters to achieve a larger collecting area. This trend is mainly driven by three parameters: (1) a better spatial resolution allows for finest details of the Sun to be resolved, (2) a narrow bandwidth allows a look at selected spectroscopic lines, and (3) timeresolved measurements (movies) which are needed to observe dynamic solar processes. On the other hand the high amount of (unused) solar radiation - especially if focused - creates severe engineering challenges for solar telescopes. Heat stops have been installed in many solar telescopes and have become a necessity as the apertures increase. In addition to atmospheric effects, solar telescopes suffer from heating by sunlight of the optics and the air within the telescope tube. This causes the image to shiver and become blurred. Mitigation of internal seeing requires minimal thermal gradients in the reflector and surface temperatures within $1 \mathrm{~K}$ of the ambient. Mirrors made of zero expansion materials will retain their shape, 
but the mirrors will heat up and glass ceramic materials are excellent thermal isolators. So how do we deal with solar heat and disturbing air turbulence above the mirrors? Modern solar telescopes are either vacuum telescopes, filled with helium, or use careful control of the optic's temperature to reduce heating of the air in the telescope. Vacuum telescopes cannot be built with very large apertures because such a design requires a vacuum window of extremely high optical quality, one that is as large as its optical aperture. This first optical component in the telescope is difficult because of the thickness needed to withstand the enormous load due to the difference in pressure between the airside and the inside of the vacuum window. The practical limit for vacuum telescopes is probably a little over one meter in diameter (or slightly larger than the SST). For the future of very large solar telescopes, the most viable technique appears to be the construction of open-air telescopes with temperature controlled optics.

\section{ZERODUR - a glass ceramic}

ZERODUR is an inorganic, non-porous glass ceramic with some extraordinary properties. It owes approximately $70-78 \%$ of its weight to micro-crystallites of $30-50 \mathrm{~nm}$ in size, embedded in a remaining glassy phase. In the first step of the production, the initially molten glass is cooled down to room temperature. The micro-crystallites result after an additional thermal treatment (called ceramization) by continually tempering the glass over a period of several months. The created high-quartz micro-crystals contract when they are subjected to heat, whereas the glass itself expands. Size and number of the micro-crystallites are adjusted to achieve an extremely low thermal expansion. In fact at ambient temperature between $0{ }^{\circ} \mathrm{C}$ and $50^{\circ} \mathrm{C}$ the net thermal expansion is nearly zero, achieved with an accuracy of typically $0 \pm 20 \mathrm{ppb} / \mathrm{K}$. In addition, thanks to careful temperature processing, the thermal expansion of ZERODUR is extremely homogeneous. About $4 \mathrm{ppb} / \mathrm{K} \mathrm{CTE}$ homogeneity values (peakto-valley) have been achieved for $1 \mathrm{~m}$ class ZERODUR blanks (Jedamzik et al. 2006). Many other properties of ZERODUR are similar to other glassy materials. Its transparency allows all in-depth inspections (to prevent unpleasant surprises). The good internal quality corresponds to only about 1 bubble per $500 \mathrm{~kg}$ of material. No seams, no pores and if any only very few striae can be detected. All ZERODUR parts are subjected to precision optical annealing in order to achieve a permanent bulk stress, which is both low and symmetrically distributed. Typically excellent stress results of $0.1 \mathrm{MPa}$ (birefringence below $4 \mathrm{~nm} / \mathrm{cm}$ ) are achieved even for huge ZERODUR components, comparable to best fine-annealed optical glass (Döhring et al. 2006). By interferometric measurements ZERODUR parts with even H1-H5 optical grade quality have been selected. Due to its excellent polishability mirrors with surface roughness of $0.2 \mathrm{~nm} \mathrm{rms}$ and an optical figure of around $\lambda / 100$ have been produced for astronomical applications. The chemical stability of ZERODUR is so high that the aluminium coating of a polished mirror substrate can be removed and restored a hundred times without affecting the optical characteristics of the mirror. 


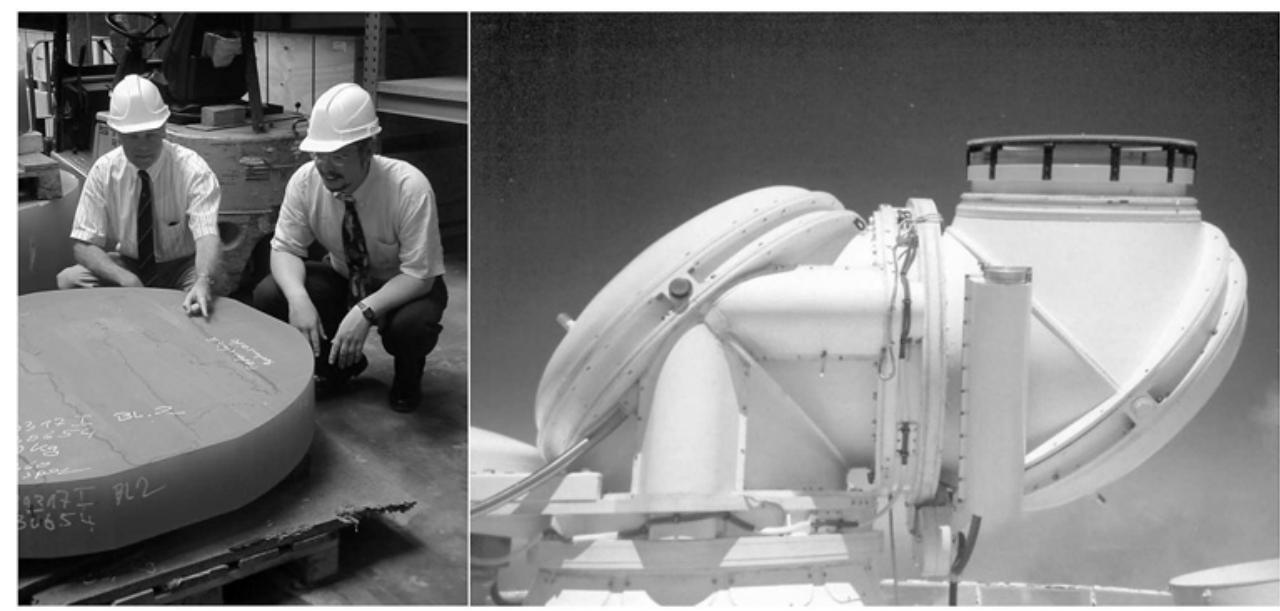

Figure 1. An initial SST blank (left) and the final SST optics (right)

\section{Production of ZERODUR components}

During the recent years, SCHOTT has ramped up its ZERODUR production capacity (Döhring et al. 2005). With a capacity for the mass production of ZERODUR, SCHOTT is now prepared for a future production of mirror blanks for extremely large telescopes with hundreds of 1-2 m class segmented mirror blanks. Utilizing economic CNC processes, ZERODUR can be manufactured to complex shapes without difficulty. Sometimes it was argued that ZERODUR blanks might be too heavy, especially when they are used as secondary mirror blanks. The density of ZERODUR is $2.53 \mathrm{~g} / \mathrm{cm}^{3}$, which is even lighter than aluminium or silicon carbide. Therefore, the argument might refer to the lightweighting capability of ZERODUR, and not the material properties per se. In the past, lightweighting of ZERODUR mirrors was performed for space optics and for secondary telescope mirrors. The secondary $1.7 \mathrm{~m}$ mirrors for MAGELLAN and MMT are examples for such large lightweighted mirror blanks, where undercut holes with hexagonal shapes have been manufactured. Their final mass is only $1 / 3$ the mass of the initial solid blanks. The technical feasibility of large lightweight mirror blanks is therefore demonstrated. An additional acid etching procedure was carried out for GEMINI-M2, achieving an even higher lightweighting percentage of $85 \%$. SCHOTT deliveries range from individual prototypes to serial production. The principal technical steps would use proven and established technology. A typical lead-time for a solid 1.5-m ZERODUR mirror blank is one month, with a corresponding lightweighted version taking about four months.

\section{Examples of ZERODUR mirrors in solar telescopes}

The Swedish $1 \mathrm{~m}$ Solar Telescope (SST) on the island of La Palma, Spain, had first light in the Spring of 2002. The SST is the largest optical solar telescope in Europe and the second largest in the world. It is operated by the Institute of Solar Physics of the Royal Swedish 

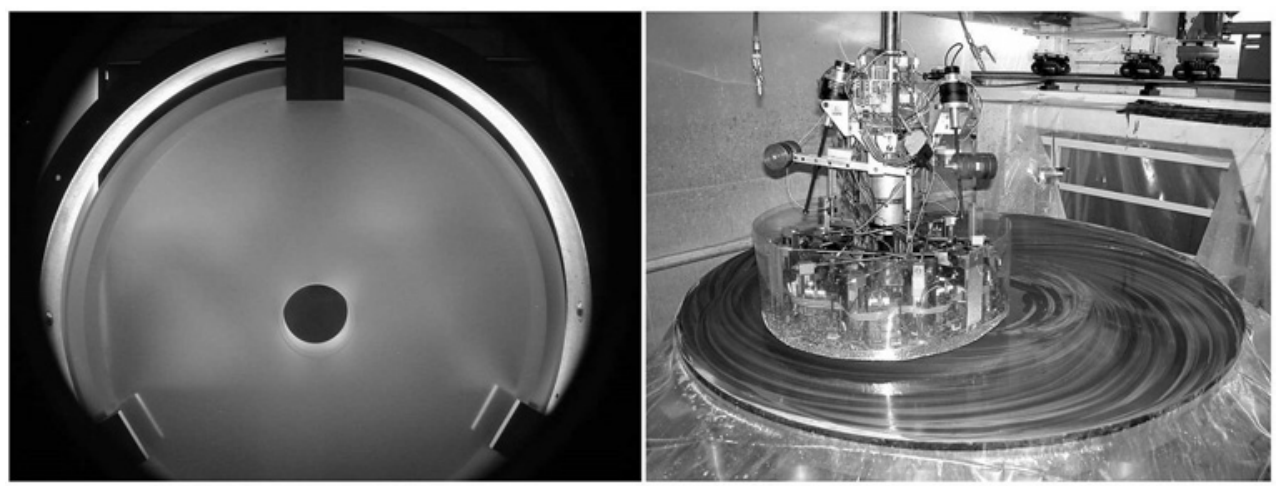

Figure 2. The preshaped SUNRISE substrate (left, courtesy of SAGEM) and polishing of the NST/BBSO ZERODUR blank (right, courtesy of Steward Mirror Lab)

Academy of Science and delivers diffraction-limited images. The SST enables astronomers to detect and observe solar details as small as $70 \mathrm{~km}$ on the solar surface (Scharmer et al. 2003). The SST is a vacuum telescope. Instead of a flat vacuum window, it uses a $1 \mathrm{~m}$ diameter fused silica lens to seal off the vacuum. In 1999 SCHOTT delivered two plano blanks of $1.4 \mathrm{~m}$ in diameter made from ZERODUR. Together with the large lens the two flat deflecting mirrors form the main optical system. It is movable as a whole in order to track the Sun during its diurnal path. Like an arm it extends on top of the telescope building, which contains the vacuum tube, some other optical components and the detectors. An adaptive optics system, a vacuum system for avoiding air turbulence, and mirrors with a mean absolute CTE of less than $5 \mathrm{ppb} / \mathrm{K}$, which is extremely low even for ZERODUR, contribute to the SST's excellent performance for solar imaging, resulting in a higher resolution than previously possible.

SUNRISE is an international project for the development, construction, and operation of a balloon-borne solar telescope, working in the UV/VIS spectral domain (Gandorfer et al. 2006). The primary mirror of the SUNRISE telescope is a Gregory-type reflector with $1 \mathrm{~m}$ clear aperture. Due to production problems, the baseline for the primary mirror material has recently been changed from silicon carbide to glass ceramic material. At the end of 2005, SCHOTT manufactured a corresponding pre-shaped solid $1.1 \mathrm{~m}$ ZERODUR blank. Out of this substrate an extremely lightweighted ZERODUR mirror is currently being manufactured under the responsibility of SAGEM, France. Prime contractor of the SUNRISE telescope is the German company Kayser-Threde. Its first long-duration balloon flight of 4-5 days is planned for the summer of 2009 from the ESRANGE station near Kiruna, Sweden. The flight trajectory is circumpolar. Flying during summer from ESRANGE has the advantage of permanent sunlight and small elevation changes of the Sun, so that observation and power generation are uninterrupted; furthermore, the thermal conditions do not vary significantly and the balloon floats at nearly constant altitude. In the real primary focus a field stop is placed, reflecting $99 \%$ of the incoming light out of the telescope. A stratospheric balloon flight offers observations of the solar surface free from seeing. At a float altitude of 35$40 \mathrm{~km}$, the remaining air pressure is only 3-7 mbar, thus reducing disturbing air turbulence 

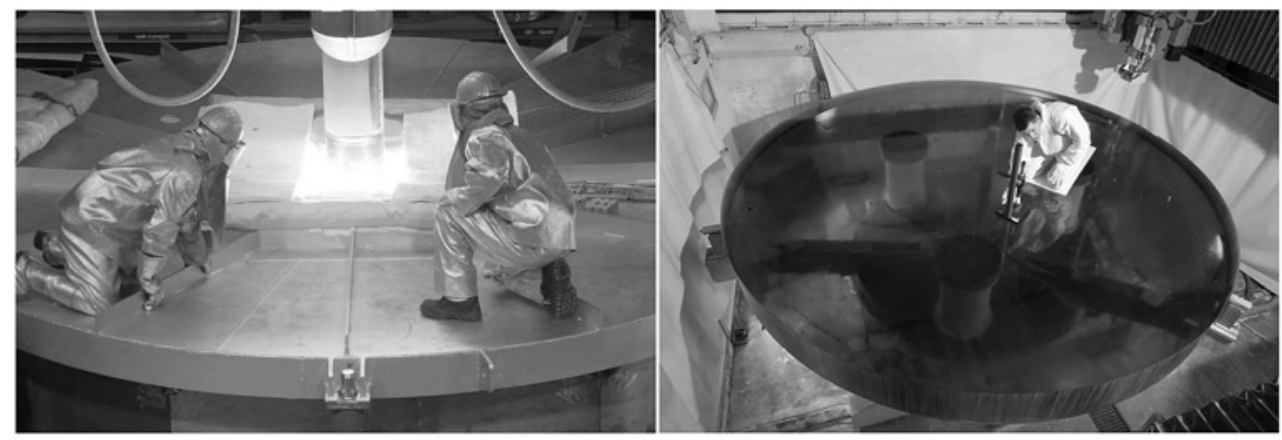

Figure 3. Casting process (left) and intermediate inspection (right) of a four-meter class ZERODUR blank

above the SUNRISE mirror. SUNRISE will provide near diffraction-limited images of the photosphere and chromosphere with an unprecedented resolution down to $35 \mathrm{~km}$ on the solar surface at wavelengths around $220 \mathrm{~nm}$.

The New Solar Telescope (NST) is under construction at the Big Bear Solar Observatory, located at Big Bear Lake's north shore (Denker et al. 2006). System integration of the NST is scheduled for the summer of 2007. The NST will be the world's largest aperture solar telescope - until 2012, when the ATST is planned to have first light. NST is an off-axis telescope with a $1.6 \mathrm{~m}$ aperture in Gregorian configuration, replacing an older and smaller solar telescope. In 2002 SCHOTT delivered a $1.7 \mathrm{~m}$ ZERODUR mirror blank for this application. The $100 \mathrm{~mm}$ thickness of the active mirror was chosen as a compromise between thermal inertia and mechanical flexibility. The Steward Observatory Mirror Lab at the University of Arizona is presently polishing the parabolic primary mirror. Since the telescope structure is open, sophisticated dome ventilation and environmental controls have to be implemented. The thermal control system includes an air-cooled optical support structure, a cooled primary mirror, forced air flows in air-knife technology at primary mirror and heat stop, and liquid coolant systems to absorb rejected light at the heat stop. Limited sunlight exposure and good dome ventilation are considered for good internal seeing conditions. An off-axis design has an additional advantage over an on-axis scheme: It allows unwanted heat to be disposed in a direction which does not introduce air turbulence inside the telescope beam. The $1.6 \mathrm{~m}$ NST telescope in combination with adaptive optics will enable the scientist to carry out spectroscopy and polarimetry with essentially diffraction-limited resolution.

In June 2006, SCHOTT cast a $4.3 \mathrm{~m}$ blank to cover the future demand from astronomy. The transformation into ZERODUR glass ceramic will be completed at the end of 2007. A potential application is the planned Advanced Technology Solar Telescope (ATST). After its first light, which is planned for 2012, ATST will be the most powerful solar telescope in the world. A site on the summit of Haleakala in Maui, Hawaii, has already been selected for this telescope. The off-axis primary mirror is designed as a solid $4.24 \mathrm{~m}$ diameter glass meniscus with a thickness of $75 \mathrm{~mm}$ (Hansen et al. 2006). Thermal performance of the optics in the ATST is critical because of the high incident flux levels a $4 \mathrm{~m}$ solar telescope can produce. Unless heat absorbed in the coatings can be efficiently removed, it has the potential to induce figure errors in the optics and produce convective heat transfer to the surrounding environ- 
ment, leading to degraded optical performance. The thickness and thermal properties of glass ceramics lead to a long thermal time constant and it is a challenge to effectively control the optical surface temperature, where absorbed heat is being removed by cooling the rear of the blank. A low and uniform coefficient of thermal expansion is required to minimize thermally induced figure errors. Finite element modelling has shown that ZERODUR provides properties that allow the error budget allocations to be met under all observing conditions. The versatile observatory design will support diffraction-limited observing at visible through infrared wavelengths.

\section{Summary and conclusion}

ZERODUR is a zero-expansion glass ceramic with extraordinary properties. More than 250 large ZERODUR mirror substrates are used in astronomical telescopes. SCHOTT has the capacity to produce large and lightweighted mirror substrates with short delivery times. Examples of ZERODUR mirror substrates for solar telescopes include the SST, BBSO/NST, and SUNRISE. If you need to keep a very close eye on the Sun, do consider "ZERODUR mirror substrates for solar telescopes".

\section{References}

Denker, C., Goode, P. R., Ren, D., Saadeghvaziri, M. A., Verdoni, A. P., et al. 2006, in Proc. SPIE, $6267,10 \mathrm{D}$

Döhring, T., Hartmann, P., Jedamzik, R., \& Thomas, A. 2005, in Proc. SPIE 5869, 5D

Döhring, T., Hartmann, P., Jedamzik, R., Thomas, A., \& Lentes, F. T. 2006, in Proc. SPIE 6148, 0G

Gandorfer, A. M., Solanki, S. K., Barthol, P., Lites, B. W., Martínez Pillet, V., et al. 2006, in Proc. SPIE 6267, 25G

Hansen, E., Price, R., \& Hubbard, R. 2006, in Proc. SPIE 6267, 131H

Hartmann, P. \& Morian, H. F. 2004, in Proc. SPIE 5382, 331H

Jedamzik, R., Müller, R., \& Hartmann, P. 2006, in Proc. SPIE 6273, 5J

Scharmer, G., Bjelskjo, K., Korhonen, T. K., Lindberg, B., \& Petterson, B. 2002, in Proc. SPIE 4853, $341 \mathrm{~S}$ 


\section{Part 2}

High-resolution and high-precision observations 



\title{
Future missions for helioseismology
}

\author{
M. Roth \\ Max-Planck-Institut für Sonnensystemforschung, Katlenburg-Lindau, Germany \\ Email: roth@mps.mpg.de
}

\begin{abstract}
In this paper I provide basic information on the scientific objectives of upcoming helioseismology missions. The launch of NASA's Solar Dynamics Observatory (SDO) in 2008 is an important opportunity for helioseismology. SDO will carry the Helioseismic and Magnetic Imager (HMI), which will have a 1 " resolution over the whole visible solar disc and is especially designed for local helioseismology. ESA's Solar Orbiter, to be launched ten years from now, will allow the study of the subsurface structure and dynamics of the Sun at high latitudes. Just recently, Hinode (Solar-B) was launched. It is designed to deliver Dopplergrams at $0.3^{\prime \prime}$ resolution. Ground-based observations are also expected to complement space projects.
\end{abstract}

\section{Introduction}

Helioseismology has been tremendously successful over the last two decades at probing the solar interior, especially since the establishment of the GONG (Global Oscillation Network Group) and the launch of SOHO (Solar Heliospheric Observatory). The GONG runs a network of six observatories distributed around the world to provide nearly continuous time series of solar oscillations. The SOHO spacecraft, in a halo orbit around the L1 Sun-Earth Lagrange point, carries the MDI (Michelson Doppler Imager) instrument. Both experiments detect solar oscillations in images of the line-of-sight velocity at the Sun's surface. These data now cover a full sunspot solar cycle. Helioseismology has provided a very good estimate of the sound speed and the large-scale differential rotation inside the Sun as function of radius and latitude and has contributed to solving the neutrino problem. Current investigations focus on the study of seismic variations that are connected with the solar magnetic cycle. In the future, helioseismology will address important question related to the origin of the solar dynamo.

Important space missions for helioseismology are planned. They will enable us to study long-standing problems in solar physics. These missions include the Japanese mission Hinode (Solar-B), the NASA space mission Solar Dynamics Observatory (SDO), and the ESA mission Solar Orbiter. In this short contribution, I gather basic information about these missions and other projects. 


\section{Future missions}

\subsection{Hinode}

Hinode (Solar-B) is equipped with three advanced solar telescopes, an X-ray telescope (XRT), an EUV imaging spectrometer (EIS) and a solar optical telescope (SOT). Hinode was successfully launched on 22 September 2006. The Sun-synchronous orbit of Solar-B allows a downlink of data nearly every orbit, hence observations will be possible 24 hours a day for about 8 months of the year. The SOT system is optimized for accurate measurements of the vector magnetic field in the photosphere and dynamics of both the photosphere and chromosphere associated with the magnetic fields. This makes the SOT instrument interesting for helioseismology, especially as SOT was designed to deliver Dopplergrams with a resolution of 0.3 arcsec. Such a resolution is unprecedented for helioseismology. The field of view of up to $328^{\prime \prime} \times 164^{\prime \prime}$ is, however, a limitation for probing the deep solar interior. Further information can be found at the Solar-B (2006) website.

\section{$2.2 \mathrm{SDO}$}

The Solar Dynamics Observatory is a major component of NASA's Living With a Star (LWS) Program, designed to understand the causes of solar variability and its impacts on Earth. Besides the Atmospheric Imaging Assembly (AIA) and the Extreme Ultraviolet Variability Experiment (EVE) instruments, SDO will carry the Helioseismic and Magnetic Imager (HMI). The HMI will provide data for helioseismology. It will make measurements of the motion of the solar photosphere (at least every $50 \mathrm{sec}$ ) to study solar oscillations. Moreover, it will measure the polarization at 5 points in a spectral line to study all three components of the photospheric magnetic field (at least every $90 \mathrm{sec}$ ). It will deliver nearly continuous full-disk images at $1^{\prime \prime}$ spatial resolution (this resolution is available with MDI only during short periods in a limited field).

Foreshortening makes it difficult to measure oscillations close to the limb with MDI. With HMI the foreshortening effect is reduced. This allows, e.g. better measurements of the Doppler signal at higher latitudes and longer tracking of active regions during their transit. Longer contiguous runs are then possible for local helioseismology.

The science goals of HMI are: the relations of convection-zone dynamics to the solar dynamo, the origin and evolution of sunspots, active regions and activity complexes, links between the internal processes and dynamics of the corona and heliosphere (together with AIA), and precursor measurements of solar disturbances for space-weather forecasts. For further information see the HMI (2006) website.

\subsection{Solar Orbiter}

Here we discuss the Solar Orbiter mission to be launched after 2015. More details on this topic can be found in Gizon (2006). The most interesting aspects of the Solar Orbiter mission clearly reside in the unique vantage points from which the Sun will be viewed. Although the exact orbit of the spacecraft is still under discussion, the orbit design will include two main characteristics, both of which offer novel perspectives for helioseismology. First, Solar Orbiter will make observations away from the ecliptic plane to provide views of the Sun's polar regions. The inclination of the spacecraft's orbit to the Ecliptic will incrementally 


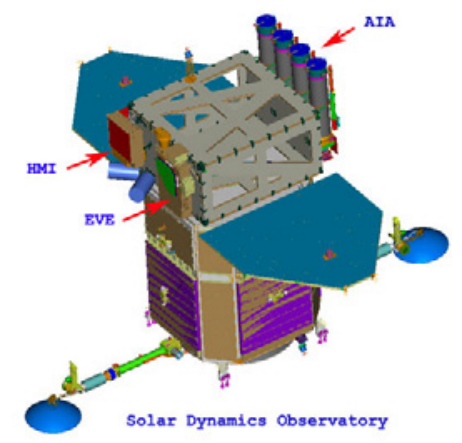

Figure 1. The Solar Dynamics Observatory (SDO) and its three instruments aboard (NASA illustration).

increase at each Venus swing-by manoeuvre to reach up to about $30^{\circ}$ toward the end of the mission. Second, Solar Orbiter will cover a large range of spacecraft-Sun-Earth angles. In combination with data collected from the ground or near-Earth orbit, Solar Orbiter will mark the advent of stereoscopic helioseismology.

The picture of the solar interior is far from being complete at high heliographic latitudes. In particular, global mode frequency inversions for rotation cease to be accurate above $70^{\circ}$ and local helioseismology above $50^{\circ}$. Out-of-the ecliptic observations will enable helioseismology to reach higher heliographic latitudes into the solar convection zone.

By definition, stereoscopy requires several observatories. Both SDO-HMI and GONG (or another ground-based facility) are expected to be operational at the time of Solar Orbiter operations. Combining Solar Orbiter with any one of them will open new windows into the Sun. Note that how well the data can be merged will depend critically on timing accuracy and geometry knowledge.

Looking at the Sun from two distinct viewing angles results in an increase of the observed fraction of the Sun's surface. Global helioseismology would naturally benefit from this situation, since the modes of oscillation would be easier to disentangle (reduction of spatial leaks).

With stereoscopic local helioseismology, new acoustic ray paths can be considered to probe deeper layers in the interior. Observations from two widely different viewing angles allow the probing of the solar interior at any depth, and in particular the solar tachocline (Ruzmaikin \& Lindsey 2003). It even becomes possible to target regions in the solar core: this is illustrated in Figure 2. This aspect of seismic stereoscopy is very promising.

\subsection{Other projects}

The CNES mission PICARD will be launched at the end of 2008. It will measure the solar oscillations with a high resolution at the solar limb. The major objective is to measure solar gravity modes and the solar shape.

Ground-based observing facilities are needed to complement the observations made by SDO and other space missions. In particular, at least two instruments are required to understand systematic errors and sources of noise in the data. Historically, this has been demon- 


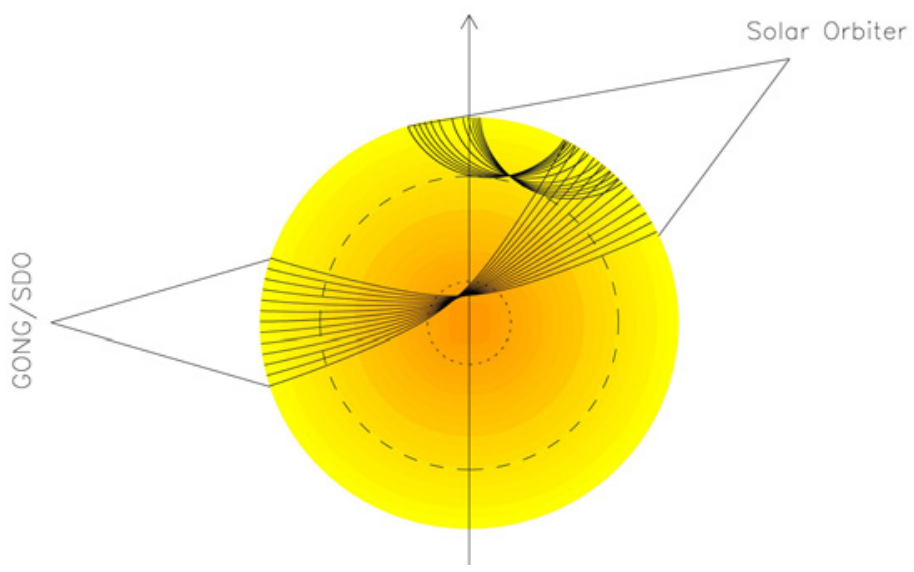

Figure 2. Sketch showing deep-focussing averaging schemes for local helioseismology. Solar Orbiter in combination with SDO or ground-based observatories will give access to local information very deep inside the Sun (stereoscopic helioseismology).

strated many times by comparing data from GONG and MDI (Schou et al. 2002, e.g.). Helioseismology needs uninterrupted time series: a second instrument guarantees continuous records. Ground-based instruments are also flexible, i.e. they can be upgraded more easily and fixed.

Other ground-based high-resolution solar observatories could turn out to be useful too, for the seismological study of the solar atmosphere by considering several lines formed at different heights in the atmosphere (Finsterle et al. 2004).

Acknowledgements. The information about the different projects was collected from the mission websites (see references below) and from Gizon (2006).

\section{References}

Finsterle, W., Jefferies, S.M., Cacciani, A., et al. 2004, Solar Phys., 220, 317

Gizon, L. 2006, in Proc. Second Solar Orbiter Workshop, ESA-SP, 641

GONG website 2006, http://gong.nso.edu/

HMI website 2006, http://hmi.stanford.edu/

MDI website 2006, http://soi.stanford.edu/

PICARD website 2006, http://smsc.cnes.fr/PICARD/

Ruzmaikin, A. \& Lindsey, C. 2003, in Proc. SOHO 12/GONG 2002, ESA SP-517, 71

Schou, J., Howe, R., Basu, S., et al. 2002, ApJ, 567, 1234

Solar-B website 2006, http://solar-b.nao.ac.jp/ 
Modern Solar Facilities - Advanced Solar Science, 89-102

F. Kneer, K. G. Puschmann, A. D. Wittmann (eds.)

(C) Universitätsverlag Göttingen 2007

\title{
Helioseismology at MPS
}

\author{
L. Gizon", R. Cameron, J. Jackiewicz, M. Roth, H. Schunker, and T. Stahn \\ Max-Planck-Institut für Sonnensystemforschung, Katlenburg-Lindau, Germany \\ *Email: gizon@mps.mpg.de
}

\begin{abstract}
Research in solar and stellar seismology at the Max Planck Institute for Solar System Research (MPS) is supported by the Junior Research Group "Helio- and Asteroseismology" of the Max Planck Society since September 2005. A presentation of the current topics of research is given, with particular emphasis on local helioseismology.
\end{abstract}

\section{Research outline}

Millions of modes of vibration, excited by turbulent convection, enable solar physicists to see inside the Sun, just as geophysicists can probe the internal structure of the Earth using records of seismic activity. Over the past twenty years, helioseismology has produced a considerable number of discoveries in solar, stellar, and fundamental physics. The best is still to come, however: three-dimensional helioseismic techniques offer unique prospects for probing complex magnetohydrodynamical processes and uncovering the mechanism of the solar cycle, while the extension of seismic investigations to distant stars will open a new era of observational stellar research.

Methods of helioseismology can be divided into two classes: global and local. The more traditional technique of global helioseismology consists of measuring the frequencies of the modes of oscillation and searching for a seismic solar model whose oscillation frequencies match the observed ones. This reveals the Sun's large-scale structure and rotation as a function of depth and latitude. To complement global helioseismology, new methods of local helioseismology are being developed to make three-dimensional images of the solar interior. The basic idea is to retrieve information at depth from the time it takes for solar waves to travel between any two surface locations.

Global helioseismology has provided by far the most precise tests for the theory of stellar structure and evolution, implying, in particular, a revision of the standard model of particle physics to solve the solar neutrino problem. Today, the most exciting aspect of helioseismology is the search for clues regarding the origin and variability of the Sun's magnetic field, possibly the most important unsolved problem in solar physics. The general belief is that a dynamo process is responsible for the solar magnetic cycle. According to this scenario, magnetic field lines are stretched and twisted by internal shearing motions. Therefore, it is essential to map internal mass motions, structural asphericities, and their temporal variations. Global helioseismology has already provided some fundamental results, revealing regions of rotational shear in the Sun's interior, solar-cycle variations in the rotation rate, and mysterious quasi-periodic changes at the base of the convection zone. 
The next advances are expected to come from local helioseismology (Gizon \& Birch 2005; Gizon 2006a), which, although still a young science, has already pinpointed a mechanism for the latitudinal transport of the magnetic flux that could determine the period of the solar cycle. Detailed 3D maps of the upper convection zone provide new insights into the structure, evolution and organization of active regions and convective flows. In yet another application, local helioseismology can be used to construct maps of active regions on the far side of the Sun. These few examples illustrate the richness of the science possible with local helioseismology. In all these cases a taste of the possibilities has been provided, but better data and further developments in the technique are required to realize the full potential.

An important technological step for helioseismology will come with the HMI instrument on the Solar Dynamics Observatory of NASA to be launched in 2008. With a high spatial resolution over the entire visible solar hemisphere, HMI is the first instrument specifically designed for local helioseismology. Later, in about one decade, ESA's Solar Orbiter should give access, for the first time, to the subsurface structure and dynamics of the Sun's polar regions. With these new observations, the need for improvements in solar modeling will come. In particular, theoretical studies and numerical simulations will be required to understand wave propagation in strongly magnetized fluids, a necessary condition for the application of local helioseismology to solar active regions. Local helioseismology is very much under development today and promises many more discoveries. Among the most ambitious goals is to directly image the magnetic field in the solar interior.

Asteroseismology, the study of global oscillations on distant stars, is entering a very exciting period of discoveries. Many stars, covering a wide range of masses and evolutionary states, are known to exhibit oscillations. Only in the last few years, however, has it been possible to detect oscillations on Sun-like stars using sophisticated spectrographs on large ground-based telescopes. Stellar oscillations have considerable diagnostic potential and allow stellar mass and age to be determined with unprecedented precision. Such knowledge for a sufficient sample of stars will revolutionize stellar evolution and galactic evolution studies. Asteroseismology has also the potential to constrain internal stellar rotation and locate the borders of convection and ionization zones. Such information would help understand dynamo-generated stellar activity cycles and the solar-stellar connection.

These exciting possibilities for the study of stellar structure, evolution, and activity will be fully realized only once observations become available for a large sample of stars. The precision on the frequencies of the global modes of stellar oscillations, however, is very much limited by available telescope time, which is why dedicated space telescopes are an attractive solution to provide long-term coverage of many types of pulsating stars. High precision photometry from space is expected in 2006 with the launch of COROT of CNES in 2006. The field of asteroseismology will make much progress in the following decades with more ambitious missions like NASA's Kepler and possibly ESA's Eddington. As in the case of helioseismology, there is a strong need to improve our understanding of the oscillations and how they interact with the magnetohydrodynamical processes in stars.

Research in solar and stellar seismology at the Max Planck Institute for Solar System Research (MPS) is supported by the Junior Research Group "Helio- and Asteroseismology" of the Max Planck Society since September 2005. A general presentation of the current topics of research is given below, with particular emphasis on local helioseismology. 


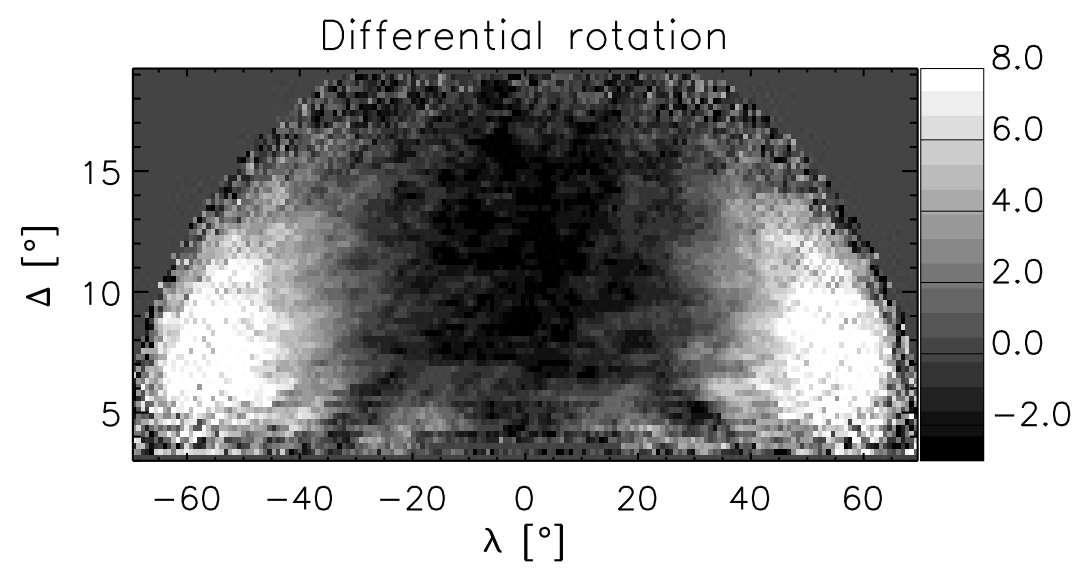

Figure 1. Difference in travel time measured in the west-east direction (travel time for westward propagating acoustic waves minus eastward propagating waves) as a function of horizontal travel distance and latitude. The travel times are measured in a frame that co-rotates with the Sun at the Carrington rotation rate. The latitudinal variation is due to solar differential rotation (faster at the equator than at high latitudes).

\section{Measurements of travel times from the MDI Structure Program}

Time-distance helioseismology consists of analyzing the travel times of acoustic wave packets that propagate through the solar interior. Flows inside the Sun break the travel-time symmetry between waves propagating in opposite directions. In order to achieve high-precision measurements of flows deep in the solar convection zone, it is important to analyze long, nearly-continuous time series of solar oscillations. We used an extensive set of velocity maps recorded by the Michelson Doppler Imager during the period 1996-2003 (MDI-SOHO Structure Program). A critical step in the data analysis is to extract the seismic travel times from the data. Travel times are determined from the correlation function of the Doppler velocity computed between any two points on the Sun's surface. Two different measurement techniques have been implemented and compared for robustness to solar noise. Figure 1 shows travel-time differences in the west-east direction as a function of horizontal travel distance and latitude. Here the travel-time shifts are caused by solar differential rotation. These measurements suggest that near-surface rotation can be infered with a precision close to $1 \mathrm{~m} / \mathrm{s}$ using one month of data.

\section{Solar-cycle variation of rotation and meridional circulation}

The temporal variations of rotation and meridional circulation have a long-term component with a period near eleven years. These small solar-cycle variations of a few $\mathrm{m} / \mathrm{s}$ are believed to be a manifestation of the evolution of the large-scale magnetic field. We have made helioseismic observations of the solar-cycle variation of flows near the solar surface and at a depth of about $60 \mathrm{Mm}$, in the latitude range $\pm 45^{\circ}$. 
a) Meridional flow residuals (surface)

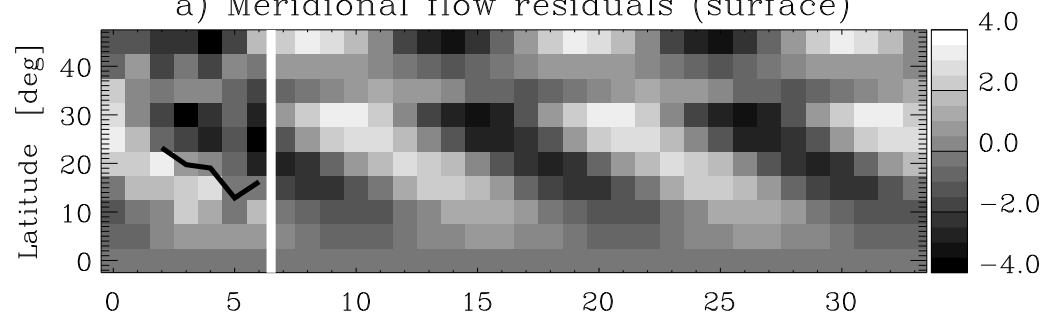

b) Meridional flow residuals (60 Mm)

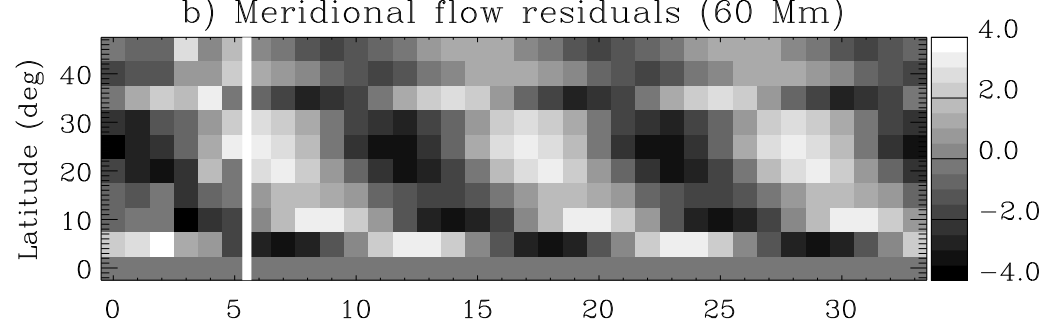

c) Zonal flow residuals (surface)

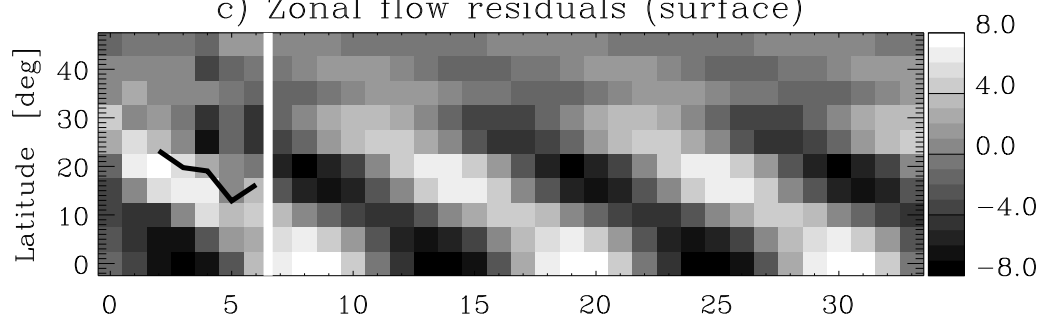

d) Zonal flow residuals (60 $\mathrm{Mm})$

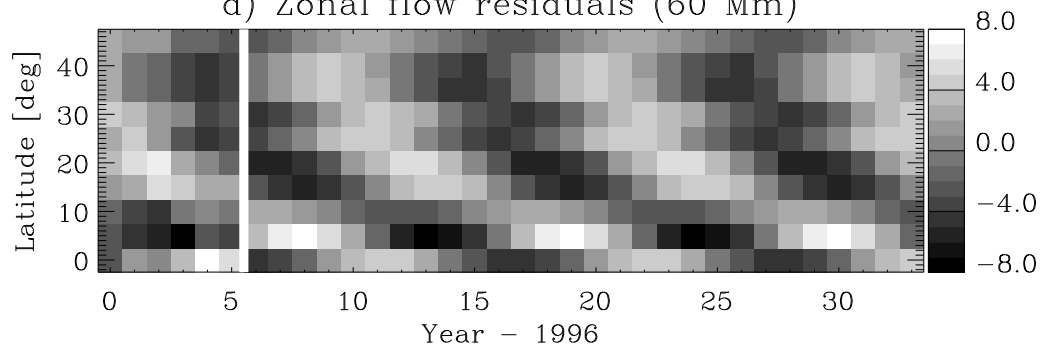

Figure 2. Time-varying components of the meridional $(a, b)$ and zonal (c,d) flows as a function of time. Panels a and $\mathrm{c}$ are for the near solar surface, while panels $\mathrm{b}$ and $\mathrm{d}$ are for a depth of about $60 \mathrm{Mm}$ below the surface. The black lines in panels a and $\mathrm{c}$ show the mean latitude of active regions. The eleven-year periodic component of the data is extrapolated into the future beyond the white vertical lines.

Acoustic ray bundles that penetrate deep inside the Sun connect surface locations that are separated by large distances. A depth of $60 \mathrm{Mm}$ corresponds to a travel distance of about $17^{\circ}$. To a first approximation, rotation and meridional circulation at this depth can be estimated 

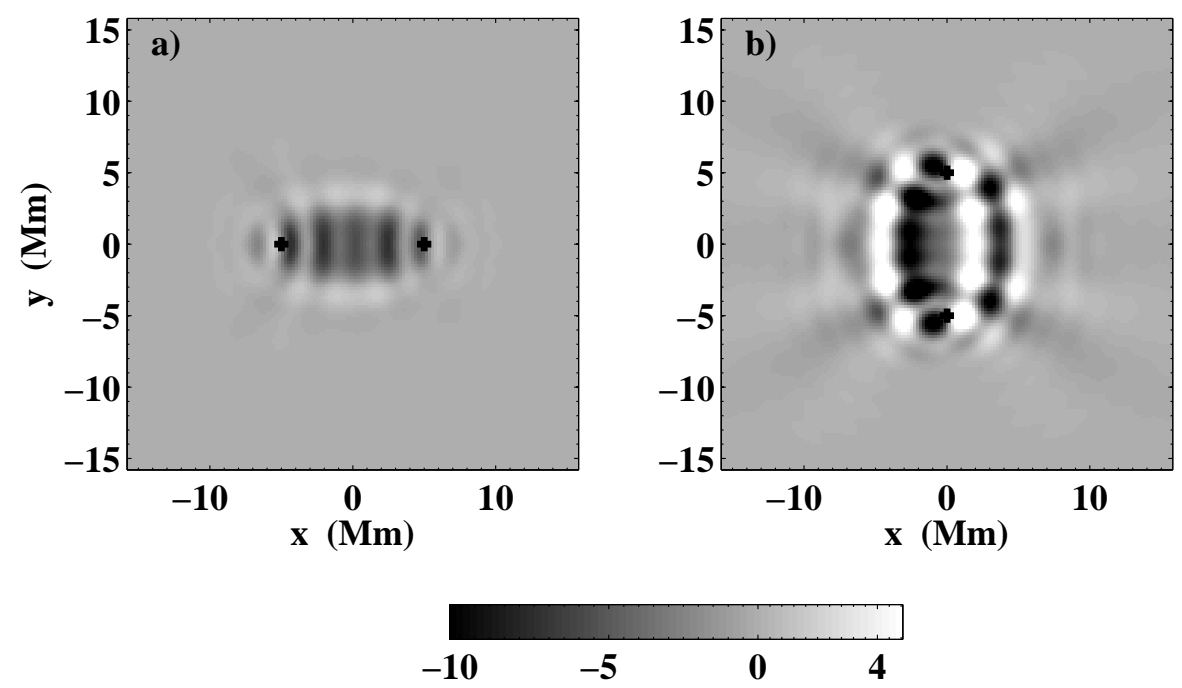

Figure 3. Two-dimensional travel-time sensitivity kernels for flows. This case is for a patch of the Sun $60^{\circ}$ towards the west limb on the equator. Each kernel gives the sensitivity of travel-time differences to flows for waves traveling in opposite directions between the two photospheric observation points, which are given by the crosses. (a) Sensitivity kernel for flows traveling in the $+x$ direction. (b) Sensitivity kernel for flows in the $+y$ direction. The units of the kernels are $\mathrm{s}(\mathrm{km} / \mathrm{s})^{-1} \mathrm{Mm}^{-2}$.

directly from the travel-time differences in the east-west and north-south directions (Sect. 2). For the near-surface layers, we used f-mode time-distance helioseismology to obtain every 12 hour a map of the convection pattern. The near-surface plasma flow is estimated from the advection of the convection pattern according to the method of Gizon et al. (2003).

Figure 2 summarizes the results. The time-varying components of the meridional flow at these two depths have opposite sign, while the time-varying components of the zonal flow are in phase. We investigated a theoretical model based on a flux-transport dynamo combined with a geostrophic flow caused by increased radiative loss in the active region belt. The model and the data are in qualitative agreement, although the amplitude of the solar-cycle variation of the meridional flow at $60 \mathrm{Mm}$ appears to be underestimated by the model. See Gizon \& Rempel (2007).

\section{High-resolution local helioseismology}

We are interested in studying the near-suface flows on the Sun, in particular supergranulation and the flows around active regions, using f-mode time-distance helioseismology. The $\mathrm{f}$ modes propagate horizontally in the top $2 \mathrm{Mm}$ of the convection zone. Determining horizontal flows accurately and with a high spatial resolution (less than the f-mode wavelength of $\approx 5 \mathrm{Mm}$ ) requires two main steps. The first step, called forward modeling, is used to derive an integral relationship between travel-time measurements and internal flows. This relationship is provided by travel-time sensitivity kernels. The second step is to then use the 


\section{Weights}

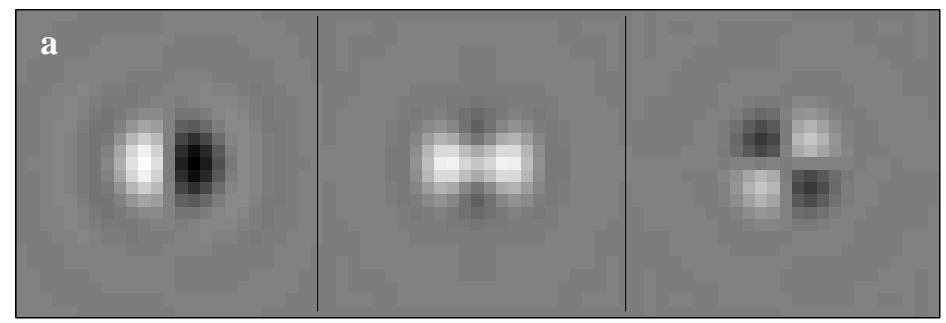

Kernels
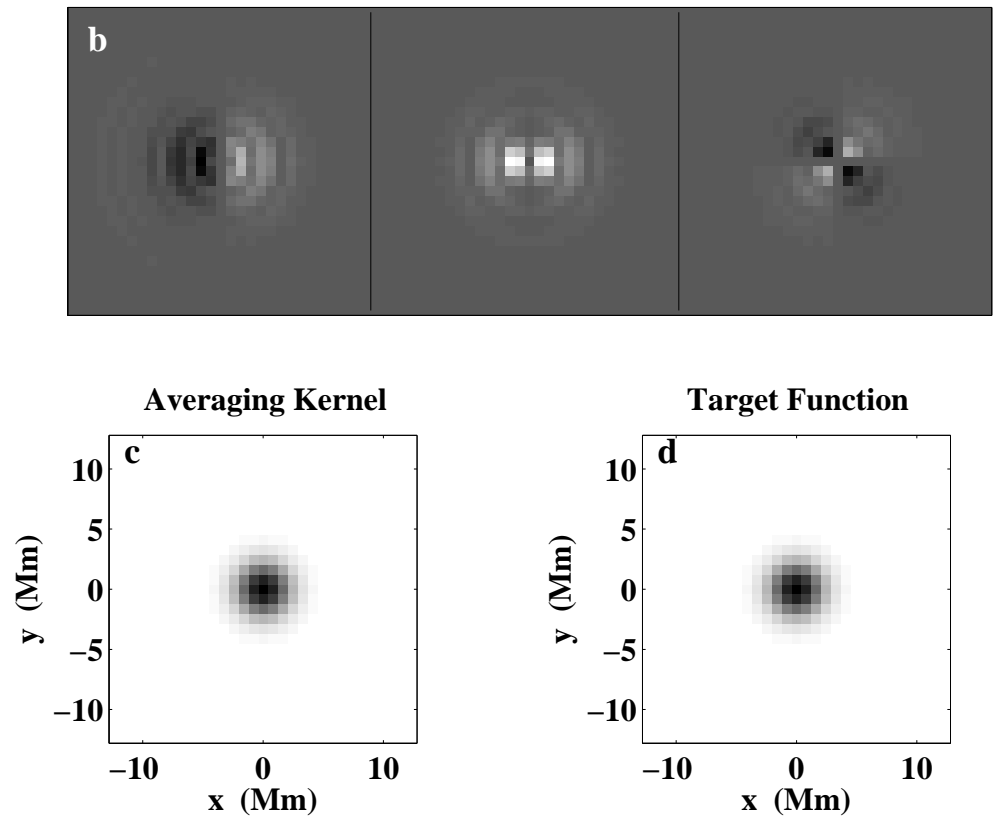

Figure 4. OLA inversion of f-mode travel times. (a) Inversion coefficients (b) Travel-time sensitivity kernels (quadrant averaging scheme). The inversion coefficients are convolved with the kernels to give the averaging kernel of panel (c). The averaging kernel matches the target function (d) well.

sensitivity kernels, along with the travel-time measurements, to infer the underlying flows. This last step is called an inversion.

We have calculated f-mode kernels for flows in the first Born approximation, which is a single-scattering approximation. Figure 3 shows examples of $2 \mathrm{D}$ kernels which give the sensitivity of f-mode travel-time differences to localized horizontal flows on the solar surface. The travel times are measured between two individual points on the solar surface. In general, the kernels depend on the position on the solar disk through the line-of-sight vector. We note that it is customary to average travel times (and kernels) over quadrants of arc in the east, west, south and north directions to reduce noise. See Jackiewicz et al. (2006a,b). 


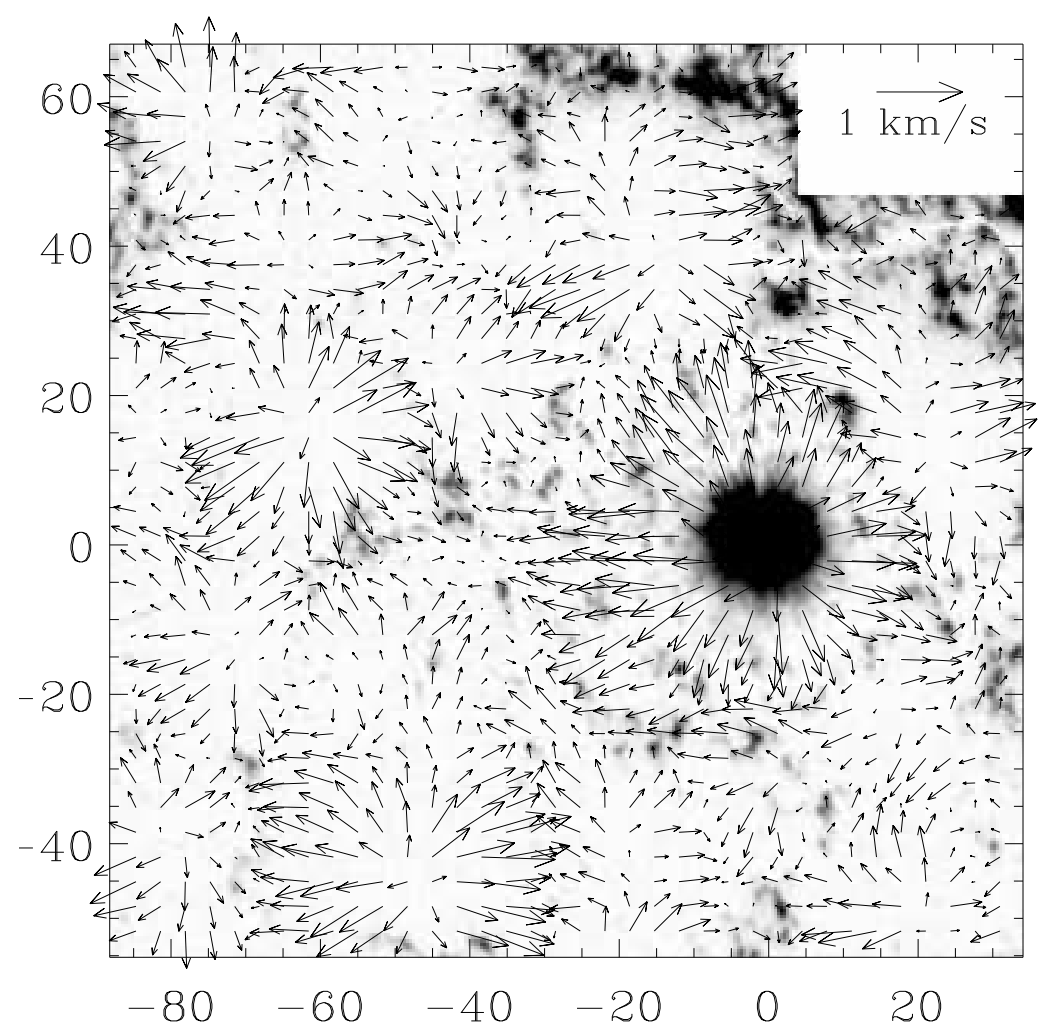

Figure 5. Map of horizontal flows around a sunspot at a depth of $1 \mathrm{Mm}$ below the surface. Flow velocities are obtained by inverting f-mode travel times (quadrant averaging). The outflow around the sunspot, with an amplitude of about $500 \mathrm{~m} / \mathrm{s}$, is called the moat. Spatial coordinates are given in units of $\mathrm{Mm}$.

The most important part of the inversion procedure is to determine a set of weights which average the measurements in some way and return the value of the local flow. In our case, the weights are found in a two-dimensional Optimally Localized Averaging (OLA) scheme, whereby one seeks a linear combination of the sensitivity kernels (an averaging kernel) that resemble a Gaussian target function. It is not always desirable to have the averaging kernel and the target function match exactly, since doing this may cause large measurement errors to propagate through the inversion. Therefore, one needs to consider a satisfactory resolution along with a permissible amount of error, which can be chosen by adjusting a trade-off parameter. In Fig. 4 we show results for an inversion. Our choice of trade-off parameter is such that the resolution is quite good, the weights are smooth, and the averaging kernel and target match well. Figure 5 gives an idea of the spatial resolution that can be achieved with only $8 \mathrm{hr}$ of data. Both supergranular flows and the moat flow outside a sunspot are resolved (even though the inversion was not quite optimal in this particular case). 


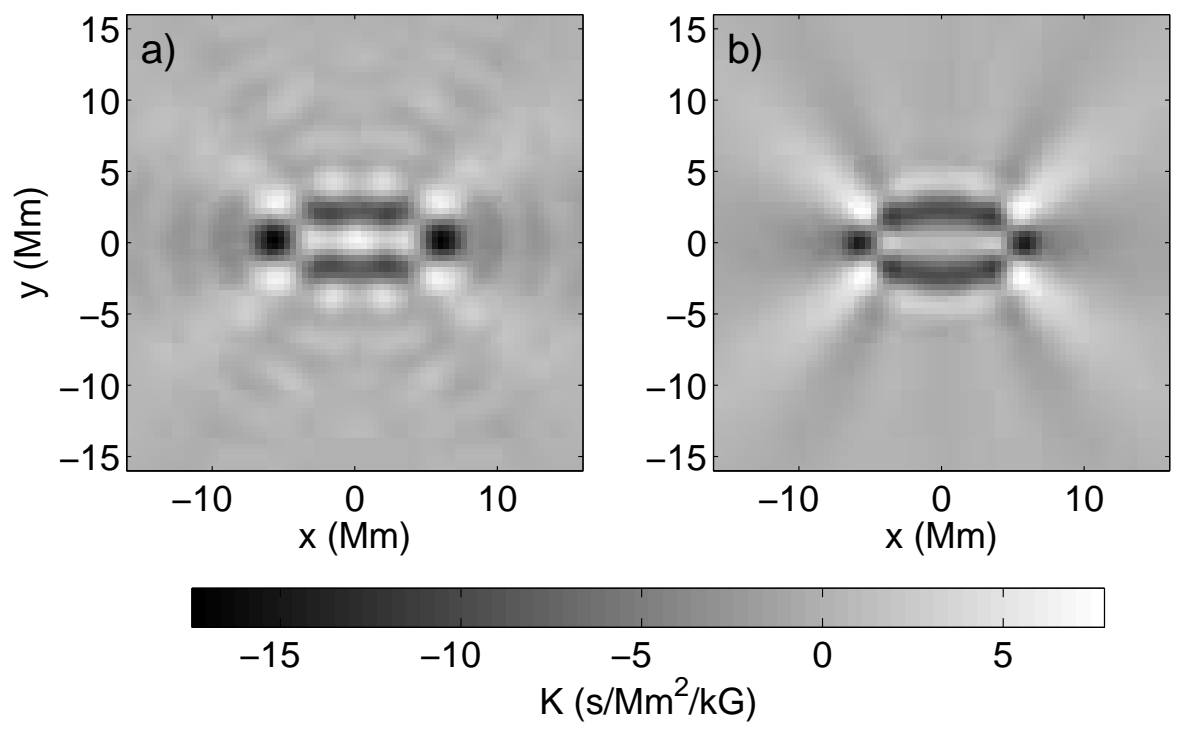

Figure 6. Sensitivity of solar surface-wave travel times to small magnetic features. (a) Observations based on MDI data from the high-resolution field of view. A magnetic feature at position $(x, y)$ causes a shift in the travel time measured between the two observation points (at $x= \pm 4.95 \mathrm{Mm}, y=0$ ). As magnetic features have a finite spatial extent, we applied a simple regularized deconvolution. The grey scale gives the shift in travel time due to a $1 \mathrm{kG}$ magnetic field covering $1 \mathrm{Mm}^{2}$. (b) Phenomenological model for a point magnetic scatterer based on single-scattering theory. A scatterer located anywhere along an ellipse (with foci at the observation points) causes travel-time shifts of the same sign, giving rise to Fresnel zones. The hyperbolic features are due to the scattering of waves generated by distant sources.

\section{Travel-time shifts caused by small magnetic features}

The solar surface magnetic field is dragged by convective motions into concentrations that form the quiet-Sun magnetic network. Because these magnetic features are smaller than the wavelengths of solar oscillations, they are ideal to study the response of finite-wavelength seismic travel times to point-like perturbations.

We used time-distance helioseismology to directly measure the spatial sensitivity of fmode travel times to a point-like magnetic perturbation. Travel-time maps reveal that the sensitivity is not restricted to the geometrical ray path, is spread on elliptical and hyperbolic curves, and oscillatory (Fig. 6a). We find that these geometrical features are mostly due to finite wavelength effects and to the fact that the sources of excitation of solar oscillations are distributed over the whole solar surface. To reach this conclusion, we developed a simple phenomenological model to explain the travel-time observations. We assumed that scattering from a magnetic feature can be described by a combination of monopole and dipole scattering. Treating solar surface-gravity waves (f modes) as deep water waves, we computed the wave field using a single-scattering approximation, and then adjusted the complex scattering amplitudes to obtain the best match with the observations (Fig. 6b). We find that the dipole 
and monopole contributions are equally important.

By studying the interaction of seismic waves with localised magnetic features on the Sun, we have provided an observational confirmation of the basic banana-doughnut theory originally developed for finite-wavelength tomography of the Earth, according to which bodywave travel times are sensitive to the wave speed in a broad region surrounding the geometrical ray path. This is the first test outside the laboratory showing the relevance of scattering theory to cross-correlation travel times (laboratory tests exist for ultrasonic waves). As in Earth seismology, we suggest that finite-wavelength modeling will be essential in revealing deep structures in the solar interior. See Duvall et al. (2006).

\section{Line profiles of fundamental modes of solar oscillations}

We have studied the asymmetry of f-mode line profiles in the power spectrum of solar oscillations, which have received less attention than for acoustic modes ( $p$ modes). Line asymmetry is interesting as it contains information about the mechanism of wave excitation.

Using MDI-SOHO data, we find that f-mode line asymmetry is pronounced in the degree range 600-1200 and has opposite signs in velocity and intensity power spectra. One may ask if the mechanism responsible for f-mode line asymmetry can be described in simple physical terms, as is done for $\mathrm{p}$ modes. An argument based on wave interference (used to explain pmode line asymmetry) has little value in the case of $\mathrm{f}$ modes, which do not propagate in the vertical direction. Is it at all conceivable that line asymmetry may occur from combinations of exponential wave functions? To investigate this question, we considered the propagation of a surface wave at the interface between two media with different constant densities, forced at a given height by a vertical momentum impulse. We find that, in the limit of a large density discontinuity, line asymmetry can occur when the source is situated above the interface. Although this toy model is not intended to approximate the Sun, it has the merit of demonstrating that line asymmetry can occur even for waves that do not propagate in the vertical direction, such as the f mode. See Gizon (2006b).

\section{Modeling wave propagation}

\subsection{Three-dimensional numerical simulations}

The propagation of waves in the near photospheric layers is being studied using numerical simulations, in order to better understand helioseismological observations. The code we have developed follows the linear evolution of perturbations in an inhomogeneous, magnetized atmosphere. We have used a box geometry, a spectral treatment in the horizontal directions, and a finite difference scheme for the vertical direction. The boundary conditions are naturally important. The side boundaries are simple: the box is periodic. For the upper boundary we currently use the condition that the Lagrangian perturbation of the vertical component of the stress tensor vanishes. A practical problem is that the solar atmosphere is superadiabatic beneath the solar photosphere, and so exponentially growing modes are present in the solutions. As we wish to focus on the effects of the convection on the waves, we currently modify the density so that, in the wave simulation, the atmosphere is nowhere unstable. The code is fully three-dimensional. See Cameron \& Gizon (2006). 


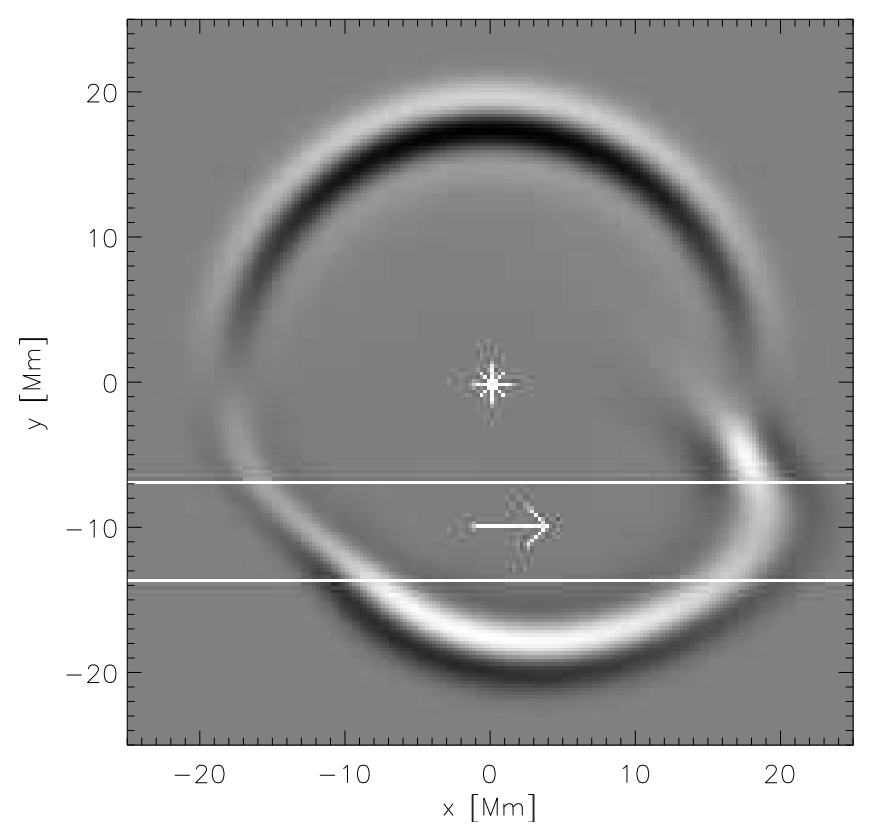

Figure 7. A snapshot from a calculation of an acoustic wave packet propagating across a jet. The background atmosphere has a uniform sound speed of $11 \mathrm{~km} / \mathrm{s}$, and is of uniform density and pressure. The two horizontal white lines indicate the presence of a jet flowing from left to right. The jet has a peak amplitude of $10 \mathrm{~km} / \mathrm{s}$ and has a profile corresponding to one half-cycle of a cosine. The white lines indicate where the velocity has fallen to $5 \mathrm{~km} / \mathrm{s}$. The source for the wave packet is a displacement in the $y$ direction centred on the asterisk.

Many applications are hoped for, in particular to study the interaction of solar waves with sunspots or near-surface convection. The code will certainly be useful to study the effects of strong perturbations. Figure 7 shows the deformation of a wave front as it traverses a $10 \mathrm{~km} / \mathrm{s}$ jet flow.

\subsection{Interaction of acoustic waves with a magnetic cylinder}

The interaction of acoustic waves with sunspot magnetic fields is strong in the near surface layers. As a result, the effect of the magnetic field on the travel times is not expected to be small near the surface. Deeper inside the Sun, however, the ratio of the magnetic pressure to the gas pressure becomes small, and it is tempting to treat the effects of the magnetic field on the waves using perturbation theory.

With the aim of studying magnetic effects in time-distance helioseismology, we used the first-order Born approximation to compute the scattering of small amplitude acoustic plane waves by a magnetic cylinder embedded in an otherwise uniform medium. Because this simple problem has a known exact solution for arbitrary magnetic field strengths, we can study the validity of the linearization of the wave field on the square of the magnetic field. We show, by comparison with the exact solution, that travel-time shifts computed in the 
single-scattering Born approximation are everywhere valid to first order in the ratio of the magnetic to the gas pressures. We conclude that, for typical values of the solar magnetic field, the Born approximation should be good at depths larger than a few hundred km below the photosphere (Gizon et al. 2006). We note that the exact solution can be used to validate the numerical simulation of Section 7.1.

\subsection{Acoustics in spherical geometry}

Methods of local helioseismolgy are also being tested using numerical simulations of wave propagation in spherical geometry. A setup in spherical geometry is necessary when wavelengths are not small compared to the solar radius. The acoustic wave field is obtained by solving the three-dimensional Euler equations in a general background solar model (no magnetic field). The excitation of the oscillations is modeled by many uncorrelated sources of excitation which are randomly distributed delta functions in a narrow spherical shell close to the solar surface. We are hoping to validate the various methods of helioseismological data analysis with this numerical code. The effect of differential rotation, meridional circulation, sound speed anomalies on the acoustic wave field will be studied. See Hanasoge et al. (2006).

\section{Farside imaging}

Space weather forecasting is important due to the impact that solar flares have on space weather. Large, complex active regions tend to be the source of strong solar flares which eject charged particles into the solar wind, and directly affect Earth operations. Farside imaging offers up to two weeks notice of active regions rotating to the nearside, providing that the active region is evident at the surface over the full term of its farside tenure. Although there are many short-term predictive tools, the importance of farside imaging is not to be under-estimated.

It is well known that magnetic fields interact with acoustic waves, in particular they absorb their energies and change their travel times in the vicinity of the magnetic field. Phasesensitive holography is used to calculate the travel time perturbations. Farside imaging is a particular application of phase-sensitive holography (Braun \& Lindsey 2001). Given observations of acoustic waves on the front side and for each target point (or focal point) on the far side, two basic computations are done: the ingression and the egression. The egression is a reconstruction of the wave field that has emanated from the focal point at time $t$ based upon the observed Doppler signal in an annular pupil. The ingression is the time reverse of the egression: the wave field in the pupil is propagated forward in time to reconstruct the wave field at the focal point. The correlation between the ingression and the egression gives the phase lag (travel time difference) between waves going into, and waves going out from, the focal point on the Sun's far surface. A time decrease (or negative phase as it is shown on this scale) indicates the presence of a strong surface magnetic field proportional to its strength.

Here we have used GONG+ data to calculate the farside image for November 12, 2003. In Fig. 8, two large active regions are clearly visible as dark patches on the farside: AR 0486 (southern hemisphere) and AR 0488 (northern hemisphere). The optical paths of waves used for farside imaging have single-skip distances in the approximate range $0.8-1.5 \mathrm{rad}$, with spherical harmonic degree up to 70 , which produces a much coarser spatial resolution than 


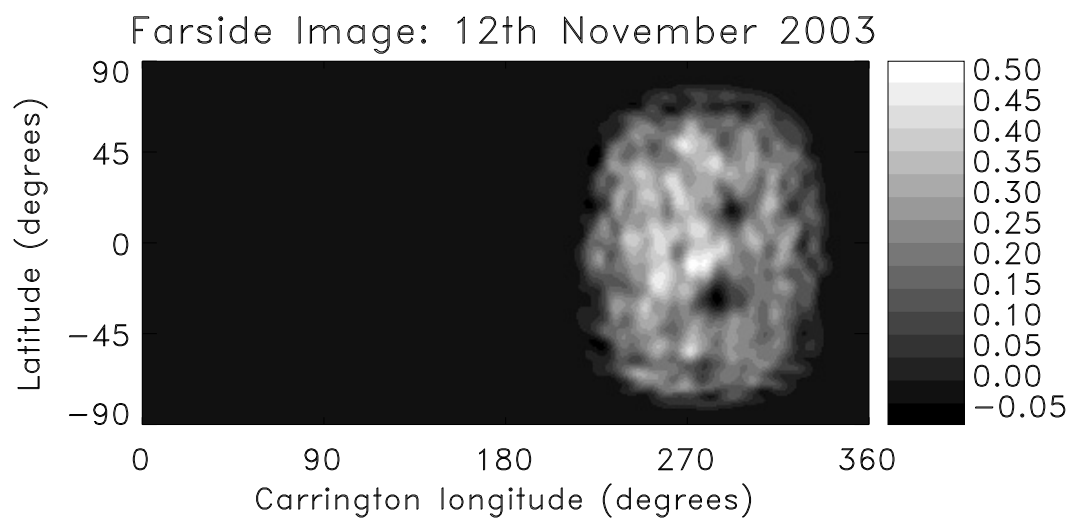

Figure 8. Farside holographic image of the Sun on the 12th November 2003 versus heliographic longitude and latitude. The greyscale gives the phase shift in radians. Two large active regions appear as dark patches on the farside: AR 0486 (southern hemisphere) and AR 0488 (northern hemisphere).

for seismic imaging of the nearside. At a frequency of $3.5 \mathrm{mHz}$ a diffraction limit of about $5^{\circ}$ is imposed which is good enough to image significant plage regions. The analysis here uses $2 \times 2$ skip holography. Therefore, this farside image is limited to approximately $50^{\circ}$ from the antipode of disk centre.

\section{Distant stars}

\subsection{Asteroseismology of K Giants}

Radial-velocity measurements of the K Giant $\alpha$ Hydra using the FEROS spectrograph at the $2.2 \mathrm{~m} \mathrm{MPG/ESA} \mathrm{telescope} \mathrm{in} \mathrm{La} \mathrm{Silla,} \mathrm{Chile,} \mathrm{reveal} \mathrm{pulsations} \mathrm{periods} \mathrm{of} \mathrm{3-4} \mathrm{days} \mathrm{(Fig.} \mathrm{9).}$ The main oscillations have periods in good agreement with theoretical expectations. The high-frequency part of the oscillation spectrum, however, is far from being understood. It has been suggested that oscillations in red giants are due to solar-like pulsations excited by turbulent convection. The possibility that all or part of the observed modes could be selfexcited Mira-like pulsations is, however, not ruled out. More observational data on K Giants will become available in the next few years to clarify the nature of the oscillations and to provide seismic constraints on the structure of these stars. See Setiawan et al. (2006).

\subsection{Analysis of time series of stellar oscillations.}

Quantitative asteroseismology requires extremely precise measurements of the frequencies, amplitudes, phases, and lifetimes of the stellar oscillations. The data analysis can be accomplished in the time domain or in Fourier space. In both cases, gaps in the data introduce complications. In the Fourier domain, the signal is convolved by the transformed of the observation window and Fourier amplitudes at different frequencies are correlated. We are 


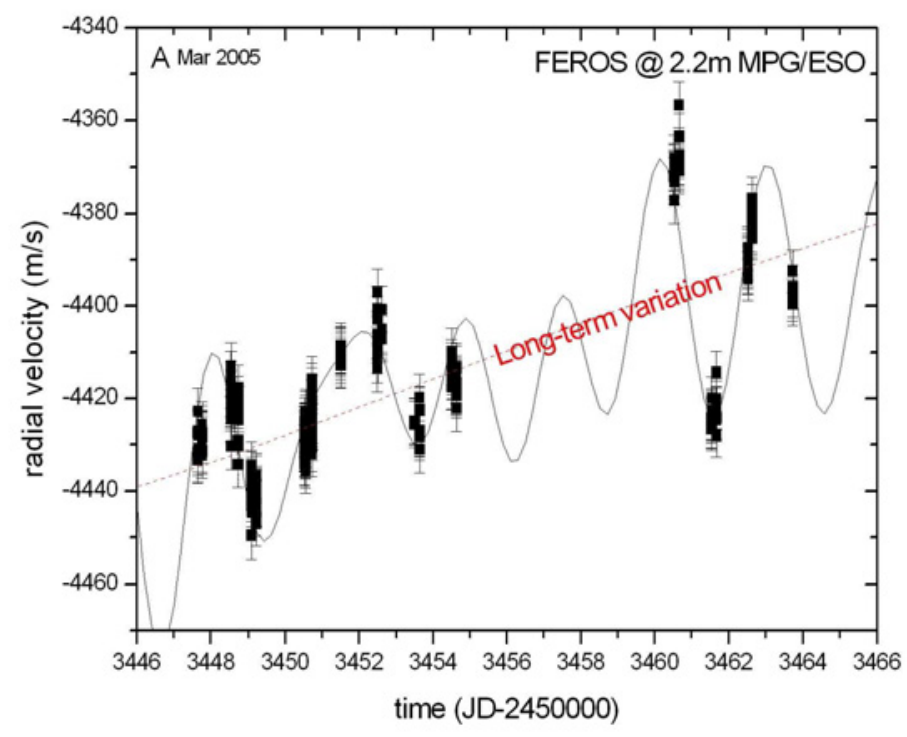

Figure 9. Radial velocity measurements of the K Giant $\alpha$ Hydra recorded during 12 days in March 2005. The solid line gives a fit of 5 superposed sine functions with periods 7.96, 3.6, 2.95, 2.48 , and 2.29 days. Besides the periodic behaviour, a long term variation is visible.

developing methods and analysis tools that take into account these correlations. In the case of solar-like oscillations, Monte-Carlo simulations show that our improved fitting method returns less noisy estimates of the line widths and amplitudes of the modes of oscillation. The improvement is less significant for long-lived, deterministic pulsations. Figure 10 shows a fit to a mode triplet in the Fourier domain (only the real part is shown) for a classical pulsator, the pre-white dwarf PG1159-035 (Winget et al. 1991).

\section{European network for helio- and asteroseismology}

HELAS is a new European network for helio- and asteroseismology, funded by the European Union for the period 2006-2010 (EU Framework Program 6). The MPS is an important component of HELAS (MR is Project Scientist, LG is Chair of the Local Helioseismology Network Activity). The objectives of the Local Helioseismology Network Activity are to (1) coordinate and consolidate European research activities in the field of local helioseismology, exchange knowledge and share experiences; (2) identify areas for which common actions are desirable; (3) promote the exchange of data for tests, comparisons, and analysis; (4) coordinate the process of developing common software tools; (5) facilitate the preparation for the SDO and Solar Orbiter missions. Three workshops are planned and one large conference will be organized in Göttingen in 2007. 


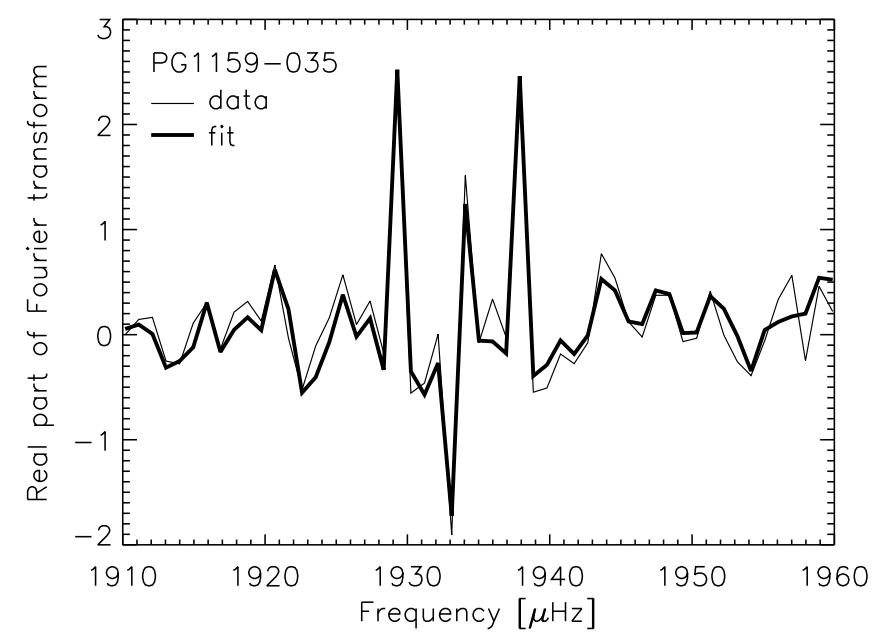

Figure 10. Section of the Fourier spectrum of PG1159-035 around the strongest dipole triplet near $1937 \mu \mathrm{Hz}$. The thin line is the real part of the Fourier transform of the data, the thick is the fit. Most of the variations away from the peaks is due to the convolution by the window function. Although it is not shown here, the imaginary part is fitted together with the real part.

Acknowledgements. The research presented in this paper is the result of work done in collaboration with others, including J. G. Beck, A. C. Birch, D. C. Braun, T. L. Duvall, S. Hanasoge, C. Lindsey, M. Rempel, and J. Setiawan.

\section{References}

Braun, D. C. \& Lindsey, C. 2001, ApJ, 560, L189

Cameron, R. \& Gizon, L. 2006, in Proc. SOHO 18, ESA SP-624

Duvall, T. L., Birch, A. C., \& Gizon, L. 2006, ApJ, 646, 553

Gizon, L. 2006a, Mod. Phys. Lett. A, 21, 1701

Gizon, L. 2006b, Cent. Eur. Astrophys. Bull., 30, 1

Gizon, L. \& Birch, A. C. 2005, Liv. Rev. Solar Phys., 2, 6 (http://www.livingreviews.org/lrsp-2005-6)

Gizon, L. \& Rempel, M. 2007, ApJ, submitted

Gizon, L., Duvall, T. L., \& Schou, J. 2003, Nature, 421, 43

Gizon, L., Hanasoge, S. M., \& Birch, A. C. 2006, ApJ, 643, 549

Hanasoge, S. M., Larsen, R. M., Duvall, T. L., DeRosa, M. L., Hurlburt, N. E., Schou, J., Roth, M., Christensen-Dalsgaard, J., \& Lele, S. K. 2006, ApJ, 648, 1268

Jackiewicz, J., Gizon, L., \& Birch, A. C. 2006a, in Proc. SOHO 17, ESA SP-617

Jackiewicz, J., Gizon, L., \& Birch, A. C. 2006b, in Proc. SOHO 18, ESA SP-624

Setiawan, J., Roth, M., Weise, P., \& Doelinger, M. P. 2006, Mem. S.A.It. 77, 510

Winget, D. E., Nather, R. E., Clemens, J. C. et al. 1991, ApJ, 378, 326 


\title{
Structure and evolution of supergranulation from local helioseismology
}

\author{
J. Hirzberger ${ }^{1, *}$, L. Gizon ${ }^{1}$, S. K. Solanki ${ }^{1}$, and T. L. Duvall, Jr. ${ }^{2}$ \\ ${ }^{1}$ Max-Planck-Institut für Sonnensystemforschung, Katlenburg-Lindau, Germany \\ ${ }^{2}$ Laboratory for Astronomy and Solar Physics, NASA/GSFC, Greenbelt,USA \\ *Email: hirzberger@mps.mpg.de
}

\begin{abstract}
Maps of the horizontal divergence of the near-surface velocity field have been calculated using local helioseismology and SOHO/MDI full-disk Dopplergrams. These maps provide a continuous coverage for two to three months each year with a cadence of 12 hours. Geometrical and evolutional properties of individual supergranular cells have been studied. Supergranular cells have sizes in a range around $650 \mathrm{Mm}^{2}$ (circular diameter of $28.77 \mathrm{Mm}$ ) with lifetimes of up to 4.5 days. We also observe a clear trend for larger cells to have stronger divergence values and larger lifetimes than smaller ones.
\end{abstract}

\section{Data}

The basic data used for this study are time series of full-disk Dopplergrams of several months length, obtained in a period between 1996 and 2002 with the Michelson Doppler Imager onboard the SOHO spacecraft. To extract information about supergranular flows the techniques of $f$-mode time-distance helioseismology (see Duvall \& Gizon 2000) have been applied. The resulting data consist of $120^{\circ} \times 120^{\circ}$ (heliographic coordinates) divergence maps of the horizontal flow field $\left(\nabla \cdot v_{\mathrm{h}}\right)$ in a depth of $1 \mathrm{Mm}$ below the solar surface (see Fig. 1). The spatial sampling rate of these maps amounts to $0.24^{\circ}$ and the cadence is $12 \mathrm{~h}$. The maps are interpolated onto a longitude-latitude $(\phi-\lambda)$ grid and structures with sizes $l>250(<17.49 \mathrm{Mm})$ are filtered out. Since the noise levels in the divergence maps increases with distance from disk center only regions within a radius of $48^{\circ}$ have been considered in the subsequent statistical analysis.

\section{Image segmentation}

Individual supergranular structures are detected by applying a segmentation algorithm to the divergence maps based on band-pass filtering in the Fourier domain (see Roudier \& Muller 1987). The obtained structures are related to regions of strong positive divergence values which are the central regions of supergranular cells. In order to capture the entire dimensions of the supergranular cells local divergence minima have been searched. These are pixels for which all surrounding pixels have higher values. Then each detected supergranular structure has been dilated against the surrounding local minima. Hence, the FOVs are fully covered by the thus obtained supergranular cells. 


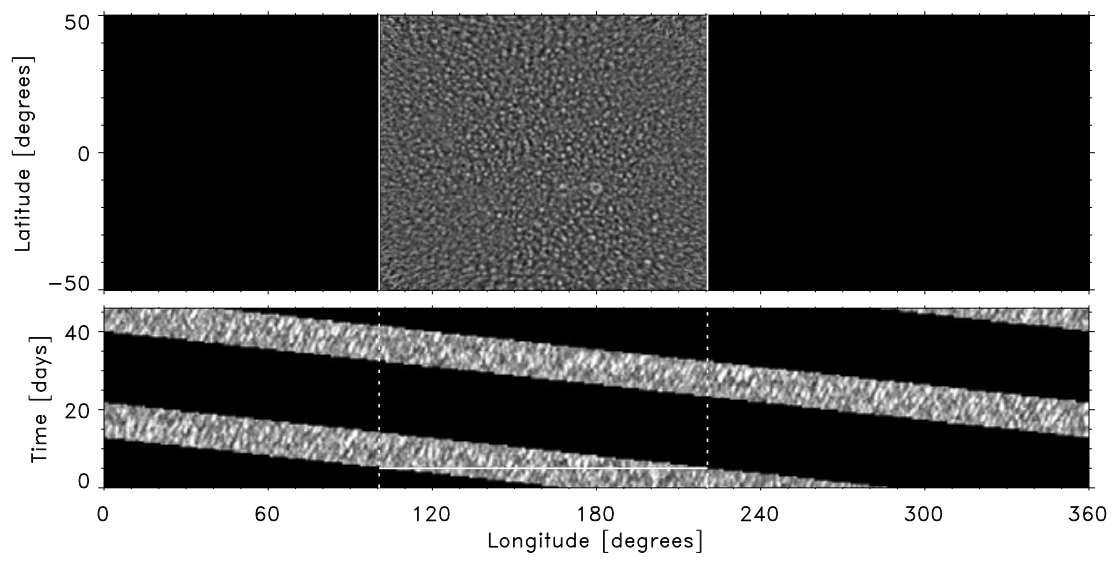

Figure 1. Time series of divergence maps obtained with $f$-mode time-distance helioseismology techniques. The upper panel shows a single map from the series. Light structures are regions where $\nabla \cdot v_{\mathrm{h}}>0$ (supergranules); in dark regions is $\nabla \cdot v_{\mathrm{h}}<0$ (inter-supergranular lanes). In the lower panel an $x$ - $t$-slice of the series from the year 2000 is displayed. At all times only one third $\left(120^{\circ}\right)$ of the entire solar longitude range is visible.

Alternatively, the Fourier-based segmentation algorithm has been also applied to structures of strong convergence $\left(\nabla \cdot v_{\mathrm{h}}<0\right)$. The thus obtained structures have larger areas and much more complex shapes which surround the divergence centers (see Fig. 2). Therefore, similar to "normal" solar granulation supergranulation defines a pattern of local divergence centers separated by a network of "inter-supergranular" lanes.

\section{Cell structure}

The distribution of detected supergranular cell areas (see Fig. 3) shows a well-defined maximum at $650 \mathrm{Mm}^{2}$ (median value of distribution) which corresponds to a circular diameter of $d=\sqrt{4 A / \pi}=28.77 \mathrm{Mm}$. The shape of the histogram is significantly different from that given in del Moro et al. (2004) who analyzed a much smaller statistical sample but have used similar data. However, it shows close similarity to distributions found by e.g. DeRosa $\&$ Toomre (2004). The mean divergence in most of the detected cells is close to zero which means that the supergranular cells are properly detected by the segmentation algorithm. The distribution of maximum divergences has a similar shape as the area histogram leading to an almost linear dependency between area and maximum divergence. Only the very largest cell structures seem to deviate from this correlation. Basically, this relation looks very similar to the area-intensity and area-velocity distributions found for "normal" granulation (see e.g. Hirzberger 2002). A close similarity in the structural properties of solar granulation and supergranulation was already stated in Berrilli et al. (2004).

\section{Lifetimes}

The time evolution of the supergranular cells has been studied by tracking the evolution of the segmented cells. The corresponding tracking algorithm is a modified version of the 

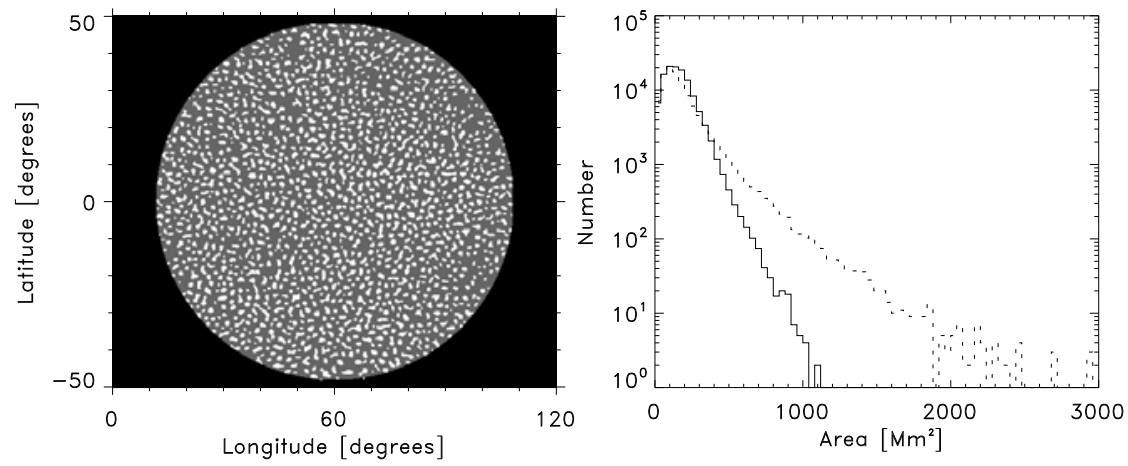

Figure 2. Fourier-based segmentation results. Left: Segmented divergence map; right: histograms of detected areas. The solid line shows the distribution of areas of divergence centers, the dotted line shows the distribution of convergence centers.
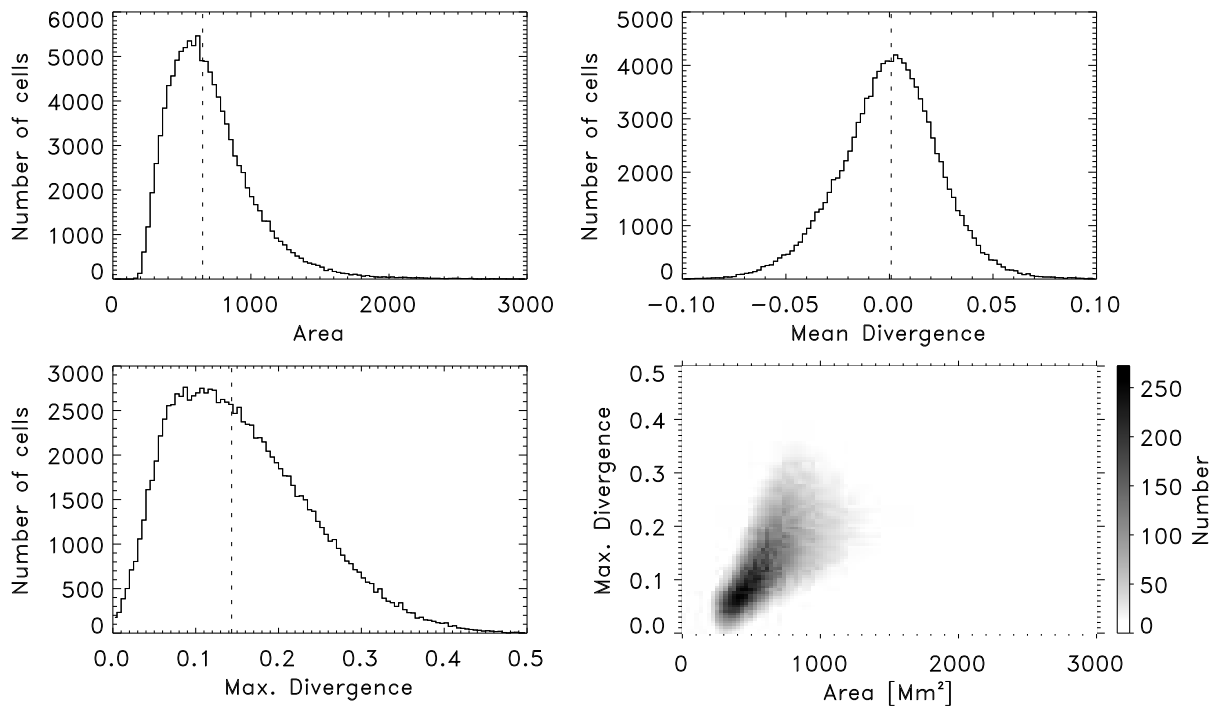

Figure 3. Histograms of areas, mean divergences, and maximum divergences of segmented supergranular cells. The vertical dotted lines represent median values of the distributions. The lower right panel shows a scatter plot of maximum divergence vs. cell area.

codes used for tracking granules (Hirzberger et al. 1999) and mesogranules (Leitzinger et al. 2005). Since the FOV is moving in longitude with time (see Fig. 1) only a small number of the detected supergranules can be followed from their "birth" to their "death". The thus estimated supergranular lifetimes and dynamical properties may be, therefore, biased by a selection effect. In addition, lifetimes below 12 hours are not resolved in the used data. The resulting lifetime histogram and variations of cell sizes and maximum divergences with supergranular lifetime is shown in Fig. 5. Lifetimes up to 4.5 days have been detected although the bulk of supergranular cells have lifetimes between 1 and 3 days. Moreover, a tendency for longer-living structures to possess larger areas and stronger divergences can 

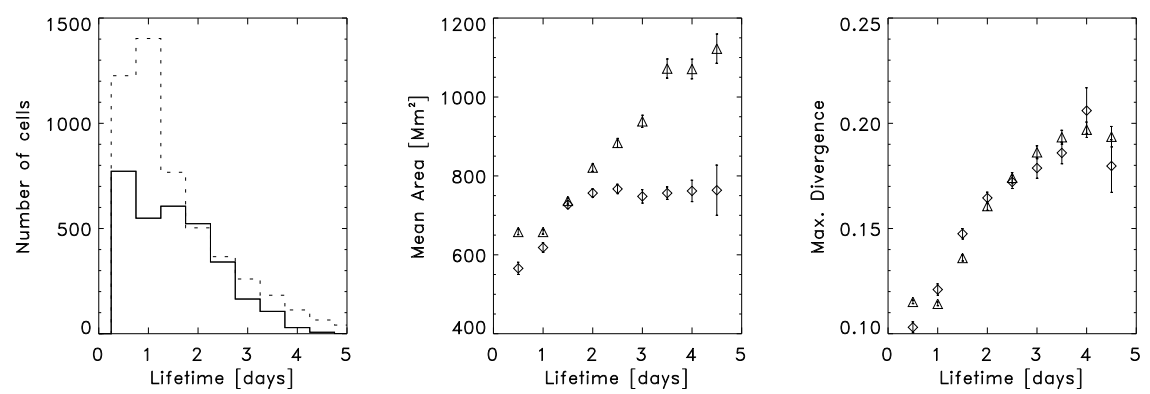

Figure 4. Evolutional parameters of supergranular cells: The left panel shows histograms of cell lifetimes (solid: feature tracking, dotted: 3d-segmentation). In the middle and right panels the timeaveraged areas and maximum divergences of the lifetime histories have been once more averaged in each bin of the lifetime histograms (diamonds: feature tracking, triangles: 3d-segmentation). The error bars denote standard deviations of the mean $(\sigma / \sqrt{N})$ in each bin of the histogram.

be detected. This result is again in close similarity to the behavior of "normal" granulation cells.

As a second method to follow time evolution we applied a 3d-version of the Fourier segmentation algorithm mentioned above. The resulting structures represent supergranules in the $\phi-\lambda$ - $t$-space. These structures have been sliced for each time step and the thus recognized supergranules have also been dilated against the surrounding local divergence minima. The results of a subsequent statistical analysis are overplotted in Fig. 4.

\section{Outlook}

The fact that the data presented here cover a period between 1996 and 2002 establishes the possibility to study variations of supergranular structure and dynamics with the solar cycle. In addition, a possible dependence with solar latitude may be studied. Both topics require a careful analysis of magnetic field maps which are also available from the MDI data archive. If such an extension of the data analysis would be successful important new insights into the interaction between solar magnetic fields and convection might be retrieved.

\section{References}

Berrilli, F., del Moro, D., Consolini, G., Pietropaolo, E., Duvall, T. L., Jr., \& Kosovichev, A. G. 2004, Solar Phys., 221, 33

del Moro, D., Berrilli, F., Duvall, T. L., Jr., \& Kosovichev, A. G. 2004, Solar Phys., 221, 23

DeRosa, M. L. \& Toomre, J. 2004, ApJ, 616, 1242

Duvall, T. L., Jr. \& Gizon, L. 2000, Solar Phys., 192, 177

Hirzberger, J. 2002, A\&A, 392, 1105

Hirzberger, Bonet, J. A., Vázquez, M., \& Hanslmeier, A. 1999, ApJ, 515, 441

Leitzinger, M., Brandt, P. N., Hanslmeier, A., Pötzi, W., \& Hirzberger, J. 2005, A\&A, 444, 245

Roudier, T. \& Muller, R. 1987, Solar Phys., 107, 11 
Modern Solar Facilities - Advanced Solar Science, 107-113

F. Kneer, K. G. Puschmann, A. D. Wittmann (eds.)

(C) Universitätsverlag Göttingen 2007

\title{
Solar adaptive optics
}

\author{
T. Berkefeld \\ Kiepenheuer-Institut für Sonnenphysik, Freiburg, Germany \\ Email: berke@kis.uni-freiburg.de
}

\begin{abstract}
We give an overview of solar adaptive optics (AO) systems, its differences to nighttime $\mathrm{AO}$ systems and some basic design considerations for the wavefront sensor and the wavefront reconstruction. As an example, we present the Kiepenheuer Adaptive Optics System (KAOS), lessons learned from four years of operation, and the various AO-related projects that use or will use KAOS.
\end{abstract}

\section{Introduction}

Ground based observations of the Sun in the visible spectral range are severely limited by wavefront aberrations caused by the Earth's atmosphere. Typically, a spatial resolution of only 1-1.5 arcseconds can be achieved, corresponding to the diffraction limit of a $10 \mathrm{~cm}$ telescope. However, in order to answer fundamental questions that occur when trying to understand the Sun, a spatial resolution approaching the diffraction limit of meter-class telescopes is required. For very short exposures this can be achieved by image reconstruction techniques such as phase diversity (van Noort et al. 2005; Tritschler \& Schmidt 2002) or speckle imaging (v.d.Lühe 1989; Mikurda \& v.d.Lühe 2006). But for high accuracy observations, e.g. spectropolarimetric measurements, long exposure times make a realtime wavefront correction mandatory. Considering observing efficiency, AO works in two ways:

- For a given spatial resolution, AO increases the amount of observing time.

- For a given observing time, AO improves the spatial resolution.

At first, image stabilisation systems were introduced (Schmidt \& Kentischer 1996; Ballesteros et al. 1996), but only by using a full compensation of the wavefront can the theoretical resolution of the telescope be restored. Although adaptive optics has been used for nighttime astronomy for more than ten years (Rousset et al. 1990), its application in solar astronomy was delayed for several years due to three reasons:

- Wavefront sensors must be able to track an arbitrary structure on an extended source, rather than an isolated point source.

- The Sun heats the ground, so the daytime seeing is usually worse than the nighttime seeing.

- Solar observations are mostly done in the visible, therefore the AO must correct in this regime. 
Table 1. Present solar AO systems.

\begin{tabular}{lcccc}
\hline & DST, Sac. Peak & SST, La Palma & VTT, Tenerife & BBSO, Big Bear \\
\hline \hline first light & 1998 & 2000 & 2002 & 2004 \\
\hline telescope diameter $[\mathrm{m}]$ & 0.76 & 0.98 & 0.70 & 0.65 \\
\hline \# corrected modes & 60 & 27 & 27 & 40 \\
\hline \# actuators & 97 & 35 & 35 & 97 \\
\hline 0 db bandwidth $[\mathrm{Hz}]$ & 130 & $?$ & 100 & 130 \\
\hline
\end{tabular}

Presently four solar AO systems are used regularly (in the order of first light): The AO at the Dunn Solar Telescope (DST) of the National Solar Observatory, Sacramento Peak (Rimmele \& Radick 1998), the AO at the Swedish solar telescope (SST), La Palma (Scharmer et al. 2000), the AO at the German Vacuum Tower Telescope (VTT) of the Kiepenheuer-Institute, Tenerife (v.d.Lühe et al. 2003), and the AO at the Big Bear Solar Observatory (BBSO) (Rimmele et al. 2004). Table 1 shows the basic parameters of these four systems. During the last years the existence of a good working AO system has become an indicator for the competitiveness of a telescope (e.g quality of the post focus instrumentation).

Section 2 gives an overview of solar wavefront sensing, in Section 3 the basic wavefront reconstruction is explained and in Section 4 the KAOS system is shown as an example for solar AO systems.

\section{Solar Shack-Hartmann wavefront sensing}

As already mentioned, the wavefront sensor (WFS) in solar AO systems has to track on arbitrary structures. Up to now, only crosscorrelating Shack-Hartmann (SH) WFSs are used. The differences to nighttime SH sensors are the larger field of view (FOV), the faster and larger CCD/CMOS WFS cameras and the way the shift vector is obtained. In order to achieve a good correlation function, the FOV should be as large as possible, but it should not exceed the isoplanatic patch $\left(\sim 10^{\prime \prime}\right.$ in the visible). The pixel scale should match the resolution of one subaperture. Crosscorrelating the image of a reference subaperture with the other subapertures leads to correlation functions that are more star-like and can be treated like stars in a stellar WFS (parabolic/gauss fit around the brightest pixel). Both FFT-based crosscorrelation and direct crosscorrelation methods are used. Solar WFS cameras typically have $\sim 250 \times 250$ pixels and operate at $1-2.5 \mathrm{kHz}$. The high framerates and the compute intensive crosscorrelation lead to a high computational load for the WFS. In contrast to nighttime AO where the wavefront reconstruction process is the most compute intensive part, this is the correlating Shack-Hartmann WFS for solar AO. As an example, Fig. 1 shows the KAOS lenslet geometry in the pupil plane, the WFS camera image and the resulting correlation functions. The KAOS WFS operates in a $10 \mathrm{~nm}$ band at about $500 \mathrm{~nm}$. The size of a subaperture corresponds to $10 \mathrm{~cm}$ in the pupil plane which results in 36 subapertures. 


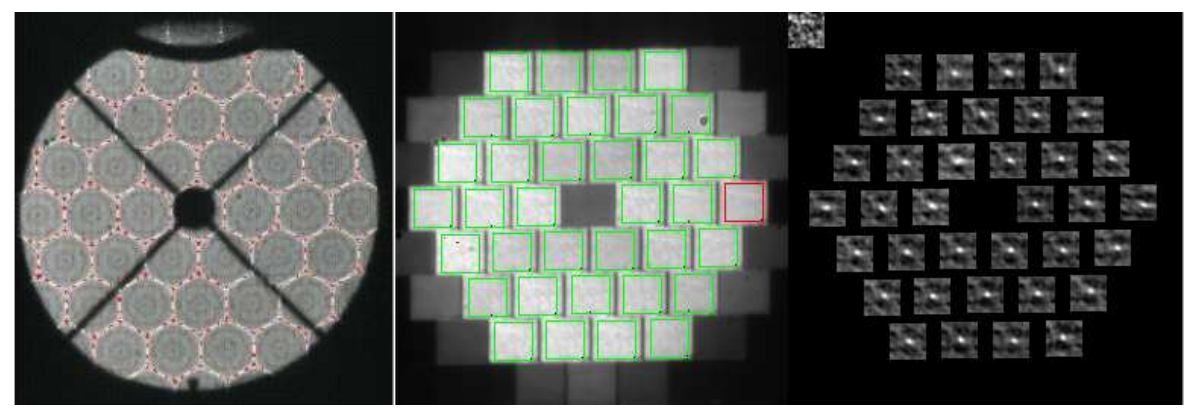

Figure 1. Screenshot from the KAOS user interface showing the WFS geometry of the pupil plane (left), image plane (centre) and the resulting correlation functions (right).

\section{Wavefront reconstruction and system control}

\subsection{Data reduction / crosscorrelation}

After the image data of a subaperture has been transferred completely from the camera into the memory of the control computer, the following data reduction steps must be performed (for each subaperture in the order given below, $n$ being the edge length of the subapertures in pixels):

- dark/flat correction,

- subtraction of the subaperture mean intensity,

- removal of the xy-intensity gradient,

- application of a Hamming window $W(x, y)=w(x) \cdot w(y)$ with $w(i)=0.54+(0.54-1) \cdot \cos (2 \pi i /(n-1)), \quad i=0 \ldots n-1$,

- addition of the former mean intensity,

- intensity normalization.

The subtraction / addition of the mean intensity and the application of the Hamming window are only advisable for FFT-based crosscorrelation. The removal of the intensity gradients and the intensity normalization are necessary to obtain a correlation peak which is related to the solar small scale structure, and not to the field edge. The reduced subaperture images are now crosscorrelated against a reference subaperture image that has undergone the same data reduction. A subsequent parabolic fit around the brightest pixel of the correlation function delivers subpixel accuracy for the correlation function position $C_{\max }$, leading to the desired xy-shift error signal $S_{\text {shift }}$ of the WFS:

$$
S_{\text {shift }}=R_{\text {ref }}-S_{\text {ref }}+R_{\text {shift }}+C_{\text {max }}
$$

$R_{\text {ref }}$ and $S_{\text {ref }}$ being the spot positions of the perfect wavefront of reference $R$ and subaperture $S$. The reference image should be updated at least once per minute by selecting the image 
with the most contrast, within the last minute. The time scale of one minute is shorter than the time scale of the evolution of solar structures resolved by a subaperture. The xy-shift $R_{\text {shift }}$ of the reference image is obtained from a crosscorrelation of the reference image with the previous reference,

$$
R_{\text {shift, new }}=R_{\text {shift,old }}+C_{\max } .
$$

Now the error shift vector can be applied to the wavefront reconstruction.

\subsection{Calibration / wavefront reconstruction}

The calibration and wavefront reconstruction is similar to nighttime $\mathrm{AO}$ and is therefore discussed only briefly.

The interaction matrix that links the wavefront sensor and the active mirrors (typically tiptilt mirror and deformable mirror (DM)) can either be obtained analytically by using models for the wavefront sensor and the mirrors, or experimentally by ramping each actuator one by one and recording the WFS response. The latter has the advantage of taking alignment errors and other deviations from the theoretical optics scheme into account but requires a pinhole that can be inserted into a focus preceding the active mirrors.

The reconstruction matrix (inverse of the interaction matrix) converts the measured shift vector from the Shack-Hartmann sensor to the desired actuator output voltage vector during closed loop. The reconstruction matrix is usually obtained by inverting the interaction matrix using a truncated Singular Value Decomposition (SVD). The SVD allows the rejection of noisy or otherwise badly behaving system eigenmodes. Alternatively, other sets of modes such as Karhunen-Loeve or Zernike modes can be used. They allow a more analytical system analysis and further improvements, but act as a spatial filter between WFS and the active mirrors, so the efficiency in terms of atmospheric wavefront error correction may go down.

The actuator output voltages are then fed to the servo control loop. Typically, this is a PI (proportional/integral) controller with distinct control parameters for the tiptilt mirror and the DM.

\section{The Kiepenheuer Adaptive Optics System (KAOS)}

The $70 \mathrm{~cm}$ VTT at the Observatorio del Teide, Tenerife, has been equipped with an adaptive optics system in 2002 which is used since then for more than $95 \%$ of the obervation campaigns. It provides diffraction limited observations in the visible for seeing better than 0.8 .

\subsection{System overview}

Table 2 gives the main parameters of KAOS: The required seeing of $0{ }^{\prime} 8$ for diffraction limited observing is the main limitation of KAOS because the median daytime seeing is well above $1^{\prime \prime}$. However, typically two to four hours after sunrise the seeing is often of the order of $0{ }^{\prime} 8$ or better, so that observations at maximum resolution are possible. The ability to work stable with 1". 8 seeing enables the observer to continue the observations without loss of lock when short "bubbles" of turbulent air cross in front of the telescope, which happens quite often. 
Table 2. KAOS parameters.

\begin{tabular}{lc}
\hline \hline minimum seeing for diffraction limited observing & $0 !^{\prime} 8$ \\
\hline minimum seeing for stable operation & $1{ }^{\prime \prime} 8$ \\
\hline minimum distance to the solar limb (with faculae points) & $15^{\prime \prime}$ \\
\hline maximum mean wind speed & $12 \mathrm{~m} / \mathrm{s}$ \\
\hline maximum number of corrected degrees of freedom & 27 \\
\hline loop frequency / bandwidth of the control loop & $2100 / 100 \mathrm{~Hz}$ \\
\hline WFS number of subapertures & 36 \\
\hline WFS field of view, pixel scale & $12^{\prime \prime}, 0 . ' 5 /$ pixel \\
\hline DM type, number of actuators & bimorph, 35 \\
\hline control computer, OS & Sun V880, Solaris \\
\hline
\end{tabular}

A key design issue during the development phase of KAOS was the capability of tracking any solar structure as long as there is enough contrast in the image. This is possible almost anywhere on the solar disk because of the ubiquitous solar granulation pattern. Only close to the solar limb (with almost no contrast to track on) a high contrast facular point is needed as a reference by the wavefront sensor. As a second requirement, the observing scientist should be able to control the AO. Therefore all stages of the WFS are motorized and moved automatically by a graphical user interface (GUI) which was designed for ease of use.

Figure 2 plots measurements of the residual RMS wavefront error of AO corrected observations as a function of the Fried parameter $r_{0}$. Diffraction limited correction is reached for seeing better than $r_{0}=13 \mathrm{~cm}$. The measurement when $r_{0}=3.5 \mathrm{~cm}$ was an extreme case and was probably done with the AO tracking on a pore. Nevertheless it shows that KAOS can be used over a wide range of observing conditions. The best ever achieved Strehl was 0.8 for extremely good seeing $\left(r_{0} \approx 40 \mathrm{~cm}\right)$.

\subsection{Lessons learned}

During the four years of KAOS operation we made the following experiences:

- 16x16 pixels per SH-subaperture are not sufficient to track reliably on granulation during average seeing $\left(1^{\prime \prime}-1{ }^{\prime \prime} 5\right)$. We therefore changed to $24 \times 24$ pixels / subaperture in 2003 and can now track during $2^{\prime \prime}$ seeing.

- It is mandatory to motorize all moving stages of the WFS in order to allow an automated setup / observing procedure for ease of use.

- A high bandwidth is very important. The biggest KAOS improvement came from the installation of a new WFS camera in 2006 that doubled the system bandwidth.

- A high order AO system that provides a stable PSF also under median seeing conditions is highly desirable, as has been also proven by the immediate success of the NSO / Sacramento Peak high order AO system (Rimmele et al. 2004). 


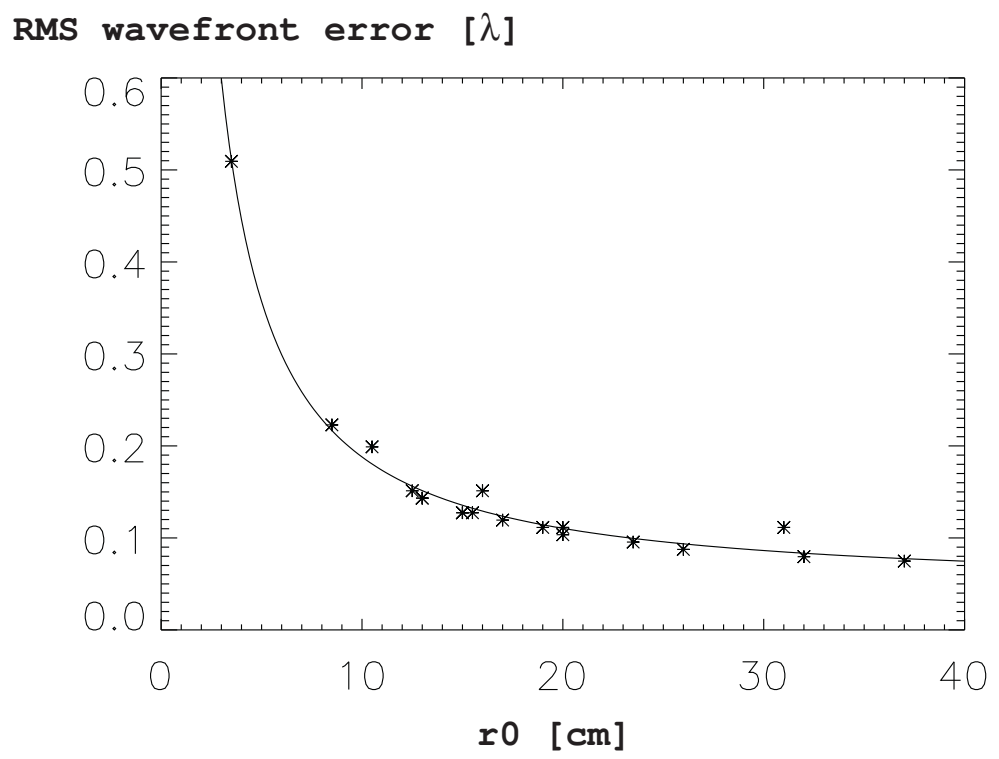

Figure 2. Residual RMS wavefront error of AO corrected observations as a function of the seeing conditions.

Table 3. Other AO projects at KIS.

\begin{tabular}{lccc}
\hline \hline & first light & \# corrected modes & bandwidth [Hz] \\
\hline GREGOR first light AO & 2008 & 55 & 100 \\
\hline GREGOR high order AO & 2009 & $250-300$ & 150 \\
\hline GREGOR MCAO & 2008 & $55+2 \times 60$ & 100 \\
\hline GREGOR nighttime AO & 2008 & 40 & 60 \\
\hline SUNRISE CWS & 2009 (flight) & 5 & 70 \\
\hline
\end{tabular}

- A dedicated realtime operating system is not necessary. Modern standard operating systems such as Solaris or Linux can assign one CPU to one process exclusively. The readout timing of the WFS camera then effectively leads to a realtime behaviour.

\section{Other AO projects at KIS}

Table 3 shows the basic parameters of other KIS AO projects, most of which are connected to the new $1.5 \mathrm{~m}$ solar telescope GREGOR. The GREGOR first light AO will be a scaled-up version of the VTT AO and will rely on proven technology, but will have less corrected degrees of freedom per aperture area than the VTT AO. This limitation will be overcome by a future high order AO (Berkefeld et al. 2004) that will allow diffraction limited observations under seeing conditions of up to 1'.3-1".5. The GREGOR MCAO (Berkefeld et al. 2006) will extend the corrected field of view to one arcminute by using two additional DMs at heights 
conjugated to strong atmospheric turbulence layers, thus giving AO-based observations another huge performance leap. The nighttime AO will use the active mirrors of the solar AO plus a very light-sensitive nighttime WFS. The last (but not least) project is the image stabilization and alignment system (CWS) for the $1 \mathrm{~m}$ balloon-borne telescope SUNRISE (Schmidt et al. 2006) that will fly in summer 2009.

\section{Conclusions}

In the last years adaptive optics systems have become a mature instrument for both improving the spatial resolution of solar observations and the observing efficiency at major solar telescopes. Future developments, e.g. at the planned $4 \mathrm{~m}$ Advanced Technology Solar Telescope, will lead to high order systems that provide a stable PSF under median seeing conditions and to multi-conjugate systems that have the capability to extend the corrected FOV considerably.

\section{References}

Ballesteros, E., Collados, M., Bonet, J. A., et al. 1996, A\&AS, 115, 353

Berkefeld, T., Soltau, D., \& von der Lühe, O. 2004, Proc. SPIE, 5490, 260

Berkefeld, T., Soltau, D., \& von der Lühe, O. 2006, Proc. SPIE, 6272, 627205

Mikurda, K. \& v.d.Lühe, O. 2006, Solar Phys., Volume 235, Issue 1-2, 31

Rimmele, T. \& Radick, R. 1998, in Adaptive Optics System Technologies, Proc. SPIE, 3353, 72

Rimmele, T., Richards, K., Hegwer, S., et al. 2004, Proc. SPIE, 5171, 179

Rousset, G., Fontanella, J., Kern, P., Gigan, P., \& Rigaut, F. 1990, A\&A, 230:L29

Scharmer, G. B., Shand, M., Löfdahl, M. G., Dettori, P. M., \& Wei, W. 2000, in Proc. SPIE, 4007, 239

Schmidt, W., Berkefeld, T., Feger, B., et al. 2006, Proc. SPIE, 6274

Schmidt, W. \& Kentischer, T. 1996, A\&A, 113, 363

Tritschler, A. \& Schmidt, W. 2002, A\&A, 382, 1093

van Noort, M., Rouppe van der Voort, L., \& Löfdahl, M. G. 2005, Solar Phys., Volume 228, Issue 1-2, 191

v.d.Lühe, O. 1989, High spatial resolution solar observations, Proceedings of the 10th Sacramento

Peak Summer Workshop, Sunspot, New Mexico, August 22-26, 1988, 147

v.d.Lühe, O., Soltau, D., Berkefeld, T., \& Schelenz, T. 2003, Proc. SPIE, 4853, 187 



\title{
Anisoplanatic optical transfer functions for solar adaptive optics from simulation using wave front sensor data
}

\author{
M. Sailer ${ }^{1, *}$ and 0 . von der Lühe ${ }^{2}$ \\ ${ }^{1}$ Institut für Astrophysik, Göttingen, Germany \\ ${ }^{2}$ Kiepenheuer-Institut für Sonnenphysik, Freiburg, Germany \\ *Email: msailer@astro.physik.uni-goettingen.de
}

\begin{abstract}
Solar observations with online compensation of atmospheric seeing is possible at the Vacuum Tower Telescope (VTT) on Tenerife (Spain) with the aid of the Kiepenheuer Adaptive Optics (AO) System Kaos. For the post-facto speckle image reconstruction methods used at our institutes, however, problems arise regarding the photometric accuracy of the intensity enhancement at each position of the reconstructed image (Puschmann \& Sailer 2006), rooting in the lack of knowledge about the field dependency of the actual residual wave front (WF) after correction and therefore of the instantaneous optical transfer functions (OTFs) at the time of each exposure.

To process more adequately particular sets of image data obtained with KAos, we simulate their OTFs and the according statistically averaged quantity for the reconstruction, the speckle transfer function (STF), with support of an activity log of the AO system during the time of data acquisition. Data regarding the wave front sensor (WFS) and the deformable mirror (DM) are analysed to model the atmospheric conditions and the average compensation power of the AO system. With this information we simulate the propagation of atmospherically distorted WFs through the optical elements of the VTT and KAOs, approaching the average WF error statistics reflected in the experimental data.
\end{abstract}

\section{Introduction}

Seeing induced wave front (WF) distortions evolve from differing optical path lengths of parallel light rays traversing each point at the telescope's entrance window. They traveled through different portions of the turbulent atmosphere with statistical distribution of the refractive index and accumulated retardations along their way, i.e. the direction along which the wave front sensor (WFS) is 'looking' through the earth's atmosphere to measure the WF deformations. Since telescope optics are designed to focus an even wave front, this cannot be optimally accomplished anymore if seeing is present. Time dependent aberrations remain.

Adaptive optics ( $\mathrm{AO}$ ) provides us with the ability to collect observational data that are realtime corrected for these wave front deformations. Own observations with the AO system KAOS (Kiepenheuer Adaptive Optics System), installed at the Vacuum Tower Telescope (VTT) on Tenerife and operated by the Kiepenheuer-Institut für Sonnenphysik (KIS), have shown that it improves the resolution of the raw images by factors of up to four, depending on the seeing, such that, occasionally, we even reach the diffraction limit. AO enlarges 
definitely the time intervals with excellent 'effective' seeing, which is of special importance both for long exposure observations and speckle applications: With an augmented number of high quality short exposures in each burst of the observational data image reconstruction manages much more consistently to extract at least 'almost' diffraction limited information for a subsequent time series.

One serious restriction to the above lies in the very principle of the conventional AO setup, i.e. the compensation of incoming wave fronts along only one single line of sight. This results in a direction dependent WF error and therefore varying resolution in the images throughout the field of view. Optimal image quality, in average, is therefore found only around the 'lockpoint', the image point which corresponds to the line of sight of the WFS. The problem for conventional speckle reconstruction, however, lies in the step of intensity amplification, which is now field dependent and also depends on structure sizes. If we need photometric accuracy, we have to measure or model this dependency to avoid overestimation around the lockpoint and underestimation in the outermost image regions. For this we have to study the changes to the purely atmospherically influenced distribution of transmitted signal strength over structure size, since the correction by AO is never perfect, but rather reduces the error contributions of only the lowest modes in a suitable decomposition of the WF (e.g. Zernike modes). For a series of short exposures this is reflected exactly by the shape of the speckle transfer function (STF), which characterises, in Fourier space, a representative point spread function (PSF) for the whole image series. With the aid of simulations and WFS measurements we intend to model this function for the whole field of view.

\section{Simulation}

The purpose of this simulation is the characterisation of the transfer properties of the VTT and KAOS with given atmospherically distorted WFs. The latter are modelled as phase screens that show the distribution of phase retardations across the entrance pupil of the telescope and are calculated with the external software package TuRBuLen $Z^{1}$ (Lane et al. 1992; Glindemann et al. 1993; Berkefeld 1998; Weiß 2003). The advantage of this code is its ability to model the atmosphere with both good accuracy and a large parameter space. For the correction of the time varying phase delays, however we model the necessary components of the $\mathrm{AO}$ system in an own simulation code described in the following.

\subsection{Deformable mirror}

To compensate the optical path differences across the incoming light beam, Kaos uses a deformable mirror with 35 actuators, arranged in keystone-geometry, as sketched in Fig. 1 (right image). By addressing the electrodes at the backside of the reflecting surface, the local curvature is changed in order to 'imitate' as well as possible the atmospherically induced WF deformation, but with half its amplitude, since after reflection, the path difference has doubled. In the image, as used in the simulation, the theoretical deflection due to a single activ actuator is superimposed to the distribution pattern in gray scale. It is the graphical representation of the solution of the Poisson equation of second degree $\nabla^{2}\left(\nabla^{2} \varphi+A \cdot V\right)=0$

\footnotetext{
${ }^{1}$ The code (version 1.0) is freely downloadable from the Max-Planck-Institut für Astronomie in Heidelberg: http://www.mpia-hd.mpg.de/AO/ATMOSPHERE/TurbuLenZ/tlz.html
} 

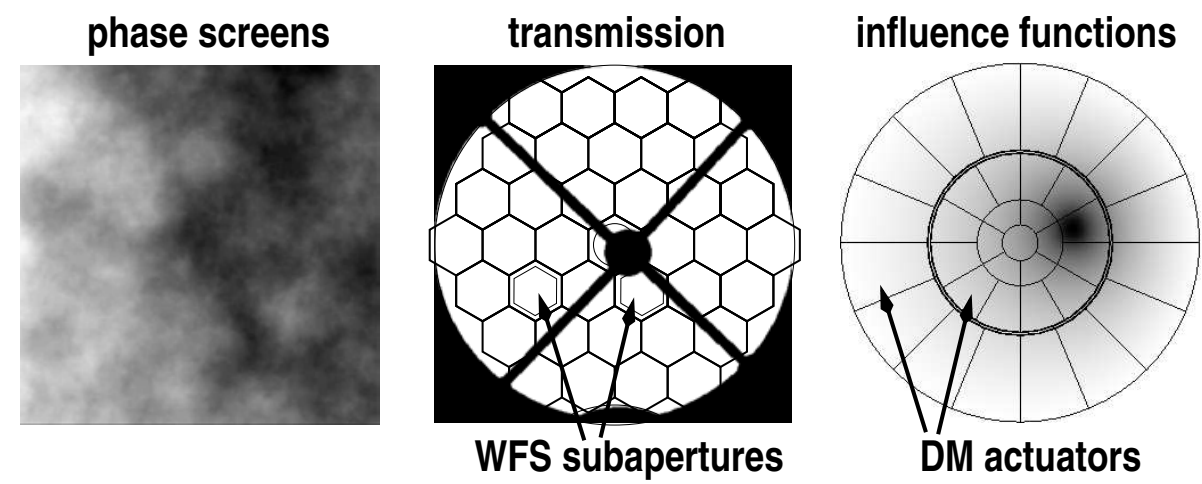

Figure 1. Left: Wave fronts are generated with the TuRbuLenZ package as phase screens. They represent the optical path differences in a cross-section of a light beam. Middle: The entrance pupil of the telescope acts as a transmission mask for the wave fronts and it is imaged on to the hexagonal lenslet array of the wave front sensor, that determines the local tilt of the wave front within each lenslet area. Right: For compensation of path differences, the shape of the deformable mirror is controlled by actuators, 35 electrodes that deform the surface similarily to the deflection shown as superimposed greyscale image. Note that the outer ring of actuators only serves to provide the boundary conditions for the illuminated inner part.

introduced by Roddier (1988), where $\nabla^{2}$ is the 2-dimensional Laplace operator, $\varphi(x, y)$ the mirror's surface and $V(x, y)$ the voltage distribution. The constant $A$ includes material constants.

\subsection{Wave front sensor}

The information, however, to control the deformable mirror, is derived from the measurements of the wave front sensor (WFS). In a Hartmann-Shack type sensor the WF's deformation is measured by the relative displacement of the images formed by a lenslet array that is placed in the image plane of the telescope's entrance pupil. In the simulation however we know the phase retardances and mimic the WFS measurements by a fit of a plane to that part of the WF which would be collected by each lenslet. The tilt angles can then be transformed into the collection of actuator signals needed to form a mirror surface that compensates best the particular WF errors. Important for the comparison with real data is the fact that in the simulation we can produce WFS signals of both the same type and relative magnitude as encountered at the telescope.

\section{Treatment of WFS data}

From the log files regarding the AO activity we want to derive estimates of the Fried parameter of the atmosphere alone, i.e. without $\mathrm{AO}$ correction, as well as the compensation performance of the AO system for the four different levels. For this we need the measured shift values and actuator signals, but only the knowledge of the actual reconstruction matrix enables to combine the two types of signals. For better visualisation we also apply a 
transformation matrix to calculate for each time series of shift values the according Zernike mode distribution. During AO compensation, only the residual differences are measured and compensated, such that the calculated Zernike modes represent an upper limit for the WF error at the lockpoint, from which we can calculate the corresponding Strehl number to characterize the image quality. Similarly, we have access to the actual correction as a WF rms error through the actuator signals, which have to be converted to equivalent shift values via the interaction matrix before deriving the Zernike decomposition. An estimate for the purely atmospheric disturbances, as would be derived in the case of an open feedback loop, is still possible during the closed loop. We just add the residual WFS shifts and the converted actuator signals to arrive at a view of the atmospheric turbulence through a 'low pass' filter, since both the DM and the WFS can, of course, not represent or sense Zernike modes of arbitrary high order. Unfortunately, we can not derive the exact WF rms errors in this case either, since the DM deformation is not exactly reconstructable from the WFS data. The comparison of the different Zernike distributions with theoretical ones derived under the premise of Kolmogorov turbulence gives us the atmospheric part of the aberration since the highest modes are not influenced by the low order AO system and must coincide for the actual value of $r_{0}$ in the atmosphere. For the simulation this is the most important parameter, since it allows to elect the phase screens representing best the atmospheric conditions for the actual image data. However, to model the anisoplanacy in the large field of view, we have to know more about the height distribution of the atmospheric turbulence (Berkefeld 1998). Here we chose a two layer atmosphere in our model and have to include therefore measurements of a second WFS in our analysis.

Acknowledgements. We thank the organising committee of this workshop for the occasion to present our ongoing studies to the community of solar physics. We thank T. Berkefeld for his efforts collecting correct logfiles of Kaos. M. Sailer thanks K. G. Puschmann for many discussions about the application of synthetic STFs to real image data as well as F. Kneer and O. von der Lühe for their stimulating support.

\section{References}

Berkefeld, T. 1998, Untersuchungen zur Messung und Korrektur einzelner Schichten der Erdatmosphäre, $\mathrm{PhD}$ thesis, Heidelberg University

Glindemann, A., Lane, R. G., \& Dainty, J. C. 1993, J. Mod. Opt., 40, 12, 2381

Lane, R. G., Glindemann, A., \& Dainty, J. C. 1992, Waves in Random Media, 2, 209

Puschmann, K. G. \& Sailer, M. 2006, A\&A, 454, 1011

Roddier, F. 1988, Appl. Opt., 27, 1223

Weiß, A. R. 2003, Point Spread Function Reconstruction for the Adaptive Optics System ALFA and its Application to Photometry, $\mathrm{PhD}$ thesis, Heidelberg University 
Modern Solar Facilities - Advanced Solar Science, 119-126

F. Kneer, K. G. Puschmann, A. D. Wittmann (eds.)

(C) Universitätsverlag Göttingen 2007

\title{
Solar image restoration
}

\author{
M. G. Löfdahl ${ }^{1, *}$, M. J. van Noort ${ }^{1}$, and C. Denker ${ }^{2,3}$ \\ ${ }^{1}$ Institute for Solar Physics of the Royal Swedish Academy of Sciences \\ AlbaNova University Center, Stockholm, Sweden \\ ${ }^{2}$ New Jersey Institute of Technology, Newark, NJ, U.S.A. \\ ${ }^{3}$ Astrophysikalisches Institut Potsdam, Potsdam, Germany \\ *Email: mats@astro.su.se
}

\begin{abstract}
Image restoration is used to repair solar images degraded by the turbulence in Earth's atmosphere. Restoration algorithms are based on models of the optical system that produce the images - from the solar source of radiation, through Earth's atmosphere and telescope/instrument optics, to the detectors recording the data. In this review, these model components are discussed in the context of two very different classes of image restoration methods, i.e., Speckle Imaging and Phase Diversity/MultiFrame Blind Deconvolution, which have been successfully used during the last two decades. The strengths and weaknesses of these two approaches are discussed, as well as some variants and recent progress.
\end{abstract}

\section{Introduction}

Atmospheric turbulence is the primary obstacle in achieving diffraction-limited resolution in modern ground-based solar observations, as it randomly distorts the wavefronts emanating from the Sun. The results are image motion, blurring, and geometrical distortions of the collected images. Adaptive optics (AO) significantly reduces low-order aberrations but residuals still remain significant. Image restoration techniques can correct higher-order aberrations because they do not suffer from the constraints involved in the AO control system. The most commonly used methods for solar image restoration are Speckle Imaging (SI) and different variants of Multi-Frame Blind Deconvolution (MFBD) and Phase Diversity (PD).

In this review, we present a data collection model for solar imaging, including seeing effects, and state the problem of image restoration in more detail. We describe, with a minimum of mathematics, the two methods including some variants that answer challenges posed by new observing methods and instruments.

\section{Data collection model}

Understanding the image formation process is necessary for algorithm developers. It is also instructive for interpreters of restored solar data, who want to understand the limitations of image restoration and what improvements are to be expected. Figure 1 illustrates a data collection model suitable for SI and MFBD methods. 
The solar image, formed on a detector, is a blurred version of the object. The detector adds random and fixed pattern noise to this blurred image.

In an image processing sense, the object is the solar scene as viewed in a certain wavelength passband and polarization state selected by the filters. Spectral lines are formed at altitudes that vary with wavelength (e.g., line core vs. wing), which results in cospatial objects with different appearance. The $\mathrm{H} \alpha$ image in Fig. 2 shows the chromosphere above a small sunspot. The inset continuum image demonstrates the multi-faceted appearance of structures in different wavelengths.

The algorithms are based on the assumption that blurring can be described as the convolution of an image with a space invariant point spread function (PSF). Therefore, the processing has to be restricted to small subfields, the approximate size of an isoplanatic patch. In the Fourier domain, the convolution with a PSF is equivalent to multiplication with an optical transfer function (OTF). The modulus of the OTF, called the modulation transfer function (MTF), measures the gain as a function of spatial frequency.

The OTF can be formulated as the autocorrelation of the pupil transmission function, which represents the entire optical system including the atmosphere as well as the actual optics. The amplitude of this complex function encodes the size and shape of the pupil and determines the diffraction-limited resolution of the telescope. The phase encodes the optical aberrations, which are the source of the solar image restoration problem. A tilt in the phase moves the image and a phase curvature lowers the MTF at high spatial frequencies and scrambles the Fourier phases of the image. Together, these effects blur the observed image and introduce false structures. A fixed or slowly varying component is related to imperfections in the telescope, re-imaging optics, and filters. However, the difficulties in restoring solar images arise from rapid, random refraction effects in the Earth's atmosphere. They can be partly compensated by AO systems, which have been operational for several years at the major high-resolution solar telescopes (see, e.g., Rimmele 2000; Scharmer et al. 2000; or Berkefeld 2007 and references therein). In addition, a minor, often ignored contribution to the MTF comes from the detector (see, e.g., Stevens \& Lavine 1994).

A wavefront emanating from the Sun expands undisturbed through empty space until it enters the Earth's turbulent atmosphere as a plane parallel wave. The atmospheric turbulence is powered by the day-night cycle, which heats and cools the Earth's surface, creating largescale atmospheric motions. Once these motions become turbulent, large-scale eddies break up, leading to temperature fluctuations across a wide range of spatial scales. The refractive index of air is temperature sensitive so that the fluctuations act as optical elements in front of the telescope aperture. The energy transfer from large- to small-scale turbulent eddies is governed by Kolmogorov's model of atmospheric turbulence (see, e.g., Roddier 1981). 
Fried (1966) introduced the parameter $r_{0}$, which is used to characterize the magnitude of atmospheric seeing. The spatial RMS of the wavefront phase over the telescope pupil, $\left(D / r_{0}\right)^{5 / 6}$, increases almost linearly with $D$, where $D$ is the diameter of the telescope aperture. The usual interpretation of $r_{0}$ has its roots in this expression: the RMS wavefront is 1 rad or $\sim 1 / 6$ wave over a telescope diameter that equals $r_{0}$. Seeing origi-

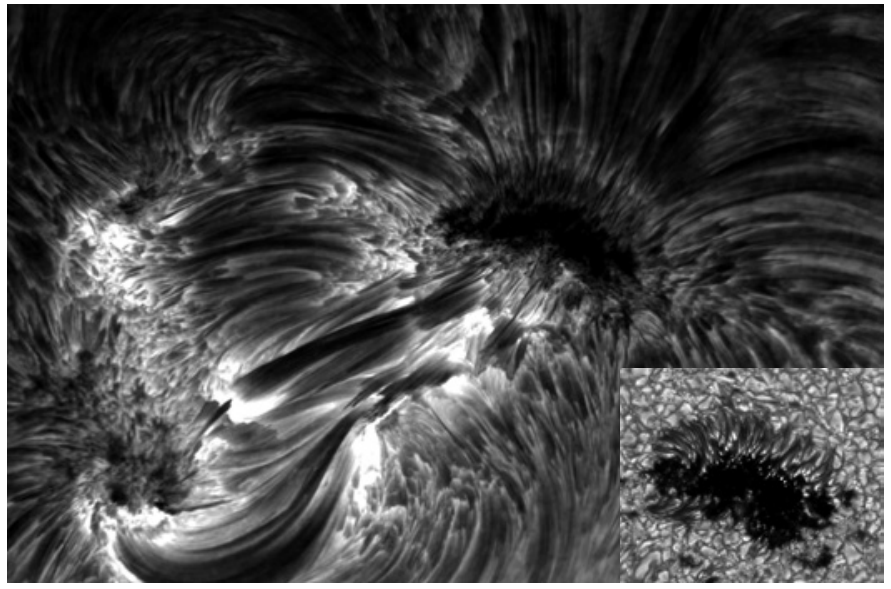

Figure 2. $\mathrm{H} \alpha$ image with blue continuum inset demonstrating the appearance of a sunspot at different wavelengths. (MFBD-restored SST images, courtesy of O. Engvold and L. Rouppe van der Voort.) nating beyond a few hundred meters from the telescope aperture causes anisoplanatic seeing, which varies across field-of-views (FOVs) typically encountered in high-resolution solar physics $\left(\sim 40^{\prime \prime}\right.$ to $\left.100^{\prime \prime}\right)$. Long exposures average over many different atmospheric states and cause a loss of resolution that cannot be undone. However, short-exposure images retain diffraction-limited information by "freezing" the wavefront aberrations (Labeyrie 1970).

\section{Methods}

\subsection{Speckle imaging}

In SI, the complex Fourier transform of the object is reconstructed from separate estimates of the amplitude (Labeyrie 1970) and the phase. $r_{0}$ is estimated from a statistical sample of the seeing (von der Lühe 1984), on the order 100 frames. An atmospheric model then yields the long-exposure MTF, which is applied to an average of the Fourier amplitudes of the data frames. The estimation of the phases requires more effort, because the phases of the data frames average to zero. This is the same reason why a long-exposure image has very little phase information. However, there are differential quantities that do not average to zero. Two such quantities, introduced by Knox \& Thompson (1974) and Weigelt (1977), are still the foundation of today's SI codes, i.e., Knox-Thompson or speckle masking algorithms. Since low-contrast objects are common in solar imaging, special adaptations and improvements were invented by von der Lühe (1993), de Boer (1993), and Mikurda \& von der Lühe (2006).

\subsection{Multi-frame blind deconvolution}

If an image is an unknown object convolved with an unknown PSF plus random noise, the blind deconvolution problem is to estimate both the unknown quantities. This problem is illposed, because an infinite number of PSF/object combinations can give a particular observed image. Therefore, we need constraints that make the correct solution more unique. 
One powerful constraint is to require that the PSF can be directly related to the telescope's pupil and $f$-number in the presence an unknown phase over the pupil. Other constraints are given by the use of multiple frames. A small number ( $~ 5)$ of frames is sufficient, although the results improve with the number of frames. The diversity of pupil phases facilitates the separation of PSFs and the common object. A special case is the introduction of a second detector, which simultaneously records defocused images. This PD technique (Gonsalves 1982) improves the identification of the aberrations in two ways: the defocus gives a known phase perturbation and the aberrations manifest themselves more clearly outside the focal plane, where the PSF wings are more pronounced.

The unknown phase is parameterized by expansion in a set of basis functions. An estimate of the expansion parameters is determined from iterative minimization of an error metric, which measures the difference between the observed images and synthetic images derived from estimated quantities (for some of the early development see, e.g., Ayers \& Dainty 1988; Paxman et al. 1992; Schulz 1993).

Solar PD was independently developed by two teams (Löfdahl \& Scharmer 1994a,b; Seldin \& Paxman 1994), who used data from the Swedish Vacuum Solar Telescope (SVST) to establish PD as a reliable method for restoring solar data. A PD-restoration example is shown in Fig. 3. After Van Kampen \& Paxman (1998) had demonstrated that solar MFBD is possible without PD, Löfdahl (2002) developed a more general formulation of the problem including PD. The new algorithm also accommodates simultaneously collected data at different wavelengths or with different polarization states. This Multi-Object MFBD (MOMFBD) approach was implemented by van Noort et al. (2005). The $\mathrm{C}++$ code is publicly available from www. momfbd.org.

\subsection{Issues common to SI and MFBD}

NoIsE. Noise in solar images is mostly shot noise that comes from the quantum nature of light and an uncertainty in the number of electrons emitted by a photodetector. It is Poisson distributed but well approximated by additive Gaussian noise for large numbers of electron emissions. Low-pass filtering is routinely used to avoid noise amplification at high spatial frequencies. Observers, who want long-exposure images in order to improve the signal-to-noise ratio (SNR), should note that combining many short-exposure data frames gives the SNR corresponding to the total exposure time. There is also a fixed pattern component from pixel-to-pixel variations in detector element sensitivity and bias. This is easily corrected with standard flat-field pre-

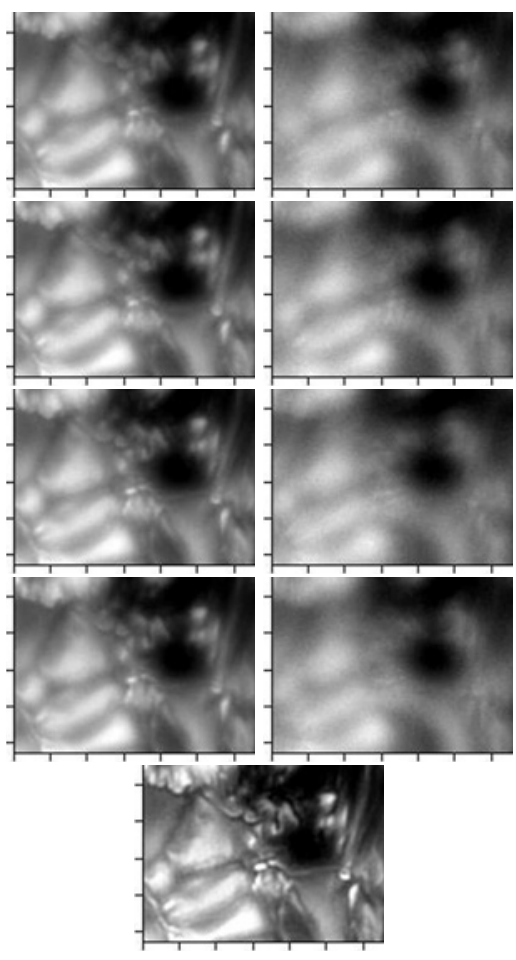

Figure 3. PD image restoration example. Left: in-focus images. Right: corresponding out-of-focus images. Bottom: restored object. Tickmarks: $1^{\prime \prime}$. processing. 
Time scales. The exposure time should be short enough so that the wavefront does not change significantly. The decorrelation time-scale of daytime seeing is $\sim 50 \mathrm{~ms}$, which sets the typical exposure time to $\sim 10 \mathrm{~ms}$ for daytime observations. It also limits statistically independent samples to about 20 frames per second. On the other hand, the photospheric sound speed is $7 \mathrm{~km} / \mathrm{s}$ or $0{ }^{\prime} 01 / \mathrm{s}$ as seen from Earth. At this speed, it takes only $5 \mathrm{~s}$ to cross half a 0 !' 1 resolution element, which corresponds to the diffraction limit of a $1-\mathrm{m}$ telescope. This leaves SI with just enough time to obtain the $\sim 100$ frames with an unchanging object, that are required for a good restoration. In the chromosphere, collection intervals should be even shorter, since the sound speed is several times larger than in the photosphere and magnetic forces can accelerate gas to even higher speeds, e.g., van Noort \& Rouppe van der Voort (2006) observed optical flows in $\mathrm{H} \alpha$ with speeds of up to $240 \mathrm{~km} / \mathrm{s}$.

AnisoplanAtism. PSFs are not space invariant, if extended scenes on the Sun are considered. Both SI and MFBD are performed on subfields with sizes corresponding to the isoplanatic patch. A mosaic of these subfields is then assembled to yield a restored image of the entire FOV. Simplified seeing models provide a rough estimate of the isoplanatic angle $\theta_{0}=0.314 r_{0} / h$, where $h$ is the distance to the seeing layer. Considering that $\theta_{0}$ is only $1^{\prime \prime}$ for $h=10 \mathrm{~km}$ and $r_{0}=15 \mathrm{~cm}$, processing subfields a few times larger than this size surprisingly still produces satisfactory results.

Data volumes AND PRocessing TIME. The most demanding observing schemes now require several hundreds of image frames from different cameras per collection interval. While SI is non-iterative and scales linearly with the number of frames, MFBD is based on iterative, nonlinear model-fitting, and scales badly with the number of frames. The difference is striking! In principle, $1 \mathrm{k} \times 1 \mathrm{k}$ pixel images can be SI restored on distributed computers in about the same time it takes to acquire the data (Denker et al. 2001). At the Dutch Open Telescope (DOT), e.g., a full day's observations are now SI restored in one night. In contrast, the Institute for Solar Physics assessed its need for computing resources. For the best possible MOMFBD restoration of $10 \mathrm{~h}$ worth of data (polarimeter in the red and $\mathrm{Ca}$ II $\mathrm{H}$ scan in the blue, both with wide-band (WB) PD) from all cameras, a 100-CPU cluster would have to work for a full year.

\subsection{Variants and new developments}

Multiple obJects. Data collected through narrow-band (NB) filters in a dark spectral line have reduced SNR, which makes image restoration algorithms less effective. Keller \& von der Lühe (1992) pioneered a technique called speckle deconvolution, using simultaneous frames collected with a WB continuum filter with better SNR. The object estimate from SI processing of the WB data together with the original frames yield the PSFs needed to deconvolve the NB data. This technique is now widely used for spectroscopy and polarimetry (see, e.g., Koschinsky et al. 2001).

While a similar scheme is possible for MFBD methods, the model fitting approach is facilitated by co-processing of WB and NB data, in order to use the available information as efficiently as possible. This MOMFBD method is illustrated in Fig. 4. The setup contains a NB camera behind the SOUP birefringent filter and two WB cameras in a PD configuration. Further, because the SOUP is not equipped with a polarizing beamsplitter, images at different polarization states have to be collected consecutively rather than in parallel. Anisoplanatic 

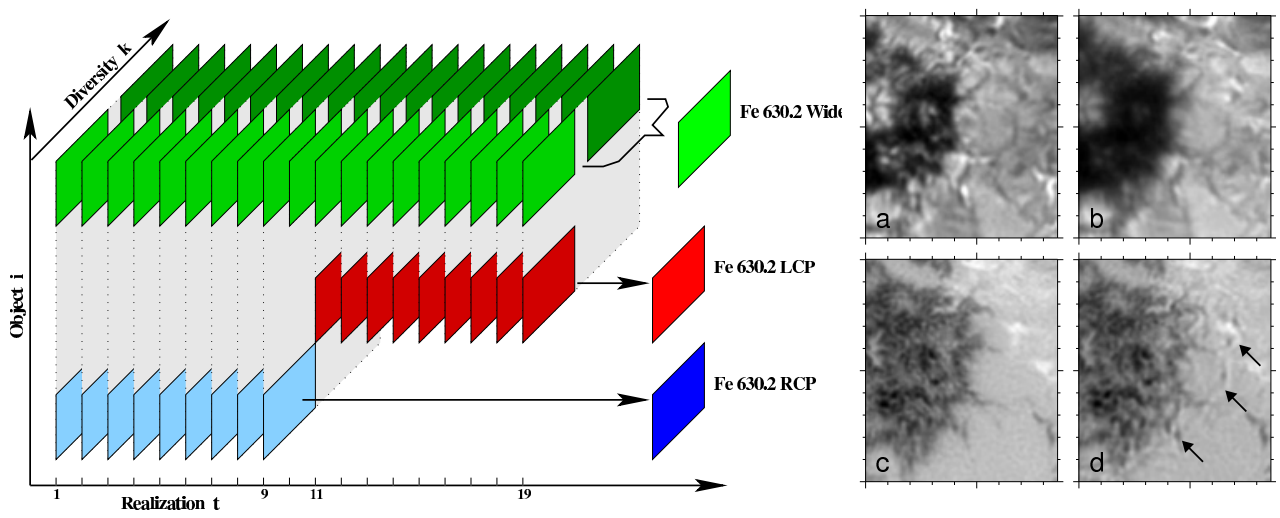

Figure 4. MOMFBD. Left: Graphic representation of constraints for a WB PD plus NB polarimetry setup. Simultaneous wavefront coefficients are required to be identical. As a consequence, the NB restorations are aligned with the WB restoration and therefore to each other. Right: (a) and (b) show restored Fe I $630.2 \mathrm{~nm}$ ( $-6 \mathrm{pm}$ from the core) right- and left-hand circularly polarized (RCP and LCP) images, respectively. (c) and (d) show line-of-sight (LOS) magnetograms made by subtracting the RCP and LCP images. In (c) the alignment is calibrated as part of the MOMFBD procedure. In (d) the alignment is based on sub-image cross-correlation and destretching. Arrows indicate artifacts from seeing-related alignment mismatches. Tickmarks: 1" (Adapted from van Noort et al. (2005)).

seeing during the collection interval makes such images imperfectly aligned, a problem that can be calibrated using a pinhole array as target. The problem is constrained by the fact that the wavefronts corresponding to the simultaneously recorded images have to be the same. Because each raw NB frame is simultaneously taken with a PD pair, both restored NB images will be aligned to the WB restoration and thus to each other. The restored NB images can then produce magnetograms without further alignment or destretching - a procedure that is prone to producing seeing-related artifacts because it involves cross-correlation of nonidentical images.

SI AND AO. SI can well estimate the Fourier phases of AO-corrected solar images (Denker et al. 2005). However, the restored power spectrum is not correctly estimated because of how AO correction modifies the statistics of the atmospheric aberrations. Puschmann \& Sailer (2006) included AO modeling in their speckle masking code (see Fig. 5). They demonstrate that traditional SI, with a single $r_{0}$, over-corrects near the AO sensor lockpoint, while allowing for a varying $r_{0}$ over-corrects with distance from the lockpoint. Including modeling of the AO correction is better in the sense that the restored "quiet sun" contrast varies less with lockpoint distance. While this appears to be a valid approach to the problem, work still remains to be done before it is a mature solution. The Swedish Solar Telescope (SST) data shown in Fig. 5 were collected when the AO was locking during almost the entire collection interval. Processing a data set with AO lock only part of the time requires logging of the AO performance as well as more modeling work.

SI AND SPECTRA. While data from a slit-spectrograph is one-dimensional, one can get two-dimensional information by scanning with the slit. The result is a data cube, which is unfortunately not only blurred by the seeing but also irregularly sampled due to anisoplanatic distortions. Keller \& Johannesson (1995) implemented a method where the cube is 

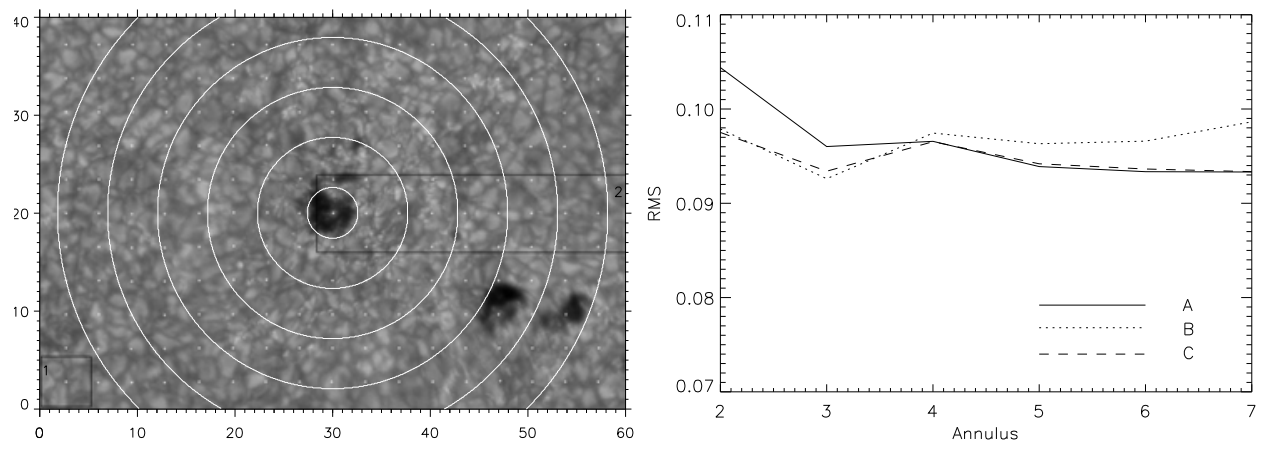

Figure 5. SI with AO correction. Restorations using three different methods: $A$ : traditional, $B$ : using a spatially varying $r_{0}$, and $C$ : with $\mathrm{AO}$ modeling. Left: Raw image with annuli centered on the pore, which was used as the AO wavefront sensor target. Right: Restored contrast in the different annuli (with magnetic structures removed) for the three different methods demonstrating that model $C$ produces the flattest contrast profile. Tickmarks: 1" (Adapted from Puschmann \& Sailer (2006)).

resampled so that the continuum is aligned to an SI restoration of the slit-jaw images. The cube is also deconvolved using information from the slit-jaw SI processing.

\section{Discussion}

Image restoration is needed to fully utilize the resolution of modern solar telescopes. AO will always leave uncorrected residuals, particularly away from the wavefront sensor FOV. There are two kinds of methods in routine use: SI and MFBD methods. Both are actively used for producing science data and both are still being developed in order to meet demands and challenges from new observations and instruments. Still, the differences between the methods give them different strengths and weaknesses.

SI does not have strict requirements for the optical setup, other than a camera fast enough to collect a statistical sample in a short time. This can lead to very large data amounts (TBytes) over an observing day. In addition, it may be difficult to collect sufficient independent samples for a quickly evolving target without violating the assumption of an unchanging object. On the other hand, SI is more robust for low contrast data than MFBD methods, which are more dependent on finding structures in the data.

In its simplest form, MFBD does also not require any extra optics and has the advantage that the sample does not have to be as large. MFBD processing is the best thing that can be done, if less images are available than required for a statistical sample. However, the number of frames needed for NB data primarily depends on SNR requirements. In addition, the inclusion of PD requires an extra camera or extra optics.

The greatest drawback of MFBD methods is the computing time. A full day's worth of DOT data can be SI restored overnight, while one day of SST data may require almost an entire year for full MOMFBD processing. While this may become less of a problem as the cost for CPU cycles continues to decrease, the choice now clearly has to be guided by whether we need high-volume, routine restoration or are aiming for a few unique data sets.

MFBD makes no assumption about seeing characteristics. This means there are no prob- 
lems with fixed aberrations or time- and space-varying AO corrections. On the other hand, MFBD restorations systematically under-estimate power spectra due to the finite expansion of the wavefronts. If the underlying atmospheric model is correct, SI should automatically obtain the correct power spectra. However, AO-corrected data are not as easily modeled, although method development is making rapid progress to address this problem.

Acknowledgements. This work was supported in part by NSF under grants IIS ITR 03-24816 (ML and CD) and ATM 02-36945 (CD). The Swedish 1-m Solar Telescope is operated by the Institute for Solar Physics of the Royal Swedish Academy of Sciences on the island of La Palma at the Spanish Observatorio del Roque de los Muchachos of the Instituto de Astrofísica de Canarias.

\section{References}

Ayers, G. R. \& Dainty, J. C. 1988, Opt. Lett., 13, 547

Berkefeld, T. 2007, (this volume)

de Boer, C. R. 1993, PhD thesis, University of Göttingen

Denker, C., Mascarinas, D., Xu, Y., et al. 2005, Solar Phys. 227, 217

Denker, C., Yang, G., \& Wang, H. 2001, Solar Phys. 202, 63

Fried, D. L. 1966, J. Opt. Soc. Amer. 54, 1372

Gonsalves, R. A. 1982, Opt. Eng., 21, 829

Keller, C. U. \& Johannesson, A. 1995, A\&A Suppl. 110, 565

Keller, C. U. \& von der Lühe, O. 1992, A\&A 261, 321

Knox, K. T. \& Thompson, B. J. 1974, ApJ 193, L45

Koschinsky, M., Kneer, F., \& Hirzberger, J. 2001, A\&A 365, 588

Labeyrie, A. 1970, A\&A 6, 85

Löfdahl, M. G. 2002, in: Proc. SPIE 4792, 146-155

Löfdahl, M. G. \& Scharmer, G. B. 1994a, in: Proc. SPIE 2302, 254-267

Löfdahl, M. G. \& Scharmer, G. B. 1994b, A\&A Suppl. 107, 243

Mikurda, K. \& von der Lühe, O. 2006, Solar Phys. 235, 31

Paxman, R. G., Schulz, T. J., \& Fienup, J. R. 1992, J. Opt. Soc. Amer. A 9, 1072

Puschmann, K. G. \& Sailer, M. 2006, A\&A 454, 1011

Rimmele, T. R. 2000, in: Proc. SPIE 4007, 218-231

Roddier, F. 1981, in: Progress in Optics 19, ed. E. Wolf (Amsterdam: North-Holland Publishing Company), 281-376

Scharmer, G. B., Shand, M., Löfdahl, M. G., Dettori, P. M., \& Wei, W. 2000, in: Proc. SPIE 4007, 239-250

Schulz, T. J. 1993, J. Opt. Soc. Amer. A 10, 1064

Seldin, J. H. \& Paxman, R. G. 1994, in: Proc. SPIE 2302, 268-280

Stevens, E. G. \& Lavine, J. P. 1994, IEEE transactions on electronic devices, 41, 1753

Van Kampen, W. C. \& Paxman, R. G. 1998, in: Proc. SPIE, Vol. 3433, Propagation and Imaging through the Atmosphere II, ed. L. R. Bissonnette, 296-307

van Noort, M., Rouppe van der Voort, L., \& Löfdahl, M. G. 2005, Solar Phys. 228, 191

van Noort, M. J. \& Rouppe van der Voort, L. H. M. 2006, ApJ 648, L67

von der Lühe, O. 1984, J. Opt. Soc. Amer. A 1, 510

von der Lühe, O. 1993, A\&A 268, 374

Weigelt, G. P. 1977, Optics Communications, 21, 55 


\title{
Probabilistic suppression of astronomical image degradations
}

\author{
M. Haindl ${ }^{1, *}$ and S. Šimberová ${ }^{2}$ \\ ${ }^{1}$ Institute of Information Theory and Automation, Academy of Sciences, Prague, Czech \\ Republic \\ ${ }^{2}$ Astronomical Institute, Academy of Sciences, Ondřejov, Czech Republic \\ *Email: haindl@utia.cas.cz
}

\begin{abstract}
A multichannel fast adaptive recursive restoration method based on the underlying spatial probabilistic image model is presented. The method assumes a linear degradation model with the unknown, possibly non-homogeneous point-spread function, and additive noise for each of mutually registered degraded observations. Pixels in the vicinity of steep image discontinuities are left unrestored to minimize restoration blurring effects. Our method is completely autonomous and does not assume any knowledge of the underlying degradation process. It is applied to a sequence of short-exposure solar photosphere images and it can be also easily and naturally generalized for multispectral (e.g. colour, multispectral satellite images) or registered images which is seldom the case for alternative methods.
\end{abstract}

\section{Introduction}

The major degradation of a ground-based telescope is caused by random fluctuations originating mostly in the Earth's atmosphere (seeing) along the optical path between the object space and the image formation device. Influences of the thermal turbulence in image formation were analyzed since the 1950s, see e.g. Fried (1966). However the image degradation by seeing is a very complicated process due to blurring, motion and distortion, and this restoration problem is still far from being solved. The image degradation is described by the changing complex point-spread-function (PSF) of the telescope, which embodies all the important behaviour of the optical image formation system. For the restoration we assume one unknown degradation function involving all degradation aspects. The degradation function model has to discriminate between different types of astronomical observations. Here we focus on modeling of degradation of short-exposure solar images, which results in recovering an undegraded image. In ground-based solar observations it is often possible to obtain several images of the same object that differ just by the component of PSF originating from seeing. For the reconstruction we adopt a short time sequence of images. The exposure time of each image is $<15 \mathrm{~ms}$, so we are allowed to group them into the "short-exposure" ones and suppose a still scene in the image. Our approach to image restoration is different to direct and indirect techniques like various types of filtering, power spectral equalization, constrained least-squares restoration, maximum entropy restoration, etc. The image restoration task is to recover an unobservable image given the whole sequence of differently corrupted images with respect to some statistical criterion. 
This work generalizes the monospectral restoration method of Haindl (2000), and Haindl (2002) to the multiversion images. It is seldom possible to obtain a degradation model analytically from the physics of the problem. More often a limited prior knowledge supports only some elementary assumptions about this process. The usual assumption, also accepted in this work, is that the corruption process can be modeled using a linear degradation model.
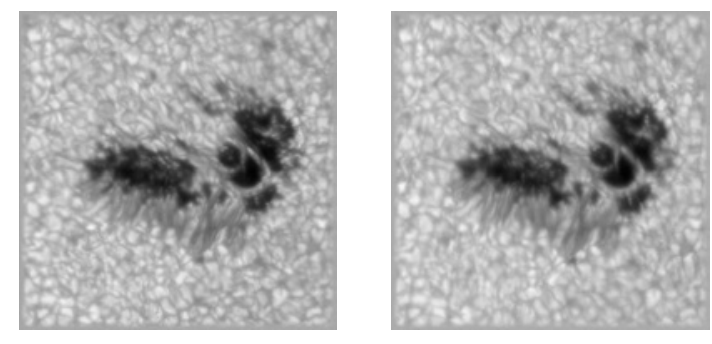

Figure 1. The measured degraded sunspot multitemporal images (courtesy of M. Sobotka).

\section{Image model}

Suppose $Y$ represents a true but unobservable monospectral image defined on the finite rectangular $N \times M$ underlying lattice $I$. Suppose further that we have a set of $d$ observable images $\mathcal{X}$ where each $X_{\bullet, i} \in \mathcal{X}$ is the i-th version of $Y$ distorted by the unknown PSF and noise independent of the signal. The notation - designates of all possible values of the corresponding multiindex (e.g. the multiindex $r=\left\{r_{1}, r_{2}\right\}$ which has the row and columns indices, respectively). We assume knowledge of all pixels from the reconstructed scene. For the treatment of the more difficult problem when some data are missing see Haindl \& Šimberová (1996), Haindl \& Šimberová (1998). The image degradation is supposed to be approximated by the linear discrete spatial domain degradation model

$$
X_{r, \bullet}=\sum_{s \in I_{r}} H_{s} Y_{r-s}+\epsilon_{r, \bullet}
$$

where $H$ is a discrete representation of the unknown point-spread function, $X_{r, \bullet}$ is the $d \times 1$ vector of the $r$-th pixel in different distortions and $Y_{r-s}$ are ideal (unobservable) image pixels. The point-spread function is assumed to be either homogeneous or it can be nonhomogeneous but in this case we assume it slowly changes relative to the size of an image. $I_{r}$ is some contextual support set, and a noise vector $\epsilon$ is uncorrelated with the true image, i.e., $E\left\{Y \epsilon_{\bullet}, i\right\}=0$. The point-spread function is unknown but such that we can assume the unobservable image $Y$ to be reasonably well approximated by the expectation of the corrupted image

$$
\hat{Y}=E\left\{X_{\bullet, i}\right\}
$$

in regions with gradual pixel value changes, and the $\mathrm{i}$-th degraded image $X_{\bullet, i} \in \mathcal{X}$ is the least degraded image from the set $\mathcal{X}$. The index $i$ of the least degraded image is excluded from the following equations (3)-(5) to simplify the corresponding notation. The above 
method (2) changes all pixels in the restored image and thus blurs discontinuities present in the scene although to much less extent than the classical restoration methods due to our restoration model (4) adaptivity. This excessive blurring can be avoided if pixels with steep step discontinuities are left unrestored, i.e.,

$$
\hat{Y}_{r}=\left\{\begin{array}{ll}
E\left\{X_{r}\right\} & \text { if } p\left(X_{r} \mid X^{(r-1)}\right)>\kappa \\
X_{r} & \text { otherwise }
\end{array},\right.
$$

where $\kappa$ is a probabilistic threshold based on the prediction density. Single matrix elements in the expectation $E\{X\}$ are approximated (Haindl \& Šimberová 2005) by the conditional expectation $E\left\{X_{j} \mid X^{(j-1)}=x^{(j-1)}\right.$ where $x^{(j-1)}$ are known past realization for $j$. Thus we suppose that all other possible realization $x^{(j-1)}$ than the true past pixel values have negligible probabilities. This assumption implies conditional expectations approximately equal to unconditional ones, i.e., then the expectation is $E\left\{X_{j}\right\} \approx E\left\{X_{j} \mid X^{(j-1)}\right\}$.

Suppose further that a noisy image can be represented by an adaptive $2.5 \mathrm{D}$ causal simultaneous autoregressive model

$$
X_{r, i}=\gamma Z_{r}+\epsilon_{r}
$$

where $\gamma=\left[A_{1}, \ldots, A_{\eta}\right], \eta=\operatorname{card}\left(I_{r}^{c}\right)$ is a $1 \times d \eta$ parameter matrix, $Z_{r}$ is a corresponding vector of $X_{r-s}, \epsilon_{r}$ is a white Gaussian noise vector with zero mean, and a constant but unknown covariance matrix $\Sigma$. The noise vector is uncorrelated with data from a causal neighbourhood $I_{r}^{c}$. $A_{s}=\left[a_{s, 1}, \ldots, a_{s, d}\right] \quad \forall s$ are parameter vectors. The model adaptivity is introduced using the exponential forgetting factor technique in parameter learning part of the algorithm. The conditional mean value can be derived under few acceptable conditions (Haindl \& Šimberová 2005) in the following form:

$$
E\left\{X_{r} \mid X^{(r-1)}\right\}=V_{z x(r-1)}^{T} V_{z z(r-1)}^{-T} Z_{r}
$$

where $V_{r-1}=\tilde{V}_{r-1}+I$ and

$$
\tilde{V}_{r-1}=\left(\begin{array}{cc}
\sum_{k=1}^{r-1} X_{k} X_{k}^{T} & \sum_{k=1}^{r-1} X_{k} Z_{k}^{T} \\
\sum_{k=1}^{r-1} Z_{k} X_{k}^{T} & \sum_{k=1}^{r-1} Z_{k} Z_{k}^{T}
\end{array}\right)=\left(\begin{array}{ll}
\tilde{V}_{x x(r-1)} & \tilde{V}_{z x(r-1)}^{T} \\
\tilde{V}_{z x(r-1)} & \tilde{V}_{z z(r-1)}
\end{array}\right) .
$$

An appropriate model support $\left(I_{r}^{c}\right)$ can be found using the Bayesian decision rule (cf. Haindl \& Šimberová 2005).
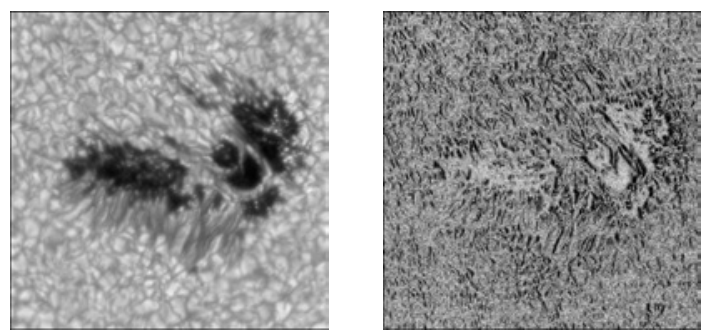

Figure 2. The reconstructed sunspot image using our method and its corresponding prediction probability image. 


\section{Results and conclusions}

A set of 4 short-exposure solar images (of which 2 are illustrated in Fig. $1 ; t_{\text {exp }}=10 \mathrm{~ms}$, blue part of spectra $\lambda=450.7 \mathrm{~nm}$, resolution $0.041 \% /$ pixel, 70s sequence capturing) were used for verification. All images were corrected by the MTF of the telescope, but no aberration and seeing corrections have been applied. As an objective evaluation measure of the restoration performance an integral of a sum of image partial derivatives (Subbarao et al. 1993; see results in Haindl \& Šimberová 2005), as well as visual evaluation has been used. The solar images in Fig. 1 were reconstructed with the threshold $\kappa=0.05$ which left $54 \%$ observation pixels unchanged. The best sunspot image (Fig. 1- left) served as reference image for the algorithm. The reconstruction result is shown in Fig. 2. Figure 2-right shows gray level coded predictor probabilities which served to control the restoration switching. The lighter shades represent higher predictor probabilities while dark areas were not changed during the reconstruction. Visual comparison of the reconstructed images with the input degraded images as well as the criterion values (Haindl \& Šimberová 2005) demonstrate clearly the deblurring effect of the presented algorithm and restoration improvement. The proposed method was superior over the classical methods (e.g. several low pass filters, pixelwise averaging; blind deconvolution not presented here) using both types of verification.

The proposed recursive multitemporal blur minimizing reconstruction method is very fast (approximately five times faster than the median filter) robust and its reconstruction results surpasses some standard reconstruction methods, which we were able to implement for the verification. Our causal model has the advantage to have the analytical solution for all needed model statistics. Possible artifacts introduced by this type of models are diminished by introducing adaptivity into the model. This novel formulation allows us to obtain extremely fast adaptive multichannel / multitemporal restoration and it can be easily parallelized as well as generalized for multispectral (e.g. colour, multispectral satellite images) or registered images which is seldom the case for alternative methods. Finally, this method enables to estimate homogeneous or slowly changing non-homogeneous degradation PSFs (not presented here).

Acknowledgements. This research was supported by the EC project no. FP6-507752 MUSCLE and CR grants No. A2075302, 1ET400750407, GACR 102/04/0155, 1M0572 DAR, 2 C06019 and partially AV CR 1QS300120506.

\section{References}

Fried, D. L. 1966, J. Opt. Soc. Amer., 56, 1372

Haindl, M. 2000, in Proceedings of the 15th IAPR Int. Conf. on Pattern Recognition, ed. A. Sanfeliu, J. Villanueva, M. Vanrell, R. Alquezar, T. Huang, \& J. Serra, Vol. III (IEEE Press), 346-349

Haindl, M. 2002, in Structural, Syntactic, and Statistical Pattern Recognition. Proc., ed. T. Caelli, A. Amin, \& R. P. W. Duin (Berlin: Springer), 617-626

Haindl, M. \& Šimberová, S. 1996, A\&A Suppl. Ser., 115, 189

Haindl, M. \& Šimberová, S. 1998, Kybernetika, 34, 423

Haindl, M. \& Šimberová, S. 2005, Lecture Notes in Computer Science, 1037

Subbarao, M., Choi, T., \& Nikzad, A. 1993, J. Optical Eng., 32, 2824 


\title{
Advances, challenges and limitations of speckle reconstruction and deconvolution
}

\author{
K. Mikurda*, O. von der Lühe, F. Wöger, and W. Schmidt \\ Kiepenheuer-Institut für Sonnenphysik, Freiburg, Germany \\ *Email: kasia@kis.uni-freiburg.de
}

\begin{abstract}
This paper presents the experiences with speckle imaging and deconvolution techniques we have made during the last five years at the Kiepenheuer-Institut für Sonnenphysik. We discuss our implementation of the above techniques, their tests and application ranges. In addition, we summarize our efforts in applying speckle techniques to the data taken with the support of the adaptive optics.
\end{abstract}

\section{Introduction}

Speckle imaging and deconvolution methods have been widely applied to the solar data for over two decades now and have proven to be an excellent tool to increase the spatial resolution of images. Here, we summarize the progress recently made in this field at the Kiepenheuer-Institut für Sonnenphysik. We also discuss the performance test of speckle techniques and their limitations and the influence of the adaptive optics on the quality of reconstructed data.

\section{Speckle reconstruction}

Speckle reconstruction techniques use a sequence of short-exposed images to obtain one high resolution (up to the diffraction limit) image. The most popular methods applied to solar data are the (Extended) Knox-Thompson (EKT, Knox \& Thompson 1974; von der Lühe 1993; Mikurda \& von der Lühe 2006) and the speckle masking (Weigelt 1977; de Boer \& Kneer 1992) algorithms.

The Kiepenheuer-Institut Speckle Imaging Package (KISIP) has been developed since the early 1980s. Recently, we have quantitatively evaluated its performance for different solar scenes and seeing conditions. For test purposes, we have created simulated data sets which allow to compare speckle reconstruction with input data. We have chosen five reconstructions of different solar scenes (e.g. granulation, pore, sunspot penumbra) taken at the German Vacuum Tower Telescope as input images ("truth images"). Then we have created five bursts of 100 frames each, for all five truth images. Individual frames are distorted by artificial random point spread functions, chosen to represent the atmospheric seeing characterized by values of the Fried parameter $r_{0}$ corresponding to $5,7,10,15$ and $20 \mathrm{~cm}$.

Probably the most interesting question is how the quality of the reconstruction depends on the seeing conditions. The left panel of Fig. 1 compares the modeled and estimated values 

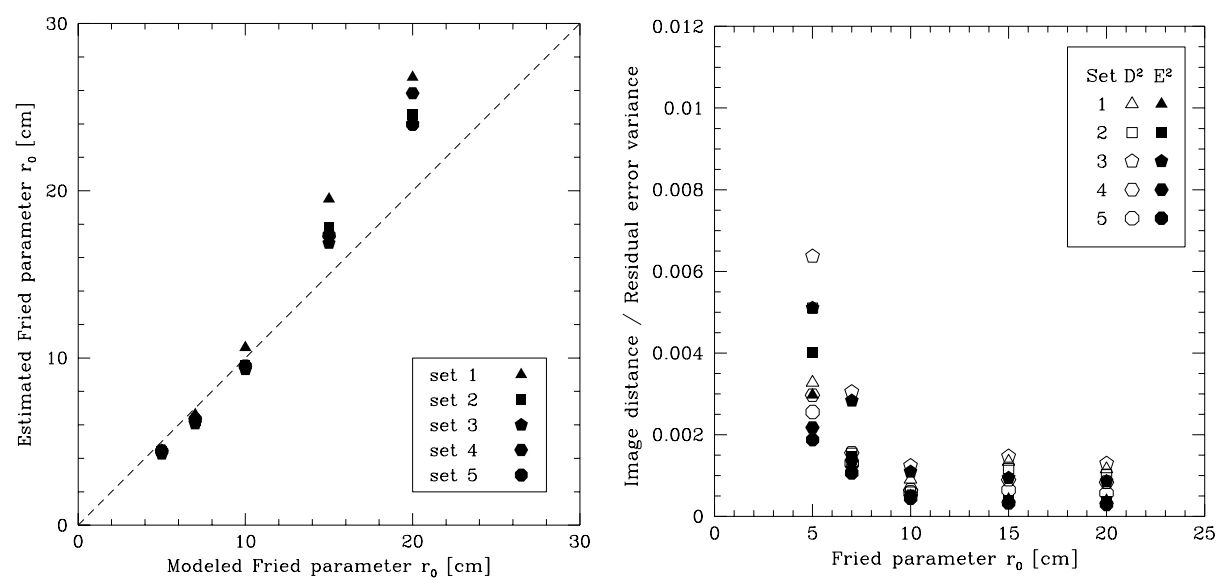

Figure 1. Left: estimated vs. modeled Fried parameter for all data sets and seeing qualities. The data sets represent: 1 - granulation with small scale structure, 2 - pore, 3 - edge of sunspot with penumbral filaments, 4 - active region granulation, 5 - quiet region granulation. Right: image distance $D^{2}$ and linear regression error $E^{2}$ for all data sets and seeing parameters.

of the Fried parameter $r_{0}$. The Fried parameter up to $r_{0}=10 \mathrm{~cm}$ seems to be very well recovered, but Fried parameters $r_{0}>10 \mathrm{~cm}$ are overestimated. It is caused by the fact that the spectral ratio technique (implemented in KISIP) is based on Korff's STF theory (Korff 1973) that is best applicable for moderate seeing conditions $\left(r_{0} / D<0.3\right)$. The right panel of Fig. 1 demonstrates the results of the Euclidean image distance $D^{2}$ calculations for all the scenes and seeing conditions. The more similar the input and reconstructed images are, the smaller this parameter becomes. For our data set the image distance is systematically lower for the reconstructions with larger Fried parameters. The sunspot penumbra scene is characterized with the largest $D^{2}$, probably because of the complexity of the reconstructed structure. More information on the tests of KISIP can be found in the recent paper of Mikurda \& von der Lühe (2006).

The application of KISIP V to "real world" observational data was mostly successful and the range of atmospheric conditions which can be successfully treated by speckle imaging turned out to be very large. Even data with a Fried parameter in the order of a few $\mathrm{cm}$ could provide a decent reconstruction. The robustness of EKT is a particular advantage for the analysis of time series which requires constant quality of the data.

\subsection{Speckle reconstruction and adaptive optics}

To date, most of the major solar ground-based telescopes are now equipped with adaptive optics (AO) systems. The AO, however, can effectively compensate only low orders of the wavefront distortions, leaving higher orders partially uncompensated. Moreover, an AO system loses efficiency with increasing distance from a lock point. A field dependent Speckle Transfer Function (STF) can correct this effect to the first order - this solution has been implemented in the IDL version of the KISIP package. The Fried parameters are calculated 


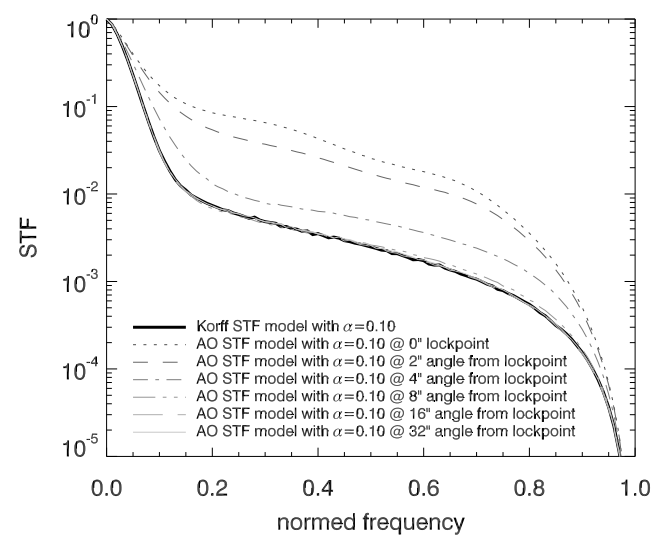

Figure 2. AO Speckle Transfer Function corresponding to different angles from a lock point, $\alpha=0, \ldots, 32$ arcsec.

individually in each isoplanatic subfield and the model STFs (Korff 1973) are applied accordingly. However, we have also studied the impact of the AO on the STF. The analytical models for the STF shown in Fig. 2 are also based on the work of Korff (1973). An AO system is taken into account in the new model presented here by subtracting phase terms that represent the AO's correction, to modify the phase structure function (see also Wang $\&$ Markey 1978). The appearing four-dimensional integrals are numerically solved using Monte-Carlo integration algorithms. An arbitrary AO can be included in the model by using performance parameters that can be measured using wave front sensor data. The main difference in the reconstructions obtained with the Korff's STF and AO-STF is that the first causes an overestimation of the image intensity contrast at and close to the lock point. The outer regions of the reconstructed images are less effected by the choice of the STF model, in fact the AO-STFs converge to the Korff's STF in regions with large enough separation from the lock point (see Fig. 2).

\section{Speckle deconvolution}

Two-dimensional spectroscopy has been gaining more importance in the last years and there are several tunable filter instruments operating currently at various solar observatories (for more details see e.g. Mikurda 2005). In general, they collect data by scanning across the spectral line and obtaining a two-dimensional filtergram at each wavelength step. The individual narrow-band filtergrams are then combined to a full 2-D spectrogram. The main limitation of this technique is the time needed to perform a scan, since the temporal evolution of the solar surface has to be taken into account. The time needed to take sufficiently large speckle bursts at every wavelength will in most cases exceed the interval of changes on the Sun and the conventional speckle reconstruction techniques cannot be applied. Speckle deconvolution techniques (Keller \& von der Lühe 1992; Krieg et al. 1999; Mikurda et al. 2006) offer a solution to this problem: simultaneous broad-band and narrow-band images, close-by in wavelength, are taken and speckle imaging is only performed for the broad-band channel data. Combining the reconstruction and the single broad-band images gives infor- 
mation on the instantaneous state of the Earth's atmosphere. This information is then used to correct the single narrow-band images.

We compared the profiles of the $\mathrm{Fe}_{\mathrm{I}}$ line at $557.6 \mathrm{~nm}$ resulting from unrestored and speckle-deconvolved data in order to understand the influence of the speckle deconvolution on the spectral line profiles. The detailed description of this procedure is beyond the scope of this paper and can be found in Mikurda et al. (2006). We found that the changes of intensity on the small scales have the correct sign: the bright granules become brighter and the dark lanes - darker in the continuum. Moreover, the speckle deconvolution causes the decrease of the line width in the bright structures and its increase in the dark ones. The line asymmetry is in most cases not altered.

The bandwidth of the tunable instruments is usually below $0.1 \mathrm{~nm}$, so the signal-to-noise ratio at the spatial frequencies close to the diffraction limit is not always sufficient. It can result in a weak grainy pattern in the reconstructed filtergrams, with a stronger effect in the dark parts of the image. Usually, it can be reduced by taking more frames at each wavelength position.

\section{Conclusions}

We have discussed the recent progress in the speckle techniques developed at the Kiepenheuer-Institut für Sonnenphysik. Our speckle imaging package - KISIP - delivers good results for a large range of atmospheric seeing conditions, and the tests made with the usage of simulated data demonstrated good photometric quality of the reconstructions. The usage of AO-STF helps to obtain even better estimation of the photometric intensities of reconstructed images. We have also investigated the influence of the speckle deconvolution on the 2-D solar spectra and demonstrated that this technique does not introduce artefacts to the line profiles. Thus, the speckle-deconvolved 2-D spectra can be used for quantitative spectroscopy.

Acknowledgements. Part of this work (KM) was supported by the DFG under grant Schm 1168/3. The Vacuum Tower Telescope on Tenerife is operated by Kiepenheuer-Institut für Sonnenphysik, Freiburg, at the Spanish Observatorio del Teide of the Instituto de Astrofísica de Canarias.

\section{References}

de Boer, C. R. \& Kneer, F. 1992, A\&A, 264, L24

Keller, C. U. \& von der Lühe, O. 1992, A\&A, 261, 321

Knox, K. T. \& Thompson, B. J. 1974, ApJL, 193, L45

Korff, D. 1973, Optical Society of America Journal, 63, 971

Krieg, J., Wunnenberg, M., Kneer, F., Koschinsky, M., \& Ritter, C. 1999, A\&A, 343, 983

Mikurda, K. 2005, Ph.D. Thesis

Mikurda, K., Tritschler, A., \& Schmidt, W. 2006, A\&A, 454, 359

Mikurda, K. \& von der Lühe, O. 2006, Solar Phys., 235, 31

von der Lühe, O. 1993, A\&A, 268, 374

Wang, J. Y. \& Markey, J. K. 1978, J. Opt. Soc. Amer. (1917-1983), 68, 78

Weigelt, G. P. 1977, Optics Communications, 21, 55 


\title{
Reversed granulation in Fe I 7090.4
}

\author{
K. Janssen* and G. Cauzzi \\ INAF Osservatorio Astrofisico di Arcetri, Firenze, Italy \\ *Email: kjanssen@arcetri.astro.it
}

\begin{abstract}
The reversal of the photospheric continuum intensity pattern is predicted by numerical simulations and has been observed in the wings of chromospheric lines. Here we present reversed granulation extracted from the photospheric $\mathrm{Fe}_{\mathrm{I}} 7090.4 \AA$ A line core intensities, after taking into account the velocity effects, in high spatial, spectral and temporal resolution. We study correlations with the underlying continuum in terms of smoothing, time delays and the temporal evolution. The latter appears to be superimposed with an oscillatory signal, that might suggest the presence of gravity waves in the observed mid-photospheric layers.
\end{abstract}

\section{Introduction and data characteristics}

The phenomenon of "reversed granulation" is referred to as the partial reversal of the granular/intergranular intensity contrast, that occurs in the mid-photosphere as consequence of a temperature decrease of granules rising in the optically thin layers due to adiabatic expansion, that is only partly cancelled by radiative heating, while an overturning element still experiences radiative heating as it begins to descend (Stein \& Nordlund 1998; Cheung et al. 2006). The reversed granulation is studied by means of a 45 minutes time series of goodquality 2D intensity maps acquired in the photospheric Fe I $7090.4 \AA$ line, supplemented by some images in the blue Ca II $8542 \AA$ line wing. We study a very quiet internetwork region, for which no obvious magnetic structures are visible in the Ca II line wing.

The data were taken with IBIS, Interferometric BIdimensional Spectrometer (Cavallini 2006), a tunable, double Fabry-Perot Interferometer at the Dunn Solar Telescope/NSO on June 2nd, 2004. The FOV shows $80^{\prime \prime} \times 80^{\prime \prime}$ of the quiet Sun at disc center on $512 \times 512$ pixels with the high order $\mathrm{AO}$ with 97 subapertures in 16 wavelength positions along the $\mathrm{Fe}$ I $7090.4 \AA$ line. A time series of 141 scans was obtained with an interval of 19 s, thus covering a time span of 45 minutes.

Dark and flat field corrections are applied, the variation of the transmission wavelength of IBIS throughout the field of view was taken into account in interpolating all spectral profiles to the same wavelength positions, creating thus monochromatic images. The images were tracked in time and a p-mode filter was applied in terms of cut above $c_{\mathrm{s}}=6 \mathrm{~km} / \mathrm{s}$ in the three-dimensional $\mathrm{k}_{\mathrm{x}}-\mathrm{k}_{\mathrm{y}}-\omega$ space. 

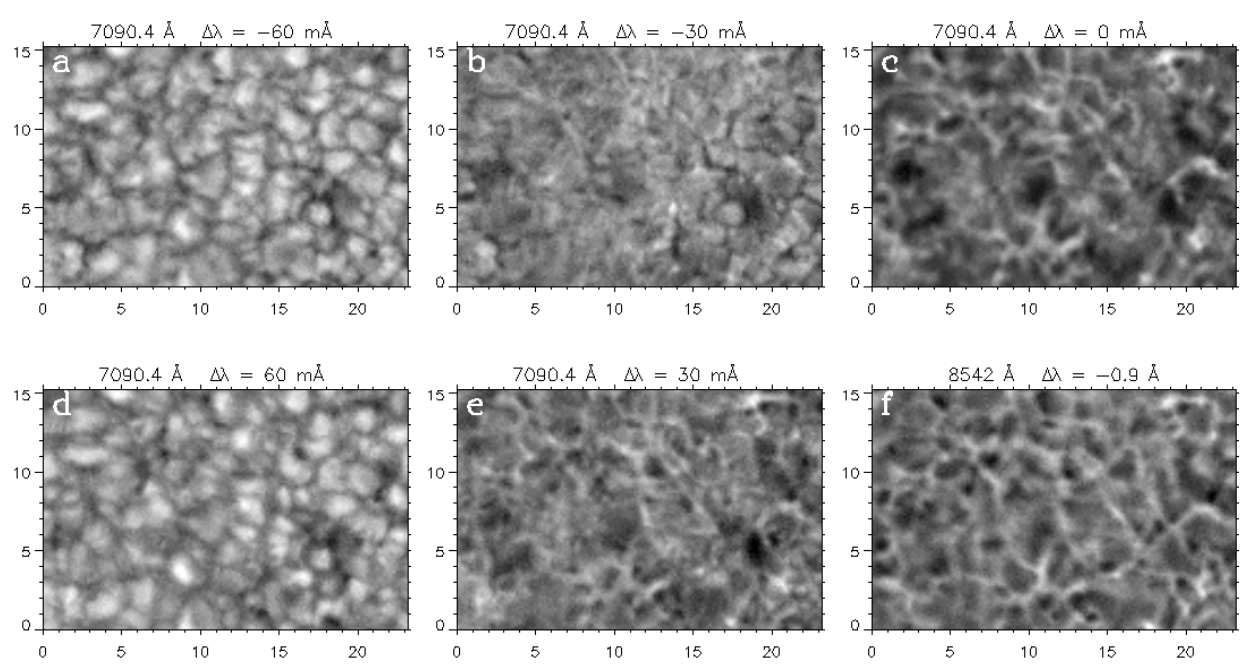

Figure 1. Intensity maps of the $\mathrm{Fe}_{\mathrm{I}} 7090.4 \AA$ line. Spectral positions of $-60 \mathrm{~m} \AA,-30 \mathrm{~m} \AA, 0 \mathrm{~m} \AA$ (upper row), $+60 \mathrm{~m} \AA$ and $+30 \mathrm{~m} \AA$ (lower row) off local line core, plus Ca II $8542 \AA$ at $0.9 \AA$ in the blue wing (lower right). The Fe I line core image (upper right) presents the 'reversed granulation', comparable to the one seen in the Ca II line wing (lower right).

\section{Reversed granulation}

The Fe I 7090.4 $\AA$ line clearly shows the transition from granular structures in the wings to reversed granulation in the core (Fig. 1). However, to reveal this phenomenon, it is crucial to take the vertical velocity (including p-mode effects) into account. Photospheric lines usually show inverted intensities in the blue wing of the line, as the velocity Doppler shift renders the brighter but upmoving and thus blueshifted granules darker than the average profile: The blueshifted profiles start to be sampled in the line wing while the average profile still shows nearly continuum intensity. The red line wing then shows granulation pattern with an enhanced contrast for the same reason. In order to extract the reversed granulation, we cancel the velocities for all pixels in the FOV, shifting all profile cores to a common zero wavelength. This results in a symmetric behaviour of the spectral positions and reveals the reversed granulation pattern in the line core as seen in Fig. 1c.

\section{Anticorrelation}

Quasi-simultaneous observations in a Ca II wing position, basically unaffected by velocity, show the same reversed granulation pattern, but reach higher anti-correlation values with the continuum. The iron line core intensity provides a weak anticorrelation with the continuum of about -0.21 , while the correlation between calcium wing and continuum reaches -0.32 .

For a spatial smoothing up to $0.5^{\prime \prime}$ the values found for these anticorrelations remain constant, but for a smoothing to lower resolution the anticorrelation decreases rapidly, indicating the typical granular scale of the reversed granulation phenomenon. 


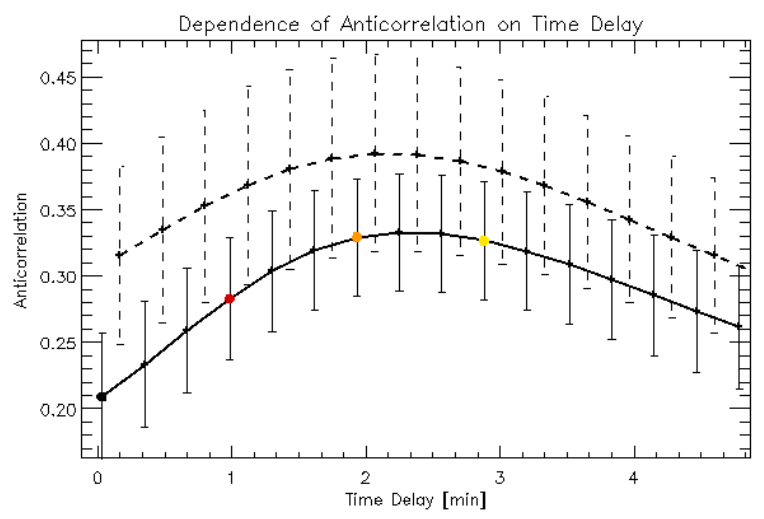

Figure 2. Anticorrelation between continuum and $\mathrm{Fe}$ I line core or Ca II wing intensities as a function of their time delay. The anticorrelation, averaged over 141 scans, follows a smooth curve with a maximum of 0.33 for $\mathrm{Fe}_{\mathrm{I}}$ at $135 \mathrm{~s}$ delay (solid line) and 0.39 for $\mathrm{Ca}$ II at $126 \mathrm{~s}$ delay (dashed-dotted).

The anticorrelation increases instead, if a time delay is allowed between the images from the layer forming the continuum and the higher atmospheric layer, see Fig. 2 and compare Rutten et al. (2004). This is consistent with the idea of upflowing granules, that are cooled in the higher layers. The time delay of about two minutes, found with the strongest anticorrelation for both $\mathrm{Fe}$ I line core and $\mathrm{Ca}$ II wing, matches the time a granule with $1-2 \mathrm{~km} / \mathrm{s}$ velocity needs to cover the 150-200 km height distance between the reversed granulation layer and the continuum. The $\mathrm{Ca}$ II wing anticorrelation reaches the maximum $10 \mathrm{~s}$ earlier than $\mathrm{Fe}$ I, confirming that the Ca II wings are formed not very high in the photosphere - around $150 \mathrm{~km}$ (Uitenbroek 1989) - and possibly even below the forming layer of the Fe I line core.

\section{Temporal variation}

The temporal evolution of the anticorrelation between continuum and Fe I line core intensities shows a variation with an oscillatory character, as seen in the upper panel of Fig. 3. The lighter symbols in the figure correspond to the 1, 2, and 3 minutes time delayed correlations (correlating the line core images with continuum images earlier observed). The period of the oscillation is about 6 minutes. The oscillation is a characteristic of the higher layer. This is demonstrated in Fig. 3 (lower panel): As in Fig. 3 upper panel the lighter curves correspond to 1, 2, 3, 4 min time delayed correlations, but here continuum images are correlated with line core images observed later. The temporal translation of the curves depends on the point in time of the core images, that are used for the correlation, leading us to the conclusion, that the oscillation resides in the higher layer. In the $\mathrm{Ca}$ II wing correlation with the continuum, we find no obvious periodic signal. The oscillation persists, if a stronger $p$-mode filter with a sound velocity of $5 \mathrm{~km} / \mathrm{s}$ is applied.

The period of the oscillation, its presence only in the higher atmospheric layers, and the stronger anticorrelation with the continuum in the lower Ca II wing, are suggestive of the presence of gravity waves in the mid-photospheric atmosphere. The reversed granulation pattern, that is strong in the Ca II images and peaks at granular scales, might get "corrupted" 

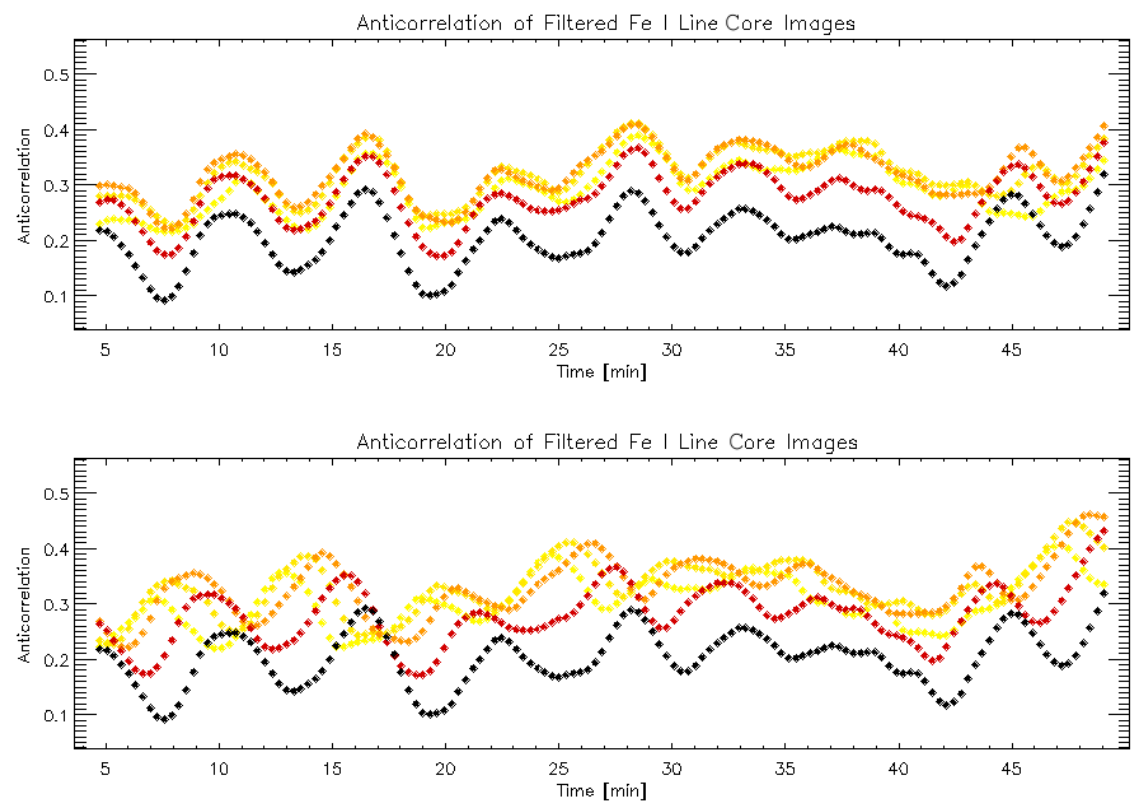

Figure 3. Time evolution of the anticorrelation between Fe I line core and continuum (black symbols). A time delay between line core and earlier recorded continuum images (lighter symbols: 1, 2, 3, 4 min delay) strengthens the anticorrelation, but does not shift the oscillation curve (upper panel), which is not the case, if one continuum image is correlated with core intensities obtained later (lower panel).

by intensity oscillations in mid-photosphere. Gravity waves are supposed to develop steeply with height (Mihalas \& Toomre 1981), so at the height of the Fe I core formation their amplitude might be sufficient to perturb the reversed granulation pattern, causing a lower anticorrelation with the continuum and a periodic behaviour (see also Janssen \& Cauzzi 2006).

As the surface of optical depth unity at any given wavelength is highly corrugated, a height definition for the phenomena is a challenge, but classical analysis employing semi-empirical 1D models provide a formation height of about $200 \mathrm{~km}$ for the Fe I core, where modern 3D hydrodynamical simulations display the reversal of granular and intergranular temperature (Nordlund 1985; Wedemeyer et al. 2004).

\section{References}

Cavallini, F. 2006, Solar Phys., 236, 415

Cheung, M. C. M., Schüssler, M., \& Moreno-Insertis, F. 2006, A\&A, 461, 1163

Janssen, K. \& Cauzzi, G. 2006, A\&A, 450, 365

Mihalas, B. W. \& Toomre J. 1981, ApJ, 249, 349

Nordlund, Å. 1985, Solar Phys., 100, 209

Rutten, R. J., de Wijn, A. G., \& Sütterlin, P. 2004, A\&A, 416, 333

Stein, R. F. \& Nordlund, Å. 1998, ApJ, 499, 914

Uitenbroek, H. 1989, A\&A, 213, 360

Wedemeyer, S., Freytag, B., Steffen, M., Ludwig, H.-G., \& Holweger, H. 2004, A\&A, 414, 1121 


\title{
The height dependence of temperature - velocity correlation in the solar photosphere
}

\author{
J. Koza ${ }^{1,2,{ }^{*}}$, A. Kučera ${ }^{2}$, J. Rybák ${ }^{2}$, and H. Wöhl ${ }^{3}$ \\ ${ }^{1}$ Sterrekundig Instituut, Utrecht University, The Netherlands \\ ${ }^{2}$ Astronomical Institute, Slovak Academy of Sciences, Tatranská Lomnica, Slovakia \\ ${ }^{3}$ Kiepenheuer-Institut für Sonnenphysik, Freiburg, Germany \\ *Email: j.koza@astro.uu.nl
}

\begin{abstract}
We derive correlation coefficients between temperature and line-of-sight velocity as a function of optical depth throughout the solar photosphere for the non-magnetic photosphere and a small area of enhanced magnetic activity. The maximum anticorrelation of about -0.6 between temperature and line-of-sight velocity in the non-magnetic photosphere occurs at $\log \tau_{5}=-0.4$. The magnetic field is another decorrelating factor along with 5-min oscillations and seeing.
\end{abstract}

\section{Introduction}

The correlative analysis proves to be an essential tool in disentangling of causal relations in the solar atmosphere. Recently, Rutten \& Krijger (2003) and Rutten et al. (2004) quantified the correlation of the reversed granulation observed in the low chromosphere and midphotosphere with surface granulation in quest for the nature of internetwork background brightness patterns in these layers. In agreement with these studies Puschmann et al. (2003) demonstrated that filtering out of the p-modes is inevitable for studying the convective structures in the solar photosphere because p-modes mostly reduce the correlation between various line parameters. Odert et al. (2005) showed that correlation coefficients can fluctuate strongly in time with amplitudes of over 0.4 due to 5-min oscillations and the amplitudes are larger for higher formed lines. In case of weak lines the situation worsens even more, because correlations derived from them are influenced stronger by seeing.

In this paper, we address the dissimilarity between non-magnetic and magnetic region seen in height variations of the correlation between temperature and line-of-sight velocity. We compare our results with a similar study by Rodríguez Hidalgo et al. (1999). Our analysis follows on the paper Koza et al. (2006, henceforth Paper I) and we invite the reader to have the paper at hand for further references.

\section{Observational data and inversion procedure}

We use a time sequence of spectrograms obtained at the German Vacuum Tower Telescope at the Observatorio del Teide on April 28, 2000. The inversion code SIR (Ruiz Cobo \& del Toro Iniesta 1992) was employed in the analysis of this observation. Thorough descriptions of the observational data, inversion procedure, and spectral lines are given in Paper I. 


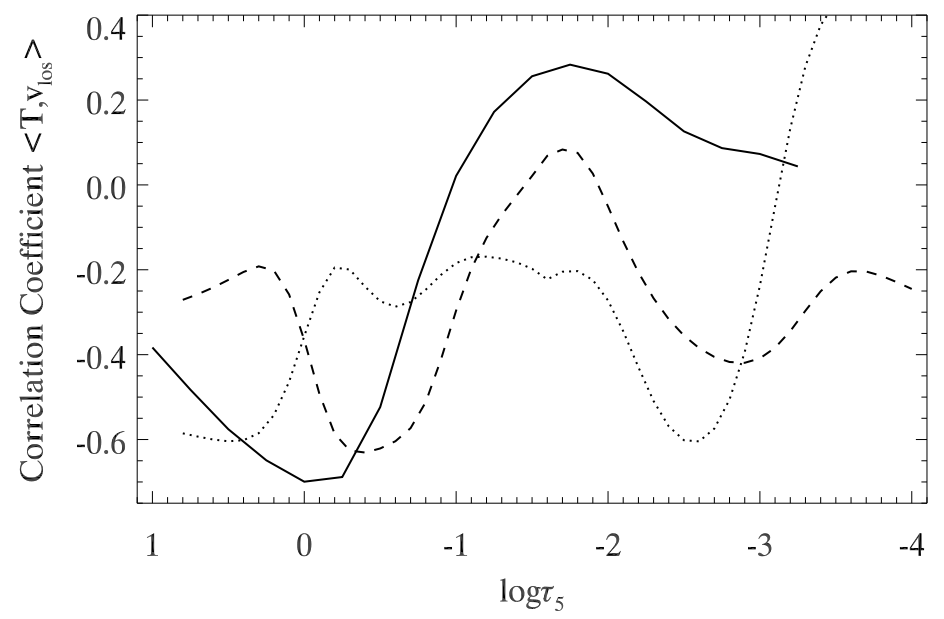

Figure 1. The height variation of the correlation between line-of-sight velocity and temperature for the results of Rodríguez Hidalgo et al. (1999) (solid) and for the non-magnetic (dashed) and magnetic (dotted) region defined in Paper I.

\section{Results}

Figure 1 shows the height variations of the correlation between temperature and line-of-sight velocity for three different sets of data. The results of Rodríguez Hidalgo et al. (1999) indicate the significant anticorrelation between granules and intergranular lanes reaching the maximum of about -0.7 at $\log \tau_{5}=-0.2$. The subsequent weakening of this anticorrelation over the $\log \tau_{5} \in\langle-0.2,-1.0\rangle$ range is followed by a rise of correlation up to 0.28 at the middle photosphere at $\log \tau_{5}=-1.75$. No significant correlation exists in the upper photosphere. In the lower layers of the non-magnetic region (Paper I) the anticorrelation decreases to -0.63 at $\log \tau_{5}=-0.4$. However, in the middle photosphere temperature and line-of-sight velocity are almost uncorrelated with a local peak value of 0.08 at $\log \tau_{5}=-1.7$. Higher up at $\log \tau_{5}=-2.9$ the anticorrelation of about -0.42 is established again. In the sub-photospheric layers of the magnetic region the anticorrelation of -0.6 was found at $\log \tau_{5}=0.5$. An approximately constant value of anticorrelation -0.2 was obtained in the low and middle photosphere. In the upper photosphere the anticorrelation reaches again -0.6 .

Figure 2 compares temperatures and line-of-sight velocities in the form of scatter correlation plots. Each plotted sample represents temperature and line-of-sight velocity specified along the $x$ and $y$ axes at a given pixel along the slit at a time within the interval of $15 \mathrm{~min}$. From the top down, the row panels show correlations of the results of Rodríguez Hidalgo et al. (1999) and our results in the non-magnetic and magnetic region in three selected optical depths $\log \tau_{5}=-0.3,-1.3$, and -1.8 . Plot saturation is avoided by showing sample density contours rather than individual points, except for the extreme outliers. The total distributions of temperatures and line-of-sight velocities are shown at the top and the left side of each panel, respectively. The first-moment curves are aligned at large correlation and become perpendicular in the absence of any correlation (Rutten \& Krijger 2003). The first column in Fig. 2 shows good agreement of correlation coefficients and positions of maxima of velocity 


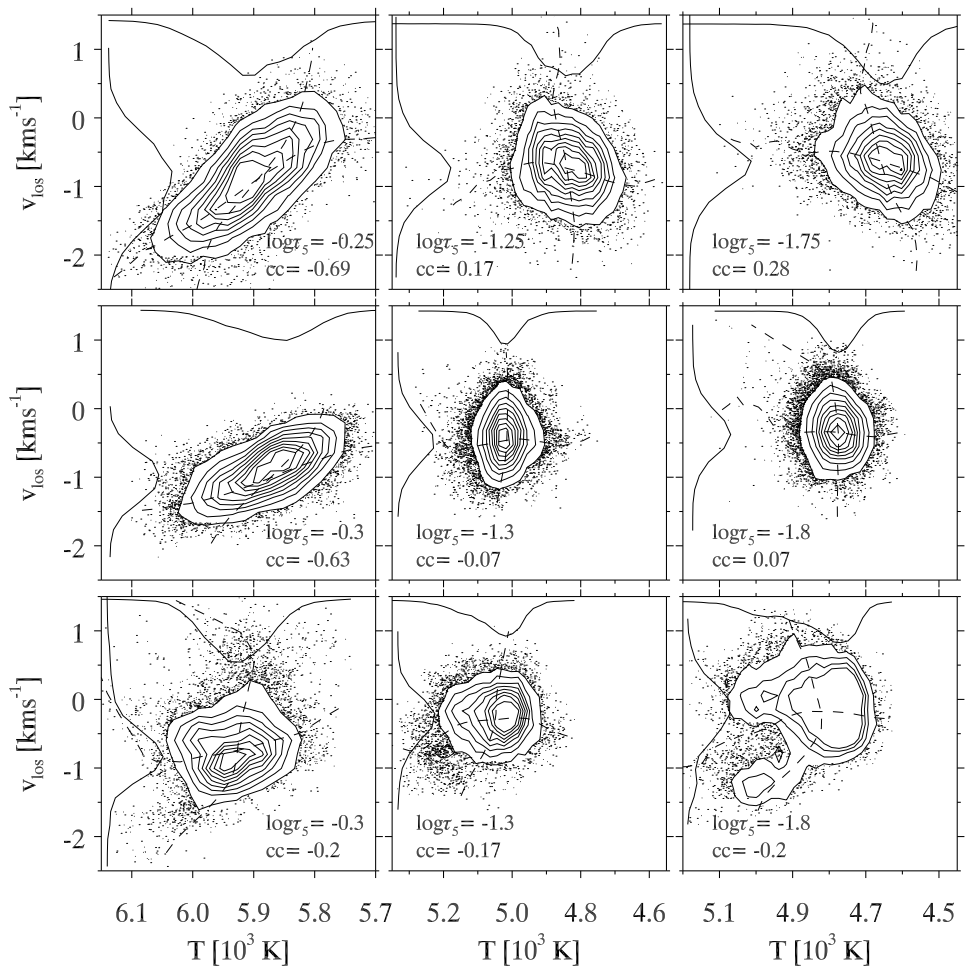

Figure 2. Height-dependent scatter correlations of the line-of-sight velocity versus temperature. Top row: data from Rodríguez Hidalgo et al. (1999, see p. 315, Fig. 1). Middle and bottom row: our data for the non-magnetic and magnetic region (Paper I), respectively. The optical depths $\log \tau_{5}$ and correlation coefficients $c c$ are specified at each panel. Negative velocities indicate upflows. The rescaled total distributions of temperatures and line-of-sight velocities are shown as solid curves at the top and the left side of each panel, respectively. The dashed curves show the first moments of the sample density distributions over temperature and velocity bins.

distributions in the non-magnetic region with the results of Rodríguez Hidalgo et al. (1999). However, the temperature distributions are dissimilar both in terms of asymmetry and also the positions of maxima. Our results indicate predominance of higher temperatures in the sample in contrast with lower temperatures prevailing in the results of Rodríguez Hidalgo et al. (1999). In the magnetic region, weak anticorrelation was found. The temperature distribution in this region is almost symmetric with maximum at higher temperatures than in the non-magnetic region. The second column of Fig. 2 corresponds to the layers where granulation is almost erased. While the temperature distributions in the non-magnetic region and in the results of Rodríguez Hidalgo et al. (1999) are symmetric, in the magnetic region the asymmetry indicates the abundant higher temperatures. The positive correlation in the results of Rodríguez Hidalgo et al. (1999) shown in the third column suggests reversed granulation. However, this is not seen in our results. In the magnetic region the asymmetries of temperature and velocity distributions indicate higher abundance of relatively hotter pixels with faster upflows. 


\section{Discussion}

Figures 1 and 2 show dissimilarities both in height variations of correlation and distributions, although we and Rodríguez Hidalgo et al. (1999) used VTT observations and the SIR code. Because the maximum of anticorrelation found at sub-photospheric layers of the magnetic region is out of the range of sensitivity of the spectral lines (Paper I), we disregard this feature. Very low anticorrelation found over $\log \tau_{5} \in\langle 0.0,-2.0\rangle$ in the magnetic region (Fig. 1) suggests that magnetic field is another important decorrelating factor along with 5-min oscillations and seeing (Puschmann et al. 2003; Odert et al. 2005). In our results, the middle layers of the non-magnetic and magnetic region lack signatures of reversed granulations (Fig. 1). The sinusoidal shape of the correlation coefficient in the non-magnetic region over the $\log \tau_{5} \in\langle-1.2,-3.5\rangle$ range can be explained as a sum of positive correlation typical for reversed granulation and negative anticorrelation characteristic for 5-min oscillations.

\section{Summary}

Using a time sequence of high-resolution spectrograms and the SIR inversion code we have inferred height variation of correlation between the temperature and line-of-sight velocity throughout the quiet solar photosphere and a small magnetic region. The most important aspect is comparison of the results with the akin study by Rodríguez Hidalgo et al. (1999). We found in agreement with Rodríguez Hidalgo et al. (1999) that the maximum anticorrelation -0.6 between the temperature and line-of-sight velocity in the non-magnetic region occurs at $\log \tau_{5}=-0.4$. The absence of signatures of reversed granulation in the middle layers of the non-magnetic region is likely to be due to 5-min oscillations, which negative anticorrelation prevails over weaker positive correlation typical for reversed granulation. Our results show that magnetic field is another decorrelating factor along with 5-min oscillations and seeing.

Acknowledgements. The VTT is operated by the Kiepenheuer-Institut für Sonnenphysik, Freiburg, at the Observatorio del Teide of the Instituto de Astrofísica de Canarias. We are grateful to B. Ruiz Cobo (IAC) for kindly providing of the original data used in Figs. 1 and 2. This research is part of the European Solar Magnetism Network (EC/RTN contract HPRN-CT-2002-00313). This work was supported by the Slovak grant agency VEGA (2/6195/26) and by the Deutsche Forschungsgemeinschaft grant (DFG 436 SLK 113/7). J. Koza's research is supported by a Marie Curie Intra-European Fellowships within the 6th European Community Framework Programme.

\section{References}

Koza, J., Kučera, A., Rybák, J., \& Wöhl, H. 2006, A\&A, 458, 941, (Paper I)

Odert, P., Hanslmeier, A., Rybák, J., Kučera, A., \& Wöhl, H. 2005, A\&A, 444, 257

Puschmann, K., Vázquez, M., Bonet, J. A., Ruiz Cobo, B., \& Hanslmeier, A. 2003, A\&A, 408, 363

Rodríguez Hidalgo, I., Ruiz Cobo, B., Collados, M., \& del Toro Iniesta, J. C. 1999, in ASP Conf. Ser. 173: Stellar Structure: Theory and Test of Connective Energy Transport, ed. A. Giménez, E. F. Guinan, \& B. Montesinos, 313

Ruiz Cobo, B. \& del Toro Iniesta, J. C. 1992, ApJ, 398, 375

Rutten, R. J., de Wijn, A. G., \& Sütterlin, P. 2004, A\&A, 416, 333

Rutten, R. J. \& Krijger, J. M. 2003, A\&A, 407, 735 


\title{
Polarimetry in the visible and near infrared
}

\author{
M. Collados \\ Instituto de Astrofísica de Canarias, La Laguna, Spain
}

\begin{abstract}
The various techniques used in the visible and near-infrared for the measurement of intrinsic polarization coming from solar regions are described. Their performance to allow for the acquisition of simultaneous spectropolarimetric data at several wavelengths is discussed. Any development of a future facility should take advantage of these capabilities to measure simultaneously different solar atmospheric layers with high spatial resolution.
\end{abstract}

\section{Introduction}

Spectropolarimetry is one of the most powerful tools to study the solar atmosphere. Magnetic fields, scattering processes and atomic polarization are usually studied, using the Zeeman and Hanle effects, via the polarized signatures they generate. Presently, much effort is devoted to the study of these signals making emphasis on two aspects: high spatial resolution and three-dimensional stratification. On the one hand, it is clear nowadays that the solar atmosphere is highly structured and that small-scale features play a crucial role when trying to understand the properties of the solar photosphere and chromosphere. Sunspot penumbrae, faculae, network and internetwork regions exhibit properties that will only be finally understandable when a spatial resolution higher than available at present will be achieved. On the other hand, the different layers of the solar atmosphere are interconnected, and a clear picture of magnetic phenomena will only be obtained when simultaneous spectropolarimetric observations at different heights are available. In this contribution, some of the most successful techniques to detect polarized light will be described, as well as the efforts to observe at different wavelengths. State-of-the-art examples will also be presented to show the capabilities of present instruments. Arguments favoring the need for large aperture telescopes will be given, since the limited number of detected photons represents the main drawback to get adequate datasets.

\section{Polarimetric techniques}

Detectors are only sensitive to the number of photons, and not to their polarization state. For that reason, all polarimetric techniques are based on the basic principle of introducing the information of polarization into the intensity of the transmitted light. To that aim, a simple polarimeter is composed by a number of retarders or waveplates (usually, one or two) followed by a linear polarizer. The retarders modify the polarization state of the incoming light in an adequate way such that the polarizer only transmits at the end the desired component of the electric field. A sequence of measurements varying the retarders (either their retardance 
or their orientation), keeping fixed the orientation of the polarizer, makes possible to obtain a set of intensity measurements from which one can retrieve efficiently the polarization state of the input light.

In astrophysics, a polarizing beamsplitter, producing simultaneously two orthogonal linearly polarized beams, is to be preferred, rather than a simple linear polarizer. This way, one can minimize the effect of intensity changes produced by seeing variations, which would otherwise be interpreted as polarization signals (see Collados 1999). These seeing-induced intensity variations, occurring at frequencies of the order of a hundred $\mathrm{Hz}$, are of a few percent, masking almost completely the true polarization coming from the Sun. For this reason, unless modulation schemes much faster than these typical atmospheric time scales are used, temporal modulation (sequence of measurements) together with spatial modulation (two simultaneous orthogonal output beams produced by the beamsplitter) are mandatory to detect the weak signals that are produced by the Zeeman and Hanle effects and scattering processes in most solar regions. For simplicity, in the rest of the text, we will refer only to one of the beams, as if only a linear polarizer were part of the device.

Mathematically, a polarimeter is described by the following equation:

$$
\boldsymbol{N}=M \cdot \boldsymbol{I},
$$

where $\boldsymbol{N}^{\dagger}=\left(N_{1}, N_{2}, \ldots, N_{n}\right)$ is a vector that represents the $n$ intensity measurements obtained with the detector, $\boldsymbol{I}^{\dagger}=\left(I_{1}, I_{2}, I_{3}, I_{4}\right)=(I, Q, U, V)$ is the input Stokes vector, and $M$ is the so-called modulation matrix, which only depends on the polarimeter itself. The symbols ' $'$ ' and ' $\dagger$ ' stand for matrix product and matrix transposition, respectively. It has to be noted that, in general, only four independent measurements are required to solve Eq. 1 and get $\boldsymbol{I}$.

Not all modulation schemes allow to recover the Stokes parameters with the same accuracy. An elegant definition of the efficiency, $\boldsymbol{\epsilon}$, of a polarimeter, that makes possible a mathematical comparison between the different techniques, has been given by del Toro Iniesta \& Collados (2000):

$$
\boldsymbol{\epsilon}=\left(n \sum_{j=1}^{n} D_{i j}^{2}\right)^{-1 / 2} .
$$

$\boldsymbol{\epsilon}$ is a four-element vector containing the efficiencies with which the four Stokes parameters are recovered, and the matrix $D$, the demodulation matrix, is given by the equation

$$
D=\left(M^{\dagger} \cdot M\right)^{-1} \cdot M^{\dagger} .
$$

For a square matrix $M$ (i.e, for $n=4$ ), this operation is equivalent to calculating its inverse.

These efficiency parameters are important because they determine how noisy the recovered Stokes parameters will be. Calling $\sigma$ the noise of the measurements $\boldsymbol{N}$, one gets

$$
\sigma_{i}=\sigma / \epsilon_{i}
$$

It can be demonstrated that $\epsilon_{1} \leq 1$ (intensity efficiency), and $\epsilon_{p}^{2}=\sum_{i=2}^{4} \epsilon_{i}^{2} \leq 1$ (squared total polarization efficiency). From here, it follows directly that, for a polarimeter that allows to measure in an optimum way the three Stokes parameters $Q, U$ and $V, \epsilon_{2,3,4}=\epsilon_{Q, U, V}=$ $1 / \sqrt{3}=0.577$. 

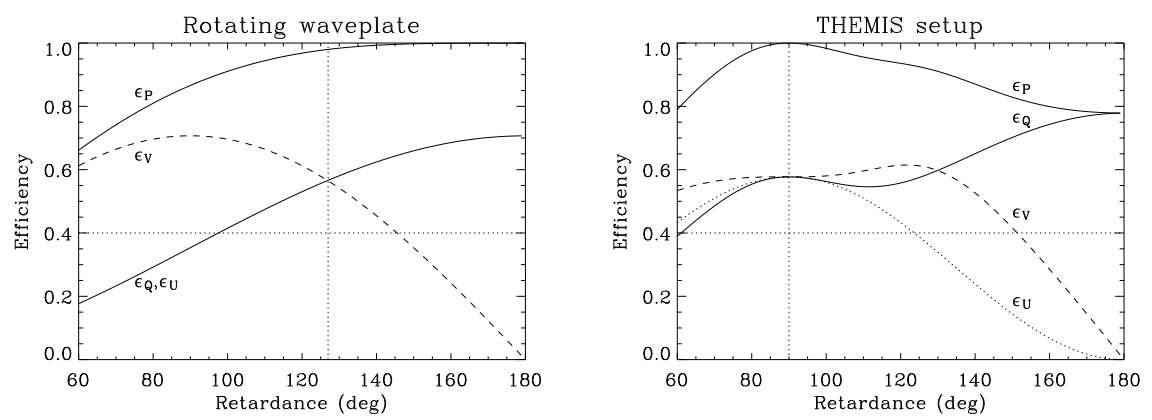

Figure 1. Left: Polarization $(Q, U$, and $V)$ efficiencies $\left(\epsilon_{Q}, \epsilon_{U}\right.$, and $\left.\epsilon_{V}\right)$ of a polarimeter using a rotating waveplate as a function of retardance. The total polarization efficiency, $\epsilon_{p}$, is also shown. The vertical dotted line gives the retardance $\left(127^{\circ}\right)$ for which all three Stokes parameters have the same efficiency. The horizontal dotted line limits the range for acceptable efficiencies in practice (larger than 0.4). Right: Polarization efficiencies of a THEMIS-like polarimeter as a function of the waveplate retardance, both assumed to be equal. The waveplate orientations are given in Table 1. The vertical and horizontal dotted lines have the same meaning as in the plot on the left. The optimum retardance, for which $Q, U$, and $V$ have the same efficiency, is $90^{\circ}$ in this case.

Presently, solar polarimeters use different techniques to modulate the output light intensity, namely,

- one rotating waveplate,

- two retarders with discrete orientations,

- two nematic liquid crystals,

- two ferroelectric liquid crystals,

- one piezoelastic modulator.

In the following, some characteristics of these polarimeters are described.

\subsection{One rotating waveplate}

This option is used, for instance, by ASP (Elmore et al. 1992), POLIS (Beck et al. 2005), and SPINOR (Socas Navarro et al. 2006). These polarimeters use a single waveplate that is continuously rotating at a fixed rate. In a half-rotation, a detector takes eight samples (the second half gives exactly the same measurements). That is, each measurement is obtained while the waveplate changes its orientation by $22.5^{\circ}$. It can be easily demonstrated that four measurements, each one corresponding to an orientation change of $45^{\circ}$, are not independent, and, consequently, they are not sufficient to solve Eq. 1. Following Eq. 2, the polarization efficiencies can be calculated (the intensity polarization is equal to one) as a function of the retardance of the waveplate. The result is plotted on the left part of Fig. 1, where the efficiencies for $Q, U$, and $V$, as well as that of the total polarization, are shown. Two aspects deserve to be mentioned here. Firstly, $Q, U$, and $V$ are all equally well recovered, with 
Table 1. Usual orientation of the waveplates used in THEMIS. $\theta_{1}$ and $\theta_{2}$ represent the orientation of the two retarders of the polarimeter. Suffix 1 refers to the retarder located closer to the polarizing beamsplitter. All angles are measured taking one of the polarizing axes of the beamsplitter as a reference. The measurements included in the third column correspond to the case where both retarders behave as ideal quarter wave plates.

\begin{tabular}{ccc}
\hline$\theta_{1}$ & $\theta_{2}$ & Measurement \\
\hline $0^{\circ}$ & $0^{\circ}$ & $I+Q$ \\
$45^{\circ}$ & $45^{\circ}$ & $I-Q$ \\
$22.5^{\circ} / 45^{\circ}$ & $22.5^{\circ} / 0^{\circ}$ & $I+U$ \\
$67.5^{\circ} /-45^{\circ}$ & $67.5^{\circ} / 0^{\circ}$ & $I-U$ \\
$0^{\circ}$ & $-45^{\circ}$ & $I+V$ \\
$0^{\circ}$ & $45^{\circ}$ & $I-V$ \\
\hline
\end{tabular}

maximum efficiency (i.e. with minimum noise) for a waveplate with a retardance of $127^{\circ}$. And, secondly, if we consider as acceptable any efficiency above 0.4 , any waveplate with a retardance in the range from $100^{\circ}$ to $145^{\circ}$ can do a good job.

Since achromatic waveplates can be constructed, these types of polarimeters can be used in a rather broad range of wavelengths. SPINOR, for instance, uses a waveplate whose retardance changes by only $\pm 16 \%$ in the full range from $400 \mathrm{~nm}$ to $1.6 \mu \mathrm{m}$, which makes it suitable for simultaneous spectropolarimetric observations from visible to near-infrared wavelengths. Note, however, that the appearance of interference fringes may limit the true usefulness of achromatic retarders in the full wavelength range (see Semel 2003, for a discussion about this topic).

\subsection{Two retarders with discrete orientations}

This is the solution adopted by THEMIS. Two achromatic waveplates are used with discrete orientations to measure sequentially the desired linear combination of the Stokes parameters (see, e.g., López Ariste et al. 2000). Usual combinations are given in Table 1. When both retarders are ideal quarter wave plates, pure Stokes parameters are measured with these combinations, and, as shown by del Toro Iniesta \& Collados (2000), maximum efficiencies are found for $Q, U$ and $V$. This setup has the advantage that one does not need to measure these three Stokes parameters. If solid reasons exist to argue that one or two of them are negligible with respect to the other, and one is not interested in them even if being small but non-vanishing, one can use only the two positions relating the parameter of interest. Since all photons will be used in that case to measure a single polarization Stokes parameter, it will be recovered with less noise (i.e., with a larger efficiency).

The right plot in Fig. 1 shows the dependence of the polarization efficiencies on the retardance of the waveplates (both assumed to be equal), taking the orientations given in Table 1 . As can be seen, there is a broad range of values (from $60^{\circ}$ to $125^{\circ}$ ) where all efficiencies are large. This is specially important when doing simultaneous observations of distant wavelengths. The same set of orientations is valid for all of them, with different modulation matrices, if the retardance is within the acceptable range. The price to pay, if the retarders 

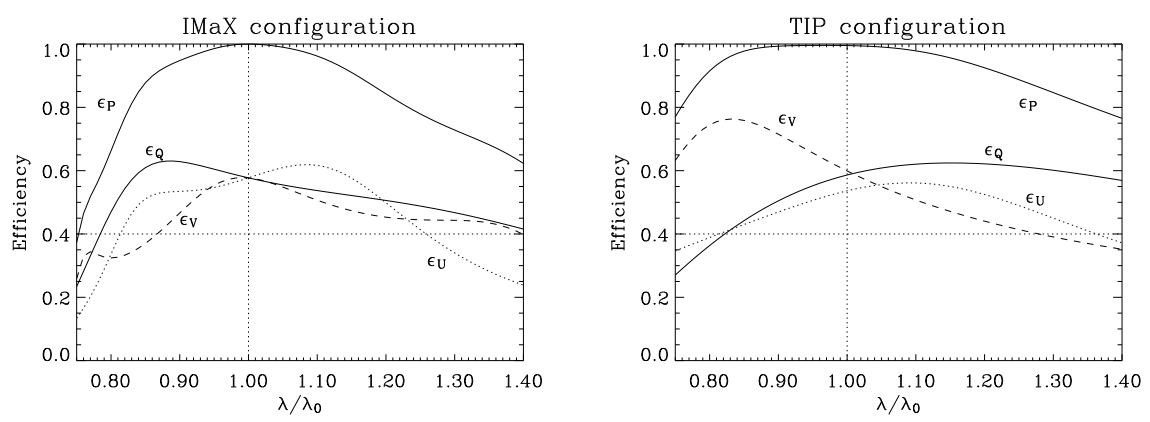

Figure 2. Left: Polarization $(Q, U$, and $V)$ efficiencies $\left(\epsilon_{Q}, \epsilon_{U}\right.$, and $\left.\epsilon_{V}\right)$ as a function of wavelength, for a polarimeter using two LCVRs with the vectorial magnetogram configuration of IMaX (see text for details). The vertical dotted line represents the optimized wavelength. The horizontal dotted line limits the range for acceptable efficiencies in practice (larger than 0.4). Right: Same as in the left plot, but for a polarimeter using two FLCs like in TIP (see text for details).

are not quarter wave plates, is that now all four Stokes parameters must be measured (i.e., with the full modulation scheme).

\subsection{Two nematic liquid crystals}

Nematic liquid crystals behave as retarders with fixed orientation and a retardance that can be varied applying an external voltage, and are usually known as Liquid Crystal Variable Retarders (LCVRs). For visible wavelengths, the retardance usually varies between a minimum value close to zero and a maximum close to one wavelength. Typical switching times are of the order of $10 \mathrm{~ms}$, although they are very much dependent on the starting and ending retardances. Increasing voltages (corresponding to decreasing retardances) are usually faster. Their large tunable retardance range makes them suitable, for example, for applications where several spectral lines are measured sequentially, optimizing their behavior for each one of them. An example might be the case when one wants to measure the circular polarization in one line and the linear polarization in another. Polarization efficiencies can then be optimized for each wavelength and desired Stokes parameter. Another advantage of liquid crystals is that the modulation is performed avoiding the motion of any optical element, which in general makes it insensitive to the presence of small dust particles or inhomogeneities. The disadvantage is that their behavior is temperature dependent, and they must be installed in a temperature-controlled environment.

This type of retarders are used, for instance, in IMaX (Martínez Pillet et al. 2004) and VIP (Bellot Rubio et al. 2007). Because of their flexibility, IMaX has implemented two observing modes to obtain longitudinal and vectorial magnetograms, using two LCVRs. The polarimeter can be tuned to one or the other observing mode just by modifying the external voltage. For instance, in the vectorial mode, the first LCVR seen by the light is oriented parallel to one of the axis of the beamsplitter and sequentially receives the voltages that correspond to $315^{\circ}, 315^{\circ}, 225^{\circ}$, and $225^{\circ}$ retardances. The second LCVR is oriented at $45^{\circ}$ and the corresponding retardances, tuned simultaneously with the first LCVR, are $305.264^{\circ}$, 
$54.736^{\circ}, 125.264^{\circ}$, and $234.736^{\circ}$. It can be easily demonstrated that maximum efficiencies in all Stokes parameters are obtained with this modulation scheme. The left plot in Fig. 2 shows the wavelength dependence of this scheme, assuming a constant birefringence. This is of interest when simultaneous observations of several spectral lines are required, and the modulation is optimized for one of them. As can be seen, all polarization efficiencies are above the 0.4 threshold for wavelengths in the range 0.87 to 1.26 , in units of the optimized wavelength.

\subsection{Two ferroelectric liquid crystals}

In opposition to LCVRs, ferroelectric liquid crystals (FLCs) behave as retarders with fixed retardance (slightly temperature dependent) and whose fast axis can alternate only between two orientations, depending on an externally applied voltage. This represents a loss of versatility, when compared to LCVRs. However, switching times are of the order of $0.1 \mathrm{~ms}$ which makes them suitable for fast modulation schemes. Their calibration is also easier, since only two states need to be determined.

Examples of polarimeters using two FLCs are TIP and LPSP (Martínez Pillet et al. 1999; Collados et al. 2007). At least, two FLCs are mandatory to get the minimum four independent measurements required to solve Eq. 1. In one of the configurations used with TIP, the first FLC seen by the light has a retardance of $155^{\circ}$, at a wavelength of $1.1 \mu \mathrm{m}$, and an orientation of $70^{\circ}$. The values for the second FLC are $75^{\circ}$ (same wavelength) and $155^{\circ}$, respectively. Both FLCs increase the orientation of their fast axis by $50^{\circ}$ with the external voltage. The right plot of Fig. 2 shows the wavelength dependence of this modulation scheme. Polarization efficiencies are within acceptable values in the range from 0.83 to 1.25 in units of the reference wavelength. Thus, despite FLCs lack degrees of freedom (they can only alternate between two states), they give rise to modulation schemes that are valid in a wavelength interval as large as those of polarimeters based on LCVRs, at least for the examples we have presented.

\subsection{One piezoelastic modulator}

ZIMPOL (Stenflo et al. 1992) is a polarimeter that deserves special attention. It was originally devised to work with one or two piezoelastic modulators, for the simultaneous measurement of three or four Stokes parameters, simultaneously. This type of modulators can reach frequencies of several $\mathrm{kHz}$ (similar to FLCs, that were not available at the time this polarimeter was constructed). Thanks to the development of specially dedicated electronics, the charge within the detector could be shifted back and forth at a large speed. After masking the adequate pixels, the modulation scheme can reach frequencies much larger than those typical of atmospheric variations, making it free from spurious polarization signals due to atmospheric variations. For that reason, it is the only polarimeter that uses a linear polarizer, instead of a polarizing beamsplitter.

\section{Future developments}

During the last years, there have been a lot of efforts to observe simultaneously several spectral regions, with polarimetric capabilities. THEMIS was originally designed to do si- 
multaneous spectropolarimetry of up to ten spectral lines. Also, recently, at the German VTT of the Observatorio del Teide, the combinations of the instruments POLIS and TIP or VIP and TIP are possible (see Beck et al. 2007).

There are two main reasons for these developments. Firstly, simultaneous observations of several photospheric spectral lines provide additional information to constrain the atmospheric model atmospheres derived from them. Several examples can be mentioned. Using visible and near-infrared lines, Martínez González et al. (2006) studied the weak signals of internetwork regions, concluding that the observed signals are compatible with well belowkG field strengths. Beck (2006) showed how visible and near-infrared lines were compatible with the uncombed penumbral model, where more or less horizontal flux-tubes are embedded in a more vertical and stronger background field. Plasma motion is required to take place in the horizontal component. Schlichenmaier et al. (2002) and Müller et al. (2006) explained in similar terms the azimuthal variation of visible and near-infrared Net Circular Polarizations observed in the penumbra of sunspots.

Secondly, different spectral lines determine the range of heights that can be probed. Specially interesting are those observations that give clues to link the photospheric and chromospheric magnetism. As an example, Centeno et al. (2006) have found that the 3-minute chromospheric oscillations in sunspot umbrae propagate from the photosphere, and they are not the result of non-linear interaction of photospheric 5-minute oscillations. Solanki et al. (2003) have derived the three-dimensional loop structure of an emerging region, where strong horizontal gradients and shears of magnetic field exist in distances of 1 " -2 ", suggesting that electric currents may play an important role in chromospheric heating.

In addition, spatial resolution is crucial for the final understanding of photospheric and chromospheric structures. Presently, using ground-based telescopes, spectropolarimetry can only be achieved, with adequate noise levels, with a resolution not better than $0.5^{\prime \prime}$. Highresolution images reveal, though, that structures with a size of $0.1^{\prime \prime}$ exist everywhere, and probably they are responsible for most of the energy channeling from the photosphere to the chromosphere. Large collecting areas will be required in the future to give us the possibility to improve the spatial resolution, with reduced integration times.

Any future development of new infrastructures must take into account these needs of multiline spectropolarimetry and high spatial resolution. Adaptive optics (and, possibly, multiconjugate adaptive optics) will be mandatory to ensure that an excellent high-spatial resolution image quality may be obtained in large fields of view. Multiline spectropolarimetry will allow for the study of the magnetic coupling of the different layers of the solar atmosphere, and the mechanisms that transfer energy upwards and give rise to a hot chromosphere. Complementary information can be obtained with the combined and simultaneous use of spectrographs and narrow-band tunable filters. Spectrographs will be necessary to ensure the integrity of the measured Stokes profiles, while tunable filters will be required to study the 2D magnetic and dynamic evolution of photospheric and chromospheric structures,

In this sense, an initiative is recently taking form to develop a large aperture European Solar Telescope (EST) that, together with the American ATST, might fulfill all these needs. EST must inherit the best of all present European facilities to minimize the risks and costs of such a large infrastructure: (i) excellent imaging capabilities, as, for instance, that of the 1-m SST, by having a simple design with excellent optics; (ii) robust and user-friendly adaptive optics like, for instance, the one operating at the VTT; (iii) multiline spectrograph capabilities like in THEMIS; (iv) visible and near-infrared simultaneous polarimetry, like at 
the VTT; (v) efficient narrow-band tunable filters like IBIS; and (vi) simultaneous control of all instruments like, for instance, at the VTT. Joint efforts of European countries can help make a reality this telescope by combining the best of all expertises. In addition, advantage can be taken of having two large aperture telescopes (EST \& ATST) located in opposite terrestrial longitudes. Observing programs requiring a large and almost continuous temporal coverage (such as the emergence of an active region) can get many benefits by using ATST and a European telescope located in the Canary Islands.

Acknowledgements. I wish to deeply thank Lena Khomenko for carefully reading the manuscript and suggesting modifications that have clearly improved this contribution. I am also grateful to the organizers of the meeting for having invited me and for their diligent editorial work. This research was funded by the Spanish Ministerio de Educación y Ciencia through project AYA2004-05792.

\section{References}

Beck, C. 2006, Ph.D. thesis. University of Freiburg (Germany)

Beck, C., Mikurda, K., Bellot Rubio, L. R., Kentischer, T., \& Collados, M. 2007, this volume

Beck, C., Schmidt, W., Kentischer, T., \& Elmore, D. 2005, A\&A 437, 1159

Bellot Rubio, L. R., Tritschler, A., Kentischer, T., Beck, C., \& del Toro Iniesta, J. C. 2007, in Solar Active Regions and 3D Magnetic Structure, 26th meeting of the IAU (in press)

Centeno, R., Collados, M., \& Trujillo Bueno, J. 2006, ApJ, 640, 1153

Collados, M. 1999, in Third Advances in Solar Physics Euroconference: Magnetic Fields and Oscillations, eds. B. Schmieder, A. Hofmann, \& J. Staude. ASP Conf. Ser. 184, 3

Collados, M., Lagg, A., Díaz García, J. J., Hernández Suárez, E. et al. 2007, in The Physics of Chromospheric Plasmas, eds. P. Heinzel, I. Dorotovic, \& R. J. Rutten, ASP Conf. Series (in press)

Elmore, D. F., Lites, B. W., Tomczyk, S., Skumanich,A. P. et al. 1992, in Polarization analysis and measurement, eds. D. H. Goldstein \& R. A. Chipman. Proceedings of the SPIE, 1746, 22

López Ariste, A., Rayrole, J., \& Semel, M. 2000, A\&A Suppl., 142, 137

Martínez González, M. J., Collados, M., \& Ruiz Cobo, B., 2006, A\&A, 456, 1159

Martínez Pillet, V., Bonet, J. A., Collados, M., Jochum, L. et al. 2004, in Optical, Infrared, and Millimeter Space Telescopes, ed. J. C. Mather. Proceedings of the SPIE, Vol. 5487, 1152

Martínez Pillet, V., Collados, M., Sánchez Almeida, J., González, V. et al 1999, in High Resolution Solar Physics: Theory, Observations, and Techniques, eds. T. R. Rimmele, K. S. Balasubramaniam, \& R. R. Radick, ASP Conf. Ser. 183, 264.

Müller, D. A. N., Schlichenmaier, R., Fritz, G., \& Beck, C., 2006, A\&A, 460, 925

Schlichenmaier, R., Müller, D. A. N., Steiner, O., \& Stix, M. 2002, A\&A, 381, L77

Semel, M. 2003, A\&A, 401, 1

Socas-Navarro, H., Elmore, D. F., Pietarila, A., Darnell, A. et al. 2006, Solar Phys. 235, 55

Solanki, S. K., Lagg, A., Woch, J., Krupp, N., \& Collados, M. 2003, Nature, 425, 692

Stenflo, J. O., Keller, C. U., \& Povel, H. P. 1992, in LEST Foundation Technical Report 54, eds. O. Engvold \& $\varnothing$. Hauge

del Toro Iniesta, J. C. \& Collados, M. 2000, Appl. Opt. 39, 1637 


\title{
Dynamics of the quiet photosphere and its magnetic field under high spatial resolution
}

\author{
K. G. Puschmann ${ }^{1,}{ }^{*}$ F. Kneer ${ }^{1}$, and I. Domínguez Cerdeña ${ }^{2}$ \\ ${ }^{1}$ Institut für Astrophysik, Göttingen, Germany \\ ${ }^{2}$ Instituto de Astrofísica de Canarias, La Laguna, Spain \\ *Email: kgp@astro.physik.uni-goettingen.de
}

\begin{abstract}
An excellent $1 \mathrm{~h} 10 \mathrm{~min}$ time series of a quiet solar region near disk centre was acquired on $4^{\text {th }}$ of May 2006 at the German Vacuum Tower Telescope. Using the new "Göttingen" FabryPerot interferometer together with the Kiepenheuer Adaptive Optics system spectropolarimetric images were obtained, scanning the $\mathrm{Fe}_{\text {I }} 5247 \AA$ and $\mathrm{Fe}_{\text {I }} 5250 \AA$ lines with a temporal cadence of $17 \mathrm{~s}$ each. Thanks to speckle reconstruction methods the achieved spatial resolution of the obtained intensity, velocity and magnetic field maps is $\sim 0 .{ }^{\prime \prime} 3$. First results on the dynamics of the quiet photosphere and magnetic fields reveal strengthening and weakening of the polarisation signal in interaction to granular convection processes indicating advection and diffusion of magnetic flux at smallest scales. Field structures, very elongated along intergranular lanes, show high dynamics, combine and split, and leave strong fingerprints at high photospheric layers where related temperatures fluctuate. Recent numerical MHD simulations come close to our findings. Possible indications of magnetic flux annihilation, flux tube evacuation or signs of upward-propagating shock fronts are illustrated.
\end{abstract}

\section{Introduction}

Small-scale magnetic fields in the quiet solar photosphere and their interaction with the ionised gas may play a key role in the heating of the chromosphere and corona. The total magnetic flux in quiet solar areas is larger than that in all active regions together. Thus, the quiet Sun may play a significant role in the physical processes governing the global solar magnetism (e.g., Domínguez Cerdeña et al. 2006, and references therein). Most of the flux at photospheric level is assigned to the interior of network cells (Stenflo 1994; Trujillo Bueno et al. 2004), whose boundaries harbour kilo-Gauss fields (Stenflo 1973). It is still under discussion if internetwork magnetism is dominated also by kilo-Gauss fields (Domínguez Cerdeña et al. 2003, 2006) or by weaker fields in the $300 \mathrm{G}$ range (Trujillo Bueno et al. 2004; Khomenko et al. 2005; Martínez González et al. 2006), located mainly in the intergranular areas, and even weaker fields $(\sim 10 \mathrm{G})$ in the granules proper (Trujillo Bueno et al. 2004). The topology and the scale of the variation of quiet Sun magnetic fields are not yet clear. There is evidence for the existence of mixed polarities (Sánchez Almeida et al. 1996; Sigwarth et al. 1999; Sánchez Almeida \& Lites 2000; Lites 2002; Khomenko et al. 2003). A further question is whether internetwork fields are very entangled, "turbulent", and thus only detectable by sophisticated Hanle diagnostics of polarised light, or, at sufficient angular and temporal resolution, the Zeeman effect can be used to reveal most of the magnetic flux. 


\section{Observations and data processing}

On $4^{\text {th }}$ May 2006, an excellent $1 \mathrm{~h} 10$ min time series of a quiet solar region near disk centre has been observed using the upgraded "Göttingen" Fabry-Perot spectrometer (Puschmann et al. 2006) at the German Vacuum Tower Telescope, Observatorio del Teide, Tenerife. The observations have been supported by the Kiepenheuer Adaptive Optics System (KAOS). The magnetically sensitive Fe I $5247 \AA$ and $\mathrm{Fe}_{\mathrm{I}} 5250 \AA$ lines have been scanned quasi simultaneously with a temporal cadence of $17 \mathrm{~s}$ each, taking short exposures of $3 \mathrm{~ms}$. The spectral resolution was $\sim 27 \mathrm{~m} \AA$. Thanks to speckle reconstruction methods (Puschmann \& Sailer 2006; Krieg et al. 1998) the broadband and narrowband images possess an angular resolution of $0 .{ }^{\prime \prime} 27$ and $0 .{ }^{\prime \prime} 3$, respectively. The reconstructed images of left and right circularly polarised light were carefully co-aligned to sub-pixel accuracy. From these, at each pixel in the field of view (FOV), Stokes I and V profiles of the spectral lines were obtained. The flux densities $\left(\mathrm{B}_{\text {eff }}\right.$; line-of-sight components averaged over the resolution element) were determined in the weak-field approximation (Landi Degl'Innocenti 1992). Velocities $\left(V_{c o g}\right)$ were measured from the local shifts of the centre of gravity of the absorption lines. Additionally, intensities at the local line minima $\left(\mathrm{I}_{\mathrm{lc}}\right)$ were determined. A subsonic filter has been applied to the time sequence, eliminating features with phase velocities $>4 \mathrm{~km} / \mathrm{s}$. Apart from their Landé factors, the two lines have nearly identical properties. Thus we treat the results below as stemming from one line. The sensitivity of the magnetic field measurements at this resolution is $\left|\mathrm{B}_{\mathrm{eff}}\right|=30 \mathrm{G}$ (corresponding to a $2 \sigma$ significance).

\section{Results}

In this contribution we present first results on small-scale magnetic field evolution in the quiet solar photosphere, focusing on three different subfields of the total observed area presented in Puschmann et al. (2007). Figures 1 and 2 demonstrate the rich action of convection on magnetic fields of opposite polarity in areas of network boundary and internetwork, respectively. From the upper to the lower rows, at a cadence of $4 \mathrm{~min} 32 \mathrm{~s}$ ( $3 \mathrm{~min} 58 \mathrm{~s}$ ), broadband images $(\mathrm{BB})$ with the scaling relative to average intensity, line minimum intensities $\left(\mathrm{I}_{\mathrm{lc}}\right)$ relative to the average continuum intensity, line of sight velocities ( $\mathrm{V}_{\mathrm{cog}}$; centre of gravity), and magnetic flux densities ( $B_{\text {eff }}$; weak-field approximation), are presented at 6 different time steps.

The good seeing conditions during the observations (Fried-parameter $r_{0}$ up to $38 \mathrm{~cm}$ ) are reflected in the high $\Delta \mathrm{I}_{\mathrm{rms}}(11 \%)$ of the reconstructed broadband images. At the high spatial resolution achieved, the granules appear eroded. We note the complex structure of the intergranular lanes, indicating cool areas related to strong downflows sometimes located in the vicinity of magnetic features. The measured magnetic flux densities amount up to $800 \mathrm{G}$ in areas with network boundary and between $50 \mathrm{G}$ and $180 \mathrm{G}$ in the internetwork. Magnetic fields are almost exclusively detected in intergranular areas. Some of them are present, in both network and internetwork areas, along the entire time sequence of $1 \mathrm{~h} 10 \mathrm{~min}$. A good, although not perfect, correlation between intergranular bright points and magnetic fields is revealed when comparing the continuum and line centre images with the magnetic field maps. While some of the strong fields in the magnetograms may be identified as tiny brightenings at the bottom of the solar atmosphere, the magnetic fields leave strong finger- 


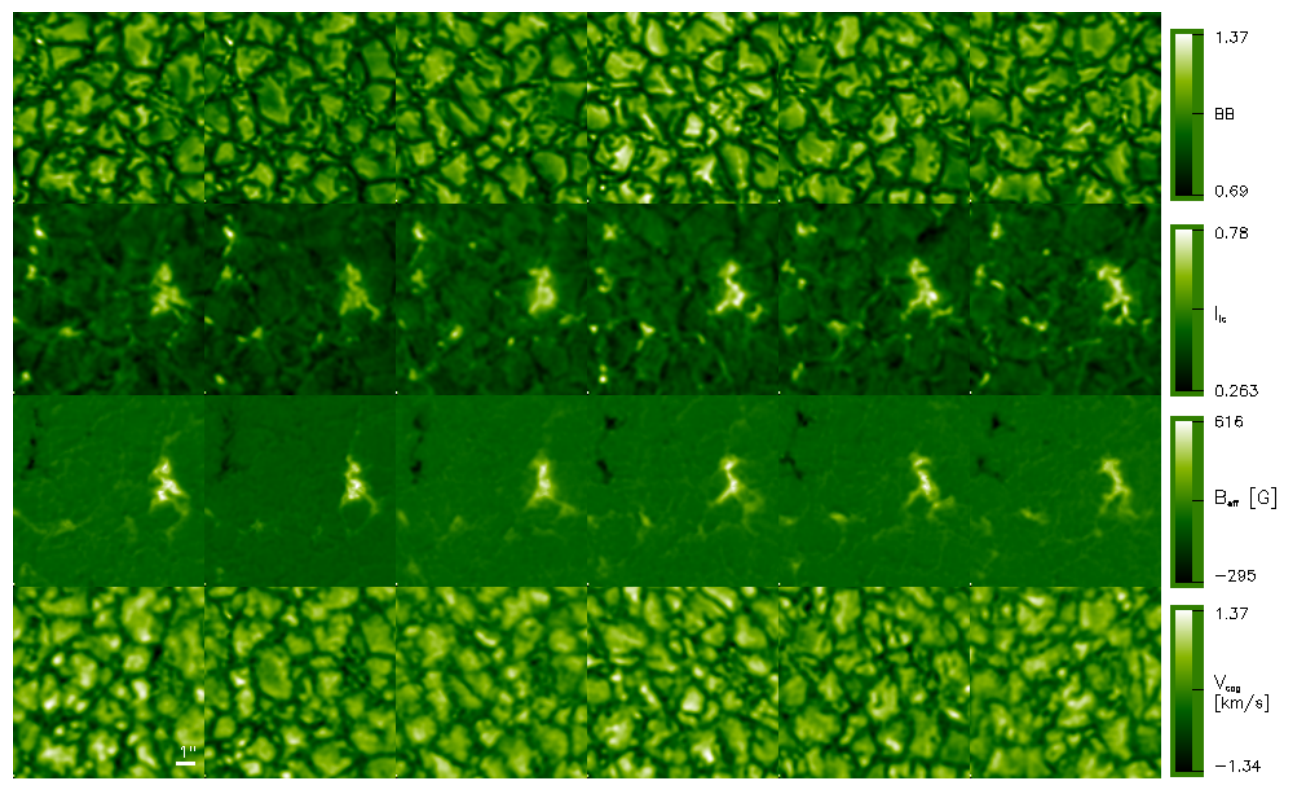

Figure 1. Time sequence of an area with network boundary magnetic fields of opposite polarity. From top to bottom: Broadband intensity $(\mathrm{BB})$, line centre intensity $\left(\mathrm{I}_{\mathrm{lc}}\right)$, magnetic flux density $\left(\mathrm{B}_{\text {eff }}\right)$ and velocity $\left(\mathrm{V}_{\mathrm{cog}}\right)$ at 6 different time steps; $\Delta \mathrm{t}=4 \mathrm{~min} 32 \mathrm{~s}$; FOV: $11^{\prime \prime} \times 11^{\prime \prime}$. Under the high spatial resolution achieved $\left(<0 .{ }^{\prime \prime} 3\right)$ granules appear eroded. Intergranular lanes show a complex structure, indicating cool areas related to strong downflows sometimes located in the vicinity of magnetic features. Network boundary magnetic fields reveal strong dynamics in interaction with granular convection, changing their fine structure at time scales $<17 \mathrm{~s}$. During the entire sequence the flux densities reach values of up to $800 \mathrm{G}$.

prints at high photospheric layers. In both network boundary and internetwork, elongated faint streaks visible in the line centre images are partially related to magnetic features, show strong dynamics at short time scales, and are likely a result of the interaction of small-scale magnetic fields with the ionised gas. Occasionally, the streaks dissolve into faint knots or faint chains.

Box 1 of Fig. 2 reveals a quite common scenario of possible flux dissipation and readvection: Following the temporal evolution of a negative polarity feature sometimes related to tiny brightenings in the line centre and broadband images, the magnetic flux undergoes strong interaction processes with convection, where the magnetic flux is pushed around, gets compressed, dissolves or disappears on short time scales, afterwards reassembling and reappearing nearby.

Box 2 of Fig. 2 shows possible indications for magnetic flux annihilation and related heat transfer to higher atmospheric layers. In the fifth frame one finds negative polarity close to strong positive polarity fields, while in the last frame, the negative polarity has disappeared and the positive field has weakened, related line centre intensities appear enhanced.

As a final example, we present in Fig. 3 a time sequence of magnetograms $\left(B_{\text {eff }}\right)$ superposed onto broadband intensity and line centre images for a conspicuous magnetic structure 


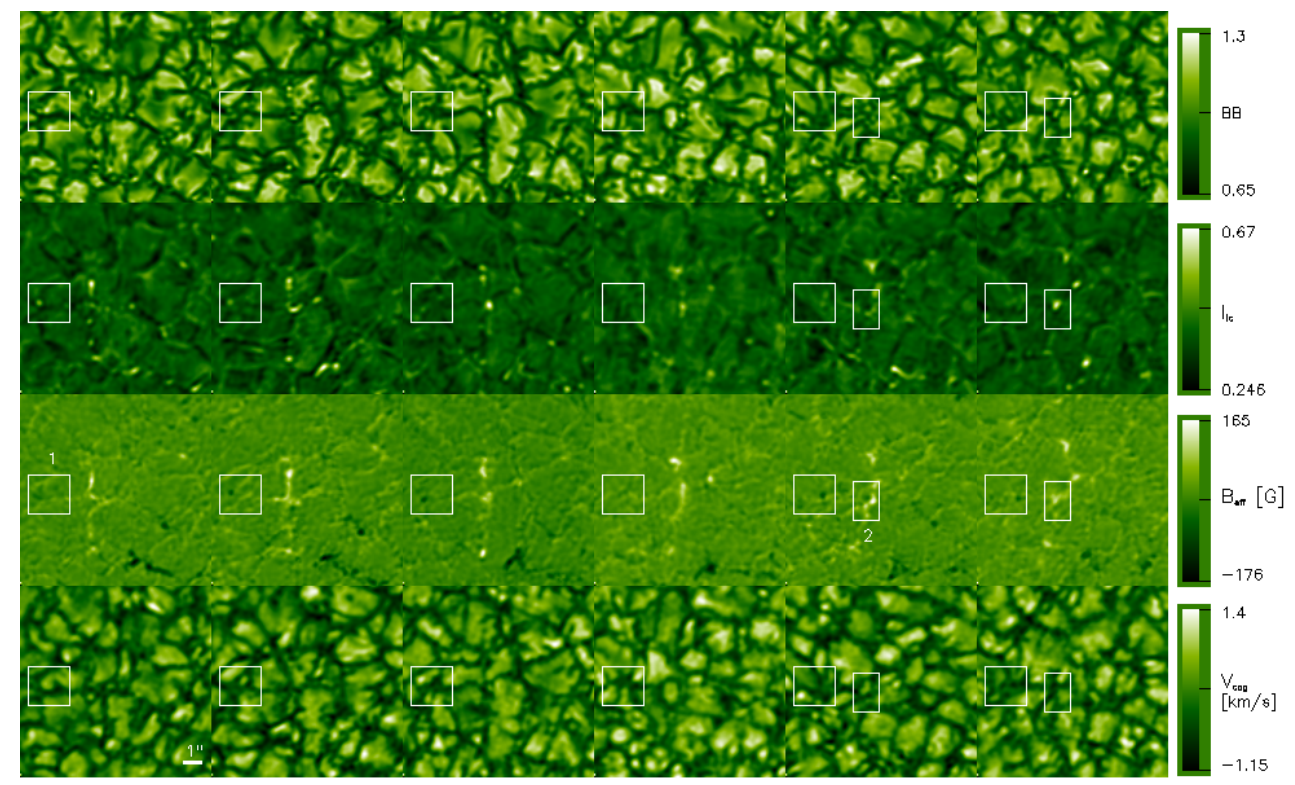

Figure 2. Time sequence of magneto-convection in quiet Sun at 6 different time steps; $\Delta t=3$ min $58 \mathrm{~s}$. FOV: $11^{\prime \prime} \times 11^{\prime \prime}$. From top to bottom: Broadband intensity, line minimum intensity, magnetic flux density, and velocity. Magnetic field structures of opposite polarity related to the internetwork appear as faint point-like structures in the broadband images and as brilliant small-scale points in the line centre images, sometimes showing faint tails. As also observed in the network boundary, faint streaks visible in the line centre images, partially related to magnetic structures, show strong dynamics at short time scales (sometimes dissolving into faint knots) and are likely an indication of magnetic field - ionised gas interaction at smallest scales. Box 1 contains negative-polarity flux, diffused and re-advected during the sequence. Box 2 gives hints on flux annihilation. Negative-polarity flux close to positive polarity has disappeared in the last frame, while related line centre intensities are enhanced.

associated with an intergranular bright point. After $3 \mathrm{~m} 58 \mathrm{~s}$ from the beginning of the sequence, the intensity related to this magnetic feature increases in both broadband and line centre images whereas the apparent magnetic signal decreases drastically. After 6 min $14 \mathrm{~s}$, the magnetic flux density grows to values larger than $100 \mathrm{G}$ while the related intensity fades. The bright point and the magnetic structure show again a clear correlation. Such a behaviour was predicted earlier by Sánchez Almeida (2000) and can possibly be explained by an evacuation of magnetic flux tubes during field enhancement. The remaining gas is heated, the Fe I lines weaken, and the magnetic signal is substantially reduced. When the tube has expanded and the field has decreased the measured magnetic signal increases again. This observation can also be explained by an upward-moving shock front within the flux tube as predicted by Steiner et al. (1998), leading to a distinct signature in Stokes V. During the passage of the shock front through the region of line formation, the corresponding measured Stokes V signal fades and augments again due to a superposition of two distinct components originating in the pre-shock and the post-shock regions, while intensities show the opposite behaviour. 

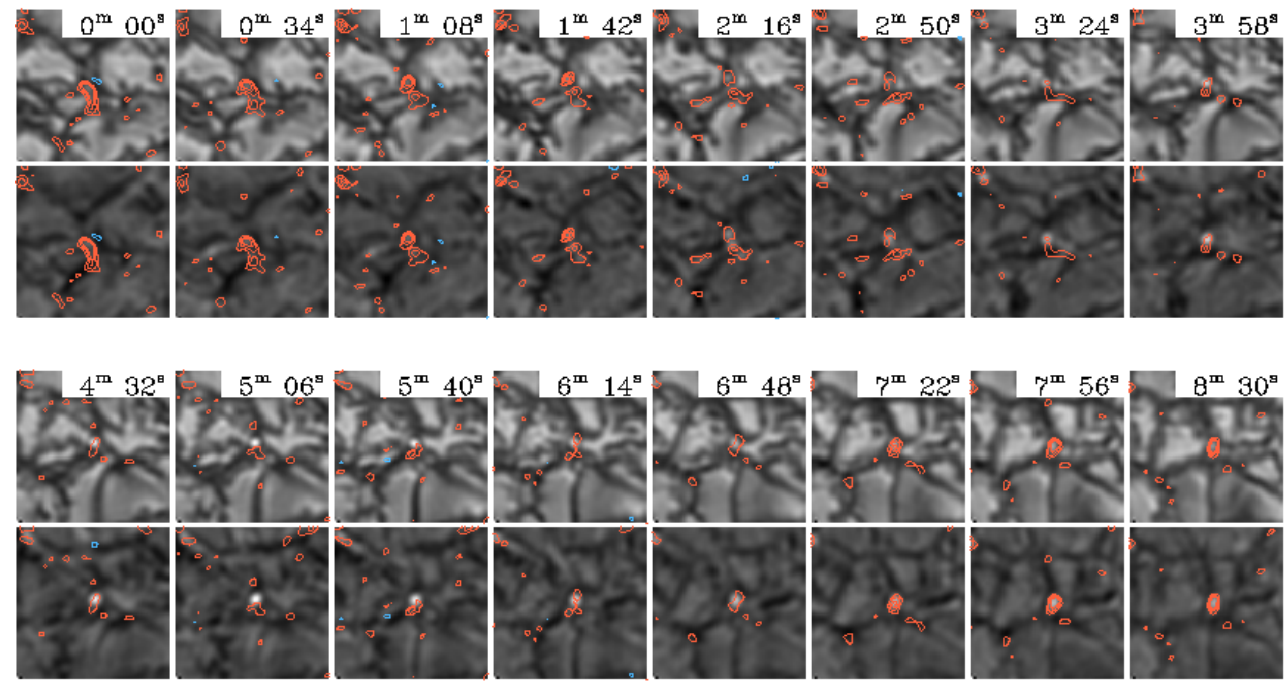

Figure 3. Reduction of magnetic signal due to flux tube evacuation or upward-propagating shock fronts. Time sequence of magnetograms superposed on broadband intensity (upper panels) and line centre intensity (lower panels) for a conspicuous magnetic structure associated with an intergranular bright point. FOV: $5^{\prime \prime} \times 5^{\prime \prime}$; time step: $34 \mathrm{sec}$. The contours belong to $\left|B_{\text {eff }}\right|=40,70,100 \mathrm{G}$. Red and blue contours (visible in the electronic version) distinguish between the two magnetic polarities. See text for a detailed description.

\section{Conclusion and outlook}

A first inspection of broadband, line centre intensity, velocity and magnetic field maps obtained from a $1 \mathrm{~h} 10 \mathrm{~min}$ time series of spectropolarimetric images of excellent quality reveals the interaction of magnetic fields with granular convection at smallest scales, bearing on the structure and dynamics of the solar atmosphere as a whole. The temporal evolution of the physical quantities resembles strongly those found in numerical simulations of magnetoconvection (Stein \& Nordlund 1996; Schaffenberger et al. 2005; Vögler et al. 2005), although one has to be careful by making direct comparisons between observations and simulations. Magnetic features are almost exclusively located in the intergranular lanes, showing opposite polarities and related flux densities of up to $800 \mathrm{G}$ in areas with network boundary and up to $180 \mathrm{G}$ in the internetwork. Very likely, our measurements give lower estimates due to the limited spatial resolution. Assuming area fillings with magnetic flux of 15-20\% the true flux densities could be partially in the kilo-Gauss range. Although the fine structure of smallscale magnetic flux concentrations changes on very short time scales, single flux elements, in both network and - noteworthy - internetwork areas, persist over the full duration of our time sequence in agreement with Rouppe van der Voort et al. (2005) pointing at a deep anchoring of at least some of the flux elements, even though numerical simulations do not seem to confirm this conjecture (Steiner 2007). Magnetic flux undergoes strong interaction processes with convection, in which the magnetic flux is pushed around, gets compressed, dissolves or 
disappears on short time scales, afterwards reassembling and reappearing nearby. Following the temporal evolution of internetwork fields at fast cadence $(\sim 17 \mathrm{~s})$ and small scales $\left(0 .{ }^{\prime \prime} 3\right)$ we find indications of flux annihilation and possibly upward-propagating shock fronts, i.e. of mechanisms to heat the upper layers of the solar atmosphere. Line centre images reveal vigorous processes at high photospheric layers related to magnetic field dynamics. More detailed descriptions and analyses will be presented in forthcoming publications. Our findings encourage to improve on the side of resolution and polarimetric sensitivity, and lend support to the efforts to apply the Zeeman effect to very high angular resolution observation with large solar telescopes, in addition to Hanle diagnostics.

Acknowledgements. We thank J. Sánchez Almeida, O. Steiner, and J. Trujillio Bueno for many fruitful discussions. KGP acknowledges support by the Deutsche Forschungsgemeinschaft through grant KN 152/29-1,2. The Vacuum Tower Telescope is operated by the Kiepenheuer-Institut für Sonnenphysik, Freiburg, at the Spanish Observatorio del Teide of the Instituto de Astrofísica de Canarias.

\section{References}

Domínguez Cerdeña, I., Sánchez Almeida, J., \& Kneer, F. 2003, A\&A, 407, 741

Domínguez Cerdeña, I., Sánchez Almeida, J., \& Kneer, F. 2006, ApJ, 646, 1421

Khomenko, E. V., Collados, M., Solanki, S. K., Lagg, A., \& Trujillo Bueno, J. 2003, A\&A, 408, 1115 Khomenko, E. V., et al. 2005, A\&A, 436, L27

Krieg, J., Kneer, F., Koschinsky, M., Ritter, C., \& Stark, J. L. 1998, in A Crossroads for European Solar and Heliospheric Physics, ed. R. H. Harris, F. Moreno Insertis, \& E. Priest, ESA SP-417, 317 Landi Degl'Innocenti, E. 1992, in Solar Observations: Techniques and Interpretation, ed. F. Sánchez, M. Collados, \& M. Vázquez, Cambridge University Press, Cambridge

Lites, B. W. 2002, ApJ, 573, 431

Martínez González, M. J., Collados, M., \& Ruiz Cobo, B. 2006, A\&A, 456, 1159

Puschmann, K. G. \& Sailer, M. J. 2006, A\&A, 454, 1011

Puschmann, K. G., Kneer, F., Seelemann, T., \& Wittmann, A. D. 2006, A\&A, 451, 1151

Puschmann, K. G., Kneer, F., Nicklas, H., \& Wittmann, A. D 2007, (this volume)

Rouppe van der Voort, L. H. M., Hansteen, V. H., Carlsson, M., et al. 2005, A\&A, 435, 327

Sánchez Almeida, J. 2000, ApJ, 544, 1135

Sánchez Almeida, J. \& Lites, B. W. 2000, ApJ, 532, 1215

Sánchez Almeida, J., Landi Degl'Innocenti, E., Martínez Pillet, V., \& Lites, B. W. 1996, ApJ, 466, 537

Schaffenberger, W., Wedemeyer-Boehm, S., Steiner, O., \& Freytag, B. 2005, in Chromospheric and

Coronal Magnetic Fields, ed. D. E. Innes, A. Lagg, \& S. K. Solanki, ESA SP-596, p.65.1

Sigwarth, M., Balasubramaniam, K. S., Knölker, M., \& Schmidt, W. 1999, A\&A, 349, 941

Stein, R. F. \& Nordlund, Å. 1996, ApJ, 642, 1246

Steiner, O. 2007, (this volume)

Steiner, O., Grossmann-Doerth, U., Knölker, M., \& Schüssler, M. 1998, ApJ, 495, 486

Stenflo, J. O. 1973, Solar Phys. 32, 41

Stenflo, J. O. 1994, Solar Magnetic Fields: Polarized Radiation Diagnostics, Kluwer Academic Publishers, Dordrecht

Trujillo Bueno, J., Shchukina, N., \& Asensio Ramos, A. 2004, Nature, 430, 326

Vögler, A. et al. 2005, A\&A, 429, 335 
Modern Solar Facilities - Advanced Solar Science, 157-160

F. Kneer, K. G. Puschmann, A. D. Wittmann (eds.)

(C) Universitätsverlag Göttingen 2007

\title{
Internetwork magnetic fields
}

\author{
M. J. Martínez González ${ }^{1, *}$, M. Collados ${ }^{2}$, and B. Ruiz Cobo ${ }^{2}$ \\ ${ }^{1}$ Laboratoire de l'Étude du Rayonnement et de la Matière en Astrophysique, Observatoire \\ de Paris-Section de Meudon, Meudon, France \\ ${ }^{2}$ Instituto de Astrofísica de Canarias, La Laguna, Spain \\ *Email: Marian.Martinez@ obspm.fr
}

\begin{abstract}
Spectropolarimetric observations of the internetwork obtained in the $1.56 \mu \mathrm{m} \mathrm{Fe}$ I doublet are used to calculate the distributions of magnetic field strength, filling factor and magnetic flux density at different positions on the solar surface. We go one step further and describe what the observations show about the three-dimensional structure of the magnetic fields at the internetwork quiet Sun.
\end{abstract}

\section{Our knowledge of the magnetic field in the internetwork ...}

... has evolved rapidly in recent years. Nowadays, concerning the Zeeman effect, the most reliable works of internetwork magnetic fields deal with the infrared Fe I doublet situated at $1.56 \mu \mathrm{m}$ (Khomenko et al. 2003; Sánchez Almeida et al. 2003; Domínguez Cerdeña et al. 2006; Martínez González et al. 2007) or with spectral lines with hyperfine structure. López Ariste et al. (2006) use the visible Mn I at $553.7 \mathrm{~nm}$ and Asensio Ramos et al. (2007) analyze the Mn I line situated at $1.53 \mu \mathrm{m}$, in the near infrared part of the spectrum. All these works are based on spectropolarimetric observations at a spatial resolution of the order of $1^{\prime \prime}$. They agree on the sub-kG nature of the magnetic features. They also find that these occupy a very small portion of the resolution element, between $1 \%$ and $10 \%$. The rest of the pixel seems to be field free, at least concerning the Zeeman data. However, recently, some works (Faurobert et al. 2001; Trujillo Bueno et al. 2004) present the Hanle effect as a very promising diagnostic technique to study the turbulent component of the photospheric magnetic field that would be undetectable by the Zeeman effect due to cancellations of circular polarization signals.

In this contribution, we present the results of a study of the distribution of field strengths and the topology of magnetic features at these low flux internetwork regions. To this aim we use spectropolarimetric observations taken at different positions of the solar disc. On July 2000 we recorded the four Stokes parameters of the Fe I $1.56 \mu \mathrm{m}$ lines scanning the solar photosphere at disc centre and at positions $\mu=0.88,0.4$ and 0.28 (with $\mu$ the cosine of the heliocentric angle). The same spectral lines were used for an observing run at disc centre on August 2003. All data sets have a spatial resolution of the order of $1^{\prime \prime}$. The last set has the lowest noise level in polarization: $5 \times 10^{-5} \mathrm{I}_{\mathrm{c}}, \mathrm{I}_{\mathrm{c}}$ being the continuum intensity. It was achieved using a Principal Components Analysis (PCA) procedure to filter the data. The noise level in the data from the year 2000, also after denoising with PCA, is $7 \times 10^{-5} \mathrm{I}_{\mathrm{c}}$ for the observation at disc centre, $9 \times 10^{-5} \mathrm{I}_{\mathrm{c}}$ for the set at $\mu=0.88$ and $10^{-4} \mathrm{I}_{\mathrm{c}}$ for both scans at $\mu=0.4$ and $\mu=0.28$. 

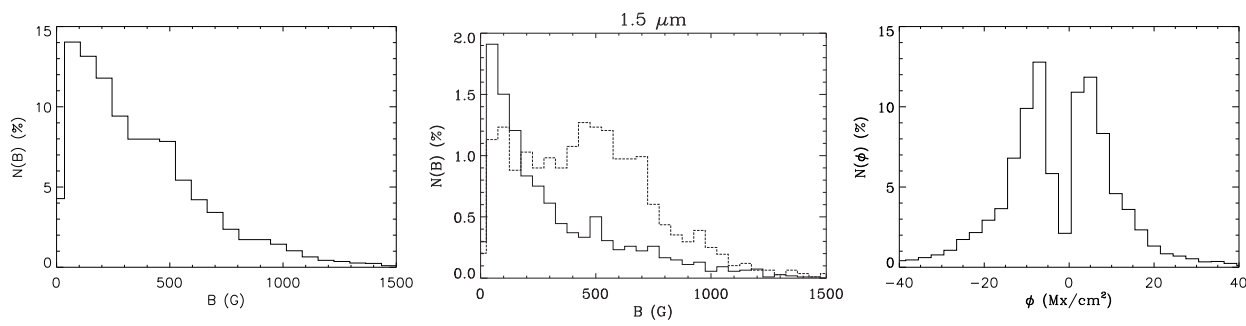

Figure 1. Magnetic field strength distribution at disc centre (left panel) and for granular (solid) and intergranular (dotted) regions (centre panel). Magnetic flux density distribution at disc centre (right panel), average values within each pixel.

In order to analyze the data we selected only those points where the Stokes $V$ signal was regular (presented only two lobes) and showed a Stokes $V$ amplitude above $5 \times 10^{-4} \mathrm{I}_{\mathrm{c}}$. With these requirements, $29 \%$ of the observed profiles in the 2003 data were selected, while $48 \%$, $47 \%, 59 \%$ and $64 \%$ were selected in the 2000 sets at disc centre for $\mu=0.88,0.4$ and 0.28 , respectively. We inverted the full Stokes vector using the $\operatorname{SIR}^{1}$ code under the hypothesis of a magnetic atmosphere and a field free one mixed in the resolution element.

The spatial distribution of the magnetic field strength at the internetwork region observed in 2003 is plotted in the left panel of Fig. 1. In accordance with previous works, the field strengths are in the range of a few hundred Gauss. The histogram reveals two different behaviours: the lowest values have a steep fall towards higher field strengths while there is an accumulation around $500 \mathrm{G}$ that is approximately the equipartition field in the photosphere. Kilogauss field strengths are very rare. A similar analysis has been done after separating granules from intergranules in the field of view, using a criterion based on the continuum intensity. Granules are defined as those areas where the continuum intensity is higher than the mean and intergranules those where it is lower. In the centre panel of Fig. 1 we can see how the magnetic field distribution in intergranules is centered around the equipartition field. In granules, smaller strengths are found and the distribution follows an exponential decrease. These two different behaviours, already observed in the total distribution, can be due to a real physical mechanism since they are associated to two distinct areas of the solar photosphere. In the right panel of Fig. 1, we show the distribution of the magnetic flux density. Both polarities cancel out almost perfectly: the mean value of the magnetic flux density is -0.075 $\mathrm{Mx} / \mathrm{cm}^{2}$. The unsigned mean magnetic flux density turns out to be $5 \mathrm{Mx} / \mathrm{cm}^{2}$, a value that is around 20 times smaller than what is typically found in network regions.

The filling factors of these sub-kG structures are rather small. The majority occupy only $2 \%$ of the resolution element, as seen in the distribution plotted in the left panel of Fig. 2. However, as shown in the right panel of the same figure, this small filling factor corresponds to the higher values of the field strengths. The smallest values (below 300-350 G) have larger filling factors. In these cases, the weak field regime applies and only the magnetic flux can be determined. Nonetheless, it can be stated that the weakest field strengths occupy larger areas than the strongest ones. They seem to represent a more diffuse component, and perhaps we are starting to detect some of the turbulent magnetic fields to what the Hanle effect is

\footnotetext{
${ }^{1}$ Stokes Inversion based on Response functions
} 

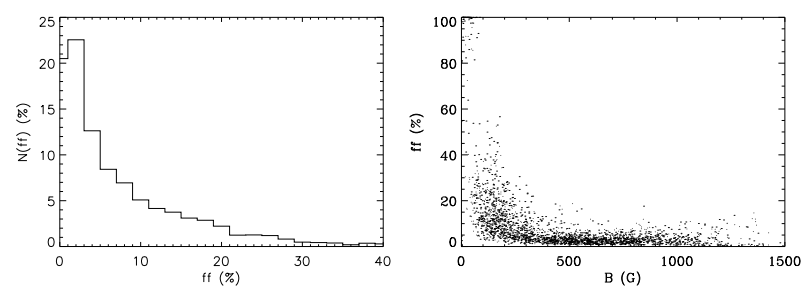

Figure 2. Magnetic filling factor distribution at disc centre (left panel) and magnetic field strength as a function of the filling factor (right panel).
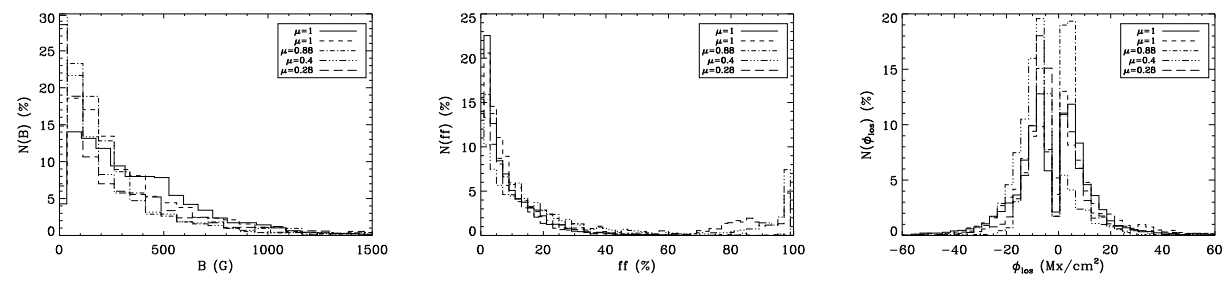

Figure 3. Magnetic field strength distribution at the 4 studied positions of the solar surface (left panel). Magnetic filling factor (central panel) and magnetic flux density (right panel) distributions at the same positions.

sensitive.

The same analysis has been done for the other data sets, since very little is known of the variation of the internetwork magnetism over the solar surface. The distributions of magnetic field strength, filling factor and magnetic flux density retrieved from all these observations are presented in Fig. 3. As can be seen, none of these quantities seem to vary with the position on the Sun. Only the observation at $\mu=0.4$ has a dominant negative polarity, which can be due to a contamination of a small network patch, even if we verified that no magnetic activity is detected in the simultaneously recorded $\mathrm{Ca} \mathrm{K}$ image. For the 2000 data, the mean magnetic flux density is $0.24,0.75,-3.47$ and $-0.74 \mathrm{Mx} / \mathrm{cm}^{2}$ for the observations at $\mu=1,0.88,0.4$ and 0.28 respectively, while the unsigned flux density is $6,4,5$ and $6 \mathrm{Mx} / \mathrm{cm}^{2}$, respectively. Consequently, the magnetism of the internetwork seems not to change over the solar surface, at least in the positions we have studied. Our observations were taken in North and East directions, so more observations covering the rest of the solar surface are needed in order to carefully study the variation of the magnetic properties of the internetwork at different positions in the Sun.

With the amount of information that the spectropolarimetric observations provide, we can go one step further and try to investigate also the topology of the magnetic structures in the internetwork. At disc centre observations, a particular feature is rather common: linear polarization signals are found between two circular polarization signals with opposite polarities. This may be an indication of loop-like structures. Away from disc centre, the identification of these loops becomes extremely difficult due to perspective and transport effects. For this reason, we have only studied the two observations at disc centre in the 2000 data. We have found 6 clear cases of such loops in the map at $\mu=1$ and 5 in the map at $\mu=0.88$. All have the same characteristics but we only detail the study of one of them.

The azimuth of the magnetic field vector recovered from the inversions is shown in the 


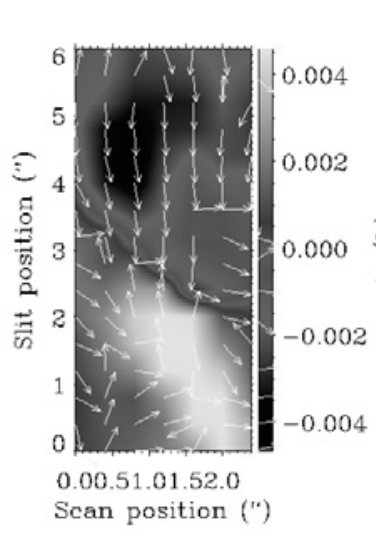

0.00 .51 .01 .52 .0
Scan position (")

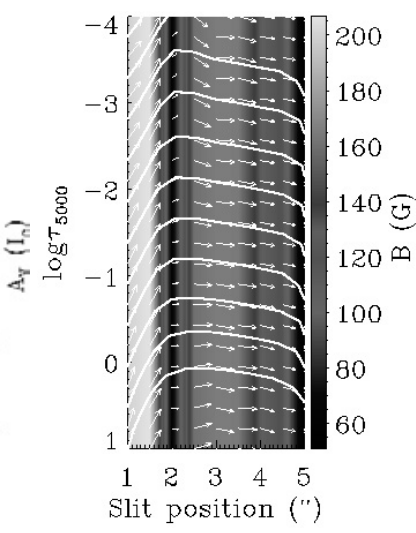

Figure 4. Left: map of the amplitude of circular polarization of the loop selected as an example and, overplotted with arrows, the azimuth of the magnetic field vector. Right: magnetic field strength (background) along the line joining the opposite polarities and along the height in the atmosphere. Overplotted, the magnetic field lines and the inclination of the field vector (arrows).

left panel of Fig. 4, overplotted to the Stokes $V$ amplitude for the loop that we have selected, as illustration. It must be noted that this azimuth has a $180^{\circ}$ ambiguity. Independently of this indetermination, the magnetic field vector is directed along the line that joins the two opposite polarities. In the right panel of Fig. 4, the magnetic field lines computed along the line that connects the two polarities are plotted, together with their variation with height. In this case the loop structure is evident: the magnetic field is almost vertical at the footpoints and turns out to be almost horizontal in the transition between both polarities. It must be noted that, if the opposite sense of the magnetic field inclination had been chosen, a dip would have been obtained, instead of a loop.

From our analysis of spectropolarimetric observations in the near infrared, the weak polarization signals measured with the Zeeman effect at internetwork regions come from organized structures in the form of loops or dips. At disc centre, these magnetized structures have field strengths well below $\mathrm{kG}$ and fill only a small fraction of the resolution element, around $2 \%$ in the case of field strengths higher than 300-350 G. Lower magnetic fields have higher occupation fractions, maybe corresponding to the top of the iceberg of the turbulent component of the photospheric magnetism to which the Hanle effect is also sensitive. The magnetic flux density at these regions is $5 \mathrm{Mx} / \mathrm{cm}^{2}$ and both positive and negative polarities of the magnetic field cancel perfectly in the whole analyzed field of view. These magnetic properties of internetwork regions do not seem to depend on the position on the solar surface, at least at the light of our datasets.

\section{References}

Asensio Ramos, A., Martínez González, M. J., López Ariste, A., Trujillo Bueno, J., \& Collados, M. 2007, ApJ, in press

Domínguez Cerdeña, I., Sánchez Almeida, J., \& Kneer, F. 2006, ApJ, 646, 1421

Faurobert, M., Arnaud, J., Vigneau, J., \& Frisch, H. 2001, A\&A, 378, 267

Khomenko, E. V., Collados, M., Solanki, S. K., Lagg, A., \& Trujillo Bueno, J. 2003, A\&A, 408, 1115

López Ariste, A., Tomczyk, S., \& Casini, R. 2006, A\&A, 454, 663

Martínez González, M. J., Collados, M., Ruiz Cobo, B., \& Beck, C. 2007, A\&A, in preparation

Sánchez Almeida, J., Domínguez Cerdeña, I., \& Kneer, F. 2003, ApJ, 597, L177

Trujillo Bueno, J., Shchukina, N., \& Asensio Ramos, A. 2004, Nature, 430, 326 
Modern Solar Facilities - Advanced Solar Science, 161-164

F. Kneer, K. G. Puschmann, A. D. Wittmann (eds.)

(C) Universitätsverlag Göttingen 2007

\title{
High-resolution CN spectroscopy of small-scale solar magnetic features
}

\author{
V. V. Zakharov*, A. Gandorfer, and S. K. Solanki \\ Max-Planck-Institut für Sonnensystemforschung, Katlenburg-Lindau, Germany \\ *Email: zakharov@mps.mpg.de
}

\begin{abstract}
High-resolution spectroscopic observations of small-scale magnetic elements in the solar photosphere were carried out in the spectral region 387.5-388.4 nm with the 1-m Swedish Solar Telescope (SST). This part of the spectrum covers not only the violet CN band-head, but also contains some lines of the $\mathrm{CH}$ molecule. The analysis of the line-core intensity contrasts of the $\mathrm{CN}$ and $\mathrm{CH}$ lines in bright points (BPs) yielded that on average the BPs appear brighter, thus providing a higher rms contrast, in the $\mathrm{CN}$ than in the $\mathrm{CH}$ lines in the same spectral band.
\end{abstract}

\section{Observations}

a)

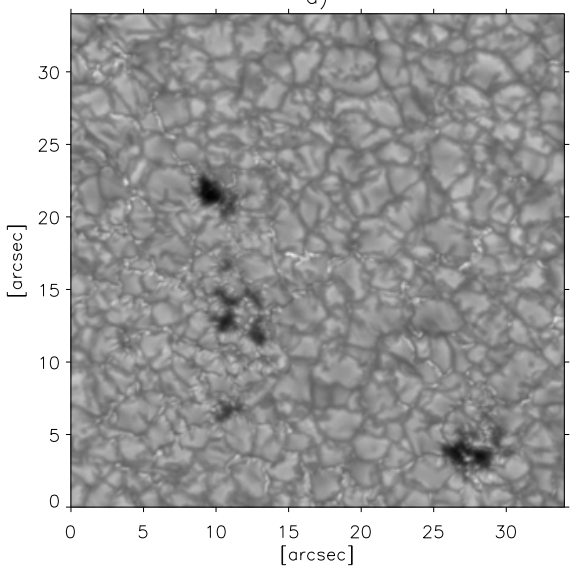

b)

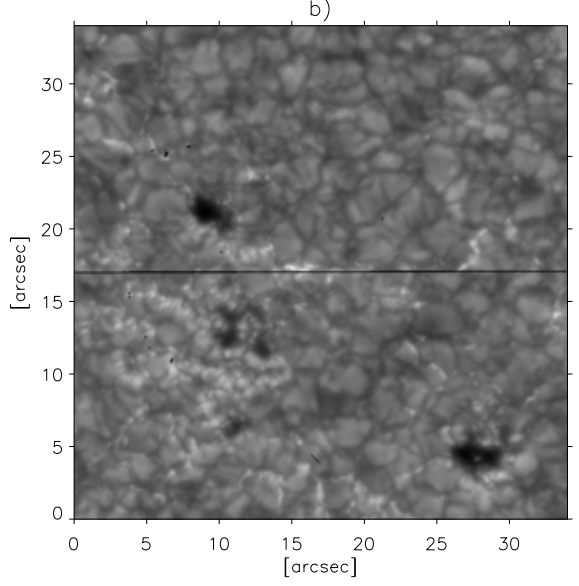

Figure 1. Active region NOAA0753 observed near $\mu=0.95$. The left panel (a) shows a high resolution red "continuum" image (at $\lambda=705.7 \mathrm{~nm}$ ) restored with MFBD. The right panel (b) represents an example of a slit-jaw image taken in the violet $\mathrm{CN}$ band.

The spectroscopic dataset presented here was collected with the TRIPPEL (TRI-Port Polarimetric Echelle-Littrow) spectrograph (Kiselman et al. 2007) installed at the SST. It was used simultaneously with the tip-tilt and the adaptive optics (AO) system and has a slit width of $25 \mu \mathrm{m}$, which corresponds to about 0.11 arcsec in the image plane. We have used a 
narrow-band interference filter $(\mathrm{CWL}=387.9 \mathrm{~nm}, \mathrm{FWHM}=0.7 \mathrm{~nm}$ with $60 \%$ peak transmission) in front of the CCD camera as an order sorter. The light reflected by the slit box was used to obtain slit-jaw images in the violet $\mathrm{CN}$ band simultaneously with the spectra (e.g. Fig. 1 - right). The exposure time for spectra and slit-jaw images was $1.5 \mathrm{~s} .10 \%$ of the total incoming sunlight were separated by a 90/10 grey beamsplitter in the light path before the spectrograph in order to obtain high resolution images in a red "continuum" window using a narrow-band interference filter centered at $705.7 \mathrm{~nm}$ with a FWHM of $0.7 \mathrm{~nm}$. These images, collected with exposure times of $10 \mathrm{~ms}$, are uncorrelated in time with the spectroscopic dataset and are processed by the Multi Frame Blind Deconvolution (MFBD) routine (Löfdahl 2002), thus providing almost diffraction limited quality (e.g. Fig. 1 - left). All employed CCD cameras were Kodak Megaplus 1.6, 10 bit with 1536×1024 pixels of $9 \mu \mathrm{m}$ each.

We have observed on 14 April 2005 an active region NOAA0753 located near $\mu=0.95$. Strong seeing variations and rotation of the FOV allowed us only to record few individual slit spectra, rather than full scans over some solar area. The analysed spectroscopic data were estimated to have 0.3 arcsec spatial resolution and a resolving power of 130000 .

\section{Results}

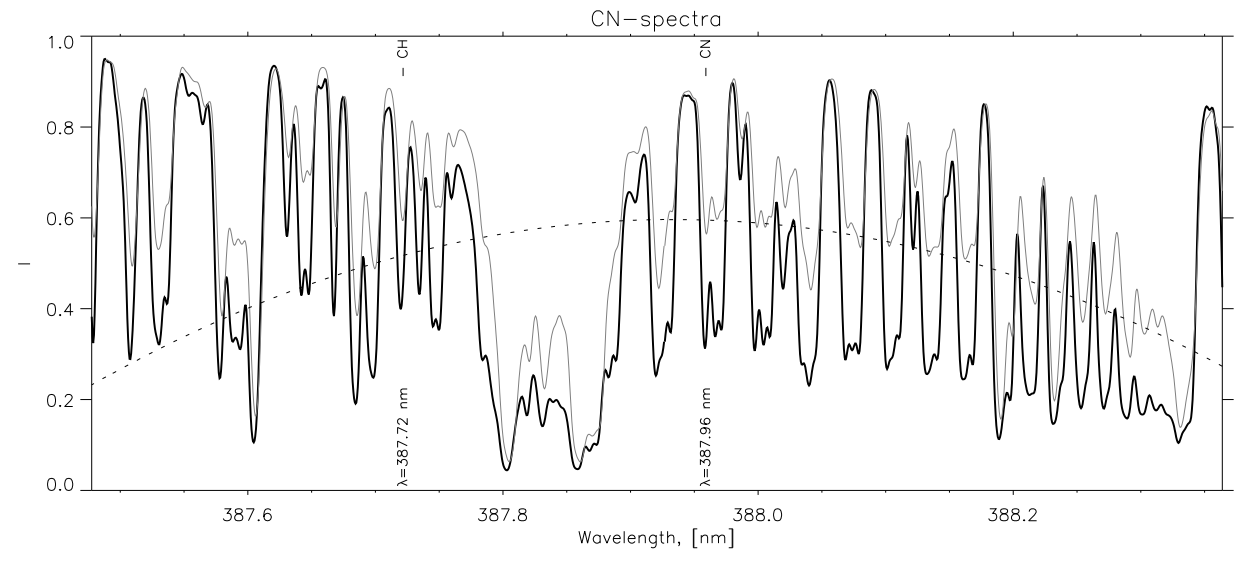

Figure 2. Example of the observed $\mathrm{CN}$ spectrum of a BP (grey line) near $\mu=0.95$ and of the quiet Sun (black line). The filter curve (dashed line) as obtained in the data reduction is also shown.

In Fig. 2 a sample of observed $\mathrm{CN}$ spectra is shown. There are individual $\mathrm{CH}$ lines among the forest of $\mathrm{CN}$ lines in the observed wavelength band. In a BP (grey line) the spectrum shows a considerably reduced absorption in spectral lines of $\mathrm{CN}$ and $\mathrm{CH}$ as compared to that in a quiet Sun area. We have defined the contrast of intensity $I_{\lambda}$ as $C_{\lambda}=I_{\lambda} /\left\langle I_{\lambda}\right\rangle_{q s}-1$, where $\left\langle I_{\lambda}\right\rangle_{q s}$ means the intensity averaged over a quiet Sun area in the immediate vicinity of the observed active region. In the present analysis we measured:

- the line-core intensities $I_{C H}$ of a $\mathrm{CH}$ line at $387.72 \mathrm{~nm}$ and $I_{C N}$ of a $\mathrm{CN}$ line at $387.96 \mathrm{~nm}$ (marked in Fig. 2), 


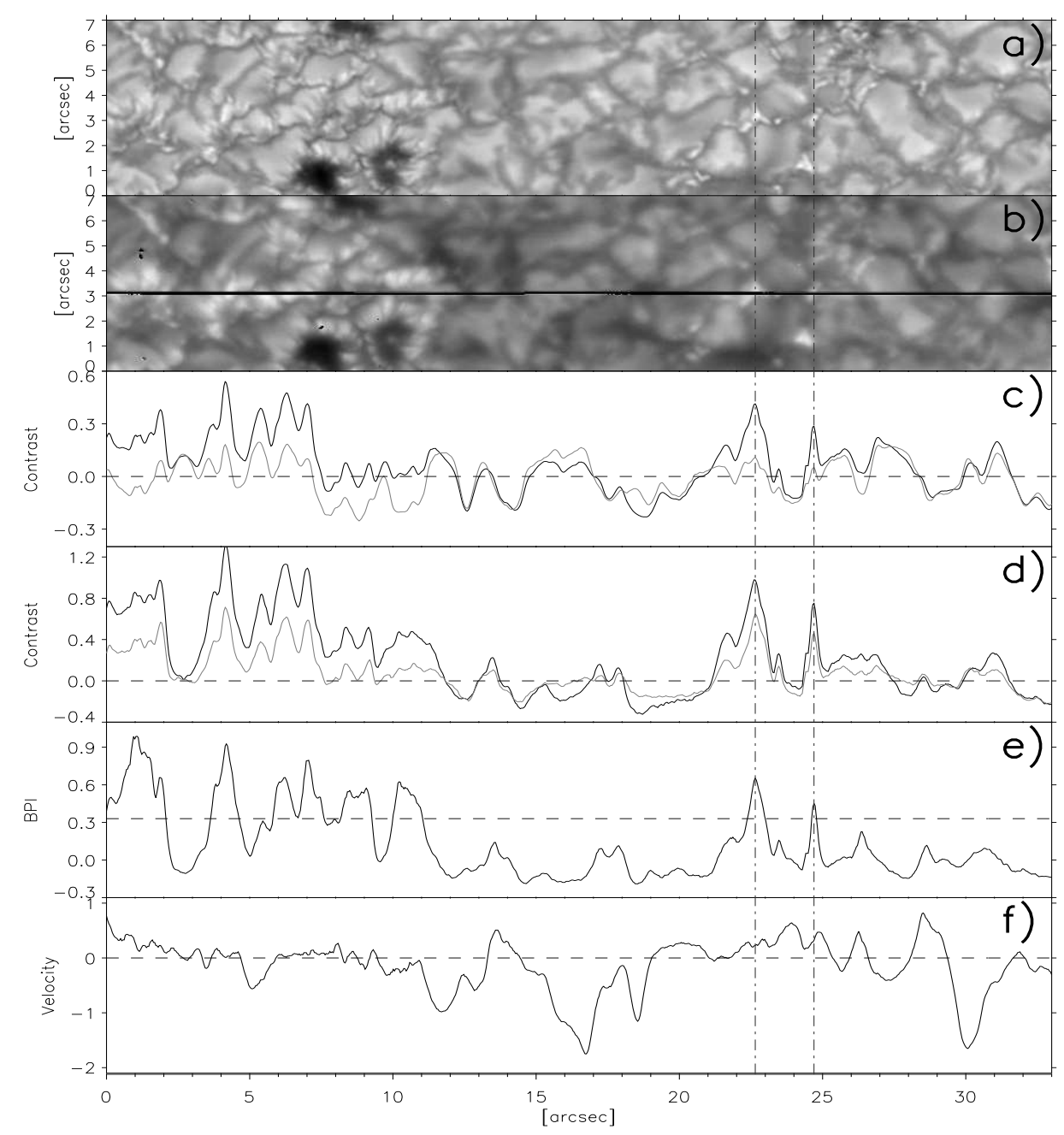

Figure 3. Spectroscopic data observed in the active region NOAA0753 at $\mu=0.95$ on 14 April 2005 at 14:56 (UT) : a) High-resolution red "continuum" image; b) slit-jaw image; c) integrated and continuum intensity contrasts $C\left(I_{\text {int }}\right)$ - black, $C\left(I_{\text {cont }}\right)$ - grey; d) line-core intensity contrasts $C\left(I_{C N}\right)$ - black, $C\left(I_{C H}\right)$ - grey; e) Bright Point Index; f) velocity measurements $v_{C N}$ in $[\mathrm{km} / \mathrm{s}]$. The horizontal black line in b) marks the position of the slit. All the images as well as the spatial domain of the spectrum were coaligned by cross correlation.

- 'quasi' -continuum brightness $I_{\text {cont }}$, averaging it over wavelength positions in the spectra which are the least affected by absorption,

- integrated intensities $I_{\text {int }}$, calculated by integration over $\lambda$ of the whole obtained spectral domain multiplied with the prefilter profile, 
- the LOS velocities, $v_{C N}$, which were computed from measured Doppler shifts in the $\mathrm{CN}$ line at $\lambda_{C N}=387.73 \mathrm{~nm}$ with a relative accuracy of around $60 \mathrm{~m} / \mathrm{s}$. Upflows were assigned negative velocities and downflows positive.

In order to determine the positions of bright small-scale magnetic features and automatically distinguish them from the non-magnetic bright features the Bright Point Index (BPI) was calculated (Langhans et al. 2004). Here we use line-core intensities of the Fe I line at $387.80 \mathrm{~nm}$ and of a CN line at $387.73 \mathrm{~nm}$. A threshold of $\mathrm{BPI}=0.33$ is used to separate magnetic bright features from the non-magnetic ones.

In Fig. 3 we present one example of a calibrated spectroscopic dataset. BPs, as the rule, have a high value of the BPI $>0.33$, enhanced continuum contrast $C\left(I_{\text {cont }}\right)>0$ and have a compact shape. Also the integrated intensity always has a higher contrast than the continuum, i.e. $C\left(I_{\text {int }}\right)>C\left(I_{\text {cont }}\right)$. The line-core contrast in such structures is always as $C\left(I_{C N}\right)>C\left(I_{C H}\right)$. In many photospheric structures showing low BPI, like granules and many intergranular lanes, assumed to be non-magnetic in nature, the continuum and linecore intensities tend to show $C\left(I_{\text {int }}\right)<C\left(I_{\text {cont }}\right)$ and $C\left(I_{C N}\right)<C\left(I_{C H}\right)$. The latter is probably caused by the difference in temperature sensitivity of the molecules. Basically all magnetic brightenings are located in the intergranular downflow area. Many observed BPs show a reduced vertical velocity surrounded by downflows (e.g. at 22.7 arcsec in Fig. 3).

\section{Conclusions}

Considering all 25 individual imaging positions of the slit we estimate that in BPs the ratio of contrasts in $\mathrm{CN}$ line cores to those in $\mathrm{CH}$ line cores lies in a range between 1.4 and 5.4 with a mean value around 2, i.e $\mathrm{CN}$ lines on average show twice the contrast as the $\mathrm{CH}$ lines in the same wavelength band. This is in line with the imaging observations by Zakharov et al. (2005).

Acknowledgements. We are grateful to the SST technical staff and G. Scharmer for help with the instrumentation. The SST is operated by the Royal Swedish Academy of Sciences at the Spanish Observatorio del Roque de los Muchachos of the Instituto de Astrofísica de Canarias.

\section{References}

Kiselman, D. et al. 2007, in preparation

Langhans, K., Schmidt, W., \& Rimmele, T. 2004, A\&A, 423, 1147

Löfdahl, M. G. 2002, in Image Reconstruction from Incomplete Data II, ed. P. J. Bones, M. A. Fiddy, \& R. P. Millane, Proc. SPIE, Vol. 4792, 146

Zakharov, V., Gandorfer, A., Solanki, S. K., \& Löfdahl, M. G. 2005, A\&A, 437, L43 


\title{
Magnetic properties of G-band bright points
}

\author{
C. Beck ${ }^{1, *}$, K. Mikurda ${ }^{1}$, L. R. Bellot Rubio ${ }^{2}$, R. Schlichenmaier ${ }^{1}$, and P. Sütterlin ${ }^{3}$ \\ ${ }^{1}$ Kiepenheuer-Institut für Sonnenphysik, Freiburg, Germany \\ ${ }^{2}$ Instituto de Astrofísica de Andalucía (CSIC), Granada, Spain \\ ${ }^{3}$ Sterrekundig Instituut, Utrecht, The Netherlands \\ *Email: cbeck@kis.uni-freiburg.de
}

\begin{abstract}
Bright points (BPs) visible in the $\mathrm{G}$ band at $430 \mathrm{~nm}$ are commonly used as tracers of magnetic fields, indicating the location of $\mathrm{kG}$ flux concentrations. To study the actual magnetic properties of G-band BPs, we took observations in 2003 and 2005, employing simultaneously a speckle setup in the $\mathrm{G}$ band and vector spectropolarimetry to derive the magnetic field vector. From the analysis of the co-aligned polarimetric data we find that the BPs show a broad range of field strengths, magnetic fluxes, and field inclinations. Many G-band BPs are not co-spatial with the central part of the nearby flux concentrations. Even at the small heliocentric angle of only $12^{\circ}$, the BPs appear projected on adjacent granules, whereas the magnetic field is concentrated in the intergranular lanes. Our findings support the view that the G-band BPs are a result of the "hot wall effect". The downward shift of the optical depth scale in the presence of magnetic fields allows to see deeper and hotter layers in the hot granules next to the field concentrations, where $\mathrm{CH}$ dissociates. Thus, information drawn from imaging observations of BPs has limited use to investigate the actual magnetic field structure, when the BPs are not co-spatial with the central part of the flux concentrations.
\end{abstract}

\section{Introduction}

The solar spectrum around $430 \mathrm{~nm}$ shows numerous spectral lines. Many of them are due to absorption by the $\mathrm{CH}$ molecule, which forms at low temperature. Images of the photosphere taken with a broadband interference filter in the $\mathrm{G}$ band show isolated brightenings located inside and near the intergranular lanes with high contrast to their surroundings. It was found that these bright points (BPs) are caused by the presence of strong magnetic field concentrations. Several authors found the BPs to be co-spatial with the flux concentrations, e.g., Berger \& Title (2001). However, BPs are only indirect tracers of the field. The presence of the field leads to a downward shift of the optical depth scale due to the evacuation of the flux concentration. $\mathrm{CH}$ dissociates in the deep layers with higher temperature, and hence, the intensity in the $\mathrm{G}$ band increases due to the disappearance of the molecular spectral lines. Similar effects take place in the $\mathrm{CN}$ band at $388 \mathrm{~nm}$ (Zakharov et al. 2005), whereas the BPs visible in chromospheric lines like $\mathrm{H} \alpha$ or $\mathrm{Ca}$ II $\mathrm{H}$ and $\mathrm{K}$ also indicate magnetic fields (Leenaarts et al. 2006), although their origin is probably different. At present, there is an ongoing discussion on which tracer is best suited for the detection of magnetic fields (Zakharov et al. 2005; Leenaarts et al. 2006; Uitenbroek \& Tritschler 2006), with Sánchez Almeida (2000) even claiming that indirect tracers are to be preferred over the direct measurements of the field via the Zeeman effect. However, none of the observational studies carried out so far 

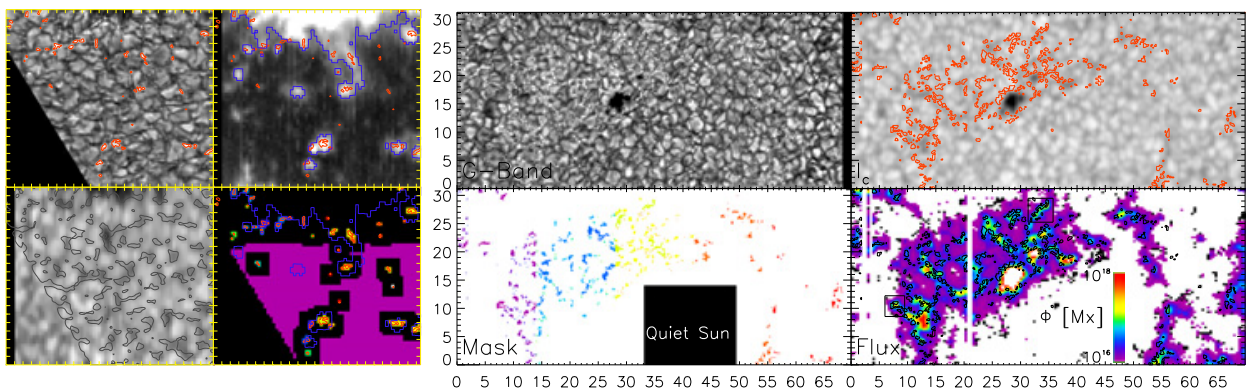

Figure 1. Left, counterclockwise: intensity in G-band, intensity at $1.5 \mu \mathrm{m}$, mask, polarization degree; 2003. Right, counterclockwise: intensity in G-band, mask, magnetic flux, intensity at $1.5 \mu \mathrm{m} ; 2005$. Tickmarks are in arcsec.

combined simultaneous measurements of G-band intensities (as a proxy of magnetic fields) and vector Stokes polarimetry (for a direct measurement). In this contribution, we present the results of simultaneous G-band proxy magnetometry and vector spectropolarimetry to investigate the relation between G-band BPs and magnetic fields in the solar photosphere.

\section{Observations \& data analysis}

For the present study we employ two data sets, taken in 2003 and 2005. On August, 9, 2003, we observed a region close to the sunspot NOAA 10425 from 09:36 to 10:34 UT with the Tenerife Infrared Polarimeter (TIP, Martínez Pillet et al. 1999) at $1.5 \mu \mathrm{m}$ and the POlarimetric LIttrow Spectrograph (POLIS, Beck et al. 2005) at $630 \mathrm{~nm}$ attached to the Vacuum Tower Telescope (VTT) on Tenerife. The same area, located at a heliocentric angle of $27^{\circ}$, was observed by the Dutch Open Telescope (DOT) on La Palma. The section shown in the left panel of Fig. 1 was scanned in total 8 times. On 11 October 2005 between UT 10:35 and 10:58, we used TIP together with a speckle channel in the G band at the VTT to observe a network region close to a pore at a heliocentric angle of $12^{\circ}$ south-west of the disc center. In both cases the same data analysis was performed: the G-band data were speckle reconstructed and spatially aligned to the polarimetric data; a mask with locations of BPs was derived from the G-band intensities by hard thresholding $\left(I>1.2 \cdot I_{c}\right)$, and the polarization profiles were inverted with the SIR code (cf. Bellot Rubio \& Beck 2005).

\section{Results}

\subsection{Statistics of bright point properties}

A statistical analysis of the magnetic properties of BPs in the 2003 data (see also Beck et al. 2007) allows us to support the magnetic nature of BPs: more than $90 \%$ of the BPs are co-spatial with significant polarization signal within $1^{\prime \prime}$, which corresponds to the spatial resolution of the polarimetric data of 2003. In the data taken in 2003, the BPs show redshifts of $\sim 400 \mathrm{~m} / \mathrm{s}$ inside the magnetic flux, as derived from the inversion of the spectral lines. The G-band intensity increases slightly with field strength and slightly decreases for large fluxes. The BP intensity scales strongly with the inclination of the field to the line of sight. 


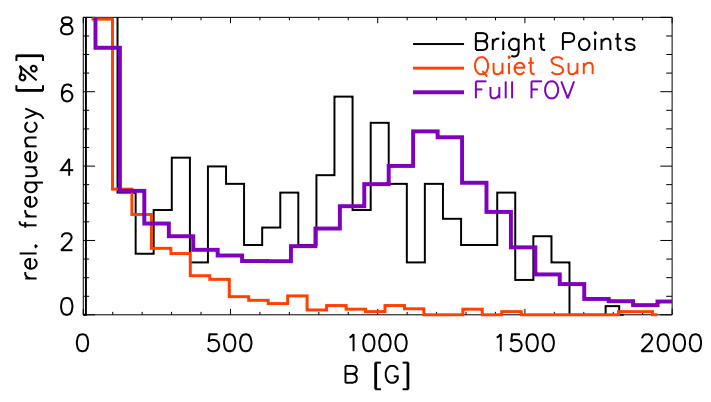

Figure 2. Histogram of field strength of quiet Sun, BPs, and the remaining FOV in the 2005 data.

For the 2005 data, the field strength associated with BPs ranges from 0 to about $1.5 \mathrm{kG}$ with a uniform distribution and no preferred value (cf. Fig. 2). The BP fields below $\sim 0.5 \mathrm{kG}$ are presumably due to the erroneous inclusion of the hot centers of granules into the BP mask; the BP field distribution is identical to that of the quiet Sun within that range. The very conspicuous peak at $1.3 \mathrm{kG}$ in the distribution of the full field of view (FOV), which is due to the network fields, is completely absent for the BPs. This indicates an intrinsic difference between the network and the BP fields. Together with the large range of variation of BP parameters in the 2003 data, this casts some doubt on the generally accepted view that every $\mathrm{BP}$ corresponds to a stable $\mathrm{kG}$ flux tube.

\subsection{Single cases}

The G-band data of 2005 were taken at the VTT, whose 70-cm primary mirror is a factor 1.55 larger than that of the DOT. The spatial resolution thus is significantly better than that of the 2003 observations. A closer inspection of the three examples shown in Fig. 3 reveals the reason why the field strength distributions of BPs and network regions differ: there is a slight offset in the locations of BPs and flux concentrations. One finds that the intergranular lanes (IGLs) in the G-band and infrared continuum intensity are co-spatial within the alignment accuracy of about 0.5 , and that the magnetic flux is concentrated in the IGLs. The BPs however are not co-spatial with the flux concentrations, but flank them on either one (3rd example) or two (2nd example) of their sides. In the 3rd example it can also be clearly seen that the brightening appears projected onto the center side of a granule, even at the small heliocentric angle of $12^{\circ}$.

\section{Conclusions}

G-band BPs are widely used as tracers of magnetic fields and are generally assumed to indicate strong concentrations of magnetic flux with kG fields. In Beck et al. (2007) we demonstrated that at the locations co-spatial with BPs various field configurations are found. Here we have only used the field strength distribution as an example. Even if nearby network elements show preferentially field strengths of $\mathrm{kG}$ or more, the BPs show a uniform field 

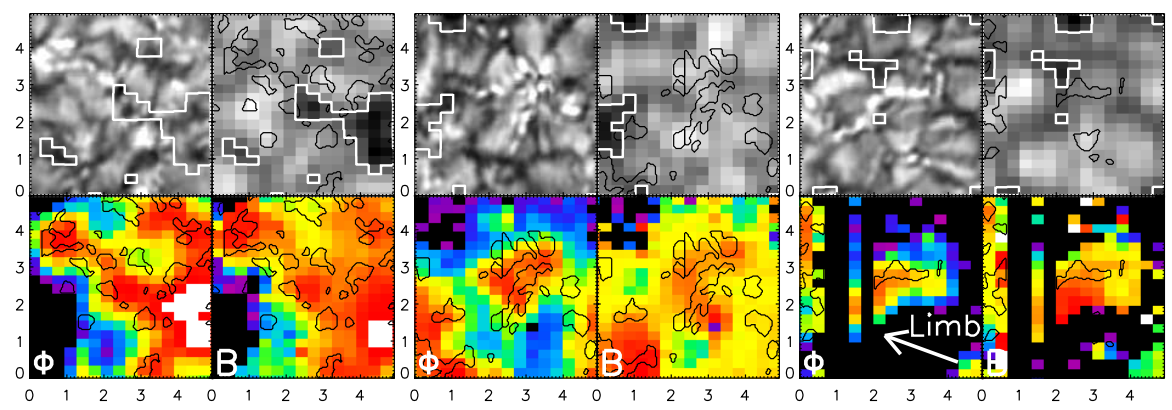

B: $1.8 \mathrm{kG}$ $\Phi: 8 * 10^{17} \mathrm{Mx}$

Figure 3. Enlarged sections of the data of 2005. Clockwise: intensity in G band, intensity at $1.5 \mu \mathrm{m}$, field strength, magnetic flux. White contours outline the darkest intergranular lanes in the infrared intensity, black contours BPs in the G band. Scales are in Mm.

strength distribution ranging from 0 to $1.5 \mathrm{kG}$. We suggest that the reason for this discrepancy is the actual location of the BPs relative to the fields: the BPs are not co-spatial with the nearby flux concentrations, but are in many cases slightly displaced.

We suggest that the generation of BPs by the magnetic field strongly depends on geometrical effects. The line of sight has to penetrate to deep and hot layers through a shift of the optical depth scale, to reach plasma, where enough $\mathrm{CH}$ molecules are dissociated to produce a significant line weakening. An accurate treatment of BPs and their relation with magnetic fields thus requires at least a two-dimensional model of flux concentrations inside IGLs. The multitude of different types of spatial structures ("ribbons", "crinkles", or "flowers") found in recent high-resolution observations by Berger et al. (2004) may well be due to the same mechanism: the structure of the magnetic fields (topology, field strength, flux) and their appearance when viewed from different directions. We conclude that BPs in the G band have a limited use as tracers, as they miss weaker flux concentrations and only indirectly reflect the properties or temporal evolution of the central part of the flux concentrations.

Acknowledgements. This work was supported by the DFG under grant SCHL 514/2-3, and by the Spanish MEC under Programa Ramón y Cajal and project ESP2003-07735-C04-03. The DOT is operated by Utrecht University at the Spanish Observatorio del Roque de los Muchachos of the Instituto de Astrofísica de Canarias (IAC). The VTT is operated by the Kiepenheuer-Institut für Sonnenphysik at the Spanish Observatorio del Teide of the IAC.

\section{References}

Beck, C., Bellot Rubio, L. R., Schlichenmaier, R., \& Sütterlin, P. 2007, A\&A, submitted

Beck, C., Schmidt, W., Kentischer, T., \& Elmore, D. 2005, A\&A, 437, 1159

Bellot Rubio, L. \& Beck, C. 2005, ApJ, 626, L125

Berger, T. E., Rouppe van der Voort, L. H. M., Löfdahl, M. G., et al. 2004, A\&A, 428, 613

Berger, T. E. \& Title, A. M. 2001, ApJ, 553, 449

Leenaarts, J., Rutten, R. J., Sütterlin, P., Carlsson, M., \& Uitenbroek, H. 2006, A\&A, 449, 1209

Martínez Pillet, V., Collados, M., Sánchez Almeida, J., et al. 1999, in ASP Conf. Ser. 183, 264

Sánchez Almeida, J. 2000, ApJ, 544, 1135

Uitenbroek, H. \& Tritschler, A. 2006, ApJ, 639, 525

Zakharov, V., Gandorfer, A., Solanki, S. K., \& Löfdahl, M. 2005, A\&A, 437, L43 


\title{
Photospheric magnetic field and chromospheric emission
}

\author{
R. Rezaei*, R. Schlichenmaier, C. Beck, and W. Schmidt \\ Kiepenheuer-Institut für Sonnenphysik, Freiburg, Germany \\ *Email: rrezaei@kis.uni-freiburg.de
}

\begin{abstract}
We present a statistical analysis of network and internetwork properties in the photosphere and the chromosphere. For the first time we simultaneously observed (a) the four Stokes parameters of the photospheric iron line pair at $630.2 \mathrm{~nm}$ and (b) the intensity profile of the $\mathrm{Ca} \mathrm{H}$ line at $396.8 \mathrm{~nm}$. The vector magnetic field was inferred from the inversion of the iron lines. We aim at an understanding of the coupling between photospheric magnetic field and chromospheric emission.
\end{abstract}

\section{Observations and data reduction}

We observed a series of 13 maps of a network region and the surrounding quiet Sun at a heliocentric angle of $53^{\circ}$, close to the active region NOAA 10675 on September 27, 2004, with POLIS (Schmidt et al. 2003; Beck et al. 2005) at the German VTT in Tenerife. The Kiepenheuer Adaptive Optics System (KAOS) was used to improve spatial resolution to about 1 arcsec (von der Lühe et al. 2003).

Using the average profile of each map, we normalized the intensity at the line wing at $396.490 \mathrm{~nm}$ to the FTS profile (Stenflo et al. 1984). From the intensity profile of Ca H we define line properties like, e.g., the H-index, which is the integral around the line core from $396.8 \mathrm{~nm}$ to $396.9 \mathrm{~nm}$. The separation between network and internetwork is based on (i) the maps of magnetic flux density, (ii) the presence of Stokes- $V$ signals and emission in $\mathrm{Ca} \mathrm{H}$.

\section{Inversion}

An inversion was performed for the two iron lines at $630 \mathrm{~nm}$ using the SIR code (Ruiz Cobo \& del Toro Iniesta 1992). To mimic unresolved magnetic fields, we used a model atmosphere with one magnetic and one field-free component, plus stray light. The inversion yields a magnetic field vector, a line-of-sight velocity, and the magnetic flux per pixel. These quantities are assumed constant along the line of sight. Using the flux density maps, we created a mask to separate network and internetwork regions.

\section{Magnetic field distribution}

The polarization signal in $Q(\lambda), U(\lambda)$, and $V(\lambda)$ is normalized by the local continuum intensity, $I_{c}$, for each pixel. The rms noise level of the Stokes parameters in the continuum 

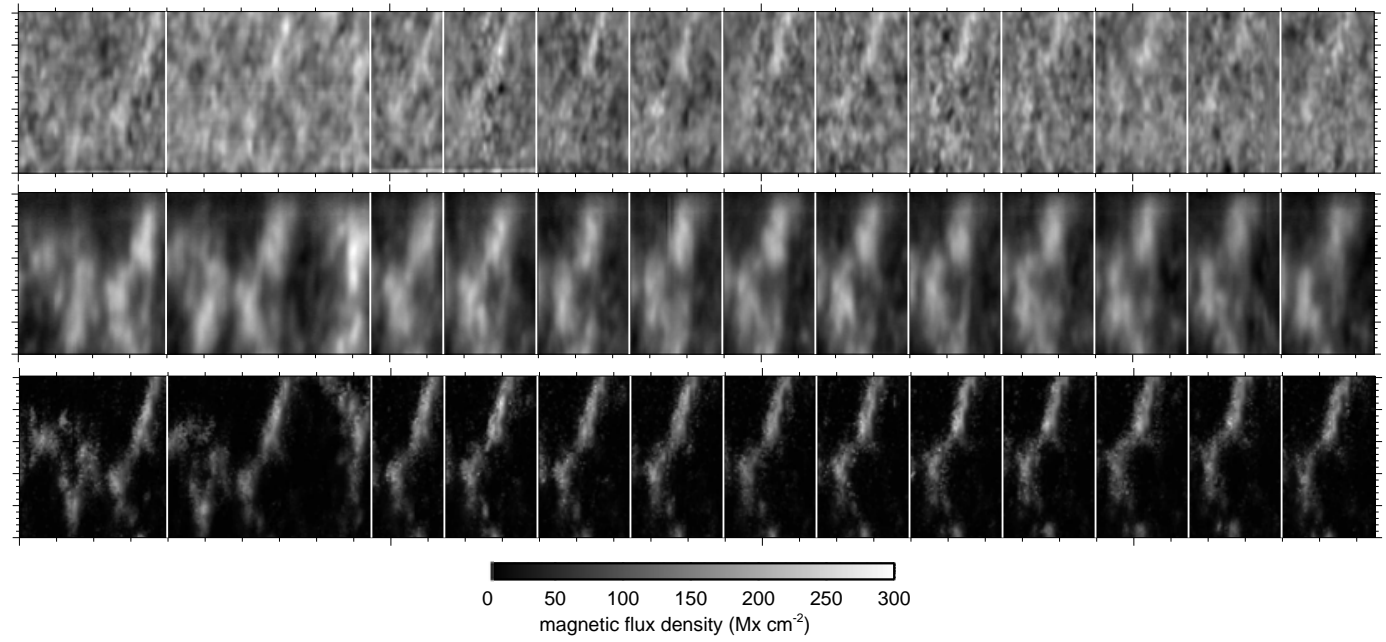

Figure 1. Top to bottom: the continuum intensity close to $630 \mathrm{~nm}$, the H-index, and the magnetic flux density obtained from the inversion.

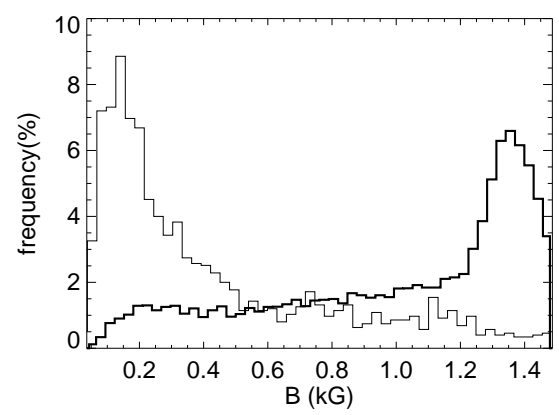

Figure 2. Distribution of the magnetic field strength for the network (thick) and internetwork (thin).

was $\sigma=8.0 \times 10^{-4} I_{c}$ for the Fe I $630 \mathrm{~nm}$ lines. Only pixels with $V$ signals greater than $3 \sigma$ were included in the profile analysis. We obtain a magnetic field distribution which peaks at some $1.4 \mathrm{kG}$ for the network elements and at about $200 \mathrm{G}$ for the internetwork elements in agreement with previous infrared observations, but in contradiction with results from visible lines (Collados 2001; Lites 2002).

\subsection{The effect of noise in the weak-field limit}

Our finding of weak rather than strong fields can be explained by the high spatial resolution and high polarimetric accuracy that we have achieved in our measurements. Since the internetwork magnetic fields are in the weak-field limit, the amount of noise strongly influences the outcome of the inversion. Figure 3 shows two different cases:

1. A fit of an original set of Stokes profiles yields $B=840 \mathrm{G}$ with a filling factor of $7.6 \%$ (left panel, Fig. 3). 

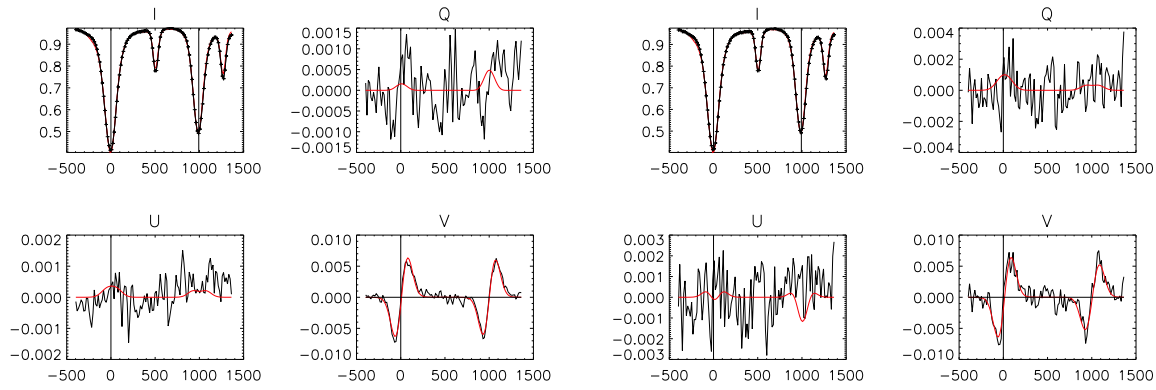

Figure 3. The left (right) four panels show the inversion results of the original (noisy) data.
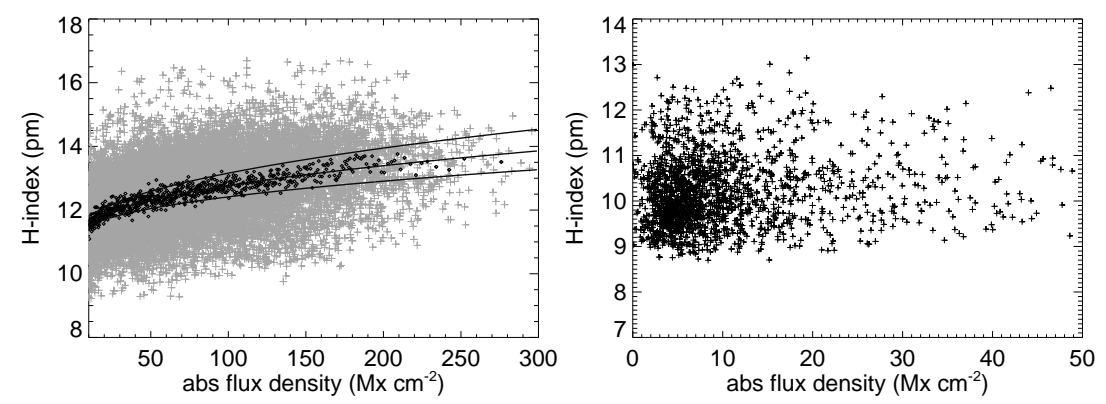

Figure 4. The H-index vs. the magnetic flux density in the network (left) and internetwork (right). Black squares show the binned data. The curves show the fit to the original data along with $1 \sigma$ confidence level.

2. A fit on the same profile with added noise (such that the rms noise level is twice as large) delivers $B=1.5 \mathrm{kG}$ and a filling factor of only $5.3 \%$ (right four panels, Fig. 3).

The reduction of noise and improvement of spatial resolution may resolve the existing discrepancy between visible and infrared magnetic field measurements.

\section{The H-index vs. magnetic flux density}

The left panel of Fig. 4 shows the H-index versus photospheric magnetic flux, $\Phi$, for the network. A power law fit, $H=a \Phi^{b}+c$, yields $b=0.3$ and $c=10 \mathrm{pm}$. In the internetwork the $\mathrm{H}$-index does not correlate with the magnetic flux density (Fig. 4, right panel). The average value of the $H$-index in the internetwork is about $H=10 \mathrm{pm}$. The offset value, $c=10 \mathrm{pm}$, can be interpreted as the non-magnetic heating contribution and the stray light.

For the first time the H-index and simultaneously measured V-profile parameters can be compared. We find no correlation between these parameters in the internetwork (Fig. 5, plus signs). In the network, a correlation exists, and the $\mathrm{H}$-index peaks at a small positive value for the area asymmetry and at vanishing V-profile Doppler shift (Fig. 5, squares). 

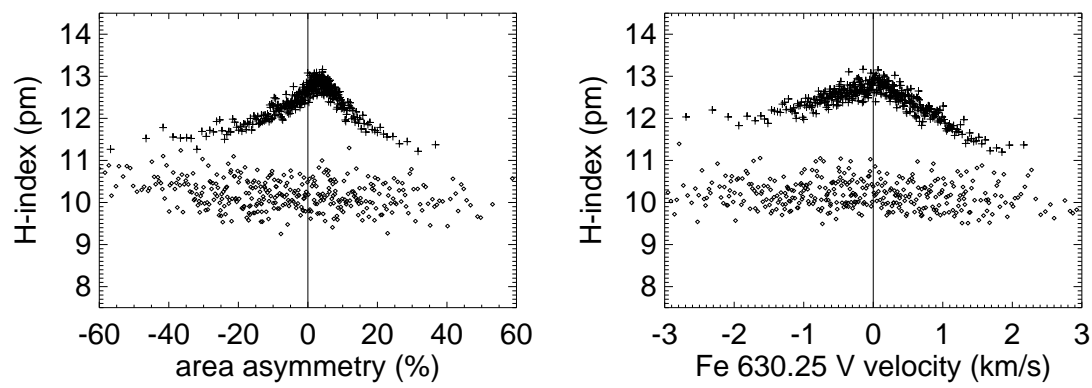

Figure 5. Scatter plot of the area asymmetry and Stokes- $V$ velocity against the H-index.

\section{Conclusions}

- The internetwork magnetic field distribution peaks around $200 \mathrm{G}$ and its mean absolute flux density amounts to $9 \mathrm{Mx} \mathrm{cm}^{-2}$. The finding of weak rather than strong fields is a consequence of the high spatial resolution and high polarimetric accuracy.

- The H-index in the network is correlated to the magnetic flux density, approaching a value of $H=10 \mathrm{pm}$ for vanishing flux.

- The H-index in the internetwork is not correlated to any property of the photospheric magnetic field implying that the chromospheric brightenings in the internetwork are nonmagnetic.

- For high values of the H-index, the network shows small positive area (and amplitude) asymmetry, being consistent with the scenario of a line-of-sight crossing the magnetic boundary (canopy) of flux tubes that fans out with height (Steiner 1999).

\section{References}

Beck, C., Schlichenmaier, R., Collados, M., Bellot Rubio, L., \& Kentischer, T. 2005, A\&A, 443, 1047 Collados, M. 2001, in ASP Conf. Ser. 236: Advanced Solar Polarimetry - Theory, Observation, and Instrumentation, ed. M. Sigwarth, 255

Lites, B. W. 2002, ApJ, 573, 431

Ruiz Cobo, B. \& del Toro Iniesta, J. C. 1992, ApJ, 398, 375

Schmidt, W., Beck, C., Kentischer, T., Elmore, D., \& Lites, B. 2003, Astron. Nachr., 324, 300

Steiner, O. 1999, in ASP Conf. Ser. 184: Third Advances in Solar Physics Euroconference: Magnetic

Fields and Oscillations, 38-54

Stenflo, J. O., Solanki, S., Harvey, J. W., \& Brault, J. W. 1984, A\&A, 131, 333

von der Lühe, O., Soltau, D., Berkefeld, T., \& Schelenz, T. 2003, in Innovative Telescopes and Instrumentation for Solar Astrophysics. Proceedings of the SPIE, Volume 4853, ed. S. L. Keil \& S. V. Avakyan, 187-193 


\title{
Velocity distribution of chromospheric downflows
}

\author{
R. Aznar Cuadrado*, S. K. Solanki, and A. Lagg \\ Max-Planck-Institut für Sonnensystemforschung, Katlenburg-Lindau, Germany \\ *Email: aznar@mps.mpg.de
}

\begin{abstract}
Infrared spectropolarimetric observations were obtained with the Tenerife Infrared Polarimeter (TIP) at the German Vacuum Tower Telescope (VTT) of the Spanish observatory of Izaña, Tenerife. We present the velocity distributions of a large dataset composed of maps of the Stokes I, $\mathrm{Q}, \mathrm{U}$, and V profiles of active and quiet sun regions obtained in the chromospheric $\mathrm{He}$ I $1083.0 \mathrm{~nm}$ triplet. The line-of-sight velocities were determined by applying a multi-Gaussian fit to the intensity profiles. Single and double component fits were carried out for all datasets. We find that $18.7 \%$ of all observed pixels show strong downflows as evidenced by a second line profile component, generally shifted by more than $8 \mathrm{~km} \mathrm{~s}^{-1}$ relative to the rest wavelegth. The distribution of these strong downflows displays two distinct populations. The slower one (near sonic and weakly supersonic flows) has line-of-sight velocities up to $17 \mathrm{~km} \mathrm{~s}^{-1}$ and is associated with moderate to strong magnetic signal (up to $\sqrt{Q^{2}+U^{2}+V^{2}} / I_{\mathrm{c}}=0.08$ ). Strongly supersonic downflows (reaching up to $60 \mathrm{~km} \mathrm{~s}^{-1}$ ) are found at places with weak to moderate magnetic signal, with $\sqrt{Q^{2}+U^{2}+V^{2}} / I_{\mathrm{c}}$ values mainly between 0.01 and 0.03 .
\end{abstract}

\section{Observations}

Infrared spectroscopic observations were carried out with the Tenerife Infrared Polarimeter (TIP; Martínez Pillet et al. 1999) mounted on the German Vacuum Tower Telescope (VTT) at the Observatorio del Teide (Spain), during May 2001, October 2002, and August 2003. The spectrograph spectral resolution was $30 \mathrm{~m} \AA$ per pixel, and the pixel size was $0.38^{\prime \prime}$. The observed wavelength range, from 1082.5 to $1083.3 \mathrm{~nm}$, contains the chromospheric $\mathrm{He}$ I multiplet (He Ia at $1082.909 \mathrm{~nm}, \mathrm{He}$ Ib at $1083.025 \mathrm{~nm}$ and He Ic at $1083.034 \mathrm{~nm}$ ).

Our observations consisted of 35 scans of 13 different active regions, and 4 scans of quiet sun regions.

\section{Determination of the line-of-sight velocity}

We determine the line-of-sight (LOS) velocities by applying a multi-Gaussian fit to the intensity profiles. A Voigt profile, free to vary in amplitude and in a restricted wavelength interval, accounting for the telluric line at $1083.21 \mathrm{~nm}$, and a linear background were also included in the fit. Single and double component fits were carried out to the chromospheric He I $1083.0 \mathrm{~nm}$ line for all datasets, but a second component was only considered when its amplitude exceeded $20 \%$ of that of the primary component. The existence of a second mag- 


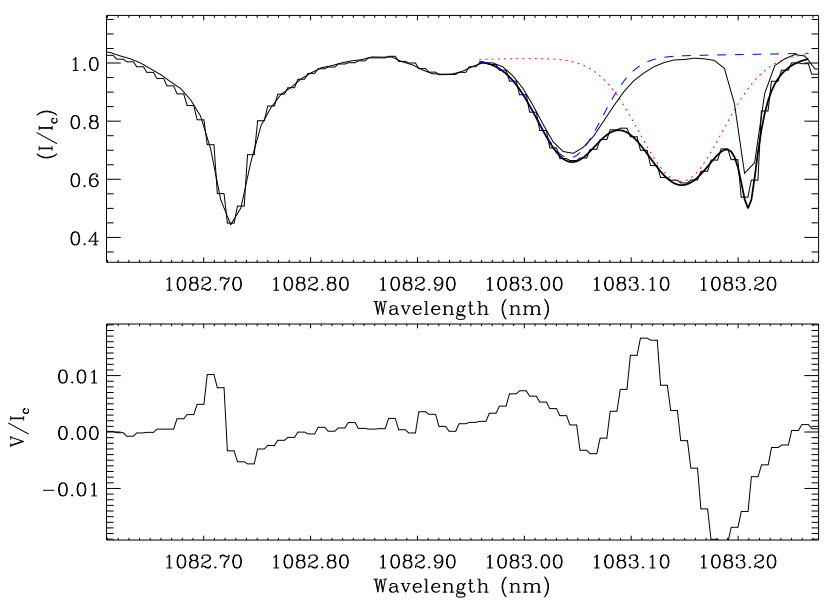

Figure 1. Stokes I and V profiles showing two different components in the chromospheric He I $1083.0 \mathrm{~nm}$ line of active region NOAA 10436 recorded 27 August 2003. Observed profiles are shown as histogram, dashed (blue in online version) and dotted (red) lines represent fits to the slow and fast components, respectively. The thick solid line is the fitting profile, the thin solid line in the upper frame is the average profile over the whole dataset. LOS-velocities of the two magnetic components are: $\mathrm{v}_{\text {slow }}=-0.4 \mathrm{~km} \mathrm{~s}^{-1}$ and $\mathrm{v}_{\text {fast }}=29.2 \mathrm{~km} \mathrm{~s}^{-1}$.

netic component was also confirmed by the presence of a magnetic signal at the position of the high velocity component in at least one of the Stokes parameters Q, U, and V.

The Doppler shifts were measured relative to the wavelength of the chromospheric He I $1083.0 \mathrm{~nm}$ line averaged over the whole dataset for every scanned region. The LOS velocity range is limited by the available spectral range, given basically by the size of the detector. Although occasionally strong upflows are also seen, we study here only the downflows.

In Fig. 1 we present an example of Stokes I and V profiles showing a second component in the chromospheric He I $1083.0 \mathrm{~nm}$ line.

\section{LOS velocity distribution}

In order to characterise all scanned regions, every observed dataset was divided into regions showing magnetic activity (magnetic regions) and no magnetic activity ('field free' regions). Hence, the parameter $M$ was derived from the Stokes parameters as $M=\sqrt{Q^{2}+U^{2}+V^{2}} / I_{\mathrm{c}}$ to account for the strength of the magnetic signal. The threshold for the magnetic activity classification was taken as the $3 \sigma$ level of the parameter $M$.

In Table 1 we report the fraction of all observed pixels showing a second component, both in magnetic regions $(M R, M>3 \sigma)$ and 'field free' regions $(F R, M<3 \sigma)$, averaged over all observed active regions $(A R)$ and quiet sun $(Q S)$ scans. We also show the fraction of observed pixels with downflow velocities above values from one to five times the sound speed in the chromosphere $\left(C_{\mathrm{s}} \approx 10 \mathrm{~km} \mathrm{~s}^{-1}\right.$, assuming a formation temperature of $10000 \mathrm{~K}$ for the He I $1083.0 \mathrm{~nm}$ line). 
Table 1. Fraction of observed pixels with a second component and fraction of observed pixels showing downflow velocities above values from one to five times the sound speed in the chromosphere, in magnetic $(M R)$ and 'field free' regions $(F R)$, averaged over all active regions $(A R)$ and quiet sun $(Q S)$ scans.

\begin{tabular}{ccrccccc}
\hline & & $2-$ comp & $>10 \mathrm{~km} \mathrm{~s}^{-1}$ & $>20 \mathrm{~km} \mathrm{~s}^{-1}$ & $>30 \mathrm{~km} \mathrm{~s}^{-1}$ & $>40 \mathrm{~km} \mathrm{~s}^{-1}$ & $>50 \mathrm{~km} \mathrm{~s}^{-1}$ \\
\hline \multirow{2}{*}{$M R$} & $A R$ & $17.97 \%$ & $11.69 \%$ & $2.58 \%$ & $0.28 \%$ & $0.03 \%$ & $0.01 \%$ \\
& $Q S$ & $0.57 \%$ & $0.35 \%$ & $0.06 \%$ & $0.00 \%$ & $0.00 \%$ & $0.00 \%$ \\
\hline \multirow{2}{*}{$F R$} & $A R$ & $0.13 \%$ & $0.06 \%$ & $0.00 \%$ & $0.00 \%$ & $0.00 \%$ & $0.00 \%$ \\
& $Q S$ & $0.01 \%$ & $0.01 \%$ & $0.01 \%$ & $0.00 \%$ & $0.00 \%$ & $0.00 \%$ \\
\hline
\end{tabular}
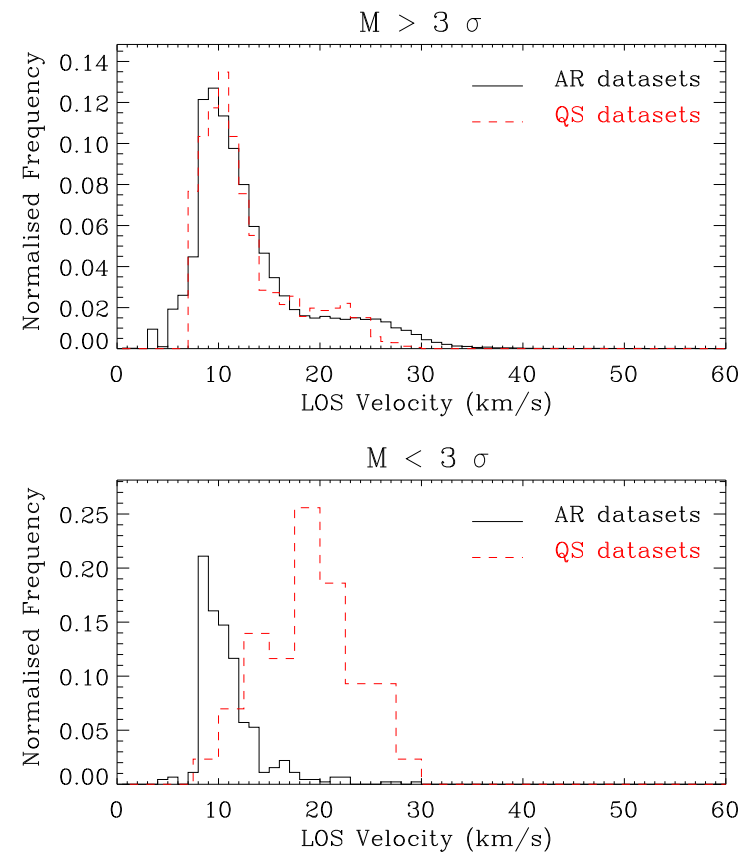

Figure 2. Histograms representing the LOS velocity distribution of downflows measured in the second component of the chromospheric $\mathrm{He}$ I $1083.0 \mathrm{~nm}$ line. Top panel: regions showing a magnetic signal $(M>3 \sigma)$, bottom panel: regions not showing a magnetic signal $(M<3 \sigma)$.

Fig. 2 shows the velocity distribution of downflows measured in the second component of the chromospheric He I $1083.0 \mathrm{~nm}$ line in magnetic regions (top) and 'field free' regions (bottom), both separately analysed in active region (solid) and quiet sun (dashed) scans. The sharp lower boundary of the histograms is due to the uncertainty in fitting 2 very close components with $\mathrm{v}<8 \mathrm{~km} \mathrm{~s}^{-1}$, where a single Gaussian fit is also valid.

We find that $18.7 \%$ of all observed pixels show downflows as evidenced by a second line 


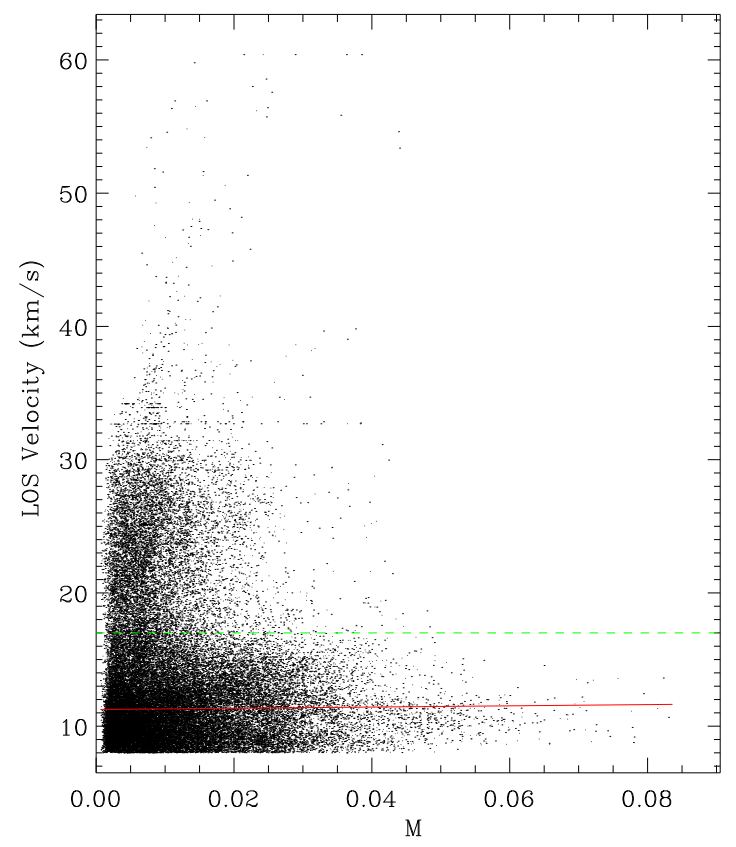

Figure 3. Scatter plot of the LOS velocities measured in the second component of the chromospheric $\mathrm{He}$ I $1083.0 \mathrm{~nm}$ line versus the magnetic field signal $M$. The dashed line (green in online version) represents an imaginary boundary of the two distinct populations of the chromospheric downflows distribution. The solid line (red in online version) shows a linear fit to the slower population.

profile component, while $12.1 \%$ of all observed pixels show supersonic downflows above $10 \mathrm{~km} \mathrm{~s}^{-1}$. The distribution of these supersonic downflows displays two distinct populations. The slower populations peaks at around $10 \mathrm{~km} \mathrm{~s}^{-1}$ with line-of-sight velocities up to $17 \mathrm{~km} \mathrm{~s}^{-1}$, and is associated with moderate to strong magnetic signal (up to $M=0.08$; see Fig.3). The faster population peaks at $20-25 \mathrm{~km} \mathrm{~s}^{-1}$. LOS velocities found in regions with no magnetic activity (i.e., $M<3 \sigma$ ) reach values of $30 \mathrm{~km} \mathrm{~s}^{-1}$. Strongly supersonic downflows (reaching up to $60 \mathrm{~km} \mathrm{~s}^{-1}$ ) are found at places with weak to moderate magnetic signal, with $M$ mainly ranking between 0.01 and 0.03 .

\section{References}

Martínez Pillet, V., Collados, M., Sánchez Almeida, J., et al. 1999, in High Resolution Solar Physics: Theory, Observations, and Techniques, ASP Conf. Ser., 183, 264 


\title{
Off-limb spectroscopy of the He I $10830 \AA$ multiplet: observations vs. modelling
}

\author{
B. Sánchez-Andrade Nuño ${ }^{1,2, *}$, R. Centeno ${ }^{3}$, K. G. Puschmann ${ }^{2}$, \\ J. Trujillo Bueno ${ }^{1,3,4}$, and F. Kneer ${ }^{1}$ \\ ${ }^{1}$ Institut für Astrophysik, Göttingen, Germany \\ ${ }^{2}$ Max-Plank-Institut für Sonnensystemforschung, Katlenburg-Lindau, Germany \\ ${ }^{3}$ Instituto de Astrofísica de Canarias, La Laguna/Tenerife, Spain \\ ${ }^{4}$ Consejo Superior de Investigaciones Científicas, Spain \\ *Email: bruno@astro.physik.uni-goettingen.de
}

\begin{abstract}
Spectropolarimetric observations of spicules were carried out with the new optical setup of the Tenerife Infrared Polarimeter (TIP 2) at the VTT, showing the variation of the He I $10830 \AA$ multiplet off-limb emission profiles with increasing distance to the solar visible limb. The ratio between the intensities of the blue and the red components of this triplet $\left(\mathcal{R}=I_{\text {blue }} / I_{\text {red }}\right)$ is an observational signature of the optical thickness along the light path, which, at the same time, is proportional to the population of the lower (metastable) level that takes part in these transitions. Our observational results show a variation of $\mathcal{R}$ as a function of the distance to the limb. In agreement with recent theoretical results we conclude that $\mathcal{R}$ could be used as a diagnostic tool for downward UV coronal irradiance, which is believed to be responsible for the population of the metastable level of the He i $10830 \AA$ triplet. We have compared our observational results with the ratio obtained from detailed radiative transfer calculations in semi-empirical models of the solar atmosphere (assuming spherical geometry) finding a fairly poor agreement. We argue that future models of the solar chromosphere and transition region should account for the observational constraints presented here.
\end{abstract}

\section{Introduction}

Solar spicules have been observed for more than 100 years. Many theoretical models have been developed to understand their nature, using a wide variety of motion triggers and developing mechanisms. A key impediment to develop a satisfactory understanding has been the lack of reliable observational data, as already Sterling (2000) pointed out in his review. In this work we focus our interest on understanding the overall scenario that produces the emission line in the He r $10830 \AA$ triplet using the latest technological improvements in observational facilities. We are able to provide observational evidence of the link between the corona and the infrared emission of this line, in the frame of the current theoretical models.

\section{Observations}

Observations were carried out on December $4^{\text {th }}, 2005$ at the Vacuum Tower Telescope (VTT) at Observatorio del Teide, supported by the Kiepenheuer Adaptive Optics System (KAOS). The instrument used was the Tenerife Infrared Polarimeter (TIP) with the new camera, TIP2. 
Full Stokes profiles were measured between 10826 and $10837 \AA$ to cover the He I $10830 \AA$ multiplet, with a spectral resolution of $10^{-6}$. The slit was placed parallel to the NE limb at $59^{\circ} .8$ from North, with a FOV of $40^{\prime \prime}$. We scanned the full height (up to 7 " off the visible solar limb) of the spicules with a step of 0 .'35. On each position, 5 consecutive spectra were measured with 10 accumulations of $250 \mathrm{~ms}$ each, with a total integration time of $2.5 \mathrm{~s}$ per off-limb slit position.

\section{Data reduction and processing}

We applied usual flat fielding and dark corrections. The polarimetric calibration was done using calibration optic measurements. To remove the scattered light on each distance to the limb, we set the continuum intensity to zero using a series of averaged spectra of nearby solar disc profiles. A high frequency electronic noise was detected and removed using a low-pass Fourier filter, leaving only the full power at lower frequencies.

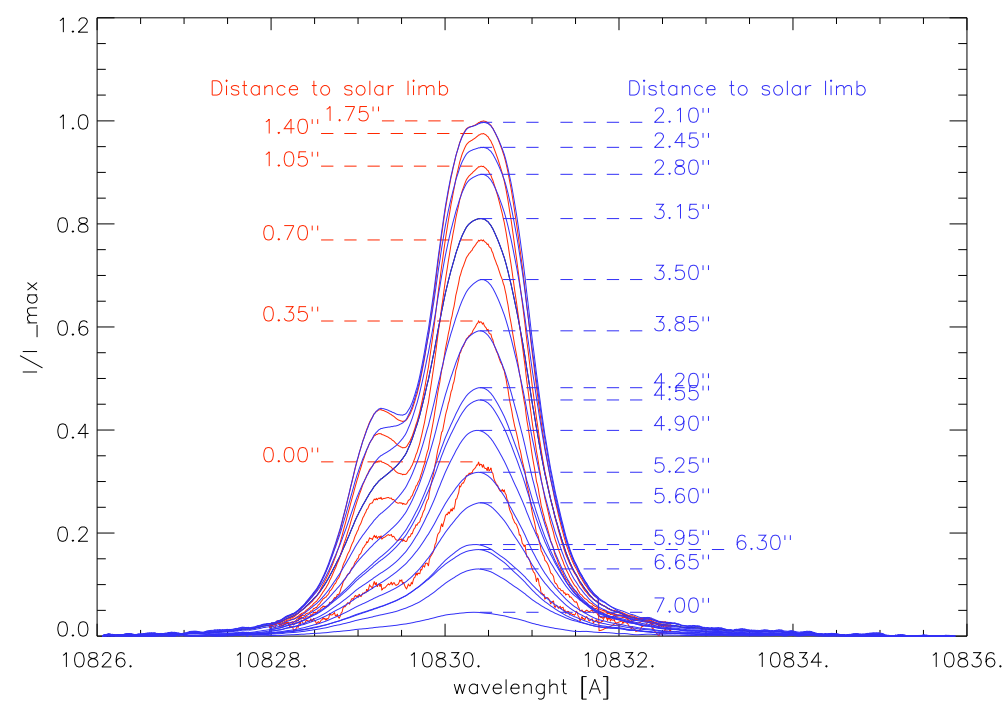

Figure 1. He I $10830 \AA$ emission profiles for increasing distances to the solar visible limb, scanning the full range of the spicules height.

Figure 1 shows the intensity profiles with increasing distances to the limb, as indicated by the labels. They correspond to the three transitions between the terms ${ }^{3} P_{2,1,0}$ and ${ }^{3} S_{1}$. The two transitions from the $\mathrm{J}=2$ and $\mathrm{J}=1$ levels of the ${ }^{3} P$ term are blended and form the so-called red component, at $10830.3 \AA$. The blue component, at $10829.1 \AA$, corresponds to the transition from the upper level with $\mathrm{J}=0$.

\section{Results}

The chromospheric temperature is not high enough to populate the ortho-helium levels. The radiation coming from the corona ionizes the para-helium, and the subsequent recombinations lead to an overpopulation of all the ortho-helium levels, in particular of those involved in the $10830 \AA$ transitions (Avrett et al. 1994). 
As explained by Trujillo Bueno et al. (2002), the relative strength of the blue and red components of the triplet $\left(\mathcal{R}:=I_{\text {blue }} / I_{\text {red }}\right)$ depends on the optical thickness along the ray. In the optically thin regime $\mathcal{R} \sim 0.12$. As the optical thickness (in the core of the red component) grows, this ratio also increases until it reaches a saturation value just over 1 for $\tau \sim 10$. Thus, $\mathcal{R}$ allows us to trace the population of the lower level involved in the $10830 \AA$ transitions and, consequently, can serve as an indicator of the amount of EUV coronal irradiance that reaches the chromosphere (see Centeno 2006). The values of $\mathcal{R}$ obtained from our observational data are shown in Fig. 2.

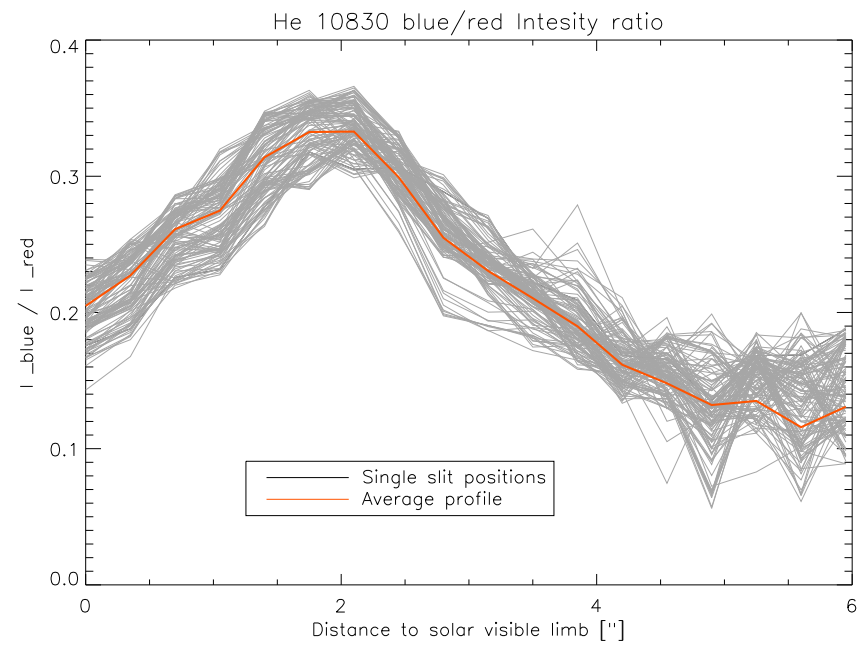

Figure 2. Observational ratio $\mathcal{R}=I_{\text {blue }} / I_{\text {red }}$. This observational signature traces the population of the lower level of the $10830 \AA$ multiplet with distance to the solar limb.

In order to make a quantitative comparison with theoretical modelling, a general non-LTE radiative transfer code (developed by $\mathrm{H}$. Uitenbroek) was used to synthesize off-the-limb $\mathrm{He}$ I $10830 \AA$ profiles in different standard model atmospheres (FAL-C, FAL-P and FAL-X). The code assumes a nominal value of the EUV coronal irradiation penetrating the chromosphere (see Centeno 2006 for details). The recombination process that follows the ionizations due to this incoming radiation populates the levels involved in the $10830 \AA$ transitions. In the outer layers of the chromosphere the density is so low that the transitions stay in the optically thin regime. With decreasing altitude the ratio $\mathcal{R}$ increases (proportionally with density) until a maximum optical thickness is reached. At even lower layers the increased extinction of the coronal irradiance leads to a reduction in the number of ionizations, and this to a decrease in optical thickness. This behavior agrees, in a qualitative manner, with that found from observations. However, the calculations using different models of the solar atmosphere are unable to reproduce the observational ratio from a quantitative point of view. In Fig. 3 we present some preliminary calculations of the comparison of the observed ratio with the one obtained from the numerical calculations. 
180 B. Sánchez-Andrade Nuño et al.: Off-limb spectroscopy of the He I 10830 A multiplet

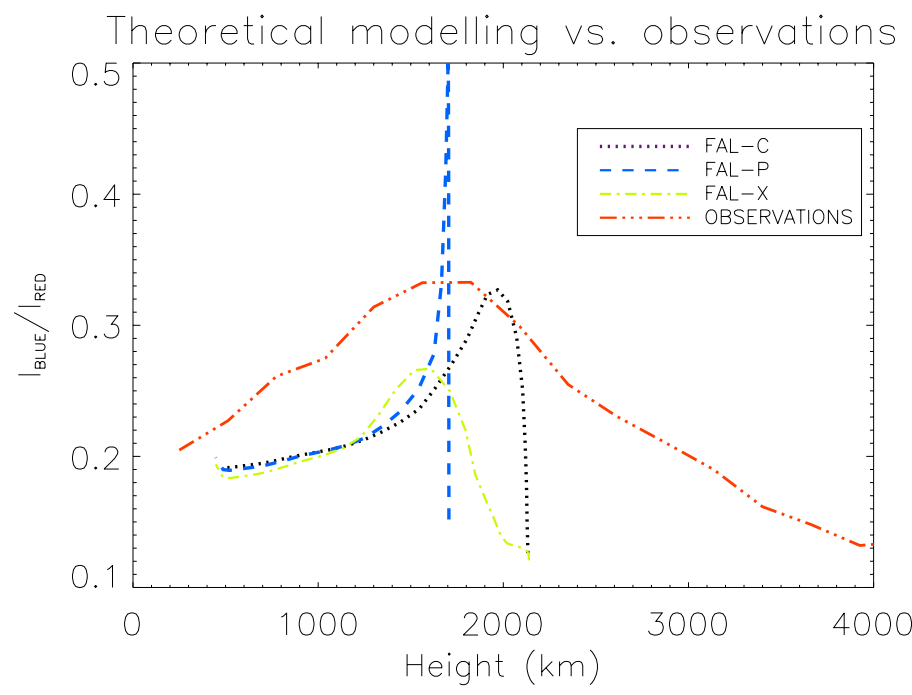

Figure 3. Observational vs. theoretical ratio $\mathcal{R}=I_{\text {blue }} / I_{\text {red }}$. This observational signature traces the population of the lower level of $10830 \AA$ multiplet with distance to the solar limb.

\section{Conclusion and future plans}

It is clear that the theoretical behavior of the ratio $\mathcal{R}$ agrees qualitatively well with observations. Quantitative comparison shows poor agreement probably due to the density profile not being adequate for spicule modelling, and the limited vertical extension of the models. New data of spicule regions near the poles and the equator, below coronal holes or coronal active regions would help to understand the detailed relation and the behavior of the He I $10830 \AA$ lines. We plan to extend this study to the full Stokes vector, in order to see the variation of the linear polarization - or even the variation of the Hanle effect - with height. Future models of the solar atmosphere should be constrained by these new observational evidences.

Acknowledgements. We would like to thank M. Collados for the extensive help and discussions, as well as for the reduction software. The help from A. Lagg was also really valuable during the reduction phase. KGP thanks the Deutsche Forchungsgemeinschaft for support through grant KN 152/29. RCE and JTB acknowledge the support of the Spanish Ministerio de Educación y Ciencia through project AYA2004-05792. Last, but not least, JTB would like to thank the Akademie der Wissenschaften zu Göttingen for having granted him the Gauss-Professur for a sabatical stay at the Institut für Astrophysik of the University of Göttingen.

\section{References}

Avrett, E. H., Fontela, J. M., \& Loeser, R. 1994, IAUS154, 35A

Centeno, R. 2006, PhD thesis, University of La Laguna.

Sterling, A. C. 2000, Solar Phys., 196, 79

Trujillo Bueno, J., Merenda, L., Centeno, R., Collados, M., \& Landi Degl'Innocenti, E. 2005 ApJ, 619, 191 


\title{
Study of polar faculae with north pole coverage of the Sun
}

\author{
J. Blanco Rodríguez ${ }^{1, *}$, O. Okunev ${ }^{1,2}$, K. G. Puschmann ${ }^{1}$, and F. Kneer ${ }^{1}$ \\ ${ }^{1}$ Institut für Astrophysik, Göttingen, Germany \\ ${ }^{2}$ Central Astronomical Observatory at Pulkovo, St. Petersburg, Russia \\ *Email: blanco@astro.physik.uni-goettingen.de
}

\begin{abstract}
We present here a description of the main characteristics of faculae at the poles of the Sun (polar faculae - PFe) observed in a series of observations covering the north solar pole. Statistics of a group of these PFe are used to estimate the contrast behaviour from $\mu=0.6$ towards the limb as well as their magnetic field. $\mathrm{H} \alpha$ is also observed to analyse the behaviour of PFe in the chromosphere.
\end{abstract}

\section{Introduction}

Polar faculae ( $\mathrm{PFe}$ ) have been observed since a long time although their properties are not yet well known. With newer instrumentation and techniques, new characteristics are discovered along with new estimations of the known ones. Due to their small scale, of the order of few $100 \mathrm{~km}$, very high spatial resolution is needed to resolve their smallest substructures. Also polarimetric observations are necessary to study their magnetic field - having a strength in the kilo-Gauss range - and polarity. In order to observe many $\mathrm{PFe}$, the best epoch is the minimum of sunspot activity since PFe have an activity cycle shifted 5-6 years with respect to the sunspot cycle. During sunspot minimum, as in these years 2005-2007, they can be observed at their maximum of occurrence and at lower latitudes than at their minimum of activity, appearing as low as $|\phi|=60^{\circ}$.

\section{Observations}

The observations for this study were made using the Göttingen Fabry-Perot interferometer (FPI) in the Vacuum Tower Telescope (VTT) at the Observatorio del Teide, Tenerife, in August 2005. The instrument was upgraded in 2005 (Puschmann et al. 2006) improving highly its performance thanks to new CCD detectors, a new Fabry-Perot etalon including controler and new software. A Stokes V polarimeter was used for obtaining spectropolarimetric data, and the Kiepenheuer Adaptive Optics System (KAOS) provided enhanced spatial resolution.

The data studied here were taken around the solar north pole. A total of 15 different subfields of $46^{\prime \prime} \times 28^{\prime \prime}$ each were observed to have a high coverage of the polar cap. The observations were carried out by wavelength scanning through two different spectral lines quasi-simultaneously, with a scan duration of 15 seconds for each line. The two lines were 
Fe I $6173 \AA$, with a Landé factor of 2.5 , and H $\alpha$ (6563 ̊). The lines were scanned through 22 spectral positions in Fe I with a step width of $23.65 \mathrm{~mA}$ and 23 spectral positions in $\mathrm{H} \alpha$ with a step width of $100.67 \mathrm{~m} \AA$ taking 15 narrowband images at each spectral position. Strictly simultaneously to these data, broadband data were taken to allow reconstruction techniques after the observations and enhance the spatial resolution of the data. The data were observed under good seeing conditions during the whole campaign, with values of the Fried parameter, $r_{0}$, around $13 \mathrm{~cm}$.

\section{Data reduction}

Before starting to analyse the data, some techniques were applied to improve the spatial resolution further than that provided by KAOS and to remove small distortions. The Göttingen speckle reconstruction code (de Boer 1996) was improved by Puschmann \& Sailer (2006) to take into account the field dependence correction of the adaptive optics providing this way a better and more constant resolution enhancement over the whole field of view. The narrowband data were reconstructuted using the method by Keller \& von der Lühe (1992) which uses the speckle reconstructed images to deconvolve the narrowband ones. Careful alignment and destretching were applied to both channels of the polarimeter (which correspond to the two circular polarisation states of light) in order to remove remaining local distortions of the images, before calculating the Stokes I and V profiles.

\section{Results}

Thanks to the use of KAOS together with the speckle reconstruction and the good seeing, we can observe PFe at very high spatial resolution, up to the diffraction limit of the VTT, around $0.2^{\prime \prime}$. At this high resolution, some conglomerates of PFe are resolved into smaller components which could not be seen this way with worse resolution. An example of the high resolution achieved are presented in Fig. 1 and Fig 3 (right panel).

Also, the high spatial resolution combined with the high coverage of the polar area, allows us to analyse the variation of the contrast, $C=\left(I_{\max }-<I>\right) /<I>$, of PFe from $\cos \vartheta=\mu \approx 0.6$ to $\mu \approx 0.2$ in this data set. The result of this study is shown in Fig. 2 where the contrast of PFe is plotted together with the contrast of the brightest granules in the quiet Sun and the rms contrast of the quiet Sun.

In this area, it can be seen that towards disk centre, the PFe contrast tends to the contrast of the brightest granules thus making PFe very difficult to be seen closer to the centre. From $\mu=0.6$ towards the limb there exists an increase of the contrast of PFe with a possible maximum around $\mu=0.4$. More data will be analysed to obtain the variation of the PFe contrast closer at the limb with higher statistical significance.

The magnetic field of PFe was studied by means of the separation of Stokes V profiles, giving approximately the same results as in previous works (Okunev \& Kneer 2004), with a value for the magnetic field strength of around 1500 Gauss. The main difference is that we have found PFe with polarity opposite to that of the global field at the solar poles. Whether these represent some isolated cases or occur regularly will be seen from the full analysis of 


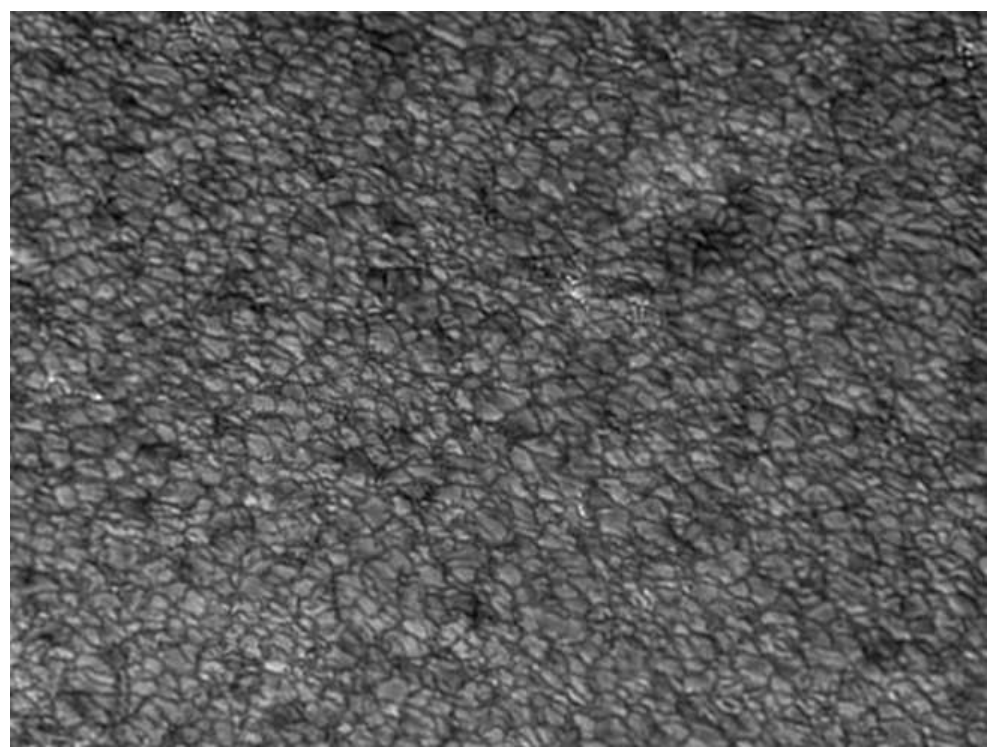

Figure 1. Speckle reconstructed image. The field of view (FOV) is $73^{\prime \prime} \times 55^{\prime \prime}$ and its centre part is located at a heliocentric angle $\vartheta=53^{\circ}$, i.e. at $\cos \vartheta=\mu=0.60$.

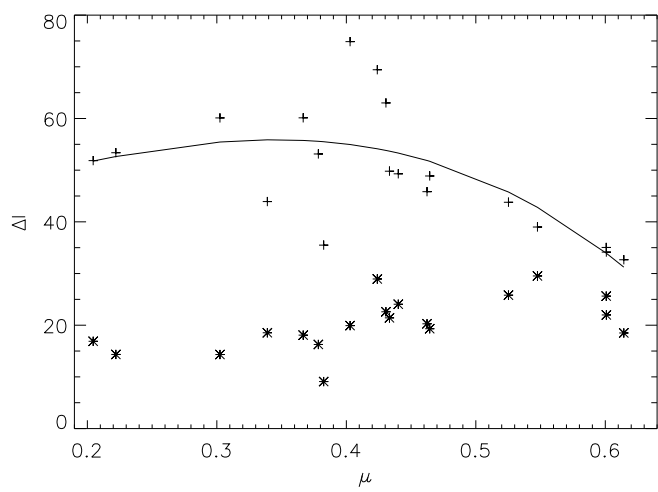

Figure 2. Measurements of contrast $\Delta I /<I>v s . \mu$. Crosses give the facular contrast and asterisks the contrast of the brightest granules.

these data with high coverage of the polar area.

Simultaneous observations in $\mathrm{H} \alpha$ had allowed us to examine PFe in higher layers of the solar atmosphere. One of the conclusions is that PFe can be clearly seen as bright structures in the blue wing of $\mathrm{H} \alpha$, making this a good proxy for finding them, although some times the brightenings in $\mathrm{H} \alpha$ are hidden due to the complex structures present in the chromosphere. 

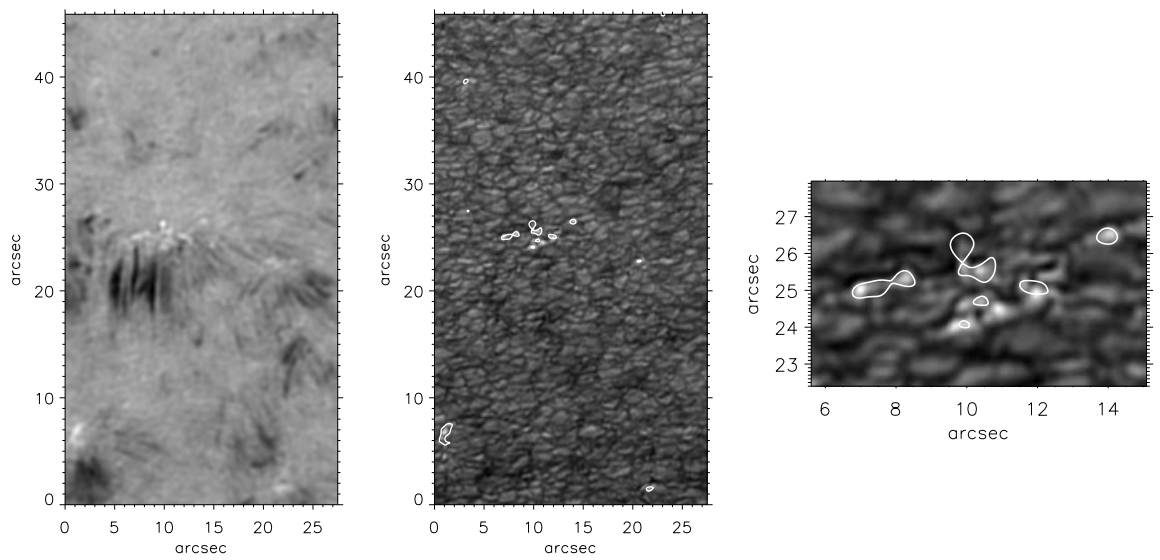

Figure 3. $\mathrm{H} \alpha$ image in the blue wing at $800 \mathrm{~m} \AA$ from line centre (left) and continuum image overlaid with contours of the brightest areas of the $\mathrm{H} \alpha$ image (centre). A zoom of the central conglomerate is presented on the right with the corresponding position of the central image.

\section{Future work}

A more in-depth analysis of the data observed in August 2005 is being performed, along with different data and ideas which will be worked out in the near future. These new studies will include the following steps:

- Analysis of observations from December 2005 in the infrared lines of Fe I at $1.56 \mu$ taken with TIP II.

- Analysis of data in Fe I $6173 \AA$ and H $\alpha$ lines observed with the Göttingen FPI in April 2006, consisting of a long time series of a specific PF in order to determine its life time and evolution.

- Comparison between data from $\mathrm{PFe}$ and equatorial faculae with data from the Göttingen FPI and TIP II.

- Study of the evolution of PFe through higher layers of the solar atmosphere with our data of $\mathrm{H} \alpha$ and images from SoHO, TRACE and Mauna Loa K-Coronameter taken at times as close as possible to our data.

- Investigation of the possible relation of PFe with the fast solar wind from the polar coronal holes.

Acknowledgement. JBR, OO, and KGP thank the Deutsche Forschungsgemeinschaft for financial support through grants 418 SPA-112/15/04, 436 RUS 17/61/05 and Kn152/29, respectively.

\section{References}

de Boer, C. R. 1996, A\&AS, 120, 195

Keller C. U. \& von der Lühe O. 1992, A\&A, 261, 321

Okunev, O. \& Kneer, F. 2004, A\&A, 425, 321

Puschmann, K. G. \& Sailer, M. 2006, A\&A, 454, 1011

Puschmann, K. G., Kneer, F., Seelemann, T. \& Wittmann, A. D. 2006, A\&A, 451, 1151 


\title{
Effects of the "false" magnetic-field imbalance in solar active regions
}

\author{
I. V. Oreshina* and B. V. Somov \\ Sternberg Astronomical Institute of Moscow State University, Moscow, Russia \\ *Email: ivo@sai.msu.ru
}

\begin{abstract}
The imbalance of magnetic fluxes of opposite polarities in solar active regions (ARs) is investigated. On the base of a topological magnetic-field model, we reveal some causes leading to the observations of the imbalance even if real magnetic fluxes are balanced.
\end{abstract}

\section{Introduction}

The question of whether or not an imbalance of the magnetic field is related to solar flares and coronal mass ejections (CME) is widely debated. Conclusions are often contradictory (Shi \& Wang 1994; Gaizauskas et al. 1998; Tian et al. 2002; Wang et al. 2002; Tian \& Liu 2003; Green et al. 2003; Chumak et al. 2004).

The aims of our study are (1) to demonstrate several effects of "false" magnetic imbalance, i.e. to reveal some causes leading to the observation of imbalance even if real magnetic fluxes are balanced in solar ARs, (2) to propose an explanation of some observational data.

\section{Model}

We assume that the magnetic field is potential and use the magnetic-field topological model (see, for example, Sweet 1958; Gorbachev \& Somov 1989; Mandrini et al. 1991; Somov et al. 2005), where the field is created by "effective sources" located below the photosphere.

The imbalance value $I$ of an AR is estimated with the formula (Choudhary et al. 2002)

$$
I=\frac{\left|\Phi_{n}\right|-\left|\Phi_{p}\right|}{\left|\Phi_{n}\right|+\left|\Phi_{p}\right|} \cdot 100 \%,
$$

where $\Phi_{n}$ and $\Phi_{p}$ are the integrated negative (away from the observer) and positive (toward the observer) magnetic fluxes, respectively.

\section{Dependence of the imbalance on the location of active regions on the solar disc}

Green et al. (2003) have studied magnetic field changes in four ARs during seven days of their disc passage. On the base of MDI/SOHO magnetograms, they have revealed two ef- 


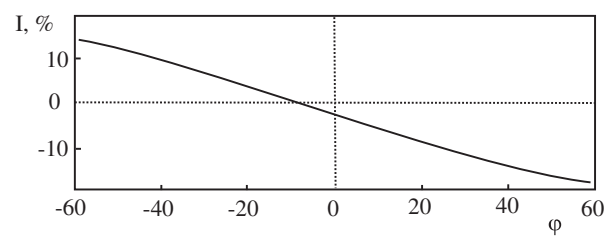

Figure 1. The imbalance change during the AR 8086 passage through the solar disc.

fects: (1) the imbalance increases when ARs move toward the solar limb and (2) the imbalance sign changes when ARs cross the central meridian. As only line-of-sight magnetograms were used in their work, the above authors could not explain the observations. But they supposed that the observed effects could be due to the presence of horizontal field components (i.e. parallel to the photospheric surface). Let us test this hypothesis using the topological model.

Let us consider the AR 8086 which passed the solar disc during September 15-21, 1997 at the latitude of $27^{\circ} \mathrm{N}$. We model the observed MDI/SOHO magnetogram using 25 magnetic sources and calculate the imbalance change during the AR passage across the solar disc (i.e. imbalance dependence on the longitude $\varphi$ ). The results are presented in Fig. 1.

The topological model can explain the dominating negative flux in the eastern hemisphere, the imbalance decrease as the AR 8086 moves towards the central meridian, the change of the imbalance sign in the vicinity of the central meridian and its new increase as the AR moves towards the western limb. The maximum imbalance values are $14.4 \%$ near the eastern limb and $18.6 \%$ near the western limb. Green et al. (2003) report about $15 \%$ for AR 8086 . So, both results (observed and theoretical ones) are in good agreement. This allows us to conclude that imbalance effects, reported by Green et al. (2003), can be observed even if the real fluxes are balanced. These effects are due to the 3D structure of the photospheric magnetic field.

\section{Imbalance dependence on the size and location of the computational box}

Investigating the connection of imbalance with flares and CMEs, many authors carried out their calculations in only small regions representing a part of an AR where the magnetic field is the most intense (Wang et al. 2002; Green et al. 2003; Yurchishin et al. 2004). So, it is important to estimate the imbalance dependence on the size and location of the computational box.

Choudhary et al. (2002) have studied 137 ARs located near the disc center and found that the average imbalance value is about $9.5 \%$, i.e. in good agreement with our examples. Moreover, $70 \%$ of ARs show imbalance smaller than $10 \%$. The topological model reveals one of the possible causes of this effect: When a computational box is small, some part of the magnetic flux does not cross it and this flux is not included in the calculations and leads to the observation of "false" imbalance (Fig. 2). 

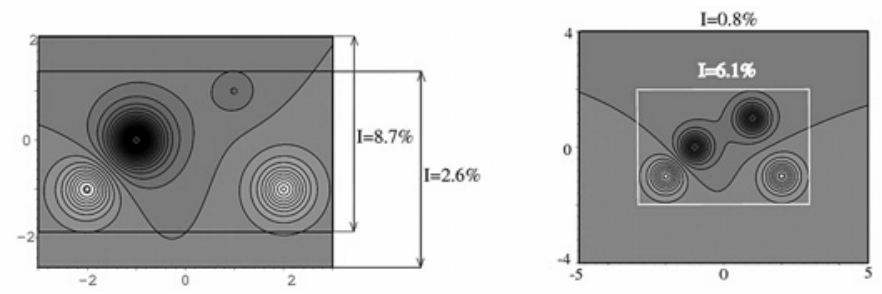

Figure 2. Imbalance dependence on choice of the computational box. The left panel illustrates a change of the imbalance as a result of the box displacement with respect to the sources: A small displacement leads to $6 \%$-change in the imbalance value. The right panel shows that an enlargement of the box leads to a significant imbalance decrease even if all the sources are covered by the computational box in both cases (for white and black rectangle as a computational box).
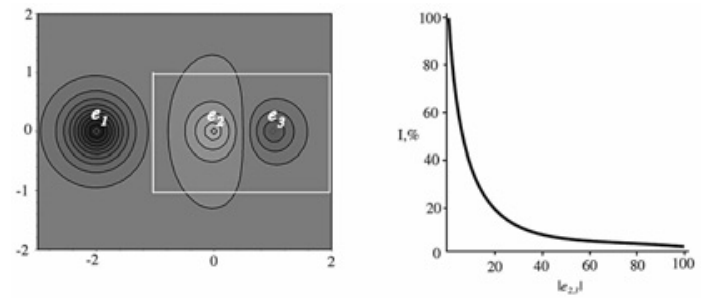

Figure 3. Imbalance change during emergence of a new balanced magnetic flux $e_{2}=-e_{3}$ in the field of the source $e_{1}=-50$. The white rectangle on the left panel shows the computational box.

\section{Emergence of a new balanced magnetic flux in a non-homogeneous back- ground field}

Let the non-homogeneous background field be created by the source $e_{1}=-50$. A new balanced flux is modelled by two sources $e_{2}=-e_{3}$ (Fig. 3).

Let us gradually change the source intensities from 0 to 100 for the source $e_{2}$ and from 0 to -100 for the source $e_{3}$ so that the total flux of these two sources is always equal to zero. Figure 3 presents a magnetogram for the case $e_{2}=-e_{3}=20$. The white rectangle on the left panel shows the computational box. The imbalance value is shown on the right panel. Note that the imbalance is a non-linear function of the magnetic-flux value and changes within large limits in the area of flux emergence.

Because magnetic fields of real ARs have much more complex configurations, this model demonstrates that it is very difficult to estimate the real magnetic-field imbalance of new emerging fluxes.

\section{Summary}

On the base of the topological magnetic-field model, the imbalance of magnetic fluxes of opposite polarities in solar active regions (ARs) is investigated. We reveal some causes leading to the observation of the imbalance even if the real magnetic fluxes are balanced: 
- An explanation of the MDI data showing an imbalance increase when ARs move toward the solar limb as well as an explanation of the fact that the imbalance sign changes when ARs cross the central meridian are proposed. The effects can be due to the non-radial structure of the photospheric magnetic field.

- Several AR models created by balanced magnetic sources are developed. It is shown that even in this case, the imbalance value can be significant (about several percents) depending on the size and location of the computational box. Such imbalance values are observed in $70 \%$ of ARs by Choudhary et al. (2002).

- The emergence of a new balanced magnetic flux in a non-homogeneous background field is studied. It is shown that the imbalance is a non-linear function of the magneticflux value and changes within large limits in the area of flux emergence.

These properties of the magnetic-field imbalance in ARs must be taken into account when one analyses magnetographic observations of the Sun.

Acknowledgements. I. V. Oreshina would like to thank the Deutsche Forschungsgemeinschaft for a travel grant and the organizers of the Workshop "Modern Solar Facilities - Advanced Solar Science" for the hospitality in Göttingen. This research was supported by the RFBR grant 04-02-16125-a.

\section{References}

Choudhary, D. P., Venkatakrishnan, P., \& Gosain, S. 2002, ApJ, 573, 851

Chumak, O., Zhang, H., \& Gou, J. 2004, Astron. Astrophys. Transactions, 23(6), 525

Gaizauskas, V., Mandrini, C. H., Démoulin, P., et al. 1998, A\&A, 332, 353

Gorbachev, V. S. \& Somov, B. V. 1989, Solar Phys.,117, 77

Green, L. M., Démoulin, P., Mandrini, C. H., \& Van Driel-Gesztelyi, L. 2003, Solar Phys., 215, 307

Mandrini, C. H., Démoulin, P., Hénoux, J.-C., \& Machado, M. E. 1991, A\&A, 250, 541

Shi, Z. \& Wang, J. 1994, Solar Phys., 149, 105

Somov, B. V., Kosugi, T., Oreshina, I.V., \& Lyubimov, G. P. 2005, Advances in Space Research, 35, 1712

Sweet, P. A. 1958, in IAU Symp. 6: Electromagnetic Phenomena in Cosmical Physics, ed. B. Lehnert (Cambridge University Press), 123

Tian, L., Liu, Y., \& Wang, J. 2002, Solar Phys. 2002, 209, 361

Tian, L. \& Liu, Y. 2003, A\&A, 406, 337

Wang, H., Spirock, T. J., Qiu, J., Ji, H., Yurchishin, V., Moon, Y.-J., Denker, C., \& Goode, P. R. 2002, ApJ, 576, 497

Yurchyshyn, V., Wang, H., Abramenko, V., Spirock, T., \& Krucker, S. 2004, ApJ, 605, 546 


\title{
Magnetic source of the solar cycle variation of the Mn I $539.4 \mathrm{~nm}$ line
}

\author{
S. Danilovic ${ }^{1,3, *}$, S. K. Solanki ${ }^{1}$, W. Livingston ${ }^{2}$, N. Krivova ${ }^{1}$, and I. Vince ${ }^{3}$ \\ ${ }^{1}$ Max-Planck-Institut für Sonnensystemforschung, Katlenburg-Lindau, Germany \\ ${ }^{2}$ National Solar Observatory, Tucson, USA \\ ${ }^{3}$ Astronomical Observatory, Belgrade, Serbia \\ *Email: danilovic@mps.mpg.de
}

\begin{abstract}
As a part of the long term program at KPNO, the Mn I $539.4 \mathrm{~nm}$ line has been observed for nearly three solar cycles using the McMath telescope and the $13.5 \mathrm{~m}$ spectrograph in double pass mode. These full-disk spectrophotometric observations revealed an unusually large amplitude change of its parameters over the solar cycle and its correlation with Ca II K intensity. One of the proposed explanations for this phenomenon is the optical pumping by the Mg II $\mathrm{k}$ line. With this work we would like to show that this may not be the main mechanism behind the change. We reconstructed the changes of the line parameters using a model that takes into account only changes of the daily surface distributions of magnetic field. This model has already been used to successfully model total solar irradiance. We now apply it for modelling the Mn I line, as well as its neighboring Fe I line using exactly the same value of the free parameter as used for the reconstruction of total solar irradiance. We reproduce well the Mn I and Fe I line changes over the cycle purely with LTE modelling. This indicates that optical pumping of the Mn I line by Mg II $\mathrm{k}$ is not the main cause of its solar cycle change and sets an independent constraint on solar irradiance models.
\end{abstract}

\section{Introduction}

The Mn I $539.47 \mathrm{~nm}$ line is interesting for several reasons. The hyperfine broadening, due to interaction of the electronic shell with the nuclear spin, makes it insensitive to non-thermal motions and hence more sensitive to the temperature in the photosphere (Elste \& Teske 1978; Elste 1987). Observations show that the line becomes stronger in sunspots and weaker in plage and network (Andriyenko 2004; Vince et al. 2005a; Malanushenko et al. 2004; Vince et al. 2005b). Although calculations confirm its photospheric origin (Gurtovenko \& Kostyk 1989; Vitas 2005), the 'Sun-as-a-star' observations (Livingston \& Wallace 1987; Livingston 1992) exhibit a significant cyclic dependence, not typical for photospheric lines. As a possible explanation Doyle et al. (2001) proposed optical pumping by Mg II k, which could be the reason why this Mn line mimics the change seen in Mg II k. On the other hand, this high correlation may be a consequence of the common source of change in both cases: bright magnetic elements - faculae and network (Danilovic \& Vince 2005). This work aims to test the latter hypothesis. 


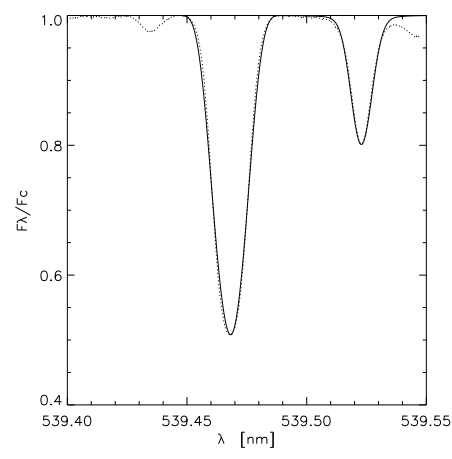

Figure 1. Comparison of Mn I 539.47 and Fe I $539.52 \mathrm{~nm}$ line profiles observed on May 19th 1999 (dotted) with the synthesized ones (solid).

\section{Observations and modelling}

Observed 'Sun-as-a-star' spectra obtained with the Kitt Peak 13.5 m scanning spectrometer in double pass mode are used (Livingston \& Wallace 1987). The first set of observations was taken from 1979 to 1992, after which the grating was changed. Implementation of the new, larger grating eliminated the loss of light from the Sun's poles. This introduced a 'jump' in equivalent width (EW) and central depth (CD) of lines. There followed an experimental period with the new grating and the changing of instrumental parameters. This lasted till 1996 when the system was fixed and observations are performed regularly again (Penza et al. 2006).

To reconstruct the time variability of the Mn I line, the SATIRE (Spectral And Total Irradiance Reconstruction) model has been used (Fligge et al. 2000; Krivova et al. 2003; Wenzler et al. 2005, 2006). This model gave excellent agreement of calculated and observed total solar irradiance (TSI) on both short and long time scales in the period of 1978 to 2003. In the model, the position and flux density of magnetic features are extracted from fulldisk magnetograms and continuum images. These magnetic features are classified as either sunspot or faculae. Every feature is represented by a plane-parallel model atmosphere with a unique temperature stratification (Unruh et al. 1999). To account for the unresolved magnetic elements, the model introduces a filling factor parameter, which describes the fractional coverage of a pixel by a facular model, which linearly increases with magnetogram signal. The limiting value for which the pixel is completely covered by faculae is a free parameter. We stress that in the present analysis exactly the same value of this free parameter has been used as employed in earlier studies to model total solar irradiance change for the same period.

Time independent emergent intensities of the Mn I line and the neighboring Fe I line are calculated for each model atmosphere for various heliocentric angles in LTE using the SPINOR code (Frutiger et al. 2000). No magnetic field is introduced in the calculations. The hyperfine structure of the Mn I line is included as blends with displacement calculated using the hyperfine constants from Davis et al. (1971) and relative intensities of the components from Condon \& Shortley (1963). Components, that are closely spaced with respect to the total splitting, have been combined to reduce the computing effort. 

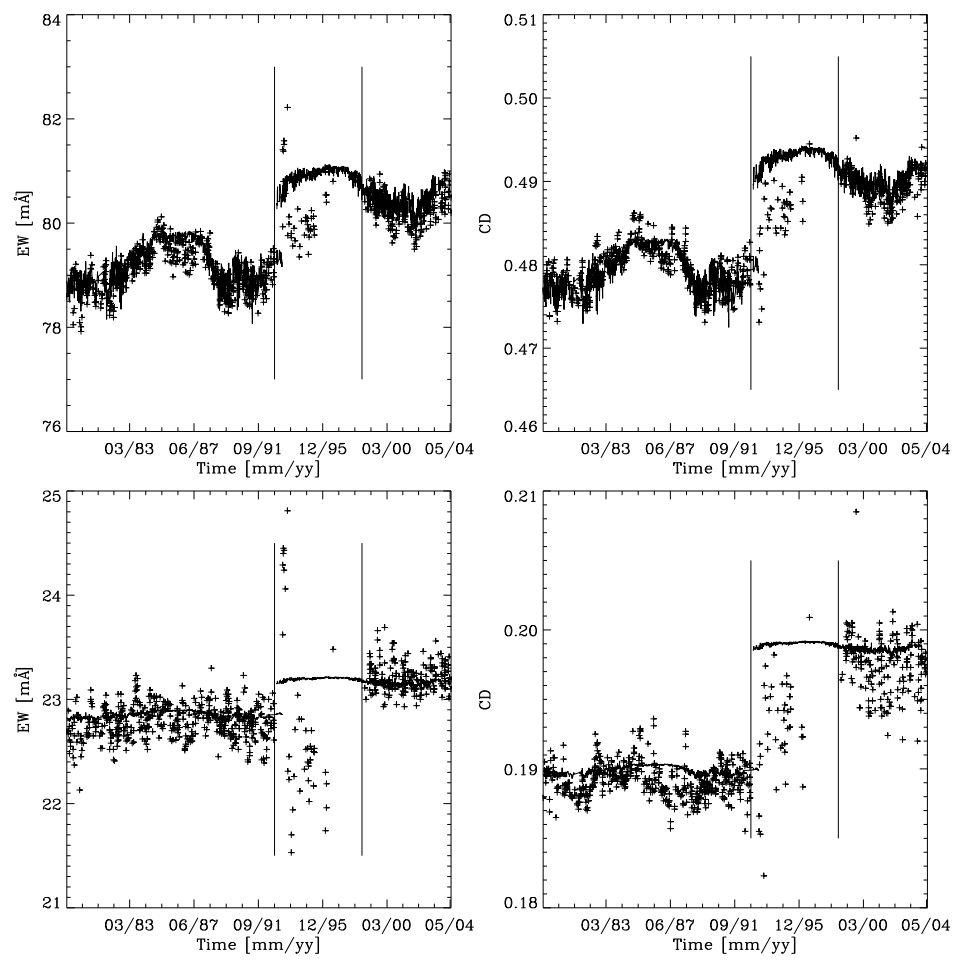

Figure 2. Equivalent widths (left) and central depths (right) of the Mn I $539.47 \mathrm{~nm}$ (up) and its neighboring Fe I $539.52 \mathrm{~nm}$ line (down) extracted from KPNO observations (crosses) and calculated using our model (solid line). Experimental period is bounded by vertical solid lines.

\section{Results}

In order to be consistent with the instrument setup, in the period before 1992 the flux is computed neglecting contributions from the poles, which were missed by the old grating. By fitting the line parameters for several days during cycle minimum we estimated what portion of the disk is missed. The obtained fit of the synthesized to the observed spectrum of Mn and its neighboring Fe line is shown in Fig. 1. The whole interval covered by the observations is given in Fig. 2. Vertical solid lines mark the period during which the instrumental setup experimentation lasted. Overplotted on the data are the modelled values, which also display the jump in CD of both lines because of different influence of the rotation on the line profiles before and after 1992. The behavior of both lines is well reproduced. Correlation coefficients for EW and CD of the Mn I 539.47 line are 0.94 and 0.96, respectively. 


\section{Conclusion}

The solar cycle variation of Mn I $539.47 \mathrm{~nm}$ line parameters have been modelled in LTE taking into account only changes of the surface distribution of the solar magnetic features. As the solar disk coverage by bright magnetic features increases toward solar cycle maximum, the Mn I line becomes weaker in the 'Sun-as-a-star' spectrum. In this way it mimics the behavior of $\mathrm{Ca}$ II K and Mg II k lines which are well known plage/faculae indicators. Good correlation with observations is obtained without taking into account the influence of the $\mathrm{Mg}$ II $\mathrm{k}$ line. This puts the optical pumping hypothesis in the background. Although energetic transfer between Mn I and Mg II exists, we do not expect it to be significant for the solar cyclic variations of the Mn I $539.47 \mathrm{~nm}$ line.

Acknowledgements. This work was partly supported by the Deutsche Forschungsgemeinschaft, $D F G$ (project number SO-711/1-1) and by the Ministry of Science and Environmental Protection of the Republic of Serbia (project number 146003).

\section{References}

Andriyenko, O. V. 2004, in Multi-Wavelength Investigations of Solar Activity, IAU Symposium, Vol. 223. Cambridge, UK: Cambridge University Press, p. 85

Condon, E. U. \& Shortley, G. H. 1963, The Theory of Atomic Spectra, University Press, Cambridge, p. 242

Danilovic, S. \& Vince, I. 2005, Mem. Soc. Astron. Ital., 76, 949

Davis, S. J., Wright, J. J., \& Balling, L. C. 1971, PhysRev A, 3, 1220

Doyle, J. G., Jevremovic, D., Short, C. I., Hauschildt, P. H., Livingston, W., \& Vince I. 2001, A\&A, 369, L13

Elste, G. 1987, Solar Phys., 107, 47

Elste, G. \& Teske, R. G. 1978, Solar Phys., 59, 275

Fligge, M., Solanki S. K., \& Unruh, Y. C. 2000, A\&A, 353, 380

Frutiger, C. C., Solanki, S. K., Fligge, M., \& Bruls, J. H. M. J. 2000, A\&A, 358, 1109

Gurtovenko, E. A. \& Kostyk, R. I. 1989, Fraunhofer Spectrum and System of Solar Oscillator Strengths, Naukova dumka, Kiev

Krivova, N. A., Solanki S. K., Fligge, M., \& Unruh Y. C. 2003, A\&A, 399, L1

Livingston, W. \& Wallace, L. 1987, ApJ, 314, 808

Livingston W. 1992, in Proceedings of the Workshop on the Solar Electromagnetic Radiation Study for Solar Cycle 22 (ed. R. F. Donnelly), 11

Malanushenko, O., Jones, H. P., \& Livingston, W. 2004, in Multi-Wavelength Investigations of Solar Activity, IAU Symposium, Vol. 223. Cambridge, UK: Cambridge University Press, p.645

Penza, V., Pietropaolo, E., \& Livingston, W. 2006, A\&A, 454, 349

Unruh, Y. C., Solanki, S. K., \& Fligge, M. 1999, A\&A, 345, 635

Vince, I., Ludmany, A., Vince, O., \& Andriyenko, O. V. 2005a, Solar Phys., 229, 273

Vince, I., Gopasyuk, O., Gopasyuk, S., \& Vince, O. 2005b, Serb. Astron. J., 170, 115

Vitas, N. 2005, Mem. Soc. Astron. Ital. Suppl., 7, 164

Wenzler, T., Krivova, N. A., \& Solanki, S. K. 2005, A\&A, 432, 1057

Wenzler, T., Solanki, S. K., Krivova, N. A., \& Fröhlich, C. 2006, A\&A, 460, 583 


\title{
Reconstruction of irradiance from 2D magnetograms and modification of the $1 / \mu$ - correction paradigm
}

\author{
A. Reiners ${ }^{1,2, *}$, G. Basri ${ }^{3}$, K. Soto ${ }^{3}$, F. Ramos Stierle ${ }^{3}$, and T. Lewis ${ }^{3}$ \\ ${ }^{1}$ Hamburger Sternwarte, Hamburg, Germany \\ ${ }^{2}$ Max-Planck-Institut für Sonnensystemforschung, Katlenburg-Lindau, Germany \\ ${ }^{3}$ Astronomy Department, University of California at Berkeley, USA \\ *Email: areiners@hs.uni-hamburg.de
}

\begin{abstract}
The key to understanding total Solar Irradiance (SI) variability is understanding the mechanisms by which continuum contrast is affected by the distribution of magnetic flux across the surface of the Sun. The Michelson Doppler Imager (MDI) instrument on the Solar and Heliospheric Observatory ( $\mathrm{SOHO}$ ) has measured full-disk magnetograms along with contemporaneous continuum images. These pairs of images can be used to study the correlation of continuum irradiance changes with magnetogram signal over the entire disk. To study these effects, however, we must first correct MDI magnetograms for geometrical effects near the limb. We derive this correction using the assumption that the distribution of true magnetic flux density (MFD) in the active latitudes should exhibit the same average behavior irrespective from the viewing angle. In a series of 106 magnetograms, we analyze annular rings at various limb angles, $\mu$, and follow individual active regions over their full disk passage. We are able to find a simple function which successfully corrects the magnetograms over the full disk, with a minimal number of overcorrected points at the extreme limb. It is a major improvement over the standard $1 / \mu$ correction. We then examine the relationship between continuum images (with limb darkening removed) and contemporaneous corrected magnetograms over all limb angles. Using a 2D function, we can predict the continuum contrast at any given disk position as a function of measured magnetic flux. We demonstrate the extent to which this procedure reproduces actual solar features.
\end{abstract}

\section{Introduction}

Solar irradiance variations are caused by magnetic fields on the solar surface. Large spots appear darker than the continuum while faculae and other small-scale features appear bright. The brightness of solar faculae becomes strongly pronounced at the limb where they become more easily visible, given their 3D granular nature (Keller et al. 2004; Carlsson et al. 2004; De Pontieu et al. 2006), thus it is the very limb that has to be investigated if one wants to understand the global irradiance variation of the Sun and active stars. The observational connection between SI and magnetic fields is achieved through the comparison of contemporaneous observations of the solar disk between an intensity image and a magnetogram (an MFD image of the Sun). The 2D disk, however, is a projection of the 3D surface, and since a magnetogram only measures the component along the line of sight, different parts of the magnetic flux are observed across the disk. 
194 Reiners et al.: Irradiance from 2D magnetograms \& modification of the $1 / \mu$-paradigm
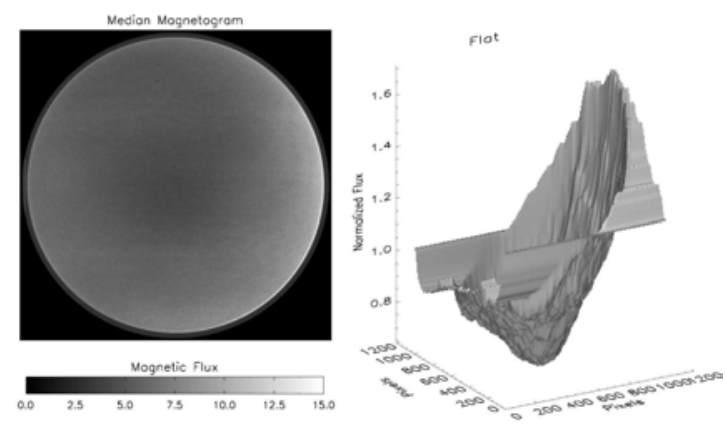

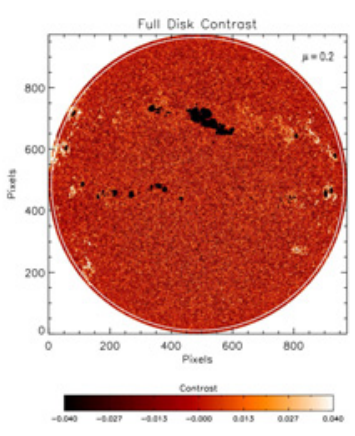

Figure 2. Solar irradiance image. The boundary of $\mu<0.2$ is indicated by a white ring (area outside: $4 \%$ of the disk)

Figure 1. The median magnetogram produced from 363 magnetograms in a quiet period of 1997.

The projection effect is usually accounted by dividing the magnetogram values by $\mu=\cos \theta$, where $\theta$ is the angle between the surface normal and the line of sight. The " $1 / \mu-$ correction" assumes that all field lines are radially directed. This is clearly not true for active periods, and it overcorrects the magnetic field lines near the limb (the reason why former studies had neglected limb-near regions on the solar disk). We started an effort to investigate a large number of $2 \mathrm{D}$ irradiance and magnetogram images from the SOHO satellite with the goal of extending analyses of magnetograms and solar faculae to the very limb.

\section{Flat field and limb area}

We investigate $2 \mathrm{D}$ continuum images together with full disk magnetrograms from the SOHO MDI. In order to follow irradiance variations over the full disk, we aim to correct for the geometric projection in the magnetograms (the continuum images are corrected for limb darkening). As a first step, we construct a "flat field magnetogram" from 363 magnetograms in a quiet period in 1997. The result is shown in Fig. 1.

After correcting for the detector sensitivity by flat fielding, one has to correct for projection effects on the solar surface. Classically, a " $1 / \mu$ - correction" is applied to the data assuming that the magnetic field lines are radial. This approach is known to overcorrect the very limb regions, and areas with $\mu<0.2$ are generally cut off. We show in Fig. 2 the area that is neglected by such an approach. The neglected area covers $4 \%$ of the disk, and it is the region where faculae have the highest contrast. The classical approach cuts off the part of the disk where the crucial information on facular contrast and the relation between magnetic flux and continuum intensity is contained.

\section{A new limb correction}

We searched for a different way to correct for the projection of the magnetic field lines. We construct a subset of a data set from 106 active period magnetograms that ensures a consistent distribution of low and high MFDs at all bins of $\mu$. Our requirement for the correction is that the distribution of MFDs should be equal within all bins of $\mu$. 

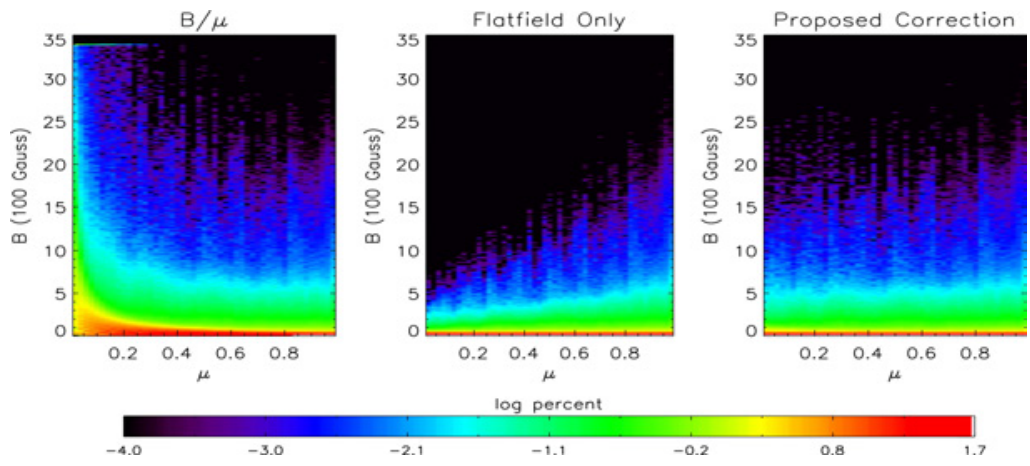

Figure 3. Distribution of magnetic field strength after different corrections (see text).

We found the following correction that works much better than the standard approach:

$$
B=\left\{\begin{array}{cl}
B_{\text {Obs }} \text { no correction } & B_{\text {Obs }}<15 \mathrm{G} \\
\frac{B_{\text {Obs }}}{0.9+0.1 \mu} & 15 \mathrm{G}<B_{\text {Obs }}<30 \mathrm{G} \\
\frac{B_{\text {obs }}-30 \mathrm{G}}{0.25+0.75 \mu}+30 \mathrm{G} & B_{\text {Obs }}>30 \mathrm{G}
\end{array}\right.
$$

In Fig. 3 we show the distribution of magnetic field strength for three different correction methods. The center panel shows the observed distribution after correcting for the flat field. The projection effect is clearly visible; weaker field strengths are observed at the limb (low $\mu$ ), and fields tend to be stronger in the center $(\mu=1)$. In the left panel, the result for the " $1 / \mu$ - correction" is shown. While this method provides a magnetic field distribution that is nearly flat at the center, it overcorrects the fields near the limb, which is clearly visible in the very strong values at low limb angle. Using the empirical correction proposed in this study rather than the " $1 / \mu$ - correction" paradigm, the distribution of magnetic field strength becomes flat over the entire disk (right panel in Fig. 3). This distribution complies with the assumption that the field strength should not depend on the projection angle.

\section{Irradiance contrast as a function of the magnetic flux over the full disk}

After correcting for the projection effect, we calculate the contrast

$$
C=\frac{I-<I_{\text {quiet }}>}{<I_{\text {quiet }}>}
$$

More than 25 million points from the data set are used. We neglect points with high negative contrast $(C<-0.8)$ since magnetograms are known to fail here, and we neglect points with zero contrast and high MFD since these are likely errors in the continuum measurement. Only $0.007 \%$ of the points are neglected. In Fig. 4, our results are plotted as a function of $\mu$ and MFD similar to the plots by Ortiz et al. (2002). We show the entire distribution in $\mu$ without neglecting the limb. A clear dependence on both MFD and $\mu$ appears in the plots. Finally, we fit a polynomial surface to the contrast as a function of $\mu$ and MFD. This function is used to predict the continuum contrast from 2D magnetograms. Figure 5 shows the results for a spot group observed at a mean $\mu=0.24$; the left panel shows the corrected magnetogram, the right panel shows the observed continuum, and in the center panel we show our continuum prediction calculated from the magnetogram. 
196 Reiners et al.: Irradiance from 2D magnetograms \& modification of the $1 / \mu$-paradigm
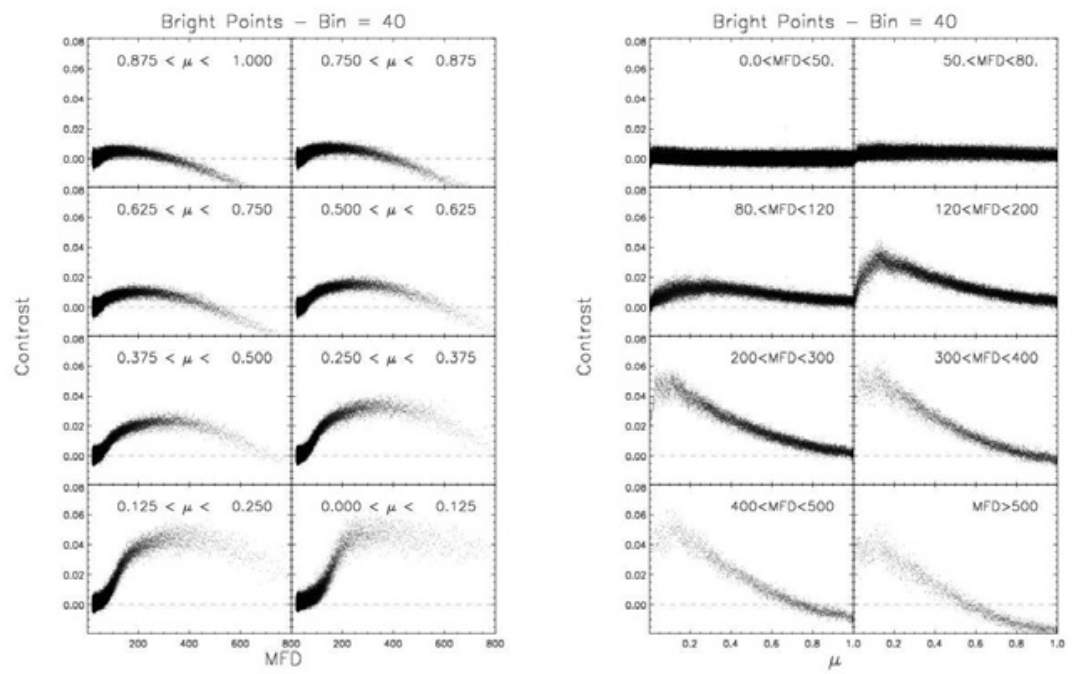

Figure 4. (Left) Contrast as a function of MFD and (right) as a function of all $\mu$ 's (full disk).
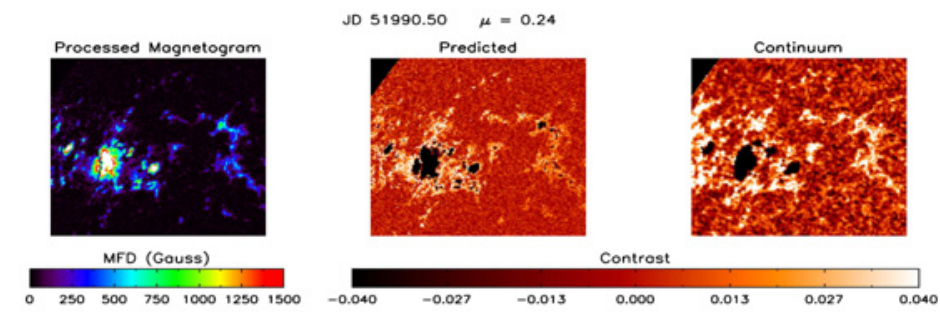

Figure 5. Left: Magnetogram; Right: Continuum; Center: Continuum predicted from magnetogram.

This methodology will be useful for predicting stellar microvariability, given assumed magnetic configurations. Additional analysis of the relations between magnetic field, contrast, and limb angle for umbrae, penumbrae, faculae and pores is needed. These relations will provide a more accurate tool to generate light curves of stars, it will give insight into stellar activity that will cause to hide the detection of Super-Earths and Earth-like planets in the data set of the missions COROT and Kepler.

Acknowledgements. AR has received research funding from the European Commission's Sixth Framework Programme as an Outgoing International Fellow (MOIF-CT-2004-002544).

\section{References}

Carlsson, M., Stein, R. F., Nordlund, Å, \& Scharmer, G. B. 2004, IAUS, 223, 233

De Pontieu, B., Carlsson, M., Stein, R. F., et al. 2006, ApJ, 646, 1405

Keller, C. U., Schüssler, M., Vögler, A., \& Zakharov, V. 2004, ApJ, 607, L59

Ortiz, A., Solanki, S. K., Domingo, V., Fligge, M., \& Sanahuja, B. 2002, A\&A, 388, 1036 


\title{
Variations in solar indices during the $23^{r d}$ solar cycle
}

\author{
N. Oklay ${ }^{1,2}$ \\ ${ }^{1}$ Istanbul University, Department of Astronomy \& Space Sciences, Istanbul, Turkey \\ ${ }^{2}$ Max-Planck-Institut für Sonnensystemforschung, Katlenburg-Lindau, Germany \\ Email: nilda@gmx.com.tr, oklay@mps.mpg.de
}

\begin{abstract}
The $23^{\text {rd }}$ solar cycle is investigated using 6 different activity indices. The variations and comparisons of these indices are presented, and the dates of beginning, first, second maximum, and minimum between maxima are calculated to see how the cycle behaves in each index. The date $01 / 01 / 1995$ is the beginning date for the study of all indices, but the ending dates vary from index to index.
\end{abstract}

\section{Introduction}

The Sun, which is the nearest star to Earth, is affecting our natural human life. Understanding the underlying mechanism of the variations of the Sun and their effects to Earth has vital importance. The solar cycle is one of the phenomena which show us that the Sun varies. Some solar activity indices are determined to reveal the characteristics of solar activity.

\section{Relative sunspot numbers (RSN)}

The $\mathrm{RSN}^{1}$ reaches its first maximum 3.9 yrs after the beginning with an increase 15.27 times the initial value 12. After the first maximum, the index values decrease by $13.70 \%$ (of that maximum), but they begin to rise again after the $57^{\text {th }}$ month until the second maximum. After the second maximum, the RSN values decrease by $27.20 \%$ (of that maximum) by the end of the first year, $44.62 \%$ for the second year, and $17.70 \%$ for the third year. The two maxima of this index have the same amplitude. I applied Fast Fourier Transform (FFT) to daily RSN data to calculate the periodicity of the cycle and to determine other small periodicities in this index.

\section{Flare index (FI)}

The $\mathrm{FI}^{2}$ has a higher first maximum. This shows that the flares can be generated during the first maximum, and it seems that the generation is decreasing towards the end of the cycle

\footnotetext{
${ }^{1}$ from Istanbul University Observatory (IUO)

${ }^{2}$ from Boğazici University Kandilli Observatory
} 


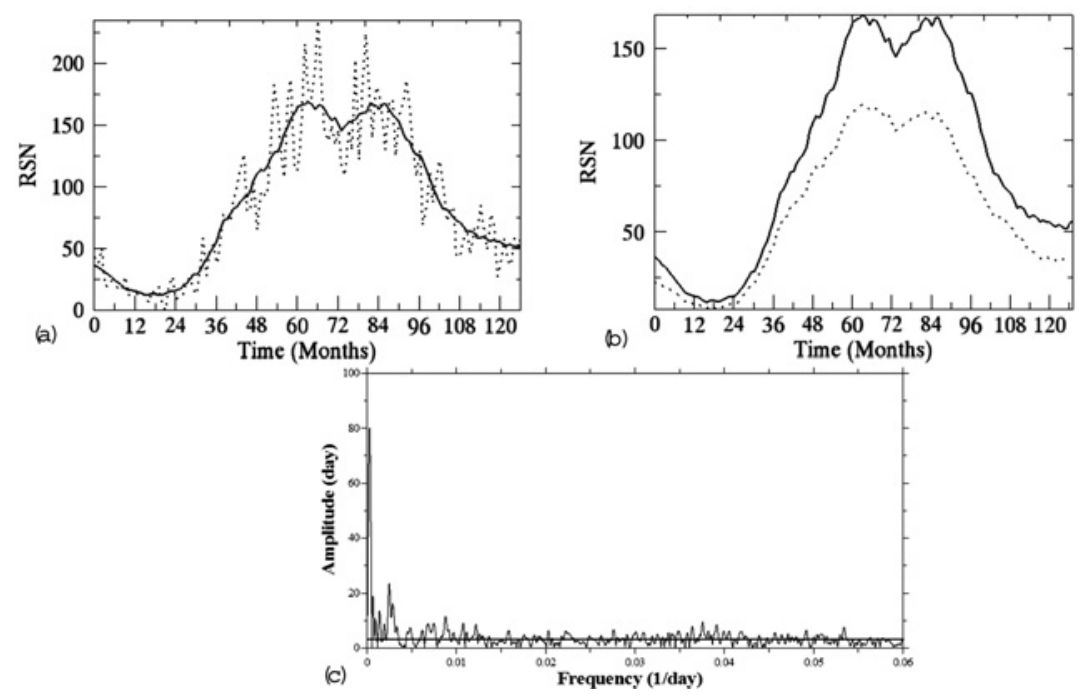

Figure 1. (a) Variations of the RSN during the $23^{\text {rd }}$ solar cycle. The dotted line shows monthly mean values; the solid line shows smoothed monthly mean values. (b) Comparison of IUO's RSN data with Solar Influences Data Centers (SIDC) data. The solid line shows the smoothed monthly means of the SIDC data; the dotted line shows the smoothed monthly means of the IUO data. (c) Power spectrum, which is obtained from FFT.

because the minimum between maxima is really faint.

\section{The total area of sunspot groups (TSA)}

The TSA ${ }^{3}$ index gives us some information about lifetime, size changes and evolution of the sunspot groups. The amplitude of the second maximum in this index is $24 \%$ higher than the first one. Thus, we can see that the sunspot groups of the second maximum are more stable than those of the first maximum and will break up into smaller groups to a lesser extent; they also include bigger, more stable spots.

\section{The mean solar magnetic fields (MMF)}

The beginnining value of $\mathrm{MMF}^{4}$ is $9 \mu \mathrm{T}$ and rises 4 times within 40 months up to $36 \mu \mathrm{T}$. Then it attains a minimum between maxima with a decrease of $17 \%$ in the $56^{\text {th }}$ month to $30 \mu \mathrm{T}$, but it increases with $86.6 \%$ to $56 \mu \mathrm{T}$ at month $79^{t h}$. Interestingly, during the time 24 months after the first maximum the index values change only $6 \mu \mathrm{T}$, and this situation needs more investigation.

\footnotetext{
${ }^{3}$ from Mt Wilson Observatory

${ }^{4}$ from Wilcox Solar Observatory
} 

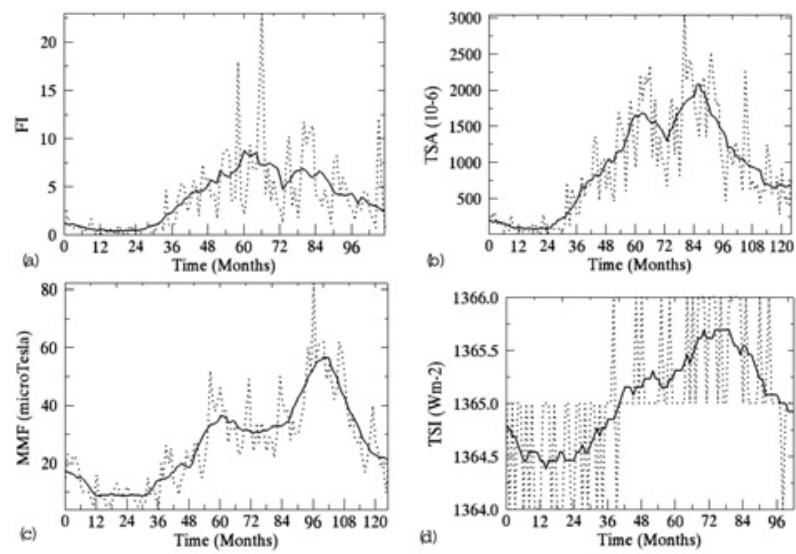

Figure 2. Variations of the FI, TSA, MMF and TSI indices during the cycle. All solid lines are smoothed monthly mean values; all dotted lines are monthly mean values of each index. (a) Flare index. (b) The total area of sunspot groups. (c) The mean solar magnetic field. (d) The total solar irradiance.
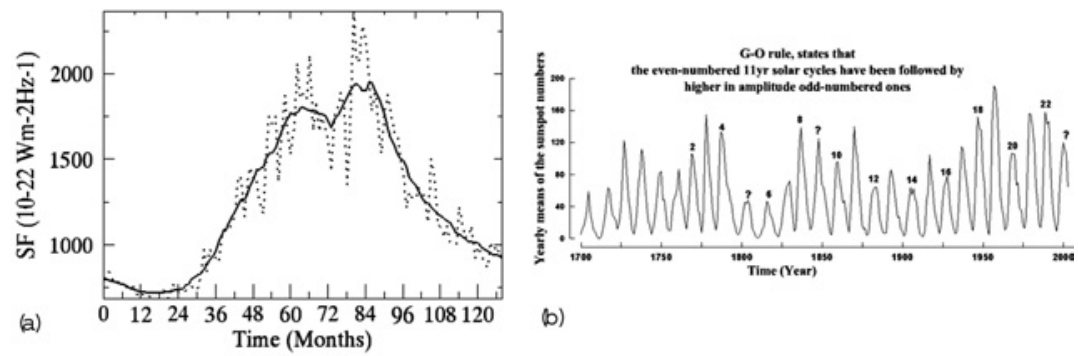

b)

Figure 3. (a) Variation of the SF during $23^{r d}$ solar cycle. The dotted line are monthly mean values, the solid line are smoothed monthly mean values. (b) Violations of the G-O rule.

\section{Total solar irradiance (TSI)}

The $\mathrm{TSI}^{5}$ maxima are fainter than those of the other indices because the solar irradiance variation of the $23^{r d}$ solar cycle is just $0.14 \%$. This value seems very small but is normal.

\section{Solar $10.7 \mathrm{~cm}$ flux (SF)}

In $\mathrm{SF}^{6}$ index the second maximum is $8.4 \%$ stronger than the first one. The relation between RSN, TSA and SF can be seen clearly in Table 1. This relation is also evident in Fligge and Solanki (1997).

\footnotetext{
${ }^{5}$ from Earth Radiation Budget Satellite

${ }^{6}$ from Dominion Radio Astrophysical Observatory
} 
Table 1. Calculated times of the maxima and the minimum between the maxima for each index.

\begin{tabular}{|c|c|c|c|c|}
\hline Indices & $\begin{array}{r}\text { Beginning } \\
\text { Date }\end{array}$ & $1^{s t}$ Max. & $\begin{array}{r}\text { Min. between } \\
\text { Maxima }\end{array}$ & $2^{\text {nd }}$ Max. \\
\hline RSN & $\begin{array}{l}\text { May } \\
1996\end{array}$ & $\begin{array}{r}47^{\text {th }} \text { Month } \\
\text { Apr. } 2000\end{array}$ & $\begin{array}{l}57^{t h} \text { Month } \\
\text { Feb. } 2001\end{array}$ & $\begin{array}{l}70^{t h} \text { Month } \\
\text { Mar. } 2002\end{array}$ \\
\hline TSA & $\begin{array}{l}\text { Aug. } \\
1996\end{array}$ & $\begin{array}{r}44^{\text {th }} \text { Month } \\
\text { Apr. } 2000\end{array}$ & $\begin{array}{l}54^{\text {th }} \text { Month } \\
\text { Feb. } 2001\end{array}$ & $\begin{array}{l}67^{\text {th }} \text { Month } \\
\text { Mar. } 2002\end{array}$ \\
\hline MMF & $\begin{array}{r}\text { Oct. } \\
1996\end{array}$ & $\begin{array}{r}40^{t h} \text { Month } \\
\text { Feb. } 2000\end{array}$ & $\begin{array}{l}54^{t h} \text { Month } \\
\text { Apr. } 2001\end{array}$ & $\begin{array}{l}79^{\text {th }} \text { Month } \\
\text { May } 2003\end{array}$ \\
\hline FI & $\begin{array}{l}\text { May } \\
1996\end{array}$ & $\begin{array}{r}44^{\text {th }} \text { Month } \\
\text { Jan. } 2000\end{array}$ & $\begin{array}{r}57^{\text {th }} \text { Month } \\
\text { Feb. } 2001\end{array}$ & $\begin{array}{l}64^{\text {th }} \text { Month } \\
\text { Sep. } 2001\end{array}$ \\
\hline TSI & $\begin{array}{l}\text { Mar. } \\
1996\end{array}$ & $\begin{array}{l}38^{\text {th }} \text { Month } \\
\text { May } 1999\end{array}$ & $\begin{array}{r}42^{\text {nd }} \text { Month } \\
\text { Sep. } 1999\end{array}$ & $\begin{array}{l}60^{t h} \text { Month } \\
\text { Mar. } 2001\end{array}$ \\
\hline SF & $\begin{array}{l}\text { May } \\
1996\end{array}$ & $\begin{array}{l}48^{\text {th }} \text { Month } \\
\text { May } 2000\end{array}$ & $\begin{array}{l}57^{\text {th }} \text { Month } \\
\text { Feb. } 2001\end{array}$ & $\begin{array}{l}70^{\text {th }} \text { Month } \\
\text { Mar. } 2002\end{array}$ \\
\hline
\end{tabular}

\section{Results: General characteristics of the $23^{r d}$ solar cycle}

1) The $23^{r d}$ solar cycle has begun at the same date in the indices RSN, FI and SF.

2) This cycle has a double maximum in each index as a characteristic feature.

3) Studying of indices and comparisons between them show the two maxima phenomenon (Gnevyshev, 1967,1977) and need further investigation.

4) The first maximum of this cycle occurs nearly at the same date in every index except TSI.

5) The minimum between the maxima occurs exactly at the same date in every index except TSI and MMF.

6) The $23^{r d}$ cycle violates the Gnevyshev-Ohl rule, which states that even-numbered 11-yr cycles are followed by higher-amplitude odd-numbered ones (Fig. 3b).

7) All second maxima have higher amplitude than the first maxima in every index except RSN and FI.

8) The period of the cycle is calculated as 10.15 year from our observatory's RSN index after a result of FFT (Oklay 2006).

9) The 27 days periodicity can be shown clearly by the result of the FFT (Fig. 1c).

Acknowledgements. I thank my supervisor, Prof. Dr. Adnan Ökten, for his patience and cooperation, Assistant Prof. Dr. Selçuk Bilir for technical support, and Associated Prof. Dr. Tansel Ak for help with the FFT.

\section{References}

Oklay, N. 2006, M.Sc. Thesis, Istanbul University.

Fligge, M. \& Solanki, S. K. 1997, Solar Phys., 173, 427-439

Gnevyshev, M. N. 1967, Solar Phys., 1, 107-120

Gnevyshev, M. N. 1977, Solar Phys., 51, 175-183 


\title{
Structure and flattening of the solar corona
}

\author{
M. T. Özkan ${ }^{1,2, *}$, A. Ökten ${ }^{1,2}$, N. Oklay ${ }^{2}$, A. Gültekin ${ }^{2}$, M. Kara ${ }^{2}$, Z. F. Bostancı ${ }^{2}$, \\ M. Başal ${ }^{2}$, and N. Al Erdoğan ${ }^{1,2}$ \\ ${ }^{1}$ University Observatory of Istanbul, Research \& Application Centre, University-Istanbul, \\ Turkey \\ ${ }^{2}$ Istanbul University, Faculty of Science, Department of Astronomy \& Space Sciences, \\ University-Istanbul, Turkey \\ *Email: ozkant@istanbul.edu.tr
}

\begin{abstract}
In this work, preliminary results of the investigation of the white-light coronal structure and the measurement of flattening parameters are presented. They were obtained using photographic eclipse observations performed during the total solar eclipse of March 29, 2006, in Ilica, Antalya, Turkey. This eclipse practically coincided with the minimum of the $23^{\text {rd }}$ solar cycle. Ludendorff flattening parameters were computed as a function of the distance from the disc centre. The results obtained within the range between 1.1 and 1.83 solar radii seem to be in satisfactory agreement with previous works by Saito (1956), Hata \& Saito (1966) and Gulyaev et al. (1994).
\end{abstract}

\section{Introduction}

The observation of the total solar eclipse was performed at Ilica observing site in Antalya. All solar photographs of the corona in integrated light were obtained by a camera and telescope. The white-light corona (WLC) is created by photospheric light scattered by electrons and dust; so it consists of light from the $\mathrm{K}$ and $\mathrm{F}$ corona. It shows many structures of different shapes and sizes. At the Ilica observing site, one of our experiments was focused on studying the morphological structure and the flattening of the WLC. Preliminary results from our observation of the total solar eclipse of March 29, 2006, are presented in this work.

\section{Observation and reduction}

The corona was photographed with an equatorial telescope camera. The main tube of this instrument has an aperture of $100 \mathrm{~mm}$ and a focal length of $1000 \mathrm{~mm}$. A Kodak T-Max 100 ASA $35 \mathrm{~mm}$ film was used to obtain photographic images of the WLC. The photographs were taken with different exposures varying from $1 / 1000 \mathrm{sec}$ to $1 / 2 \mathrm{sec}$ which also show the variety of details in the coronal structure caused by the solar magnetic field. All of the films were developed at the University Observatory of Istanbul.

The developed eclipse films were then photometrically processed with a Microtek Artix Scan $4000 t$ film scanner with a dynamic range of 16 bit. The density matrices, consisting of $2000 \times 2000$ pixels, were recorded, and we produced a composite (Figure 1) of 10 digital images which differ in exposure times between $1 / 1000 \mathrm{sec}$ and $1 / 2 \mathrm{sec}$. 


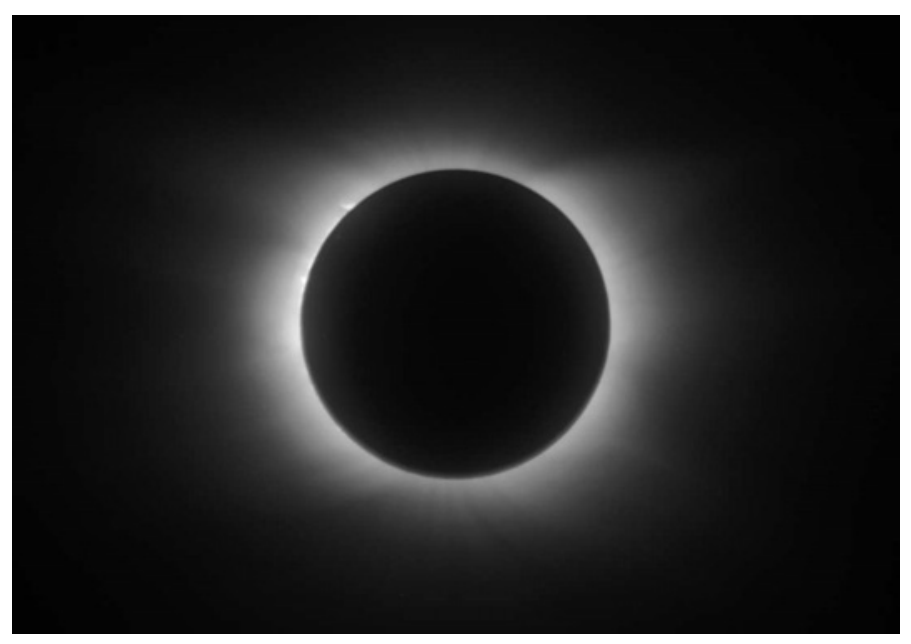

Figure 1. Composite image of the solar corona in white light obtained from the total solar eclipse of March 29, 2006.
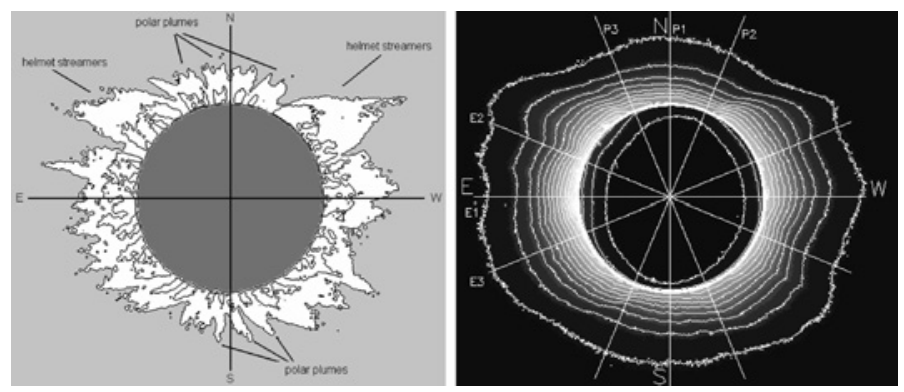

Figure 2. Left: Structure of the white-light corona. Right: Illustration of the method for computing the flattening of the corona observed during the eclipse.

\section{Results and discussion}

The total solar eclipse of March 29, 2006, occurred during the $23^{\text {rd }}$ solar cycle's minimum phase. The composite image shows that the structure of the corona is in concord with the cycle's minimum phase. For nearly a century the variations of structures of the white-light solar corona have been studied and were found to be related to the solar cycle (Koutchmy et al. 1991).

Although the general appearance of the corona is complicated, it is possible to extract detailed structures using the composite image after processing by a computer. All the basic types of coronal formations such as helmet streamers, polar plumes and coronal beams can be seen in this processed image (Figure 2, left). The helmet type streamers are believed to be related to the chromospheric structural elements (Stoev et al. 2002). Coronal streamers show a higher intensity on the eastern side of the sun than on the western side. 
Table 1. The calculated diameters of the isodensity curves. The last column gives the distance from the limb of the solar disc.

\begin{tabular}{cccccccc}
\hline $\begin{array}{c}\text { Isodensity } \\
\text { Curves }\end{array}$ & $\begin{array}{c}\mathrm{E}_{1} \\
\text { (Pixel) }\end{array}$ & $\begin{array}{c}\mathrm{E}_{2} \\
\text { (Pixel) }\end{array}$ & $\begin{array}{c}\mathrm{E}_{3} \\
\text { (Pixel) }\end{array}$ & $\begin{array}{c}\mathrm{P}_{1} \\
\text { (Pixel) }\end{array}$ & $\begin{array}{c}\mathrm{P}_{2} \\
\text { (Pixel) }\end{array}$ & $\begin{array}{c}\mathrm{P}_{3} \\
\text { (Pixel) }\end{array}$ & $\mathrm{r} / \mathrm{R}_{\odot}$ \\
\hline 1 & 234.5 & 223.0 & 227.0 & 202.0 & 203.0 & 212.0 & 1.12 \\
2 & 244.5 & 232.0 & 236.5 & 211.0 & 211.5 & 223.0 & 1.17 \\
3 & 256.0 & 242.0 & 247.5 & 219.5 & 218.0 & 232.5 & 1.22 \\
4 & 268.8 & 260.0 & 261.5 & 230.0 & 222.5 & 244.0 & 1.29 \\
5 & 287.5 & 272.5 & 282.5 & 245.0 & 242.0 & 259.0 & 1.37 \\
6 & 316.0 & 303.0 & 313.0 & 270.5 & 267.5 & 281.5 & 1.52 \\
7 & 377.5 & 375.0 & 375.5 & 327.0 & 327.0 & 329.0 & 1.83 \\
\hline
\end{tabular}

We produced a contour map from this composite image. In this way, the maps of isodensities are used for determining the oblateness of the solar corona (Figure 2, right). The Ludendorff parameter (Petrov et al. 2002) is described by

$$
\varepsilon=\frac{E_{1}+E_{2}+E_{3}}{P_{1}+P_{2}+P_{3}}-1
$$

where $E_{1}$ and $P_{1}$ are the diameters of the isodensity curves in the equatorial and polar direction, respectively. $E_{2}$ and $E_{3}$ are the equatorial diameters placed at angles of $\pm 22^{\circ} .5$ from $E_{1}$. $\mathrm{P}_{2}$ and $\mathrm{P}_{3}$ are the polar diameters tilted with respect to $\mathrm{P}_{1}$ at angles of $\pm 22^{\circ} .5$.

The isodensity diameters derived from Figure 2 (right) are given in Table1. The Ludendorff parameter was calculated using this table and is displayed as a function of the distance from the solar disc centre in Figure 3. The flattening increases linearly from the limb of the solar disc towards the outer solar corona.

The calculated $\varepsilon$ (flattening parameter) values from Table 1 are lying between $r=1.12$ and $1.83 \mathrm{R}$. To proceed with our investigation of the oblateness, we need to calculate the flattening parameter in the range of $r=2$ to $3 R_{\odot}$. But here, we face the problem that the maximum distance in our images is $1.83 \mathrm{R}_{\odot}$. On the other hand, taking into account that we performed our observation with a refractor with an aperture of $100 \mathrm{~mm}$ and that the size of our images was $35 \mathrm{~mm} \times 24 \mathrm{~mm}$ with the diameter of the moon being $9.66 \mathrm{~mm}$, we used the approximation given below to solve this problem.

Since the flattening parameter increases linearly with distance from the solar disc, the Ludendorff parameter can be given in linear form (Saito 1956) by

$$
\varepsilon=a+b\left(r / R_{\odot}-1\right)
$$

where $\mathrm{r}$ is the mean equatorial radius of an isophote. The value $a+b$ equals $\varepsilon$ at $\mathrm{r}=2 \mathrm{R}_{\odot}$. We could not derive this value from our composite image. For this reason, we calculated it according to Eq. (2). On the other hand, a correlation between the phase of the cycle and $\varepsilon$ has been known for a long time (Saito 1956). For this eclipse, the cycle's phase can be found from the equation given below:

$$
\phi=\frac{T-T_{\min }}{T_{\max }-T_{\min }}
$$




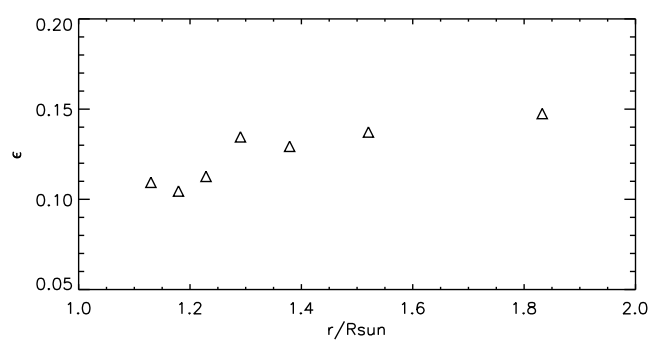

Figure 3. Dependence of the oblateness $\varepsilon$ of the solar corona on the radius.

where $\mathrm{T}$ is the date of the observation of the eclipse, $\mathrm{T}_{\min }$ and $\mathrm{T}_{\max }$ are the nearest minimum and maximum date of the cycle, respectively. Taking $\mathrm{T}_{\min }$ and $\mathrm{T}_{\max }$ from (http://www.ips.gov.au/Educational/2/4/6) and (http://www.sec.noaa.gov/SolarCycle/), $\phi$ is found to be 0.11 according to Eq. (3). $\phi$ values vary between 1.0 and 0.0 according to the cycle phase. While the flattening parameter is 0.103 at $2 \mathrm{R}_{\odot}$, the observational flattening value corresponding to the same cycle phase 0.11 taken from Figure 6 of Saito (1956) is 0.23 . As long as we lack further observational evidence from our second set of observations (see below), we think the difference between these two values to be due to the linear approximation. However, it may be added that our calculated flattening values within the range from 1.12 to $1.83 \mathrm{R}_{\odot}$ are in agreement with the minimum corona presented in the work of Allen (Hata \& Saito 1966).

We are confident that we will be able to check our approximation using another set of observations we obtained with a refractor with an aperture of $130 \mathrm{~mm}$ and a focal length of $1500 \mathrm{~mm}$, giving a diameter of the moon of $14.28 \mathrm{~mm}$ on a film of the size of $60 \mathrm{~mm} \times 60 \mathrm{~mm}$. Therefore, the analysis of this piece of photographic film may result in the derivation of oblateness parameters up to a distance of $3.20 \mathrm{R}_{\odot}$ which may be considered to be equally reliable.

Acknowledgements. The present work resulted from Project No. 470/27122005 supported by the Research Fund of Istanbul University.

\section{References}

Gulyaev, R. A., Vanyarkha, N. Ya., \& Vanyarkha, E. S. 1994, IAU Colloq. 144, 111

Hata, S. \& Saito, K. 1966, Annals Tokyo Astron. Obs., second series, vol. X, No. 1,16

Koutchmy, S., Zirker, J. B., Steinolfson, R. S., \& Zhugzhda, J. D. 1991, Solar Interior and Atmosphere, ed. A. N. Cox, W. C. Livingston, \& M. S. Mathews, The University of Arizona Press, p. 1044

Petrov, N., Özkan, M. T., Dermendjiev, V. N., Ökten, A., \& Rompolt, B. 2002, in Proceedings of the International Conference, Varna, Bulgaria, Sept. 11-15, 2000, ed. D. N. Mishev and K. J. H. Philips, p. 57

Saito, K. 1956, Publ. Astron. Soc. Japan, vol. 8, no. 3-4, 126

Stoev, A., Shopov, Y., Muglova, P., Kiskinova, N., Varbunova, Yu., Michaylova, Z. I., \& Velkov, R. 2002, in Proceedings of the International Conference, Varna, Bulgaria, Sept. 11-15, 2000, ed.

D. N. Mishev and K. J. H. Philips, p. 143 


\title{
Fine structure in a dark umbra
}

\author{
M. Sobotka ${ }^{1,{ }^{*}}$ and K. G. Puschmann ${ }^{2}$ \\ ${ }^{1}$ Astronomical Institute AS CR, Ondřejov, Czech Republic \\ ${ }^{2}$ Institut für Astrophysik, Göttingen, Germany \\ *Email: msobotka@asu.cas.cz
}

\begin{abstract}
An excellent-quality time series of images of a large dark umbra of the leading sunspot of NOAA 10634 was acquired on 18 June 2004 with the 1-m Swedish Solar Telescope at La Palma, simultaneously in blue, red, and $G$-band channels. The temporal and spatial resolutions are $20 \mathrm{~s}$ and 0 ' 14 . A 2-hour long series of the red continuum images is analysed, showing the faintest umbral fine structures. In addition to umbral dots, often clustered to more stable "granules" or aligned to short chains, we observe large, low-intensity elongated structures with dark central channels, resembling extremely faint light bridges. At the periphery of the umbra, bright umbral dots move inwards, showing a similarity to penumbral grains. Kinematic properties of umbral fine structures are studied.
\end{abstract}

\section{Introduction}

Sunspot umbrae, when observed under high spatial resolution and sufficient signal-to-noise ratio, show inhomogeneities in brightness. Chevalier (1916) detected a small-scale granularlike pattern in the umbra in his collection of sunspot photographs obtained under a resolution of 0 ! $7-1$ ". The existence of "umbral granulation" was reported later by several observers (Thiessen 1950; Bray \& Loughhead 1964; Bumba et al. 1975). Bumba \& Suda (1980) claimed that the spatial distribution of "granules" inside the umbra is identical with that in the photosphere. The term "umbral dots" (UDs) was introduced by Danielson (1964). In the set of photographs from the balloon-borne experiment Stratoscope he detected very small, bright point-like features. The spatial distribution of UDs was different from the photospheric granulation pattern. It is accepted now that the "umbral granules" correspond to unresolved groups and clusters of UDs observed with moderate spatial resolution. Referring to observations with the 91-cm telescope at Kitt Peak, Livingston (1991) claimed that the umbra has a radial filamentary structure with dark "voids" (dark nuclei) free of UDs between bright filaments. So, UDs might be only seeing-induced artifacts. However, the existence of UDs was confirmed by many posterior observations and UDs have been considered as typical representatives of the bright umbral component, embedded in the dark, "diffuse" umbral background with smoothly varying intensity (Sobotka 1999).

\section{Observations and data processing}

The large leading sunspot of NOAA 10634 was observed on 18 June 2004 from 07:43 to 15:30 UT with the 1-m Swedish Solar Telescope (SST, Scharmer et al. 2003) equipped with 
adaptive optics. The spot, located near disk centre (N13, E6), was in the phase of growth. The images were acquired in a frame-selection mode (selection interval $20 \mathrm{~s}$ ), simultaneously in three wavelength bands: blue $(4507.5 \pm 4.6) \AA$, red $(6020.0 \pm 13.0) \AA$, and $G$ band $(4308.6 \pm$ 5.8) $\AA$. Exposure times were 11-14 ms and the pixel size corresponded to $0{ }^{\prime} 0405$.

After dark- and flatfield corrections, the stray light was eliminated following Martínez Pillet (1992). Parameters of the scattering were determined for each wavelength from the shapes of photometric profiles across the solar limb. The level of stray light, originating mostly in the instrument, was $8.5 \%$ in blue, $6.5 \%$ in red, and $8.7 \%$ in $G$ band. The deconvolution of the instrumental profile of the diffraction-limited 1-m telescope and the noise filtering was carried out simultaneously, applying Wiener filters with noise suppression starting at 0.11 (blue), $0 . ' 14$ (red), and $0 . ' 13$ ( $G$ band). Regarding the correction of wavefront aberrations done by the adaptive optics, these values characterise the spatial resolution in the best frames. No other restoring techniques were applied.

The image rotation was compensated and the frames were aligned and de-stretched. Finally, a subsonic filter with a cutoff at $4 \mathrm{~km} \mathrm{~s}^{-1}$ was applied to the series of images. For further analyses we selected the best part of the time series taken in the red channel from $12: 15$ to $14: 12$ UT, containing 350 frames. The field of view was reduced to 20 ' $25 \times 20$ ' 25 $(500 \times 500$ pixels $)$, covering most of the umbra. For the purpose of visualisation and feature tracking, it is good to eliminate large-scale intensity variations. We calculated a smooth, time-dependent intensity "background" using 2D spline fits to local intensity minima in the umbra for each frame, averaging them over a period of $140 \mathrm{~s}$ (7 frames). This interpolated "background" intensity was subtracted from the images. The motions of umbral features were studied using the methods of local correlation tracking (LCT, November \& Simon 1988) and feature tracking (Sobotka et al. 1997).

\section{Results}

The observed umbra was very dark. After the stray-light correction, the minimum intensity in the umbra was 0.05 (blue) and 0.09 (red) in units of the average intensity of the undisturbed photosphere $\left(I_{\mathrm{ph}}\right)$. In Fig. 1 (left) we present one of the images with subtracted large-scale intensity variations, showing the complex internal structure of the umbra. An MPEG movie, covering 90 minutes of the time series, can be found at http://www.asu.cas.cz/ sdsa/galleryastropictures.html.

Bright isolated UDs are not very frequent in this dark umbra. Many of them are located at the periphery and move inwards. These UDs are often followed by faint bright tails, resembling tails of penumbral grains, or by dark "wakes" (resembling dark cores of penumbral filaments). Some of the bright UDs are static or slowly moving. They can be found both in the peripheral and central parts of the umbra and are often clustered.

At the bottom left and upper right regions in the umbra, clusters of faint UDs create granule-like features, separated by dark lanes. These non-moving features with diameters of about $0 .{ }^{\prime} 6-0.7$ rapidly change their shape but live substantially longer (about 40 minutes) than individual UDs. They are sometimes surpassed by bright inward-moving UDs.

The central part of the umbra is dominated by two faint filamentary structures with dark central channels, resembling extremely weak light bridges. They are composed of small aligned point-like features that show an organised motion. The average intensity of the 

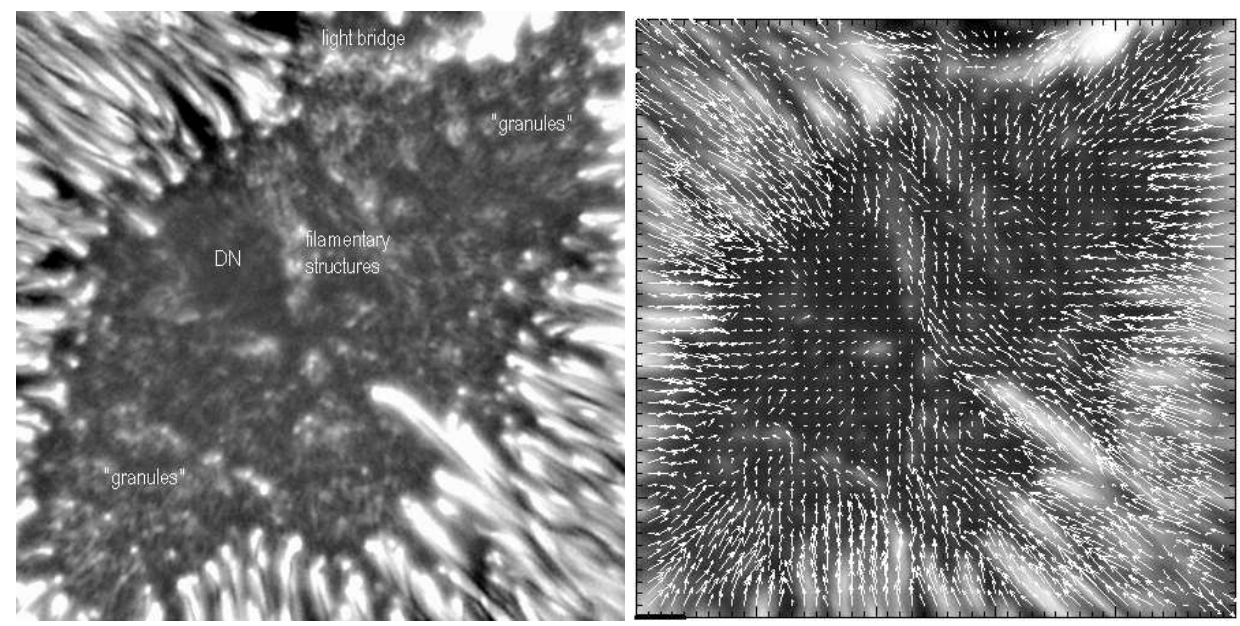

Figure 1. Left: Image of the umbra, showing various morphological structures. DN denotes a dark nucleus. Large-scale intensity variations are subtracted. The field of view is approximately $20^{\prime \prime} \times 20^{\prime \prime}$. Right: Map of horizontal velocities calculated by LCT. The length of the black horizontal bar at the bottom left corner represents $1 \mathrm{~km} \mathrm{~s}^{-1}$.

filamentary structures is by $0.1 I_{\mathrm{ph}}$ higher than the intensity of the interpolated "background".

In addition to bright UDs and the granule-like and filamentary structures mentioned above, the whole umbra is covered by a relatively stable small-scale grainy and/or filamentary (alignments of unresolved UDs?) pattern with rms fluctuations of only $0.01-0.02 I_{\mathrm{ph}}$. The bright inward-moving UDs travel in this background pattern. Even the dark nucleus, the less intense part of the umbra, where the strongest magnetic field is expected, shows a very faint grainy and filamentary structure. Identical patterns were detected also in the blue bandpass, proving that we observe real structures. So, the umbral background does not have the diffuse character as it was expected in the past.

The LCT map of horizontal velocities, calculated with the tracking window of 0.3 and averaged over a period of 117 minutes, is shown in Fig. 1 (right). We can see that the motion field of penumbral grains merges into the motion field of inward-moving peripheral UDs. The typical LCT speed of these UDs is $500 \mathrm{~m} \mathrm{~s}^{-1}$. The global motion of bright features along each of the two faint filamentary structures has opposite direction, with a typical LCT speed of $300 \mathrm{~m} \mathrm{~s}^{-1}$. This gives an impression of a large-scale organised flow in the central part of the umbra, possibly connected with the horizontal motions in the extended bright penumbral filament seen at the bottom right part of the field of view.

Positions, horizontal velocities, lifetimes, and sizes of features brighter by $0.06 I_{\mathrm{ph}}$ than the interpolated "background", larger than 0.14 , and living longer than 2 minutes, were measured using the feature tracking method. The mean horizontal velocities of UDs and bright features in the faint filamentary structures are equal to $370 \mathrm{~m} \mathrm{~s}^{-1}$. The median speed of UDs $\left(330 \mathrm{~m} \mathrm{~s}^{-1}\right)$ is slightly higher than that in the faint filamentary structures $\left(300 \mathrm{~m} \mathrm{~s}^{-1}\right)$ due to the presence of fast-moving peripheral UDs. The average lifetimes of UDs and of the bright features in the faint filamentary structures are similar, 9 and 10 minutes, respectively, 
and their mean diameters are equal to $0.18(130 \mathrm{~km})$. To compare the positions of features with different horizontal velocities, let us term "slow" features all features with average speeds below $300 \mathrm{~m} \mathrm{~s}^{-1}$ and "fast" features those with speeds above $500 \mathrm{~m} \mathrm{~s}^{-1}$. The "slow" features can be found everywhere in the umbra, while the "fast" ones are mostly located at the peripheral parts of the umbra and inside the faint filamentary structures.

\section{Conclusions}

An-excellent quality 2-hour time series of images of a large and dark sunspot umbra acquired with the $1-\mathrm{m}$ SST in the red continuum around $6020 \AA$ was studied. Thanks to the spatial resolution of $0{ }^{\prime} 14$ and the signal-to noise ratio around 200 , we detected the faintest pointlike, granule-like, and filamentary features in the umbra.

Bright peripheral UDs show morphological (bright tails, dark "wakes") and kinematic (inward-directed speeds of $500 \mathrm{~m} \mathrm{~s}^{-1}$ ) similarity to penumbral grains. Static or slowly moving UDs are often clustered in relatively stable granule-like structures separated by dark lanes. Large, low-intensity filamentary structures with dark central channels are probably extremely faint light bridges. They consist of small features, similar in size to UDs, that move in an organised way along the axis of the structure. The whole umbra shows a slowly evolving grainy and/or filamentary low-contrast "background" pattern, which is surpassed by the inward-moving peripheral UDs.

Acknowledgements. We thank C. Möstl, R. Kever, and R. Henderson for assisting the observations. This work was supported by the Grant Agency of the Academy of Sciences of the Czech Republic (grant IAA 3003404), the OPTICON Trans-national Access Programme, the Deutsche Forschungsgemeinschaft (grant KN 152/29-1,2) and the European Solar Magnetism Network (contract HRPNCT-2002-00313). The SST is operated by the Royal Swedish Academy of Sciences in the Spanish Observatorio del Roque de los Muchachos of the Instituto de Astrofísica de Canarias.

\section{References}

Bray, R. J. \& Loughhead, R. E. 1964, Sunspots (Chapman and Hall, London)

Bumba, V., Hejna, L., \& Suda, J. 1975, Bull. Astr. Inst. Czechosl., 26, 315

Bumba, V. \& Suda, J. 1980, Bull. Astr. Inst. Czechosl., 31, 101

Chevalier, S. 1916, Ann. Obs. Astron. Zô-Sé, Tome IX, B1

Danielson, R. 1964, ApJ, 139, 45

Livingston, W. 1991, Nature, 350, 45

Martínez Pillet, V. 1992, Solar Phys., 140, 207

November, L. J. \& Simon, G. W. 1988, ApJ, 333, 427

Scharmer, G. B., Bjelksjö, K., Korhonen, T. K., Lindberg, B., \& Petterson, B. 2003, in Innovative Telescopes and Instrumentation for Solar Astrophysics, ed. S. Keil \& S. Avakyan, Proc. SPIE, 4853, 341

Sobotka, M. 1999, in Motions in the Solar Atmosphere, ed. A. Hanslmeier \& M. Messerotti, Kluwer, 71

Sobotka, M., Brandt, P. N., \& Simon, G. W. 1997, A\&A, 328, 682

Thiessen, G. 1950, Observatory, 70, 234 


\title{
Dynamics of a solar pore with light bridge
}

\author{
S. Giordano, F. Berrilli", D. Del Moro, and V. Penza \\ University of Rome Tor Vergata, Department of Physics, Rome, Italy \\ *Email: francesco.berrilli@ roma2.infn.it
}

\begin{abstract}
Pores are one of numerous features formed by the emerging of magnetic field from the solar surface. Their uniqueness derives from the fact that - as far as size is concerned - they lie between the tiny flux tubes associated with magnetic elements, and the complex and large magnetic regions associated with sunspots. Light bridges, in a pore or a sunspot, are bright features dividing the umbral region in a more or less complex structure. Commonly, light bridges indicate that inside the active region a process is underway: the merging of magnetic regions or, conversely, the breakup of the area. In both cases a topological reconfiguration of the emerging magnetic field is expected. In this study we investigate the velocity structure of a solar pore with light bridge and of the quiet solar photosphere around it by means of high spatial and spectral resolution data obtained with the Interferometric BI-dimensional Spectrometer (IBIS) at the Dunn Solar Telescope of the NSO. We observe that the amplitude of the LOS velocities decreases inside the pore with respect to the quiet granulation around the active region, and that the pore exhibits a downflow ring-shaped structure in the surrounding region. We also observe an average downward flow from the light bridge into the umbra.
\end{abstract}

\section{Introduction}

The magnetic field appears at the solar surface with a wide variety of magnetic structures, ranging from the largest sunspots (tens of Mm across), down to the $0.1 \mathrm{Mm}$ scale magnetic elements. In this family of solar magnetic structures pores represent a link between small solar flux tubes and larger structures. They are small $(2-4 \mathrm{Mm})$ and contain intense concentrations of magnetic field, with strengths of $2000 \mathrm{G}$ and flux contents up to $7 \mathrm{TWb}$ (Hulburt et al. 2000). Normally, solar pores are distinguishable from sunspots as they do not develop a penumbra. Pore 'umbrae', like sunspot umbrae, contain a large variety of bright structures like umbral dots or light bridges. Two models explain the presence of these bright structures: the monolithic flux-tube model, which relates these structures to the not completely inhibited convective motions (Chouduri 1986), and the cluster model, where these structures are columns of field-free gas penetrating the vertical field lines inside the umbra (Parker 1979). From an evolutional point of view pores can form sunspots or end their life as pores. The possible evolution of pores into sunspots depends on a dynamic stability criterion (Bray \& Loughhead 1964; Wang \& Zirin 1992; Rucklidge el al. 1995). Regarding the dynamics around solar pores, several studies show the presence of an annular structure of strong downflows in the surrounding region. The horizontal flow field around solar pores appears to be dominated by rosetta-like divergence centers.

In this work we study the dynamics of a light bridge in comparison with a quiet granular field using IBIS observations. We also investigate the radial structure of the pore, giving an 
estimate of its dimension.

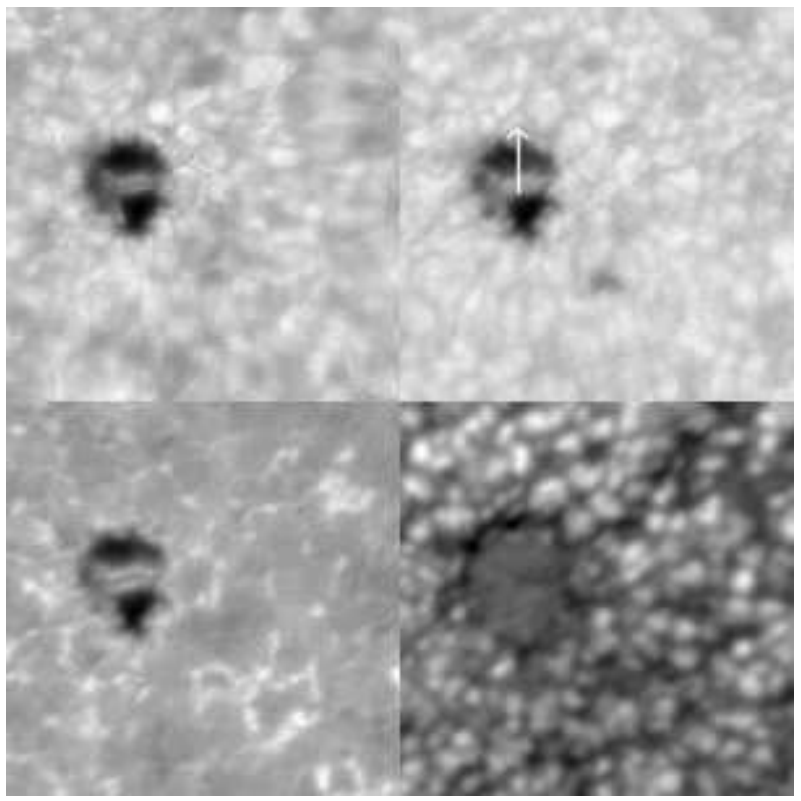

Figure 1. Upper left panel: $\mathrm{G}$ band intensity image; upper right panel: continuum image; lower left panel: Ca II $854.2 \mathrm{~nm}$ wing intensity image; lower right panel: Fe I 709.0 nm Doppler velocity field.

\section{Observations and Data Analysis}

The observations were performed on September 28, 2005, with the IBIS 2-D spectrometer (Cavallini 2006) in the spectral lines Fe I $709.0 \mathrm{~nm}$, Fe II $722.4 \mathrm{~nm}$ and Ca II $854.2 \mathrm{~nm}$. We observed a central region of the solar disk including a pore with light bridge. The exposure time for each monochromatic image was $25 \mathrm{~ms}$. The CCD camera was rebinned to $512 \times 512$ pixels, so that the final pixel scale for the images was $0.17^{\prime \prime} /$ pixel. The time interval between two successive images and two successive spectral series was $0.3 \mathrm{~s}$ and $19 \mathrm{~s}$, respectively. In addition to the narrow-band images, G-band images were simultaneously recorded. The observational setup prevented us from applying restoring procedures to monochromatic images. Each monochromatic image was corrected for CCD non-linearity effects, dark current, gain table, monochromatic flat field and instrumental blue shift. Velocity maps are obtained applying a line-profile Gaussian fit to monochromatic cubes of calibrated images and transforming Doppler shifts into velocity units. Absolute values of the Doppler velocities have been defined by setting the mean velocity in granular subfields equal to zero. We removed acoustic patterns by applying a $k-\omega$ filter with a cut-off velocity of $7.0 \mathrm{kms}^{-1}$ on the time series of continuum and velocity maps.

By examining high resolution MDI continuum images, corresponding to three days before and after our observation run, we established that the analyzed region results from the merging of two different magnetic structures and ends its life as a pore. Our paper concentrates on 
the velocity structure of this pore with light bridge (LB). By analyzing the intensity-velocity plot in the light bridge and in a rather quiet granular field, we found that the light bridge intensity is reduced by about $20 \%$ with respect to a quiet granular field. Concerning the velocity, we remark that we set to zero the mean LOS velocity of a quiet granular subfield. In this way the velocity inside the umbra is negative with values of the order of $-200 \mathrm{~ms}^{-1}$. The velocity inside the light bridge is about $-250 \mathrm{~ms}^{-1}$ with an RMS in the order of $50 \mathrm{~ms}^{-1}$. The averaged Doppler map shows persistent downflows around the pore in the range -(400-500) $\mathrm{ms}^{-1}$. This result is in agreement with previous findings, e.g. Keil et al. (1999).

In Fig. 2 the $\mathrm{Ca}$ II $\lambda 8542$ intensities and the Fe I $\lambda 7090$ velocity are plotted for different profiles along the light bridge. The Ca II $\lambda 8542$ profile shows the presence of a dark lane in the light bridge, which is not visible in the continuum, where only a plateau is present. This is probably due to a lack of resolution inside the light bridge, where we are able to measure only an increase of the intensity with respect to the umbral part. There is a clear correspondence between this dark lane and a strong downflow. This dark central lane seems to be a common feature of LBs: It has been observed and inferred in other LB studies. As the pore is of almost circular shape, we investigated its radial structure by computing the mean radial velocity and intensity as shown in Fig. 3. The velocity is quasi-constant in the center of the pore with values decreasing moving away from the center, until it reaches a strong downflow that surrounds it. This downflow corresponds to the boundary of the pore. From this point the velocity increases to typical granular values (set to zero, by convention).

By considering the evolution of the analyzed structure as observed in MDI data and taking our cue from a simulation by Hulburt et al. (2000), we model the pore (Fig. 3) as a result of the merging of two different magnetic structures, of the same polarity, both surrounded by downward flows. These downflow structures persist in the quenching region, where the convection results strongly modified by the presence of magnetic field. Our scheme, supported by velocity measures, is similar to the one reported by Jurčák et al. (2006) to explain the magnetic canopy above light bridges.
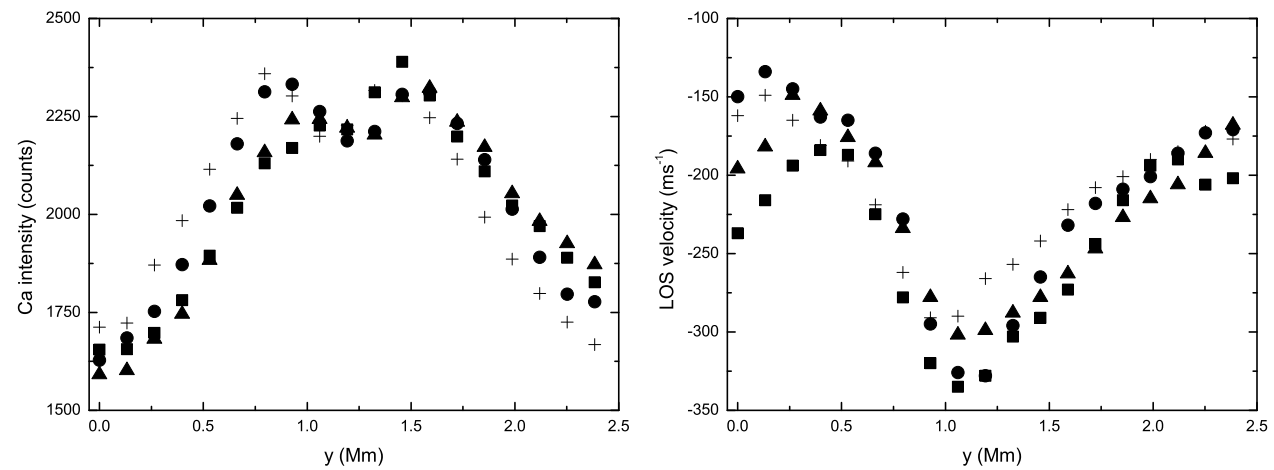

Figure 2. Plots of the Fe 709.0 nm LOS velocity and of Ca II 854.2 intensity for different traces along the light bridge, in the direction of the arrow shown in Fig. 1. 


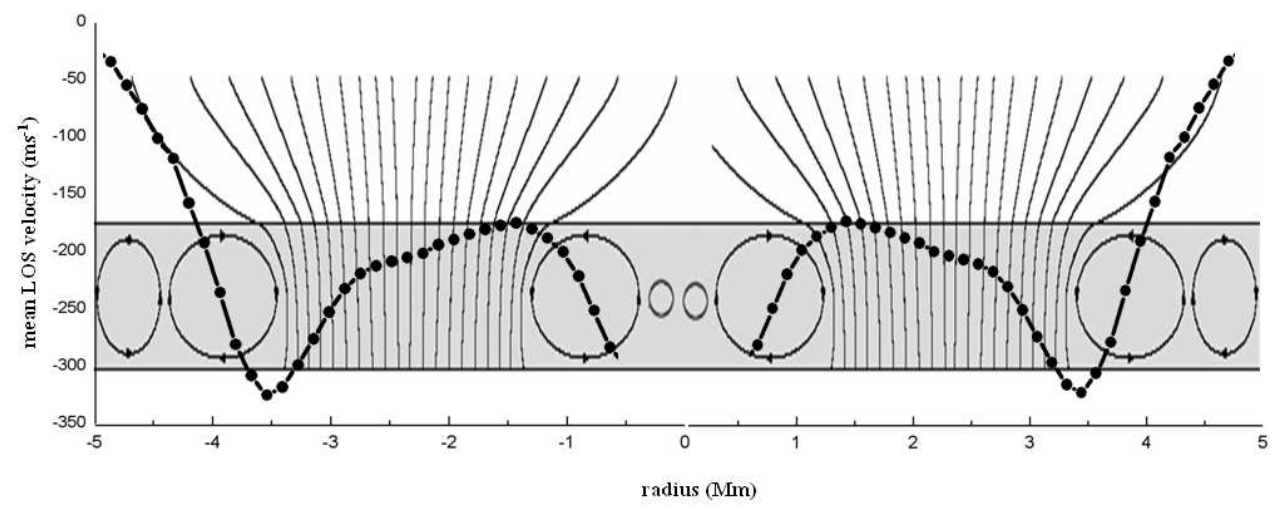

Figure 3. Schematic view of the analyzed structure. In the background a sketch of the flows around the pore: the model is obtained by merging two magnetic structures, each derived from the simulation by Hulburt et al. (2000). Superimposed is the computed mean radial velocity (dot+spline).

\section{References}

Bray, R. J. \& Loughhead, R. E. 1964, Sunspots (Dover: New York)

Cavallini, F. 2006, Solar Phys. 263, 415

Choudhuri, A. R. 1986, ApJ, 302, 809

Hulburt, N. E. \& Rucklidge, A. M. 2000, MNRAS, 314, 793

Jurčák, J., Martínez Pillet, V., \& Sobotka, M. 2006, A\&A 453, 1079

Keil, S. L., Balasubramaniam, K. S., Smaldone, L. A., \& Reger, B. 1999, ApJ, 510, 422

Parker, E. N. 1979, ApJ, 234, 333

Rimmele, T. R. 1997, ApJ, 490, 458

Rucklidge, A. M., Schmidt, H. U., \& Weiss, N. O. 1995, MNRAS, 273, 491

Wang, H. \& Zirin, H. 1992, Solar. Phys., 140, 41 


\title{
Ca II H line wing images of sunspot penumbrae recorded with the Swedish 1-m Solar Telescope
}

\author{
G. Narayan ${ }^{1,2, *}$, M. J. van Noort ${ }^{1}$, and G. Scharmer ${ }^{1}$ \\ ${ }^{1}$ Institute for Solar Physics of the Royal Swedish Academy of Sciences, Stockholm, \\ Sweden \\ ${ }^{2}$ Stockholm Observatory, Stockholm, Sweden \\ *Email: gautam@astro.su.se
}

\begin{abstract}
.
We present recent Ca II H images of sunspot penumbrae taken with the Swedish $1 \mathrm{~m}$ Solar Telescope (SST) during June-July 2006 and restored to a resolution close to $0{ }^{\prime} 1$. Images were recorded at different $\mathrm{Ca}$ II $\mathrm{H}$ line positions using one tunable filter, one fixed wing filter and a wide band quasi-continuum filter. Apart from the images recorded at line center (and formed under non-LTE conditions), this provides temperature information from approximately the first $200-300 \mathrm{~km}$ above the photosphere.
\end{abstract}

\section{Introduction}

The $\mathrm{Ca}$ II H and $\mathrm{K}$ wings provide a large sampling space from the adjacent continuum to their core for probing different heights, ranging from the very deep photospheric layers up to about the lower chromosphere. As calcium is predominantly in a singly ionized state, LTE is a good approximation for the $\mathrm{Ca}$ II H source functions and line opacities (Sheminova et al. 2005). Thus in the visible spectrum, the $\mathrm{Ca}$ II $\mathrm{H}$ and $\mathrm{K}$ line wings provide unique diagnostics of the photospheric temperature stratification. $\mathrm{Ca}$ II $\mathrm{H}$ has also been used for studying umbral flashes (Rouppe van der Voort 2002) and for chromospheric studies (Grigoryeva \& Turova 1998).

\section{Observations}

Figure 1(a) shows an intensity versus wavelength plot of the $\mathrm{Ca}$ II $\mathrm{H}$ and $\mathrm{K}$ lines. The four wavelength bands that have been used for recording data are marked in this image. The tunable filter alternated between the line core at $396.85 \mathrm{~nm}$ (Band A) and the wing position at $396.74 \mathrm{~nm}$ (Band B) of the Ca II H line. The fixed filter was centered at the wing position of $396.47 \mathrm{~nm}$ (Band C) and the wide band continuum filter was centered at $395.38 \mathrm{~nm}$ (Band $\mathrm{D})$, a wavelength midways between the $\mathrm{Ca}$ II H and $\mathrm{K}$ lines. The positions were chosen to minimize blends with other lines. The optical setup, which is shown in Fig. 1(b), consisted of two Redlake MegaPlus 1.6i and two Redlake MegaPlusII es1603 cameras, strictly synchronized by means of an external shutter. A defocused camera was used in a phase diversity 


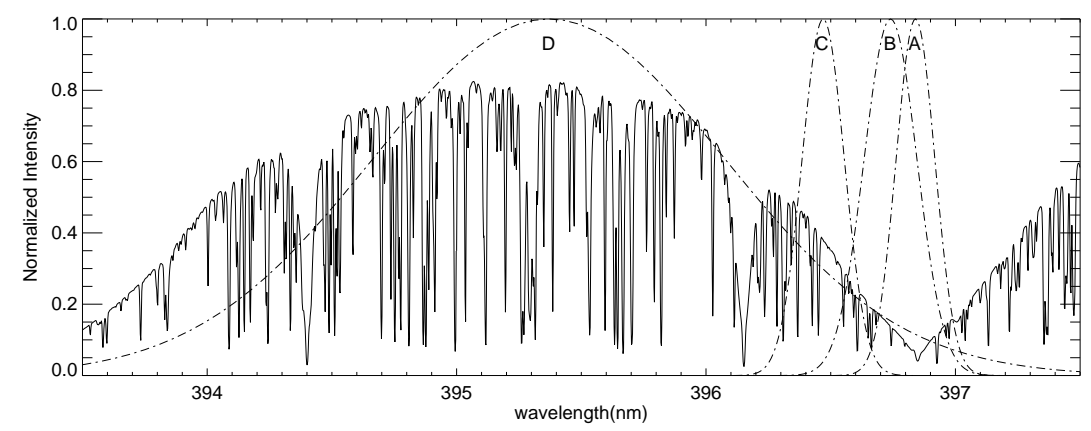

(a)

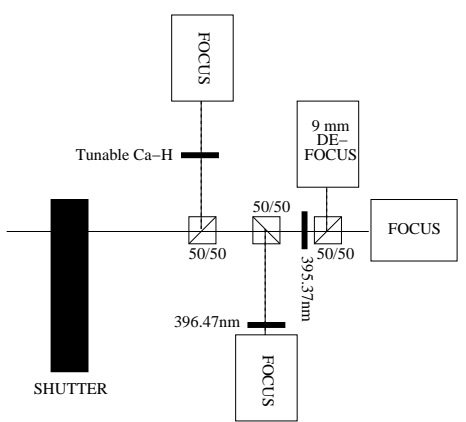

(b)

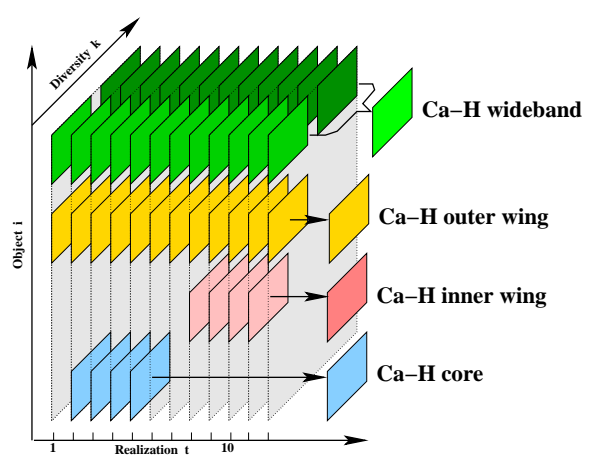

(c)

Figure 1. (a) Intensity versus wavelength plot of the $\mathrm{Ca} I \mathrm{H}$ and $\mathrm{K}$ lines, superposed are the four wavelength bands (A, B, C, D) used for observations. (b) Optical setup. (c) Observation scheme. See the text for details.

setup with the wide band continuum camera. The tunable filter camera though synchronized, took alternately few frames in bands A and B, as shown in Fig. 1(c). The frame rate of the cameras was 3.5 frames per second, at an exposure time of $10 \mathrm{~ms}$.

The data set was then MOMFBD (van Noort et al. 2005) processed, assuming that simultaneous exposures are blurred by the same seeing and thus have identical wavefront abberations. This is illustrated in Fig. 1(c). Every plane represents a unique wavefront, shared by the various frames in that plane. Even though some objects do not have any exposures in common, they can be aligned with sub-pixel accuracy through other objects that have frames in common with both.

\section{Results}

Figure 2 shows the image set recorded on June 26 2006. It features a small spot (AR0897) near the solar limb. Figure 3 recorded on July 42006 features a large spot (AR0898) near 


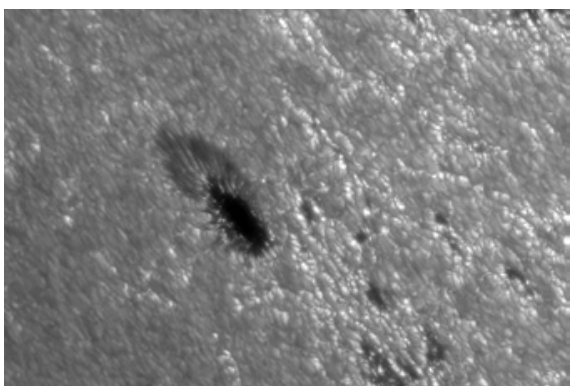

(a) Band D

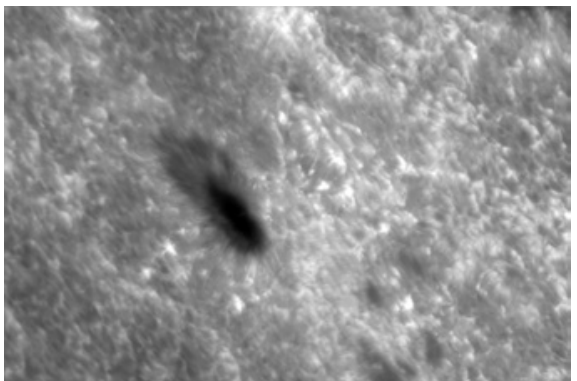

(c) Band B

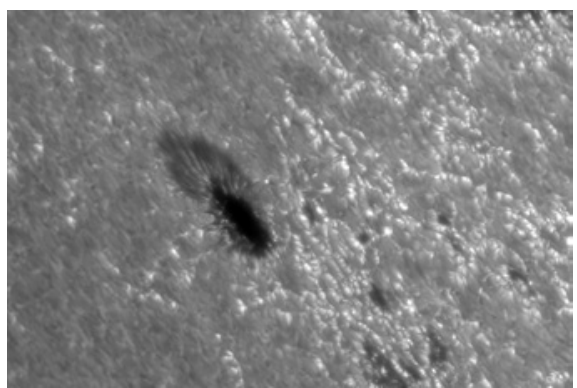

(b) Band C

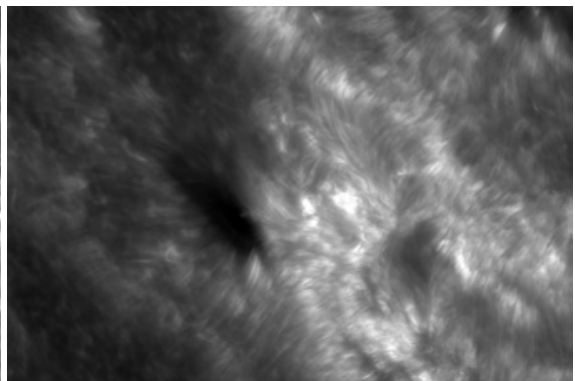

(d) Band A

Figure 2. Small spot (AR0897) near solar limb in 4 different wavelength bands (see Fig. 1(a)).

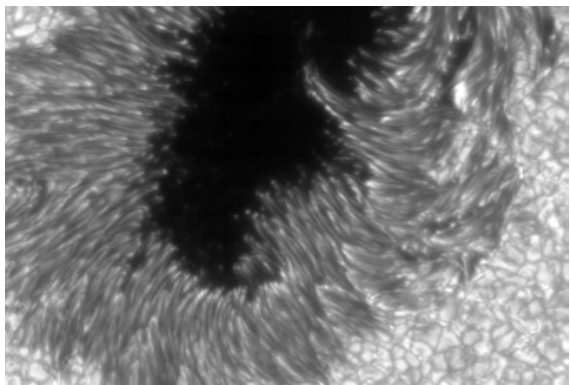

(a) Band D

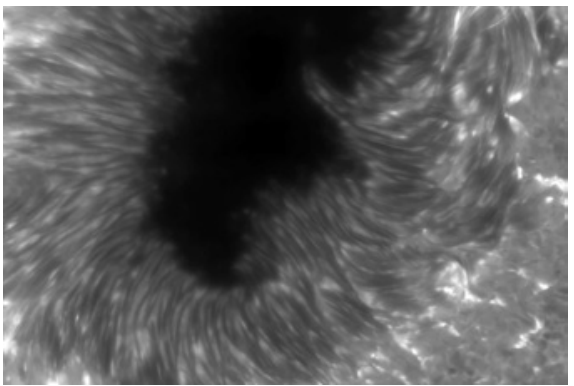

(c) Band B

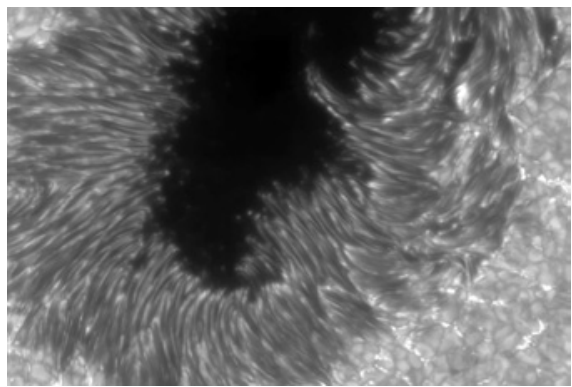

(b) Band C

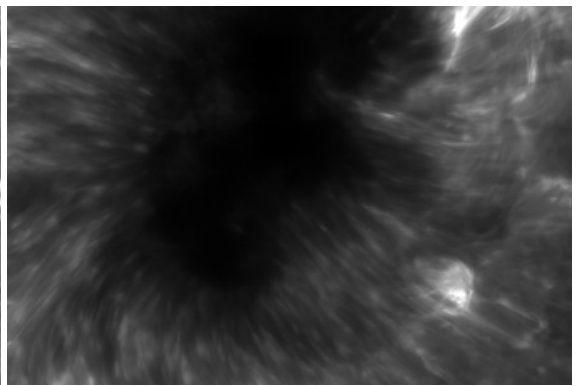

(d) Band A

Figure 3. Large spot (AR0898) near solar disk center in 4 different wavelength bands (see Fig. 1(a)). 
the solar disk center. Figures 4 and 5 are co-spatial subfields extracted from the images of Figs. 2 and 3, respectively, showing from right to left, bands $\mathrm{A}, \mathrm{B}, \mathrm{C}$ and $\mathrm{D}$ (see Fig. 1(a)). In Fig. 4, the variation in height as we move from the continuum to the core of the line is clearly evident. This effect is much less pronounced in Fig. 5 as expected as height variations are most prominent in limb observations.
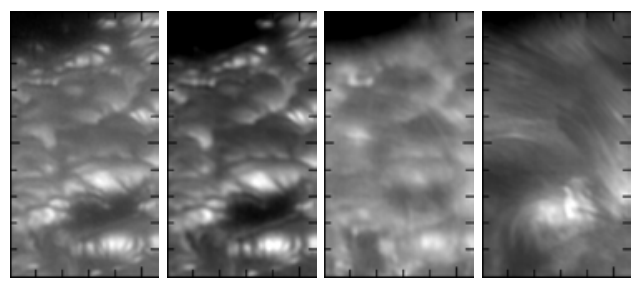

Figure 4. Co-spatial subfields extracted from the images presented in Fig. 2; from right to left: Bands $\mathrm{A}, \mathrm{B}, \mathrm{C}$ and D.

\section{Conclusions}

By combining multiple images recorded simultaneously at different wavelength positions and using short exposure times, MOMFBD image restoration can be used to perfectly align all images and to restore temperature information down to scales approaching the diffraction limit. It is thus possible to analyse resolved small-scale structures and their dynamics in the solar photosphere using the $\mathrm{Ca}$ II $\mathrm{H}$ wings. The next step is to derive the temperature structure above the photosphere by applying inversion techniques.
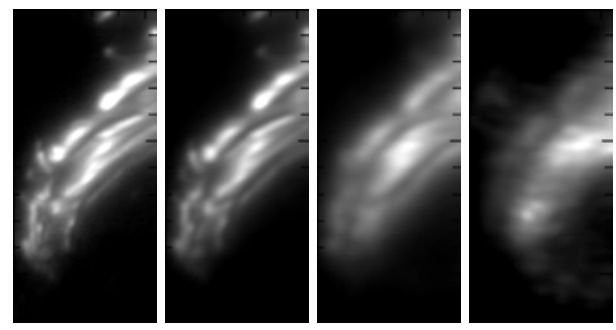

Figure 5. Co-spatial subfields extracted from the images presented in Fig. 3; from right to left: Bands $\mathrm{A}, \mathrm{B}, \mathrm{C}$ and D.

Acknowledgements. The Swedish 1-m Solar Telescope is operated by the Institute for Solar Physics of the Royal Swedish Academy of Sciences on the island of La Palma at the Spanish Observatorio del Roque de los Muchachos of the Instituto de Astrofísica de Canarias.

\section{References}

Grigoryeva, S. A. \& Turova, I. P. 1998, Solar Phys., 179, 17

Rouppe van der Voort, L. H. M. 2002, PhD thesis, Stockholm University

Sheminova, V. A., Rutten, R. J., \& Rouppe van der Voort, L. H. M. 2005, A\&A, 437, 1069

van Noort, M., Rouppe van der Voort, L. H. M., \& Löfdahl, M. G. 2005, Solar Phys., 228, 191 


\title{
Temporal evolution of intensity, velocity and magnetic field of sunspots at high spatial resolution
}

\author{
N. Bello González*, F. Kneer, and K. G. Puschmann \\ Institut für Astrophysik, Göttingen, Germany \\ *Email: nazaret@astro.physik.uni-goettingen.de
}

\begin{abstract}
We present results of sunspot observations obtained in April 2006 with the new 'Göttingen' Fabry-Perot spectrometer. Thanks to the large field of view $\left(77^{\prime \prime} \times 58^{\prime \prime}\right)$ of the new optical setup it has been possible to perform 2D-spectropolarimetric observations of a small sunspot and ist surroundings at a heliocentric angle $\theta \sim 40^{\circ}$. A long time series of about one hour has been taken scanning along the magnetic $\mathrm{Fe}_{\mathrm{I}} 6173 \AA$ and the non-magnetic $\mathrm{Fe}_{\mathrm{I}} 5576 \AA$ spectral lines quasi-simultaneously. Hence, with the help of image reconstruction techniques, the temporal evolution of the sunspot fine-structure in intensity as well as in velocity and magnetic field is analysed at high spatial resolution.
\end{abstract}

\section{Introduction}

After one century of investigations on their magnetic and dynamical nature, sunspots remain enigmatic. Their evolution from pores to sunspots with developed umbra and penumbra and their stability at small scales is not well understood. There is growing evidence that, even at high spatial resolution of current spectroscopic observations, the sunspot fine-structure is still unresolved. The key for the study of the sunspot magnetic fields and the Evershed flow in the penumbra consists in spectropolarimetry at high spatial resolution. We need to obtain and to analyse the information carried by suitable spectral lines and to measure and interpret the polarised radiation coming from the solar atmosphere. Therefore, our work aims at contributing to the understanding of the sunspot fine-structure from the analysis of spectropolarimetric observations with spatial resolution better than 0.'5.

\section{Observations and data analysis}

The observations of sunspots and surroundings were obtained with the optical setup based on the upgraded Göttingen Fabry-Perot interferometer (Puschmann et al. 2006), a Stokes $V$ polarimeter and the Kiepenheuer Adaptive Optics System (KAOS), attached to the Vacuum Tower Telescope (Observatorio del Teide, Tenerife). The data consist of a time series ( $65 \mathrm{~min}$ ) of 2D-imaging of both circularly polarised components of the light, i.e. $\frac{1}{2}(I+V)$ and $\frac{1}{2}(I-V)$, while scanning almost simultaneously along the magnetic sensitive Fe I $6173.3 \AA(g=2.5)$ and the non-magnetic Fe I 5576.1 $(g=0)$ lines. The active region NOAA 0867 was observed on April 6, 2006, when it was located at a heliocentric angle of 
$\theta \sim 40^{\circ}$. As shown in Fig. 1, the field of view (FOV) includes a round, symmetric sunspot accompanied by two pores nearby, at $\sim 3^{\prime \prime}$ distance from the penumbral border, and some others farther away (at $\sim 15^{\prime \prime}$ distance).

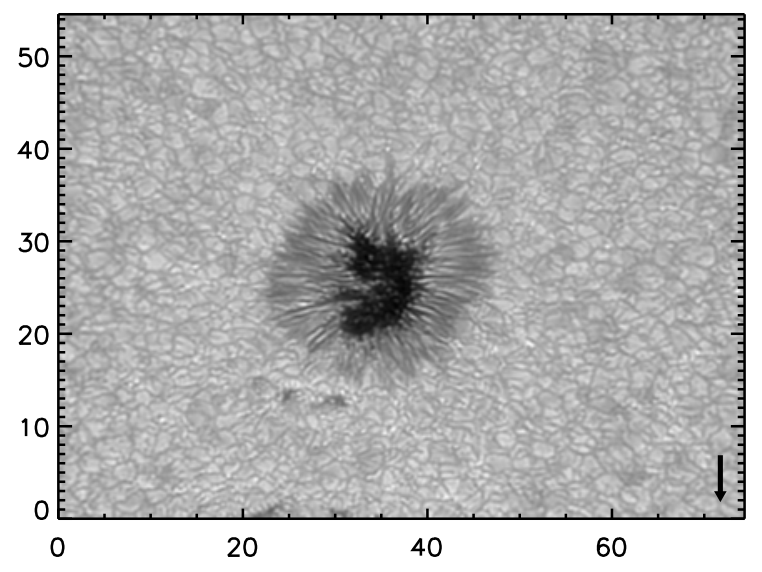

Figure 1. Speckle reconstructed image of NOAA 0867 and surroundings observed on April 6, 2006. Arrow points towards disc centre and scale is given in arcseconds.

Thanks to the strictly simultaneous observations of broad-band images, speckle reconstruction techniques can be applied to achieve a spatial resolution of 0.25 for the broadband data $\left(\mathrm{FOV} \sim 73^{\prime \prime} \times 55^{\prime \prime}\right)$. A similar procedure to that proposed by Puschmann $\&$ Sailer (2006) to account for the effects of the adaptive optics correction has been used. The narrowband data (FOV $\sim 29^{\prime \prime} \times 50^{\prime \prime}$ ) are then restored (Keller \& von der Lühe 1992) achieving a spatial resolution better than 0 .' 5 for magnetograms and velocity maps.

The magnetic and velocity field calculations for the whole time series are similar to that described in Bello González et al. (2005). They consist of magnetic field measurements in the Fe I 6173 line, using the Zeeman splitting visible in the Stokes $I$ profiles and the separation of the centre of gravity (COG method) of both circularly polarised components. The methods yield, respectively, the total magnetic field strength and the line of sight (LOS) component of the magnetic field averaged over the atmospheric region where the line is formed, i.e. mid photosphere. To estimate the velocity field, the COG method has also been applied. In this case it consists of measuring Doppler shifts from the average COG position of the $\frac{1}{2}(I+V)$ and $\frac{1}{2}(I-V)$ profiles of Fe I 6173 and from the Stokes $I$ profiles of Fe I 5576 , both with respect to a reference wavelength position, i.e. the surrounding non-spot photosphere.

\section{Results on temporal evolution}

An example of the resulting maps is given in Fig. 2. There, the intensity at continuum level, at the line minimum of Fe I 6173, LOS magnetic maps and LOS velocities are shown. We summarise the main observational characteristics visible from individual maps and from the temporal evolution: 
- The well-known apparent inward motion of the fibrils in the inner penumbra and outward motions in the outer penumbra seen in the intensity maps (Sobotka \& Sütterlin 2001) are also visible in both the magnetic and velocity maps.

- The penumbral filaments remain stable within the 65 min sequence not only in intensity but also in magnetic and velocity maps (see also Langhans et al. 2005).

- In the continuum images, the granulation pattern, the highly structured filaments of the penumbra and the umbral dots are seen with high spatial resolution. The nearby pores show enhanced-intensity structures at their limb-side borders. The interpretation is that the magnetic and therefore depressed and cooler atmosphere of pores is more transparent to the radiation. Then we see the hot walls of the surrounding granules on their limb side.

- With regard to the intensity maps of the minimum of Fe I 6173, the typical granulation pattern is not visible. However, the filamentary structure of the penumbra appears the same as in the continuum image except for the ring of enhanced intensity visible in the inner to middle penumbra. This effect is also visible in the wings of Fe I 5576. This has recently been explained by Bellot Rubio et al. (2006) in terms of the smaller line asymmetries and line widths from the inner penumbra compared to the outer penumbra (where the Evershed flow is stronger). The explanation does not apply to the enhanced intensities observed in the line minimum of $\mathrm{Fe}_{\mathrm{I}}$ 6173. Its increased (raised) core intensity is due to the Zeeman effect broadening by the strong magnetic fields in the inner penumbra.

- From the stronger LOS component of the magnetic field in the inner centre-side penumbra and the opposite polarity shown in the outer limb-side penumbra, the general picture of the magnetic field configuration in sunspots arises: strong and more vertical magnetic fields in the umbra and inner penumbra and more horizontal fields towards the outer penumbra. At small scales, penumbral magnetic fields also organise in filaments which show stability within one hour. Features of both magnetic polarities close to each other appear in the limb-side penumbra. This does not occur on the centre side. This is again due to projection effects: The highly dynamic penumbra seen at $\theta \sim 40^{\circ}$ shows at the limb side mainly magnetic polarity opposite to that of the umbra, i.e. inclination larger than $50^{\circ}$ with respect to the vertical, with some slightly more vertical components, i.e. inclination smaller than $50^{\circ}$. The structures surrounding the spot possess low LOS magnetic field components and evolve moving away (Vrabec 1974). The penumbra-pore interaction always occurs such that the penumbra seemingly 'feeds' the pore with magnetic flux. A similar connection is not evident in the LOS velocity maps.

- Direct comparison shows that bright structures in the line-minimum maps are good tracers of the magnetic elements surrounding the spot. It is caused by the hotter atmospheres associated with such magnetic knots. This is the well known line-gap phenomena caused by increased temperatures in the atmospheres of magnetic knots. Similarly as in the G-band bright points (e.g. Steiner et al. 2001), high temperatures lead to ionisation of iron. Thus, the atmosphere becomes more transparent and intensities from lower layers are seen. 

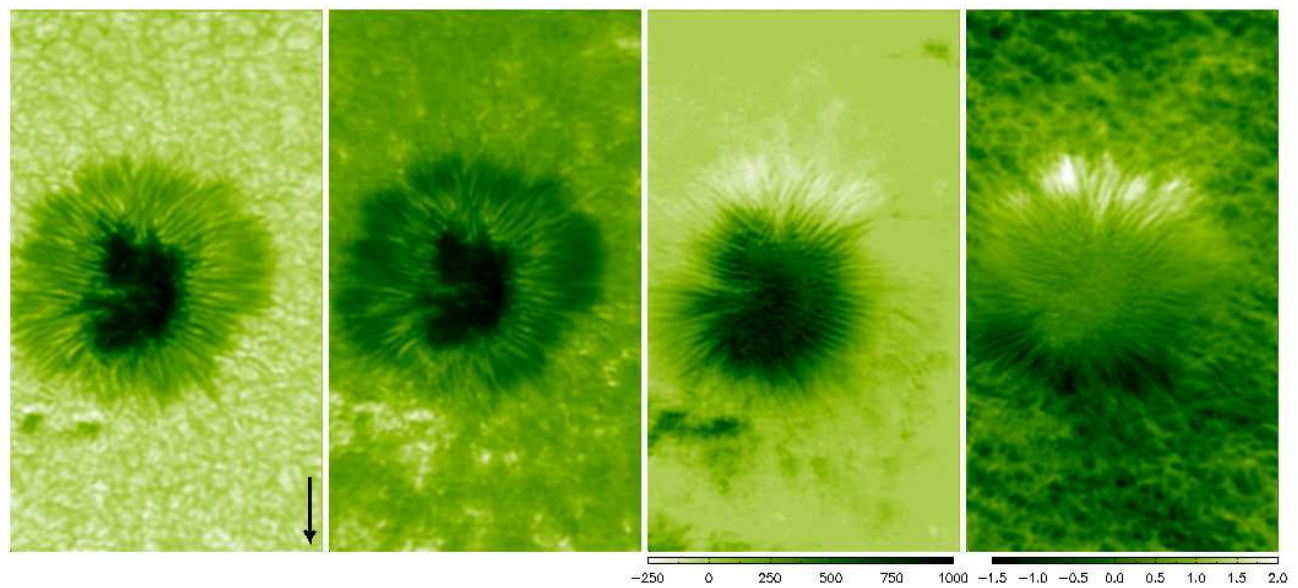

Figure 2. From left to right: Intensity map at continuum level, intensity map at the line minimum of $\mathrm{Fe}_{\mathrm{I}}$ 6173, LOS magnetic field (values in Gauss) and LOS velocity field (values in $\mathrm{km} \mathrm{s}^{-1}$ ). The FOV is $29^{\prime \prime} \times 50^{\prime \prime}$ and the arrow points towards disc centre.

- Detailed comparison of intensity and LOS velocity maps confirm the finding by Bello González et al. (2005), that the strongest LOS components of the Evershed flow are correlated with bright structures in the centre-side penumbra and with dark structures on the limb side. Such a correlation is not a fortuitous phenomenon since it is stable and visible during the whole time sequence.

In conclusion, from observations at high spatial resolution much information can be inferred on the small-scale structures of the solar photosphere, where magnetic fields and mass flows interact. Many other observable processes taking place in sunspots and their close surroundings, together with those described here, will be addressed in a forthcoming paper which will include the online material on the time evolution.

Acknowledgements. NBG and KGP thank the Deutsche Forschungsgemeinschaft for financial support through grants Kn 52/31-1 and Kn 152/29, respectively. The Vacuum Tower Telescope is operated by the Kiepenheuer-Institut für Sonnenphysik, Freiburg, at the Spanish Observatorio del Teide of the Instituto de Astrofísica de Canarias.

\section{References}

Bello González, N., Domínguez Cerdeña, I., Okunev, O., Kneer, F., \& Puschmann, K. G. 2005, A\&A, 434, 317

Bellot Rubio, L. R, Schlichenmaier, R., \& Tritschler, A. 2006, A\&A, 453,1117

Keller, C. U. \& von der Lühe, O. 1992, A\&A, 261, 321

Langhans, K., Scharmer, G. B., Kiselman, D., Löfdahl, M. G., \& Berger, T. E. 2005, A\&A, 436, 1087

Puschmann, K. G. \& Sailer, M. 2006, A\&A, 454, 1011

Puschmann, K. G., Kneer, F., Seelemann, T., \& Wittmann, A. D. 2006, A\&A, 451, 1151

Sobotka, M. \& Sütterlin, P. 2001, A\&A, 380,714

Steiner, O., Hauschildt, P. H., \& Bruls, J. 2001, A\&A, 372, L13

Vrabec, D. 1974, IAUS, 56, 201 


\title{
Magnetic vector field above a sunspot
}

\author{
P. Gömöry ${ }^{1,2}$ and H. Balthasar ${ }^{1}$ \\ ${ }^{1}$ Astrophysikalishes Institut, Potsdam, Germany \\ ${ }^{2}$ Astronomical Institute of the Slovak Academy of Sciences, Tatranská Lomnica, Slovakia \\ *Email: gomory@astro.sk, hbalthasar@aip.de
}

\begin{abstract}
We present an analysis of the spectro-polarimetric measurements of the active region NOAA 10886 taken with the Tenerife Infrared Polarimeter in the Fe I $10783 \AA$ Apectral line. The magnetic field strength and the magnetic inclination and azimuth were inferred from the data using the inversion code SIR. The vertical current densities and the vertical derivative of the vertical component of the magnetic field were then calculated for the leading spot of the AR 10886. We revealed that typical values of the vertical current densities vary in the range $\pm 40 \mathrm{~mA} \mathrm{~m}^{-2}$ inside the spot. Moreover, we found an extended area of enhanced vertical current densities in the umbra (values up to $-80 \mathrm{~mA} \mathrm{~m}^{-2}$ ). Our analysis also showed that the vertical component of the magnetic field strength decreases by 0.6 $1.6 \mathrm{G} \mathrm{km}^{-1}$ and $0.5-0.8 \mathrm{G} \mathrm{km}^{-1}$ in the umbra and inner penumbra; on the other hand we found an increase of the vertical component of the magnetic field in the outer penumbra (typical values varying between $0.1-0.5 \mathrm{G} \mathrm{km}^{-1}$ ).
\end{abstract}

\section{Introduction}

The low value of the plasma $\beta$ in the corona implies that the processes in the outer solar atmosphere are driven by magnetic fields (Walsh \& Ireland 2003). But with the present instruments it is almost impossible to measure coronal magnetic fields directly. Therefore, extrapolations of the photospheric magnetic fields are commonly used for a better description of the coronal structures. The most recent extrapolation methods assume a force-free magnetic field (Régnier et al. 2002; Valori et al. 2005). A good knowledge of the full magnetic vector $\mathbf{B}$ and/or the electric current densities $\mathbf{j}$ is crucial for such extrapolations. Moreover, better determinations of the electric current densities can help to understand the fine structure of sunspots (e.g. penumbral filaments). Another important and still not fully solved question is how fast the magnetic field strength decreases with height.

Following these points we investigate the magnetic vector field of a sunspot in order to determine the vertical component of the electric current densities and the vertical derivative of the vertical component of the magnetic field strength. For the calculation of these parameters we used the method described in the paper of Balthasar (2006).

\section{Data and data reduction}

The data discussed here were taken on May 27, 2006 with the Tenerife Infrared Polarimeter (Martínez Pillet et al. 1999) attached to the spectrograph of the German Vacuum Tower Telescope. In addition, the KAOS system (Kiepenheuer Adaptive Optic System) was used 


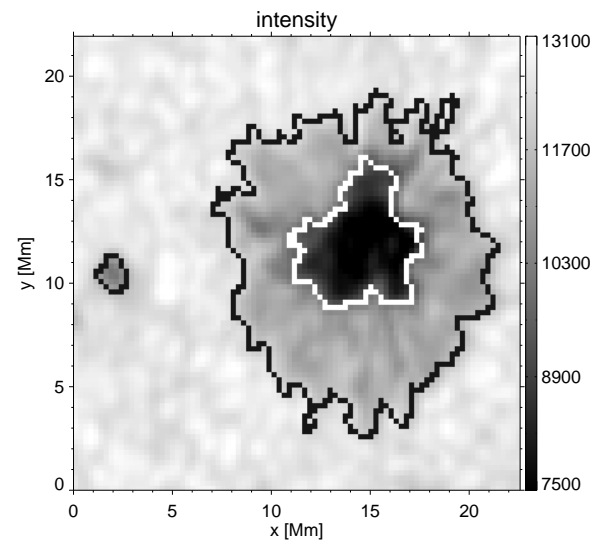

Figure 1. Infrared intensity map of the leading spot of the active region NOAA 10886 composed of the local continuum near the observed spectral line. Scanning was done from left to right. The contour lines mark the outer boundaries of umbra and penumbra.

to ensure stable conditions during the whole observing sequence. The full Stokes vector measurements of the active region NOAA 10886 were performed near disk center in the $\mathrm{Fe}$ I $10783 \AA$ spectral line. To obtain the final components of the Stokes vector (i.e. $I, Q, U$, and $V$ ), 10 sets of single exposures of $200 \mathrm{~ms}$ were added up to increase the signal-to-noise ratio. Therefore, the exposure time was $2 \mathrm{~s}$ for each Stokes parameter. The observed AR was scanned with a step width of 0.34 arcsec. The pixel size along the slit corresponded to 0.35 arcsec. Totally 3 scans, each with 360 steps, were performed to cover the whole AR. Note, that in this paper we will focus only on results which were determined for the leading spot of the AR 10886 (Fig. 1).

The standard photometric reduction (i.e. removing of the dark counts, applications of the flatfields etc.) of the raw measurements was performed, and the instrumental crosstalk was removed from the data. The data were then corrected for geometrical foreshortening and interpolated so that a pixel size corresponds to 0.34 arcsec in both spatial directions.

\section{Results and Discussion}

The components of the magnetic vector $\mathbf{B}$ (magnetic field strength, and magnetic field angles) were determined through an inversion of the Stokes parameters. The inversion was performed using the SIR code (Ruiz Cobo \& del Toro Iniesta 1992) in the same way as it is described in Balthasar \& Collados (2005). Vertical components of the electric current densities $j_{z}$ were then calculated using the following formula

$$
\mu j_{z}=\frac{\partial B_{y}}{\partial x}-\frac{\partial B_{x}}{\partial y},
$$

where $\mu$ is the magnetic permeability. The partial derivatives were determined from the difference of the two neighboring pixels. The obtained values of the $j_{z}$ are shown in Fig. 2 (left). Typical values of the vertical current densities vary between $\pm 40 \mathrm{~mA} \mathrm{~m}^{-2}$ in the 

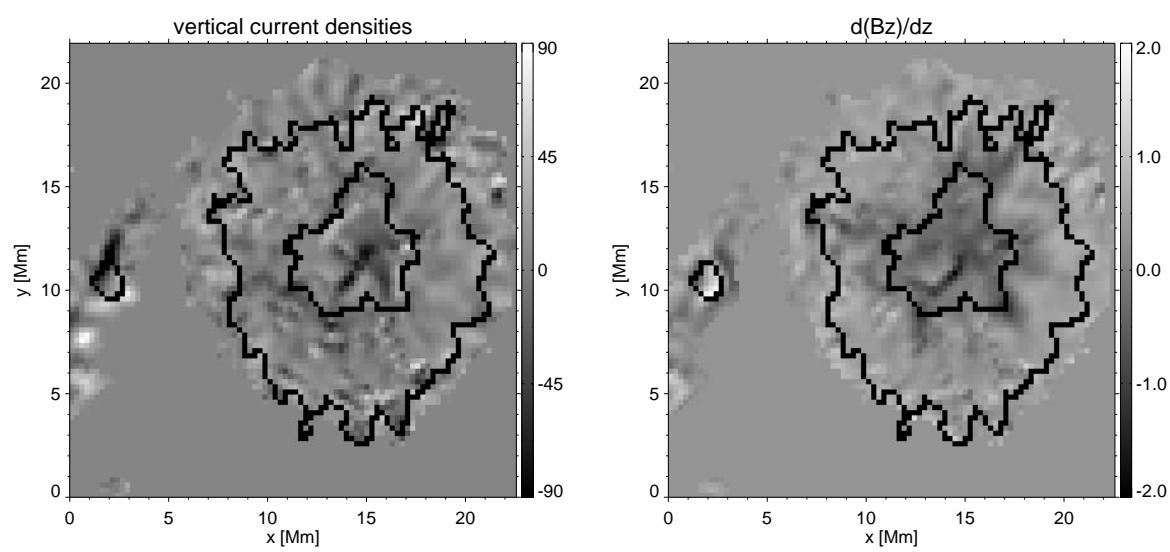

Figure 2. Left: Vertical current densities calculated for the leading spot of the AR 10886. The displayed values are given in $\mathrm{mA} \mathrm{m}^{-2}$. Right: Vertical derivative of the vertical component of the magnetic field strength for the leading spot of the AR 10886. The displayed values are given in $\mathrm{G} \mathrm{km}^{-1}$. Note that if the integrated absolute circular and linear polarization both drop below a treshold value, the remaining polarization signal is regarded to be noise and the corresponding areas are suppressed in the figures. The contour lines indicate outer and inner penumbral boundary.

umbra. The values of the $j_{z}$ in the penumbra are somewhat smaller and vary in the range $\pm 30 \mathrm{~mA} \mathrm{~m}^{-2}$. Note that the estimated errors of the values mentioned above are of the same order of magnitude. These findings are in good agreement with results of Balthasar (2006). He analyzed a set of eight sunspots and came to the conclusion that the typical values of the vertical current densities vary between $\pm 40 \mathrm{~mA} \mathrm{~m}^{-2}$ inside the sunspots. Beside of this we found also areas with enhanced values of the vertical current densities extending up to $-75 \mathrm{~mA} \mathrm{~m}^{-2}$ in the umbra and up to $-60 \mathrm{~mA} \mathrm{~m}^{-2}$ in the penumbra. Unfortunately, limited spatial resolution of the VTT did not allow us to associate the position of the enhanced vertical current densities with the fine structures of the sunspot. On the other hand, relatively high values of the $j_{z}$ detected near the outer penumbral boundary are rather unrealistic as only a very weak polarization signal was detected in that area.

As the Fe $\mathrm{I} 10783 \AA$ spectral line comes from a rather narrow atmospheric layer, the vertical stratification of the magnetic field strength obtained directly from the inversion code could be unreliable. Therefore, we calculated only the vertical derivative of the vertical component of the magnetic field strength using partial derivatives of the horizontal magnetic components, i.e.

$$
\frac{\partial B_{z}}{\partial z}=-\left(\frac{\partial B_{x}}{\partial x}+\frac{\partial B_{y}}{\partial y}\right) \Longleftrightarrow \operatorname{div} \boldsymbol{B}=0 .
$$

The same method was used by Hofmann \& Rendtel (1989) and Balthasar (2006). The determined gradients of the $B_{z}$ are shown in Fig. 2 (right). We revealed that the magnetic field strength decreases with height by $0.6-1.6 \mathrm{G} \mathrm{km}^{-1}$ in the umbra. These values are higher than the $0.3-0.4 \mathrm{G} \mathrm{km}^{-1}$ obtained by Hofmann \& Rendtel (1989) but comparable with the findings of Balthasar (2006). We found negative gradients of the vertical component of the 
magnetic field strength also in the inner penumbra. But the decrease of the $B_{z}$ with height is slower in the inner penumbra than in the umbra (typical values vary between $-0.5 \mathrm{G} \mathrm{km}^{-1}$ and $-0.8 \mathrm{G} \mathrm{km}^{-1}$ ). In contrast, we found an increase of the magnetic field strength with height in the outer penumbra and outside the spot. The typical values are in the range between $0.1 \mathrm{G} \mathrm{km}^{-1}$ and $0.5 \mathrm{G} \mathrm{km}^{-1}$. The increase of the magnetic field strength with height can be explained if the magnetic field is less inclined in higher layers, as reported by Westendorp Plaza et al. (2001). But recently Borrero et al. (2004) found an increase of the inclination with height while results of Sánchez Cuberes et al. (2005) reveal that the inclination is independent of height.

\section{Conclusions}

We examined the vertical current densities and the height dependence of the vertical component of the magnetic field strength in a sunspot. Our analysis showed that typical values of $j_{z}$ vary between $\pm 40 \mathrm{~mA} \mathrm{~m}^{-2}$ inside the spot. We also revealed a decrease of the vertical component of the magnetic field strength with height in the umbra and inner penumbra and positive height gradients of $B_{z}$ in the outer penumbra and outside the spot. But we should remind here that the results presented in this paper depend on two assumptions: $(i)$ the neighboring magnetic components belong to the same height layer, and (ii) they belong to the same magnetic finestructure. If this is not true then the obtained values become meaningless as the determined partial derivatives (see Eqs. (1) and (2)) and were not calculated for the same structure of the spot. Data with much higher spatial resolution (as they will be provided with new solar telescopes like GREGOR or ATST) are therefore necessary for the future verification of our results.

Acknowledgements. The Vacuum Tower Telescope in Tenerife is operated by the KiepenheuerInstitut für Sonnenphysik (Germany) in the Spanish Observatorio del Teide of the Instituto de Astrofísica de Canarias. This research is part of the European Solar Magnetism Network (EC/RTN contract HPRN-CT-2002-00313).

\section{References}

Balthasar, H. 2006, A\&A, 449, 1169

Balthasar, H. \& Collados, M. 2005, A\&A, 429, 705

Borrero, J. M., Solanki, S. K., Bellot Rubio, L. R. et al. 2004, A\&A, 422, 1093

Hofmann, A. \& Rendtel, J. 1989, Astron. Nachr., 310, 61

Martínez Pillet, V., Collados, M., Sánchez Almeida, J. et al. 1999, in High Resolution Solar Physics: Theory, Observations and Techniques, eds. T. R. Rimmele, K. S. Balasubramaniam, \& R. R. Radick, ASP Conf. Ser., 183, 264

Régnier, S., Amari, T., \& Kersale, E. 2002, A\&A, 392, 1119

Ruiz Cobo, B., \& del Toro Iniesta, J. C. 1992, ApJ, 398, 375

Sánchez Cuberes, M., Puschmann, K. G., \& Wiehr, E. 2005 A\&A, 440, 345

Valori, G., Kliem, B., \& Keppens, R. 2005, A\&A, 433, 335

Walsh, R. W. \& Ireland, J. 2003, A\&AR, 12, 1

Westendorp Plaza, C., del Toro Iniesta, J. C., Ruiz Cobo, B. et al. 2001, ApJ, 547, 1130 


\title{
The observational counterpart of the rising flux tube model?
}

\author{
J. Jurčák ${ }^{*}$ and M. Sobotka \\ Astronomical Institute of the Academy of Sciences, Ondřejov, Czech Republic \\ *Email: jurcak@asu.cas.cz
}

\begin{abstract}
An analysis of Stokes observations of a penumbra in active region NOAA 8990 is presented. The observations were recorded with the La Palma Stokes Polarimeter attached to the 1-m Swedish Solar Telescope. The stratification in the solar atmosphere of different physical parameters is retrieved from these data using the Stokes Inversion based on Response functions (SIR). Our results confirm previous findings, that with increasing distance from the umbra the magnetic field becomes weaker and more horizontal and the line-of-sight velocities increase. The results suggest the existence of unresolved filamentary structure in the spatial distributions of temperature, magnetic field strength and inclination. The maps of temperature and magnetic field strength along the vertical cuts through the penumbra indicate the presence of rising flux tubes, predicted theoretically by Schlichenmaier et al. (1998).
\end{abstract}

\section{Introduction}

The fine structure of the penumbra was studied, among earlier investigations, by e.g. Beckers \& Schröter (1969), who found stronger and more vertical magnetic field in dark filaments than in the bright ones. Recently, many authors agreed on the picture of the uncombed structure of the penumbral magnetic field, which was proposed by Solanki \& Montavon (1993) to explain the broadband circular polarisation of sunspots and further developed by Martínez Pillet (2000). This uncombed structure is created from horizontal flux tubes (with diameters around $100 \mathrm{~km}$ ) embedded in a more vertical and stronger background field. Evidences for this empirical model were found in recent analyses of observations (see e.g. Bello González et al. 2005; Bellot Rubio et al. 2006, and references therein).

This complex magnetic fine structure of the penumbra can be caused by the convective exchange of the flux tubes. One of the possible scenarios starts with a flux tube which is initially positioned at the penumbra-quiet sun boundary. It is heated by the field-free convection and rises, developing a flow along the tube that points upwards beneath and outwards in the photosphere. The part of the flux tube emerging above the $\log \tau=0$ surface is filled with hot gas and creates a bright grain. At higher layers, the flux tube cools down by radiative losses and becomes more horizontal and the outward flow resembles the observed Evershed effect. Up to this point, the scenario has been confirmed by the two-dimensional simulations made by Schlichenmaier et al. (1998). The theoretical maps of plasma parameters in and around the rising flux tube can be found in that article. In the present work, these theoretical maps are compared with the maps of plasma parameters found in the penumbra using the 


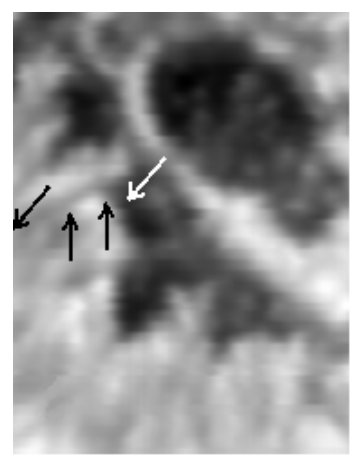

Figure 1. The continuum intensity in the part of the active region observed on May 13, 2000. The arrows are explained in Section 3.

observations and data processing described in the following section.

\section{Observations and data processing}

The data were retrieved with the La Palma Stokes Polarimeter (LPSP) attached to the 1-m Swedish Solar Telescope. The resulting spatial resolution is of about 0." 7 .

The object of the observations was an irregular leading sunspot in the active region NOAA 8990. One of the two fields, which were scanned in magnetically sensitive lines Fe I $630.15 \mathrm{~nm}$ (Landé factor $g=1.67)$ and Fe I $630.25 \mathrm{~nm}(g=2.5)$, is shown in Fig. 1 . The field covers a limbward part of a penumbra at heliocentric position $14^{\circ} \mathrm{N}$ and $17^{\circ} \mathrm{W}$.

In each pixel of this field all Stokes profiles of the two mentioned lines were measured and used as an input to the inversion code SIR (Ruiz Cobo \& del Toro Iniesta 1992). A one-component model of atmosphere was used with two different settings of numbers of nodes for the inversion. The numbers of nodes, which should correspond to the expected complexity of the stratifications of the appropriate plasma parameters, are listed in Table 1. Other details about the observation, data reduction, and inversion can be found in Jurčák et al. (2006).

\section{Results}

Our results confirm previous findings: The magnetic field becomes weaker and more horizontal and the line-of-sight velocities increase with increasing distance from the umbra.

Table 1. The numbers of nodes in two different settings of the inversion process.

\begin{tabular}{lcccc}
\hline & Temperature & Mag. field strength & Inclination & LOS velocity \\
\hline Inversion 1 & 9 & 7 & 5 & 5 \\
Inversion 2 & 9 & 9 & 9 & 9 \\
\hline
\end{tabular}



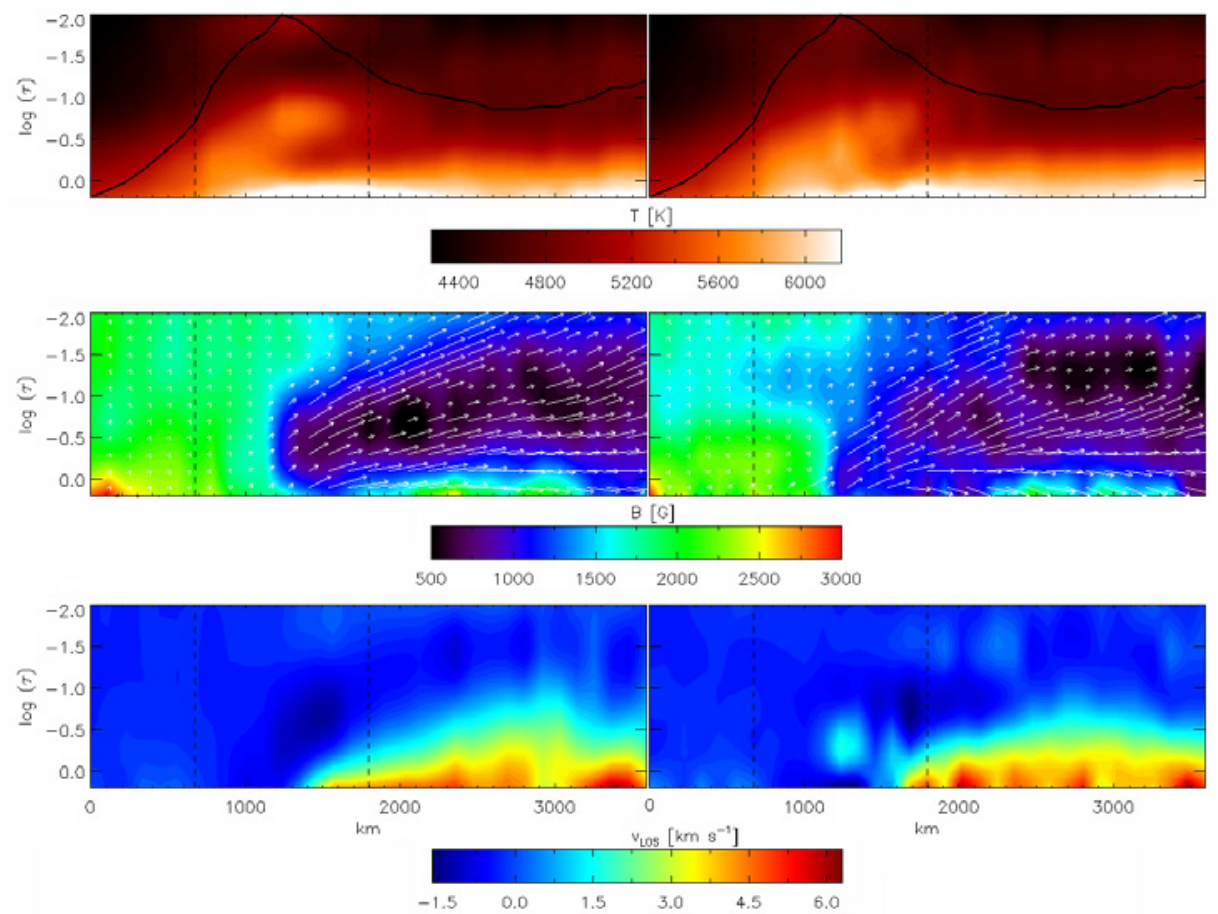

Figure 2. The stratifications of temperature (upper row), magnetic field strength together with velocities along the field lines marked by white arrows (middle row), and line-of-sight velocity (lower row) along the vertical cut through the penumbra. The stratifications in the left and right column correspond to inversion 1 and 2, respectively with regard to the different settings in the numbers of nodes (see Table 1).

These general findings are not discussed due to the limited space and we concentrate on the expected fine structure of the penumbra.

We made a vertical cut through the measurements of the bright grain in the penumbra. The position of the cut is marked in Fig. 1 by the inclined arrows starting in the umbra and ending at the edge of the scanned area. The vertical arrows in Fig. 1 enclose the bright grain and correspond to the dashed black lines in Fig. 2, where the stratifications of plasma parameters along the cut are shown. The solid black lines in the temperature stratifications (Fig. 2, upper row) correspond to the continuum intensity along the cut. The difference between the right and left columns consists of the different numbers of nodes (see Table 1).

The temperature stratifications along the cut show the rising filamentary structure hotter than the surrounding plasma, which is, in the direction away from the umbra, rapidly cooled down and becomes horizontal. Moreover, this cooled flux tube can be followed in the plot of magnetic field strength (middle row), as the field is weak inside it. In the lower row are shown the line-of-sight (LOS) velocities obtained by the SIR code. The upflows can be found in areas of the bright grain, despite the limbward part of the penumbra is studied, where the horizontally oriented Evershed flow should be observed as a flow away from the 
observer. The height of the maximal LOS velocities does not correspond to the one of weak magnetic field. The velocities along the field lines were computed from the retrieved values of inclination and LOS velocities and are shown as white arrows in the maps of the magnetic field. The orientation of the arrows thus shows the magnetic field inclination and the length is proportional to the velocity magnitude.

In all bright grains in the analysed part of the penumbra the retrieved stratifications of temperature and magnetic field strength correspond to the theoretical predictions of the rising flux tube model, i.e. the hot filamentary structures are located in places of the bright grains in the penumbra, and the weak magnetic field is found in these bright grains and in the dark areas which can be traced back as the extensions of the bright filaments. Moreover, higher values of inclination are reached at the heights of the weak magnetic field if higher numbers of nodes are used for the inversion. The LOS velocities, however, reach maximal values at deepest layers irrespective of the numbers of nodes. Velocities along the magnetic field lines are uncertain and only rarely correlated with areas with inclined and weak magnetic field.

\section{Conclusions}

With the current spatial resolution $(500 \mathrm{~km})$ we cannot observe single flux tubes, but only their conglomerates, so that the presented results of the inversion can be influenced by complex unresolved structures. The agreement between the theoretical predictions and obtained stratifications of plasma parameters is nonetheless promising. However, observations with higher spatial resolutions are needed to clarify whether we really observe rising flux tubes or some fine structures in the background field which are not connected with the Evershed flow.

Acknowledgements. This work was supported by the research projects IAA3003404 of the GAAV of the Czech Republic and GACR 205/04/2129.

\section{References}

Beckers, J. M. \& Schröter, E. H. 1969, Solar Phys., 10, 384

Bello González, N., Okunev, O. V., Domínguez Cerdeña, I., Kneer, F., \& Puschmann, K. G. 2005, A\&A, 434, 317

Bellot Rubio, L. R., Schlichenmaier, R., \& Tritschler, A. 2006, A\&A, 453, 1117

Jurčák, J., Martínez Pillet, V., \& Sobotka, M. 2006, A\&A, 453, 1079

Martínez Pillet, V. 2000, A\&A, 361, 734

Ruiz Cobo, B. \& del Toro Iniesta, J. C. 1992, ApJ, 398, 375

Schlichenmaier, R., Jahn, K., \& Schmidt, H. U. 1998, A\&A, 337, 897

Solanki, S. K. \& Montavon, C. A. P. 1993, A\&A, 275, 283 


\title{
Simultaneous polarimetric observations with VTT and THEMIS
}

\author{
H. Balthasar ${ }^{1, *}$ and V. Bommier ${ }^{2}$ \\ ${ }^{1}$ Astrophysikalisches Institut Potsdam, Potsdam, Germany \\ ${ }^{2}$ Observatoire de Meudon, Meudon, France \\ *Email: hbalthasar@aip.de
}

\begin{abstract}
Spectro-polarimetric observations of sunspot groups have been performed simultaneously with the VTT and with THEMIS. At the VTT we observed a group of iron and silicon lines at $1078 \mathrm{~nm}$ with the Tenerife Infrared Polarimeter, and at THEMIS we observed in five spectral channels (MTRmode): $\mathrm{Fe} 630 \mathrm{~nm}, \mathrm{NaD}, \mathrm{H}_{\alpha}, \mathrm{Fe} 557.6 \mathrm{~nm}$ and alternatively $\mathrm{Cr} 578 \mathrm{~nm}$ or Fe $525 \mathrm{~nm}$. The inversion of the Stokes-profiles was done with the SIR-code for the infrared lines and for Fe $630 \mathrm{~nm}$, for the other spectral ranges the inversion is still pending. So far we derived that the magnetic field strength decreases with height at most locations inside the spots, in contrast to some recently published results of other investigations.
\end{abstract}

\section{Introduction}

The height dependence of the magnetic vector field has been investigated many times, but recent results are still controversial, see Balthasar \& Collados (2005), Sánchez Cuberes et al. (2005), Orozco Suárez et al. (2005), Balthasar (2006) and Jurčák et al. (2006). In order to obtain reliable results we observed several lines originating in the photosphere and in the chromosphere simultaneously with two telescopes next to each other: the German Vacuum Tower Telescope (VTT) and the French-Italian Télescope Héliographique pour l'Étude du Magnétisme et des Instabilités Solaires (THEMIS) on the island of Tenerife. Once the magnetic vector field is known, investigations of electric currents and electromagnetic forces become possible, and such investigations belong to our future plans.

\section{Observations and data reductions}

Two observing campaigns were performed in the years 2005 and 2006 . Here we present results from an active region observed on May 29, 2005. At the VTT we recorded the Stokes profiles of an iron and a silicon line in the near IR with the Tenerife Infrared Polarimeter (TIP II), both are Zeeman triplets with a splitting factor of 1.5. The Fe $1078.3 \mathrm{~nm}$ line probes the deep layers of the photosphere, while the Si $1078.6 \mathrm{~nm}$ line is formed in the mid photosphere.

At THEMIS we observed in the MulTi Raies (MTR) mode the chromium line Cr $578.2 \mathrm{~nm}$ from the deep photosphere, the iron lines Fe 610.1/630.2 nm from the mid photosphere and 


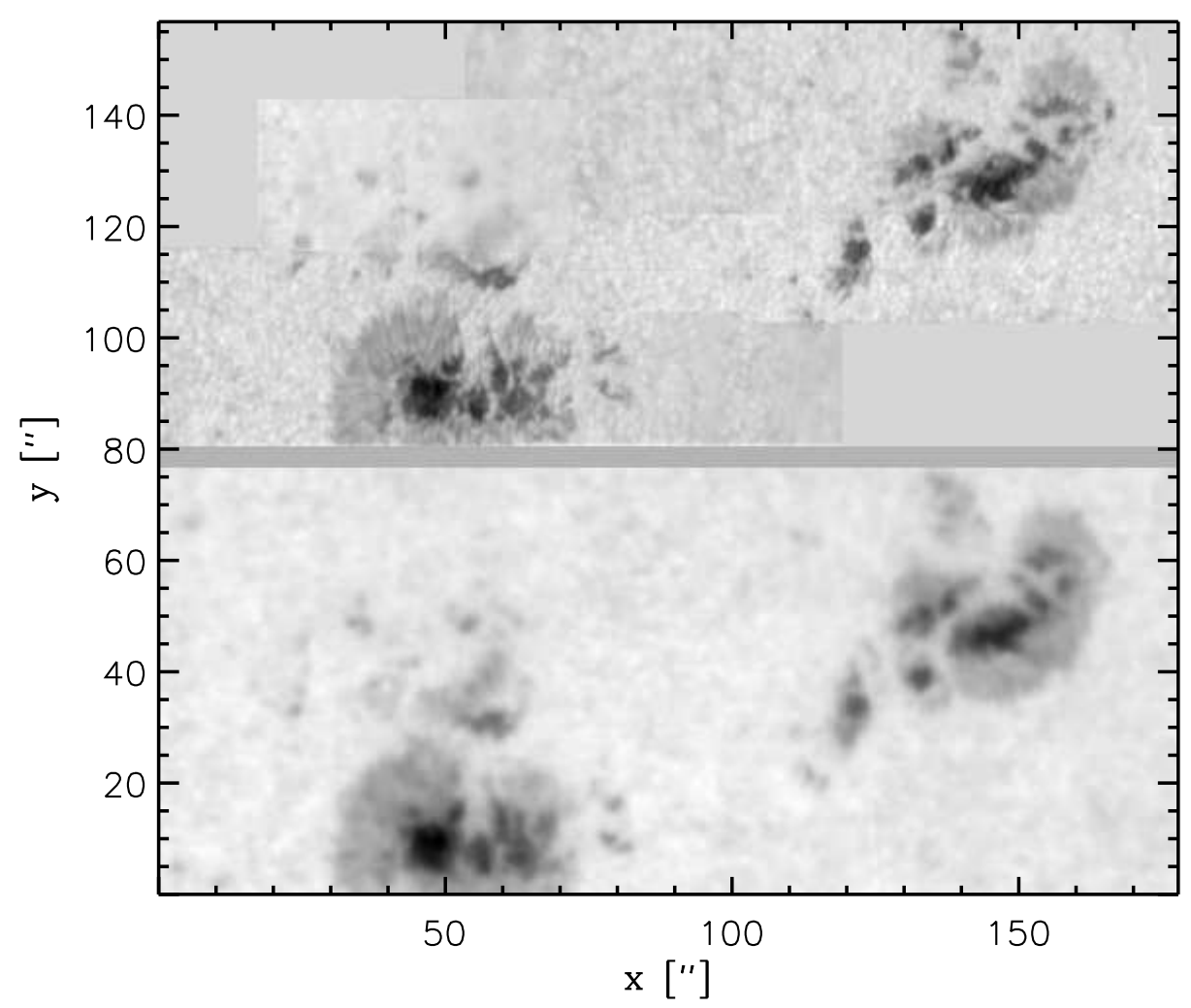

Figure 1. Intensity maps of the sunspot group, recombined from the spectral continua. the upper panel is obtained at the VTT in the near infrared using the adaptive optics system, the lower panel is from THEMIS near $630 \mathrm{~nm}$.

the sodium $\mathrm{D}_{1}$ line from the chromosphere. Besides, we observed $\mathrm{H} \alpha$ and $\mathrm{Fe} 557.6 \mathrm{~nm}$ for calibration purposes.

Figure 1 shows the mosaic scans from the VTT and the corresponding area extracted from the THEMIS-maps.

In order to derive the physical quantities magnetic field strength, inclination and azimuth, the SIR-code (Stokes Inversion based on Response functions) of Ruiz Cobo \& del Toro Iniesta (1992) was used. The magnetic filling factor was forced to unity, but a variable amount of stray light was permitted. Heights of formation of the different lines were calculated for a quiet sun model atmosphere and an umbral one. Then the temperature maps obtained from the SIR-inversion were used to interpolate the formation heights. Fe 1078.3 originates between 180 and $220 \mathrm{~km}, \mathrm{Fe} 630.2$ between 250 and $300 \mathrm{~km}$ and Si 1078.6 between 300 and $350 \mathrm{~km}$, except for the umbra, where the line weakens so that it is formed in almost the same layers as Fe 1078.3. 


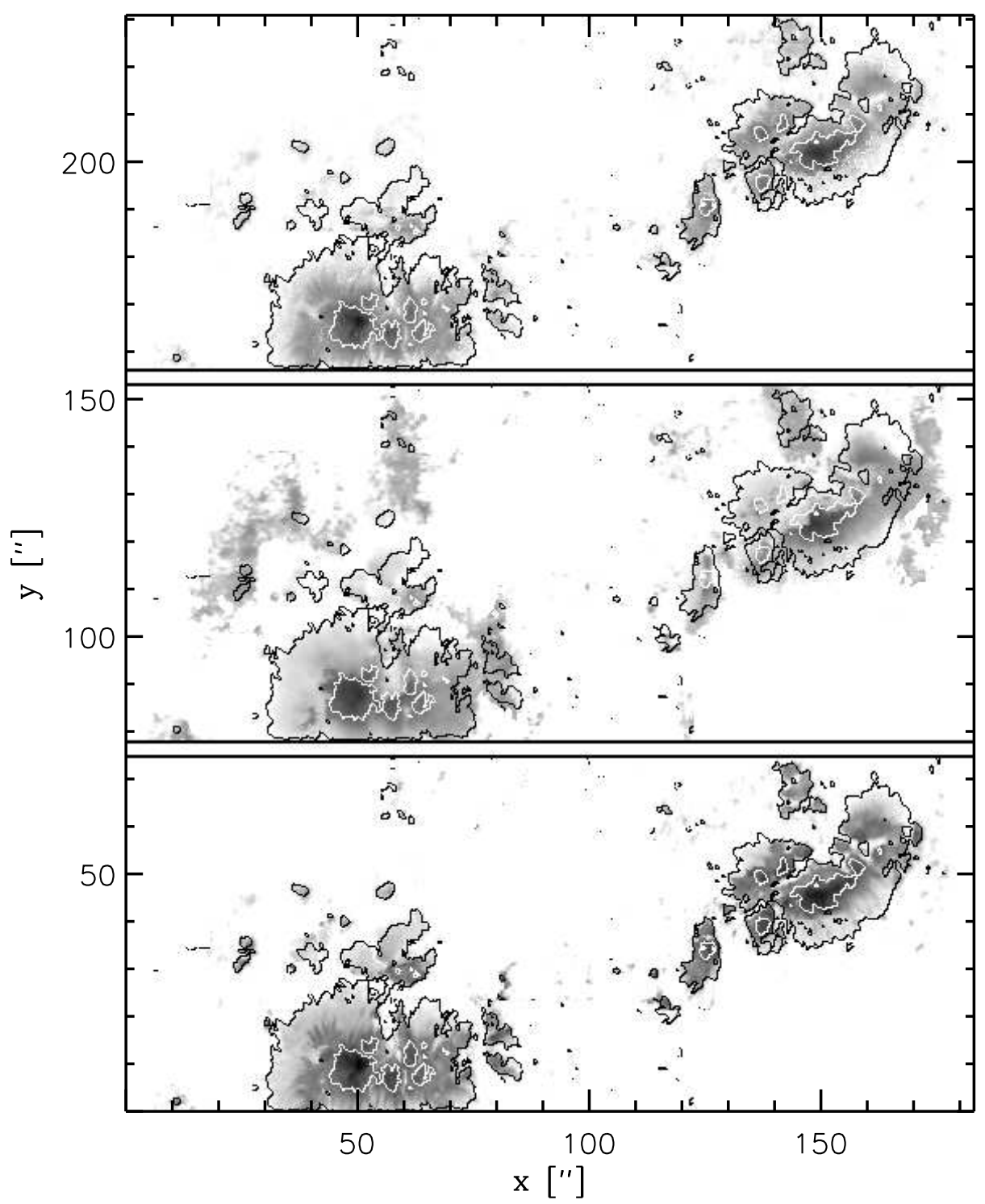

Figure 2. Maps of the total magnetic field strength obtained from the three different lines: Si 1078.6 in the upper panel, Fe 630.2 in the middle and Fe 1078.3 in the lower panel. Values below $600 \mathrm{G}$ are suppressed, the maximum (black) corresponds to $2600 \mathrm{G}$. The contour lines indicate the boundaries of umbra and penumbra.

\section{Results}

Since the investigation of some lines is still pending, we present here results obtained from three lines: Fe 1078.3, Si 1078.6 and Fe 630.2. The inversions were performed indepen- 
dently for the three lines without allowing gradients of the magnetic field in order to avoid artifacts due to discontinuities as that discussed by Martínez Pillet (2000).

The total magnetic field strength obtained for the three lines is displayed in Fig. 2.

Comparing the two lines in the infrared, obtained at the VTT, we find a decrease of the magnetic field strength with height for most locations inside the spots, except for a light bridge between sub-umbrae and a ring-like structure in the middle of the penumbra on the left of the main spot. Typical values for the decrease are around $1 \mathrm{G} / \mathrm{km}$ in the penumbra and $2-2.5 \mathrm{G} / \mathrm{km}$ in the umbra. Outside the spots and pores we find an increase of the magnetic field strength of about $1 \mathrm{G} / \mathrm{km}$. This might be an indication of canopies where the magnetic field is very small or even absent in deep layers. Nevertheless the magnetic inclination is less in higher layers, inside and outside of the spots!

Estimates of the magnetic flux observed in these two lines show a good agreement.

The other iron line observed at THEMIS exhibits a somewhat higher magnetic flux, mainly for some areas outside the dark features in the continuum images. This indicates instrumental differences between THEMIS and VTT. The highest field strengths are somewhat lower than those obtained from the infrared iron line as to be expected if the field decreases with height, but the values obtained for the penumbra do not fall between those from the infrared lines as they are expected to do.

\section{Conclusions}

Our results indicate that the magnetic field decreases with height inside spots, not only in the umbra but also in most parts of the penumbra. Outside the spots we observe canopies: In the higher layer we find a higher magnetic field strength. The smaller magnetic inclination in the upper photosphere could be explained by a goblet-like structure of the magnetic field, as discussed for sunspots by Balthasar \& Collados (2005). For regions outside spots the picture would be that the magnetic field is concentrated to small structures in the continuum forming layers, then in the lower photosphere it widens. Finally, neighboring magnetic structures approach in the upper photosphere.

In the present state of the data reductions it is hardly possible to draw a conclusion from the comparison of the results from the two telescopes.

Acknowledgements. This work is part of the TMR-ESMN (European Solar Magnetism Network) supported by the European Commission under contract HPRN-CT-2002-00313.

\section{References}

Balthasar, H. 2006, A\&A, 449, 1169

Balthasar, H. \& Collados, M. 2005, A\&A, 429, 705

Jurčák, J., Martínez Pillet, V., \& Sobotka, M. 2006, A\&A, 453, 1079

Martínez Pillet, V. 2000, A\&A, 361, 734

Orozco Suárez, D., Lagg, A., \& Solanki, S. K. 2005, in Proceedings of the International Scientific

Conference on Chromospheric and Coronal Magnetic Fields, ESA SP-596

Ruiz Cobo, B. \& del Toro Iniesta, J. C. 1992, ApJ, 398, 375

Sánchez Cuberes, M., Puschmann, K. G., \& Wiehr, E. 2005, A\&A, 440, 345 


\title{
On the inhomogeneities of the sunspot penumbra
}

\author{
R. Schlichenmaier ${ }^{1, *}$, D. A. N. Müller ${ }^{2}$, and C. Beck ${ }^{1,3}$ \\ ${ }^{1}$ Kiepenheuer-Institut für Sonnenphysik, Freiburg, Germany \\ ${ }^{2}$ European Space Agency, c/o NASA Goddard Space Flight Center, Greenbelt, MD, USA \\ ${ }^{3}$ Instituto de Astrofísica de Canarias, La Laguna, Tenerife, Spain \\ *Email: schliche@kis.uni-freiburg.de
}

\begin{abstract}
The penumbra is ideally suited to challenge our understanding of magnetohydrodynamics. The energy transport takes place as magnetoconvection in inclined magnetic fields under the effect of strong radiative cooling at the surface. The relevant processes happen at small spatial scales. In this contribution we describe and elaborate on these small-scale inhomogeneities of a sunspot penumbra. We describe the penumbral properties inferred from imaging, spectroscopic and spectropolarimetric data, and discuss the question of how these observations can be understood in terms of proposed models and theoretical concepts.
\end{abstract}

\section{Introduction}

Almost hundred years ago, Hale (1908a,b) performed the first spectropolarimetric measurements on the Sun and discovered that sunspots are manifestations of magnetic fields with strengths of up to $3000 \mathrm{G}$ in their centers. He proposed the tornado theory to explain both the darkness of a spot by dust that is whirled up into the solar atmosphere and the magnetic field by the circular current by electrons. Investigating Doppler shifts in sunspots, Evershed (1909) measured radial rather than circular movements in sunspot penumbrae and concluded that his findings were "entirely out of harmony with the splendid discovery of the Zeeman effect in sun-spots, made by Prof. Hale."

Until today, we lack a complete understanding of sunspots and penumbrae, but it seems obvious that the Evershed flow plays an essential role in the latter. The presence of the Evershed flow is intimately linked to the mere existence of the penumbra as demonstrated by the observations of Leka \& Skumanich (1998). The filamentary bright and dark structure should be related to the flow field, in a similar way as the granular pattern of the quiet Sun is related to the granular flow field of hot up- and cool downflows.

The magnetic field can - in principle - be inferred from the spectropolarimetric imprints of the Zeeman effect on photospheric absorption lines. However, this leads to unambiguous results only if the magnetic and velocity fields are homogeneous along the line-of-sight. Considerable complications in interpreting the line profiles arise if gradients or discontinuities are present in the volume of the solar atmosphere which is sampled by one resolution element. Such variations may be present laterally due to insufficient spatial resolution, but also along the line-of-sight from the depth layers that contribute to the observed line profile. 
In this contribution, we make the case that the latter must be assumed to reconstruct some of the observed profile asymmetries. We summarize our knowledge of the inhomogeneities in the magnetic and velocity field of a sunspot penumbra, aiming at an understanding of radiative magneto-convection in inclined magnetic fields.

\section{Penumbral properties}

The photospheric penumbral properties may be characterized by three different types of measurements: (1) imaging, (2) spectroscopy of lines that do not show the Zeeman effect, and (3) spectropolarimetric measurements of Zeeman sensitive lines.

\subsection{Imaging}

At a spatial resolution of 1 " or worse, the penumbra appears as a gray ring that surrounds the umbra. The radial bright and dark filaments only become apparent at a spatial resolution of about $0 .{ }^{\prime \prime} 5$. At a spatial resolution of about $0 .{ }^{\prime \prime} 2$ bright filaments show internal intensity variations: In the inner penumbra, predominantly on the center side, bright filaments show a dark elongated core (Scharmer et al. 2002; Sütterlin et al. 2004; Langhans et al. 2007). Such dark-cored bright filaments have a width of some $0 . " 2$.

\subsection{Spectroscopy}

As for the intensity, the length scale of variations in a Doppler map decreases with improving spatial resolution. At 1" no flow filaments are visible and the Doppler shifts only show a transition from red shifts on the limb side to blue shifts on the center side. To infer the flow vector at this spatial resolution, azimuthal cuts at constant distance from the spot center are constructed. The azimuthal mean reflects the vertical velocity component, while the amplitude of the variation measures the horizontal component which is known to be dominant. It is found that the flow field has an upward component in the inner and a downward component in the outer penumbra (Schlichenmaier \& Schmidt 2000; Schmidt \& Schlichenmaier 2000).

A filamentary structure becomes visible in velocity maps of the penumbra if the spatial resolution is of the order of $0 .{ }^{\prime} 5$ or better. However, flow filaments are not always co-spatial with intensity filaments (Tritschler et al. 2004; Schlichenmaier et al. 2005). Individual flow and intensity filaments typically have a joined starting point in the inner penumbra. Yet, further outwards the flow filament is not always co-spatial with a bright filament.

The Evershed flow in the penumbra is characterized not only by a line shift, but also by a line asymmetry. The line asymmetry is such that the wing is more strongly shifted than the core (Bumba 1960; Schröter 1965a; Wiehr et al. 1984; Degenhardt 1993; Wiehr 1995). The asymmetry decreases with formation height of the spectral line (Degenhardt \& Wiehr 1994; Balthasar et al. 1997). Studying the line asymmetry, i.e., the shape of the bisector, one can show that the measurements are consistent with the assumption that the flow is concentrated in the deepest photospheric layers (Maltby 1964). Line asymmetries could in principle be assigned to laterally separated unresolved components (Schröter 1965b), but as they are still present in high spatial resolution observations down to 0." 3 (see Bellot Rubio et al. 2005), the line asymmetries can only be explained by velocity gradients or discontinuities 
along the line-of-sight (Schlichenmaier et al. 2004; Bellot Rubio et al. 2006). Therefore, the measurements seem to require a flow field that is concentrated in the deepest layers of the photosphere beneath $\tau \approx 0.1$.

Spectroscopy at 0.' 3 spatial resolution resolves dark-cored bright filaments. Bellot Rubio et al. (2005) found that the dark cores are associated with strong predominantly horizontal flows, while the lateral bright edges are less Doppler shifted. Yet the lateral bright edges are still more shifted than the dark surroundings in which the dark-cored penumbral filaments are often embedded in the inner penumbra. The inner end of a dark-cored bright filament is typically bright and is associated with an upflow. Rimmele \& Marino (2006) find that these hot upflows have a diameter of about $0 ., 3$ and turn horizontal within $1^{\prime \prime}$. They continue in the dark core of a bright filament.

\subsection{Magneto-convetive modelling}

The first attempt to describe quantitatively (i) the dynamic evolution of penumbral filaments and (ii) the Evershed effect consisted of a 1D (thin) magnetic flux tube that evolved in a 2D time-independent background (Schlichenmaier et al. 1998a,b). Such tubes develop a flow that brings up hot plasma into the photosphere which radiatively cools as it flows outwards, yielding a flow topology that is consistent with the findings by Rimmele \& Marino (2006). As long as the plasma is hot, the magnetic field strength is reduced and can be weaker by $10^{3} \mathrm{G}$ as compared to the background. In this framework, magneto-convective instabilities cause solutions in the form of sea serpents (Schlichenmaier 2002), in which the hot upflow is followed by a cool downflow further outwards. Such a flow arch is conceptually very similar to the time-independent siphon flows (e.g., Degenhardt 1991; Thomas \& Montesinos 1993). In both cases a pressure gradient accelerates the flow. The pressure gradient in the moving tube model is sustained by radiative cooling between the inner hot footpoint (high gas pressure) and the cool flow channel further outwards (low gas pressure). This configuration is a natural consequence of magneto-convective instabilities and radiative cooling.

In contrast, Weiss et al. (2004) have proposed that magnetic field lines associated with the downflows in the outer penumbra are pumped downwards by small-scale granular convection outside the sunspot. They demonstrate that turbulent transport of mean magnetic fields by convective motions, i.e., magnetic pumping, is a robust phenomenon. However, in this scenario it remains obscure how the magnetized penumbra with supposedly coherent flows and field lines is connected to the turbulent convection that is needed to produce topological pumping effects. It seems more promising to perform 3D simulations of inclined magnetoconvection including the effects of radiation. First results have been presented by Heinemann et al. (2006) which confirm the flow topology that we describe above of a hot upflow followed by a cooler downflow (cf. their Fig. 6). Yet, in these simulations the flow is not strictly radial, but has a small azimuthal component towards the lateral edges of the filament. Eventually such simulations will clarify whether or not the energy transport takes place due to convection in field free gaps as it was suggested recently (Spruit \& Scharmer 2006; Scharmer \& Spruit 2006) or by flows along magnetic tubes as suggested by the moving tube model. In any model, the magnetic field strength in bright penumbral features is certainly less than $1000 \mathrm{G}$, i.e., much smaller than the average surrounding magnetic field strength. In accordance with the latter expectation, Bellot Rubio et al. (2005) measure that dark-cored filaments have smaller magnetic field strengths than the surroundings of the fil- 


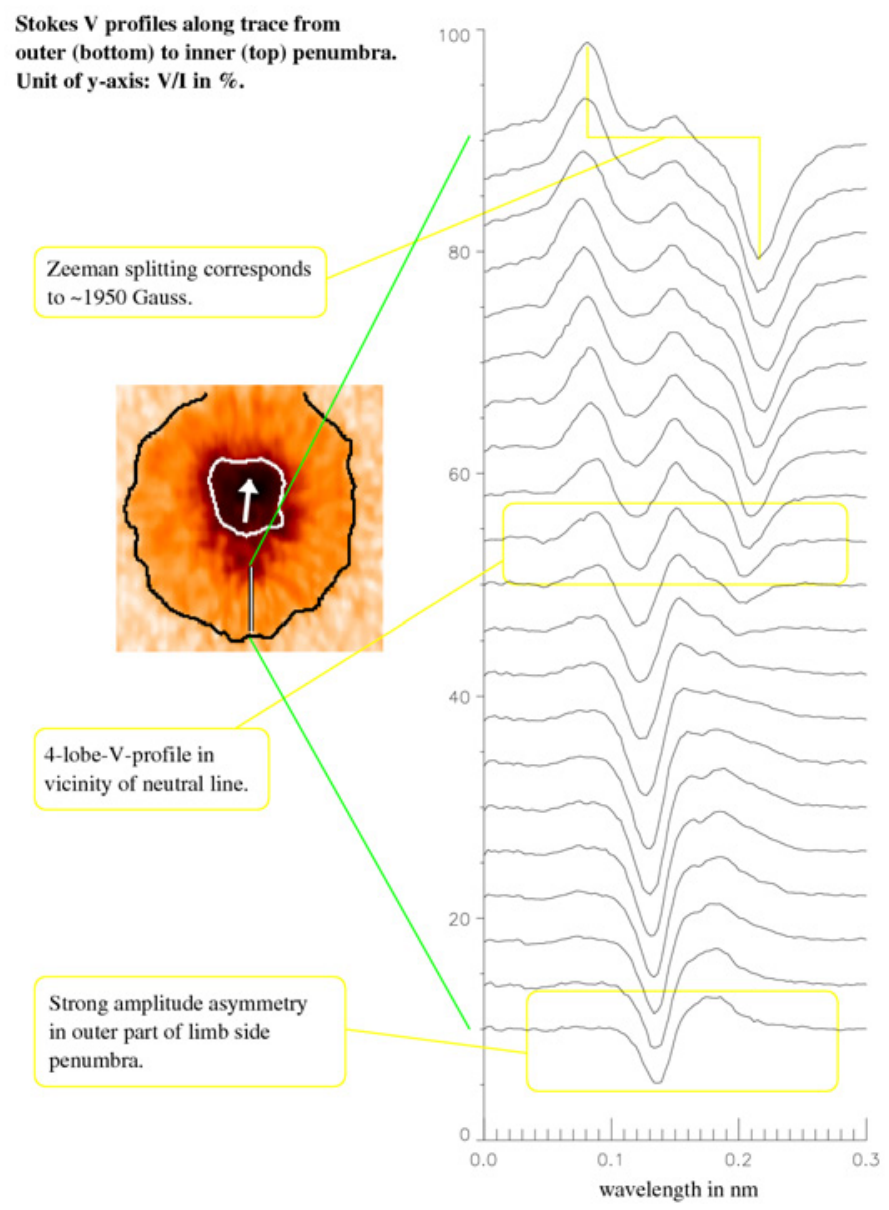

Figure 1. Stokes-V profiles of $\mathrm{Fe}_{\mathrm{I}} 1564.8 \mathrm{~nm}$ along a radial cut for the limb-side penumbra of a spot at $30^{\circ}$ heliocentric angle. From the inner to the outer penumbra, the V-profiles undergo a transition from one polarity to the other. In the central penumbra, along the so-called magnetic neutral line, both polarities seem to be present. This is clear observational evidence for an inhomogeneous magnetic field. Such a 4-lobe-profile needs at least two different orientations of the magnetic field within one resolution element.

aments. They use the Zeeman sensitive line Fe II $6149 \mathrm{~nm}$ and even find a tendency that the strength is slightly larger in dark cores than in the bright lateral edges, as one expects due to contraction of the plasma during the cooling phase.

\subsection{Spectropolarimetry}

Spectroscopic measurements establish the inhomogeneous flow field within the sunspot penumbra. Direct observational evidence for an inhomogeneous magnetic field consists of 4lobe V-profiles as presented in Fig. 1: At the location within the penumbra where the viewing angle to the average magnetic field lines is perpendicular, i.e., in the vicinity of the magnetic neutral line, one expects vanishing $\mathrm{V}$-signal if the field were homogeneous. V-profiles with up to four lobes, as observed, imply that at least two directions (components) of the magnetic field must be present in the resolution element. Another strong indication of non-trivial topologies of the velocity and magnetic field are given by the observations of amplitude and area asymmetries of Stokes-V (e.g., Sánchez Almeida \& Lites 1992; Schlichenmaier \& 

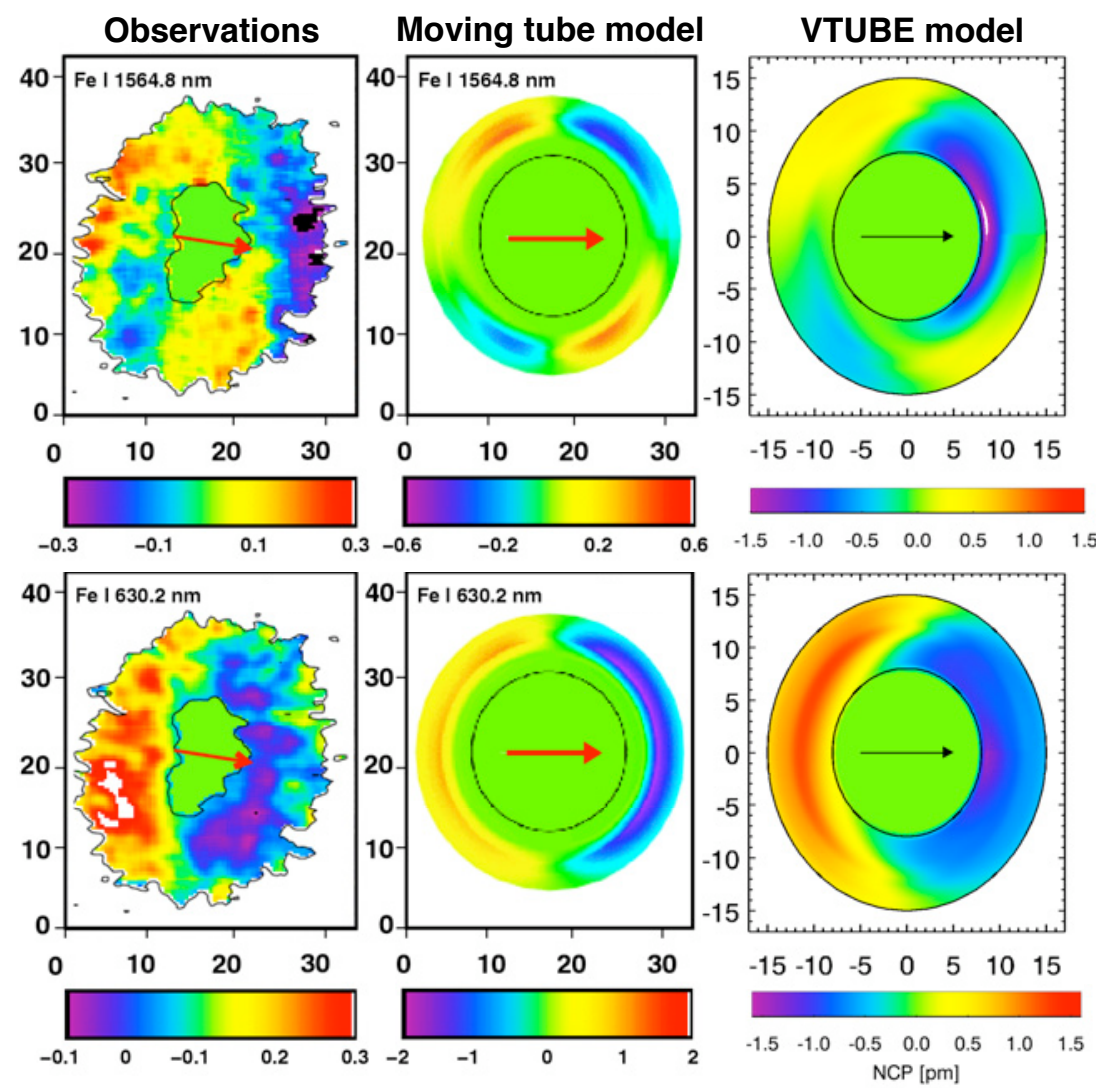

Figure 2. Observed and synthesized sunspot maps of net circular polarization for Fe I $15648 \mathrm{~nm}$ (top row) and Fe $630.2 \mathrm{~nm}$ (bottom row), respectively. The synthesized maps in the middle column are based on a snapshot from the moving tube model (Müller et al. 2002), and the maps in the right colum are based on the VTUBE tool (see text), where inversion results are used. The spot is at a heliocentric angle of $30^{\circ}$ and the arrows point towards disk center.

Collados 2002). These findings motivate that reconstructions of the (magnetic and velocity) field topology should consider at least two components or strong gradients or discontinuities along the line-of-sight. Such attempts have been made using inversion techniques (e.g., Bellot Rubio et al. 2004; Borrero et al. 2006). In such inversions, synthetic Stokes parameters resulting from polarized radiative transfer based on model atmospheres are compared with observed profiles of the four Stokes parameters.

The assumption of two interlaced components with constant velocity and magnetic fields give reasonable fits to the observed profiles. Yet, the pure existence of net circular polarization, $\mathcal{N}=\int V(\lambda) \mathrm{d} \lambda$, proves that velocity gradients along the line-of-sight are present (Sánchez Almeida \& Lites 1992; Landolfi \& Landi degl'Innocenti 1996). Sunspot maps of the net circular polarization are displayed in Fig. 2. The first row shows observed and synthesized maps of Fe I $15648 \mathrm{~nm}$, and the second row shows the same for Fe I $630.2 \mathrm{~nm}$. The 


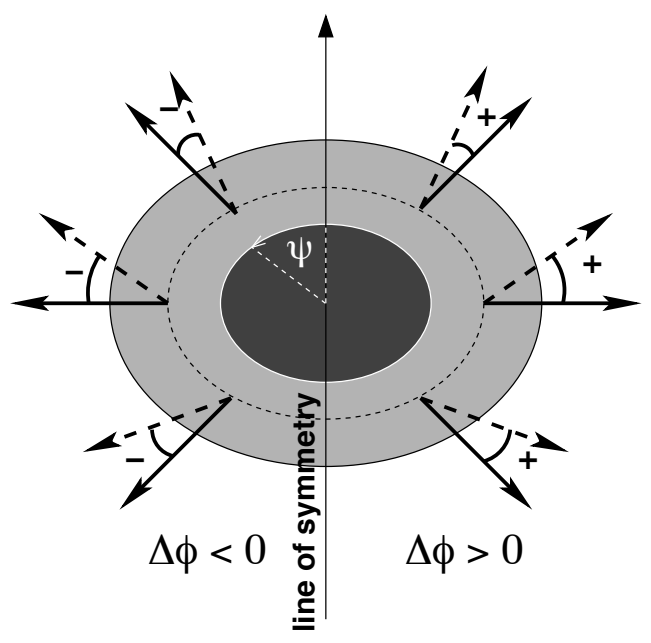

Figure 3. Difference in the azimuth, $\Delta \phi$, of horizontal and inclined magnetic field when viewed off disk center. The sign of $\Delta \phi$ changes from one side of the penumbra to the other. The dashed vectors represent the less inclined background magnetic field being at rest, and the solid vectors represent the horizontal magnetic components that is coaligned with the flow.

observed maps of $\mathcal{N}$ (left column) are simultaneous measurements of the same sunspot at $30^{\circ}$ heliocentric angle (Müller et al. 2006). The symmetry properties of the two observed maps are different. The middle column shows synthetic maps that are based on a snapshot of the moving tube model (Müller et al. 2002). The synthetic maps reproduce the observations quite convincingly. The main ingredient for reproducing these symmetry properties are two components along the line-of-sight with the following properties (1) They have two different inclinations of the magnetic field and (2) they are Doppler shifted relative to each other (Schlichenmaier et al. 2002). Figure 3 sketches how the two different inclinations of the magnetic field produce a sign change in the difference of the magnetic field azimuth, which is crucial to reconstruct the observed maps. It is important to realize that these maps cannot be reproduced with a mixture of two laterally separated (unresolved) components with each having constant magnetic and velocity fields along the line-of-sight!

\section{A diagnostic tool: VTUBE}

In an attempt to bring together snapshots from simulations and results from inversions, a tool to diagnose spectropolarimetric measurements was developed (Müller et al. 2006). We constructed a 3D geometric model (VTUBE) of a magnetic flux tube embedded in a background atmosphere that can be used to gain a better understanding of the different factors that determine $\mathcal{N}$ and its spatial variation within the penumbra. This model serves as the frontend for a radiative transfer code (DIAMAG, Grossmann-Doerth 1994). Combining the two, we can generate synthetic Stokes spectra for any spectral line and construct maps of suited diagnostic quantities, like $\mathcal{N}$-maps, for any desired axisymmetric magnetic field configuration and arbitrary properties of flux tubes being embedded in an arbitrary atmosphere. The model has been built to offer a high degree of versatility, e.g. the option to calculate several parallel rays along the line-of-sight that intersect the tube at different locations with arbitrary viewing angles. One can then average over these rays to model observations of flux tubes at different spatial resolutions and for different magnetic filling factors of the atmosphere. Furthermore, one can also take into account radial variations of the physical properties of the flux tube. 
Doing so, one can e.g. model the interface between the flux tube and its surroundings.

To generate a realistic $\mathcal{N}$-map with the VTUBE model, we extracted the radial dependence of azimuthally averaged quantities from results of inversions based on two components as mentioned above, thereby assuming that the penumbral fine structure is axially symmetric. From the two components, one is assigned to be the background component, and the other serves as the flow component. This is in accordance with inversion results with one component being essentially at rest and the other the other carrying the flow. While the magnetic field strength decreases with penumbral radius, the difference between the weaker flow component and the stronger one decreases outwards and vanishes at the outer penumbral edge. The flow velocity along the channel (flow component) increases from some $6 \mathrm{~km} / \mathrm{s}$ at the inner to $8 \mathrm{~km} / \mathrm{s}$ in the outer penumbra. The inclination of the flow changes gradually from an upflow with $20^{\circ}$ in the inner to a downflow with $-10^{\circ}$ in the outer penumbra. The resulting maps (right column in Fig. 2) compare well with the observed ones, but, especially in the radial dependence, differences are seen. Possibly this difference may be explained by recent results (Bellot Rubio et al. 2003; Beck 2006) that suggest that the background field is not at rest in the outermost penumbra, but shows flow velocities of up to $1 \mathrm{~km} / \mathrm{s}$. This may modify the $\mathcal{N}$-map and may reconcile the model with the observations.

\section{Conclusions}

We have described the properties of a sunspot penumbra, including the topology of the magnetic and velocity field. The main conclusion is that the penumbra is inhomogeneous. In order to explain the mentioned spectroscopic and spectropolarimetric measurements at least strong gradients if not discontinuities must be present along the line-of-sight. We believe that low lying channels $(\tau>0.1)$ that carry a magnetized flow and are embedded in a magnetized background at rest must be assumed in order to understand the measurements, like the observed bisectors of unmagnetic lines, and the symmetry properties of net-circularpolarization maps. Yet, we cannot offer a complete picture as the spatial resolution is not yet good enough to present unambiguous model fits to the observed spectropolarimetric measurements. A great step forward may be accomplished by the recently launched satellite Hinode, as it will allow for spectropolarimetric observations at 0. ' 25 . The best ground based observations today are not much better than $1^{\prime \prime}$. At the same time, simulations of magnetoconvection in inclined magnetic field like the ones presented recently by Heinemann et al. (2006) will significantly enhance our understanding of the penumbral fine structure.

\section{References}

Balthasar, H., Schmidt, W., \& Wiehr, E. 1997, Solar Phys. 171, 331

Beck, C. 2006, PhD thesis, Universität Freiburg (Kiepenheuer-Institut für Sonnenphysik)

Bellot Rubio, L. R., Balthasar, H., \& Collados, M. 2004, A\&A, 427, 319

Bellot Rubio, L. R., Balthasar, H., Collados, M., \& Schlichenmaier, R. 2003, A\&A, 403, L47

Bellot Rubio, L. R., Langhans, K., \& Schlichenmaier, R. 2005, A\&A, 443, L7

Bellot Rubio, L. R., Schlichenmaier, R., \& Tritschler, A. 2006, A\&A, 453, 1117

Borrero, J. M., Solanki, S. K., Lagg, A., Socas-Navarro, H., \& Lites, B. 2006, A\&A, 450, 383

Bumba, V. 1960, Izv. Krymk. Astrofiz. Observ., 23, 253

Degenhardt, D. 1991, A\&A, 248, 637 
Degenhardt, D. 1993, A\&A, 277, 235

Degenhardt, D. \& Wiehr, E. 1994, A\&A, 287, 620

Evershed, J. 1909, MNRAS, 69, 454

Grossmann-Doerth, U. 1994, A\&A, 285, 1012

Hale, G. E. 1908a, ApJ, 28, 315

Hale, G. E. 1908b, ApJ, 28, 100

Heinemann, T., Nordlund, A., Scharmer, G. B., \& Spruit, H. C. 2006, ArXiv Astrophysics e-prints, astro-ph/0612648

Landolfi, M. \& Landi degl'Innocenti, E. 1996, Solar Phys. 164, 191

Langhans, K., Scharmer, G. B., Kiselman, D., \& Löfdahl, M. G. 2007, A\&A, accepted

Leka, K. D. \& Skumanich, A. 1998, ApJ, 507, 454

Maltby, P. 1964, Astrophysica Norvegica, 8, 205

Müller, D. A. N., Schlichenmaier, R., Fritz, G., \& Beck, C. 2006, A\&A, 460, 925

Müller, D. A. N., Schlichenmaier, R., Steiner, O., \& Stix, M. 2002, A\&A, 393, 305

Rimmele, T. \& Marino, J. 2006, ApJ, 646, 593

Sánchez Almeida, J. \& Lites, B. W. 1992, ApJ, 398, 359

Scharmer, G. B., Gudiksen, B. V., Kiselman, D., Löfdahl, M. G., \& Rouppe van der Voort, L. H. M. 2002, Nature, 420, 151

Scharmer, G. B. \& Spruit, H. C. 2006, A\&A, 460, 605

Schlichenmaier, R. 2002, AN, 323, 303

Schlichenmaier, R., Bellot Rubio, L. R., \& Tritschler, A. 2004, A\&A, 415, 731

Schlichenmaier, R., Bellot Rubio, L. R., \& Tritschler, A. 2005, Astron. Nachr., 326, 301

Schlichenmaier, R. \& Collados, M. 2002, A\&A, 381, 668

Schlichenmaier, R., Jahn, K., \& Schmidt, H. U. 1998a, ApJL, 493, L121

Schlichenmaier, R., Jahn, K., \& Schmidt, H. U. 1998b, A\&A, 337, 897

Schlichenmaier, R., Müller, D. A. N., Steiner, O., \& Stix, M. 2002, A\&A, 381, L77

Schlichenmaier, R. \& Schmidt, W. 2000, A\&A, 358, 1122

Schmidt, W. \& Schlichenmaier, R. 2000, A\&A, 364, 829

Schröter, E. H. 1965a, Z. Astrophys., 62, 228

Schröter, E. H. 1965b, Z. Astrophys., 62, 256

Spruit, H. C. \& Scharmer, G. B. 2006, A\&A, 447, 343

Sütterlin, P., Bellot Rubio, L. R., \& Schlichenmaier, R. 2004, A\&A, 424, 1049

Thomas, J. H. \& Montesinos, B. 1993, ApJ, 407, 398

Tritschler, A., Schlichenmaier, R., Bellot Rubio, L. R., \& the KAOS Team 2004, A\&A, 415, 717

Weiss, N. O., Thomas, J. H., Brummell, N. H., \& Tobias, S. M. 2004, ApJ, 600, 1073

Wiehr, E. 1995, A\&A, 298, L17

Wiehr, E., Koch, A., Knölker, M., Küveler, G., \& Stellmacher, G. 1984, A\&A, 140, 352 


\title{
Modified $p$-modes in penumbral filaments
}

\author{
D. S. Bloomfield ${ }^{1, *}$, A. Lagg $^{1}$, S. K. Solanki ${ }^{1}$, and J. M. Borrero ${ }^{2}$ \\ ${ }^{1}$ Max-Planck-Institut für Sonnensystemforschung, Katlenburg-Lindau, Germany \\ ${ }^{2}$ High Altitude Observatory, Boulder, Colorado, U.S.A. \\ *Email: bloomfield@mps.mpg.de
}

\begin{abstract}
A time series analysis was performed on velocity signals in a sunspot penumbra to search for possible wave modes. The spectropolarimetric photospheric data obtained by the Tenerife Infrared Polarimeter were inverted using the SPINOR code. An atmospheric model comprising two magnetic components and one stray-light component gave an optimal fit to the data. Fourier phase difference analysis between line-of-sight velocities of both magnetic components provided time delays between the two atmospheres. These delays were combined with the speeds of atmospheric wave modes and compared to height separations derived from velocity response functions to determine the wave mode.
\end{abstract}

\section{Introduction}

Disentangling the signatures of various magnetoatmospheric (MA) waves that are supported by magnetic atmospheres is a difficult, even daunting, task. However, information may be extracted from spatially unresolved structures by spectropolarimetry. This approach uses the full Stokes polarization spectra (I, Q, U, V), allowing physical properties of the emitting plasma to be inferred through the application of appropriate model atmospheres. Here we present a method that may identify the form of wave which exists in a magnetic environment.

\section{Observations}

Active region NOAA 10436 (Fig. 1) was observed on 21 August 2003 with the Tenerife Infrared Polarimeter (Martínez Pillet et al. 1999) attached to the German VTT. Full Stokes profiles were recorded for the spectral lines Fe I $15662.018 \AA$ (effective Landé factor $\bar{g}=1.50$ ) and $\mathrm{Fe}_{\mathrm{I}} 15665.245 \AA(\bar{g}=0.75)$ over the period 14:39-15:41 UT, yielding a time series of 250 stationary image positions on the slit at a cadence of one exposure every $14.75 \mathrm{~s}$. Given the small amount of circular polarization asymmetry in the inner limbside penumbra, it is likely that these lines are not affected by gradients in the magnetic field vector or line-of-sight (LOS) velocity. Therefore, the data were inverted using the SPINOR inversion code (Frutiger 2000) and the height-independent two magnetic component model of Borrero et al. (2004). The inversion yields a magnetic field geometry consisting of a near-horizontal component (flux tube, FT) and a closer-to-vertical component (magnetic background, MB). Since the velocity variations were most strongly observed in Stokes Q the response functions (RFs) of Stokes Q to LOS velocity (Fig. 2) were calculated. The RFs of the magnetic components overlap over most part of the atmosphere, but their center-of-gravity (COG), 


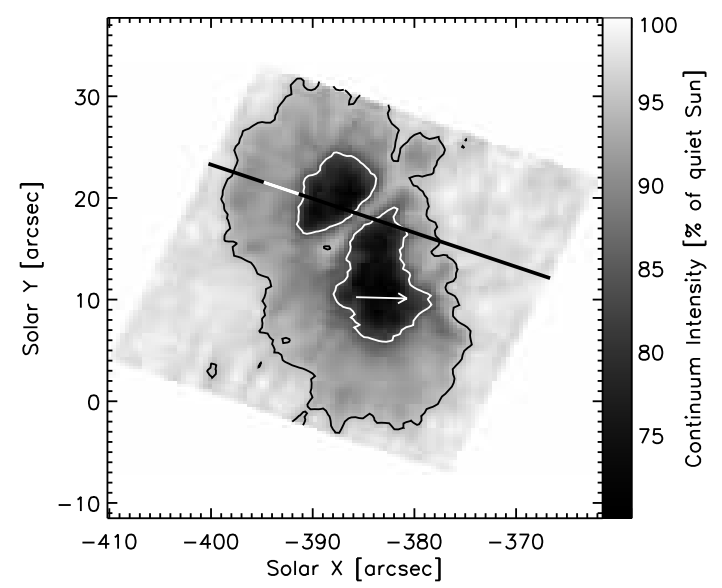

Figure 1. Continuum intensity image of NOAA 10436. White (black) contours mark the umbral/penumbral (penumbral/quiet Sun) boundaries. The straight black line marks the slit position during the time series, the white portion the region studied, while the arrow points to disk centre.

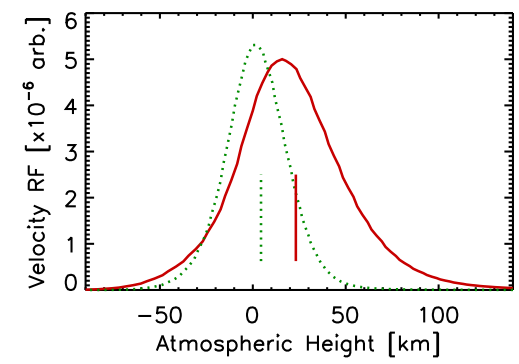

Figure 2. Height variation of combined $15662 \AA$ and $15665 \AA$ Stokes Q velocity response for the MB (solid) and FT (dotted) atmospheres. Vertical lines mark the COG in each component.

displayed as vertical lines, reveal a distinct separation between the components. Height differences between the LOS velocity signals were taken as the separation of the COGs, while wave propagation speeds were calculated over these height ranges from the output inversion atmospheres.

\section{Time Series Analysis}

A Fourier phase difference analysis was performed between the MB and FT velocity signals following Krijger et al. (2001). Spectra from the eleven analyzed pixels of the inner limbside penumbra are overplotted in the left panel of Figure 3. Approximately constant phase difference values were recorded in the range $2.5-4.5 \mathrm{mHz}$, with the probability distribution function (PDF) centred on $-5.5^{\circ}$. Negative phase differences mean that the FT velocity leads the MB, agreeing with the COG heights in Figure 2 for upward wave propagation. The centroid value was converted into time delay between the signals, resulting in values ranging 

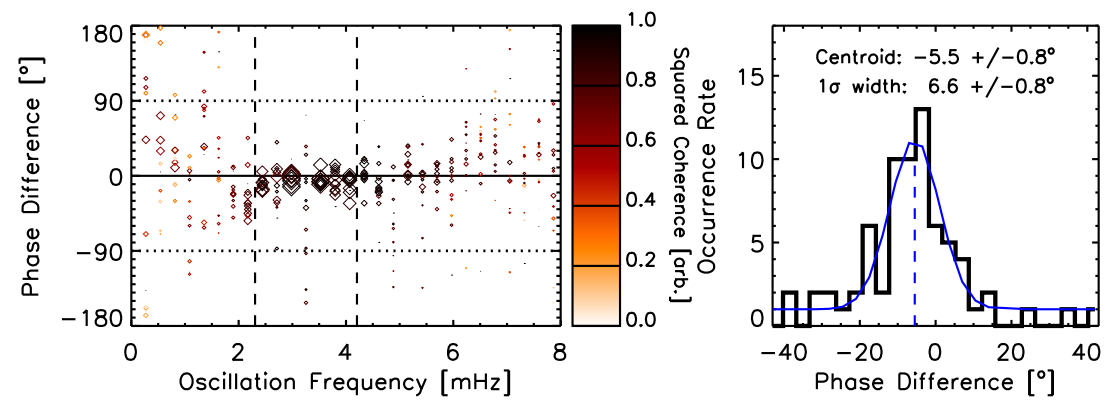

Figure 3. Left: Fourier phase difference spectra between the MB and FT velocities from the eleven analyzed pixels. Darker shading denotes greater Fourier coherence and larger symbol size greater cross-spectral power. Right: PDF of phase difference values over the frequency range $2.5-4.5 \mathrm{mHz}$. The thick curve displays the measured values and the thin curve the best-fit Gaussian profile.

from $-6.3 \mathrm{~s}$ to $-3.8 \mathrm{~s}$. Attributing these phase differences to propagating waves conflicts with the evanescent behaviour of vertical waves below the acoustic cutoff $(5.2 \mathrm{mHz})$. However, $p$-modes may travel at angles away from the vertical at these heights (e.g., $40^{\circ}-60^{\circ}$ for $l=600-800$ at $3.5 \mathrm{mHz}$; Cally 2006, private communication) and, for inclinations of $50^{\circ}$, frequencies $\geqslant 3.2 \mathrm{mHz}$ may propagate.

\section{Height Separation Comparison}

The low-photospheric sampling of the spectral lines means that both components are mostly gas dominated (Fig. 2; $\beta \geqslant 1$ below $70 \mathrm{~km}$ and $90 \mathrm{~km}$ in the MB and FT, respectively). As such, differing forms of wave will have certain properties in terms of propagation speed and direction: acoustic waves propagate isotropically at the sound speed, $c_{S}$; Alfvén waves are restricted to the direction of the field and propagate at the Alfvén speed, $v_{A}$; MA slow modes propagate along the field at either $v_{A}$ or the tube speed, $c_{T}$, if the sunspot is thought of as a large "flux tube"; MA fast modes propagate at a speed between $c_{S}$ and $\left(c_{S}^{2}+v_{A}\right)^{1 / 2}$ depending on the angle between the direction of the field and that of wave propagation.

Taking these considerations into account, the previously obtained vertical height separations are converted into path lengths along the direction of propagation: $50^{\circ}$ to the vertical for acoustic and fast-mode waves; along the field for Alfvénic and slow-mode waves. Note that the fast-mode speed will differ between waves moving toward the sunspot (ingressing) and those moving away (egressing). Probability distribution functions of the RF-predicted path lengths are given as solid curves in Fig. 4, while values calculated by combining time delays, wave speeds and propagation angle to the field are overplotted as dotted and dashed curves. The best correspondence is observed for ingressing fast-mode waves: this case has the smallest difference between the RF-predicted and calculated path length COGs (Table 1).

\section{Conclusions}

This is the first time that spectropolarimetric data have been used in this manner to identify a MA wave mode. The fact that a fast-mode wave best fits the observational data makes 

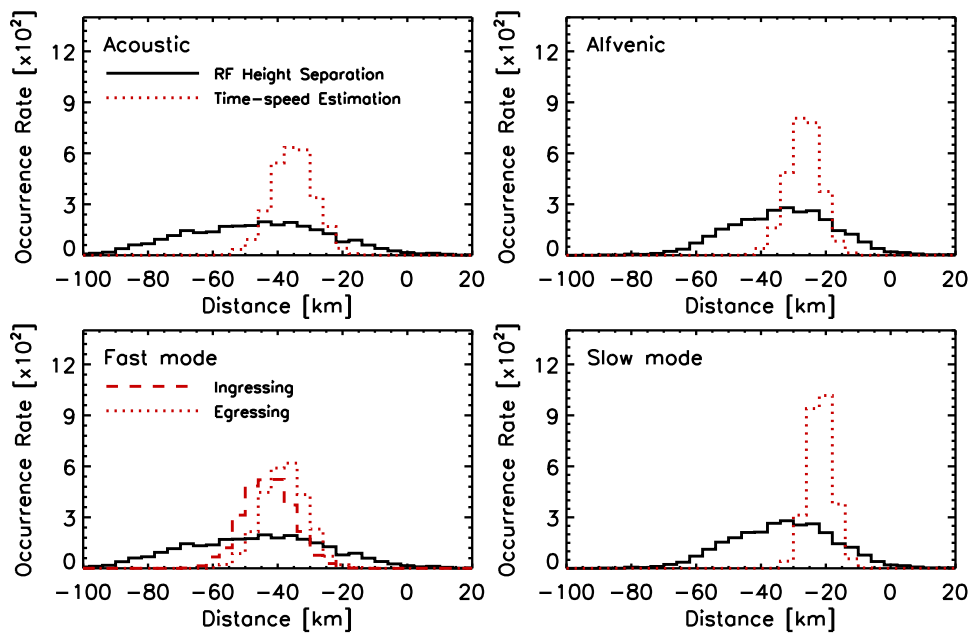

Figure 4. Comparison of RF-predicted (solid lines) and calculated (dotted and dahed lines) wave travel distances. Cases are presented for waves propagating at $50^{\circ}$ to the vertical at the acoustic (top left) and fast-mode speeds (bottom left), as well as field-aligned waves propagating at the Alfvén (top right) and slow-mode tube speeds (bottom right). In all panels, vertical height separations were converted into path length along the direction of propagation.

Table 1. Differences between RF-predicted and calculated path length COG values.

\begin{tabular}{lc}
\hline Wave Type & Absolute Separations [km] \\
\hline Acoustic & 8 \\
Alfvénic & 4 \\
Slow mode & 9 \\
Fast mode (ingressing) & 0 \\
Fast mode (egressing) & 5 \\
\hline
\end{tabular}

qualitative sense as the spectral lines sample a high- $\beta$ region of the low photosphere where $p$-mode waves should be modified into a fast-mode form by the magnetic field. It will be interesting to see if the detected form of MA wave changes from the case where the velocity response of a spectral line is formed below $\beta=1$ to one where it is formed above this level.

\section{References}

Borrero, J. M., Solanki, S. K., Bellot Rubio, L. R., Lagg, A., \& Mathew, S. K. 2004, A\&A, 422, 1093 Cally, P. S. 2006, private communication

Frutiger, C. 2000, Ph.D. Thesis, Institute of Astronomy, ETH, Zürich, No. 13896

Krijger, J. M., Rutten, R. J., Lites, B. W., Straus, Th., Shine, R. A., \& Tarbell, T. D. 2001, A\&A, 379, 1052

Martínez Pillet, V., et al. 1999, in High Resolution Solar Physics: Theory, Observations, and Techniques, ed. T. R. Rimmele, K. S. Balasubramaniam, \& R. R. Radick, ASP Conf. Ser., 183, 264 


\title{
Wave propagation and shock formation in diverse magnetic structures
}

\author{
R. Centeno ${ }^{1,2, *}$, M. Collados ${ }^{1}$, and J. Trujillo Bueno ${ }^{1,3,4}$ \\ ${ }^{1}$ Instituto de Astrofísica de Canarias, La Laguna/Tenerife, Spain \\ ${ }^{2}$ High Altitude Observatory, Boulder CO, USA \\ ${ }^{3}$ Institut für Astrophysik, Göttingen, Germany \\ ${ }^{4}$ Consejo Superior de Investigaciones Científicas, Spain \\ *Email: rce@hao.ucar.edu
}

\begin{abstract}
Velocity oscillations measured simultaneously at the photosphere and the chromosphere of different solar magnetic features (sunspots, pores and facular regions) allow us to study the properties of wave propagation as a function of the magnetic flux of the structure. While photospheric oscillations are similar everywhere, oscillations measured at chromospheric heights show different amplitudes, frequencies and stages of shock development depending on the observed magnetic feature. The analysis via power and phase spectra, together with simple theoretical modeling, lead to a series of results concerning wave propagation within the range of heights of this study. We find that, while the atmospheric cut-off frequency and the propagation properties of the different oscillating modes depend on the magnetic feature, in all the cases the power that reaches the high chromosphere comes directly from the photosphere by means of linear wave propagation rather than from non-linear interaction of modes.
\end{abstract}

\section{Introduction}

The study of the generation and propagation of waves in the solar atmosphere is a hot topic of research in astrophysics, since it provides information about the atmospheric structure and dynamics (Lites 1992; Bogdan \& Judge 2006), while at the same time it helps us identify the key mechanisms of chromospheric and coronal heating. Simultaneous time-series observation of various spectral lines that sample different regions of the solar atmosphere is one of the most useful techniques for studying wave propagation.

For the analysis that follows we measure the full Stokes vector of the photospheric Si I $10827 \AA$ line and of the chromospheric He I $10830 \AA$ A multiplet. The analysis of the photospheric and chromospheric LOS velocity oscillations and the relation between them give us information about the behavior of the atmosphere: the propagation of photospheric disturbances, the amplification of the oscillations as they reach higher layers of the atmosphere, the cut-off frequency below which the oscillation modes do not propagate, the development of shocks and so on. In this work we carry out a comparative study among 4 magnetic structures with different magnetic fluxes: a rather big sunspot, a smaller one, a pore that has developed no penumbra and a facular region. 

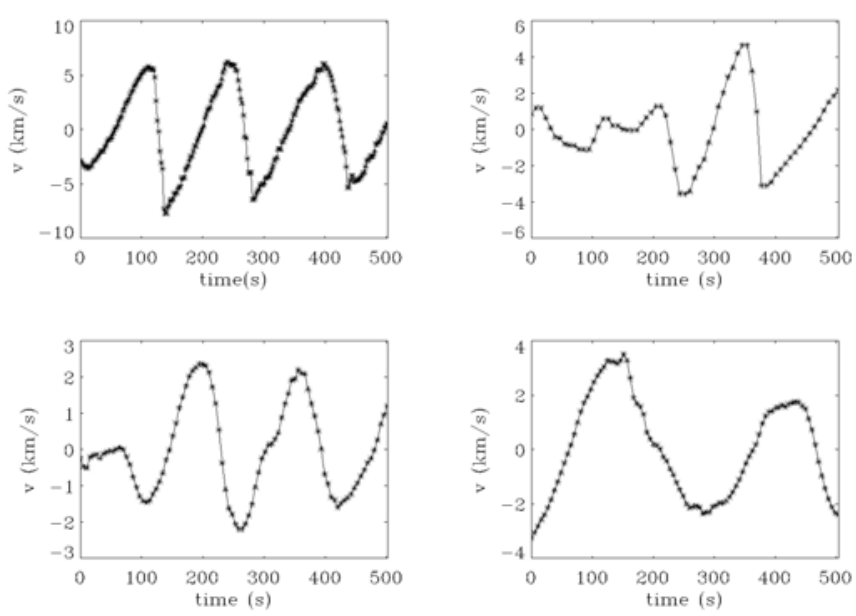

Figure 1. Chromospheric oscillations in different magnetic features. From left to right and top to bottom we show a typical velocity profile for one position inside: the umbra of a big sunspot (May 9, 2001), the umbra of small sunspot, a pore and a facular region. In all the cases, asterisks ('stars') represent the measured values, equispaced in time.

\section{Observations}

Observations were carried out at the German Vacuum Tower Telescope (VTT) of the Observatorio del Teide between October 2000 and June 2002, using the Tenerife Infrared Polarimeter (TIP). The spectrograph slit ( $0 .{ }^{\prime} 5$ wide and $40^{\prime \prime}$ long) was placed over the targets and was kept fixed during the entire observing run (approx. 1 hour). The image stability throughout the temporal series was achieved by using a correlation tracker device which compensates for the image motion induced by the Earth's high frequency atmospheric variability, as well as for solar rotation.

The observed spectral range spanned from 10825 to $10833 \AA$, with a spectral sampling of 31 $\mathrm{m} \AA$ per pixel. This spectral region is a powerful diagnostic window for the solar atmospheric properties since it contains valuable information coming from two different regions in the atmosphere. It includes a photospheric Si I line at $10827 \AA$ and a chromospheric He I triplet centered around $10830 \AA$.

In order to infer the physical parameters of the magnetized atmosphere in which the measured spectral lines were generated we carried out the full Stokes inversion of both the silicon line and the helium triplet for the whole time series of observations and for all four data-sets. Thus, we were able to obtain the temporal variability of several physical quantities (line-of-sight velocity, magnetic field intensity and orientation, ...) at the photospheric and chromospheric regions where the observed spectral line radiation has originated, though here we will focus only on the results concerning the line-of-sight velocity oscillations. 

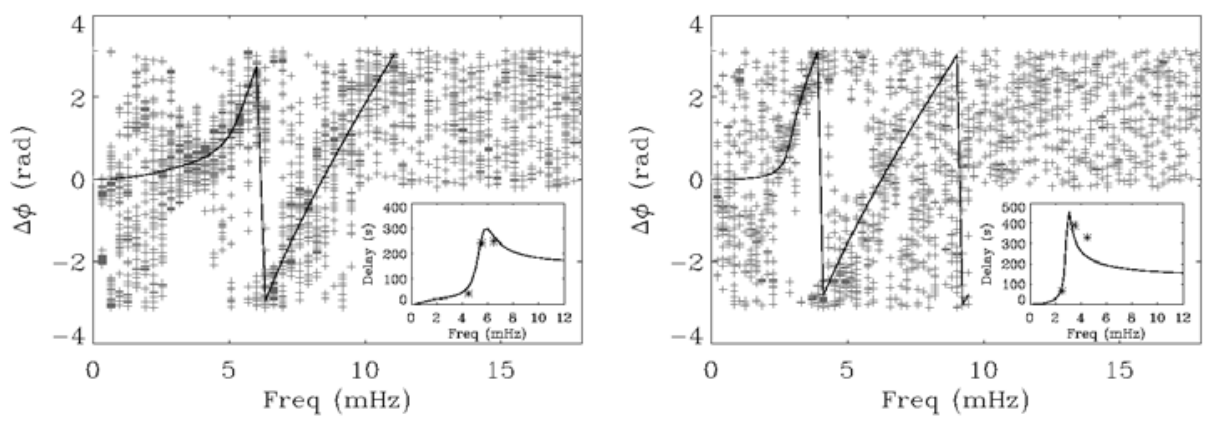

Figure 2. Phase difference between photospheric and chromospheric oscillations as a function of frequency. Left: for the umbra of the big sunspot (May 9, 2001), representative of sunspot-like structures. Right: for the facular region. The small boxes on the bottom right corner of each figure show the expected time delay between photospheric and chromospheric oscillations as a function of frequency. Asterisks represent the measured values obtained from the cross-correlation of the filtered velocity maps.

\section{Analysis}

Photospheric velocity oscillations (retrieved from the inversion of the $\mathrm{Si}$ I line) show the same characteristics everywhere: 5-min period, 300-400 $\mathrm{m} \mathrm{s}^{-1}$ peak to peak amplitudes and fairly sinusoidal patterns. Contrarily, chromospheric velocity oscillations are very different depending on the magnetic structure (Fig. 1). In case of the big sunspot of May 9, 2001, chromospheric oscillations have a 3-min period and peak to peak variations of $\sim 15 \mathrm{~km} \mathrm{~s}^{-1}$. They also show a constant and clear saw-tooth shape typical of shock wave trains (top left panel of Fig. 1). As the magnetic flux of the structure decreases (going from the big sunspot to the pore), the amplitude of the oscillations grows smaller, along with the steepness and frequency of appearance of the shocks. The period of the oscillations, however, remains the same. The case of the facular region is somewhat different. Again, while photospheric oscillations remain the same, the chromospheric oscillations have the smallest amplitudes of all the cases (1-2 $\mathrm{km} \mathrm{s}^{-1}$ ) and shocks are barely detectable, although a slight asymmetry in the oscillation pattern can easily be seen. Interestingly, the chromospheric main period is now 5 minutes instead of 3 minutes.

Following Centeno et al. (2006), Fig. 2 shows the phase difference between the photospheric and the chromospheric oscillations for the big sunspot (left) and for the facular region (right). The solid line corresponds to the best fit to a model of upward linear wave propagation in an isothermal stratified atmosphere that allows for radiative losses. The solid lines in the small boxes inside both panels of Fig. 2 show the expected time delays, as a function of frequency, between the oscillations measured at the photosphere and the ones measured at the chromosphere, obtained from the theoretical modeling. The expected time delay shows a very strong dependence with frequency. To make an estimation - directly from the observations - of the time that it takes for a photospheric perturbation to reach the high chromosphere, we have to take this fact into account. We have filtered both the photospheric and the chromospheric velocity maps in very narrow frequency ranges (so that the expected 
time delay does not vary within the bandwidth) and have compared the external modulation schemes of each pair of filtered velocity maps.

In the case of the sunspot-like structures, we filtered the velocity maps in 3 narrow ranges around $6 \mathrm{mHz}$ (where the bulk of the chromospheric power is mainly concentrated): 4-5, 5-6 and 6-7 mHz. We then compared each pair of filtered maps finding that they resembled each other, and, in order to make them match, a time shift between them that depended on the frequency range had to be applied. This shift corresponds to the time that it takes for a perturbation originated at photospheric levels (where the Si line is formed) to reach the chromosphere (at the height where the He triplet is generated). In the case of the facular region we filtered the velocity maps around $3 \mathrm{mHz}$ (where both photospheric and chromospheric power spectra have their main contributions). The rest of the analysis remained the same. Asterisks superposed on the theoretical time delays in the small boxes in Fig. 2 correspond to the measured values. Even though the theoretical curves predict a very strong variation of the time delay within the $1 \mathrm{mHz}$ filtering bands, the measured delays agree surprisingly well with what was expected.

\section{Discussion and conclusions}

In the case of sunspot-like features the cut-off frequency is $\sim 4 \mathrm{mHz}$, so the modes with frequencies below this one will not be able to reach the high chromosphere. Many authors have argued before about the possibility of the non-linear interaction among 5-min modes being the source of the chromospheric 3-min oscillations (see, e.g. Fleck \& Schmitz 1993). But if this were the case, the photospheric and chromospheric filtered maps would show no correlation between them. As a matter of fact, this correlation exists and is very clear, indicating that most of the $6 \mathrm{mHz}$ power observed at chromospheric heights in sunspot-like structures comes directly from the same frequency range in the photosphere via upward linear wave propagation.

In the case of facular regions, the cut-off frequency stays around $2 \mathrm{mHz}$, due to the radiative losses and the higher temperature, allowing the 5-min power propagate through the atmosphere and reach the high chromosphere. Again, a clear correlation between photospheric and chromospheric filtered velocity maps can be found, indicating that the propagation is mainly linear within the $2-5 \mathrm{mHz}$ range.

As the photopheric perturbations propagate upwards, their amplitude increases due to the rapid decrease in density and they eventually develop asymmetries that steepen more or less depending on the magnetic structure (the lower the magnetic flux, the smaller the steepnes of the developing shock). In all the cases, the time delay between photospheric and chromospheric oscillations is around several minutes in the frequency range near where the chromospheric power peaks.

\section{References}

Bogdan, T. J. \& Judge, P. G. 2006, Phil. Trans. R. Soc. London A, 364, 313

Centeno, R., Collados, M., Trujillo Bueno, J. 2006, ApJ, 640, 1153

Fleck, B. \& Schmitz, F. 1993, A\&A, 273, 671

Lites, B. W. 1992, in Sunspots: Theory and Observations, ed. J. H. Thomas \& N. O. Weiss, NATO ASI ser. C 375, Kluwer, Dordrecht, 261 


\title{
Evolution of coronal hole boundaries seen in EIT $195 \AA$ A and TRACE $171 \AA$ images
}

\author{
M. S. Madjarska* and T. Wiegelmann \\ Max-Planck-Institut für Sonnensystemforschung, Katlenburg-Lindau, Germany \\ *Email: madjarska@mps.mpg.de
}

\begin{abstract}
We aim at studying the spatial and temporal scales of the small-scale evolution of coronal hole boundaries using EUV observations from TRACE (171 ̊) and EIT (195 ^) on-board SoHO with a spatial resolution of $1^{\prime \prime}$ and $5.5^{\prime \prime}$, respectively, and various time cadences. We found that small-scale loops known as bright points may play an essential role in coronal hole boundaries evolution. Their emergence and disappearance continuously expand or contract coronal holes. The changes appear to be random on a time scale comparable with the lifetime of the loops seen at these temperatures. No signature was found for a major energy release during the evolution of the loops.
\end{abstract}

\section{Introduction}

Coronal holes (CHs) are large regions on the Sun that are magnetically open and were identified as the source of the fast solar wind $\left(\sim 800 \mathrm{~km} \mathrm{~s}^{-1}\right.$, Krieger et al. 1973). They are visible in coronal lines $\left(\mathrm{T} \geq 6 \cdot 10^{5} \mathrm{~K}\right)$ as regions with a reduced emission relative to the quiet Sun (Stucki et al. 2002). There are two types of coronal holes: polar and mid-latitude CHs. During the minimum of the solar activity the solar atmosphere is dominated by two large $\mathrm{CHs}$ situated at both polar regions. The mid-latitude $\mathrm{CHs}$ can be either 'isolated' or connected with a channel of open magnetic flux to a polar $\mathrm{CH}$. The latter $\mathrm{CHs}$ are called equatorial extensions of polar CHs (EECHs). Navarro-Peralta \& Sanchez-Ibarra (1994) showed that isolated CHs have a typical differential rotation, while EECHs maintain two types of rotational rates: differential below latitude of $40^{\circ}$ which becomes almost rigid while approaching the poles.

The small-scale $\mathrm{CH}$ boundaries evolution was a subject of several studies during the last few decades (Kahler \& Moses 1990 and the references therein). Kahler \& Moses (1990) studied SKYLAB X-ray images of EECH boundaries with a time resolution of 90 minutes aimed at looking for discrete changes of $\mathrm{CH}$ boundaries. They found that X-ray bright points (BPs, Madjarska et al. 2003) play an important role in both the expansion and contraction of the CH. Kahler \& Hudson (2002) made the first systematic morphological study of the boundaries of $\mathrm{CHs}$ as viewed in soft X-ray images from the Yohkoh Soft X-ray telescope. The studied coronal holes represent equatorial extensions of polar coronal holes observed during solar maximum phases of cycles 23 and 24. They found that the CHs evolve slowly, and neither large-scale transient X-ray events nor coronal bright points appeared significant factors in $\mathrm{CH}$ boundaries development. They suggested that open-close magnetic field reconnection is more likely to describe the actual physics at the $\mathrm{CH}$ boundaries. 

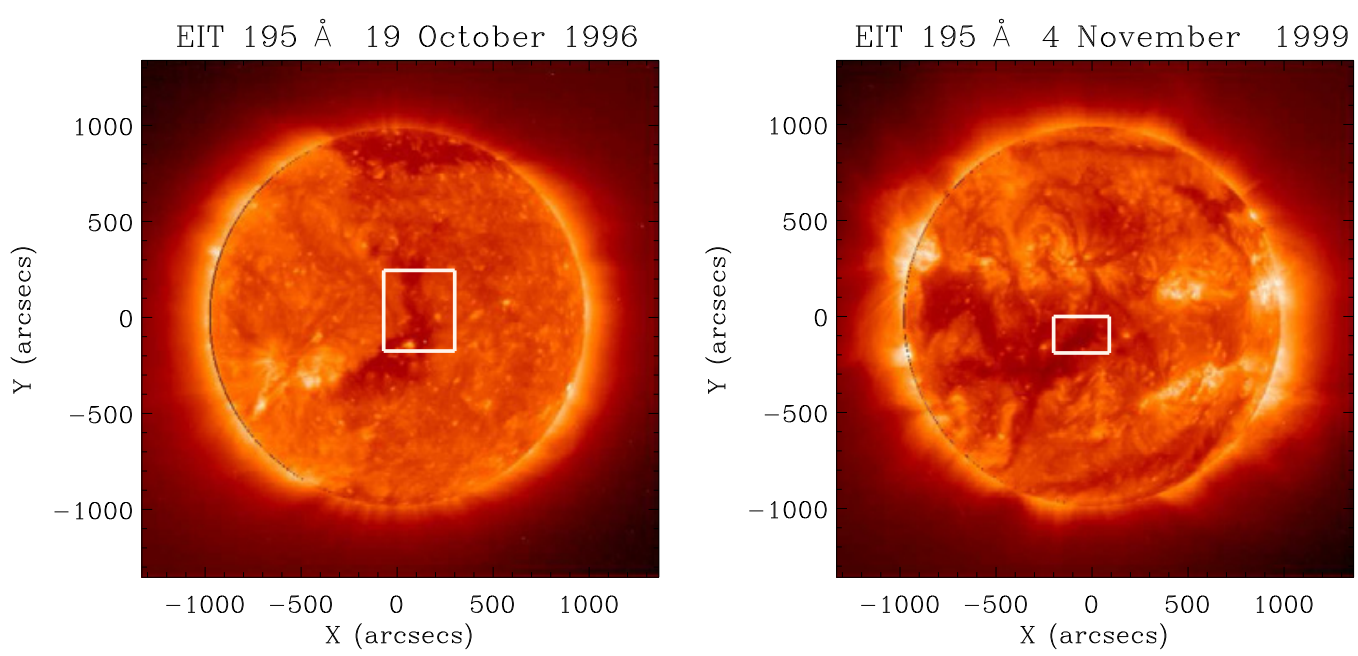

Figure 1. Full-disk EIT $195 \AA$ images obtained on October 19, 1996 and November 4, 1999. The images are derotated to 15:00 UT on October 20, 1996 and November 5, 1999. The over-plotted boxes outline the field-of-view subject to a detailed study.

\section{Observational material}

We selected two long-lived coronal holes: an EECH (during this rotation the channel connecting the equatorial part of the $\mathrm{CH}$ with the polar $\mathrm{CH}$ was closed) in October 1996 (CH1) and an isolated $\mathrm{CH}$ in November 1999 (CH2) (Fig. 1). We analysed Extreme-ultraviolet Imaging Telescope (EIT; 2.62" pixel size) and Transition Region And Coronal Explorer (TRACE; 0.5" pixel size) data obtained in the Fe XII $195 \AA$ and Fe Ix/x $171 \AA$ 年 passbands, respectively. After the necessary data reduction, a derotation to a reference time was applied to all images in this study. The images have different cadences ranging from 15 min to $6 \mathrm{hrs}$. In the present work we paid special attention to the data reduction in order to get information on the evolution of the $\mathrm{CH}$ boundaries using a contour plot. This method is more reliable than any visual inspection and ensures the detection of any small-scale changes.

\section{Data analysis and results}

In order to follow the evolution of the $\mathrm{CHs}$ for the longest possible period of time we produced movies from the full-disk $2 \times 2$ binned images obtained with EIT in the $195 \AA$ passband. Their visual inspection shows that after $48 \mathrm{hrs}$ the boundaries have significantly changed which in the case of $\mathrm{CH} 1$ results in a full displacement of part of the coronal hole. In order to determine the cause for these changes a more detailed analysis of the full-resolution EIT and TRACE images was done. Figure 2 presents examples of the full-resolution images of $\mathrm{CH} 1$ used in this study. Figures 3 and 4 give a closer look on selected areas from $\mathrm{CH} 1$. The arrows point at some of the BPs whose evolution appears to have expanded or contracted the coronal hole. We could not identify any flaring event related to this evolution. The time 

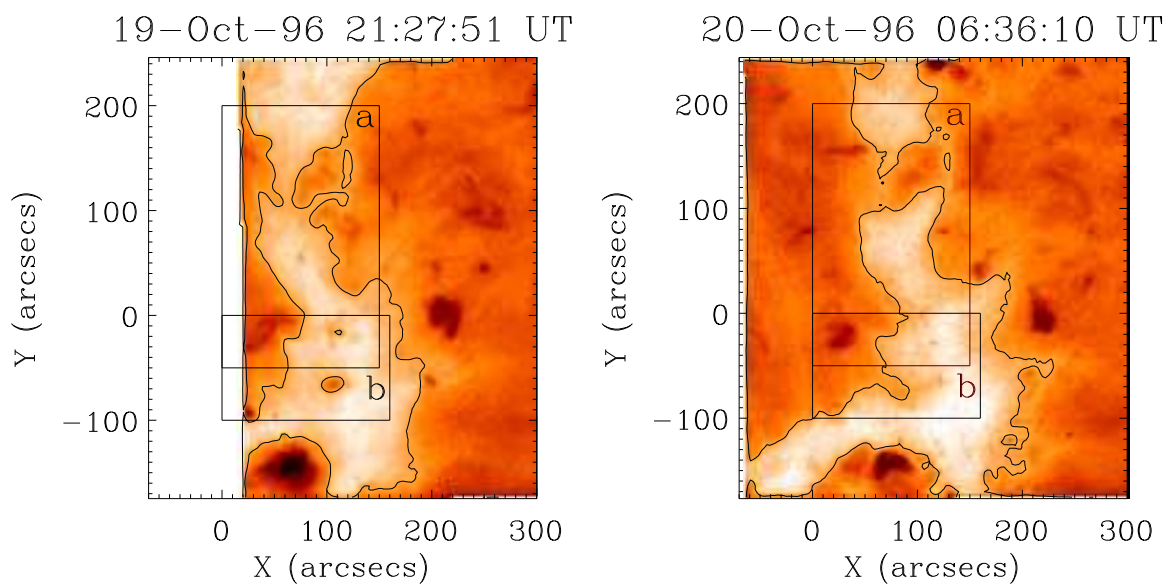

Figure 2. Intensity reversed EIT $195 \AA$ Amages of the coronal hole observed on October 19-20, 1996. The evolutions within the over-plotted rectangular areas ( $\mathrm{a}$ and $\mathrm{b}$ ) are shown in Figs. 3 and 4.
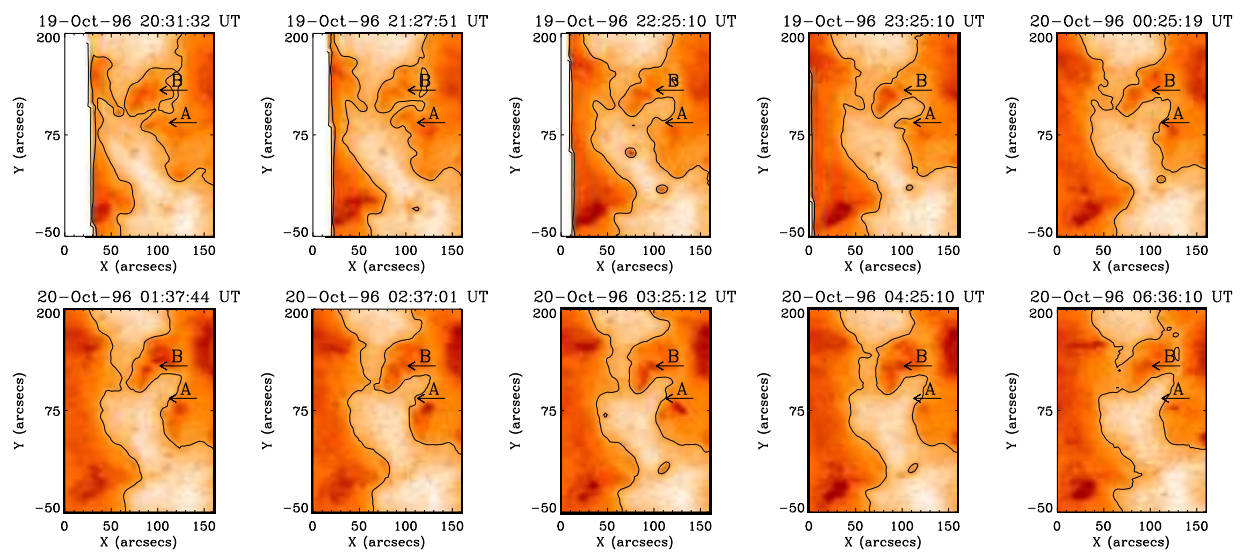

Figure 3. Intensity reversed EIT $195 \AA$ A images of the area marked with 'a' in Fig. 2. The arrows point at the BPs whose evolution led to the expansion (arrow A) and the contraction (arrow B) of the $\mathrm{CH}$.

scale of the changes is defined by the lifetime of the BPs which is around $20 \mathrm{hrs}$ at these temperatures (Zhang et al. 2001).

We believe that our results do not contradict Kahler \& Hudson's (2002) result and the discrepancy could be explained with the fact that the visibility of BPs in X-ray (also EUV) images, especially during the solar maximum activity, is strongly diminished by the presence of active regions and bright loops seen in X-ray. The BPs number and size, and also their lifetime strongly depend on the temperature in which they are observed with more and longer living BPs seen in spectral lines formed at lower temperatures. However, predominantly large loops exist at temperatures above $1.4 \times 10^{6} \mathrm{~K}$ (Feldman et al. 1999) which are closing at large distances showing slow evolution. 

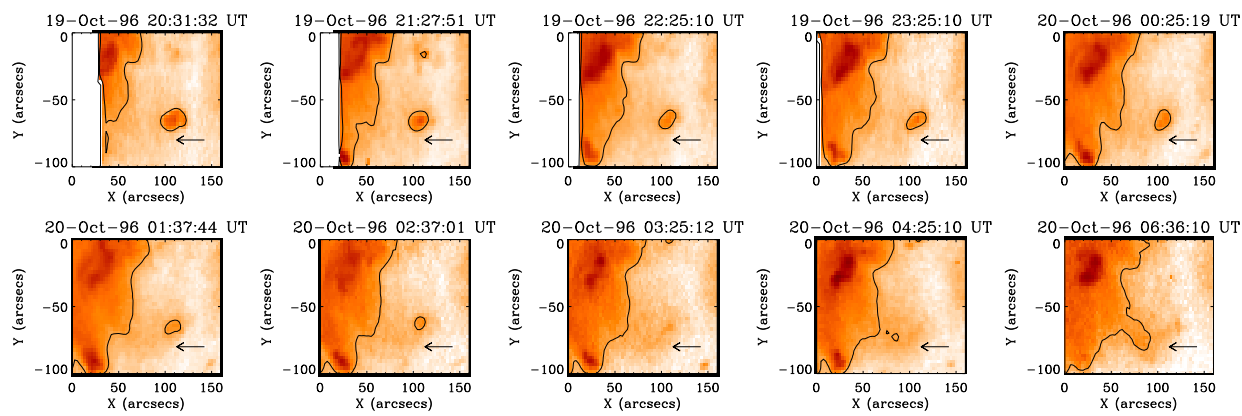

Figure 4. Intensity reversed EIT $195 \AA$ A images of the area marked with 'b' in Fig. 2. The arrows point at a bright point whose evolution led to the contraction of the coronal hole.

Due to the different rotational profiles at coronal (Insley et al. 1995 and the references therein) and photospheric levels (Meunier 2005) and the fact that $\mathrm{CH}$ boundaries separate two topologically different (open and closed) magnetic field configurations, coronal hole boundaries are presumably the regions where processes that open and close magnetic field lines take place. The possible mechanism of the $\mathrm{CH}$ boundaries evolution could, therefore, be a continuous magnetic reconnection (Kahler \& Hudson 2002) between the open magnetic field of the $\mathrm{CH}$ and the closed field of the small-scale loops located along $\mathrm{CH}$ boundaries.

Acknowledgements. The work of TW was supported by DRL-grant 50 OC 0501. SoHO is a mission of international collaboration between ESA and NASA.

\section{References}

Insley, J. E., Moore, V., \& Harrison, R. A. 1995, Solar Phys., 160, 1

Feldman, U., Widing, K. G., \& Warren, H. P. 1999, ApJ, 522, 1133

Kahler, S. W. \& Hudson, H. S. 2002, ApJ, 574, 467

Kahler, S. W. \& Moses, D. 1990, A\&A, 362, 728

Krieger, A. S., Timothy, A. F., \& Roelof, E. C. 1973, Solar Phys., 29, 505

Madjarska, M. S., Doyle, J. G., Teriaca, L., \& Banerjee, D. 2003, 398, 775

Meunier, N. 2005, A\&A, 443, 309

Navarro-Peralta, P. \& Sanchez-Ibarra, A. 1994, Solar Phys., 153, 169

Stucki, K., Solanki, S. K., Schühle, U., Rüedi, I., Wilhelm, K., Stenflo, J. O., Brković, A., \& Huber, M. C. E. 2002, A\&A, 381, 653

Zhang, J., Kundu, M. R., \& White, S. M. 2001, Solar Phys., 198, 347 


\title{
Magnetic field structure and dynamics in coronal hole - active region interface zone
}

\author{
I. A. Bilenko \\ Sternberg State Astronomical Institute, Moscow, Russia \\ Email: bilenko@sai.msu.ru
}

\begin{abstract}
We study the changes of magnetic field structure in active regions (AR), coronal holes $(\mathrm{CH})$, and coronal hole - active region ( $\mathrm{CH}-\mathrm{AR})$ interface zones. Zones of coronal hole - active region interface are characterized by relatively fast fluctuations of the photospheric magnetic fields. Interaction between the closed AR magnetic fields and the open $\mathrm{CH}$ fields can cause or facilitate some eruptive events.
\end{abstract}

\section{Introduction}

It is often assumed that $\mathrm{CH}$ boundaries are sources of slow solar wind. $\mathrm{CH}$ boundaries have also been suggested as important factors in coronal eruptive events, especially when they are associated with ARs. Webb et al. (1978) found that most of the transients are related to large-scale changes in $\mathrm{CH}$ areas and tend to occur on the borders of evolving equatorial holes. Kahler \& Moses (1990) found that the boundary changes are characterized by events of length scales $<2 \times 10^{4} \mathrm{~km}$ and time scales of $<3 \mathrm{~h}$. They interpret the boundary changes in terms of magnetic reconnection. There are different types of $\mathrm{CH}$ boundaries (Kahler \& Hudson 2002). The boundaries of CHs are quite irregular, reflecting the structure of adjacent photospheric magnetic fields. Small-scale changes in the structure of a $\mathrm{CH}$ boundary could be seen from SOHO/EIT images to occur on a time scale of a few hours. However, the main structure may be observed for several months and appears to rotate quasi-rigidly. The maintenance of such a rigid open-field structure against differential AR rotation could be achieved by continuous reconnection between open and closed field lines at the boundary of the $\mathrm{CH}$ (Wang et al. 1996).

It is interesting to compare the evolution of the photospheric magnetic field at $\mathrm{CH}-\mathrm{AR}$ interface zones as seen by MDI (Scherrer 1995) to that of the solar corona observed by EIT (Delaboudinière et al. 1995) and Yohkoh SXT (Tsuneta et al. 1991).

\section{Analysis and results}

CHs are known to be the regions of open magnetic field, low coronal density, and low temperature. They are associated with weak, predominantly unipolar photospheric magnetic fields (Bohlin 1997).

In Figure 1 the process of formation and evolution of a positive-polarity $\mathrm{CH}$ near the $\mathrm{AR}$ NOAA 9026 during the period 2000, 5-7 June is presented. The top row shows magne- 
tograms from SOHO/MDI overlaid with the $\mathrm{CH}$ boundaries observed in the He $\mathrm{I} 10830 \AA$ line (from Kitt Peak National Solar Observatory/KP-NSO). The middle row represents the $\mathrm{AR}$ and $\mathrm{CH}$ evolution in X-ray observed by Yokoh/SXT, and the bottom row shows their evolution in the Fe XII $195 \AA$ line observed by EIT. In this area, there was no $\mathrm{CH}$ in the He I line detectable on June 5. In some small regions of reduced density overlapping, large faint arc structures were seen. In X-ray a large region of reduced density (X-ray coronal hole) surrounded the AR. It forms sharp arc boundary structures. The arcs connect the AR negative-polarity fields with the positive-polarity photospheric fields at the boundary of the $\mathrm{X}$-ray $\mathrm{CH}$. The general magnetic configuration changed little until June 6. On June 6, X11 (13:30 UT), X23(14:58 UT) class flares, and several minor M and C class events occurred in NOAA 9026. These events resulted in changes of magnetic field configuration. The Fe XII arc structures were destroyed, probably they reconnected with closed magnetic fields of the $\mathrm{AR}$ and an open magnetic structure appeared (cf. Fig. 1 middle row, second image). A new $\mathrm{CH}$ in the chromospheric He I line was observed (second image in the top row in Fig. 1). The post-flare X-ray arc increased and connected boundaries of the new positive-polarity $\mathrm{CH}$ and a negative one on the other side of the AR, forming a large helmet structure above the AR. Such structures are thought to be the sources of some eruptive events. Six CMEs were observed during June 6 and three CMEs on the next day including two halo CME. Possibly, the halo CMEs were produced in that magnetic structure. Some brightenings were observed in the footpoints of the X-ray arc at the $\mathrm{CH}$ boundary. Changes in coronal arcs occur, and the brightness could be attributed to changes in the photospheric magnetic field (Howard \& Ŝvestka 1977).

An analogous process was observed during the period 1-7 November 1998. An X-ray $\mathrm{CH}$ was present during the whole period. The magnetic configuration indicated by arcs was stable until November 3. A great number of $\mathrm{M}$ and $\mathrm{C}$ class events occurred in the AR NOAA 8375. These events changed completely the magnetic field configuration. A new positivepolarity $\mathrm{CH}$ appeared in $\mathrm{He}$ I on November 3 on the unipolar region near NOAA 8375. A new large structure of arcs was formed which connected the boundaries of X-ray CHs at the leading and the following parts of the AR. Possibly, the halo CME on November 4 originated in that structure, and such an eruptive event resulted in the formation of an open magnetic field structure on the following negative-polarity unipolar region, and a $\mathrm{He}$ I chromospheric $\mathrm{CH}$ appeared on November 5. A huge helmet arc structure overlapping an AR and joining positive-polarity and negative-polarity $\mathrm{CH}$ boundaries was created. The two halo CMEs which were observed on November 5 were possibly associated with that magnetic structure.

The structure of photospheric magnetic network elements in the interface zone differs strongly from that in the other part of the adjacent $\mathrm{CH}$ or the quiet region. Figure 2 shows the magnetic field evolution in NOAA 9026, in the adjacent $\mathrm{CH}$, and in the $\mathrm{CH}-\mathrm{AR}$ interface zone. MDI magnetograms at $96 \mathrm{~m}$ cadence are used to study magnetic field changes. Daily CH maps in He 10830 were obtained from the Kitt Peak National Solar Observatory. The MDI data were converted to magnetic field strength according to $\mathrm{H}$ (Gauss) $=1.232+$ 2.816(pixel value). The positive-polarity magnetic field average strength (thick line) and the negative one (thin line) in the CH (Fig. 2b), in AR (Fig. 2c), and in the zone between them (Fig. 2a) for magnetic fields greater than 20 Gauss are presented. In the zone of CH-AR interface fluctuations are significant, especially after the coronal hole appearance. In this region positive-polarity magnetic fields dominate as in the $\mathrm{CH}$. In Figure $2 \mathrm{~d}$, the evolution of the ratio of the average magnetic field strength of positive-polarity and the absolute value 

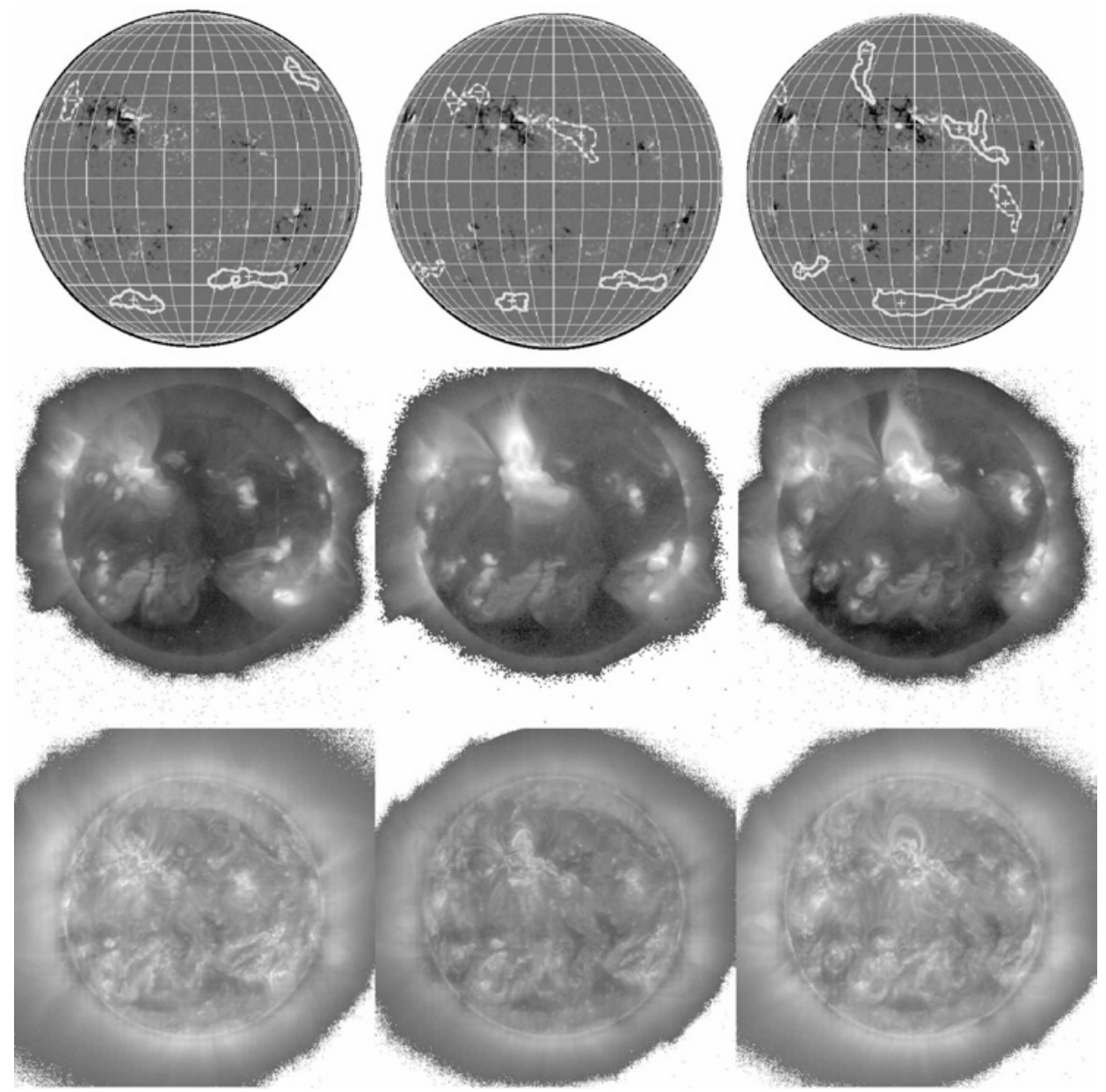

Figure 1. Formation and evolution of the $\mathrm{CH}$ during the period 2000, 5-7 June. Top row: magnetograms from KP-NSO overlaid with CH boundaries observed in $\mathrm{He}_{\mathrm{I}} 10830 \AA$ (KP-NSO); middle row: $\mathrm{AR}$ and $\mathrm{CH}$ evolution in X-ray (SXT); bottom row: evolution in Fe XII $195 \AA$ (EIT).

of the negative-polarity for all values of magnetic fields in the AR (3), in the $\mathrm{CH}$ (2), and in the zone of $\mathrm{CH}-\mathrm{AR}$ interface (1) is shown. In the $\mathrm{CH}$ and $\mathrm{CH}-\mathrm{AR}$ zone the imbalance is much stronger than that in the $\mathrm{AR}$, and the maximum coincides with the time of the $\mathrm{CH}$ creation above the photospheric region with unipolar, positive-polarity magnetic field. Some simultaneous variations are observed at the time of X-class flares on June 6.

\section{Conclusion}

The photospheric magnetic field structure in the $\mathrm{CH}$ part connected with the AR differs significantly from that in the other parts of the $\mathrm{CH}$. A CH - AR interface zone is characterized 
$|\mathrm{H}|$

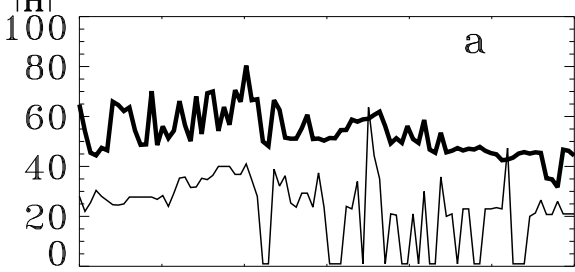

$|\mathrm{H}|$

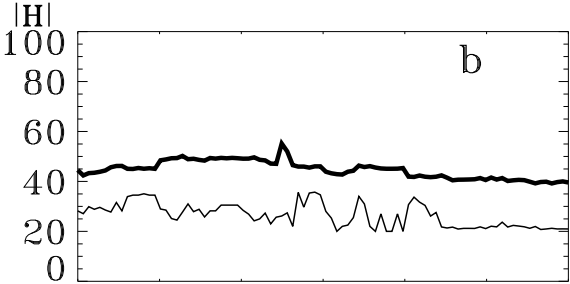

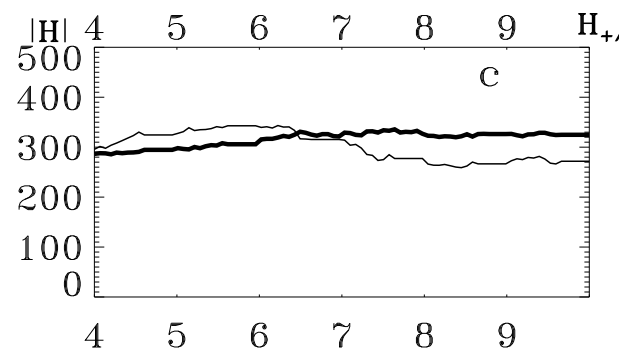

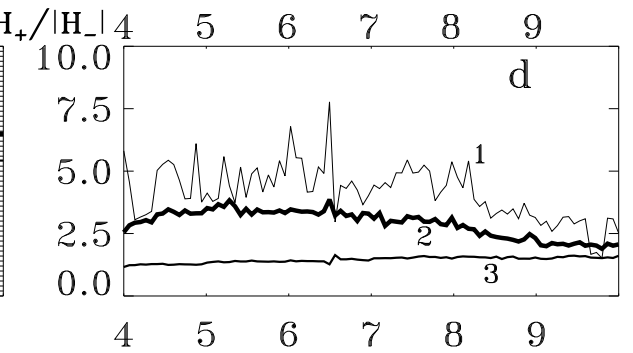

Figure 2. Evolution of averaged line-of-sight magnetic field strength in $\mathrm{CH}-\mathrm{AR}$ interface zone of NOAA 9026 (a), in the $\mathrm{CH}$ (b), and in the AR itself (c) for magnetic fields greater than 20 Gauss; thick: positive polarity, thin: negative polarity; Panel d: magnetic field strength imbalance in $\mathrm{CH}$ (2), in zone of CH-AR interface (1), and AR (3) for all magnetic fields. Abscissae: day in June 2000; ordinates: absolute field strengths in Gauss ( $a, b, c)$, relative units (d).

by relatively fast fluctuations of the photospheric magnetic fields. Emerging or cancellation of magnetic flux, intensive motion of small photospheric magnetic fields may yield increased instability, and shear or twist the footpoints of the coronal magnetic fields. Such dynamic processes increase the magnetic energy in the solar corona. Interaction between the closed AR fields and the open $\mathrm{CH}$ fields can cause or facilitate some eruptive events. And vice versa, magnetic fields eruption can form large-scale patterns, which contain open magnetic fields. $\mathrm{CH}$ were formed within these open-field regions. The deepest layers of the solar atmosphere are involved in such processes.

Acknowledgements. SOHO is a project of international cooperation between ESA and NASA. $\mathrm{NSO} /$ Kitt Peak data used here were produced cooperatively by NSF/NOAO, NASA/GSFC, and NOAA/SEL. We are grateful to the Yohkoh team for supplying the SXT data.

\section{References}

Bohlin, J. D. 1997, in Coronal Holes and High Speed Wind Streams, ed. J. B. Zirker, Boulder, Colorado Associated University Press, p.27

Delaboudinière, J.-P., Artzner, G. E, Brunaud, J., et al., 1995, Solar Phys., 162, 291

Howard, R. \& Ŝvestka, Z. 1997, Solar Phys., 54, 65

Kahler, S. W. \& Hudson, H. S. 2002, ApJ, 574, 467

Kahler, S. W. \& Moses, D. 1990, ApJ, 362, 728

Scherrer, P. H. 1995, Solar Phys., 162, 129

Tsuneta, S., Acton, L., Bruner, M., et al. 1991, Solar Phys., 136, 37

Wang, Y.-M., Hawley, S. H., \& Sheeley, N. R., Jr. 1996, Science, 271, 417

Webb, D. F., McIntosh, P. S., Nolte, J. T., \& Solodyna, C. V. 1978, Solar Phys., 58, 389 


\title{
Evolution of the photospheric magnetic field in the source regions of coronal mass ejections
}

\author{
V. Bothmer ${ }^{1, *}$ and D. Tripathi ${ }^{2}$ \\ ${ }^{1}$ Institut für Astrophysik, Göttingen, Germany \\ ${ }^{2}$ Department of Applied Mathematics and Theoretical Physics, University of Cambridge, \\ Cambridge, United Kingdom \\ *Email: bothmer@astro.physik.uni-göttingen.de
}

\begin{abstract}
Six coronal mass ejections associated with erupting quiescent filaments on the visible solar disk were identified in data from SoHO (Solar and Heliospheric Observatory) LASCO (Large Angle and Spectrometric Coronagraph), EIT (Extreme ultraviolet Imaging Telescope) and MDI (Michelson Doppler Imager) data and ground-based $\mathrm{H} \alpha$ observations from Big Bear and Meudon observatories. These events were analysed to investigate whether their initiations could be related to changes of the underlying photospheric field. The results show that in five out of the six events, substantial changes in the photospheric magnetic field occurred in the source regions prior and around the CME's lift-off times as identified from emerging/diminishing flux detected by MDI. In one event large magnetic flux changes could be identified not in the source region itself, but in a neighbouring active region. The results demonstrate that new missions, such as STEREO and Hinode (Solar-B) in conjunction with SoHO and ground-based measurements, will provide joint data sets that have the potential to provide new insight into the physical causes of CMEs.
\end{abstract}

\section{Introduction}

Large-scale transient releases of solar matter into interplanetary space occur in the form of coronal mass ejections (CMEs) (e.g., Hundhausen 1999). CMEs are the causes of interplanetary shock waves, large-scale disturbances of the heliosphere, major geomagnetic storms and solar energetic particle events (Webb 2000, Bothmer 1999, 2003). The physical causes of CMEs are not understood so far and a variety of theoretical models exist having their own advantages and drawbacks (e.g., Lin et al. 2003, Aschwanden 2004). CMEs are often associated with the disappearance of a filament, as visible in H $\alpha$, EUV and micro-wave observations (e.g., Bothmer \& Rust 1997, Bothmer \& Schwenn 1998, Gopalswamy et al. 2003, Tripathi et al. 2004).

Rather few studies have been undertaken so far using simultaneous space- and groundbased multi-wavelength observations (e.g., Song et al. 2002). A study based on the combined analysis of coronal and photospheric data suggests that newly emerging magnetic flux in the photosphere can lead to the onset of a CME if the flux has a magnetic polarity which is favourable for magnetic reconnection with the pre-existing field near the filament site (Feynman \& Martin 1995). However, it seems that eruptions can also occur in the absence of any observable flux emergence (Wang \& Sheeley 1999). In addition to the scenario of 
emerging flux, cancellation of photospheric magnetic flux has also been suggested as a trigger for prominence eruptions and CMEs (e.g., Linker et al. 2003). Alternatively, CMEs may be caused by large-scale instabilities of the corona, as in the case of instabilities of trans-equatorial loops connecting different active regions (e.g., Zhou et al. 2005).

In a detailed analysis of SoHO data Tripathi et al. (2004) and Tripathi (2005) found that transient post-eruptive arcades (PEAs) visible at EUV-wavelengths can be considered as unique tracers of CMEs on the solar disk. From the database ${ }^{1}$ of this study we have selected those CME events which were associated with disappearing filaments exceeding heliographic lengths of 10 degrees. Our set of six events comprises CMEs associated with quiescent filaments from decaying active regions with less complex magnetic field structures, contrary to the study of CMEs from active regions by Muglach \& Dere (2005). The less complex magnetic structure is useful for unambiguous interpretation of the data. With our selection criterium we tried to minimize the problem of making false associations between coronal and photospheric changes because the photospheric flux is ever varying on different spatial- and time-scales, e.g., as visible from the observations of the small-scale bipoles of the magnetic carpet, the appearances of X-ray bright points and active regions or the large changes apparent in sunspot groups.

\section{Data}

For the present study, data from SoHO/LASCO/EIT/MDI (Fleck et al. 1995) were used. Complementary, $\mathrm{H} \alpha$ ground-based data from Paris/Meudon ${ }^{2}$ and Big Bear ${ }^{3}$ were studied.

The source regions of the six selected CME events were defined by the heliographic position of the associated EUV PEAs visible at $195 \AA$ (Tripathi et al. 2004) overlying the magnetic inversion lines separating the areas of opposite polarity magnetic fields in the photosphere. A rectangular box was fit to each region including the bipolar field regions and quiescent filament. The evolution of the photospheric flux was investigated in this area and also in the surroundings of the source region from a day before until a day after the onset of the CME using MDI magnetograms with a time cadence of 96 min and thresholds of $\pm 200 \mathrm{G}$ allowing to identify significant changes of the field. The onset times of CMEs were obtained by extrapolating the corresponding height-time diagrams based on the LASCO/C2 and $\mathrm{C} 3$ observations provided in the CME catalogue ${ }^{4}$.

\section{Summary of the observations}

On 12 of September 2000 a huge CME with a speed of of $1600 \mathrm{~km} \mathrm{~s}^{-1}$ was detected by the LASCO instruments around noon (Tripathi et al. 2004). Figure 1 left panel shows at the top the associated filament on the solar disk observed in $\mathrm{H} \alpha$ and at $195 \AA$ by SoHO EIT, followed by MDI-snapshots of the photospheric field in the CME's source region.

The white arrow in the MDI images shows one of the two locations close to the filament channel, to the East, where larger areas of magnetic flux of negative polarity (black color)

\footnotetext{
${ }^{1}$ http://vizier.u-strasbg.fr/viz-bin/VizieR?-source=J/A+A/422/337

${ }^{2}$ http://bass2000.obspm.fr/home.php

${ }^{3} \mathrm{ftp} / / / \mathrm{ftp}$. bbso.njit.edu/pub/archive/

${ }^{4}$ http://cdaw.gsfc.nasa.gov/CME_list/
} 

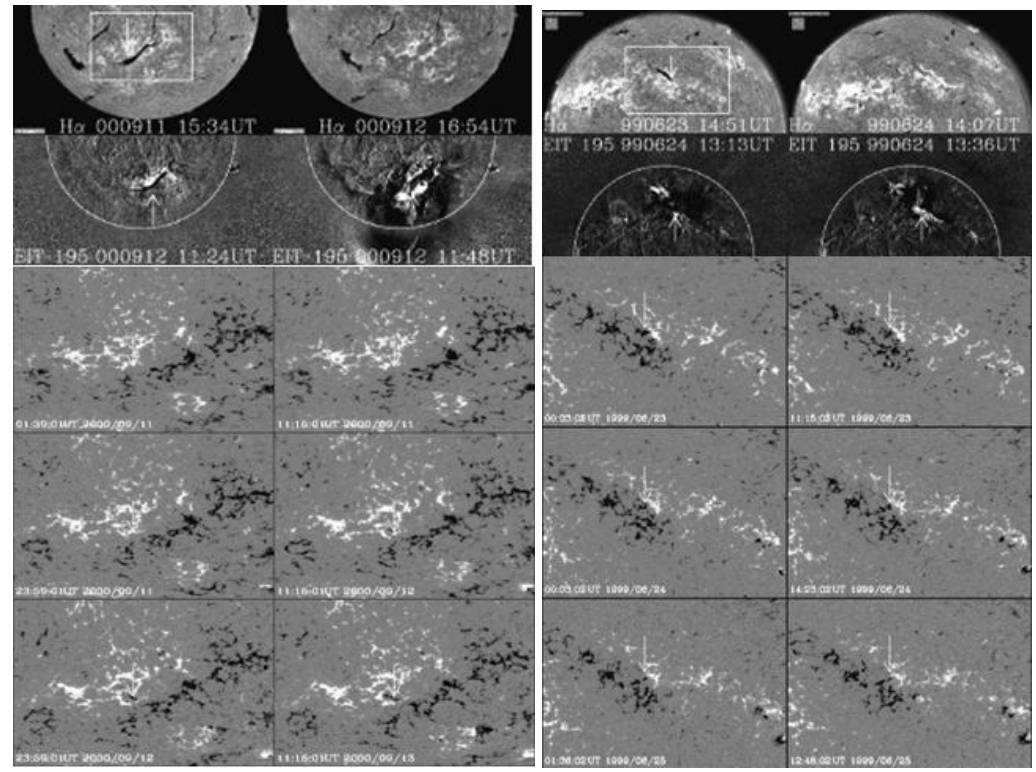

Figure 1. Left Panel: Top to bottom: $\mathrm{H} \alpha$ observations showing the location of the disappearing filament on 11/12 September 2000; EIT $195 \AA$ images showing the erupting prominence; 6 MDI snapshots showing the intensity and polarity changes of the photospheric magnetic field in the source region of the CME. Right panel: Same observations as displayed in the left panel for the CME event on June 24, 1999.

had emerged within the area of pre-existing positive polarity (white color). From the inspection of the MDI data, the opposite polarity flux started appearing near 10:00 UT, i.e. prior to the subsequent detection of the CME by LASCO/C2 after 11:30 UT. The appearance of newly emerging flux near the filament channel, with a magnetic polarity favourable to stimulate magnetic reconnection processes, corresponds to the scenario proposed by Feynman \& Martin (1995).

Similar observations are shown in the right panel of Fig. 1 for the CME event on 24 June 1999. Opposite to the previous event, no newly emerging flux could be identified in the CME's source region during the time interval from a day before until a day after the CME. A close investigation of the field intensities near the magnetic polarity inversion line showed that the flux intensities of both polarities were continuously diminishing in the course of the event, in agreement with the scenario of magnetic flux cancellation (Linker et al. 2003). In a different study of the same event by Zhang et al. (2001), the authors independently came to the same conclusion.

In another type of event such as on March 5, 2000 (not shown here) no flux changes could be identified in the source region of the CME itself, but new flux with a polarity favourable to trigger magnetic reconnection occurred in an active region bordering the filament channel. 


\section{Conclusions}

For six CME events associated with large quiescent filament disappearances, simultaneous multi-wavelength observations of the corona and photosphere were studied using data from SoHO and ground-based observatories. Remarkable changes of the photospheric flux could be identified close to the onset time of the CMEs in each of the selected events. According to the characterization of the photospheric field changes, CMEs can be triggered through emergence of newly magnetic flux with a polarity favorable to trigger reconnection processes in an area with pre-existing opposite polarity flux or through the process of continuous cancellation of magnetic flux, or through flux changes in a near-by active region neighboring the filament site which destabilize the magnetic arcade overlying the disappearing filament.

Acknowledgements. This study is part of the scientific investigations of the project Stereo/Corona, supported by the German Bundesministerium für Bildung und Forschung through the Deutsches Zentrum für Luft- und Raumfahrt e.V. (DLR) under project number 50 OC 0005. Stereo/Corona is a science and hardware contribution to the optical imaging package SECCHI for the NASA STEREO mission launched in October 2006. The work is further supported through EU-INTAS project 03-516206 'Solar and interplanetary disturbances causing severe geomagnetic storms' and through project PO20060453 with Praxis, Inc., Washington, D.C., USA as part of the SECCHI project. DT acknowledges the support from PPARC.

\section{References}

Aschwanden, M. 2004, Physics of the solar corona: An Introduction, Springer-Praxis, p. 703 et seqq. Bothmer, V. 1999, in Workshop on Space Weather, ESA WPP-155, 117

Bothmer, V. \& Rust, D. M. 1997, in Coronal Mass Ejections: Causes and Consequences, ed. N. Crooker, J. A. Joselyn, J. Feynman, AGU Geophys. Monograph, 99, 139

Bothmer, V. \& Schwenn, R. 1998, Ann. Geophys., 16, 1

Feynman, J. \& Martin, S. F. 1995, J. Geophys. Res., 100, 3355

Fleck, B., Domingo, V., \& Poland, A. I. 1995, Solar Phys., 162, 1

Gopalswamy, N., Shimojo, M., Lu, W., et al. 2003, ApJ, 586, 562

Hundhausen, A. J. 1999, in The many faces of the sun, ed. K. T. Strong, J. L. R. Saba, B. M. Haisch, \& J. T. Schmelz, Springer, 143

Lin, J., Soon, W., \& Baliunas, S. L. 2003, New Astron. Rev., 47, 53

Linker, J. A., Mikic, Z., Lionello, R., \& Riley, P. 2003, Phys. of Plasmas 10, 5, 1971

Muglach, K. \& Dere, K. 2005, in Coronal and Stellar Mass Ejections, ed. K. P. Dere, J. Wang, Y. Yan, IAUS 226, 179

Song, L., Zhang, J., Zhiliang, Y., \& Wang, J. 2002, Solar Phys., 211, 315

Tripathi, D., Bothmer, V., \& Cremades, H. 2004, A\&A, 422, 337

Tripathi, D. 2005, in EUV and Coronagraphic Observations of Coronal Mass Ejections, Copernicus, PhD Thesis, Göttingen University, 111

Wang, Y.-M. \& Sheeley, N. R. Jr. 1999, ApJ, 510, 157

Webb, D. F. 2000, in IEEE Transactions on Plasma Science 28, 6, 1795

Zhou, G., Wang, J., Zhang, J., \& Xiao, C. 2005, in Coronal and Stellar Mass Ejections, ed. K. P. Dere, J. Wang, Y. Yan, IAUS 226, 200 


\title{
Two-dimensional imaging of the $\mathrm{He}_{3} / \mathrm{H} \beta$ emission ratio in quiescent solar prominences
}

\author{
E. Wiehr ${ }^{1}$, G. Stellmacher ${ }^{2}$, and J. Hirzberger ${ }^{3}$ \\ ${ }^{1}$ Göttingen, Germany \\ ${ }^{2}$ Institut d'Astrophysique de Paris, France \\ ${ }^{3}$ Max-Planck-Institut für Sonnensystemforschung, Katlenburg-Lindau, Germany
}

\begin{abstract}
We extend our earlier studies of two-dimensional prominence imaging by simultaneous observations of the $\mathrm{He}_{3}$ and $\mathrm{H} \beta$ emissions at the $1 \mathrm{~m} \mathrm{SST}$ on La Palma. The observed small spatial variations of the $\mathrm{He}_{3} / \mathrm{H} \beta$ ratio indicate rather homogeneous physical conditions within prominences. Sub-regions do exist which show a largely uniform ratio in spite of substantial spatial intensity variations. Here, the fine-structures will exhibit almost uniform physical conditions. In other regions with variations of the ratio parallel to the emissions, the structures will have different intrinsic physical state.
\end{abstract}

\section{Introduction}

A powerful diagnostics of physical conditions in prominences is the ratio of total line emissions, i.e. the $\lambda$-integrated intensities. Model calculations of the emission ratios Ca II $8542 / \mathrm{H} \beta$ (Gouttebroze et al. 1997) and $\mathrm{He}_{3} / \mathrm{H} \beta$ (Labrosse \& Gouttebroze 2001) showed the dependences on temperature, pressure and slab-widths. The observation of such integrated intensities does not require the use of a spectrograph, but may well be done with narrow filters, just wide enough to also cover Doppler shifted profiles. This enables a substantial decrease of the exposure time, thus higher spatial resolution and, hence, the investigation of fine-structures, hardly visible in spectra. Wiehr and Stellmacher (1999) and Stellmacher and Wiehr (2000) observed simultaneous images in $\mathrm{Ca}$ II 8542 and $\mathrm{H} \beta$, here we present such observations in $\mathrm{He} \mathrm{D}_{3}$ and $\mathrm{H} \beta$.

\section{Observations}

On July 14 and 15, 2003, we observed with the $1 \mathrm{~m} \mathrm{SST} \mathrm{on} \mathrm{La} \mathrm{Palma} \mathrm{various} \mathrm{quiescent}$ prominences simultaneously in $\mathrm{He}_{3}$ and $\mathrm{H} \beta$ using a dichroitic beam splitter. The exposure time was set between $0.1 \mathrm{~s}$ and $0.25 \mathrm{~s}$, accounting for the individual prominence brightness. Image motion was minimized using the correlation tracker of the SST, set to a nearby white light limb facular grain as lock-point. The raw images were corrected for the dark and the gain matrices; the underlying aureole was determined from the neighborhood of the respective prominence and subtracted. 

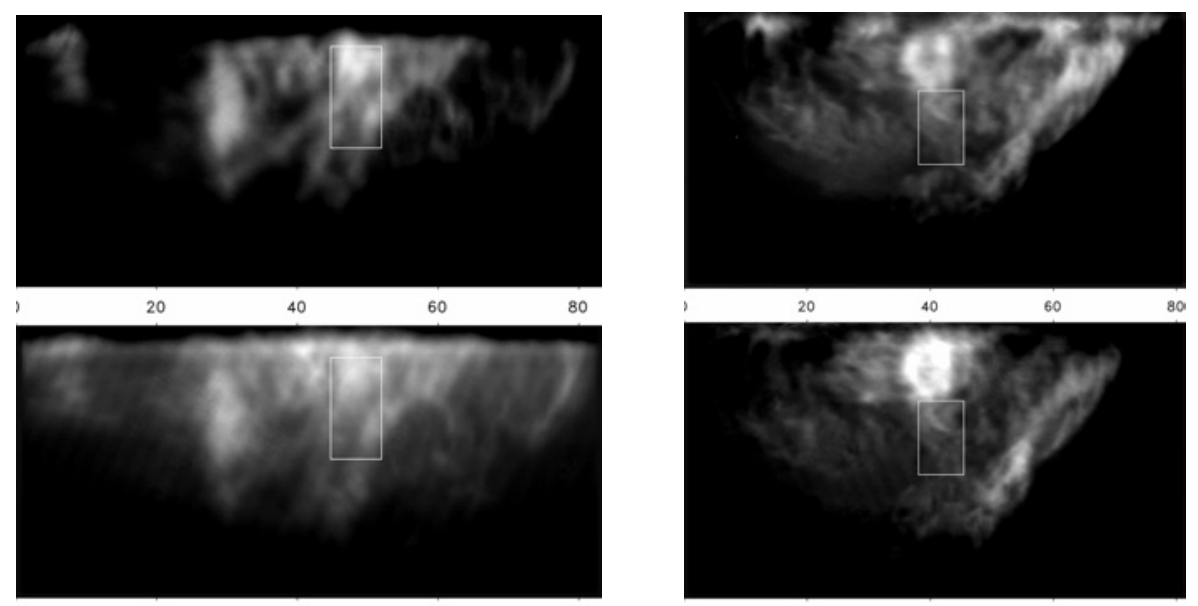

$20 \quad 40$

60

80
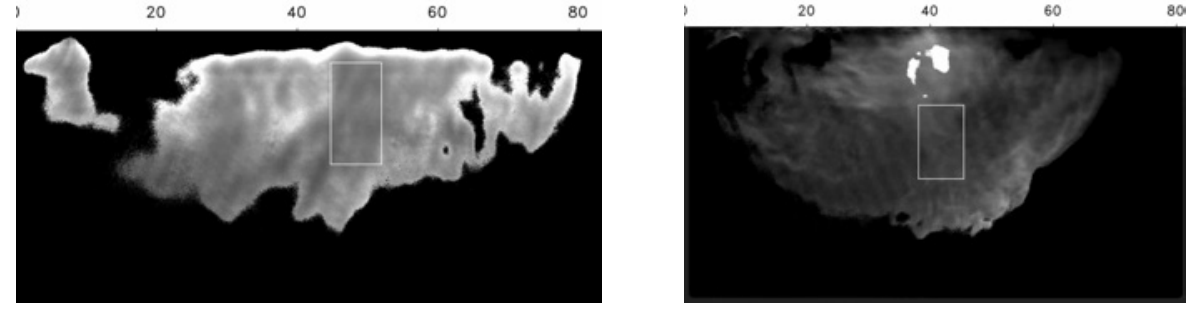

Figure 1. Prominence at the east limb, $55^{\circ} \mathrm{N}$, on July 14 (left) and 15,2003 , taken at the SST in the total emission of $\mathrm{H} \beta$ (upper), $\mathrm{He}_{3}$ (middle) together with the two-dimensional ratio maps (lower panels); the white rectangles indicate sub-areas selected for the scatterplots shown in Figs. 2 and 3.

\section{Results}

At the high spatial resolution achieved in the two-dimensional data (Fig. 1), we mostly find small spatial variations of the $\mathrm{He}_{3} / \mathrm{H} \beta$ emission ratio over the prominences.

For a more detailed discussion, we give scatterplots of the emission ratio versus the $\mathrm{He}_{3}$ and, respectively, the $\mathrm{H} \beta$ total intensities. These scatterplots show a clustering of data points indicating the spatial prominence structure in the respective sub-region.

This clustering is well seen in histograms obtained by integrating the scatterplots along their ordinate (panels on top of the scatterplots). Distinct maxima of the intensity histograms, marked by vertical lines, frequently occur in prominences (Stellmacher \& Wiehr, 2000) and are attributed to different numbers of threads along the line-of-sight.

Interestingly, such pronounced spatial brightness structure does not necessarily occur in the ratio of the emission lines which, instead, is often found to be largely constant, even in regions with substantial intensity variation. In these cases, the prominence structure is similar in both lines, thus resulting in a largely uniform emission ratio. 
Prominence: $\mathrm{E} / 55 \mathrm{~N} ; 2003$, July 14, 10:50:09UT
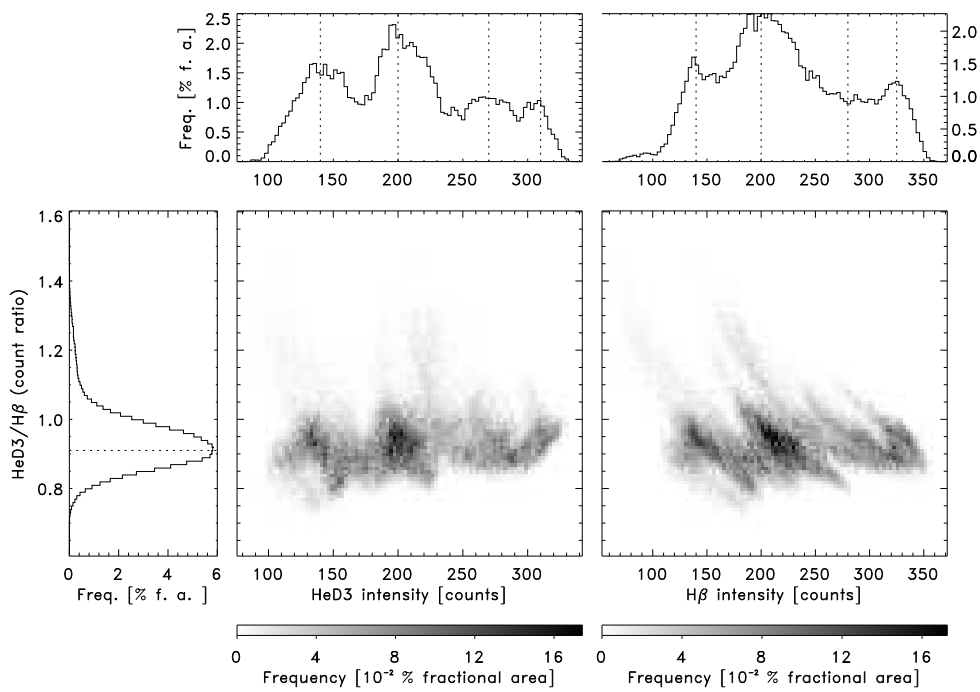

Figure 2. Scatterplot of the $\mathrm{He} \mathrm{D}_{3} / \mathrm{H} \beta$ ratio versus the total intensities of $\mathrm{He}_{3}$ (middle) and $\mathrm{H} \beta$ (right panels) for the marked sub-region in prominence $\mathrm{E} / 55 \mathrm{~N}$ from July 14, 2003; cumulations of data points are indicated by vertical lines in the corresponding number $\left[10^{-4}\right]$ histograms (at the top, obtained from integration along the Y-direction); the ratio histogram (at the left side, obtained from integration along the $\mathrm{X}$-direction) shows a rather small range, in spite of largely varying $\mathrm{He}_{3}$ and $\mathrm{H} \beta$ intensities, indicating prominence structures of almost equal intrinsic physical state .

Prominence: $\mathrm{E} / 55 \mathrm{~N} ; 2003$, July 15, 08:27:38UT
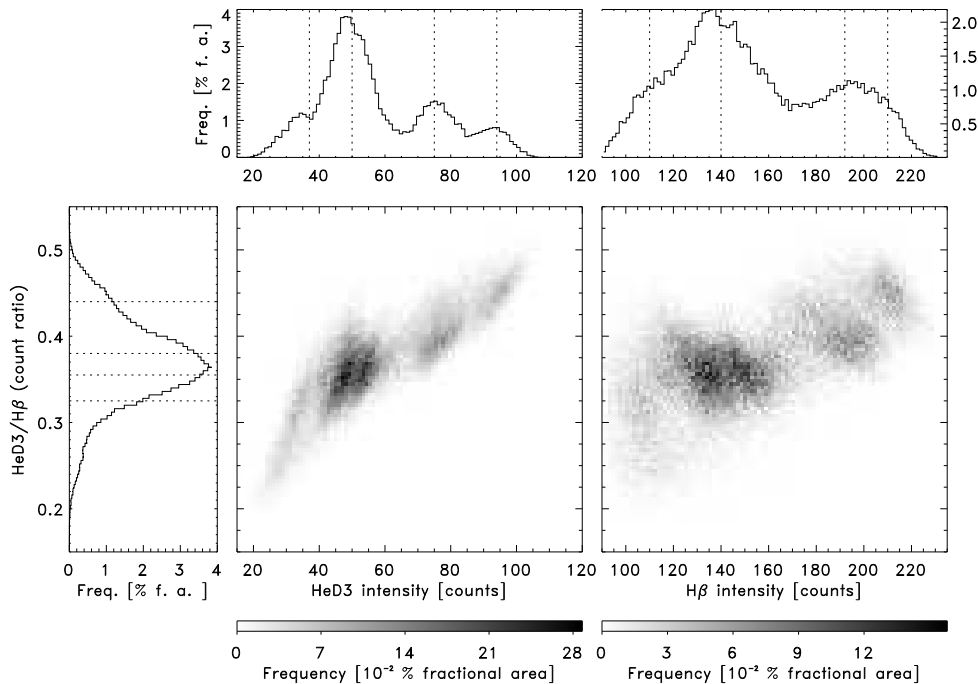

Figure 3. As Fig. 2 but for the same prominence on the next day; the ratio histogram (left) shows distinct values for each emission cumulation, indicating structures of different intrinsic physical state. 


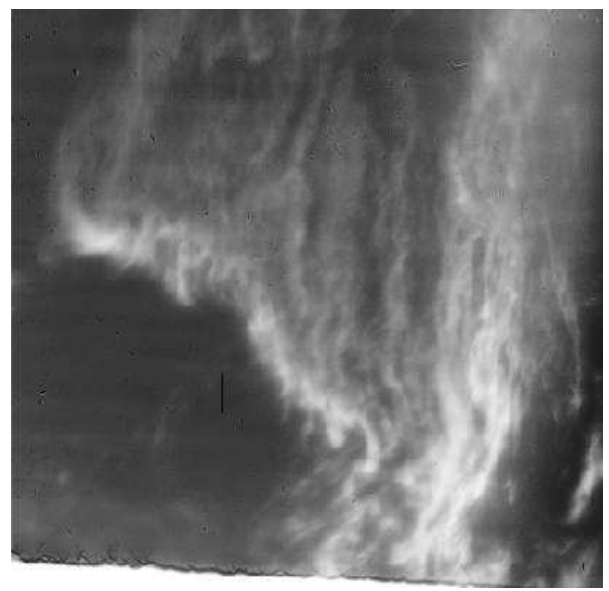

Figure 4. Frame-selected $\mathrm{H} \alpha$ image of a highly structured, faint prominence obtained at the VTT with a $2 \AA$ filter; pronounced structures only occur in faint, hot prominences (cf., Stellmacher \& Wiehr 1994) and exhibit substantial temporal variations (see Engvold 1997) .

\section{Conclusions}

The model calculations by Labrosse and Gouttebroze (2001) show that mostly the gaspressure affects the emission ratio, whereas temperature and slab-width have a much smaller influence. However, the pressure would have to vary unrealistically fast if responsible for the time variation also observed for the emission ratio. Hence, we alternately suggest an influence of the ionizing EUV radiation, required to populate the helium triplet system from the (singlet) ground state. The respective $(504 \AA)$ EUV photons will penetrate into the prominence depending on the 'packing density' of the prominence structures (cf., Fig. 4): denser structures will thus show less $\mathrm{He}_{3}$ emission. The structuring, in turn, is known to exhibit fast variations (cf., Engvold, 1997).

[The complete discussion of the observations will appear in 'Solar Physics'.]

Acknowledgements. The $1 \mathrm{~m}$ Swedish Solar Telescope is operated by the Swedish Academy of Science at the Spanish Observatorio del Roque de los Muchachos (IAC). This project has been supported by the European Commission through the Instituto de Astrofísica de Canarias to fund the European Northern Observatory, in the Canary Islands.

\section{References}

Engvold, O. 1997, 'Observations of Filament Structure and Dynamics (Review)', in Proceedings of 'New Perspectives on Solar Prominences', Aussois, eds. D. Webb, D. M. Rust, B. Schmieder, p. 23 Gouttebroze, P., Vial, J. C., \& Heinzel, P. 1997, Solar Phys. 172, 125

Labrosse, N. \& Gouttebroze, P. 2001, A\&A 380, 323

Stellmacher, G. \& Wiehr, E. 1994, A\&A 286, 302

Stellmacher, G. \& Wiehr, E. 2000, Solar Phys. 196, 375

Wiehr, E. \& Stellmacher, G. 1999, in: A. Wilson (ed.), ESA SP-448, 435 


\title{
Wavelet-based method for coronal loop oscillations analysis
}

\author{
D. Tothova*, D. E. Innes, and S. K. Solanki \\ Max-Planck-Institut für Sonnensystemforschung, Katlenburg-Lindau, Germany \\ *Email: tothova@mirage.mps.mpg.de
}

\begin{abstract}
We study the properties of Doppler shift oscillations observed in hot coronal loops by SoHO/SUMER. These oscillations have been identified as magnetoacoustic slow mode standing waves and may be an important magnetic field diagnostic for the oscillating loops. Visual inspection of the SUMER data suggests that one in three microflares trigger oscillation. So for an average active region we can expect several oscillation events per hour. To study the statistics of the oscillations, automatic identification and classification of the events is required. We have performed a Wavelet analysis on a 12 hour time series of SUMER Fe xIx data and find that almost all of the oscillation events in the data set are identified by this technique, with very few false positives. The method is expected to be useful for the analysis of future Ca XVII and Fe xxIV spectra of microflares observed with HINODE/EIS.
\end{abstract}

\section{Introduction}

SoHO/SUMER has recently revealed standing slow mode oscillations in hot (6-8 MK) coronal loops (Wang et al. 2002; Kliem et al. 2002). The search for coronal loop oscillations is motivated by the variety of information they can provide us with. Doppler shift oscillations give us information on the excitation site, the excitation mechanism, the energy dissipation and the coronal magnetic field. The magnetoacoustic waves are only seen in hot flare lines, such as Fe xIx, with a formation temperature above $6 \mathrm{MK}$, without any signature in cooler lines. Analysis and conclusions on coronal loop oscillations are limited because SUMER is only able to observe at the solar limb and is not able to raster across the loop structures. The HINODE mission will give us the possibility to make high cadence imaging and spectral observations of active region loops at any position on the Sun, and thus will provide us with the loop geometry and oscillations characteristics at different loop positions in a wave period. In order to understand the properties of these oscillations we will carry out a statististical study using the wavelet-based method. We would like to determine the percentage of active region microflares that trigger oscillations, find which modes are excited, their periods, damping times as well as the range of temperatures of the lines in which we observe them.

\section{Observations}

The spectroscopic observations of the active region AR 9176 with a hot loop system have been obtained by SUMER in the sit-and-stare-mode on September 16-17th, 2000, with an exposure time of 1.5 min (Fig. 1). Time series in five spectral lines, Fe XIX $\lambda 1118$ (6.3 MK), 


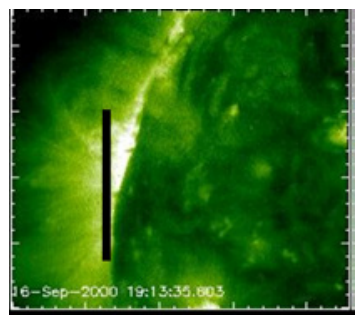

Figure 1. EIT $195 \AA$ image of the observed active region showing the position of the SUMER slit.

Ca XV $\lambda 1098$ and $\lambda 1110$ (3.5 MK), Ca XIII $\lambda 1134$ (2.2 MK), and Si III $\lambda 1113$ (0.06 K), were recorded and after processing the raw data line moments have been computed. The Doppler oscillations are seen in the hot Fe XIX line coinciding with the flare like brightenings typical for this line.

\section{Data Analysis}

We use the wavelet-based algorithm À trous ("with holes") (Fligge \& Solanki 1997; Starck et al. 1998) to help identify the oscillations in the velocity time series (Fig. 3). À trous reduces the noise by local thresholding on wavelet coefficients at each scale. First we determined the noise in shift, $\lambda_{s}$, at each position and time pixel by evaluating the error in the center of gravity of the line using the error propagation law $\sigma^{2}\left(\lambda_{s}\right)=\sigma^{2}\left(I_{\lambda}\right)\left(\partial \lambda_{s} / \partial I_{\lambda}\right)^{2}$, and assuming a Poisson distribution of the noise for the intensity $\sigma\left(I_{\lambda}\right)=\sqrt{I_{\lambda}}$. In order to find the noise wavelet coefficients at each scale, we applied the Â trous algorithm to the relative error time series, using the same parameters as for the data. After identifying those wavelet coefficients that are significantly non-zero against the noisy background, we have used the Morlet wavelet transform to find the power of the wavelet coefficients and to reconstruct the time series at each pixel, filtering out oscillations with periods under 6 , and above 15 minutes. To make this method automatic, all the criteria must be of general form giving acceptable results for any chosen time series.

\section{Conclusions}

We have developed a technique, based on the discrete wavelet algorithm À trous and a subsequent Morlet reconstruction, allowing automatic extraction of coronal loop Doppler shift oscillations. We have tested this method on a 12 hour time series and found 22 clear cases of oscillations (Fig. 3 ). Their occurrence coincides with about 33\% of the microflare events typical for the line Fe xIX (Fig. 2), they are usually not seen as oscillations in the intensity map. Our method has been shown to be reliable in the analysis of the subsequent three day long SUMER time series.

\section{References}

Fligge, M. \& Solanki, S. K. 1997, A\&AS, 124, 579 


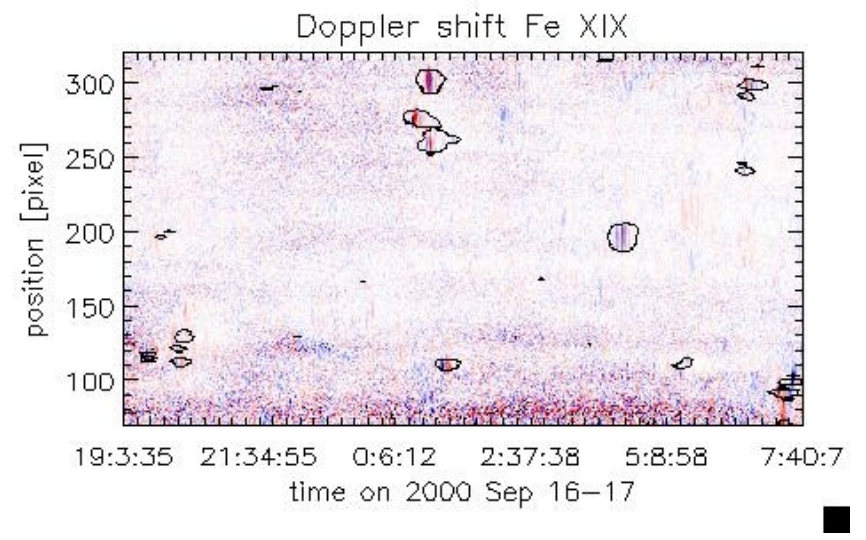

Figure 2. Intensity of the Fe xIx with the contours defining the position of the Doppler shift oscillations.

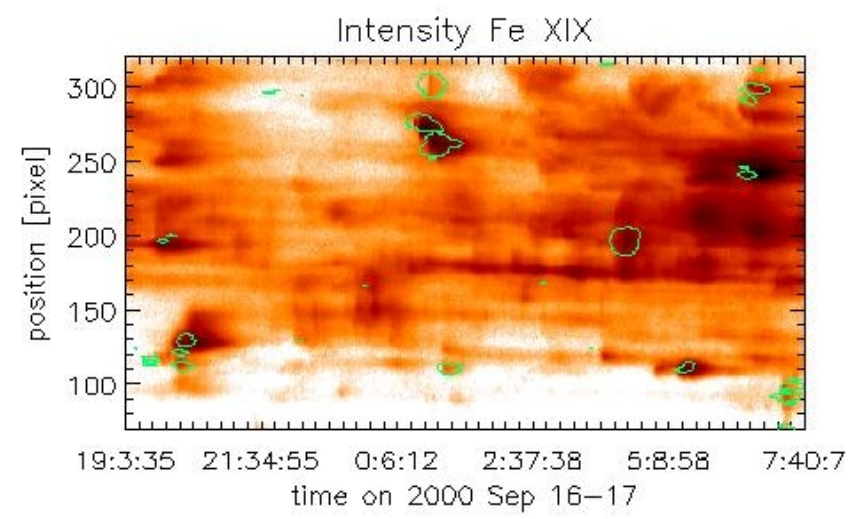

Figure 3. Corresponding Doppler shift map after denoising using À trous algorithm and after Morlet reconstruction.

Kliem, B., Dammasch, I. E., Curdt, W., \& Wilhelm, K. 2002, ApJ, 568, L61

Starck, J. L., Murtagh, F., \& Bijaou, A. 1998, Image Processing and Data Analysis: The Multiscale Approach (Cambridge University Press, New York, NY, USA)

Wang, T. J., Solanki, S. K, Curdt, W., \& Innes, D. E. 2002, ApJ, 574, L101 



\title{
Magnetic stereoscopy of coronal loops in NOAA 8891
}

\author{
L. Feng ${ }^{1,2, *}$, T. Wiegelmann ${ }^{1}$, and B. Inhester ${ }^{1}$ \\ ${ }^{1}$ Max-Planck-Institut für Sonnensystemforschung, Katlenburg-Lindau, Germany \\ ${ }^{2}$ Purple Mountain Observatory, Chinese Acedemy of Sciences, Nanjing, China \\ *Email: feng@mps.mpg.de
}

\begin{abstract}
We present an application of the magnetic stereoscopy tool to the coronal loops observed by TRACE in the active region NOAA 8891. Designed for the newly launched STEREO mission, this tool aims to reconstruct the 3D geometry of coronal loops. Taking advantage of the solar rotation, two TRACE images observed one day apart are looked as STEREO-A and STEREO-B EUVI images. Combining with the extrapolated magnetic field lines in the corona from photospheric MDI data, the $3 \mathrm{D}$ geometry is reconstructed with the identified loop pair.
\end{abstract}

\section{Introduction}

The STEREO mission for observing the Sun-Earth system simultaneously from two different viewpoints, provides us a good opportunity to reconstruct the three-dimensional geometry of coronal loops which are important to obtain more precise physical parameters along the loop, then to test loop models (Aschwanden et al. 1999). Accurate loop properties are needed to reproduce loop oscillations and to use them to measure coronal magnetic field strength. Due to the high electric conductivity in the corona, loops outline the magnetic field lines. Therefore, 3D geometry of coronal loops are of fundamental importance for studing the associated magnetic field.

Wiegelmann \& Inhester (2006) developed a magnetic stereoscopy tool which combines pure geometrical stereoscopy with the local magnetic field orientation from different magnetic field models. However, this tool has only been applied to a model active region from which they computed artificial loops as seen from two different viewpoints. In this work, we apply the magnetic stereoscopy tool to real TRACE and SOHO/MDI data. This represents a test of the method to solar data affected by noise, instrumental artifacts, etc.

\section{Observations and loop identification}

The investigated active region NOAA 8891 was a long-lived, relatively quiet one which is shown in the white box marked in Fig. 1. Figure 2 shows two EUV images of this active region recorded by TRACE in the $171 \AA$ wavelength band (Handy et al. 1999) about one day apart, one on March 1st, 1422UT, 2000 and the other on March 2nd, 1744UT. We can regard these two TRACE images as approximations of the EUVI image pair from the two 


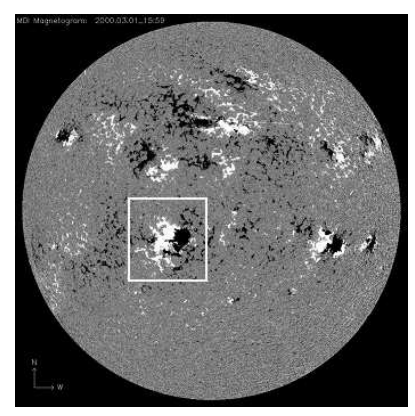

Figure 1. SOHO/MDI line of sight magnetogram observed on March 1st, 1535UT, 2000 with NOAA 8891 located in the white square.
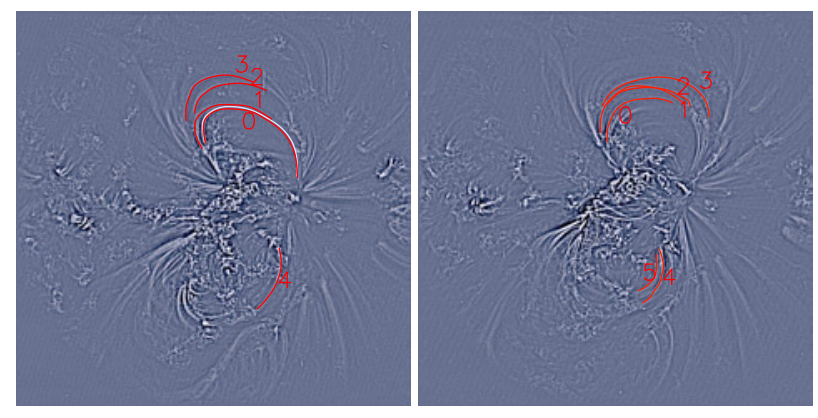

Figure 2. Hand-traced loops (dark/red lines, see internet version for colours) marked with numbers on the filtered TRACE $171 \AA$ images observed on March 1st, 2000, 1422UT (left) and March 2nd, 2000, 1744UT (right). In the left image, the white line is segmented out from another TRACE image observed on March 1st, 1351UT, and then transformed with proper coordinates onto the March 1st, 1422UT image.

STEREO spacecrafts. To enhance the faint loop structures in the two TRACE observations, high-pass filtered images are created by subtracting a smoothed image from the original image. Subsequently the loops are traced out in the filtered images and marked in Fig. 2. In fact the right part of Loop 0 in the left filtered image is invisible, thus a white line is shown as a reference of the direction of Loop 0.

\section{Magnetic field extrapolation and field line projection}

Usually, coronal magnetic fields cannot be measured directly. Therefore one has to extrapolate them from photosphere magnetic observations. Because of low $\beta$ values in the lower corona, the magnetic field can be considered force-free. We use the method of Seehafer (1978) to extrapolate the linear force-free field from SOHO/MDI line-of-sight magnetograms. We have computed the linear force free field model for 45 different values of $\alpha$ varied from $-0.0156 \mathrm{Mm}^{-1}$ to $0.0156 \mathrm{Mm}^{-1}$ (the value of $\alpha$ increases by $7.1 \times 10^{-4}$ at every 

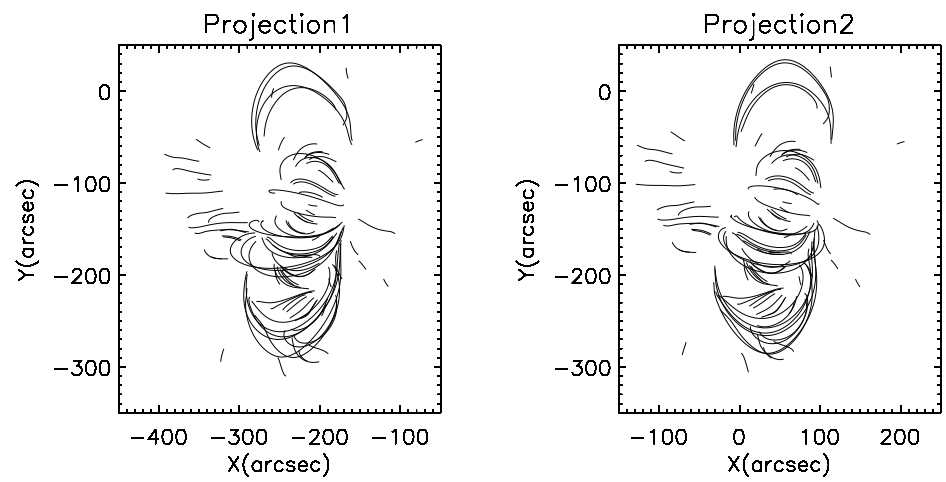

Figure 3. Projection results of the extrapolated 3D potential field lines along two TRACE view directions. The left image is for the viewpoint on March 1st, 1422UT, 2000 and the right one on March 2nd, 1744UT.

step). In total, 4183 field lines are stored in an initial coarse magnetic field group because of the randomly selected starting footpoints of the field lines. All the field lines are then projected onto both TRACE images to be compared with the identified loops shown in Fig. 2. As an example the projected potential field lines are given in Fig. 3.

\section{Magnetic stereoscopy}

A clear association of loops in two TRACE images is very important before the stereoscopic reconstruction. To quantify how good the correspondence is between a loop combination from both TRACE images, we introduce a quantity $C_{L_{A} L_{B}}(b)$ which shows the average distance of a certain field line $b$ to one loop $L_{A}$ in image A $C_{L_{A}}(b)$ and to one loop $L_{B}$ in image B $C_{L_{B}}(b)$. For each loop combination, an optimal $b$ is found from the total 4183 field lines which minimize $C_{L_{A} L_{B}}(b)$. The smaller this minimum $C_{L_{A} L_{B}}(b)$ is, the better the loop combinations associate with each other. In this way we find the initial results of possible loop pairs and their best fitting field lines to which further results are referred. Because the starting points of the calculated field lines are randomly selected and the interval of the $45 \alpha$ values is not small enough, we are aiming here to find some more precise candidates which fit the loop pairs even better. We calculate additional field lines with their footpoints focussed near the footpoint of the optimal field lines found so far and with smaller $\alpha$ interval. Then comparing all these 2585 new field lines with the loops traced from Image A and Image B, we obtain a more precise result. Possible loop pairs are listed in Table 1. A stereoscopic reconstruction for the most possible loop pair $(0,0)$ are shown in Fig. 4 with the aid of the best fitting field line $b(0,0)$. In the right panel, we also indicate the reconstruction error bars. 
Table 1. List of the possible loop pairs and $\alpha$ values of the field line best fitted with each loop pair.

\begin{tabular}{ccccc}
\hline Loop pair & Loop No.(Image A) & Loop No.(Image B) & $C_{L_{A} L_{B}}(b)(\operatorname{arcsec})$ & $\alpha \mathrm{L}$ \\
\hline 1 & 0 & 0 & 1.54 & -1.2 \\
2 & 1 & 1 & 3.08 & -1.5 \\
3 & 1 & 2 & 3.45 & -1.5 \\
4 & 2 & 3 & 4.63 & -1.2 \\
5 & 4 & 4 & 6.36 & 0.8 \\
\hline
\end{tabular}
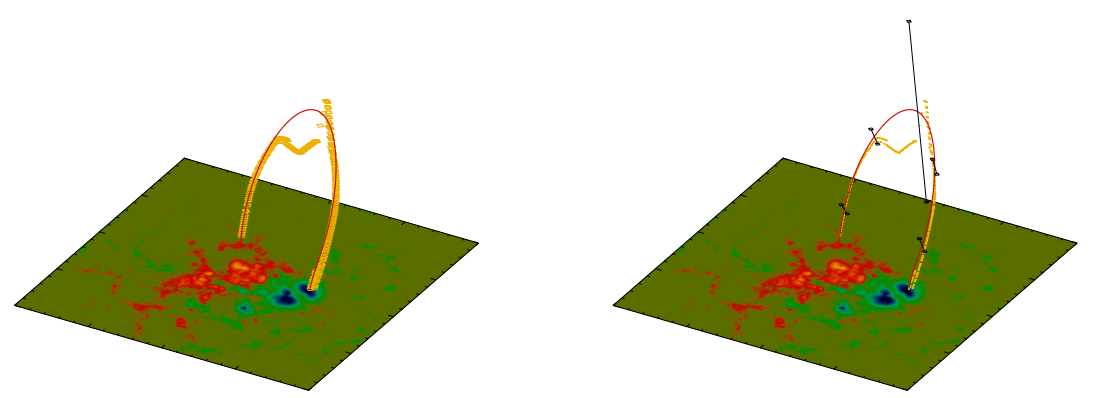

Figure 4. Magnetic stereoscopy of loop pair 0-0. Light/yellow points give the solution of the 3D reconstruction, the dark/red line is the best fitting 3D magnetic field line. The right panel shows the reconstruction error bars at five points in the $3 \mathrm{D}$ magnetic field line. (See the internet version for colours.)

\section{Conclusion}

We reconstruct the 3D loop structure for one loop pair identified from two TRACE images observed one day apart with the help of the extrapolated linear force-free magnetic field lines. This work will be applied to the STEREO data in the near future. We expect that better stereoscopic results can be obtained also with an automated loop segmentation tool and with a better magnetic field model.

Acknowledgements. The authors thank the SOHO/MDI, EIT and TRACE consortia for their data. SOHO is a joint project of ESA and NASA. The work of L. Feng was supported by the International Max-Planck Research School on Physical Processes in the Solar System and Beyond at the Universities of Braunschweig and Göttingen. The work of T. Wiegelmann was supported by DLR-grant 500C 0501.

\section{References}

Aschwanden, M. J., Newmark, J. S., Delaboudinière, J.-P., Neupert, W. M., Klimchuk, J. A., Gary, G. A., et al. 1999, ApJ, 515, 842

Handy, B. N., Acton, L. W., Kankelborg, C. C., et al. 1999, Solar Phys., 187, 229

Seehafer, N. 1978, Solar Phys., 58, 215

Wiegelmann, T. \& Inhester, B. 2006, Solar Phys., 236, 25 


\title{
Observations of a flaring active region in $\mathrm{H} \alpha$
}

\author{
B. Sánchez-Andrade Nuño ${ }^{1,2, *}$, K. G. Puschmann ${ }^{1}$, and F. Kneer ${ }^{1}$ \\ ${ }^{1}$ Institut für Astrophysik, Göttingen, Germany \\ ${ }^{2}$ Max-Plank-Institut für Sonnensystemforschung, Katlenburg-Lindau, Germany \\ *Email: bruno@astro.physik.uni-goettingen.de
}

\begin{abstract}
A 40 min time series of the active region NOAA AR10875 was obtained on April $26^{\text {th }}$, 2006 under very good seeing conditions using the new "Göttingen" Fabry-Perot interferometer at the German Vacuum Tower Telescope, Observatorio del Teide, Tenerife. The $\mathrm{H} \alpha$ line was scanned giving two-dimensional images at 21 different wavelength positions with a cadence of $\sim 20$ s per scan. After speckle reconstruction the spatial resolution achieved was better than 0.35 . The data provide a unique possibility to study the temporal evolution of a pre-flaring, flaring and post-flaring solar active region in $\mathrm{H} \alpha$. From the analysis of the present spectrometric data set we expect important advances in the understanding of Alfén waves, dynamics of fibrils and filaments and the physical nature of the sigmoid structure seen in the core of $\mathrm{H} \alpha$. Here we present first results from this data set.
\end{abstract}

\section{Introduction}

The solar chromosphere is highly dynamic. Since its discovery in the 19th century it has remained a lively field of research (e.g., Innes et al. 2005). In this part of the atmosphere the plasma $\beta$ changes from $\beta>1$ in the photosphere to $\beta<1$ at larger heights. Thus, magnetic fields, emerging from deep layers and moved around, start dominating the processes in higher layers. Especially when seen in $\mathrm{H} \alpha$, apart from being fascinating, the rich structuring and fast events as a result of magnetic field reorganisation and changes of energy are well observable. The interaction between the gas and the magnetic field is expected to be a rich source of Alfvén waves. Here, we present two-dimensional (2D) spectroscopic H $\alpha$ observations of a complex, active region exhibiting the full variety of structural dynamics in long fibrils and sigmoid structures. The high spatial and temporal resolution of our time sequence allows to scrutinize the wealth of processes occurring in the chromosphere.

\section{Observations and data analysis}

The solar AR10875 was observed at $\mu=0.59$ on $26^{\text {th }}$ April, 2006 with the upgraded Göttingen Fabry-Perot interferometer (Puschmann et al. 2006) at the Vacuum Tower Telescope (VTT) on Tenerife. The observations have been supported by the Kiepenheuer Adaptic Optics System, KAOS (von der Lühe et al. 2003). 126 spectral scans through the $\mathrm{H} \alpha$ line, taking narrowband images $(\mathrm{FWHM}=55 \mathrm{m \AA})$ at 21 individual wavelength positions. The stepwidth in wavelength was $100.7 \mathrm{~m} \AA$, the exposure time $5 \mathrm{~ms}$, and the field of view (FOV) $77^{\prime \prime} \times 58^{\prime \prime}$, with an image scale of $0.112 /$ pixel. 

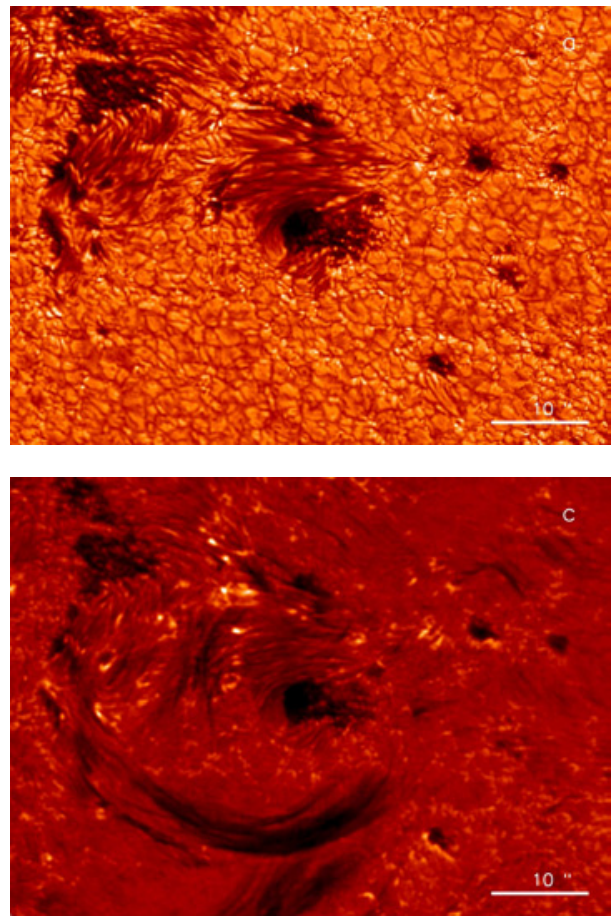

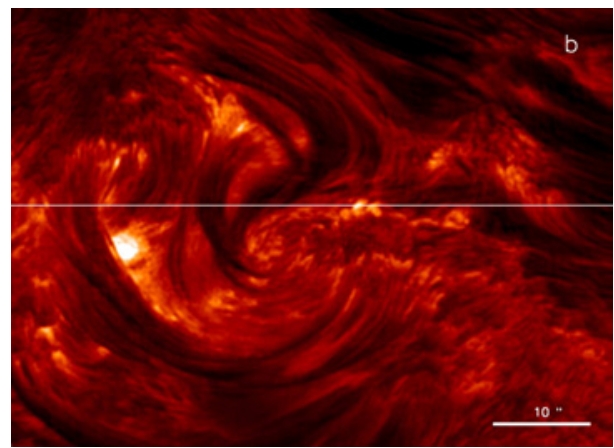

Figure 1. Active region NOAA AR10875 at $\mu=0.59$ observed with the upgraded Göttingen Fabry-Perot interferometer. a) Speckle reconstructed broadband image at $6300 \AA$. The spatial resolution is better than 0.3 , the FOV is $\sim 63^{\prime \prime} \times 47^{\prime \prime}$. b) Reconstructed narrowband image in $\mathrm{H} \alpha$ line centre (spatial resolution better than 0 ' 35 , see Fig. 3 for the white line). c) Reconstructed narrowband image at $\mathrm{H} \alpha-1 \AA$ off line centre.

Broadband data have been taken strictly simultaneously with the narrowband data by imaging through a broadband filter $(\mathrm{FWHM}=50 \AA$ ) centred around $6300 \AA$. This allows post-facto image reconstruction to achieve high spatial resolution on each spectral position. The temporal cadence between each scan was $\sim 19 \mathrm{~s}$, spanning in total $40 \mathrm{~min}$. The seeing condition during the observations was very stable with a Fried parameter up to $r_{0} \approx 30 \mathrm{~cm}$.

To obtain highest possible spatial resolution in the broadband images, we used the modified version of the Göttingen speckle reconstruction code (de Boer 1996; Puschmann \& Sailer 2006) that takes into account the field dependent correction of the adaptive optics. The narrowband data have been reconstructed following the method of Keller \& von der Lühe (1992), using the information of the broadband image (see also Bello González et al. 2005). To remove remaining jitter within the time sequence, all images in broadband and at every $\mathrm{H} \alpha$ line position were co-aligned with a correlation method.

\section{Results}

In Fig. 1 an example of a speckle reconstructed broadband image of the observed active region is presented. It harbors the lower part of a large sunspot with many bright umbral dots, several small spots with distorted penumbrae, and pores. Many intergranular bright points are visible.

A large variety of different chromospheric features, like fibrils, dark filaments and a sigmoid like structure, emphasizing their dynamics, are seen in the line core image shown in Fig. 1b. Finally, in Fig. 1c the reconstructed narrowband image near the blue continuum is 

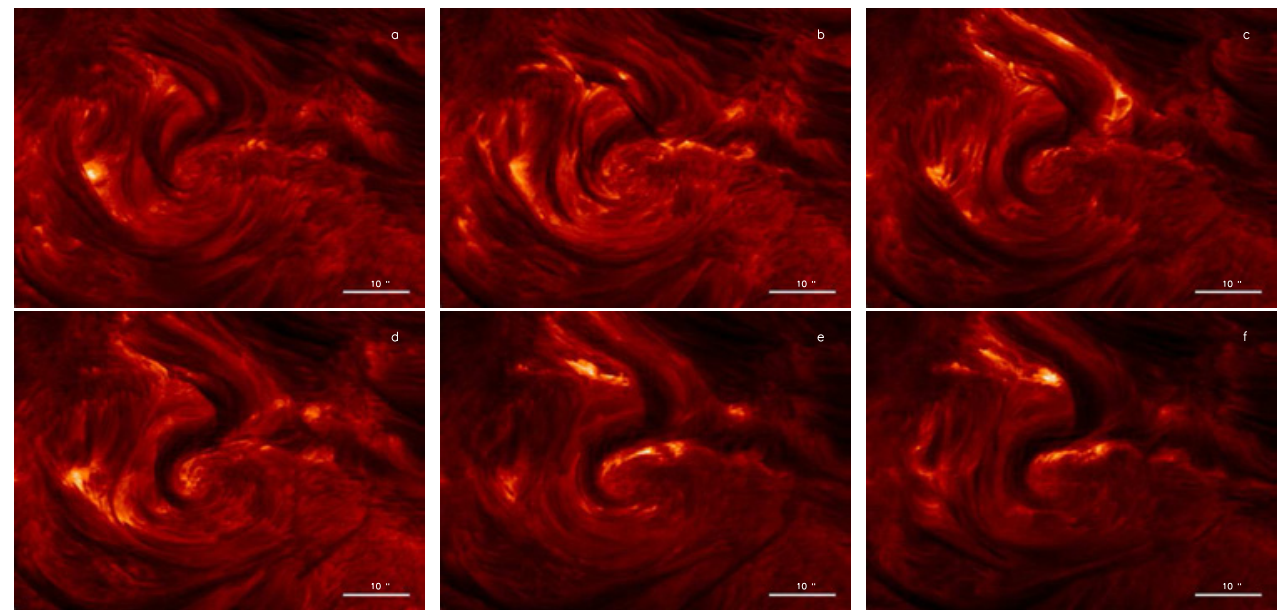

Figure 2. a)-f) Temporal evolution of the active region NOAA AR 10875 at the line centre of $\mathrm{H} \alpha$ starting at 08:53:45 UT with a cadence of $6.6 \mathrm{~min}$.

presented, where we observe network bright points and dark filament whose strong blueshifts indicate fast upward flows.

Figure 2 gives an impression of the high dynamics of chromospheric features present in the FOV. Different line centre images are shown at 6 equidistant times $(\Delta t=6.6 \mathrm{~min})$ covering the entire time series of $40 \mathrm{~min}$. The occurrence of several flare events is observed. In Fig. $3 \mathrm{a}$ we follow such en event in time, presenting the temporal evolution of the line centre intensity in the stripe indicated by the white horizontal line in Fig. 1b. 10 consecutive spectral profiles $(\Delta t=19 \mathrm{~s})$ corresponding to the part with the temporally most apparent brightening reveal the change from a normal weak umbral absorption profile to an emission profile during such a flare event. We measure an increase of the line core intensity by up to $400 \%$ compared to the starting profile in less than $40 \mathrm{sec}$ and a slow decrease of the line core intensity afterwards. At the same time of this sudden increase, the intensity also increases at some $15^{\prime \prime}$ distance (to the right in the upper panel of Fig. 3).

\section{Conclusion and outlook}

At this first inspection, the excellent quality throughout the entire $40 \mathrm{~min}$ time series is revealed. This gives an opportunity to study the different chromospheric features and their dynamics present in the FOV at smallest scales and to analyze their relation to the structures rooted more deeply in the photosphere. The motions of fibrils, filaments and the temporal changes of the sigmoid like structure as well as several observed solar flare events will be addressed in further studies. Velocities will be determined from the Doppler effect, and with the help of models, physical properties will be inferred. The search for Alfvén waves and an in-depth analysis of "fast moving dark clouds" as found in previous works (SánchezAndrade Nuño et al. 2005) will be a further interesting topic of future research. 


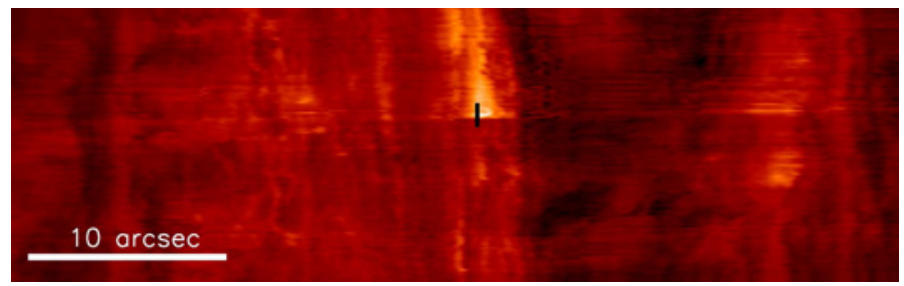

Figure 3. Upper panel: Temporal evolution (ordinate) of $\mathrm{H} \alpha$ line centre intensity at the positions marked by the white horizontal line in Fig.1b. The area with the temporally most apparent brightening is marked in black. It is located at the outer umbra of the sunspot visible in the centre part of the broadband image. Right panel: $\mathrm{H} \alpha$ profiles at 10 consecutive times $(\Delta t=19 \mathrm{~s})$ related to the section marked in black in the upper panel, revealing the emission during the solar flare. The profile changes in less than $40 \mathrm{~s}$ from a weak umbral absorption profile to a strong emission profile (increase by $\sim 400 \%$ ) and is gradually fading later on.

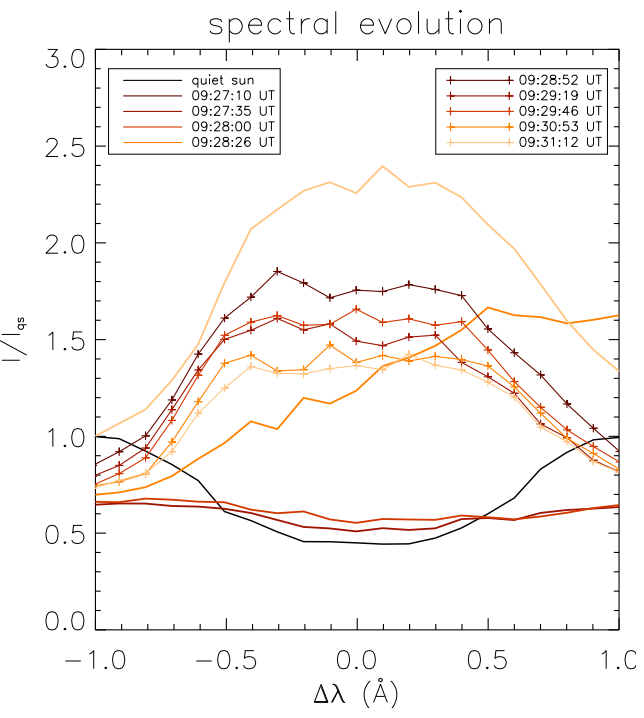

\section{References}

Al, N., Bendlin, C., Hirzberger, J., Kneer, F., \& Trujillo Bueno, J. 2004, A\&A, 418, 1131

Bello González, N., Domínguez Cerdeña, I., Okunev, O., Kneer, F., \& Puschmann, K. G. 2005, A\&A, 434, 317

de Boer, C. R. 1996, A\&AS, 120, 195

Innes, D. E., Lagg, A., \& Solanki, S. K. (eds.) 2005, Proceedings of the International Scientific Conference on Chromosphereic and Coronal Magnetic Fields, ESA-SP 596

Keller, C. U. \& von der Lühe, O. 1992, A\&A, 261, 321

Labeyrie, A. 1970, A\&A, 6, 85

Puschmann, K. G. \& Sailer, M. 2006, A\&A, 454,1011

Puschmann, K. G., Kneer, F., Seelemann, T., \& Wittmann, A. D. 2006, A\&A, 451, 1151P

Sánchez-Andrade Nuño, B., Puschmann, K. G., Sánchez Cuberes, M., \& Blanco Rodríguez, J., Kneer, F. 2005, Proceedings of the 11th European Solar Physics Meeting "The Dynamic Sun: Challenges for Theory and Observations", ESA SP-600, 70S

von der Lühe, O. 1984, J. Opt. Soc. Am., A1, 510

von der Lühe, O., Soltau, D., Berkefeld, T., \& Schelenz, T. 2003, Proc. SPIE, 4853, p. 187 


\title{
On the thermal and non-thermal excitation effects as studied in the $\mathrm{H} \alpha, \mathrm{H} \beta$ and Ca II $8542 \AA$ line profiles in a solar flare
}

\author{
P. Kotrč ${ }^{1, *}$ and L. K. Kashapova ${ }^{2}$ \\ ${ }^{1}$ Astronomical Institute AS CR, Ondřejov, Czech Republic \\ ${ }^{2}$ Institute of Solar-Terrestrial Physics, Irkutsk, Russia \\ *Email: pkotrc@asu.cas.cz
}

\begin{abstract}
Spectral observations of the 26 June 1999 flare (Kotrč et al. 2004) presented a unique opportunity to compare effects of thermal and non-thermal mechanisms of excitation on profiles of Balmer series and Ca II $8542 \AA$ lines. The radio spectra and HXR flux demonstrated indirect evidence of the non-thermal beam effect on the chromosphere during the flare (Kashapova et al. 2005). We compared the line profiles in the flare kernel associated with the influence of non-thermal electron beams with the "only thermal" kernels. Results are discussed both from their observational and theoretical aspects.
\end{abstract}

\section{Introduction}

Nowadays it is accepted that the solar flare energy is initially released in the corona and then transported by various means from the primary release site to chromosphere. The high-energy particle beams are the fastest and effective transportation agents. This type of emission excitation is called non-thermal. However, diagnostics of the lower atmosphere response to the particle beams still remains an unsolved problem of solar flare physics.

Many of the investigators (Fang et al. 2004; Heinzel 2003) considered the hydrogen and Ca lines as dominating diagnostic tools to study the energy mechanisms in the solar atmosphere. However, the obtained results are not based on any well-defined method. Thus, simultaneous observations in $\mathrm{H} \alpha$ and in Ca II $8542 \AA$ revealed the fact of opposite asymmetries at the same moments of the flare (Mein et al. 1997). The theoretical simulations (Ding \& Fang 1996) showed that under downwards motion the $\mathrm{H} \alpha$ line profiles will be sensitive to the location of the moving region (from top to deeper layers) while the Ca II $\mathrm{K}$ line will not. The deeper the moving region the more significant was the effect.

The solar flare on 26 June 1999 consisted of three phases. The fact that we found an indirect evidence of accelerated non-thermal particle beams for only the second phase of the flare, allowed us to distinguish the emission kernels according to mechanisms of excitation and compare the characteristics of profiles of the Balmer and Ca II $8542 \AA$ lines observed by the Ondřejov Multichannel Flare Spectrograph, hereafter MFS. 

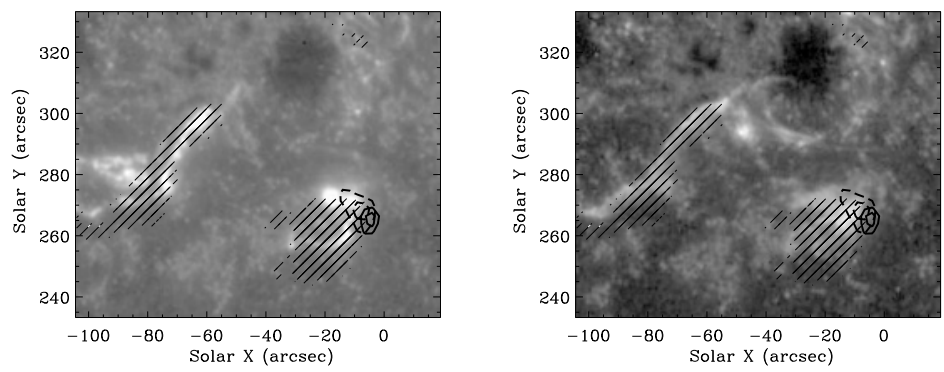

Figure 1. Position of HXR source obtained by Yohkoh Hard X-ray Telescope (from 07:17:12.214 to $07: 17: 39.714 \mathrm{UT}$ ) and the $\mathrm{H} \alpha$ slit-jaw image of the flare taken at 07:23:18 UT (shaded area) as projected on images in the 1600 $\AA$ band at 07:17:15 UT and 07:28:45 UT (from left to right). The $\mathrm{L}$ band $(13.9-22.7 \mathrm{keV})$ and the M1 band $(22.7-32.7 \mathrm{keV})$ are marked by dashed and full lines, respectively.
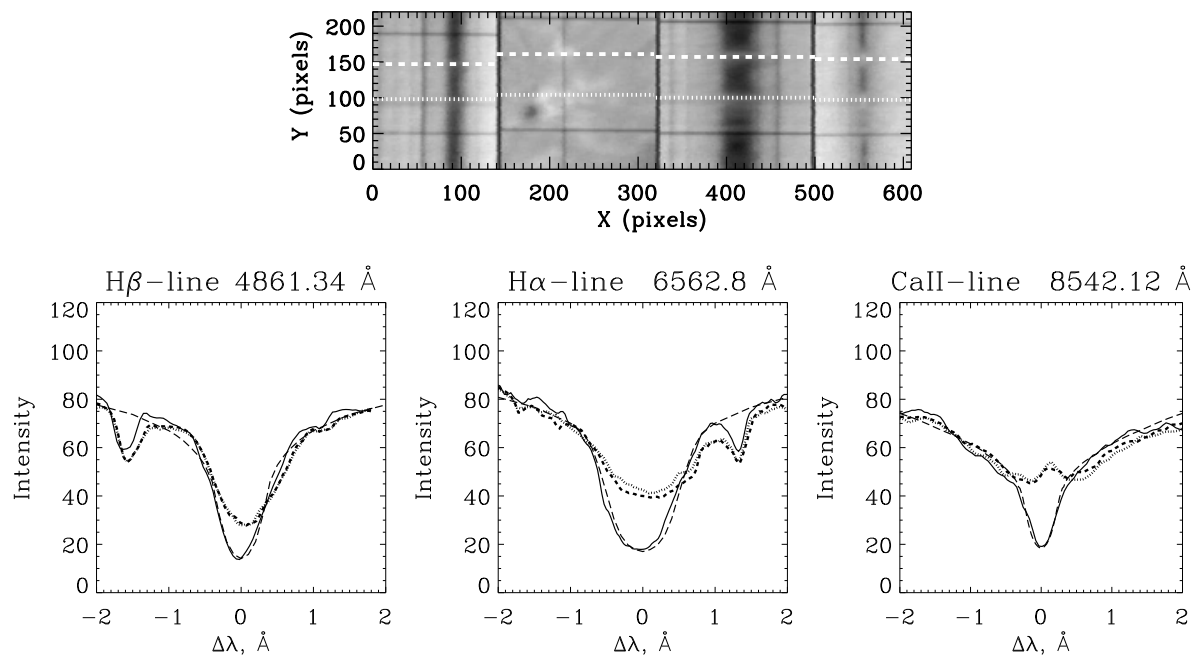

Figure 2. Top: Composed $\mathrm{H} \beta$ line spectrogram, $\mathrm{H} \alpha$ slit-jaw filtergram, $\mathrm{H} \alpha$ line and Ca II $8542 \AA$ line spectrograms (from left to right) taken by MFS at 07:08:53 UT (the first phase of the flare - thermal). Bottom: $\mathrm{H} \alpha, \mathrm{H} \beta$ and $\mathrm{Ca}$ II $8542 \AA$ intensity profiles. The type of line corresponds to the scan position as marked on the top panel. The solid and dashed lines are the quiet region and tabulated profiles, correspondingly.

\section{Effect of non-thermal electrons: discussion and preliminary conclusions}

As can be seen in Figure 1 at the $1600 \AA$ image the emission kernels associated with the HXR source were compact and bright at 07:17:15 UT (the moment of HXR flux rising). But at 07:28:45 UT the intensity of the kernel decreased and the structure became diffuse. We can see only one bright point in this kernel. Possibly, this point corresponds to the real location 

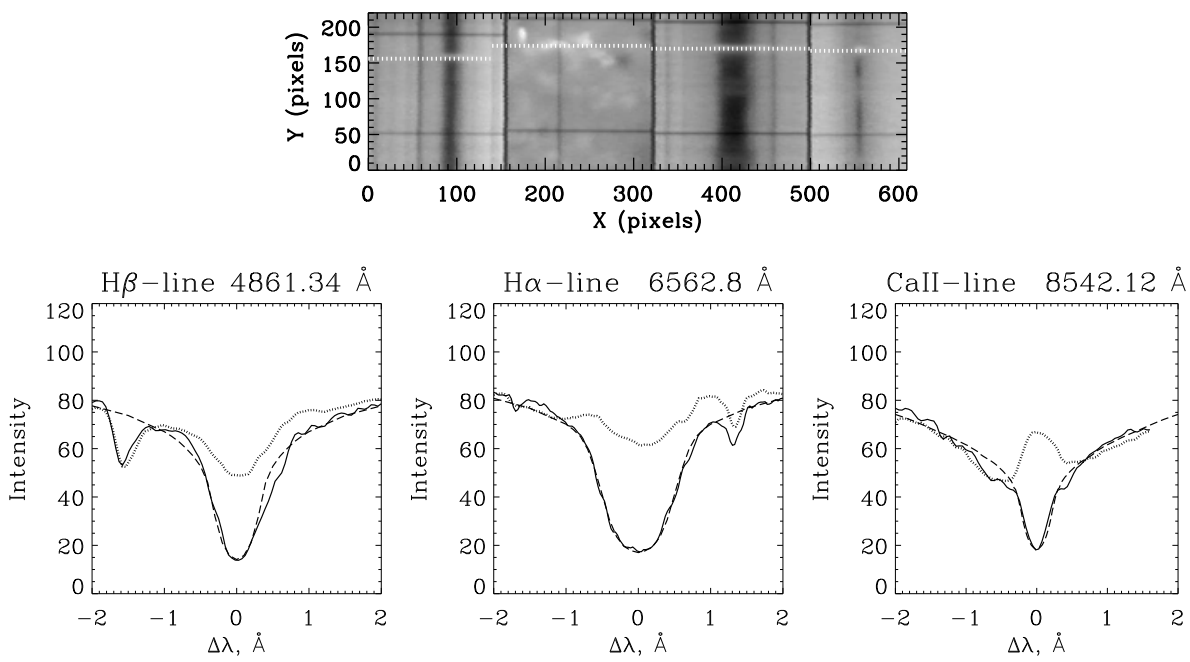

Figure 3. The same as in Figure 2 at 07:40:37 UT (the third phase of the flare - thermal again).
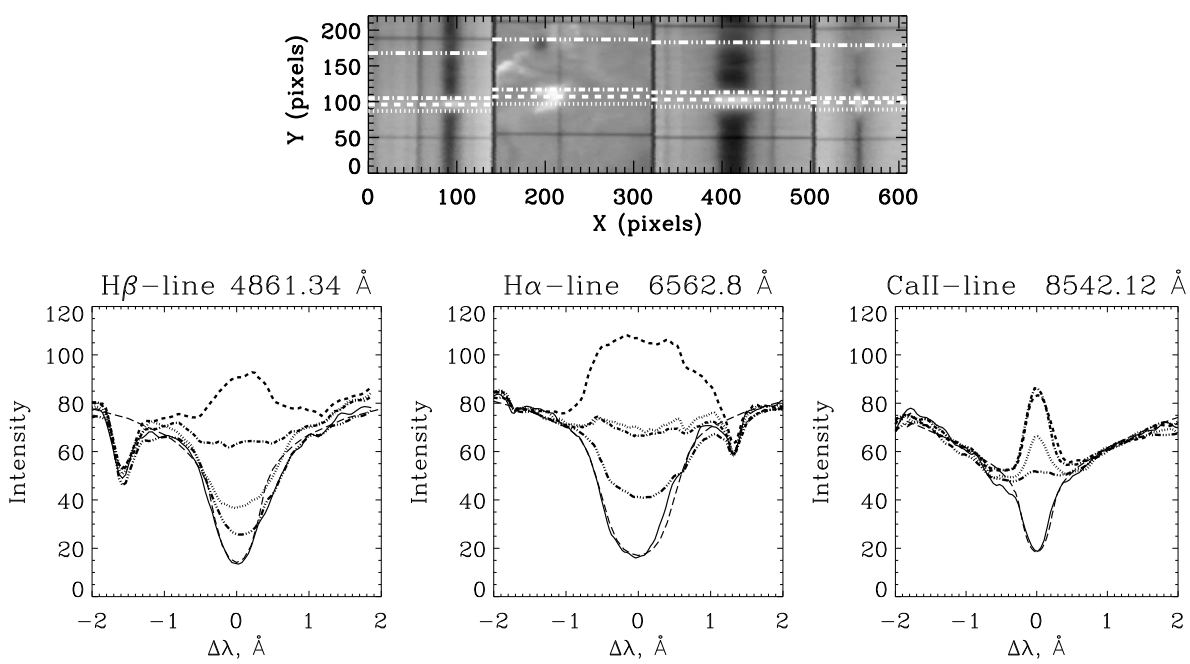

Figure 4. The same as in Figure 2 at 07:23:42 UT (the second flare phase - non thermal).

of the HXR source of electron beam with energy larger than $22 \mathrm{keV}$. The emission visible in all the studied lines is stronger in the non-thermal kernels as compared to the thermal ones. In the flare kernel associated with the thermal processes (Figures 2 and 3, we can see a red asymmetry in the Balmer series lines as well as in the Ca II $8542 \AA$ line. Possibly, it can be ascribed to the gentle evaporation of the flare plasma or to the conductive heat flux.

Apart from this fact we can observe that in the non-thermal kernels the red asymmetry can be seen only in the Balmer lines while in the Ca II $8542 \AA$ no line profile asymmetry is visible. The apparently different form of asymmetries in $\mathrm{H} \alpha$ and $\mathrm{Ca}$ II $8542 \AA$ at this flare 

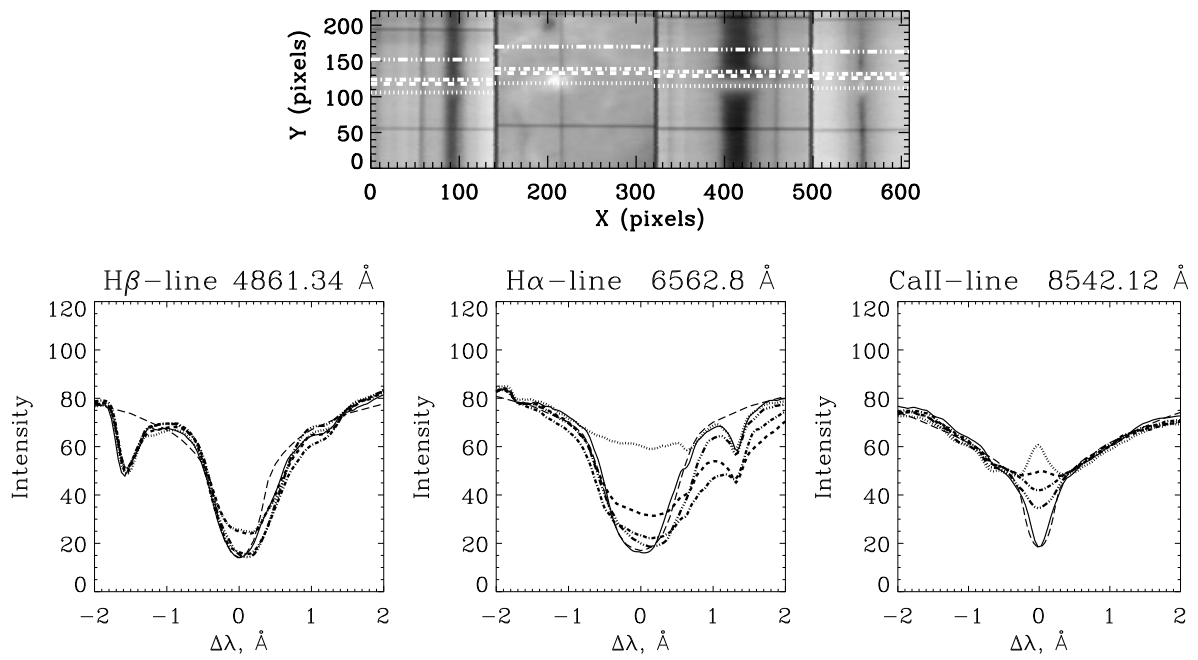

Figure 5. The same as in Figure 2, but obtained at 07:59:35 UT for the flare kernel arisen during the second flare phase.

kernel might be explained by a lower location of energy release and by a hydrodynamic process beginning in the chromosphere.

Based on the above result we can conclude that in the non-thermal kernels the red asymmetry can be seen only in the Balmer lines while the Ca II $8542 \AA$ line profiles are still symmetric. Quite the contrary to this behavior, the emission observed in the flare kernel associated with the thermal excitation processes demonstrated a red asymmetry both in the Balmer lines and in the Ca II $8542 \AA$ line. Such properties revealed from simultaneous observation are attractive tools for investigations of non-thermal processes that imply the energy transport to deep layers of solar atmosphere.

Acknowledgements. We are grateful to the Yohkoh/HXT, SOHO/MDI and TRACE teams who have provided free access to their results. PK appreciates support of the University Göttingen, the grant A3003203 of the Academy of Sciences of the Czech Republic, the ESMN project and the project AV0Z10030501 of the Astronomical Institute of the Academy of Sciences of the Czech Republic and the project No. 95 of the Czech - Slovak cooperation.

\section{References}

Ding, M. D. \& Fang, C. 1996, Solar Phys., 166, 437

Fang, C., Hénoux, J. C., \& Gan, W. Q. 1993, A\&A, 274, 917

Heinzel, P. 2003, Adv. Space Res., 32, 2393

Kashapova, L. K., Kotrč, P., Kupryakov, Yu. A., \& Kašparová J. 2005, in Proceedings of the 11th European Solar Physics Meeting, eds. D. Danesy, S. Poedts, A. De Groof, \& J. Andries, ESA, published on CDROM, 126.1

Kotrč, P., Kashapova, L. K., \& Kupryakov, J. A. 2004, in Multi-wavelength investigation of solar activity, ed. A. V. Stepanov, E. E. Benevolenskaya, \& A. G. Kosovichev, IAU Symposium, 223, 463 Mein, P. et al., 1997, Solar Phys., 172, 161 


\title{
Solar HXR- and $\gamma$-ray emission measurements in 2005 by SONG/CORONAS-F near minimum of the last activity cycle
}

\author{
I. N. Myagkova*, S. N. Kuznetsov, E. A. Muravieva, and L. I. Starostin \\ Skobeltsyn Institute of Nuclear Physics, Lomonosov Moscow State University, Moscow, \\ Russia \\ *Email: irina@srd.sinp.msu.ru
}

\begin{abstract}
The Russian solar observatory CORONAS-F (Complex ORbital Observations in Near-Earth space of the Activity of the Sun) was launched on July 31, 2001 into an orbit with inclination $82.5^{\circ}$ and altitude $500 \mathrm{~km}$. CORONAS-F operated until December 12, 2005, when its altitude had decreased to $350 \mathrm{~km}$. One of the prime objectives of the SONG (SOlar Neutrons and Gamma-rays) experiment aboard CORONAS-F was to measure neutron fluxes and hard X ray (HXR) and $\gamma$ emission produced by solar flares, providing important informations about particle acceleration processes in the Sun. Very interesting, SONG measurements ( 22 flares with the X-ray energy $>80 \mathrm{keV}$ ) in this respect were taken in 2005, close to the solar activity minimum, when a significant number of flares occurred on the Sun, which could be measured well isolated from other events. These measurements were compared with data obtained from the RHESSI mission during the same time.
\end{abstract}

\section{Introduction}

The temporal evolution of soft X-ray (SXR), hard X-ray (HXR) and $\gamma$-ray emission produced on the Sun and their energy spectrum in a wide energy interval provide us with the most direct information about particle injection and acceleration processes in solar flares. It is well known that the solar flare X-ray and $\gamma$-ray emission is the result of charged particle interaction with the solar atmosphere - the superposition of electron bremsstrahlung continuum and $\gamma$-ray line emission (e.g., Ramaty et al. 1988; Ramaty \& Mandzhavidze 1994). The observed HXR- and $\gamma$-ray spectrum showed that the charged energetic particles were accelerated up to rather high energies $(\mathrm{E}>300 \mathrm{MeV})$ during the flare. Of course, recent experimental and theoretical studies show that interplanetary shocks driven by coronal mass ejections (CMEs) play a major role in accelerating SEPs (e.g., Cane et al. 1988; Berezhko et al. 2001), but solar flare HXR and $\gamma$-ray hardness data are very important in estimating the possible damage that may be caused by a given flare to technical systems.

\section{Experiment}

One of the main goals of the Russian solar observatory CORONAS-F (Complex ORbital Observations in the near-Earth space of the Activity of the Sun) was to measure solar X-ray 
and $\gamma$-ray emission and to study its connection with solar energetic particle events and CMEs (see Kusnetzov et al. 2002).
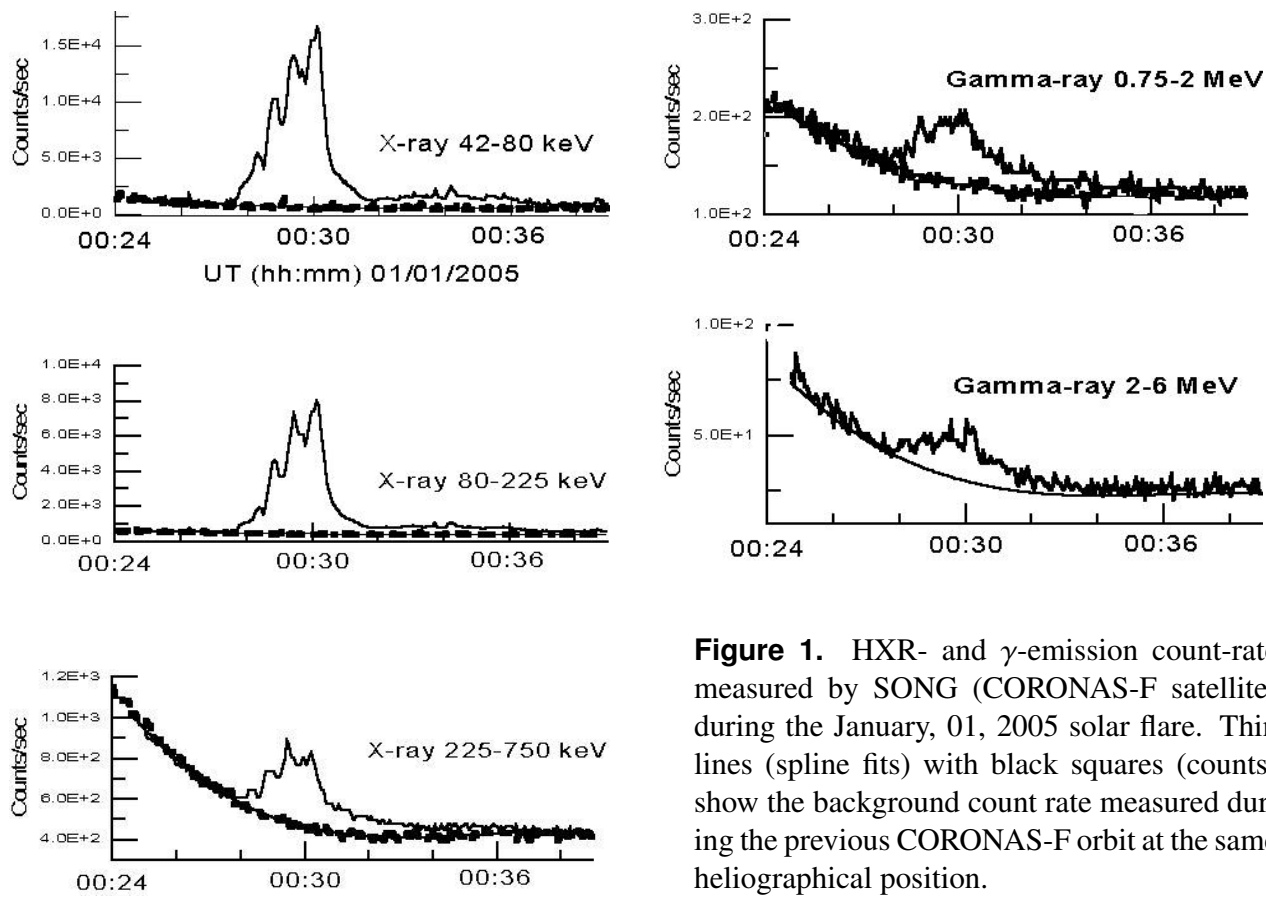

Figure 1. HXR- and $\gamma$-emission count-rate measured by SONG (CORONAS-F satellite) during the January, 01, 2005 solar flare. Thin lines (spline fits) with black squares (counts) show the background count rate measured during the previous CORONAS-F orbit at the same heliographical position.

The SONG (SOlar Neutrons and $\gamma$ rays) instrument, consisting of a large CsI(Tl) crystal, detected X-ray and $\gamma$-ray emission over a wide energy range: $0.03-200 \mathrm{MeV}$. Neutrons are detected due to their interactions with Cs and I nuclei. For the identification of neutrons on the background of $\gamma$-ray flux the dependence of the pulse shape in CsI(Tl) on the relative ionization of the particle is used (Kusnetzov et al. 2004). In this paper we present results related to this goal obtained near the minimum of the last solar activity cycle. Many powerful solar flares took place during the year 2005 when the sunspot number was relatively low (near 4560). Information about flare high energy electromagnetic emission during such conditions in the solar atmosphere is very important for better understanding solar flare physics.

\section{Observations and data analysis}

It is well-known that CMEs are among the main drivers of space weather (e.g., Gopalswamy 2006). Though a large part of CMEs are associated with flares, their connection is not yet clear. It is also known that the Sun is the most energetic particle accelerator in the solar system, producing ions of up to tens of $\mathrm{GeV}$ and electrons of up to tens of $\mathrm{MeV}$. So, the data obtained by the SONG-experiment about HXR and $\gamma$ emission, which permit us to estimate the flux and spectra of charged particles accelerated in the solar atmosphere, are useful.

The duty cycle for the detection of solar flares on board CORONAS-F was about $40 \%$ - a result of its orbit parameters. So some major flares during 2005, January - September were lost or measured only partly. However, 22 flares were detected, with HXR emis- 
sion with energy $>80 \mathrm{keV}$, eight of them with $\gamma$ emission with energy $>750 \mathrm{keV}$. Not all of them were measured in detail by the RHESSI experiment, see the RHESSI flare list http://hesperia.gsfc.nasa.gov/hessidata/dbase/hessi_flare_list.txt,

e.g., the powerful flare of January 1, 2005 (SXR-class X1.7). Its temporal evolution in various energy bands is presented as an example in Fig. 1. Comparing and using SONG and RHESSI solar flare catalogues together may be useful for detailed investigations of high energy neutral emission of solar flares.

$\gamma$-ray observations of the solar flare observed by the SONG and RHESSI experiments during 20 January 2005 provided two different signatures of the interaction of high energy ions in the solar atmosphere: $\gamma$-line emission and $\gamma$ emission due to $\pi^{0}$ decay, like for the October 28, 2003 and November 4, 2003 flares (e.g., Veselovsky et al. 2003).

Characteristics of the SONG flare (detection time, highest $\gamma$-ray energy channel) as well as SXR GOES characteristics are presented in Table 1 (here Fig. 3). We can see that $\gamma$ emission was observed not only in major flares of GOES X-class in SXRs. About $30 \%$ of the flares with HXR and $\gamma$ emission were only GOES M-class (less than M5) in SXR. Such hard spectra of energetic neutral emission show that the charged energetic particles were accelerated in these flares up to rather high energies. With respect to the HXR emission detected during some of C-class flares, especially the C9.3 flare on May 06, 2005 was impressive. The time evolution of HXR emission obtained by SONG for this flare is presented in Fig. 2. This flare and its possible role in space weather effects were discussed in Myagkova et al. (2006). We note that significant flux of HXR-emission is rather unusual for C-class flares and during more than four years of CORONAS-F operation the significant flux of HXR-emission was detected only in two C-class flares.

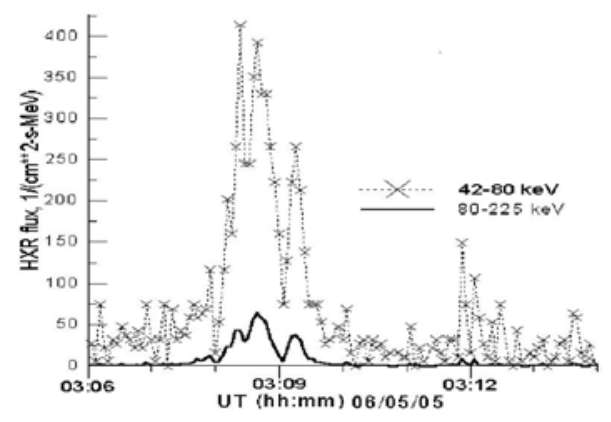

Figure 2. HXR emission measured by SONG experiment aboard CORONAS-F satellite during the solar flare of May 6, 2005 (background subtracted).

\section{Summary and conclusions}

In this paper we have presented observational results regarding HXR and $\gamma$-emission observations obtained by the CORONAS-F spacecraft (SONG instrument) during the year 2005.

Measurements of solar flare HXR- and $\gamma$-ray emission permits one to estimate the hardness of the flare emission in order to better predict the possible damage of technical systems that can be caused by flares. In four of the events $\gamma$ emission with energy up to $100 \mathrm{MeV}$ and more was detected. We have observed several major solar flares during January and September 2005 in hard X ray and $\gamma$ ray onboard the CORONAS-F satellite (SONG experiment) and presented most of them. We observed two distinguished phases in $\gamma$-ray emission in the 
powerful flare 2005, January, 20 SONG data, demonstating that solar HXR- and $\gamma$-emission monitoring is important during all phases of the solar cycle.

\begin{tabular}{|c|c|c|c|c|c|c|c|c|c|}
\hline${ }^{\mathrm{N}} \mathrm{d}^{\mathrm{d}}$ & $\begin{array}{c}\mathrm{dd} / \mathrm{m} \\
\mathrm{m}\end{array}$ & \begin{tabular}{|c|} 
SHR-flare UT \\
(COES) start-mas- \\
finish, hhtmm
\end{tabular} & \begin{tabular}{|c|} 
SONG UT \\
$(42-80 \mathrm{keV})$
\end{tabular} & $\begin{array}{l}\text { SXR- } \\
\text { class }\end{array}$ & $\begin{array}{l}\text { Coordin } \\
\text { ates }\end{array}$ & AR & \begin{tabular}{|c|}
$\mathrm{Max} . \mathrm{E}$ \\
$\mathrm{MeV}$
\end{tabular} & $\begin{array}{c}\text { RHESSI } \\
\text { data }\end{array}$ & $\begin{array}{l}\text { SONG data } \\
\text { comments }\end{array}$ \\
\hline 10 & 0101 & $00: 01-0031-00: 39$ & $00: 27-0: 37$ & $\mathrm{X1.7}$ & N06E34 & 0715 & 2.6 & no & \\
\hline 21 & 1401 & $21: 08-2126-21: 39$ & $21: 16-2124$ & M1.9 & N14E10 & 0720 & $.08-0225$ & yes & some shirt peaks \\
\hline 3 & 1501 & $00: 22-00: 43-01: 02$ & $00: 39-00.42$ & $\mathrm{X} 1.2$ & N14D08 & 0720 & $.225-0.75$ & yes & middle latitudes \\
\hline 4 & 15.01 & $05: 54-0638-07: 17$ & \begin{tabular}{|l|}
$06: 24-0632$ \\
\end{tabular} & M8.6 & N16D24 & 0720 & $.08-0225$ & partly & ERB till 05:24 \\
\hline 5 & 1501 & $22: 25-23: 02-23: 31$ & $20: 38-20: 55$ & $\mathrm{X} 2.6$ & N1swos & 0720 & .225 .0 .75 & yes & in polar cap \\
\hline 6 & 1701 & 06:59-09:52-10:07 & $09: 06-10: 00$ & 83.8 & N15W25 & 0720 & $2-6$ & partly & $\begin{array}{l}\text { ERB after 09:16, } \\
\text { shade till09:S2 }\end{array}$ \\
\hline 71 & 1901 & $08: 03-0822-08: 40$ & 08:08-08:15 & $\mathrm{X} 1.3$ & N15wS1 & 0720 & $.08-0225$ & partly & shade till 0808 \\
\hline 8 & 20.01 & 06:35-07:01-07:25 & $09: 47-09: 58$ & $\mathrm{x} 7.1$ & N14W61 & 0720 & 90.150 & yes & \\
\hline 9 & 2101 & $10: 10-10: 16-10: 19$ & \begin{tabular}{|l|}
$10: 14-10: 16$ \\
\end{tabular} & M1.7. & N19W81 & 0720 & $.225-0.75$ & yes & \\
\hline 100 & 0605 & $03: 05: 03: 14-03: 21$ & 03:07-03:12 & C9.3 & S04W71 & 0756 & $.08-0225$ & yes & hard HXR \\
\hline 111 & 1606 & $20: 01-2022-20: 42$ & $20: 50-20: 53$ & M4.0. & N08W90 & 0775 & $.08-0225$ & yes & After SXR peak \\
\hline 121 & 14.07 & $05: 57-0725-07: 43$ & $07: 22-0724$ & M9.1 & No9w90 & 0786 & $2-6$ & no & \\
\hline 132 & 2807 & $21: 39-22: 08-22: 24$ & $21: 49-21: 55$ & M4.8 & N09D32 & 0792 & $.08-0225$ & no & \\
\hline $14 \sqrt[3]{2}$ & 30.07 & 06:17-0635-の7:01 & 06:12-0828 & $\mathrm{X} 1.3$ & N12ES0 & 0792 & $.225-0.75$ & yes & \\
\hline 152 & 2208 & 16:46-1727-18:02 & $17: 00-1724$ & MS.6 & S13Ws & 0798 & $.08-0225$ & yes & ERB 17:07-17:11 \\
\hline 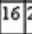 & 2308 & $14: 19-14: 44-16: 08$ & \begin{tabular}{|l|}
$14: 36-14: 40$ \\
\end{tabular} & $\mathrm{M} 2.7$ & S14W90 & 0798 & $.225-0.75$ & yes & \\
\hline 170 & 0709 & 17:17-17:40-18:03 & 17:50-18:29 & $\mathrm{X} 17$ & S11E7? & 0808 & $2-6$ & yes & shade till17:50 \\
\hline 180 & 0809 & 16:49-1703-17:11 & 16:59-1701 & M2.1 & S12E74 & 0808 & .2250 .75 & yes & \\
\hline 190 & 08092 & $20: 52-2106-21: 17$ & 21:04-21:06 & $\mathrm{X} 5.4$ & S12E7S & 0808 & $.08-0225$ & no & small peak \\
\hline 200 & 0909 & 19:13-2004-20:36 & $20: 00-20: 16$ & $\mathrm{x} 6.2$ & S12E67 & 0808 & $2-6$ & partly & shade till 2000 \\
\hline 21 & 10092 & $21: 30-22: 11-22: 43$ & $21: 45-22: 15$ & $\mathrm{X} 2.1$ & S13E4? & 0808 & $0.75-2$ & yes & \\
\hline \begin{tabular}{|l|l|}
22 & 1 \\
\end{tabular} & 12090 & $08: 37-0903-00: 20$ & \begin{tabular}{|l|l|}
$08: 45-08: 49$ \\
\end{tabular} & M6.2 & S11E2S & 0808 & $2-6$ & partly & \\
\hline
\end{tabular}

Figure 3. (= Table 1). Solar HXR- and $\gamma$-ray flares detected by SONG (CORONAS-F) from January to September 2005.

We emphasize that CORONAS-F and RHESSI data of solar HXR and $\gamma$ emission are a good database for HARD NEUTRAL EMISSION INDEX of solar flares creation. Comparing such new HXR-index with SXR one will be very useful at least for the priliminary estimation of spectral shape of total solar neutral emission.

Acknowledgements. This work has been partly supported by grant N 05-02-17487 of the Russian Foundation for Basic Research. I. N. Myagkova thanks the organizers of the workshop for collaboration and understanding.

\section{References}

Berezhko, E. G., Petukhov, S. I., \& Taneev, S. N. 2001, Izvestiya RAN, Phys. Ser., 64 (3), 339

Cane, H. V., Reames, D. V., \& von Rosenvinge, T. T. 1988, J. Geophys. Res., 93 (A9), 9555

Gopalswamy, N. 2006, J. Astroph. \& Astron., 27, 243

Kuznetzov, S. N., Kudela, K., Ryumin, S. P., \& Gotselyuk, Yu. V. 2002, Adv. Space Res., 30, 1857

Kuznetzov, S. N., Kudela, K., \& Myagkova, I. N., et al. 2004, Indian J. Radio Space Phys. 33, 353

Myagkova, I. N., Kuznetsov, S. N., \& Panasyuk, M. I. et al. 2006, Sun and Geosphere, 1(2), 34

Ramaty, R., Dennis, B. R., \& Emslie, A. G. 1988, Solar Phys. 118, 17

Ramaty, R. \& Mandzhavidze, N. 1994, in High Energy Solar Phenomena, New Era of Spacecraft Measurements, ed. J. M. Ryan \& M. T. Vestrand, Proc. AIP Conf. 294, Amer. Inst. Phys., New York, 26

Veselovsky, I. S., Panasyuk, M. I., Avdyushin, S. I., Bazilevskaya, G. A., et al. 2004, Cosmic Research $42(5), 435$ 


\section{Part 3}

\section{Theory and interpretation}





\title{
Inversion techniques: From observations to atmospheres
}

\author{
B. Ruiz Cobo \\ Instituto de Astrofísica de Canarias, Tenerife, Spain \\ Email: brc@iac.es
}

\begin{abstract}
Inversion techniques are presently the most powerful tools to deduce magnetic, dynamic, and thermal properties of the solar plasma from polarization line profiles. The reliability and robustness of Stokes inversions have been confirmed many times by means of numerical tests. Part of the solar physics community, however, is still worried about the uniqueness of the obtained solutions. In this presentation I clarify the scope and the limitations of different Stokes inversion techniques and discuss the challenges for inversion techniques for the near future.
\end{abstract}

\section{Introduction}

Inversion techniques (IT), i.e., the automatic way to reach from observations to atmospheres, have been frequently used during the last three decades, thus a lot of information about IT is available in the literature. Among others, the following excellent reviews about IT are to be mentioned: Wood \& Fox (1995), a general review about inversion methods in spectropolarimetry; del Toro Iniesta \& Ruiz Cobo (1995, 1996, 1997), revisions of least square IT with special weight on error estimation and the gradient determination problem; Socas-Navarro (2001), a revision of most recent developments of Stokes inversion techniques and projects, currently under development; del Toro Iniesta (2003), a comprehensive review on IT; and finally I would like to refer to the fantastic review of Bellot Rubio (2006) who summarizes the main results of inversion techniques and pays special attention to the gradient determination problem.

In this contribution I will try to address two important topics. Firstly, the uniqueness problem of Stokes inversions, and secondly, the establishment of criteria for the selection of the most adequate IT for each problem. Therefore I classify the IT in three main groups, analyzing the advantages and shortcomings of each of them. Finally, I present recent advances and most exciting promises for the next future.

\section{On the uniqueness problem}

In the literature one easily finds definitions of forward modelling or inversions similar to those reflected here: "Our way of representing the solar atmosphere is through a set of model parameters which is assumed to describe completely such a physical system. These parameters are not directly measurable but determine the values of the observables: the Stokes spectrum. The forward problem consists in predicting the unknown Stokes profiles 
by solving the radiative transfer equation (RTE), once a set of values of the model parameters is chosen. The inverse problem consists in inferring the values of the model parameters in such a way that the solution of the RTE optimally matches the observed spectra". Of course these expressions are true and beautiful (in fact some of them can be found in del Toro Iniesta $\&$ Ruiz Cobo 1996). But, following these ideas, one could conclude that inversion methods are able to retrieve an atmospheric model just from observed spectra. However, many authors (see for instance Sankarasubramanian \& Rimmele 2002; McMillan et al. 2004) have noticed that this is not the case, claiming that inversion methods are unable to produce a unique solution from a set of Stokes spectra. Consequently, a strong criticism against inversion methods was born.

Sabatier (2000) describes inversion techniques as the analysis of a set of observational results in order to retrieve as much information as possible for a model which is proposed to represent the system in the real world. With this definition in mind, it is clear that the usual criticism against inversion methods, in the sense of their non-uniqueness, is really baseless. Before doing an inversion, an a priori model is needed whose parameters will be fitted afterwards. An inversion procedure does not intend to find a new model, rather it tries to find the proper values for the free parameters of a given model.

For clarification purposes, let me introduce two definitions: let us denote "model atmosphere", or simply "atmosphere", a table containing values of several physical quantities (for instance, temperature, electronic pressure, density, line of sight velocity, magnetic field vector) discretised as function of depth (geometrical or optical). And let us call "additional information" (AI) all information needed, in addition to the model atmosphere itself, in order to synthesize the proper Stokes spectra. Such an AI includes atomic parameters (excitation potentials, transition rates, etc.), a table of abundances, some physical approximations (for instance LTE or Statistical Equilibrium, Hydrostatic Equilibrium, stationarity, etc.) and a scenery (geometry of structures present in our atmosphere, sizes or magnetic probability density function, etc.). In some particular cases some information of the AI can be included in the model atmosphere directly.

When comparing forward modelling and inversions, we can say that the forward problem consists in obtaining the Stokes profiles from both, model atmosphere and additional information, while inversion consists in retrieving the model atmosphere from Stokes profiles and additional information. The AI can neither be obtained from inversion nor through forward modelling and must be introduced as an input to both approaches. Different inversion methods applied to the same set of spectral profiles would produce different results only, as long different AI values are used. It has been proven, that, once the additional information is fixed, the output model atmosphere becomes unique (e.g., Ruiz Cobo \& del Toro Iniesta 1992; Westendorp Plaza et al. 1998; Socas-Navarro et al. 1998; Bellot Rubio et al. 2000). Of course, each parameter of the model atmosphere will be indeterminate inside a certain error bar, which in some cases may be extremely large. Here I would like to mention, e.g., the problem of magnetometry of the quiet Sun internetwork from Stokes profiles of the $630 \mathrm{~nm}$ Fe I lines (Martínez González et al. 2006) which is not a non-uniqueness problem. Simply, the information at low polarization signal, encoded in the Stokes spectra used, is insufficient to produce acceptable error bars. 


\section{Validation criteria}

Once a model atmosphere has been obtained after applying IT, three main validation criteria can be outlined which can be sequentially used. Firstly, the synthetic profiles must match the observed ones inside the observational error. In many cases (for instance when flexible enough IT are used) the inversion is always able to find a model which, together with the AI chosen, can reproduce the observational data. Is the resulting model atmosphere the real one? Not necessarily. We can only be sure that our model is compatible with the AI adopted. Any error or inadequacy inside the AI will be translated to erroneous values of the atmosphere. E.g., an LTE inversion of lines harboring large departure coefficients will produce well fitted profiles adopting erroneous temperature values; a large set of neutral iron lines can be perfectly fitted by an erroneous pressure stratification the effects of which on the lines are compensated by an erroneous temperature stratification; asymmetric Stokes profiles stemming from solar faculae are easily fitted by one-component inversions, but the velocity and magnetic field gradients will be the opposite to the real ones. In this cases additional validation criteria are needed. The second one is the reliability of the model atmosphere: For instance, strong line of sight variations of the physical quantities can in general be rejected because they are simply translating to the atmospheric model errors in the AI set chosen. The third validation criterion is the capability of the obtained atmosphere to reproduce spectral profiles which have not been used in the inversion. Figure 1 (adapted from Figs. 3 and 7 of Allende Prieto et al. 1988) illustrates how a quiet Sun model obtained after inversion of 20 spectral lines taken from the Solar Flux Atlas of Kurucz et al. (1984) is able to reproduce two spectral regions not included in the inversion. Other examples of the application of these validation criteria can be found in Bellot Rubio et al. (1997).

\section{Classification of inversion methods}

The Radiative Transfer Equation (RTE) relates Stokes vector and atmosphere through the absorption matrix $K$ and the source function vector $S$. In order to simplify the solution of the RTE different standard approximations are used: a) the Milne-Eddington (ME) approach considers $K$ as a constant through the atmosphere and $S$ as a linear function of optical depth; b) LTE approach, in which $K$ is evaluated from ionization and excitation equilibrium equations (Saha and Boltzmann equations) while $S$ is approximated by the Planck function at local temperature; c) NLTE, in which $K$ and $S$ are evaluated by solving the stationary Statistical Equilibrium equations. Each of these approximations gives rise to a different inversion technique. Even more, an IT always works in minimizing the merit function which tells us about the misfit between the synthetic and the observed Stokes profiles. The way in which the minimization procedure is carried out, least squared, by genetic algorithms, principal component analysis or neural network, gives raise to different IT.

Reading the abundant literature about IT, one easily concludes that the authors chose a particular IT for strange reasons: e.g., because it is available, easy or user-friendly. Before proposing proper IT selection criteria I would like to make a classification of the IT. Therefore, I will group the most important IT in three main families: the ME family; the SIR family; and the PCA family. For each family I will cite seminal papers, outline the advantages and shortcomings of each IT, and refer to some very recent papers in which such IT have been used. 

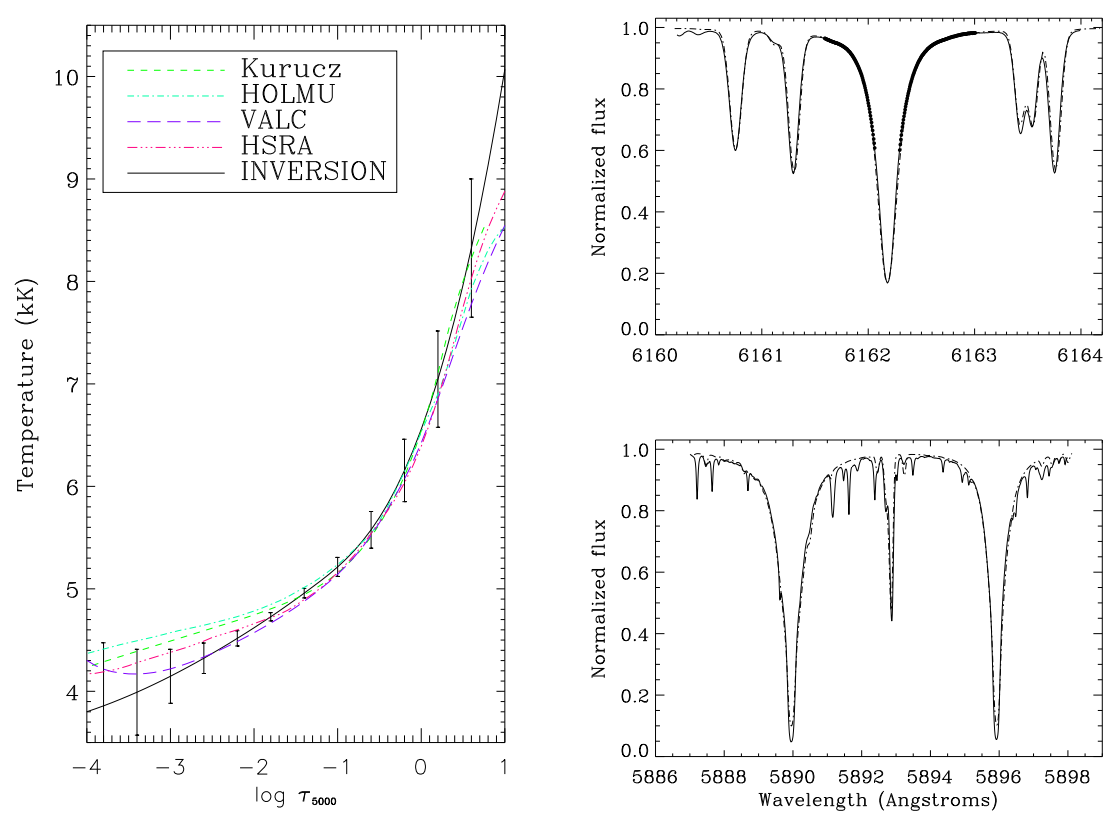

Figure 1. Left: The solid line represents the quiet Sun temperature stratification obtained after inversion of 20 spectral lines taken from the Solar Flux Atlas of Kurucz et al. (1984). Right, upper panel: Observed profile (solid line) of the Ca I $6162 \AA$ lines whose wings (enhanced in the graph) were included in the inversion. The dot-dashed lines show the synthesized profile obtained from the resulting model. The observed profile is nicely reproduced. A good agreement is also found for neighboring lines not included in the inversion. Lower panel: Very good agreement between observed and synthesized profiles was also found for the wings of the $\mathrm{Na}$ I $\mathrm{D}$ lines (not included in the inversion). Figure adapted from Figs. 3 and 7 of Allende Prieto et al. (1988).

\subsection{ME family}

\subsubsection{Some important papers}

The first Stokes inversion technique found in the literature was based on the Milne-Eddington approximation neglecting magneto-optical effects (Harvey et al. 1972). Auer et al. (1977) carefully tested the behavior of their IT with simulated observations. This code was generalized by Landolfi, Landi Degl'Innocenti, \& Arena (1984) to account for damping wings and magneto-optical effects. In the High Altitude Observatory a strong impulse was given to IT after the papers of Skumanich \& Lites $(1984,1987)$. This ME code was extensively used for the analysis of the Advanced Stokes Polarimeter data (Elmore et al. 1992) producing groundbreaking scientific results. Among other most recent developments of ME IT, I would like to mention the work of Lagg et al. (2004), who present an ME code specifically designed to invert He I $1083.0 \mathrm{~nm}$ Stokes profiles induced by the Zeeman effect, and taking into account a simplified treatment of the Hanle effect similar to that used by Collados et al. (2003). In addition I mention the code of Socas-Navarro et al. (2004), where the importance 
of doing the radiative transfer calculations for He I $1083.0 \mathrm{~nm}$ within the framework of the incomplete Paschen-Back effect theory is demonstrated.

\subsubsection{Advantages}

ME inversion codes are very fast: a typical map of Stokes spectra takes around 4-5 CPU hours (see for instance Socas-Navarro 2005). Additionally, these codes are easy to handle. Provided that the output consists in some few parameters which are constant throughout the atmosphere, the interpretation of the results becomes straightforward. ME inversion codes are robust, in the sense that the results are user independent. Besides, LTE approximation or Hydrostatic Equilibrium are not needed, thus ME IT can be even applied when the knowledge of the atmospheric structure or about the physics involved in the line formation is poor.

\subsubsection{Shortcomings}

ME IT are unable to fit asymmetric Stokes profiles, which are extremely common in real observations. This fact is caused by the assumption of a constant absorption matrix. Several strategies have been proposed in order to circumvent this problem building second-order ME codes capable to fit gradients (see for instance Skumanich 2001, and references therein), but in any case, these solutions need more free parameters than other techniques (as SIR for instance, see next subsection) and produce larger error bars in order to reach poorer fits. ME codes do not supply temperature, pressure or density information. Finally, ME codes are only adequate to attack simultaneous inversion of one or two spectral lines because the number of free parameters grows with the number of spectral lines, while, in more sophisticated strategies, the number of free parameters is mostly independent of the number of spectral lines.

\subsubsection{Some recent papers}

Among other papers using ME published in 2006, one finds the beautiful work of Centeno et al. (2006), where thanks to an ME inversion of the He I line at $1083 \mathrm{~nm}$ shock formation at chromospheric layers in sunspot umbrae has been found. Nagata et al. (2006) studied the dynamical properties of photospheric flux tubes at the foot points of coronal loops.

\subsection{SIR family}

\subsubsection{Some important papers}

Least square inversion techniques based on response functions suffer a big inflation since the 1990s. The additional information for these codes can be changed very easily and, consequently, a lot of different methods are presently operative corresponding to different geometries, approximations or even spatial distributions of magnetic structures. Among others, as the most important contributions I mention: the SIR method (Ruiz Cobo \& del Toro Iniesta 1992) that has been extensively used to study the structure of many photospheric features (see for instance Ruiz Cobo 1999, 2001); the SPINOR code (Frutiger et al. 2000; see also Bernasconi \& Solanki 1996) which is inspired by the same philosophy as SIR. Bellot Rubio et al. (1997) designed an inversion code that takes into account a flux-tube geometry (see 


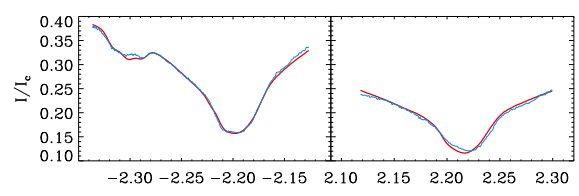

Wavelength $-852(\mathrm{~nm})$
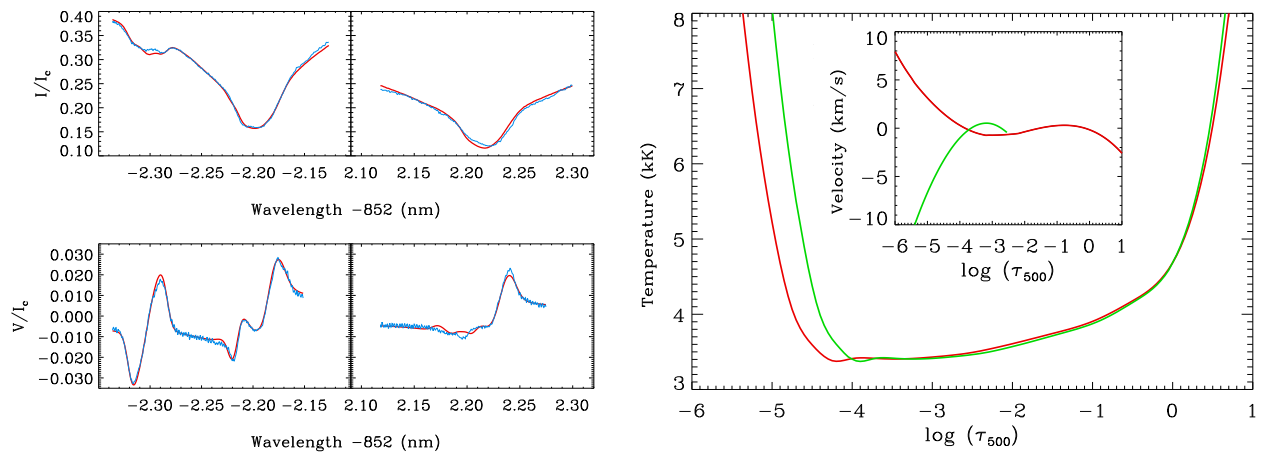

Figure 2. Left panels: Stokes I (top) and V (bottom) profiles of two lines of the Ca II infrared triplet observed in a sunspot umbra and corresponding synthesized profiles provided by the SIR-NLTE code of Socas-Navarro et al. (2000b). Note the normal Stokes I and anomalous Stokes V profile characteristics related to an umbral flash. Right panel: Temperature and velocity stratifications in the resulting two component umbra model during the occurrence of anomalous Stokes V profiles (figure adapted from Socas-Navarro et al. 2000b).

also Frutiger \& Solanki 1998); the IT of Bellot Rubio (2003), see also Borrero et al. (2005), has been specially designed to study the uncombed penumbral model; the MISMA code (Sánchez Almeida 1997) was built to study the properties of micro-scale magnetic structures; the MISS code (Allende Prieto et al. 1988; see also Frutiger et al. 2005) uses the same strategy as SIR to invert stellar spectra; the SIR-NLTE code (Socas-Navarro et al. 2000a) was the first successful Stokes inversion code running outside the LTE approximation. Figure 2, adapted from Socas-Navarro et al. (2000b), illustrates the mechanism involved in the occurrence of umbral flashes, observed in the lines of the Ca II infrared triplet. This result has been obtained from the application of the above mentioned SIR-NLTE code.

\subsubsection{Advantages}

The SIR family admits an arbitrary complexity of the atmosphere, thus accounting for any variation of physical quantities along the line of sight; also it permits the consideration of many atmospheric components or different treatments of the physics involved in the line formation (LTE or NLTE, etc.). As the free parameters correspond to real atmospheric values, the inversion of many spectral lines (including blends) can be attacked. The IT output includes thermodynamic (temperature, pressures, density, etc.) information. Besides, these codes are in general very flexible, allowing to adopt different geometries or scenarios. Finally, they provide the user with reliable error estimations. Figure 3, adapted from Figs. 5 and 6 of Ruiz Cobo et al. (1997) shows results from the SIR inversion of a temporal series of $85 \mathrm{~min}$ in the $\mathrm{K}_{\mathrm{I}} 769.9 \mathrm{~nm}$ line observed at quiet Sun center. Error bars represent the uncertainties evaluated by the SIR code. To study the reliability a Monte Carlo simulation has been carried out: The shaded areas in this figure represent the resulting standard deviations (for details, see Ruiz Cobo et al. 1997). 

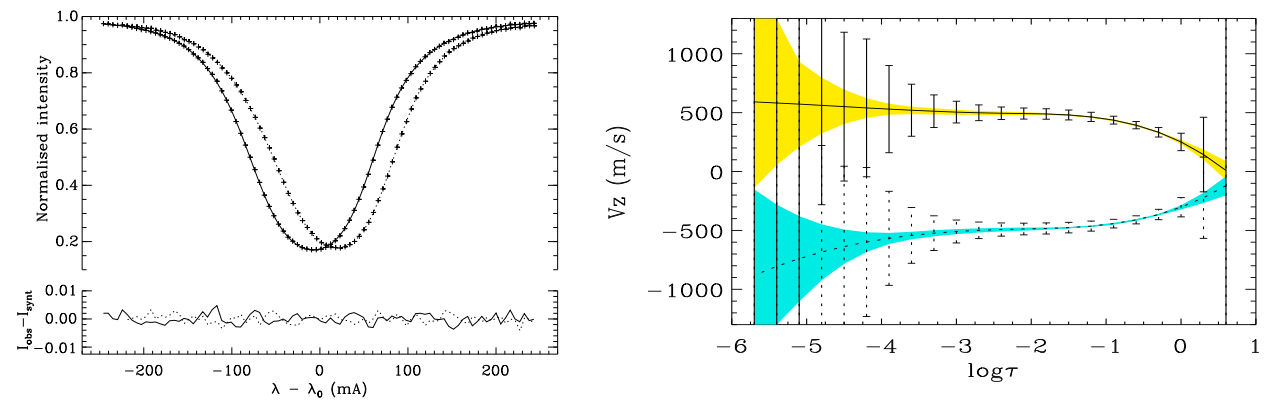

Figure 3. Left panel: Two individual profiles of $\mathrm{K}_{\mathrm{I}}$ at $769.9 \mathrm{~nm}$ corresponding to opposite phases of the velocity oscillation (separated $145 \mathrm{~s}$ in time). Crosses represent the observations $I_{\mathrm{obs}}$ and solid and dotted lines show the synthesized profiles $I_{\text {syn }}$. In the lower part of this panel differences between observed and synthesized profiles are plotted. Right panel: Line-of-sight velocity stratification resulting from the inversion of the profiles shown in the left panel. Positive and negative velocities correspond to redshift (solid line) and blueshift (dotted line), respectively. Error bars correspond to uncertainties calculated by the inversion code (SIR). Shaded bands represent uncertainties coming from a Monte Carlo simulation. Figure adapted from Figs. 5 and 6 of Ruiz Cobo et al. (1997).

\subsubsection{Shortcomings}

These codes are slow: a typical run takes around 20 or 30 times longer than an ME inversion (Bellot Rubio 2006). SIR family inversion codes are, as mentioned above, very flexible, however, their use becomes extremely difficult. Uniqueness problems may appear, because the additional information set can be changed very easily and, consequently, different users could obtain different results inverting the same observational data.

\subsubsection{Some recent papers}

Among other papers published during 2006 I mention: the work of Socas-Navarro et al. (2006), in which the study of Ellerman Bombs is carried out through NLTE inversion of the Ca II infrared triplet; Jurčák et al. (2006) found a higher temperature magnetic canopy above a sunspot light bridge using SIR; Borrero et al. (2006) attacked the inversion of neutral iron lines at $630 \mathrm{~nm}$ and $1560 \mathrm{~nm}$ using the uncombed penumbral model inversion code; Balthasar (2006) searched for vertical electric currents in sunspot umbrae using SIR inversion; Bellot Rubio et al. (2006) studied, through SIR inversion, the structure of penumbral filaments at a spatial resolution of 0."5; Domínguez Cerdeña et al. (2006) quantified magnetic field strengths in quiet Sun regions using MISMA inversion of visible and infrared lines.

\subsection{PCA family}

\subsubsection{Some important papers}

The simplest inversion strategy is one that uses a look-up table. From a large number of known model atmospheres a database of synthetic Stokes profiles is built. The Principal 
Component Analysis (PCA) allows a fast search in the look-up table. The most important papers related to the PCA inversion development are those of Rees et al. (2000), SocasNavarro et al. (2001), and López Ariste \& Casini (2002).

\subsubsection{Advantages}

PCA inversion techniques are extremely fast: A typical map takes around ten minutes to be inverted (Bellot Rubio 2006). In 2004, at the French telescope THEMIS, a PCA IT allowed for the first time real-time inversions of Stokes spectra.

\subsubsection{Shortcomings}

The main limitation of these techniques is related to the size of the database. To keep the latter small, only few free parameters can be fitted, and the table grid step must be coarse. This implies that the accuracy of PCA inversion becomes slightly lower than the ME one.

\subsubsection{Some recent papers}

A beautiful paper using PCA inversion is the work of López Ariste et al. (2006), in which the 3D magnetic field topology of solar filaments is studied.

\section{Recent advances and next future}

Besides the preceding classical inversions other techniques have been developed during the last decade. Significant progress may arise from the inversion of Stokes profiles affected by the Hanle effect. There is in fact a new generation of inversion codes taking into account both Zeeman and Hanle effects which will produce in the next future groundbreaking scientific results (see for instance Asensio Ramos \& Trujillo Bueno 2007; Trujillo Bueno et al. 2006, and references therein; Merenda et al. 2006).

To break the degeneracy between magnetic field strength and filling factor apparent in the weak magnetic field regime it is possible to take advantage of some properties of lines showing hyperfine structure, such as Mn I $553.7 \mathrm{~nm}$ and Mn I $874.1 \mathrm{~nm}$ (López Ariste et al. 2002, 2006).

Another recently developed inversion technique is based on artificial neural networks (Carroll \& Staude 2001; Socas-Navarro 2005). This technique is extremely fast but, at the moment, the results show significantly larger errors than classical inversion methods.

Finally, major progress on inversions rate could come from hardware inversion on Field Programmable Gate Arrays, which is about thousand times faster than software inversions (see Bellot Rubio 2006).

\section{Conclusions}

The uniqueness problem is caused by a poor definition of the additional information needed for the inversion. Once the scenery has been defined (i.e. all the geometry, approximations used, atomic parameters, abundances, etc.) the inversion methods produce unique results. 
As a summary one can establish the following rule for an optimum selection of the proper inversion technique: a) ME must be used in cases of unknown physics controlling the line formation; b) PCA to obtain very fast results; c) a SIR family inversion method when the knowledge about the line formation mechanisms is appropriate and the entire information hidden in the spectral lines shall be retrieved.

Acknowledgements. I would like to thank Axel D. Wittmann: his synthesis code is the core of SIR; to Franz Kneer for his kind invitation and for being so patient; to my teachers M. Collados, J. C. del Toro Iniesta, V. Martínez Pillet \& J. Trujillo Bueno; to my students L. Bellot Rubio, H. Socas-Navarro, C. Westendorp Plaza, M. J. Martínez; and, specially, to Klaus G. Puschmann. This work has been supported by the Spanish Ministerio de Educación y Ciencia through project AYA2004-05792.

\section{References}

Auer, L. H., House, L. L., \& Heasly, J. N. 1977, Solar Phys., 55, 47

Allende Prieto, C., Ruiz Cobo, B., \& García López, R. J. 1998, ApJ, 502, 951

Asensio Ramos, A. \& Trujillo Bueno, J. 2007, in Coimbra Solar Physics Meeting on The Physics of Chromospheric Plasmas, ed. P. Heinzel, I. Dorotovic, \& R. J. Rutten, ASP Conf. Ser., in press

Balthasar, H. 2006, A\&A, 449, 1176

Bellot Rubio, L. R. 2003, in Solar Polarization, ed. J. Trujillo Bueno \& J. Sánchez Almeida, ASP Conf. Ser., 307, 301

Bellot Rubio, L. R. 2006, ASP Conf. Ser., 358, in press (astro-ph/0601483)

Bellot Rubio, L. R., Ruiz Cobo, B., \& Collados, M. 1997, ApJ, 478, L45

Bellot Rubio, L. R., Ruiz Cobo, B., \& Collados, M. 2000, ApJ, 535, 475

Bellot Rubio, L. R., Schlichenmaier, R., \& Tritschler, A. 2006, A\&A, 453, 1117

Bernasconi, P. N. \& Solanki, S. K. 1996, Solar Phys., 164, 277

Borrero, J. M., Lagg, A., Solanki, S. K., \& Collados, M. 2005, A\&A, 436, 333

Borrero, J. M., Solanki, S. K., Lagg, A., Socas-Navarro H., \& Lites, B. W. 2006, A\&A, 450, 383

Carroll, T. A. \& Staude, J. 2001, A\&A, 378, 316

Centeno, R., Collados, M., \& Trujillo Bueno, J. 2006, ApJ, 640, 1153

Collados, M., Trujillo Bueno, J., \& Asensio Ramos, A. 2003, in Solar Polarization 3, ed. J. Trujillo Bueno \& J. Sánchez Almeida, ASP Conf. Series, 307, 468

Domínguez Cerdeña, I., Sánchez Almeida, J., \& Kneer, F. 2006, ApJ, 646, 1421

Elmore, D. F., et al. 1992, in Polarization analysis and measurement, Proc. SPIE, 1746, 22

Frutiger, C. \& Solanki, S. K. 1998, A\&A, 336, L65

Frutiger, C., Solanki, S. K., Fligge, M., \& Bruls, J. H. M. J. 2000, A\&A, 358, 1109

Frutiger, C., Solanki, S. K., \& Mathys, G. 2005, A\&A, 444, 549s

Harvey, J., Livingston, W. L., \& Slaughter, C. 1972, in Line Formation in the Presence of Magnetic Fields. Manuscripts presented at a conference held in Boulder, CO (USA) 30 August, 1971, ed. R. G. Athay, L. L. House, \& G. A. Newkirk, Jr.

Jurčák, J., Martínez Pillet, V., \& Sobotka, M. 2006, A\&A, 453,1079

Kurucz, R. L., Furenlid, I., Brault, J., \& Testerman, L. 1984, NOAO Atlas No. 1, The Solar Flux Atlas from 296 to $1300 \mathrm{~nm}$, (Sunspot: NSO)

Lagg, A., Woch, J., Krupp, N., \& Solanki, S. K. 2004, A\&A, 414, 1109

Landolfi, M., Landi Degl'Innocenti, E., \& Arena, P. 1984, Solar Phys., 93, 269

López Ariste, A., Aulanier, G., Schmieder, B., \& Sáinz Dalda, A. 2006, A\&A, 456, 725

López Ariste, A. \& Casini, R. 2002, ApJ, 575, 529

López Ariste, A., Tomczyk, S., \& Casini, R. 2002, ApJ, 580, 519

López Ariste, A., Tomczyk, S., \& Casini, R. 2006, A\&A, 454, 663 
Martínez González, M. J., Collados, M., \& Ruiz Cobo, B. 2006, A\&A, 456, 1159

McMillan, M. T., Sankarasubramaniam, K., \& Uitenbroek, H. 2004, American Astronomical Society Meeting 205, 1003, Bulletin of the American Astronomical Society, 36, 1349

Merenda, L., Trujillo Bueno, J., Landi Degl'Innocenti, E., \& Collados, M. 2006, ApJ, 642, 554

Nagata S., Bellot Rubio, L. R., \& Katsukawa, Y. 2006, ApJ, 638, 539

Rees, D. E., López Ariste, A., Thatcher, J., \& Semel, M. 2000, A\&A, 355, 759

Ruiz Cobo, B. 1999, Ap\&SS, 263, 331

Ruiz Cobo, B. 2001, in Highlights of Spanish Astrophysics II, ed. J. Zamorano, J. Gorgas, \& J. Gallego, Kluwer Ac. Publ., p. 241

Ruiz Cobo, B., Rodríguez Hidalgo, I., \& Collados, M. 1997, ApJ, 488, 462

Ruiz Cobo, B. \& del Toro Iniesta, J. C. 1992, ApJ, 398, 375

Sabatier, P. C. 2000, Journal of Mathem. Phys., 41, 4082

Sánchez Almeida, J. 1997, ApJ, 491, 993

Sankarasubramaniam, K. \& Rimmele, T. 2002, ApJ, 576, 1048

Skumanich, A. 2001, in Advanced Solar Polarimetry - Theory, Observation and Instrumentation, ed. M. Sigwarth, ASP Conf. Ser., 236, 543

Skumanich, A. \& Lites, B. W. 1984, in Measurements of Solar Vector Magnetic Fields, ed. M. J. Hagyard, NASA Conf. Publ., 2374, 341

Skumanich, A. \& Lites, B. W. 1987, ApJ, 322, 473

Socas-Navarro, H. 2001, in Advanced Solar Polarimetry - Theory, Observation and Instrumentation, ed. M. Sigwarth, ASP Conf. Ser., 236, 487

Socas-Navarro, H. 2005, ApJ, 621, 545

Socas-Navarro, H., Ruiz Cobo, B., \& Trujillo Bueno, J. 1998, ApJ, 507, 470

Socas-Navarro, H., Trujillo Bueno, J., \& Ruiz Cobo, B. 2000a, ApJ, 530, 977

Socas-Navarro, H., Trujillo Bueno, J., \& Ruiz Cobo, B. 2000b, Science, 288, 1396

Socas-Navarro, H., López Ariste, A., \& Lites, B. W. 2001, ApJ, 553, 949

Socas-Navarro, H., Trujillo Bueno, J., \& Landi Degl'Innocenti, E. 2004, ApJ, 612, 1175

Socas-Navarro, H., Martínez Pillet, V., Elmore, D., Pietarila, A., Lites, B. W., \& Manso Sainz, R. 2006, Solar Phys., 235, 75

Trujillo Bueno, J., Asensio Ramos, A., \& Shchukina, N. 2006, ASP Conf. Ser., 358, in press (astro$\mathrm{ph} / 0612678)$

del Toro Iniesta, J. C. 2003, Astron. Nachr., 324, 383

del Toro Iniesta, J. C. \& Ruiz Cobo, B. 1995, in La Polarimétrie, outil pour l'étude de l'activité magnétique solaire et stellaire, ed. N. Mein \& S. Sahal-Bréchot, 127

del Toro Iniesta, J. C. \& Ruiz Cobo, B. 1996, Solar Phys., 164, 169

del Toro Iniesta, J. C. \& Ruiz Cobo, B. 1997, in Forum Themis, ed. N. Mein \& S. Sahal-Bréchot, 93

Westendorp Plaza, C., del Toro Iniesta, J. C., Ruiz Cobo, B., Martínez Pillet, V., Lites, B. W., \& Skumanich, A. 1998, ApJ, 494, 453

Wood, K. \& Fox, G. K. 1995, Inverse Problems, 11, 795 


\title{
Stokes profile inversion in Meso-Structured Magnetic Atmospheres
}

\author{
T. A. Carroll \\ Astrophysikalisches Institut Potsdam, Germany \\ Email: tcarroll@aip.de
}

\begin{abstract}
Based on the Meso-Structured Magnetic Atmosphere (MESMA) approximation (Carroll \& Kopf 2007) we present first results of an inversion of spectropolarimetric observations obtained from internetwork regions. To cope with the inherent complexity of the mostly unresolved magnetic field in the solar photosphere the MESMA approach provides a statistical description of the underlying atmosphere in terms of a random Markov field. This statistical model allows us to derive a stochastic transfer equation for polarized light. The stochastic transfer equation explicitly accounts for the spatial correlation - the characteristic length scale - of the underlying magnetic and non-magnetic structures. We use this new diagnostic parameter in an inversion approach to demonstrate that the magnetic flux structures in the solar internetwork possess a finite correlation length which is not compatible with the classical flux tube picture.
\end{abstract}

\section{Introduction}

The entire solar photosphere exhibits a rich structure of large- and small-scale magnetic features like sunspots, pores faculae or plages. But except for sunspots and pores these magnetic fields cannot be spatially resolved with present telescopes, although these fields clearly manifest themselves in high resolution spectropolarimetric observations.

With the improvement of spectropolarimetric sensitivity and spatial resolution over the last years it became clear that these unresolved magnetic fields are much more ubiquitous than previously thought. This raises the question of the significance of these elusive and complex magnetic fields for the solar magnetism in general (Schrijver \& Title 2003; Sánchez Almeida 2004) and how these magnetic fields can be appropriately investigated by spectropolarimetric observations.

The interpretation of Stokes profiles in the context of the thin flux tube model relies on the basic picture of an embedded cylindrical magnetic structure surrounded by a quasi field-free medium. Based on that assumption a so called 1.5-dimensional radiative transfer is applied where a number of rays piercing through the underlying 2- or 3-dimensional geometry of the model to obtain the spectral 'signature' of the underlying magnetic structure (Solanki 1993). But if the underlying structures are much more dynamic, disrupted and intermittent, the conventional static flux tube model will allow only a poor representation of the real magnetic field structure. In this sense the flux tube modeling provides a rather macroscopic treatment of the problem - in the 1.5 dimensional sense - since the averaging process for all line-of-sights (LOS) is performed after the actual integration of the transfer equation. 
The other extreme, in contrast to the macroscopic view, is the MISMA approximation. The assumption here is that the atmospheric conditions along the line-of-sight are rapidly changing. The fluctuation of the atmospheric parameters occurs on very short scales, such that a micro-structured or micro-turbulent approach is justified. This allows an averaging over all atmospheric parameters at each spatial position before the actual transfer equation is integrated. Despite its appealing simplicity in the way this approach treats the radiative transfer, the idealized assumptions about the underlying atmosphere strongly limit the application of this approach. Structures in the solar photosphere whether magnetic or non-magnetic are in general not in a microturbulent state. Magneto-convective simulations suggest that neither predefined static macro-structures nor pure micro-structures are present in the solar photosphere, the possible structuring seems much more to comprise a broad range of different scales (Schaffenberger et al. 2005; Vögler et al. 2005; Stein \& Nordlund 2006).

This paper is organized as follows: In Sect. 2 I briefly summarize the basic concept of line formation in stochastic media and present the stochastic polarized transfer equation. In Sect. 3 I give an overlook of the first results of an inversion of spectropolarimetric observations obtained from internetwork regions. Sect. 4 concludes with a summary of the here presented analysis.

\section{The stochastic transfer equation for polarized light}

The approach described here is based on a statistical model of the atmosphere in terms of a random Markov field, the MEso-Structured Magnetic Atmosphere (MESMA) which was introduced by Carroll \& Kopf (2007). In this contribution I will just give a brief summary of the basic concept of the MESMA approach, for a more detailed presentation of the statistical model and derivation of the stochastic transfer equation the reader is referred to Carroll \& Staude (2003, 2005a) and Carroll \& Kopf (2007).

The atmospheric volume of interest (the actual resolution element) is assumed to be characterized by an a-priori unknown structuring along the line-of-sight. The only assumption we make about the underlying atmosphere is that the structures have a finite spatial extent and can be described in terms of a Markov random field. This allows us to neglect all higher order spatial correlation effects to use a first order approximation to describe the spatial correlations.

We begin by introducing a random atmospheric vector $\boldsymbol{B}$ which comprises all relevant atmospheric parameters such as temperature, pressure, velocity, magnetic field strength, magnetic field inclination, etc. If we move then along an arbitrary line-of-sight we obtain a series of realizations at different positions $s$ for the random vector $\boldsymbol{B}$. This spatial dependency allows us to describe $\boldsymbol{B}$ in terms of a stochastic process (a Markov process) along the line-of-sight and for which we can specify a suitable conditional probability density or transition probability from one spatial point $s$ to another $s+\Delta s$,

$$
p\left(\boldsymbol{B}_{s+\Delta s} \mid \boldsymbol{B}_{s}\right)=e^{-\frac{\Delta s}{\lambda}} \delta\left(\boldsymbol{B}_{s}-\boldsymbol{B}_{s+\Delta s}\right)+\left(1-e^{-\frac{\Delta s}{\lambda}}\right) p\left(\boldsymbol{B}_{s+\Delta s}\right) .
$$

This conditional probability which specifies the so called Kubo-Anderson process (Frisch $\&$ Frisch 1976) describes how the probability for a transition changes as we move along the line-of-sight from a given position $s$ and the associated atmospheric conditions $\boldsymbol{B}_{s}$ at this position. The probability for staying in the same regime $\boldsymbol{B}_{s}$ for the entire trajectory $\Delta s$ decays 
exponentially while the probability for a sudden jump into another atmospheric regime $\boldsymbol{B}_{s+\Delta s}$ rapidly grows. The conditional probability density therefore describes the correlation of the individual structures between two spatial positions $s$ and $s+\Delta s$. The degree of correlation is controlled by the parameter $\lambda$, the characteristic length (correlation length) of the structures. Based on that particular Markov process we can derive the following stochastic transport equation (see Carroll \& Kopf 2007), for the so called mean conditional Stokes vector $\boldsymbol{Y}$

$$
\frac{\partial \boldsymbol{Y}_{B}(s)}{\partial s}=-\boldsymbol{K} \boldsymbol{Y}_{\boldsymbol{B}}+\boldsymbol{j}+\int_{\hat{\boldsymbol{B}}} \lambda_{B^{\prime \prime}}^{-1} \boldsymbol{Y}_{\boldsymbol{B}^{\prime \prime}} p\left(\boldsymbol{B}^{\prime \prime}, s\right) d \boldsymbol{B}_{s}^{\prime \prime}-\int_{\hat{\boldsymbol{B}}} \lambda_{B}^{-1} \boldsymbol{Y}_{\boldsymbol{B}} p\left(\boldsymbol{B}^{\prime \prime}, s\right) d \boldsymbol{B}_{s}^{\prime \prime},
$$

The mean conditional Stokes vector $\boldsymbol{Y}$ is defined as

$$
\boldsymbol{Y}_{B}(s)=\int_{\hat{I}} \boldsymbol{I} p(\boldsymbol{I}, s \mid \boldsymbol{B}, s) d \boldsymbol{I} .
$$

It is important to realize that $\boldsymbol{Y}_{\boldsymbol{B}}$ is given by a statistical equation and it is conditioned on one particular atmospheric regime $\boldsymbol{B}$. The transport Eq. (2) describes the evolution of $\boldsymbol{Y}_{\boldsymbol{B}}$ through the atmosphere along the LOS. There are four basic processes that govern the transport of $\boldsymbol{Y}_{\boldsymbol{B}}$, the two processes of absorption and emission and two more processes that describe the statistical inflow and outflow of intensity to and from the regime $\boldsymbol{B}$ under consideration. The degree of statistical scattering and absorption is controlled by the correlation length $\lambda$ of the particular atmospheric structures. The observable of our problem - the expectation value of the Stokes vector - at the top of the atmosphere $s_{t}$ can easily be obtained from a final integration of the mean conditional Stokes vector over the entire atmospheric state space $\hat{\boldsymbol{B}}$,

$$
<\boldsymbol{I}\left(s_{t}\right)>=\int_{\hat{\boldsymbol{B}}} \boldsymbol{Y}_{B}\left(s_{t}\right) p\left(\boldsymbol{B}, s_{t}\right) d \boldsymbol{B} .
$$

It is this extra degree of freedom in the stochastic transfer equation, given by the correlation length, which provides the additional diagnostic capability of the stochastic approach (Carroll \& Staude 2005b, 2006; Carroll \& Kopf 2007). As could be shown by Carroll \& Kopf (2007) the asymmetry of the Stokes $V$ profiles directly depends upon the underlying correlation length and allows to estimate the characteristic length scale of the magnetic field from the net-circular-polarization (NCP) of Stokes $V$ profiles.

\section{Analysis of internetwork magnetic fields}

Based on the stochastic mesostructured approach we have analyzed observations of full Stokes profiles of the iron line pair Fe I at $630 \mathrm{~nm}$, taken at the High Altitude Observatory/National Solar Observatory Advanced Stokes Polarimeter (ASP). The quiet sun data were obtained by B. Lites on 1994 September 29 (Lites et al. 1996). In the following we are in particular interested to gain some insight into the characteristic length scale of the magnetic structures in the internetwork. Our inversion routine is based on the LevenbergMarquardt algorithm (Press et al. 1992) and incorporates the stochastic transport equation as the forward kernel. In a first step we analyzed granular and intergranular regions in order to determine the convective characteristics of the non-magnetic components from Stokes $I$ profiles. 


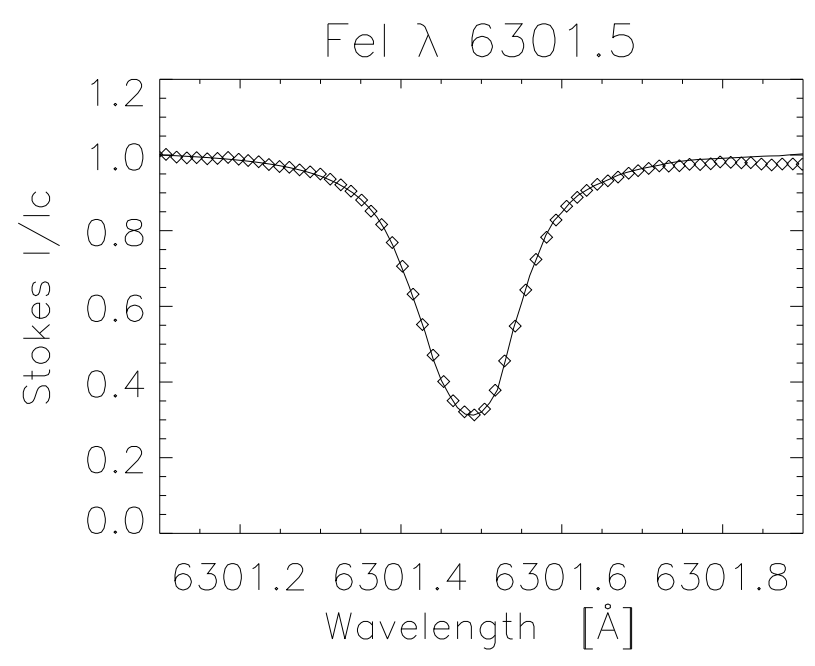

Figure 1. A fitted Stokes $I$ profile of the Fe I $6301.5 \AA$ line. Diamonds represent the observation, the solid line the profile fit.

For the granular and intergranular components we adopted the granular and intergranular model atmospheres from Borrero \& Bellot Rubio (2002). Based on the temperature and pressure stratification of these models we assumed a simple 3-type stochastic velocity field. Please note that the stochastic approach makes no assumption about the numbers of individual structures in the resolution element, the only assumption made here is that there are three different types of structures present.

The free parameter of the fitting routine are the three single-valued velocities for each atmospheric component, the probability values of the individual types (comparable to the conventional filling factor) and the correlation lengths of the individual components. For an upflow (granular) region a fit of a Stokes $I$ profile is shown in Fig. 1. The remarkable result here is the fact that there is no need to use nonphysical parameters like micro- or macroturbulence to fit the profiles. The convective velocity structure exhibits a clear mesostructured behavior on scales between $200 \mathrm{~km}$ and $400 \mathrm{~km}$. These results are in agreement with earlier investigations of mesoturbulent velocity fields in the solar photosphere by Gail et al. (1976).

As we could place tight constraints upon the characteristics of the ambient flow pattern we proceed by analyzing the magnetic field by inverting the respective Stokes $V$ profiles. We adopted a stochastic model of two different types of structures (non-magnetic and magnetic). The free parameters of the inversion are the velocities, magnetic field strengths as well as the correlation lengths of the individual structures. Two fits of the Stokes $V$ profiles are shown in Fig. 2 and Fig. 3. The observed Stokes $V$ profiles could be well reproduce by the stochastic two-ensemble model. In particular the asymmetries of the Stokes $V$ profiles are well reproduced. The magnetic structures in the analyzed internetwork region have surprisingly small correlation lengths between $50 \mathrm{~km}$ and $125 \mathrm{~km}$ for structures in upflow elements and $150 \mathrm{~km}$ to $230 \mathrm{~km}$ for structures in downflow elements. These results clearly indicate 


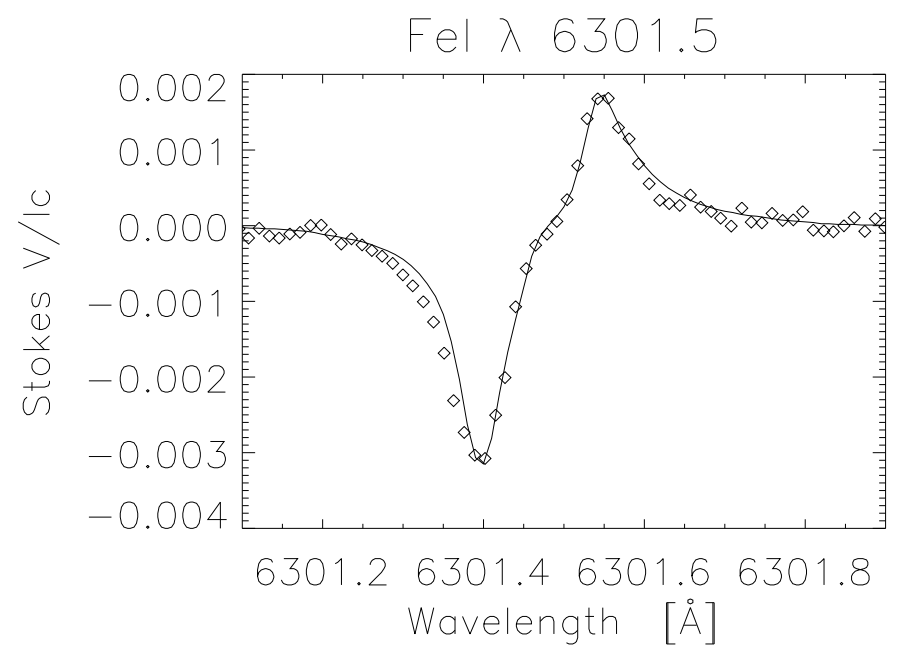

Figure 2. Fit of a typical Stokes $V$ profile of the Fe I $6301.5 \AA$ line, again the diamonds represent the observation and the solid line the fit to the profile.

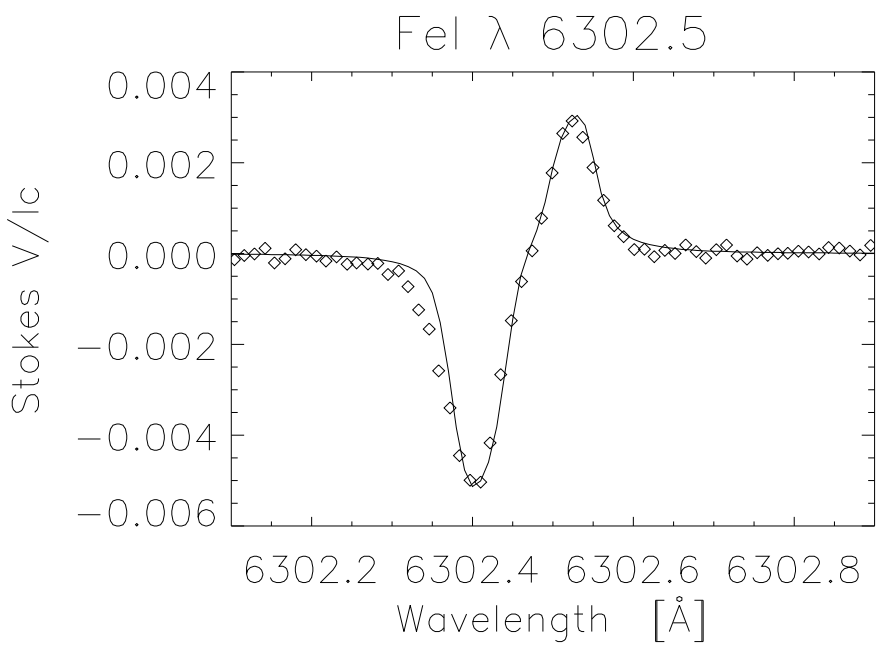

Figure 3. A typical fit for the Stokes $V$ profile of the Fe I $6302.5 \AA$ A line. Diamonds: observations, solid: line fit.

that the magnetic field - as well as the velocity field - in the internetwork can neither by described by macroscopic structures like flux tubes nor can they be described in terms of a microstructured or microturbulent field. An interesting result here is the clear trend for stronger field structures to have larger correlation lengths and for weaker structures to have shorter correlation lengths. This seems to be the result of the increased buoyancy forces of 
strong magnetic structures which results in a preferred vertical alignment and gives rise - for disc center observations - to an increase of the line-of-sight correlation lengths.

\section{Summary}

This analysis of magnetic field structures in the internetwork clearly demonstrates the feasibility of an inversion under the MESMA concept. Moreover, we found that the characteristic length scales in the solar internetwork are relatively small, but clearly far from being microstructured or microturbulent. However, the length scales found in this work are also not consistent with the classical flux tube picture (Stenflo 1994) which would require correlation lengths larger than $350 \mathrm{~km}$. Another intriguing result here is the fact that the obtained correlation lengths of the magnetic structures agrees very well with the autocorrelation (along a vertical direction) of magnetic structures in magnetohydrodynamic simulations (Schaffenberger et al. 2005) which have a mean value of approximately $220 \mathrm{~km}$. This surely deserves further investigations but one can already say that advanced magnetoconvective simulations and (polarized) radiative transfer modeling provide a fruitful combination to gain further insight into the surface magnetism of the sun.

Acknowledgements. We gratefully acknowledge support of this work by the Deutsche Forschungsgemeinschaft (DFG) under the grant CA 475/1-1.

\section{References}

Borrero, J. M. \& Bellot Rubio, L. R. 2002, A\&A, 385, 1056

Carroll, T. A. \& Staude, J. 2003, Astron. Nachr., 324, 392

Carroll, T. A. \& Staude, J. 2005a, Astron. Nachr., 326, 296

Carroll, T. A. \& Staude, J. 2005b, ESA SP-596: Chromospheric and Coronal Magnetic Fields

Carroll, T. A. \& Staude, J. 2006, Solar Polarization 4, eds. R. Casini and B. W. Lites, 19-23 September 2005, National Center for Atmospheric Research, Boulder, Colorado, USA, ISBN: 978-1-58381226-6

Carroll, T. A. \& Kopf, M. 2007, A\&A, submitted

Frisch, H. \& Frisch, U. 1976, MNRAS, 175, 157

Gail, H.-P., Sedlmayr, E., \& Traving, G. 1976, A\&A, 46, 441

Lites, B. W., Leka, K. D., Skumanich, A., Martínez Pillet, V., \& Shimizu, T. 1996, ApJ, 460, 1019

Press, W. H., Teukolsky, S. A., Vetterling, W. T., \& Flannery, B. P. 1992, Cambridge: University Press, c1992, 2nd ed.

Sánchez Almeida, J., Landi Degl'Innocenti, E., Martínez Pillet, V., \& Lites, B. W. 1996, ApJ, 466, 537

Sánchez Almeida, J. 2004, ASP Conf. Ser. 325: The Solar-B Mission and the Forefront of Solar Physics, 325, 115

Schaffenberger, W., Wedemeyer-Böhm, S., Steiner, O., \& Freytag, B. 2005, ESA SP-596: Chromospheric and Coronal Magnetic Fields

Schrijver, C. J. \& Title, A. M. 2003, ApJ, 597, L165

Solanki, S. K. 1993, Space Science Reviews, 63, 1

Stein, R. F. \& Nordlund, Å. 2006, ApJ, 642, 1246

Stenflo, J. O. 1994, Solar magnetic fields: polarized radiation diagnostics, Astrophysics and Space Science Library, Dordrecht; Boston: Kluwer Academic Publishers

Vögler, A., Shelyag, S., Schüssler, M., Cattaneo, F., Emonet, T., \& Linde, T. 2005, A\&A, 429, 335 


\title{
Line ratio method applied to inter-network magnetic fields
}

\author{
E. Khomenko ${ }^{1,2, *}$ and M. Collados ${ }^{1}$ \\ ${ }^{1}$ Instituto de Astrofísica de Canarias, Tenerife, Spain \\ ${ }^{2}$ Main Astronomical Observatory, NAS, Kyiv,Ukraine \\ *Email: khomenko@iac.es
}

\begin{abstract}
We investigate the validity of the Stokes $V$ amplitude ratio as an indicator of the magnetic field strength in solar inter-network regions with the help of MHD simulations. We show that the Stokes $V$ amplitude ratio of the Fe I 15652-15648 $\AA$ lines and Fe I 5247-5250 A lines show a good correlation with the magnetic field strength even for magnetic fields with a complex internal structure like those in MHD simulations. However, in the latter case, the amplitude ratio sub-estimates the magnetic field strength, always revealing sub-kG values. The Stokes $V$ amplitude ratio of the Fe I 6301-6302 A lines shows no correlation with the magnetic field strength. The reasons of this behaviour are explained.
\end{abstract}

\section{Introduction}

It is an open question whether the characteristic field strength in solar inter-network regions lies in the kG or sub-kG range (see, e.g., Lin 1995; Socas-Navarro \& Sánchez Almeida 2002; Khomenko et al. 2003; Domínguez Cerdeña et al. 2006; López Ariste et al. 2006). Since Stenflo (1973), the Stokes $V$ amplitude ratio of the the Fe I 5257, $5250 \AA$ A pair of lines was used as an indicator of the intrinsic field strength of the unresolved network elements. A common assumption used a priory in many observational works about inter-network fields is that the ratio of the Stokes $V$ amplitudes of the Fe I 6301, 6302 and Fe I 15648, 15652 lines is directly related to the field strength contained in the resolution element, i.e. the same as for the Fe I 5257, $5250 \AA$ pair. However, this approach is valid if the pair of spectral lines used for the analysis have exactly the same sensitivity to all atmospheric parameters, except for the magnetic field, and form at the same height. The purpose of this paper is to check the validity of the Stokes $V$ line ratio method for the Fe I 5247-5250 $\AA$, 6301-6302 $\AA$ and 15652-15648 $\AA$ pairs of lines, as applied to the complex fields obtained via MHD simulations, assuming that these fields provide an adequate representation of the fields in solar inter-network regions.

\section{MHD simulations, Stokes spectra synthesis and calibration of the line ratio}

We have used a snapshot of realistic 3D simulations of solar magneto-convection with a bipolar structure of the magnetic field and $\langle|B|>=30 \mathrm{G}$ (see Vögler et al. 2005, for details). The horizontal spatial resolution is $20 \mathrm{~km}$. The Stokes spectra of the Fe I 5247, 5250, 6301, 6302, 15648, and $15652 \AA$ lines formed at solar disc centre $(\mu=1)$ were calculated for 

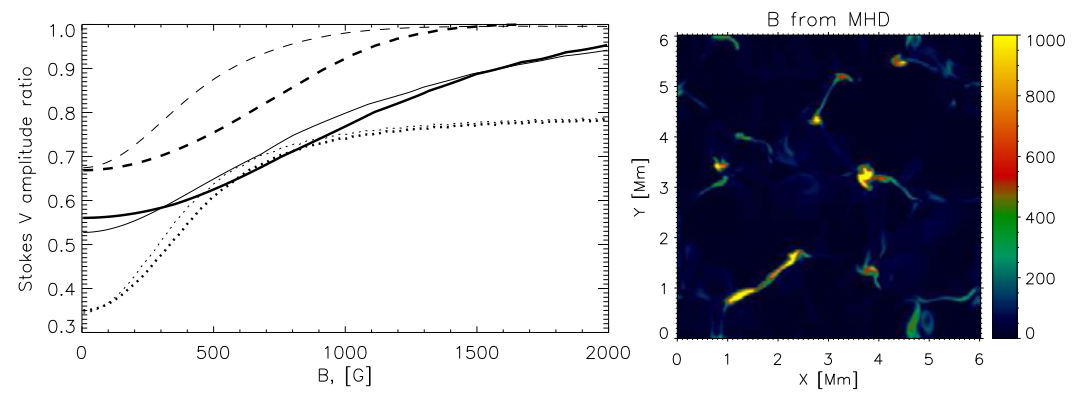

Figure 1. Left: Calibration curves for the line ratio. Thin lines: $V_{\mathrm{mic}}=0$ and $V_{\mathrm{mac}}=0$ (applied to the profiles with original resolution); Thick lines: $V_{\text {mic }}=0.3 \mathrm{~km} \mathrm{~s}^{-1}, V_{\mathrm{mac}}=0.7 \mathrm{~km} \mathrm{~s}^{-1}$ (applied to the profiles with reduced resolution). Solid lines: Fe I 6301, 6302; dashed lines: Fe I 5247, 5250; dotted lines: Fe I 15648, 15652 lines. Right: The map of the longitudinal magnetic field component at $\log \tau_{5}=-1$ in the $30 \mathrm{G}$ flux snapshot from the simulations of Vögler et al. (2005).
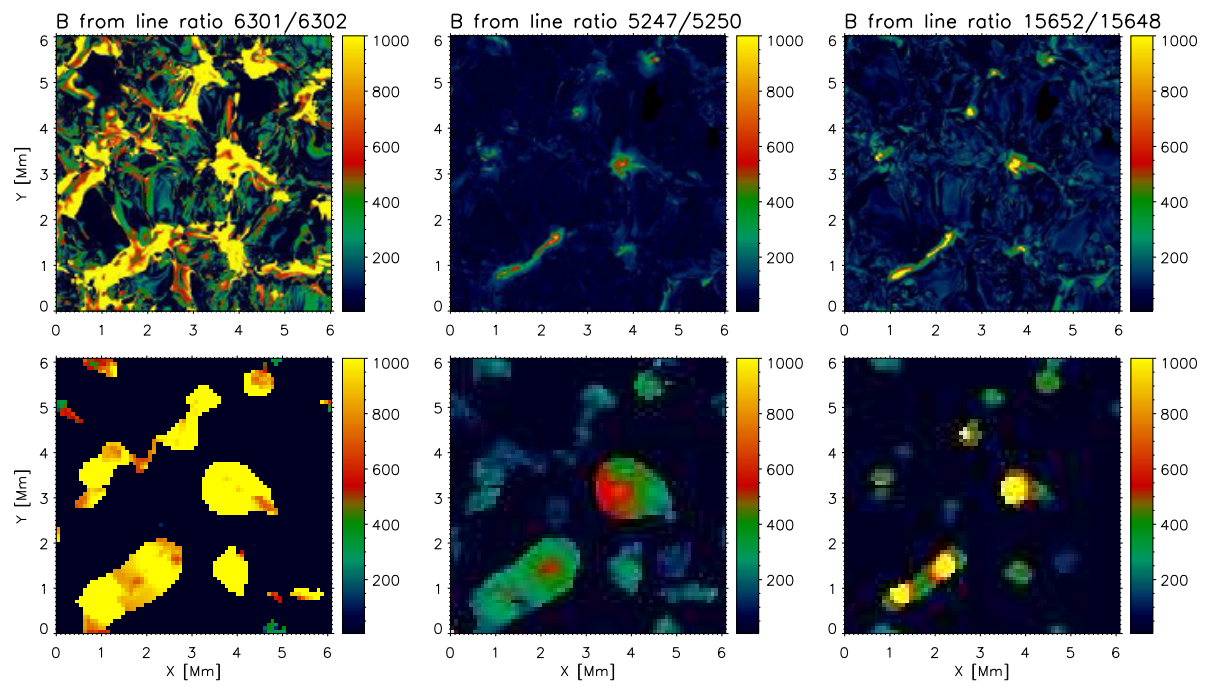

Figure 2. Magnetic field strength obtained from the line ratio of the Fe I 6301 and $6302 \AA$ lines (left), Fe I 5247 and $5250 \AA$ lines (middle) and Fe I 15652 and $15648 \AA$ lines (left). Top panels: Spatial resolution of $20 \mathrm{~km}$. Bottom panels: Spatial resolution of $0.6-0.7$ arcsec. Only profiles with amplitudes above a threshold of $5 \times 10^{-3}$ in units of $I_{c}$ were used.

every vertical column of the selected snapshot. In order to make realistic the comparison of the synthetic spectra with observations, we performed a convolution of the two-dimensional snapshot with an adequate point-spread function decreasing the spatial resolution to about 0.6-0.7 arcsec (Khomenko et al. 2005).

The line ratio is defined as the amplitude of the blue lobe of Stokes $V$ of the line with the smaller Landé factor divided by the amplitude of the line with the larger one. The calibration curves for the line ratio are given in Fig. 1 obtained by radiative transfer calculations in the HSRA model atmosphere varying the constant vertical magnetic field strength. 

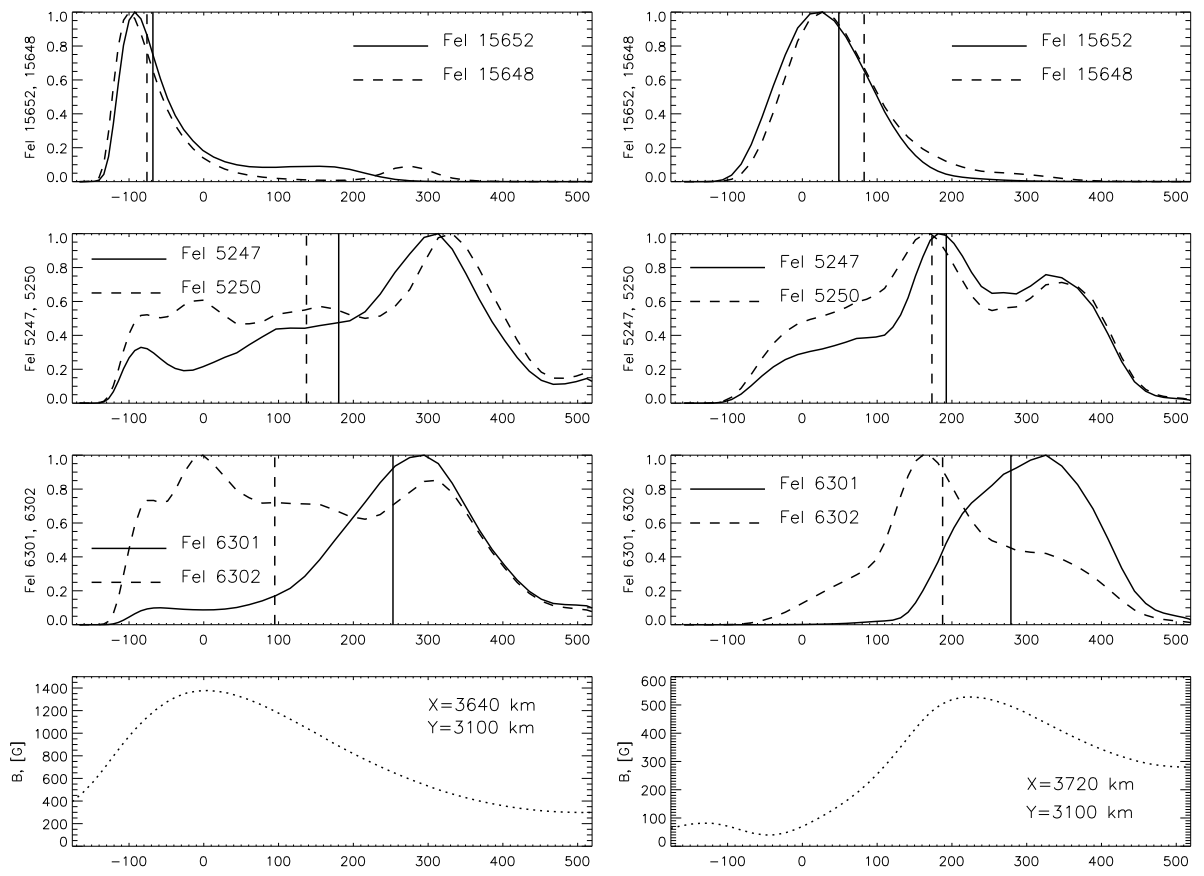

Figure 3. Height variation of the RFs at the wavelength of the line core intensity inside a strong magnetic field concentration (left panels) and in the canopy zone (right panels) at points with coordinates $X$ and $Y$ marked in the bottom panels. The vertical lines are the centroids of the RF's. The bottom panels represent the height variations of $B_{z}$ at the selected points.

\section{Line ratio with full and reduced spatial resolution}

Figure 2 gives maps of the magnetic field strength obtained after the calibration of the line ratio for the different pairs of lines at the original numerical resolution of $20 \mathrm{~km}$ (top panels) and at the reduced resolution (bottom panels). For comparison, the right panel of Fig. 1 gives the longitudinal magnetic field strength at $\log \tau_{5}=-1$.

The locations with strong fields in the original snapshot correspond rather well to locations with the maximum field derived from the line ratio of the Fe I 5247, 5250 and 15648, $15652 \AA$ line pairs. The patches with enhanced line ratio being larger than the actual field concentrations in the case of the reduced spatial resolution. The field strength obtained from the Fe I 5247, 5250 line ratio does not reach $\mathrm{kG}$ values in any point of the simulation snapshot, staying within $600-800 \mathrm{G}$ at those pixels corresponding to $\mathrm{kG}$ fields in the original snapshot. In the case of the Fe I 6301, $6302 \AA$ lines, the field strength derived from the line ratio is largest not where the field is largest, but rather in the canopy regions surrounding the magnetic field concentrations and in the granular-intergranular borders (at the original resolution). The line ratio of the smoothed Fe I 6301-6302 $\AA$ profiles corresponds to kG fields in most of the pixels of the simulation box. The correlation between the "true" field strength and the one derived from the line ratio of the Fe I 6301, 6302 lines is absent. 


\section{Response Functions and regions of formation}

The reason for such a behavior of the line ratio becomes clear in terms of the region of formation of the considered set of lines calculated by means of Response Functions (RFs, see Ruiz Cobo \& del Toro Iniesta 1994). Some examples of the RF's of the Stokes I to temperature at the wavelength of the line core intensity are given in Fig. 3. They are calculated at two points inside a strong magnetic field concentration and at the canopy region, close to it.

According to Fig. 3, the RFs of the infrared lines (top panels) are similar and are rather narrow. This is a very useful property since the magnetic field does not change much within the formation region. The formation regions of the Fe I 5247 and 5250 lines are nearly the same, but the significant contribution comes from a very large portion of the solar atmosphere. The maximum of the contribution appears to come from upper layers, where the magnetic field is already small even inside the $\mathrm{kG}$ fluxtube, thus explaining the absence of the $\mathrm{kG}$ line ratio of these lines in Fig. 2. For the Fe I 6301, 6302 lines, the formation regions are also very extended. In addition, the shapes of the RFs are rather different. Thus, the gradients in physical quantities affect these lines in a different way, leading to Stokes $V$ amplitude ratios that do not carry information about the magnetic field strength. In particular, in the canopy regions (right panels of Fig. 3), the field strength increases with height. It makes the Stoke $V$ amplitude of the Fe I 6301 line to increase with respect to the Fe I 6302 line, leading to the $\mathrm{kG}$ line ratio in the region where the field is sub-kG.

\section{Conclusions}

The Stokes $V$ amplitude ratio of the Fe I 15648-15652 and Fe I 5247-5250 lines give reasonable estimate of the field strength even for fields with complex internal structure as those from MHD simulations. In the conditions appropriate for inter-network, the kG Fe I 52475250 line ratios are improbable due to the maximum sensitivity of these lines in the upper photosphere, where the field is already below $\mathrm{kG}$. Due to the large difference in the formation regions of Fe I 6301 and $6302 \AA$ lines, their Stokes $V$ amplitude ratio is affected by the gradients of the magnetic field, velocity and thermodynamic parameters and does not carry information about the magnetic field strength, showing an excess of $\mathrm{kG}$ values.

Acknowledgements. The authors are grateful to A. Vögler for the kind permission to use 3D model atmosphere for the purposes of our study. This research was funded by the Spanish Ministerio de Educación y Ciencia through project AYA2004-05792.

\section{References}

Khomenko, E. V., Collados, M., Solanki, S. K., Lagg, A., \& Trujillo Bueno, J. 2003, A\&A, 408, 1115

Khomenko, E. V., Shelyag, S., Solanki, S. K., \& Vögler, A. 2005, A\&A, 442, 1059

Lin, H. 1995, ApJ, 446, 421

Domínguez Cerdeña, I., Sánchez Almeida, J., \& Kneer, F. 2006, ApJ, 646, 1421

López Ariste, A., Martínez González, M. J., \& Ramírez, J. 2007, A\&A, in press

Ruiz Cobo, B. \& del Toro Iniesta, J. C. 1994, A\&A, 283, 129

Socas-Navarro, H. \& Sánchez Almeida, J. 2002, ApJ, 565, 1323

Stenflo, J. O. 1973, Solar Phys., 32, 41

Vögler, A., Shelyag, S., Schüssler, M., et al. 2005, A\&A, 429, 335 


\title{
The Ba II $\lambda 4554$ resonance line and solar granulation
}

\author{
V. L. Olshevsky* and N. G. Shchukina \\ Main Astronomical Observatory, National Academy of Sciences, Kyiv, Ukraine \\ *Email: sya@mao.kiev.ua
}

\begin{abstract}
We present the results of an investigation of the impact of NLTE effects and of granulation inhomogeneities on the solar Ba II $\lambda 4554 \AA$ line. Our analysis is based on both the classical one-dimensional (1D) solar atmosphere models and on the new generation of three-dimensional (3D) hydrodynamical models. We show that NLTE and 3D effects have to be taken into account for reliable diagnostics of the solar atmosphere using this line. We analyse the influence of different parameters on the line shape. It turns out to be most sensitive to collisional broadening and barium abundance. Uncertainties in the oscillator strength, micro- and macroturbulence (in 1D-case) have a secondary importance. We have derived the barium abundance assuming NLTE. We find $A_{\mathrm{Ba}}=2.16$ in good agreement with the recent result of Asplund et al. (2005).
\end{abstract}

\section{Introduction}

There are at least two attractive features of the Ba II $\lambda 4554 \AA$ resonance line as a diagnostic tool. Firstly, the line shows conspicuous linear polarization signal around $0.6 \%$ in the atlas of Gandorfer (2002). Apparently, this line seems to be suitable for investigating the Sun's hidden magnetism via the Hanle effect. Secondly, the Ba II $\lambda 4554 \AA$ line is sensitive to the non-thermal motions because its thermal broadening is small. The line formation heights "trace" the photosphere and the lower chromosphere making this line a good indicator of the evolution of granulation structure with height. An example of such a diagnostic is the analysis by Sütterlin et al. (2001) of observations obtained with a barium filter at DOT (Skomorovsky et al. 1976).

Diagnostic usage of the Ba II resonance line requires understanding of its NLTE formation in inhomogeneous models of the solar atmosphere. We stress that after the NLTE analyses made by Rutten $(1977,1978)$ and Rutten \& Milkey (1979), interest on the solar barium spectra has in fact faded. Note that more numerous stellar studies (see, e.g., Mashonkina \& Gehren 2000; Mashonkina et al. 2003; Mashonkina \& Zhao 2006) are restricted by the narrow constraints of $1 \mathrm{D}$ classical modeling and by using NLTE abundance corrections without careful analysis of the physics of formation of the barium spectrum. This is precisely the aim of our contribution where we show results of our multilevel radiative transfer simulations of the Ba II $\lambda 4554 \AA$ line both in 1D and 3D models of the solar atmosphere. 


\section{The method and atomic model}

We carry out our investigation using the NLTE numerical code "NATAJA". The code was developed by Shchukina \& Trujillo Bueno (2001) to facilitate radiative transfer simulations with complex atoms in realistic solar and stellar atmospheric models.

Our atomic model includes 40 energy levels of $\mathrm{Ba}$ I and Ba II. The levels are interconnected by 99 bound-bound and 39 bound-free radiative transitions. We have also taken into account the hyperfine structure and isotopic shift for the Ba II $\lambda 4554 \AA$ line.

\section{One-dimensional case}

We performed our calculations using different "standard" 1D atmospheric models: MACKKL (Maltby et al. 1986), VALC (Vernazza et al. 1981), HOLMUL (Holweger \& Müller 1974). We found that the line shape depends insignificantly on the choice of the model. Thus we present our results only for the MACKKL model.

Figure 1 (right panel) shows that the departure coefficiens $\beta$ of the upper levels of the Ba II resonance doublet ( $\lambda 4554 \AA$ and $\lambda 4934 \AA$ ) and the upper level of the Ba I $\lambda 5535$ resonance line drop rapidly below unity as the height increases. This level underpopulation is mainly due to resonance line scattering and photon losses. Concerning the lower (ground) level of the Ba I $\lambda 5535$ line its underpopulation is caused by another NLTE mechanism known as ultraviolet overionization (Shchukina \& Trujillo Bueno 2001). At the same time the lower level of the Ba II resonance doublet is insensitive to this mechanism. So its population is close to the LTE value. The levels with excitation potential of more than $2 \mathrm{eV}$ are overpopulated because of ultraviolet line pumping. The main consequence of such a behaviour of the departure coefficients is significant deviation of the line source function of the Ba II $\lambda 4554$ resonance line from the Planck function. As a result the line profile becomes deeper than in LTE. Figure 1 (left panel) shows that the NLTE effects are more pronounced around the core of the line, with the LTE approximation producing weaker profiles. The observed line wings could be reproduced under the LTE assumption.

Besides the NLTE effects there are two other parameters which cause appreciable broadening of the wings of the Ba II $\lambda 4554$ resonance line. They are the collisional damping constant and the abundance. The former effect is more important. The main contribution to the damping is given by van der Waals broadening $\gamma_{6}$. We estimated $\gamma_{6}$ using the Deridder $\&$ van Rensbergen (1976) approach with enhancement factor $E=1.5$. In agreement with the conclusion of Mashonkina \& Zhao (2006) we found that the recent calculations of the collisional damping constant made by Barklem \& O'Mara (2000) overestimate $\gamma_{6}$ for this line by a factor 1.5. The near line wings are sensitive as well to the value of microturbulent velocity, while the line core is affected by the macroturbulence. For our calculations we have adopted a "typical" choice of $V_{\text {micro }}=1 \mathrm{~km} \mathrm{~s}^{-1}$ and $V_{\text {micro }}=2 \mathrm{~km} \mathrm{~s}^{-1}$. The line profile appeared to be non-sensitive to the uncertainties in the oscillator strength. Using abovementioned parameters and the abundance $A_{\mathrm{Ba}}=2.13$ (on a logarithmic scale with $A_{\mathrm{H}}=12.0$ ) we can reach a good agreement with the observed profile, except for the line core. 
V. L. Olshevsky and N. G. Shchukina: Ba II $\lambda 4554$ resonance line and solar granulation 309
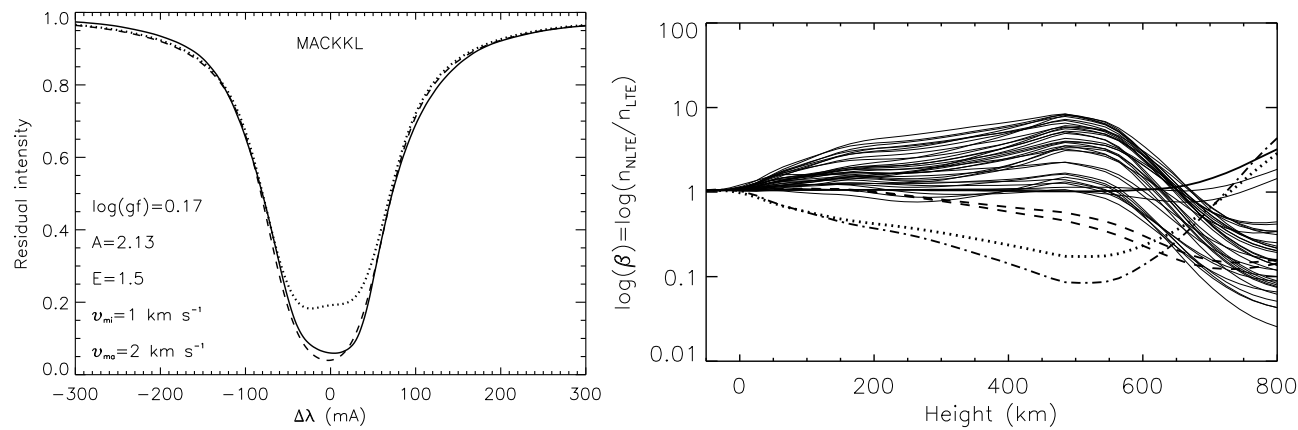

Figure 1. Left panel: line profiles for the MACKKL model calculated under LTE assumption (dotted line), NLTE (dashed line). Solid thick line: observations taken from Liège Atlas (Delbouille et al. 1973). Right panel: Departure coefficients $(\beta)$ vs. height in the 1D atmospheric model MACKKL. The thick solid and dashed lines are the $\beta$ coefficients for the lower (ground) and the upper levels of the Ba II resonance doublet $\lambda 4554$ and $\lambda 4934 \AA$, respectively. Dotted and dashed-dotted lines: $\beta$ coefficients for the lower and upper levels of the $\mathrm{Ba}$ I resonance line $\lambda 5535 \AA$. Thin solid lines: $\beta$ coefficients of $\mathrm{Ba}$ II levels with excitation potential above $2 \mathrm{eV}$.
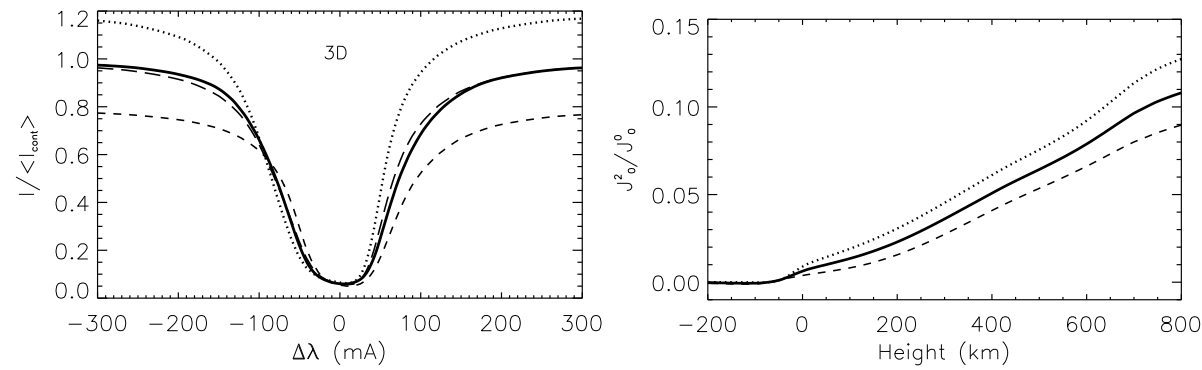

Figure 2. Left panel: the profiles in 3D-model spatially averaged over granules(dotted), over intergranules (dashed) and over the whole surface (long dashes). Solid curve: observations taken from the Liège Atlas. Right panel: the mean anisotropy in 3D-model vs. height in granules (dotted); intergranules (dashed) and the total anisotropy.

\section{Three-dimensional case}

We have used a 3D snapshot model taken from realistic radiation hydrodynamical simulations of the solar surface convection by Asplund et al. (2000). Such simulations remove the need for fudge parameters like micro- and macroturbulence.

In the 3D-case the Ba II $\lambda 4554$ line profile suffers most from uncertainties in the damping enhancement factor $E$ followed by the abundance uncertainties. We can reach an excellent agreement with observations even in the line core (left panel in Fig. 2). The best fitted parameters are $A_{\mathrm{Ba}}=2.16$ and $E=1.5$. Figure 2 shows that granular and intergranular profiles differ from each other. Granules are brighter and blueshifted, the opposite effect taking place for the intergranules. The Doppler shifts cause an "inversion" effect in the left wing of the profile between $\Delta \lambda=-20$ and $-80 \mathrm{~m} \AA$ : in filtergrams the intergranules can be 
brighter than the granules.

Another difference between granules and intergranules reveals itself in the intensity anisotropy parameter: $J_{0}^{2} / J_{0}^{0}$ (right panel in Fig. 2). The anisotropy in the granules is higher than in the intergranules. Since the Stokes $\mathrm{Q}$ value is proportional to this parameter, the effect must be taken into account in the analysis of the solar small-scale magnetic fields.

\section{Conclusions}

The solar granulation inhomogeneities and NLTE effects are found to be important for the Ba II $\lambda 4554$ line formation. Granular and intergranular profiles are very different. Departures from LTE are larger in the hot granules than in the cooler intergranules. The intensity anisotropy appears to be higher in granules than in intergranules, which is important for the Stokes diagnostics. The wings of this line are sensitive to the uncertainties in the collisional damping and the abundance. More comprehensive analyses of all these results will be presented in future publications.

Acknowledgements. V.L.O. acknowledges financial support by the Deutsche Forschungsgemeinschaft for participation in the workshop.

\section{References}

Asplund, M., Ludwig, H.-G., Nordlund, Å., \& Stein, R. F. 2000, A\&A, 359, 669

Asplund, M., Grevesse, N., \& Sauval, A. J. 2005, in Cosmic Abundances as Records of Stellar Evolution and Nucleosynthesis, ed. T. G. Barnes III \& F. N. Bash., ASP Conf. Ser., 336, 25

Barklem, P. S. \& O’Mara, B. J. 2000, MNRAS, 311, 535

Delbouille, L., Roland, G., \& Neven, L. 1973, Atlas photométrique du spectre solaire de $\lambda 3000$ à $\lambda 10000$ (Liège: Institut d'Astrophysique de l'Université de Liège)

Deridder, G. \& van Rensbergen, W. T. 1976, A\&AS, 23, 147

Gandorfer, A. 2002, The Second Solar Spectrum: A high spectral resolution polarimetric survey of scattering polarization at the solar limb in graphical representation. Volume II: $3910 \AA$ to $4630 \AA$ (Zürich: Hochschulverlag)

Holweger, H. \& Müller, E. A. 1974, Solar Phys., 39, 19

Maltby, P., Avrett, E. H., Carlsson, M., Kjeldeseth-Moe, O., Kurucz, R. L., \& Loeser, R. 1986, ApJ, 306, 284

Mashonkina, L. \& Gehren, T. 2000, A\&A, 364, 249

Mashonkina, L. \& Zhao, G. 2006, A\&A, 456, 313

Mashonkina, L., Gehren, T., Travaglio, C., \& Borkova, T. 2003, A\&A, 397, 275

Miles, B. M. \& Wiese W. L. 1969, Atomic Data, 1, 1

Rutten R. J. 1977, Solar Phys., 51, 3

Rutten R. J. 1978, Solar Phys., 56, 237

Rutten R. J. \& Milkey R. W. 1979, ApJ, 231, 277

Skomorovsky, V., Merkulenko, V., \& Poliakov, V. 1976, Solnechnye dannye, 5, 73

Shchukina, N. G. \& Trujillo Bueno, J. 2001, ApJ, 550, 970

Sütterlin, P., Rutten, R. J., \& Skomorovsky, V. I. 2001, A\&A, 378, 251

Vernazza, J. E., Avrett, E. H., \& Loeser, R. 1981, ApJS, 45, 635 
Modern Solar Facilities - Advanced Solar Science, 311-316

F. Kneer, K. G. Puschmann, A. D. Wittmann (eds.)

(C) Universitätsverlag Göttingen 2007

\title{
Magnetic mappers of the quiet solar atmosphere
}

\author{
J. Trujillo Bueno ${ }^{1,2}$ \\ ${ }^{1}$ Instituto de Astrofísica de Canarias, La Laguna, Tenerife, Spain \\ ${ }^{2}$ Institut für Astrophysik, Göttingen, Germany \\ Email: jtb@iac.es
}

\begin{abstract}
The magnetic sensitivity of the solar spectrum is caused by the Zeeman effect and by a variety of less familiar physical mechanisms by means of which a magnetic field can create and destroy spectral line polarization. This paper highlights how plasma diagnostic tools based on them could help us to explore the magnetism of the "quiet" regions of the solar photosphere and chromosphere. A few suggestions for increasing the discovery potential of some telescopes and polarimeters are also given.
\end{abstract}

\section{Introduction}

Solar magnetic fields leave their fingerprints in the state of polarization of the emergent electromagnetic radiation. This occurs through a variety of rather unfamiliar physical mechanisms, not only via the Zeeman effect. In particular, in stellar atmospheres there is a more fundamental mechanism producing polarization in spectral lines. There, where light escapes through the stellar "surface", the atoms and molecules are illuminated by an anisotropic radiation field. The ensuing radiation pumping produces population imbalances among the magnetic substates of the energy levels (that is, atomic level polarization), in such a way that the population of substates with different values of $|M|$ are different. This is termed atomic level alignment. As a result, the emission process can generate linear polarization in spectral lines without the need for a magnetic field. This is known as scattering line polarization. However, light polarization components will also be selectively absorbed when the lower level of the transition is polarized (Trujillo Bueno \& Landi Degl'Innocenti 1997; Manso Sainz \& Trujillo Bueno 2003). Thus, the medium becomes dichroic simply because the light itself is escaping from it.

In summary, the mere presence of atomic level polarization can generate spectral line polarization. The interesting point is that a magnetic field modifies the atomic level polarization via the Hanle effect, where the magnetic field $B$ (in $\mathrm{G}$ ) that produces a significant change is (e.g., Landi Degl'Innocenti \& Landolfi 2004)

$$
B_{H} \approx 1.137 \times 10^{-7} /\left(t_{\text {life }} g_{J}\right)
$$

( $t_{\text {life }}$ and $g_{J}$ being the lifetime, in seconds, of the $J$-level under consideration and its Landé factor, respectively). Since the lifetimes of the upper levels $\left(J_{u}\right)$ of the transitions of interest are usually much smaller than those of the lower levels $\left(J_{l}\right)$, it is clear that diagnostic tech- 
niques based on the lower-level Hanle effect are sensitive to much weaker fields than those based on the upper-level Hanle effect.

\section{The Zeeman effect vs. the Hanle effect}

The most important mechanisms for magnetic field diagnostics in the atmospheres of the Sun and of other stars are the Zeeman effect, scattering processes and the Hanle effect.

The advantage of the Zeeman effect as a diagnostic tool is that the mere detection of polarization implies the presence of a magnetic field. The bad news are the following. First, it is of limited practical interest for the determination of magnetic fields in hot (chromospheric and coronal) plasmas because the Zeeman polarization scales with the ratio between the Zeeman splitting and the Doppler-broadened line width. Second, the Zeeman effect is blind to magnetic fields that are tangled on scales too small to be resolved.

Concerning the Hanle effect, one of the good news is that it is sensitive to weaker magnetic fields than the Zeeman effect: from at least $1 \mathrm{mG}$ to hundreds of gauss (see Eq. 1). Moreover, it is sensitive to the presence of hidden, mixed-polarity fields at sub-resolution scales. Finally, it is important to point out that the diagnostic use of the Hanle effect is not limited to a narrow solar limb zone. In particular, in forward scattering at disk center, the Hanle effect can create linear polarization, in the presence of inclined magnetic fields. The bad news is that the Hanle effect signal saturates for magnetic strengths $B>10 B_{H}$. For example, for the typical case of the Sr I $4607 \AA$ line (whose $B_{H} \approx 23 \mathrm{G}$ ) we have that a tangled magnetic field of $250 \mathrm{G}$ produces an amount of Hanle depolarization similar to that of a tangled magnetic field of $1000 \mathrm{G}$. In other words, the Hanle effect does not allow us to determine the fraction of quiet Sun volume occupied by kG fields.

Finally, it is also useful to consider the Zeeman broadening of the intensity profiles, which is proportional to the squared modulus of the magnetic field strength - that is, it can also give us information on the presence of tangled magnetic fields at subresolution scales. The main disadvantage is that it is difficult to disentangle the Zeeman line broadening effect from that due to the thermal and convective motions. It is also rather insensitive to weak magnetic fields.

\section{Magnetic mappers of the quiet solar photosphere}

An important point is that the Hanle effect can be suitably complemented with the Zeeman effect, not only with the Zeeman-induced polarization (which, as mentioned above, is invisible to magnetic fields that are tangled on scales too small to be resolved), but also with the Zeeman broadening of the intensity profiles of some carefully selected near-IR lines (for instance, the Mn I line at $15262.70 \AA$ considered in Fig. 1). The combination of the Hanle and Zeeman effects (including the Zeeman line broadening) offers an attractive tool to obtain information on the distribution of tangled magnetic fields at sub-resolution scales in the quiet regions of the solar photosphere (see the recent review by Trujillo Bueno et al. 2006).

For example, a detailed theoretical analysis of observations of the Hanle effect in $\mathrm{C}_{2}$ lines

and in the Sr I $4607 \AA$ line has led to the conclusion that the strength of the hidden magnetic field fluctuates on the spatial scales of the solar granulation pattern, with rather weak fields in the (granular) upflowing regions and much stronger fields in the (intergranular) downflowing 

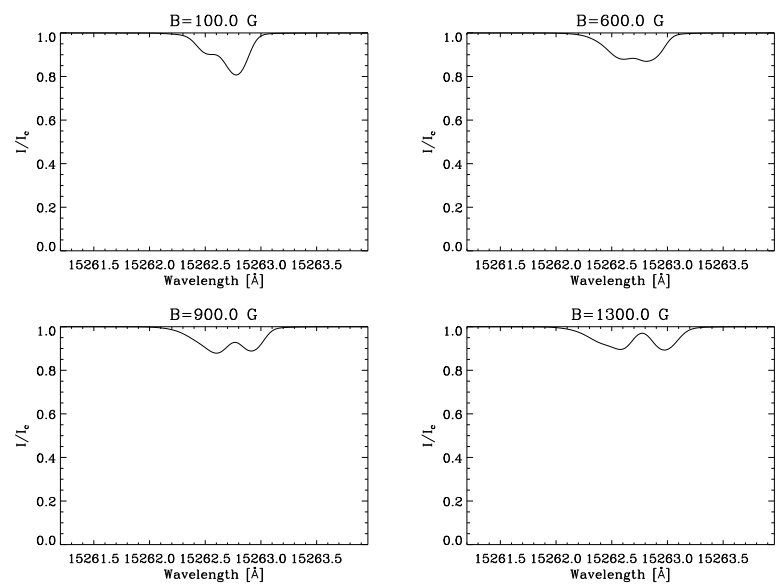

Figure 1. Synthetic Stokes I profiles of the Mn I line at $15262.7 \AA$ calculated in a magnetized MilneEddington atmosphere for 100, 600, 900 and $1300 \mathrm{G}$. Note the presence of distortions in Stokes $I$, and how these features change as the magnetic field strength is increased. While the Stokes $V$ profile of this line is insensitive to magnetic fields that are tangled on scales too small to be resolved, the Zeeman broadening of its Stokes I profile (which is seen clearly in this near-IR line thanks to the presence of level crossings between the hyperfine structured sublevels of $\mathrm{Mn} \mathrm{I}$ ) is sensitive to such hidden fields, especially for $100<B<600$ G. For more information see Asensio Ramos et al. (2007).

plasma. The inferred mean strength of the hidden field is $\langle B\rangle \sim 100 \mathrm{G}$, which implies an amount of magnetic energy density that is more than sufficient to compensate the energy losses of the solar outer atmosphere (Trujillo Bueno et al. 2004; 2006).

\section{Magnetic mappers of the quiet solar chromosphere}

Most of the chromospheric lines that can be observed from the ground are broad, which implies that the magnetic fields of the quiet solar chromosphere are difficult to diagnose via the only consideration of the Zeeman effect. Let us show some more suitable tools.

\subsection{The Hanle effect in the Ca II IR triplet}

An important diagnostic window for investigating the magnetism of the quiet solar chromosphere is the Hanle effect in the Ca II IR triplet (Manso Sainz \& Trujillo Bueno 2007). Interestingly, while the scattering polarization signal $(Q / I)$ in the $8498 \AA$ line shows a strong sensitivity to inclined magnetic fields with strengths between $1 \mathrm{mG}$ and $10 \mathrm{G}$, the emergent $Q / I$ in the $8542 \AA$ and $8662 \AA$ lines is sensitive to magnetic fields in the milligauss range (i.e., between $10^{-3}$ and $10^{-1} \mathrm{G}$ ). The reason for this very interesting behavior is that the $Q / I$ in the $8498 \AA$ line is dominated by the selective emission processes that result from the atomic polarization of the short-lived upper level, while the $Q / I$ in the $8542 \AA$ and $8662 \AA$ lines is dominated by the selective absorption processes that result from the atomic polarization of the long-lived lower levels (Manso Sainz \& Trujillo Bueno 2003). It is also important 


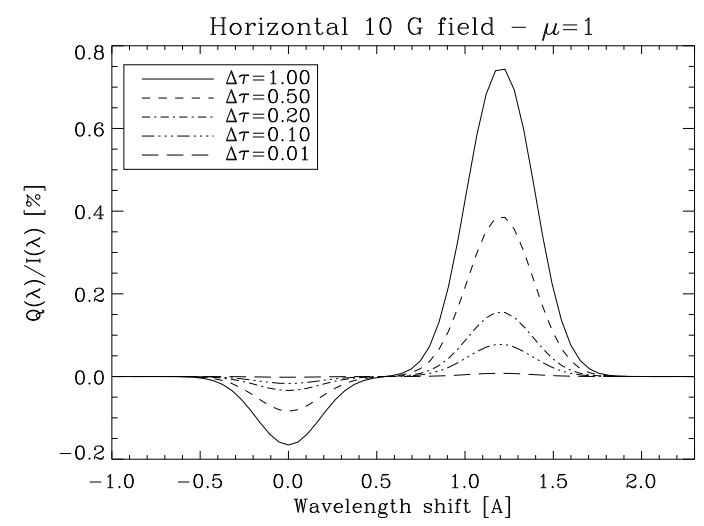

Figure 2. The emergent fractional polarization at the solar disk center in the lines of the He I 10830 $\AA$ multiplet, assuming that a constant-property slab of helium atoms at a height of about $2000 \mathrm{~km}$ is permeated by a horizontal magnetic field of $10 \mathrm{G}$. The various $Q / I$ profiles correspond to the slab's optical thickness indicated in the inset, calculated at the wavelength of the red blended component. The positive reference direction for the definition of the Stokes $Q$ parameter is along the magnetic field vector. For more information see Trujillo Bueno \& Asensio Ramos (2007).

to mention that the calculated polarization amplitudes are very sensitive to the assumed thermal model of the solar chromosphere (see Fig. 4 of Manso Sainz \& Trujillo Bueno 2001). In conclusion, simultaneous observations of the scattering polarization in the Ca II IR triplet should be carried out in order to use them as a sensitive thermometer and magnetometer of the quiet regions of the solar chromosphere.

Suggestion-1: Develop the instrument SUPOS of SUNRISE thinking in measuring simultaneously at least any of the following two line pairs of the Ca II IR triplet (i.e., not only the $8542 \AA$ line): $8498 \AA$ and $8542 \AA$ or $8498 \AA$ and $8662 \AA$.

\subsection{Forward scattering in the $\mathrm{He}_{\mathrm{I}} 10830 \AA$ A multiplet}

As mentioned in Section 2, in the presence of an inclined magnetic field forward scattering processes can produce linear polarization signatures in spectral lines. In this geometry, the polarization signal is created by the Hanle effect, a physical phenomenon that has been clearly demonstrated via spectropolarimetry of solar coronal filaments in the He $10830 \AA$ multiplet (Trujillo Bueno et al. 2002a).

Figure 2 shows theoretical examples of the emergent fractional linear polarization in the lines of the He I $10830 \AA$ multiplet, assuming a constant-property slab of helium atoms permeated by a horizontal magnetic field of $10 \mathrm{G}$. As expected, the smaller the optical thickness of the assumed plasma structure the smaller the fractional polarization amplitude. In principle, the Tenerife Infrared Polarimeter allows the detection of very low amplitude polarization signals, such as those corresponding to the $\Delta \tau=0.1$ case of Fig. 2. However, in order to be able to achieve this goal without having to sacrifice the spatial and/or temporal resolution we need a large aperture solar telescope. Certainly, a promising investigation would be to explore the topology of the chromospheric magnetic field via this novel diagnostic window. 
Suggestion-2: Develop a polarimeter capable of measuring simultaneously the spectral lines of the following two multiplets of He r: $10830 \AA$ and $5876 \AA$. The reasons for including the $\mathrm{D}_{3}$ multiplet at $5876 \AA$ can be found in Trujillo Bueno et al. (2005a).

\subsection{Forward scattering in the $\mathrm{K}$ line of $\mathrm{Ca}$ II}

There are more opportunities for mapping the magnetic fields of the solar chromosphere via the Hanle effect in forward scattering at the solar disk center. For example, a spectral line of great interest for chromospheric diagnostics is the $\mathrm{K}$ line of Ca II, whose forward scattering polarization has already been measured (see Stenflo 2006). Since the K line at $3934 \AA$ has $J_{l}=1 / 2$ and $J_{u}=3 / 2$ its scattering polarization is totally caused by the selective emission processes from the polarized upper level. Its fractional linear polarization at the line center should be sensitive to inclined magnetic fields with $B \leq 10 \mathrm{G}$. On the contrary, the Ca II $\mathrm{H}$ line at $3969 \AA$ is of little practical interest for the exploration of the magnetism of the quiet solar chromosphere given that its lower and upper levels cannot be aligned (because $J_{l}=J_{u}=1 / 2$ ).

Suggestion-3: Modify the polarimeter POLIS in order to measure with the VTT and GREGOR the $\mathrm{K}$ line of $\mathrm{Ca}$ II, instead of the $\mathrm{H}$ line.

\subsection{Enhancement of scattering polarization by vertical fields}

The common belief that scattering polarization is insensitive to vertical magnetic fields does not apply to transitions between spectral terms whose $J$-levels (or $F$-levels for hyperfine structured multiplets) cross and anticross in a given range of magnetic field strengths. Thus, the scattering polarization in the $\mathrm{Na}_{\mathrm{I}} \mathrm{D}_{2}$ line increases steadily with the magnetic strength, for vertical fields between about 10 and 100 gauss (Trujillo Bueno et al. 2002b). Another very interesting example is shown in Fig. 3, which gives the emergent fractional linear polarization of the $\mathrm{BaII} \mathrm{D}_{2}$ line in $90^{\circ}$ scattering geometry for increasing values of the magnetic strength of the assumed vertical field. The $\mathrm{Ba}_{\mathrm{II}} \mathrm{D}_{2}$ line at $4554 \AA$ is particularly interesting because the emergent linear polarization has contributions from different isotopes that are easily resolved and have a different behavior in the presence of a magnetic field. As a result, there is a differential magnetic sensitivity of the emergent linear polarization at the line center (where the signal is produced by the even isotopes without level crossings) with respect to the line wings (where the signals are produced by the odd isotopes with crossings and anti-crossings between the ensuing hyperfine levels). Therefore, the measurement and modeling of spectropolarimetric observations in the Ba II $\mathrm{D}_{2}$ line could be a very useful diagnostic tool to obtain empirical information on the spatial fluctuations of the magnetic field vector in the lower solar chromosphere.

\section{Concluding comments}

As we have seen, we have novel diagnostic opportunities for mapping the hidden magnetic fields of the quiet solar photosphere and chromosphere. To this end, it is now urgent to develop a large aperture ground-based solar telescope optimized for spectropolarimetric observations. We could also have a powerful coronal magnetic mapper if a UV/EUV polarimeter is developed for a solar space telescope (see Trujillo Bueno et al. 2005b). 


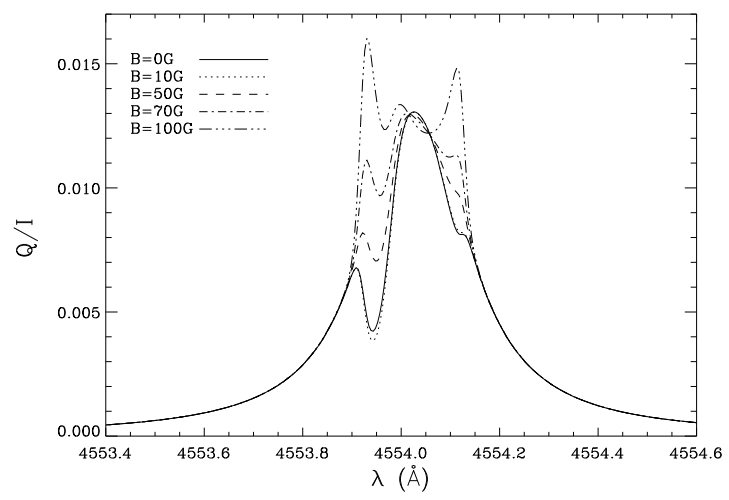

Figure 3. Theoretical calculation of the emergent linear polarization of the $\mathrm{Ba}$ II $\mathrm{D}_{2}$ line in $90^{\circ}$ scattering geometry for increasing values of the assumed vertical magnetic field. The positive reference direction for Stokes $Q$ is the parallel to the solar limb. For more information see Belluzzi et al. (2006).

Acknowledgements. I would like to thank the Akademie der Wissenschaften zu Göttingen for having granted me the Gauss-Professur for a sabbatical stay at the Institut für Astrophysik.

\section{References}

Asensio Ramos, A., González, M., López Ariste, A., Trujillo Bueno, J., \& Collados, M. 2007, ApJ, in press

Belluzzi, L., Trujillo Bueno, J., \& Landi Degl'Innocenti, E. 2006, in Solar Polarization 4, ed. R. Casini \& B. W. Lites, ASP Conf. Series, in press

Landi Degl'Innocenti, E. \& Landolfi, M. 2004, Polarization in Spectral Lines, Kluwer Academic Publishers

Manso Sainz, R. \& Trujillo Bueno, J. 2001, in Advanced Solar Polarimetry: Theory, Observation and Instrumentation, ed. M. Sigwarth, ASP Conf. Series, 236, 213

Manso Sainz, R. \& Trujillo Bueno, J. 2003, Phys. Rev. Letters, 91, 111102-1

Manso Sainz, R. \& Trujillo Bueno, J. 2007, in The Physics of Chromospheric Plasmas, ed. P. Heinzel, I. Dorotovič, \& R. J. Rutten, ASP Conf. Series, in press

Stenflo, J. O. 2006, in Solar Polarization 4, ed. R. Casini \& B. W. Lites, ASP Conf. Series, in press

Trujillo Bueno, J. \& Landi Degl'Innocenti, E. 1997, ApJ, 482, L183

Trujillo Bueno, J., Landi Degl'Innocenti, E., Collados, M., Merenda, L., \& Manso Sainz, R. 2002a, Nature, 415, 403

Trujillo Bueno, J., Casini, R., Landolfi, M., \& Landi Degl'Innocenti, E. 2002b, ApJ Letters, 566, L53

Trujillo Bueno, J., Shchukina, N., \& Asensio Ramos, A. 2004, Nature, 430, 326

Trujillo Bueno, J., Merenda, L., Centeno, R., Collados, M., \& Landi Degl'Innocenti, E. 2005a, ApJ Letters, 619, L191

Trujillo Bueno, J., Landi Degl'Innocenti, E., Casini, R., \& Martínez Pillet, V. 2005b, in Trends in Space Science and Cosmic Vision 2020, ed. F. Favata et al., ESA Publications Division Vol. SP-588, 203

Trujillo Bueno, J., Asensio Ramos, A., \& Shchukina, N. 2006, in Solar Polarization 4, ed. R. Casini \& B. W. Lites, ASP Conf. Series, 358, 269

Trujillo Bueno, J. \& Asensio Ramos, A. 2007, ApJ, 655, 642 


\title{
Molecular Hanle effect in the Paschen-Back regime: theory and application
}

\author{
A. I. Shapiro ${ }^{1, *}$, S. V. Berdyugina ${ }^{1,2}$, D. M. Fluri ${ }^{1}$, and J. O. Stenflo ${ }^{1,3}$ \\ ${ }^{1}$ Institute of Astronomy, ETH Zurich, Zurich, Switzerland \\ ${ }^{2}$ Tuorla Observatory, University of Turku, Piikkiö, Finland \\ ${ }^{3}$ Faculty of Mathematics \& Science, University of Zurich \\ *Email: shapiro@astro.phys.ethz.ch
}

\begin{abstract}
The second solar spectrum resulting from coherent scattering is a main tool for diagnostics of turbulent magnetic fields on the Sun. Scattering on diatomic molecules plays an important role in forming this spectrum and even dominates in some spectral regions. In a magnetic field electronic states of a molecule are often perturbed via the Paschen-Back effect. Sometimes this perturbation can completely change the spectrum, not only quantitatively, but even qualitatively. Here we calculate molecular scattering properties taking into account the Paschen-Back effect. We calculate the Mueller matrix for coherent scattering at diatomic molecules in the intermediate Hund's case (a-b) and look for the effects that can be caused by the Paschen-Back effect. We have found a considerable deviation from the Zeeman regime and discuss here the quantitative and qualitative effects on observed polarization signals for the CN $B^{2} \Sigma-X^{2} \Sigma$ system as an example. We show an application of the Hanle effect for the interpretation of observations of the $\mathrm{CN}$ violet system, from which we determine a turbulent magnetic field strength of about $13 \mathrm{G}$.
\end{abstract}

\section{Introduction}

The properties of the coherent scattering process are modified in a magnetic field via the Hanle effect, which provides us with a very sensitive tool for studying the distribution of weak magnetic fields on the Sun and gives the possibility to measure spatially unresolved mixed polarity turbulent magnetic fields using the second solar spectrum. Such measurements are impossible with the Zeeman effect because of the Stokes $V$ cancellation.

Scattering on molecules plays an important role in forming the second solar spectrum and even dominates in some spectral regions. Sensitivities of the molecular lines to the Hanle effect significantly vary with the total molecular angular momentum. This allows us to employ the differential Hanle effect (cf. Stenflo et al. 1998; Trujillo Bueno 2003; Berdyugina \& Fluri 2004). The recent analysis of molecular scattering polarization (Berdyugina et al. 2002; Faurobert \& Arnaud 2003; Landi Degl'Innocenti 2003; Trujillo Bueno 2003; Asensio Ramos \& Trujillo Bueno 2005; Bommier et al. 2007) and the first detection of the Hanle effect in diatomic molecular lines on the Sun (Berdyugina \& Fluri 2004) revealed the clear advantages of molecular lines for studying turbulent magnetic fields by means of the differential Hanle effect.

The Hanle effect is most sensitive when the magnetic splitting is comparable to the nat- 

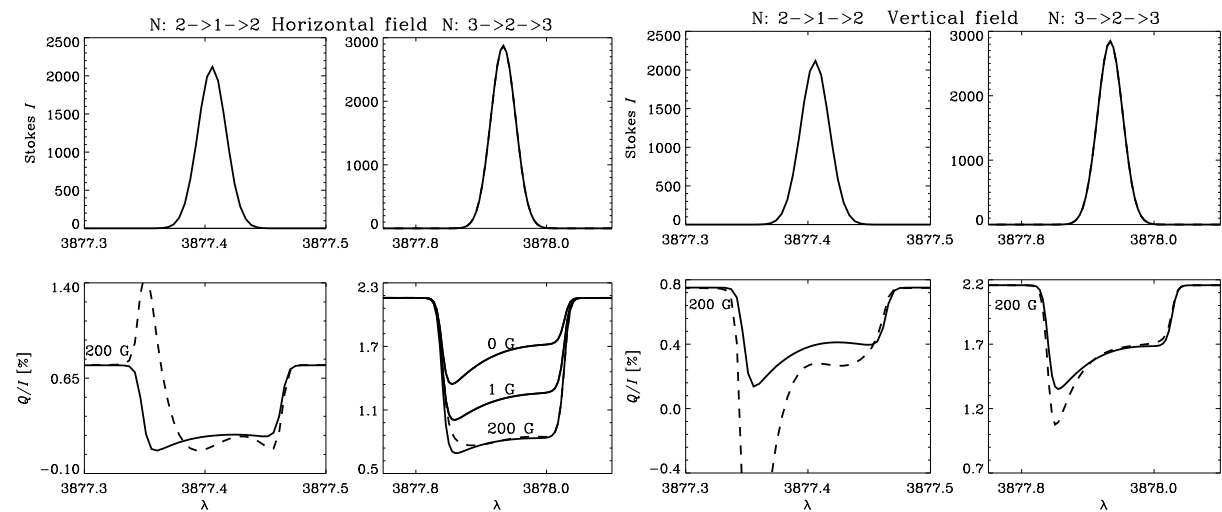

Figure 1. Scattering profiles for a whole doublet, in the $(0,0)$ band of the $\mathrm{CN}$ violet system. Solid and dashed curves represent the PBR and ZR cases, respectively. In the two left panels the magnetic field is perpendicular to the line of sight and in the plane of the solar surface (horizontal field). In the two right panels the magnetic field is perpendicular to the solar surface and to the line of sight (vertical field). We assumed a Doppler width $\Delta \lambda_{\mathrm{D}}=25 \mathrm{~m} \AA$, and a Voigt parameter $a=0.0001$. Figure from Shapiro et al. (2007b).

ural line width. For most atomic transitions this implies weak magnetic fields, so that the level splitting can be calculated in the Zeeman effect regime (ZR). For many molecular transitions even weak magnetic fields can significantly perturb the internal molecular structure and change wave functions of molecular levels. Therefore, the level splitting and amplitudes of the transitions should be calculated taking into account the Paschen-Back effect (PBE) regime (Berdyugina et al. 2005; Asensio Ramos \& Trujillo Bueno 2006).

Previously the Hanle effect in the Paschen-Back regime (PBR) was treated only for atomic transitions by Bommier (1980) and Landolfi \& Landi Degl'Innocenti (1985), who studied the Hanle effect on level-crossings for the helium $\mathrm{D}_{3}$ and sodium $\mathrm{D}$ lines of solar prominences.

For the first time we couple the PBE with the Hanle effect in molecular lines and discuss briefly the main alteration in the Hanle effect theory caused by the PBE with the example of the $\mathrm{CN} B^{2} \Sigma-X^{2} \Sigma$ system, which is strongly influenced by the PBE. We apply this theory for the interpretation of observations of the $\mathrm{CN}$ violet system and show first results of our technique.

\section{Alterations due to the PBE}

The efficiency of the Hanle effect strongly depends on the magnetic displacement and on the transition amplitudes. Both these quantities are subject to the PBE which results from the perturbation between different molecular or atomic states in the presence of a magnetic field.

In this section we present calculated scattering profiles of $\mathrm{CN}$ transitions for several values of magnetic fields. We assume an unpolarized incident radiation field and single scattering at an angle of $90^{\circ}$. Collisional depolarization and continuum opacity are neglected. We choose the magnetic field direction to be perpendicular to the line of sight such that the Stokes $U$ and $V$ signals equal to zero. 

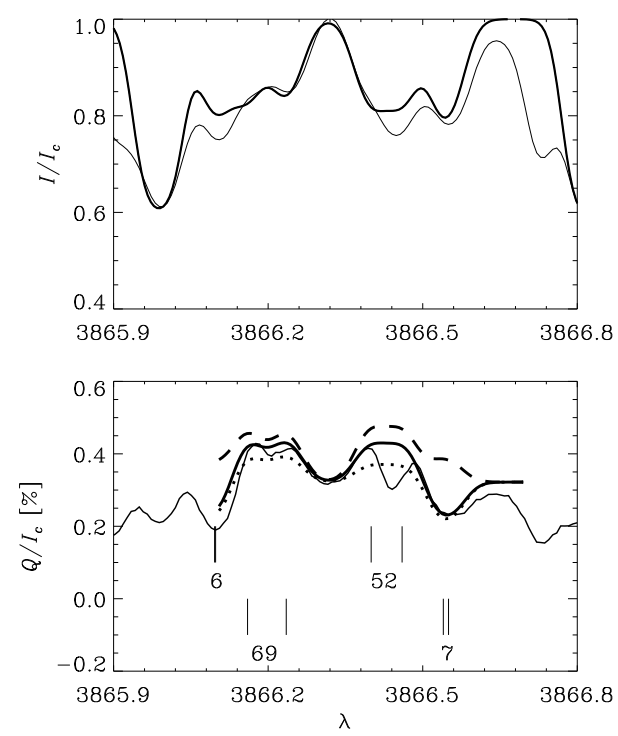

Figure 2. Fits to the observations (thin solid lines in both panels) of Stokes $I / I_{\mathrm{c}}$ and $Q / I_{c}$ in the $\mathrm{CN}$ violet system. The assumed magnetic field in the calculated spectra, going from the curves with larger to the ones with smaller $Q / I_{\mathrm{c}}$, are: $0 \mathrm{G}$ (no depolarization, thick dashed line), $13 \mathrm{G}$ (best fit result, thick solid line) and $25 \mathrm{G}$ (too much depolarization, thick dotted line).

In Fig. 1 we present doublet scattering profiles for $P(2) \quad(N=2 \rightarrow 1 \rightarrow 2)$ and $P(3)$ $(N=3 \rightarrow 2 \rightarrow 3)$ transitions in the presence of a horizontal (two left panels) and vertical (two right panels) magnetic field. The PBE profiles differ significantly from the ZR case. First of all, the PBE modifies the profile shape in polarization. Furthermore, the integrated line depolarization is considerably influenced by the PBE. In PBR, in contrast to the ZR, even the saturated polarization is magnetic field dependent (see detailed discussion in Shapiro et al. 2007b). It is well-known that the Hanle effect is absent for a vertical field direction (cf. Stenflo 1994; Landi Degl'Innocenti \& Landolfi 2004), but because of PBE the depolarization will also depend on the magnetic field strength.

Note that the line profiles of the individual doublet components significantly change due to the PBE already for a magnetic field strength of $100 \mathrm{G}$ for levels with small $J$ values. These modifications are however smeared by Doppler broadening and the influence from the second doublet component.

\section{Interpretation of the $\mathrm{CN}$ violet system in the second solar spectrum}

As application of our theory we present here the interpretation of the $\mathrm{CN}$ violet system observations in a quiet area near the solar limb presented in the atlas by Gandorfer (2005). We analyse the data only in one spectral region, for more examples see Shapiro et al. (2007a). The selected region contains four $\mathrm{CN}$ violet system doublets: with $N=6,7$ and 52 from the $(1,1)$ vibrational band and with $N=69$ from the $(0,0)$ vibrational band. Because of the relatively large Landé factor $\left(g_{N} \sim 1 / N\right)$ the lines with $N=6$ and 7 are very sensitive to the 
magnetic field and show the saturated Hanle effect already at the fields $15-20 \mathrm{G}$, while lines with $N=52$ and 69 are much less affected by the magnetic field. Therefore, this region is ideal for applying the differential Hanle effect technique, in particular since there are lines with small and big $N$-number from the same vibrational band, as the relative strength of the lines from the different bands can be affected by other factors.

As a first step we use a quite simple model for the radiative transfer calculations. We assume that the isothermal and homogeneous $\mathrm{CN}$ molecular layer is situated above the region where the continuum radiation is formed. Its lower boundary is irradiated by the solar continuum radiation. While passing through the molecular layer this initial incident radiation will be either scattered (but only once, as we adopt the single scattering approximation) or absorbed by $\mathrm{CN}$ molecules (details in Shapiro et al. 2007a).

We have made a simultaneous fit of the $Q / I_{\mathrm{c}}$ and $I / I_{\mathrm{c}}$ signals, varying the free parameters of the model and the turbulent magnetic field strength (Fig. 2). The observed doubling of the line with $N=52$ appears due to a strong titanium blend, neglected in our model. The magnetic field strength is obviously within well defined limits. It has to be stronger than 10 G to saturate the depolarization of the line with $N=7$, while the upper limit is defined by the only modest depolarization of the lines with $N=52$ and $N=69$. The best fit corresponds to $13 \mathrm{G}$, which is consistent with the examination of the other spectral features. Furthermore, this result fits well with previous work (see the discussion in Berdyugina \& Fluri 2004; Trujillo Bueno 2005, and references therein).

Acknowledgements. This work has been supported by SNF grant 20002-103696. SB acknowledges the EURYI Award, from the ESF and SNF, grant PE002-104552.

\section{References}

Asensio Ramos, A. \& Trujillo Bueno, J. 2005, ApJ, 635, L109

Asensio Ramos, A. \& Trujillo Bueno, J. 2006, ApJ, 636, 548

Berdyugina, S. V., Braun, P. A., Fluri, D. M., \& Solanki, S. K. 2005, A\&A, 444, 947

Berdyugina, S. V. \& Fluri, D. M. 2004, A\&A, 417, 775

Berdyugina, S. V., Stenflo, J. O., \& Gandorfer, A. 2002, A\&A, 388, 1062

Bommier, V. 1980, A\&A, 87, 109

Bommier, V., Landi Degl'Innocenti, E., \& Molodij, G. 2007, in Solar Polarization, proc. 4rd SPW, ed.

R. Casini \& B. W. Lites, ASP Conf. Ser., in press

Faurobert, M. \& Arnaud, J. 2003, A\&A, 412, 555

Gandorfer, A. 2005, The Second Solar Spectrum, Vol. III: $3160 \AA$ A to $3915 \AA$ (Zurich: VdF), ISBN no. 3728130184

Landi Degl'Innocenti, E. 2003, in ASP Conf. Ser., Vol. 307, Solar Polarization, proc. 3rd SPW, ed. J. Trujillo Bueno \& J. Sánchez Almeida, 164

Landi Degl'Innocenti, E. L. \& Landolfi, M. 2004, Polarization in Spectral Lines (Dordrecht: Kluwer) Landolfi, M. \& Landi Degl'Innocenti, E. 1985, Solar Phys., 98, 53

Shapiro, A. I., Berdyugina, S. V., Fluri, D. M., \& Stenflo, J. O. 2007a, A\&A, to be submitted

Shapiro, A. I., Fluri, D. M., Berdyugina, S. V., \& Stenflo, J. O. 2007b, A\&A, 461, 339

Stenflo, J. O. 1994, Solar Magnetic Fields (Dordrecht: Kluwer)

Stenflo, J. O., Keller, C. U., \& Gandorfer, A. 1998, A\&A, 329, 319

Trujillo Bueno, J. 2003, in ASP Conf. Ser., Vol. 307, Solar Polarization, proc. 3rd SPW, ed. J. Trujillo

Bueno \& J. Sánchez Almeida, 407

Trujillo Bueno, J. 2005, in ESA SP-600: The Dynamic Sun: Challenges for Theory and Observations 


\title{
Recent progresses in the simulation of small-scale magnetic fields
}

\author{
O. Steiner \\ Kiepenheuer-Institut für Sonnenphysik, Freiburg, Germany \\ Email: steiner@kis.uni-freiburg.de
}

\begin{abstract}
New high-resolution observations reveal that small-scale magnetic flux concentrations have a delicate substructure on a spatial scale of $0.1^{\prime \prime}$. Its basic structure can be interpreted in terms of a magnetic flux sheet or tube that vertically extends through the ambient weak-field or field-free atmosphere with which it is in mechanical equilibrium. A more refined interpretation comes from new three-dimensional magnetohydrodynamic simulations that are capable of reproducing the corrugated shape of magnetic flux concentrations and their signature in the visible continuum. Furthermore it is shown that the characteristic asymmetric shape of the contrast profile of facular granules is an effect of radiative transfer across the rarefied atmosphere of the magnetic flux concentration. I also discuss three-dimensional radiation magnetohydrodynamic simulations of the integral layers from the top of the convection zone to the mid-chromosphere. They show a highly dynamic chromospheric magnetic field, marked by rapidly moving filaments of stronger than average magnetic field that form in the compression zone downstream and along propagating shock fronts. The simulations confirm the picture of flux concentrations that strongly expand through the photosphere into a more homogeneous, space filling chromospheric field. Future directions in the simulation of small-scale magnetic fields are indicated by a few examples of very recent work.
\end{abstract}

\section{Introduction}

With "realistic simulations" computational physicists aim at imitating real physical processes that occur in nature. In the course of rebuilding nature in the computer, they aspire to a deeper understanding of the process under investigation. In some sense the opposite approach is taken by computational physicists that aim at separating the fundamental physical processes by abstraction from the particulars for obtaining "ideal simulations" or an analytical model of the essential physical process. Both strategies are needed and are complementary as can be seen for example in Section 4 on the physics of faculae. In this paper, however, we mainly focus on "realistic simulations" and comparison with observations.

The term small-scale flux concentration is used here to designate the magnetic field that appears in G-band filtergrams as bright tiny objects within and at vortices of intergranular lanes. They are also visible in the continuum, where they are called facular points (Mehltretter 1974), while the structure made up of bright elements is known as the filigree (Dunn \& Zirker 1973). In more recent times, the small-scale magnetic field was mostly observed in the $\mathrm{G}$ band (a technique originally introduced by Muller 1985) because the molecular band-head of $\mathrm{CH}$ that constitutes the $\mathrm{G}$ band acts as a leverage for the intensity contrast (Rutten 1999; 
Rutten et al. 2001; Sánchez Almeida et al. 2001; Shelyag et al. 2004; Steiner et al. 2001). Being located in the blue part of the visible spectrum, this choice also helps improving the diffraction limited spatial resolution and the contrast in the continuum.

Small-scale magnetic flux concentrations are studied for several reasons:

- Since they make up the small end of a hierarchy of magnetic structures on the solar surface, the question arises whether they are "elemental" or whether yet smaller flux elements exist. How do they form? Are they a surface phenomenon? What is their origin?

- Near the solar limb they can be identified with faculae, known to critically contribute to the solar irradiance variation.

- They probably play a vital role in the transport of mechanical energy to the outer atmosphere, e.g., by guiding and converting magnetoacoustic waves generated by the convective motion and granular buffeting.

\section{The basic structure of small-scale magnetic flux concentrations}

Recent observations of unprecedented spatial resolution with the $1 \mathrm{~m}$ Swedish Solar Telescope by Berger et al. (2004) and Rouppe van der Voort et al. (2005) reveal G-band brightenings in an active region as delicate, corrugated ribbons that show structure down to the resolution capability of the instrument of $0.1^{\prime \prime}$, while isolated point-like brightenings exist as well. The structure made up of these objects evolves on a shorter than granular time-scale, giving the impression of a separate (magnetic) fluid that resists mixing with the granular material. Figure 1 shows an example G-band filtergram from the former paper taken in a remnant active region plage near disk center. In this region, intergranular lanes are often completely filled with magnetic field like in the case marked by the white lines in Fig. 1. There, and in other similar cases, the magnetic field concentration is framed by a striation of bright material, while the central part is dark. Figure 1 shows examples of ribbon bands and also an isolated bright point in the lower right corner.

The graphic to the right hand side of Fig. 1 displays the emergent G-band intensity (solid curve) from the cross section marked by the white horizontal lines in the image to the left. Also shown are the corresponding magnetographic signal (dashed curve), the blue continuum intensity (dotted), and the $\mathrm{Ca} \mathrm{H}$-line intensity (dash-dotted). Note that the magnetic signal is confined to the gap between the two horizontal white lines. The intensities show a twohumped profile.

This situation reminds of the flux-sheet model and the "bright wall effect". A first quasistationary, self-consistent simulation of a small-scale flux sheet was carried out by Deinzer et al. (1984a,b), popularly known as the "KGB-models". The basic properties of this model are sketched in Fig. 2. Accordingly, a small-scale flux concentration, either of tube or sheetlike shape, is in mechanical equilibrium with the ambient atmosphere, viz., the gas plus magnetic pressure of the atmosphere within the tube/sheet balances the gas pressure in the ambient (field-free) medium at equal geometrical height. This situation calls for a reduced density in the flux concentration with respect to the environment, at least in the photospheric part, where the radiative heat exchange quickly drives the configuration towards radiative equilibrium, hence to a similar temperature at constant geometrical height. This density reduction renders the flux tube/sheet atmosphere more transparent, which causes a depression 

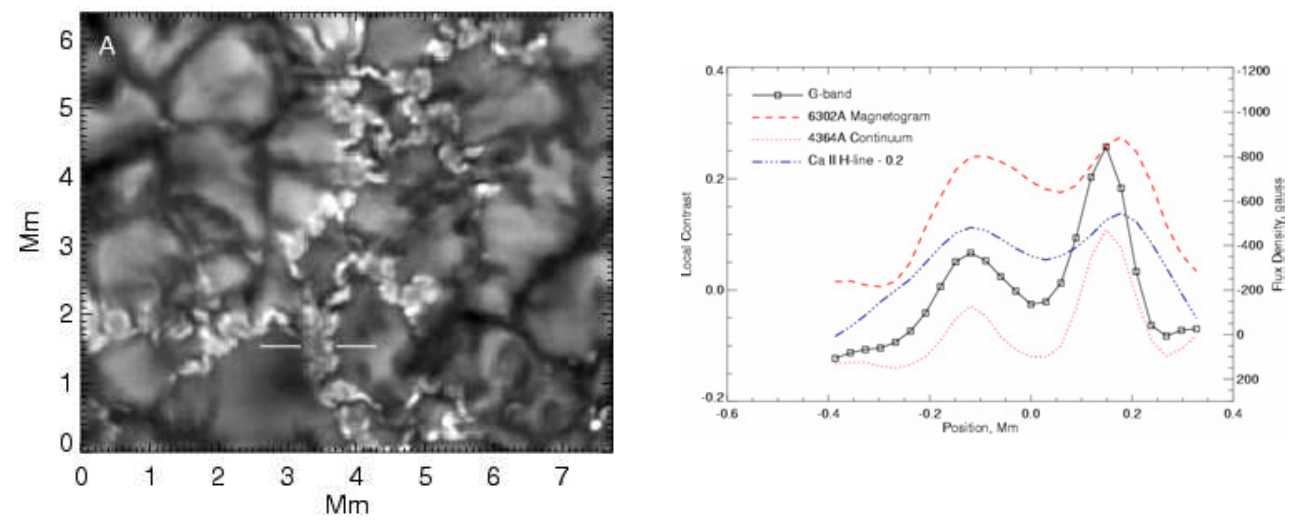

Figure 1. Left: G-band filtergram showing the ribbon-like shape of magnetic flux concentrations. Right: G-band intensity (solid curve) along the section indicated by the horizontal white lines in the image to the left. Also shown are the magnetogram signal, the continuum intensity at $436.4 \mathrm{~nm}$, and the Ca II H-line intensity down-shifted by 0.2. From Berger et al. (2004).

of the surface of constant optical depth, as indicated by the surface of $\tau_{c}=1$ in Fig. 2 . In a plage or network region, this effect increases the "roughness" of the solar surface, hence the effective surface from which radiation can escape, which increases the net radiative loss from these areas.

The graphics to the right hand side of Fig. 2 shows a sketch of the relative intensity emerging from this model, viz., the intensity of light propagating in the vertical direction as a function of distance from the flux sheet's plane of symmetry. It corresponds to the plot on the right hand side of Fig. 1. The similarity between this model and the observation is striking. Turning to a narrower flux sheet/tube would result in the merging of the two contrast peaks to a single central peak in both, model and observation, i.e., to a ribbon band or bright point, respectively. Yet, the striation of the depression wall that can be seen in the observation is of course not reproduced by the model, which is strictly two-dimensional with translational invariance in lane direction. We will see in Sect. 4 that three-dimensional magnetoconvection simulations show rudimentary striation. The physical origin of the striation is still unknown.

Accordingly, the basic properties of ribbon-like magnetic flux concentrations can be understood in terms of a magnetic flux sheet embedded in and in force balance with a more or less field-free ambient medium. This can also be said (replacing the word sheet by tube) of the rosette structure visible in other still images of Berger et al. (2004) who call it "flowerlike". Flowers can transmute to pores and vice versa. The striation of their bright collar is similar to that seen in ribbon structures. Discarding the striation, the basic properties of flowers can well be interpreted in terms of a tube shaped flux concentration like the one sketched in Fig. 2.

A $2 \mathrm{~h}$ sequence of images with a quality comparable to Figs. 1 (Rouppe van der Voort et al. 2005) reveals that the shape of the ribbon-like flux concentrations and the striation of ribbons and flowers change on a very short time-scale, of the order of the Alfvén crossing travel time. This suggests that these morphological changes and the striation itself are related to the flute instability, which small-scale flux concentrations are liable to. For an untwisted 


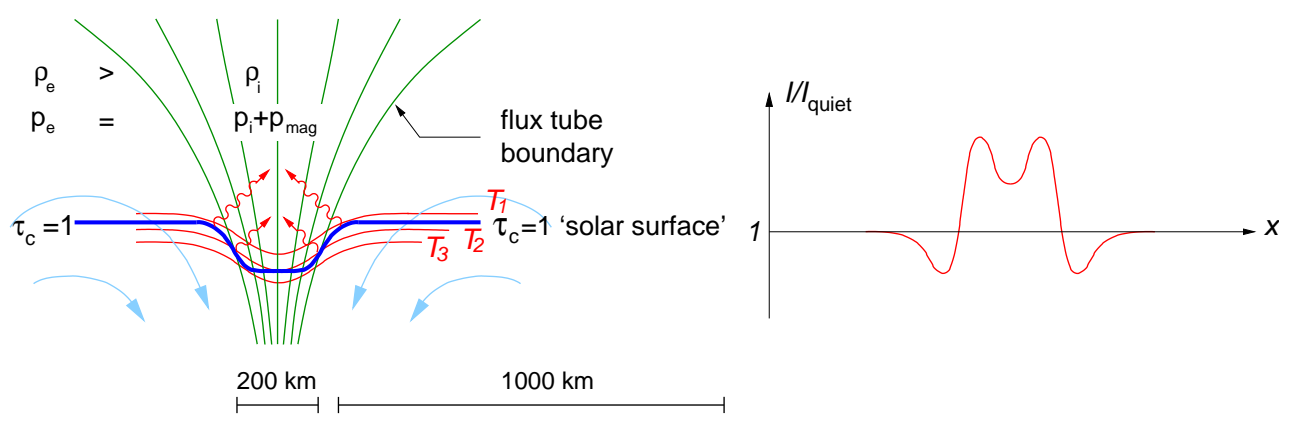

Figure 2. Sketch of a magnetic flux sheet (left) with corresponding intensity contrast (right), distilled from self-consistent numerical MHD simulations. Note that the isothermal surfaces are not exactly parallel to the surface of optical depth unity, which gives rise to the particular M-shape of the contrast profile.

axisymmetric flux tube, the radial component of the magnetic field at the flux-tube surface must decrease with height, $\mathrm{d} B_{r} /\left.\mathrm{d} z\right|_{S}<0$, in order that the flux tube is stable against the flute (interchange) instability (Meyer et al. 1977). While sunspots and pores with a magnetic flux in excess of $\Phi \approx 10^{19} \mathrm{Mx}$ do meet this condition, small-scale flux concentrations do not fulfill it (Schüssler 1984; Steiner 1990; Bünte et al. 1993). Bünte (1993) shows that smallscale flux sheets too are flute unstable, and he concludes that filament formation due to the flute instability close to the surface of optical depth unity would ensue. As the flux sheet is bound to fall apart because of the flute instability, its debris are again reassembled by the continuous advection back to the intergranular lane so that a competition between the two effects is expected to take place, which might be at the origin of the corrugation of the field concentrations and of the striation of the tube/sheet interface with the ambient medium.

Although the fine structure of small-scale magnetic flux concentrations changes on a very short time scale, single flux elements seem to persist over the full duration of the time sequence of $2 \mathrm{~h}$. They may dissolve or disappear for a short period of time, but it seems that the same magnetic flux continually reassembles to make them reappear nearby. Latest G-band time sequences obtained with the Solar Optical Telescope (SOT) on board of the Japanese space satellite HINODE (http://solarb.msfc.nasa.gov/movies.html) seem to confirm these findings even for G-band bright points of low intensity. This suggest a deep anchoring of at least some of the flux elements although numerical simulation seem not to confirm this conjecture.

As indicated in the sketch of Fig. 2, the magnetic flux concentration is framed by a downflow of material, fed by a horizontal flow that impinges on the flux concentration. Already the flux-sheet model of Deinzer et al. (1984b) showed a persistent flow of this kind. According to these authors it is due to radiative cooling from the depression walls of the magnetic flux concentrations (the "hot wall effect") that causes a horizontal pressure gradient, which drives the flow. The non-stationary flux-sheet simulations of Steiner et al. (1998) and Leka \& Steiner (2001) showed a similar persistent downflow, which, with increasing depth, becomes faster and narrower, turning into veritable downflow jets beneath the visible surface. While downflows in the periphery of pores have been observed earlier (Leka \& Steiner 2001; Sankarasubramanian \& Rimmele 2003; Tritschler et al. 2003) and also horizontal motions 

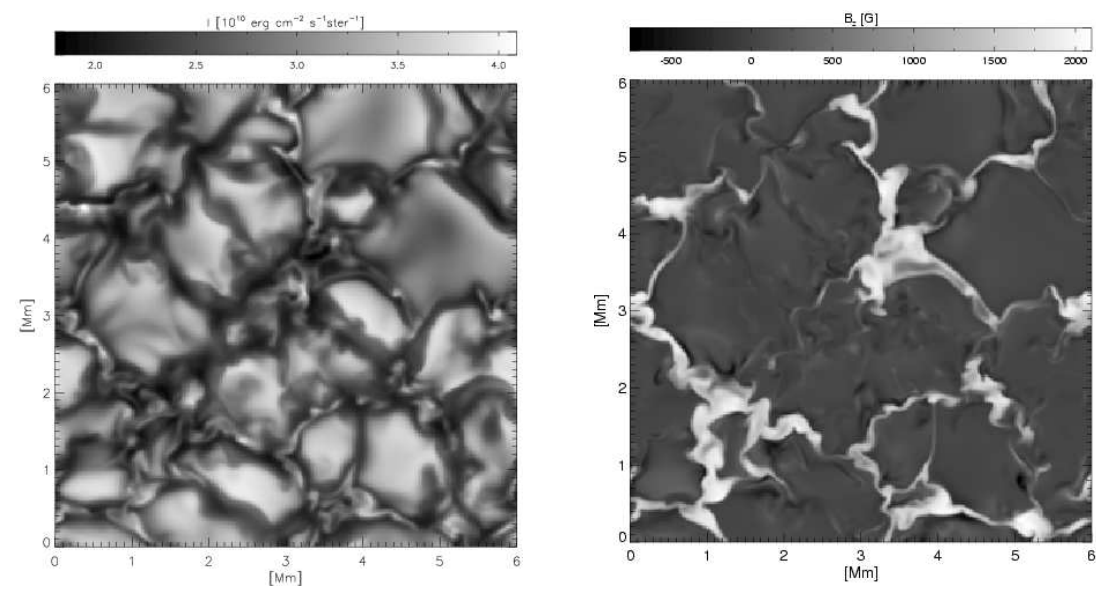

Figure 3. Simulation snapshot. Left: Frequency integrated intensity. Right: Vertical magnetic flux density at constant average geometrical height of optical depth unity. The mean flux density is $200 \mathrm{G}$. From Vögler et al. (2005).

towards a pore by Dorotovič et al. (2002), only very recently such an accelerating downflow has been observationally detected in the immediate vicinity of ribbon bands by Langangen et al. (2007).

\section{3-D simulations of small-scale magnetic flux concentrations}

New results from realistic simulations on the formation, dynamics and structure of smallscale magnetic flux concentrations have recently been published in a series of papers by Schüssler and collaborators. Vögler et al. (2005) simulate magnetoconvection in a box encompassing an area on the solar surface of $6 \times 6 \mathrm{Mm}^{2}$ with a height extension of $1400 \mathrm{~km}$, reaching from the temperature minimum to $800 \mathrm{~km}$ below the surface of optical depth unity. Although this is only $0.4 \%$ of the convection zone depth, the box still includes the entire transition from almost completely convective to mainly radiative energy transfer and the transition from the regime where the flux concentration is dominated by the convective plasma flow to layers where the magnetic energy density of the flux concentrations by far surpasses the thermal energy density. The bottom boundary in this and similar simulations is open in the sense that plasma can freely flow in and out of the computational domain, subject to the condition of mass conservation. Inflowing material has a given specific entropy that determines the effective temperature of the radiation leaving the domain at the top, while the outflowing material carries the entropy it instantly has.

Figure 3 shows a snapshot from this simulation: To the left the emergent mean intensity, to the right the vertical magnetic field strength at a constant height, viz., at the horizontally averaged geometrical height of optical depth unity. (I would like to caution that this magnetic map is not what would be seen with a magnetograph, irrespective of its spatial resolution.) The strong magnetic field in intergranular lanes is manifest in a corresponding signal in the emergent intensity very much like the snapshot discussed in Sect. 2. Also the intensity signal 
shows the same corrugated and knotted ribbon structure that is observed, and sometimes there appear also broader ribbon structures with a dark central core, like the one marked in Fig. 1. In the latter case however, the characteristic striation is absent, possibly because the flute instability is suppressed on very small spatial scales due to lack of sufficient resolution of the simulation. In the central part of the snapshot, a micro pore or magnetic knot has formed.

A comparison of the average gas plus magnetic pressure as a function of height at locations of magnetic flux concentrations with the run of the average gas pressure in weak-field regions reveals that the two are almost identical, proving that even in this dynamic regime, the thin flux tube approximation is very well satisfied (Vögler et al. 2005). This result confirms that the model discussed in Sect. 2 and sketched in Fig. 2 is indeed an acceptable first approximation to the real situation.

Simulations are not just carried out for the sake of reproducing observed quantities. Once good agreement with all kind of observations exists, simulations allow with some confidence to inform about regions not directly accessible to observations, for example about the magnetic structure in subsurface layers. In this respect the simulations of Vögler et al. (2005) show that often flux concentrations that have formed at the surface disperse again in shallow depths. This behaviour was also found by Schaffenberger et al. (2005) in their simulation with an entirely different code and further by Stein \& Nordlund (2006a). A vertical section through a three-dimensional simulation domain of Schaffenberger et al. (2005), where two such shallow flux concentrations have formed, is shown in Fig. 8. The superficial nature of magnetic flux concentrations in the simulations, however, is difficult to reconcile with the observation that many flux elements seem to persist over a long time period.

\section{The physics of faculae}

With growing distance from disk center, small-scale magnetic flux concentrations grow in contrast against the quiet Sun background and become apparent as solar faculae close to the limb. Ensembles of faculae form plage and network faculae that are as conspicuous features of the white light solar disk, as are sunspots. It is therefore not surprising that they play a key role in the solar irradiance variation over a solar cycle and on shorter time scales (Fligge et al. 2000; Wenzler et al. 2005; Foukal et al. 2006). Measurements of the center to limb variation of the continuum contrast of faculae are diverse, however, as the contrast is not only a function of the heliocentric angle, $\mu=\cos \theta$, but also of facular size, magnetic field strength, spatial resolution, etc., and as measurements are prone to selection effects. While many earlier measurements report a contrast maximum around $\mu \approx 0.2 \ldots 0.4$ with a decline towards the limb, latest measurements (Sütterlin et al. 1999; Ahern \& Chapman 2000; Adjabshirizadeh \& Koutchmy 2002; Ortiz et al. 2002; Centrone \& Ermolli 2003; Vogler et al. 2005) point rather to a monotonically increasing or at most mildly decreasing contrast out to the limb.

The standard facula model (Spruit 1976), again consists of a magnetic flux concentration embedded in and in mechanical equilibrium with a weak-field or field-free environment as is sketched in Fig, 2. When approaching the limb, the limb side of the bright depression wall becomes ever more visible and ever more perpendicular to the line of sight, which increases its brightness compared to the limb darkened environment. At the extreme limb, obscuration 

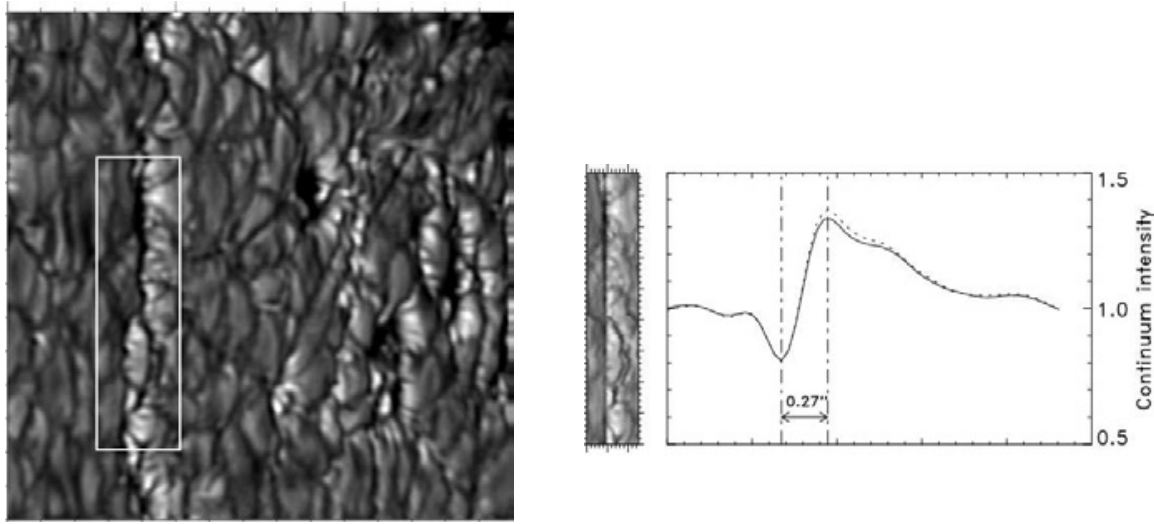

Figure 4. Left: Network faculae at a heliocentric angle of $\mu=0.48$ in the continuum at $587.5 \mathrm{~nm}$. The solar limb is to the right. Right: Faculae within the white box of the image to the left aligned according to the position of the dark lane, together with the mean spatial scan through the aligned faculae. From Hirzberger \& Wiehr (2005).

by the centerward rim of the depression starts to take place, which decreases the size and possibly the contrast of the visible limb-side wall.

Recently, Lites et al. (2004) and Hirzberger \& Wiehr (2005) have obtained excellent images of faculae with the $1 \mathrm{~m}$ Swedish Solar Telescope. Figure 4 (from Hirzberger \& Wiehr 2005) shows on the left hand side network faculae at a heliocentric angle of $\mu=0.48$ in the continuum at $587.5 \mathrm{~nm}$. The solar limb is located towards the right hand side. It is clearly visible from this image that faculae are in reality partially brightened granules with an exceptionally dark and wide intergranular lane ("dark facular lane") on the disk-center side of the contrast enhancement, which is also the location of the magnetic flux concentration. The right half of Fig. 4 shows the string of faculae within the white box of the image to the left, aligned according to the position of the dark lane. Also shown is the mean contrast profile, averaged over the alignment. Similar contrast profiles of single faculae are shown by Lites et al. (2004). Such contrast profiles pose now a new constraint that any model of faculae must satisfy.

Magnetoconvective simulations as the one discussed in Sect. 3 indeed show facular-like contrast enhancements when computing the emergent intensity along lines of sight that are inclined to the vertical direction for mimicking limb observation. Such tilted threedimensional simulation boxes are shown in the papers by Keller et al. (2004), Carlsson et al. (2004), and De Pontieu et al. (2006). Keller et al. (2004) also show the contrast profile of two isolated "faculae", which however have a more symmetric shape, rather than the observed characteristic steep increase on the disk-center side with the gentle decrease towards the limb. Also they obtain a maximum contrast of 2, far exceeding the observed value of about 1.3. It is not clear what the reason for this discrepancy might be. Interestingly, the old "KGBmodel" (Deinzer et al. 1984b; Knölker \& Schüssler 1988) as well as the two-dimensional, non-stationary simulation of Steiner (2005) do nicely reproduce the asymmetric shape and the dark lane.

Another conspicuous property of faculae that high-resolution images reveal is that they 

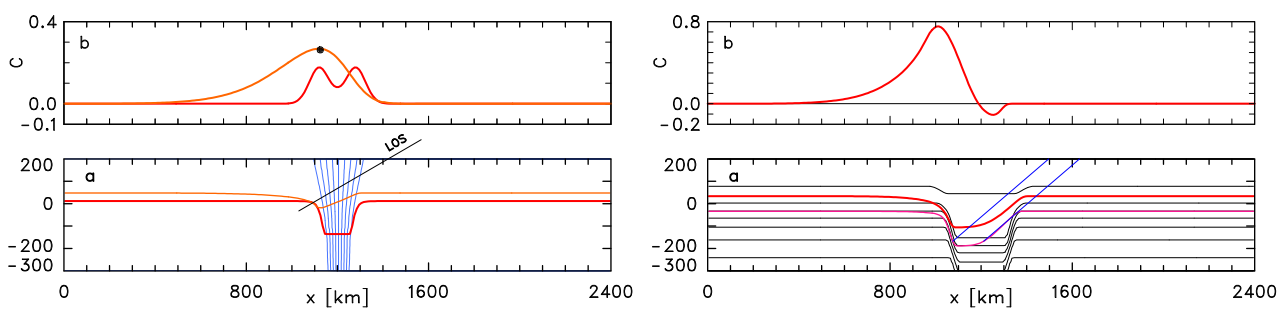

Figure 5. Left: a) Magnetic flux concentration (blue, vertically oriented lines of force, see internet version for colours) with surfaces of optical depth $\tau=1$ for vertical lines of sight (thick/red curve) and lines of sight inclined by $\theta=60^{\circ}$ to the vertical direction (thin/red curve). b) Corresponding contrast curves. All values of the light red curve left of the black dot originate from lines of sight left of the one indicated in panel a). Right: a) Surfaces of optical depth $\tau=1$ and 5 (thick/red) for lines of sight inclined by $50^{\circ}$ to the vertical, together with isotherms. b) Contrast profile. The region of negative contrast is bounded by the two oblique lines of sight indicated in panel a).

are not uniformly bright but show a striation not unlike to and possibly in connection with the one seen in G-band ribbons at disk center. While this feature cannot be reproduced in a two-dimensional model, it must be part of a satisfactory three-dimensional model. But so far 3-D simulations show only a rudimentary striation. This finding, rather surprisingly, indicates that the effective spatial resolution of present-day three-dimensional simulations is inferior to the spatial resolution of best current observations.

In an attempt to better understand the basic properties of faculae, Steiner (2005) considers the ideal model of a magnetohydrostatic flux sheet embedded in a plane parallel standard solar atmosphere. For the construction of this model the flux-sheet atmosphere is first taken to be identical to the atmosphere of the ambient medium but shifted in the downward direction by the amount of the "Wilson depression" (the depression of the surface of continuum optical depth unity at the location of the flux concentration). The shifting results in a flux-tube atmosphere that is less dense and cooler than the ambient medium at a fixed geometrical height. In the photospheric part of the flux concentration, thermal equilibrium with the ambient medium is then enforced. Denoting with index $i$ the flux-sheet atmosphere and with $e$ the ambient atmosphere and with $W$ the depth of the "Wilson depression", we therefore have

$$
T_{i}(z)=\left\{\begin{array}{l}
T_{e}(z+W) \text { for } \tau_{c} \gg 1 \\
T_{e}(z) \text { for } \tau_{c} \ll 1,
\end{array}\right.
$$

where $\tau_{c}$ is the optical depth in the visible continuum and $\rho_{i}(z)<\rho_{e}(z) \forall z$. The lower left panel of Fig. 5 shows this configuration together with two surfaces of optical depth unity, one for vertical lines of sight (disk center), the other for lines of sight running from the top right to the bottom left under an angle of $\theta=60^{\circ}$ to the vertical, like the one indicated in the figure. The upper left panel shows the corresponding continuum enhancement for disk center (double humped profile) and $\theta=60^{\circ}$. Of the curve belonging to $\theta=60^{\circ}$, all values left of the black dot belong to lines of sight left of the one indicated in the lower panel. This means that the contrast enhancement extends far beyond the depression proper in the limbward direction, exactly as is observed. The reason for this behaviour is explained with the help of Fig. 6 as follows. 


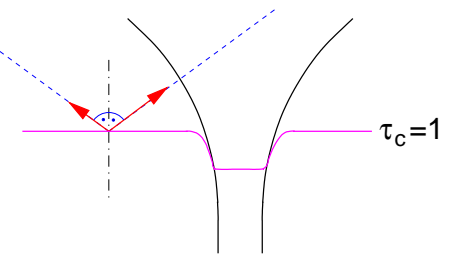

Figure 6. Photons preferentially escape along the line of sight to the right that traverses the magnetic flux sheet/tube in comparison to the line of sight to the left under equal zenith angle, because of the rarified (less opaque) atmosphere in the flux sheet/tube.

A material parcel located in the solar atmosphere and lateral to the flux sheet "sees" a more transparent atmosphere in the direction toward the flux sheet compared to a direction under equal zenith angle but pointing away from it because of the rarefied flux-sheet atmosphere. Correspondingly, from a wide area surrounding the magnetic flux sheet or flux tube, radiation escapes more easily in the direction towards the flux sheet so that a single flux sheet/tube impacts the radiative escape in a cross-sectional area that is much wider than the magnetic field concentration proper.

The right hand side of Fig. 5 shows a similar situation as to the left but for a flux sheet that is twice as wide. The continuum contrast for lines of sight inclined by $\theta=50^{\circ}$ to the vertical is shown in the top panel. It can be seen that a dark lane of negative contrast occurs on the disk-center side of the facula. It arises from the low temperature gradient of the flux-sheet atmosphere in the height range of $\tau_{c}=1$ and its downshift relative to the external atmosphere in combination with the inclined lines of sight. One could say that the dark lane in this case is an expression of the cool "bottom" of the magnetic flux sheet.

It is remarkable that this basic, energetically not self-consistent model is capable of producing both, the facular dark lane and the asymmetrically shaped contrast curve of the facula, with realistic contrast values. The results of this basic hydrostatic model carry over to a fully self-consistent model of a magnetic flux sheet in dynamic interaction with non-stationary convective motion (Steiner 2005). In this case the facular lane becomes broader and darker.

It follows from these insights that a facula is not to be identified with bright plasma that sticks, as the name may insinuate, like a torch out of the solar surface and as the "hillock model" of Schatten et al. (1986) suggests. Rather is it the manifestation of photospheric granulation, seen across a magnetic flux concentration - granulation that appears brighter than normal in the form of so called "facular granules". Interestingly, already Chevalier (1912) wondered: "La granulation que l'on voit autour des taches plus éclatante que sur les autres parties est-elle la granulation des facules ou celle de la photosphère vue à travers les facules ?" and Ten Bruggencate (1940) noted that "Sie [Photosphärengranulen und Fackelgranulen] unterscheiden sich nicht durch ihre mittlere Grösse, wohl aber durch den Kontrast gegenüber der Umgebung."

If this is true, one expects facular granules to show the same dynamic phenomena like regular granulation. Indeed, this is confirmed in a comparison of observations with threedimensional simulations by De Pontieu et al. (2006). They observe that often a dark band gradually moves from the limb side of a facula toward the disk center and seemingly sweeps over and "erases" the facula temporarily. The same phenomenon they also observed in a 
time sequence of a three-dimensional simulation, which enabled them to identify the physics behind this phenomenon.

Examination of the simulation sequence reveals that dark bands are a consequence of the evolution of granules. Often granules show a dark lane that usually introduces fragmentation of the granule. The smaller fragment often dissolves (collapses) in which case the dark lane disappears with the collapsing small fragment in the intergranular space. Exactly this process can lead to the dark band phenomenon, when a granular dark lane is swept towards the facular magnetic flux concentration. Since the facular brightening is seen in the disk-center facing side of granules, only granular lanes that are advected in the direction of disk center lead to facular dimming. This example nicely demonstrates how regular granular dynamics when seen across the facular magnetic field can lead to genuine facular phenomena.

Despite the major progress that we have achieved in understanding the physics of faculae over the past few years, open questions remain. These concern

- a comprehensive model of the center to limb variation of the brightness of faculae including dependence on size, magnetic flux, flux density, color, etc.,

- a quantitative agreement between simulation and observation with respect to measurements in the infrared and with respect to the observed geometrical displacement between line core and continuum filtergrams of faculae,

- the physical origin of the striation,

- a quantitative evaluation of the heat leakage caused by faculae, or

- the role of faculae in guiding magnetoacoustic waves into the chromosphere.

\section{3-D MHD simulation from the convection zone to the chromosphere}

For investigating the connection between photospheric small scale magnetic fields and the chromosphere, Schaffenberger et al. (2005) have extended the three-dimensional radiation hydrodynamics code $\mathrm{CO}^{5} \mathrm{BOLD}{ }^{1}$ to magnetohydrodynamics for studying magnetoconvective processes in a three-dimensional environment that encompasses the integral layers from the top of the convection zone to the mid chromosphere. The code is based on a finite volume scheme, where fluxes are computed with an approximate Riemann-solver (LeVeque et al. 1998; Toro 1999) for automatic shock capturing. For the advection of the magnetic field components, a constrained transport scheme is used.

The three-dimensional computational domain extends from $1400 \mathrm{~km}$ below the surface of optical depth unity to $1400 \mathrm{~km}$ above it and it has a horizontal dimension of $4800 \times 4800 \mathrm{~km}$. The simulation starts with a homogeneous, vertical, unipolar magnetic field of a flux density of $10 \mathrm{G}$ superposed on a previously computed, relaxed model of thermal convection. This low flux density is representative for magnetoconvection in a very quiet network-cell interior. The magnetic field is constrained to have vanishing horizontal components at the top and bottom boundary, but lines of force can freely move in the horizontal direction, allowing for flux concentrations to extend right to the boundaries. Because of the top boundary being located at mid-chromospheric heights, the magnetic field is allowed to freely expand with height through the photospheric layers into the more or less homogeneous chromospheric field.

\footnotetext{
${ }^{1}$ www.astro.uu.se/_bf/co5bold_main.html
} 

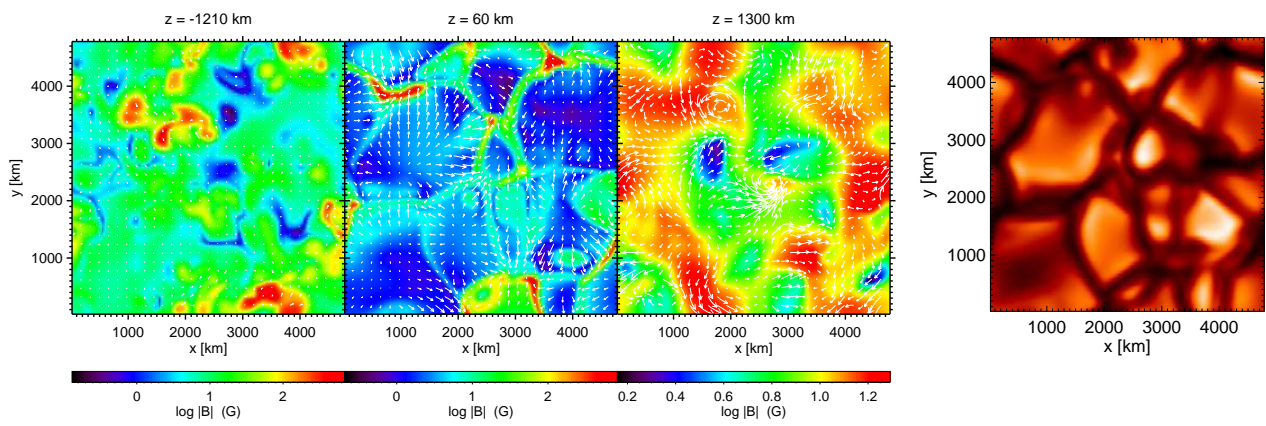

Figure 7. Horizontal sections through the three-dimensional computational domain. Color coding displays $\log |B|$ with individual scaling for each panel (see internet version for colours). Left: Bottom layer at a depth of $1210 \mathrm{~km}$. Middle: Layer $60 \mathrm{~km}$ above optical depth $\tau_{c}=1$. Right: Top, chromospheric layer in a height of $1300 \mathrm{~km}$. White arrows indicate the horizontal velocity on a common scaling. Longest arrows in the panels from left to right correspond to $4.5,8.8$, and $25.2 \mathrm{~km} / \mathrm{s}$, respectively. Rightmost: Emergent visible continuum intensity. From Schaffenberger et al. (2005).

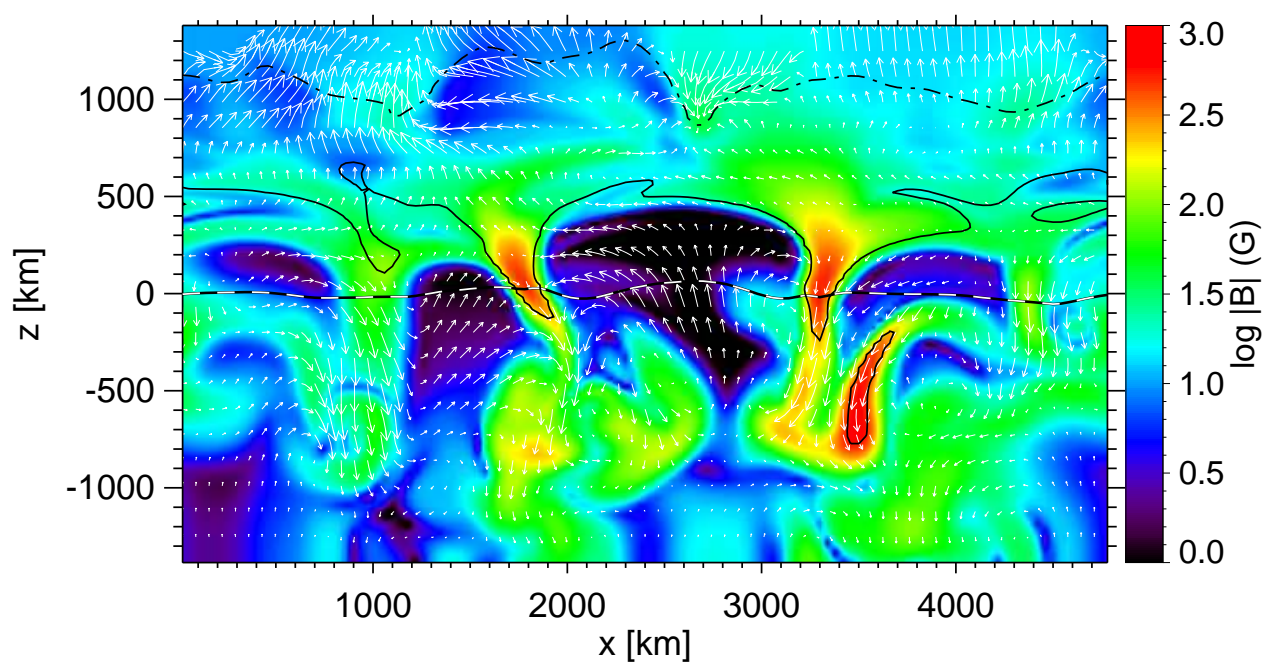

Figure 8. Snapshot of a vertical section through the three-dimensional computational domain, showing $\log |B|$ (color coded) and velocity vectors projected on the vertical plane (white arrows). The $\mathrm{b} / \mathrm{w}$ dashed curve shows optical depth unity and the dot-dashed and solid black contours $\beta=1$ and 100, respectively. See internet version for colours. From Schaffenberger et al. (2005). 

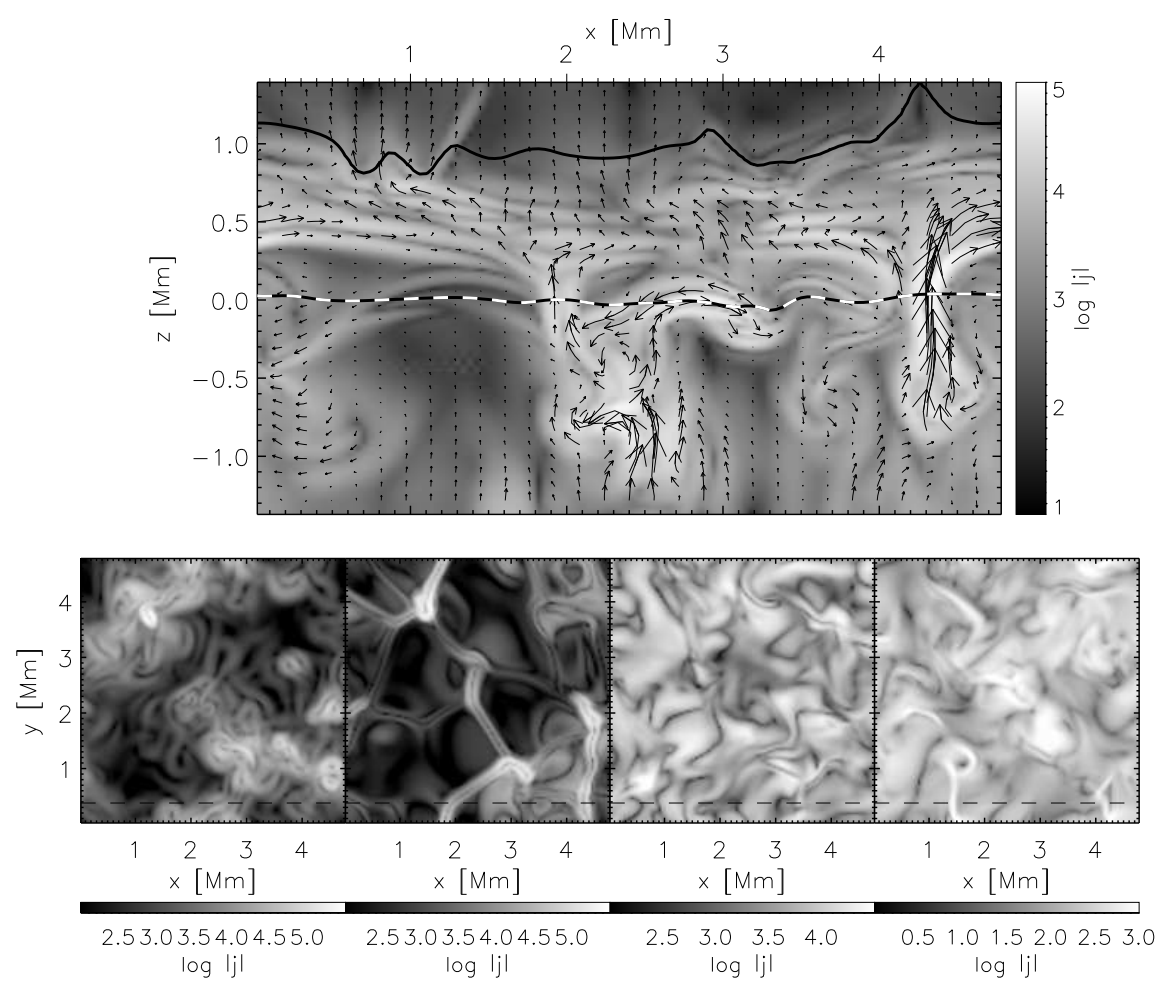

Figure 9. Logarithmic current density, $\log |j|$, in a vertical cross section (top panel) and in four horizontal cross sections in a depth of $1180 \mathrm{~km}$ below, and at heights of $90 \mathrm{~km}, 610 \mathrm{~km}$, and $1310 \mathrm{~km}$ above the mean surface of optical depth unity from left to right, respectively. The arrows in the top panel indicate the magnetic field strength and direction. The dashed line indicates the position of the vertical section. $[j]=3 \times 10^{5} \mathrm{~A} / \mathrm{m}^{2}$. From Wedemeyer-Böhm et al. (2007).

Figure 7 shows the logarithmic absolute magnetic flux density in three horizontal sections through the computational domain at a given time instant, together with the emergent Rosseland mean intensity. The magnetic field in the chromospheric part is marked by strong dynamics with a continuous rearrangement of magnetic flux on a time scale of less than $1 \mathrm{~min}$, much shorter than in the photosphere or in the convection-zone layers. There, the field has a strength between 2 and $40 \mathrm{G}$ in the snapshot of Fig. 7, which is typical for the whole time series. Different from the surface magnetic field, it is more homogeneous and practically fills the entire space so that the magnetic filling factor in the top layer is close to unity. There seems to be no spatial correlation between chromospheric flux concentrations and the small-scale field concentrations in the photosphere.

Comparing the flux density of the panel corresponding to $z=60 \mathrm{~km}$ with the emergent intensity, one readily sees that the magnetic field is concentrated in intergranular lanes and at lane vertices. However, the field concentrations do not manifest a corresponding intensity signal like in Fig. 3. This is because the magnetic flux is too weak to form a significant "Wilson depression" (as can be seen from Fig. 8) so that no radiative channeling effect takes 
place.

Figure 8 shows the logarithm of the absolute field strength through a vertical section of the computational domain. Overplotted are white arrows indicating the velocity field. The $\mathrm{b} / \mathrm{w}$ dashed curve corresponds to the optical depth unity for vertical lines of sight. Contours of the ratio of thermal to magnetic pressure, $\beta$, for $\beta=1$ (dot-dashed) and $\beta=100$ (solid) are also shown. Magnetoacoustic waves that form transient filaments of stronger than average magnetic field are a ubiquitous phenomenon in the chromosphere and are also present in the snapshot of Fig. 8, e.g., along the contour of $\beta=1$ near $x=1200 \mathrm{~km}$ and $x=2500 \mathrm{~km}$. They form in the compression zone downstream and along propagating shock fronts. These magnetic filaments that have a field strength rarely exceeding $40 \mathrm{G}$, rapidly move with the shock fronts and quickly form and dissolve with them.

The surface of $\beta=1$ separates the region of highly dynamic magnetic fields around and above it from the more slowly evolving field of high beta plasma below it. This surface is located at approximately $1000 \mathrm{~km}$ but it is corrugated and its local height strongly varies in time.

A very common phenomenon in this simulation is the formation of a "magnetic canopy field' that extends in a more or less horizontal direction over expanding granules and between photospheric flux concentrations. The formation of such canopy fields proceeds by the action of the expanding flow above granule centres. This flow transports 'shells' of horizontal magnetic field to the upper photosphere and lower chromosphere, where shells of different field directions may be pushed close together, leading to a complicated network of current sheets in a height range from approximately 400 to $900 \mathrm{~km}$.

This network can be seen in Fig. 9 (top), which shows, for a typical snapshot of the simulation, the logarithmic current density, $\log |j|$, together with arrows indicating the magnetic field strength and direction. Figure 9 (bottom) shows from left to right $\log |j|$ in four horizontal cross sections in a depth of $1180 \mathrm{~km}$ below, and at heights of $90 \mathrm{~km}, 610 \mathrm{~km}$, and $1310 \mathrm{~km}$ above the mean surface of optical depth unity. Higher up in the chromosphere (rightmost panel), thin current sheets form along shock fronts, e.g., in the lower left corner near $x=1.4 \mathrm{Mm}$.

Using molecular values for the electrical conductivity, Wedemeyer-Böhm et al. (2007) derive an energy flux of 5 to $50 \mathrm{~W} \mathrm{~m}^{-2}$ into the chromosphere caused by ohmic dissipation of these current sheets. This value is about two orders of magnitude short of being relevant for chromospheric heating. On the other hand, the employed molecular values for the conductivity might be orders of magnitude too high for to be compatible with the effective electrical conductivity of the numerical scheme determined by the inherent artificial diffusion. Therefore, the ohmic heat flux might be conceivably two orders of magnitude larger than suggested by this rough estimate, so that magnetic heating by ohmic dissipation must be seriously taken into account. More advanced simulations, taking explicit ohmic diffusion into account will clarify this issue.

\section{Future directions}

Continuously increasing power of computational facilities together with steadily improving computational methods, expand the opportunity for numerical simulations. On the one hand, more detailed physics can be included in the simulation, on the other hand either the compu- 
tational domain or the spatial and temporal resolution can be increased. In most simulations, especially when the computational domain encompasses only a small piece of a star, boundary conditions play an important role. They convey information on the outside world to the physical domain of the simulation. But this outside world is often poorly known. In order to acquire experience and intuition with respect to the influence of different types of boundary condition on the solution, one can implement and run various realizations of boundary conditions, which however also requires additional resources in computer power and time. Also the initial condition may critically determine the solution, for example the net flux and flux density of an initial, homogeneous vertical magnetic field. Boundary conditions, therefore, remain a hot topic also in future.

Most excitement in carrying out numerical simulations comes from the prospect of performing experiments with the object under investigation: experiments in the numerical laboratory. Not only that an astrophysical object can be reconstructed and simulated in the virtual world of the numerical laboratory. Once in the computer, the computational astrophysicist has the prospect of carrying out experiments with it as if the celestial body was taken to the laboratory.

The following few examples shall illustrate some aspects of this.

\subsection{More detailed physics}

In the solar chromosphere the assumption of LTE (local thermodynamic equilibrium) is not valid. Even the assumption of statistical equilibrium in the rate equations is not valid because the relaxation time-scale for the ionization of hydrogen approaches and surpasses the hydrodynamical time scale in the chromosphere (Kneer 1980). Yet, in order to take time dependent hydrogen ionization in a three-dimensional simulation into account, simplifications are needed. Leenaarts \& Wedemeyer-Böhm (2006) employ the method of fixed radiative rates for a hydrogen model atom with six energy levels in the three-dimensional radiation (magneto-)hydrodynamics code $\mathrm{CO}^{5} \mathrm{BOLD}$. Thus, additional to the hydrodynamic equations, they solve the time-dependent rate equations

$$
\frac{\partial n_{i}}{\partial t}+\nabla \cdot\left(n_{i} \mathrm{v}\right)=\sum_{j \neq i}^{n_{l}} n_{j} P_{j i}-n_{i} \sum_{j \neq i}^{n_{l}} P_{i j},
$$

with $P_{i j}$ being the sum of collisional and radiative rate coefficients, $P_{i j}=C_{i j}+R_{i j}$. The rate coefficients are now local quantities given a fixed radiation field for each transition, which is obtained from one-dimensional test calculations.

Simulations with this approach show that above the height of the classical temperature minimum, the non-equilibrium ionization degree is fairly constant over time and space at a value set by hot propagating shock waves. This is in sharp contrast to results with LTE, where the ionization degree varies by more than 20 orders of magnitude between hot gas immediately behind the shock front and cool regions further away. The addition of a hydrogen model atom provides realistic values for hydrogen ionization degree and electron density, needed for detailed radiative transfer diagnostics.

\subsection{Large box simulations}

Benson et al. (2007) have carried out first simulations with a large simulation box so as to 

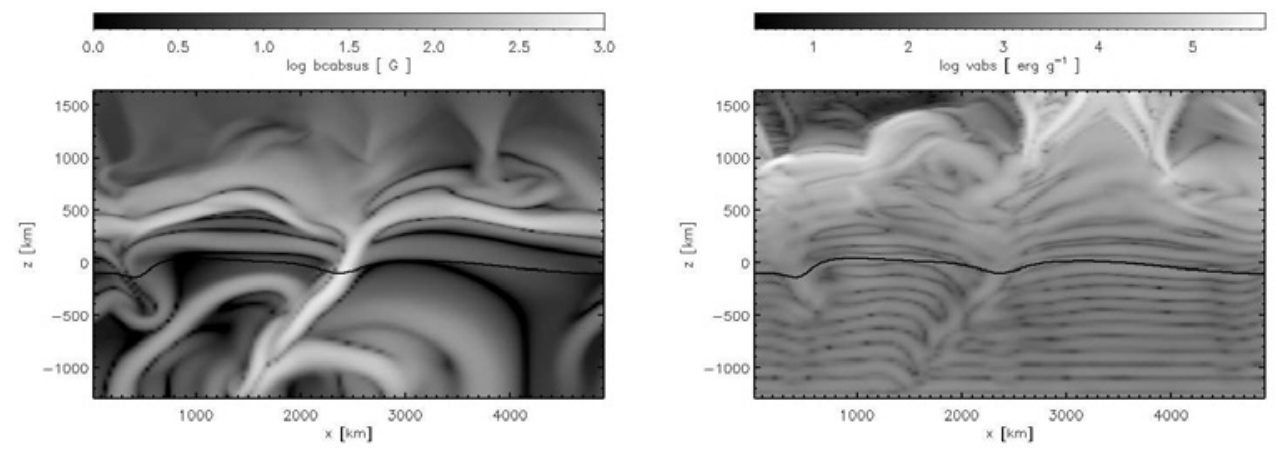

Figure 10. Left: Still image of the logarithmic magnetic flux density from a time series for the instant $t=1368 \mathrm{~s}$ after starting with an initial homogeneous vertical field of $10 \mathrm{G}$ flux density. A strong magnetic flux sheet has formed extending from $(x, z) \approx(2000,-500)$ to $(x, z) \approx(2500,0)$. Right: A plane parallel wave with frequency $100 \mathrm{mHz}$ travels through convecting plasma into the magnetically structured photosphere and further into the low $\beta$ (magnetically dominated) chromosphere. The panel shows the difference in absolute velocity between the perturbed and the unperturbed solution $212 \mathrm{~s}$ after the start of the perturbation. The wave becomes strongly refracted in the low $\beta$ region and at the location of the flux sheet.

accommodate a supergranulation cell. They started a simulation that encompasses a volume of $48 \times 48 \times 20 \mathrm{Mm}^{3}$ using $500^{3}$ grid cells. With this simulation they hope to find out more about the origin of supergranulation and to carry out helioseismic experiments (Zhao et al. 2007).

Hansteen (2004) has carried out MHD simulations comprising a vast height range from the top layers of the convection zone into the transition region and the corona. With these simulations they seek to investigate various chromospheric features such as dynamic fibrils (Hansteen et al. 2006), mottles, and spicules, which are some of the most important, but also most poorly understood, phenomena of the Sun's magnetized outer atmosphere. But also the transition zone and coronal heating mechanisms are in the focus of these kinds of "holistic" simulations.

\subsection{Improvements in boundary conditions}

Many conventional magnetohydrodynamic simulations of the small-scale solar magnetic field assume that the horizontal component of the magnetic field vanishes at the top and bottom of the computational domain (e.g. Weiss et al. 1996; Cattaneo et al. 2003; Vögler et al. 2005; Schaffenberger et al. 2005), which is a rather stark constraint, especially with respect to magnetoacoustic wave propagation and Poynting flux. Recently, Stein \& Nordlund (2006b) have introduced an alternative condition with the possibility of advecting magnetic field across the bottom boundary. Thus, upflows into the computational domain carry horizontal magnetic field of a prescribed flux density with them, while outflowing plasma carries whatever magnetic field it instantly has. With this condition an equilibrium in which equal amounts of magnetic flux are transported in and out of the computational domain is approached after some time. It should more faithfully model the plasma flow across the lower 
boundary and it allows for the effect of magnetic pumping (Tobias et al. 1998).

\subsection{Helioseismic experiment with a magnetically structured atmosphere}

With numerical experiments Steiner et al. (2007) have explored the feasibility of using high frequency waves for probing the magnetic fields in the photosphere and the chromosphere of the Sun. They track an artificially excited, plane-parallel, monochromatic wave that propagates through a non-stationary, realistic atmosphere, from the convection-zone through the photosphere into the magnetically dominated chromosphere, where it gets refracted and reflected.

When comparing the wave travel time between two fixed geometrical height levels in the atmosphere (representing the formation height of two spectral lines) with the topography of the surface of equal magnetic and thermal energy density (the magnetic canopy or $\beta=$ 1 surface) we find good correspondence between the two. These numerical experiments support expectations by Finsterle et al. (2004) that high frequency waves bear information on the topography of the 'magnetic canopy'. This simulation exemplifies how a piece of Sun can be made accessible to virtual experimenting by means of realistic numerical simulation.

\section{References}

Adjabshirizadeh, A. \& Koutchmy, S. 2002, in ESA SP-506: Solar Variability: From Core to Outer Frontiers, 415-418

Ahern, S. \& Chapman, G. A. 2000, Solar Phys., 191, 71

Benson, D., Stein, R., \& Nordlund, A. 2007, in Solar MHD Theory and Observations: a High Spatial Resolution Perspective, ed. H. Uitenbroek, J. Leibacher, \& R. F. Stein, ASP Conf. Ser., 94

Berger, T. E., Rouppe van der Voort, L. H. M., Löfdahl, M. G., et al. 2004, A\&A, 428, 613

Bünte, M. 1993, A\&A, 276, 236

Bünte, M., Steiner, O., \& Pizzo, V. J. 1993, A\&A, 268, 299

Carlsson, M., Stein, R. F., Nordlund, Å., \& Scharmer, G. B. 2004, ApJ, 610, L137

Cattaneo, F., Emonet, T., \& Weiss, N. 2003, ApJ, 588, 1183

Centrone, M. \& Ermolli, I. 2003, Memorie della Società Astronomica Italiana, 74, 671

Chevalier, S. 1912, Ann. de l'Obs. de Zô-sè, 8, C1

De Pontieu, B., Carlsson, M., Stein, R., et al. 2006, ApJ, 646, 1405

Deinzer, W., Hensler, G., Schüssler, M., \& Weisshaar, E. 1984a, A\&A, 139, 426

Deinzer, W., Hensler, G., Schüssler, M., \& Weisshaar, E. 1984b, A\&A, 139, 435

Dorotovič, I., Sobotka, M., Brandt, P. N., \& Simon, G. W. 2002, A\&A, 387, 665

Dunn, R. B. \& Zirker, J. B. 1973, Solar Phys., 33, 281

Finsterle, W., Jefferies, S., Cacciani, A., Rapex, P., \& McIntosh, S. 2004, ApJ, 613, L185

Fligge, M., Solanki, S. K., \& Unruh, Y. C. 2000, A\&A, 353, 380

Foukal, P., Fröhlich, C., Spruit, H., \& Wigley, T. M. L. 2006, Nature, 443, 161

Hansteen, V. H. 2004, in IAU Symposium, ed. A. V. Stepanov, E. E. Benevolenskaya, \& A. G. Kosovichev, 385-386

Hansteen, V. H., De Pontieu, B., Rouppe van der Voort, L., van Noort, M., \& Carlsson, M. 2006, ApJ, 647, L73

Hirzberger, J. \& Wiehr, E. 2005, A\&A, 438, 1059

Keller, C. U., Schüssler, M., Vögler, A., \& Zakharov, V. 2004, ApJ, 607, L59

Kneer, F. 1980, A\&A, 87, 229

Knölker, M. \& Schüssler, M. 1988, A\&A, 202, 275 
Langangen, Ø., Carlsson, M., van der Voort, L. R., \& Stein, R. F. 2007, ApJ, 655, 615

Leenaarts, J. \& Wedemeyer-Böhm, S. 2006, A\&A, 460, 301

Leka, K. D. \& Steiner, O. 2001, ApJ, 552, 354

LeVeque, R., Mihalas, D., Dorfi, E., \& Müller, E. 1998, in Computational Methods for Astrophysical Fluid Flow, ed. O. Steiner \& A. Gautschy (Springer-Verlag), 1-159

Lites, B. W., Scharmer, G. B., Berger, T. E., \& Title, A. M. 2004, Solar Phys., 221, 65

Mehltretter, J. P. 1974, Solar Phys., 38, 43

Meyer, F., Schmidt, H. U., \& Weiss, N. O. 1977, MNRAS, 179, 741

Muller, R. 1985, Solar Phys., 100, 237

Ortiz, A., Solanki, S. K., Domingo, V., Fligge, M., \& Sanahuja, B. 2002, A\&A, 388, 1036

Rouppe van der Voort, L. H. M., Hansteen, V. H., Carlsson, M., et al. 2005, A\&A, 435, 327

Rutten, R. J. 1999, in ASP Conf. Ser. 184: Third Advances in Solar Physics Euroconference: Magnetic Fields and Oscillations, ed. B. Schmieder, A. Hofmann, \& J. Staude, 181-200

Rutten, R. J., Kiselman, D., Rouppe van der Voort, L., \& Plez, B. 2001, in ASP Conf. Ser. 236: Advanced Solar Polarimetry - Theory, Observation, and Instrumentation, ed. M. Sigwarth, 445451

Sütterlin, P., Wiehr, E., \& Stellmacher, G. 1999, Solar Phys., 189, 57

Sánchez Almeida, J., Asensio Ramos, A., Trujillo Bueno, J., \& Cernicharo, J. 2001, ApJ, 555, 978

Sankarasubramanian, K. \& Rimmele, T. 2003, ApJ, 598, 689

Schaffenberger, W., Wedemeyer-Böhm, S., Steiner, O., \& Freytag, B. 2005, in Chromospheric and Coronal Magnetic Fields, ESA Publication SP-596, 299-300

Schatten, K. H., Mayr, H. G., Omidvar, K., \& Maier, E. 1986, ApJ, 311, 460

Schüssler, M. 1984, A\&A, 140, 453

Shelyag, S., Schüssler, M., Solanki, S. K., Berdyugina, S. V., \& Vögler, A. 2004, A\&A, 427, 335

Spruit, H. C. 1976, Solar Phys., 50, 269

Stein, R. F. \& Nordlund, ̊̊. 2006a, ApJ, 642, 1246

Stein, R. F. \& Nordlund, Å. 2006b, ApJ, 642, 1246

Steiner, O. 1990, PhD thesis, ETH-Zürich, Nr. 9292

Steiner, O. 2005, A\&A, 430, 691

Steiner, O., Grossmann-Doerth, U., Knölker, M., \& Schüssler, M. 1998, ApJ, 495, 468

Steiner, O., Hauschildt, P. H., \& Bruls, J. 2001, A\&A, 372, L13

Steiner, O., Vigeesh, G., Krieger, L., et al. 2007, Astron. Nachr./AN, in press

Ten Bruggencate, P. 1940, ZAp, 19, 59

Tobias, S., Brummell, N., Clune, T., \& Toomre, J. 1998, ApJ, 502, 177

Toro, E. 1999, Riemann Solvers and Numerical Methods for Fluid Dynamics (Springer-Verlag)

Tritschler, K., Schmidt, W., \& Rimmele, T. 2003, Astron. Nachr. Suppl., 324, 54

Vögler, A., Shelyag, S., Schüssler, M., et al. 2005, A\&A, 429, 335

Vogler, F. L., Brandt, P. N., Otruba, W., \& Hanslmeier, A. 2005, in Astronomy and Astrophysics Space Science Library, Vol. 320, Solar Magnetic Phenomena, ed. A. Hanselmeier, A. Veronig, \& M. Messerotti (Kluwer), 191-194

Wedemeyer-Böhm, S., Steiner, O., Bruls, J., \& Rammacher, W. 2007, in Coimbra Solar Physics Meeting on The Physics of Chromospheric Plasmas, ed. P. Heinzel, I. Dorotovič, \& R. Rutten, ASP Conference Series, in press

Weiss, N. O., Brownjohn, D. P., Matthews, P. C., \& Proctor, M. R. E. 1996, MNRAS, 283, 1153

Wenzler, T., Solanki, S. K., \& Krivova, N. A. 2005, A\&A, 432, 1057

Zhao, J., Georgobiani, D., Kosovichev, A. G., et al. 2007, ApJ, in press 

Modern Solar Facilities - Advanced Solar Science, 339-342

F. Kneer, K. G. Puschmann, A. D. Wittmann (eds.)

(C) Universitätsverlag Göttingen 2007

\title{
Solar mesogranulation as a cellular automaton effect
}

\author{
L. Matloch*, R. Cameron, D. Schmitt, and M. Schüssler \\ Max-Planck-Institut für Sonnensystemforschung, Katlenburg-Lindau, Germany \\ *Email: matloch@mps.mpg.de
}

\begin{abstract}
We present a simple cellular automaton model of solar granulation that captures the granular cell characteristics in terms of lifetime and size distributions. We show that mesogranulation, as defined in observational data, is an intrinsic feature of such a cell system.
\end{abstract}

\section{Introduction}

Since the first report of mesogranulation by November et al. (1981), the phenomenon has been investigated by many scientists with various approaches to the problem. Unlike granulation, which is believed to be quite well understood as a convective pattern arising from surface cooling, the nature of mesogranulation still remains elusive. The reported size of mesogranules is 3 to $10 \mathrm{Mm}$, hence deeper convection levels have been invoked to provide for the large cells, assuming that those patterns are of convective origin. Specifically, it was suggested that the first ionization of He, which occurs at depths around $7 \mathrm{Mm}$, is the driving force behind mesogranulation. However, this ionization level is not accompanied by a peak in the specific heat and therefore cannot give rise to significant increase of buoyancy. Furthermore, in contrast to granulation and supergranulation, the reported mesogranular characteristics vary greatly depending on the analysis methods applied (Rieutord et al. 2000) and the power spectra of the horizontal velocity field do not show a distinctive mesogranular peak, but rather a continuous spectrum of granular sizes which extend to the meso-domain (Chou et al. 1991; Straus et al. 1992; Straus \& Bonaccini 1997; Lawrence et al. 2001). As indications of mesogranular structure in shallow HD simulations were reported (Ploner et al. 2000), this suggests that mesogranulation can be a surface self-arrangement effect of granulation, without reaching deeper into the convection zone. We have developed a simple model, which retains the mutual influence of granules through the horizontal pressure as the only means of interaction. We show that such a simple model reproduces quite well the granulation features as well as mesogranulation when analyzed in the way the observational data and numerical simulations are.

\section{One-dimensional model}

The first version of the model has one spatial dimension and traces the evolution of artificial granules in time. It can be represented by $2 \mathrm{D}$ plots of granule positions versus time (see 
Fig. 1). The granules are defined as the space between the intergranular lanes, which are simple points in this model. These points start from a random initial distribution and move along the spatial dimension in each time step, therefore producing lines that run along the time axis. Thus at any given time there is a number of granules present in the simulation, their sizes being the distances between neighboring points. The spatial domain is periodic. The movement of any given point (intergranular lane) at each time step is determined by a 'pressure' difference exerted on it by the granules that the point separates. The pressure is assumed to be proportional to the granule size; the point moves towards lower pressure, i.e. small granules tend to be squeezed out of existence. We call it a 'cell competition' model, since this scheme makes cells 'compete' for space. When two intergranular lanes meet, they merge, marking the disappearance of a granule. This accounts for 'dissolving' granules. On the other hand, when an expanding granule reaches a critical size, it splits, becoming a fragmenter, with a new point (intergranular lane) appearing. The splitting parameters, which include random components for critical splitting size and new intergranular lane position, are chosen such that the cell lifetime and size distributions correspond to the observed properties of granulation.

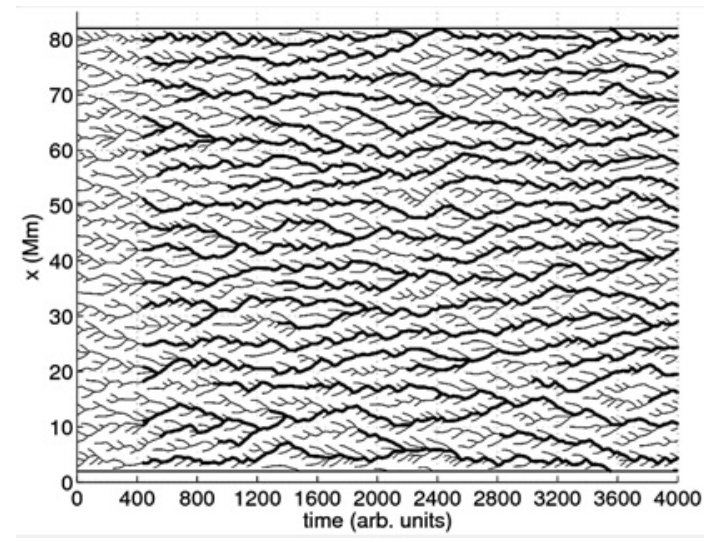

Figure 1. Example of the granule evolution plot, with intergranular lanes (black lines) evolving in time from left to right. Mesogranular lanes are marked as thick lines for averaging time $t_{0}=440$ timesteps (corresponding to $50 \mathrm{~min}$ ). Timespan of the domain is $445 \mathrm{~min}$. The $(x, y)$ units are arbitrary.

A common way to detect mesogranulation in observational data and MHD simulations is by means of 'corks', test particles being passively advected by the flow. The corks are introduced onto the surface of the fluid in each timestep and, after a few granule lifetimes, they tend to gather in the long-lived intergranular lanes, which mark places of horizontal velocity convergence (feeding downflows). The patterns of such long-lived intergranular lanes outline areas of horizontal fluid divergence which are identified with mesogranules (Simon et al. 1991; Ploner et al. 2000; Rieutord et al. 2000; Cattaneo et al. 2001; Roudier et al. 2003). In our model, there are no explicit fluid velocities, but we can define mesogranular lanes as intergranular lanes living longer than a given time, $t_{0}$, which is significantly longer than the average granule lifetime. Whenever two lanes merge, we keep the lifetime of the older one. Figure 1 shows an example of a granule evolution plot, with the mesogranular 

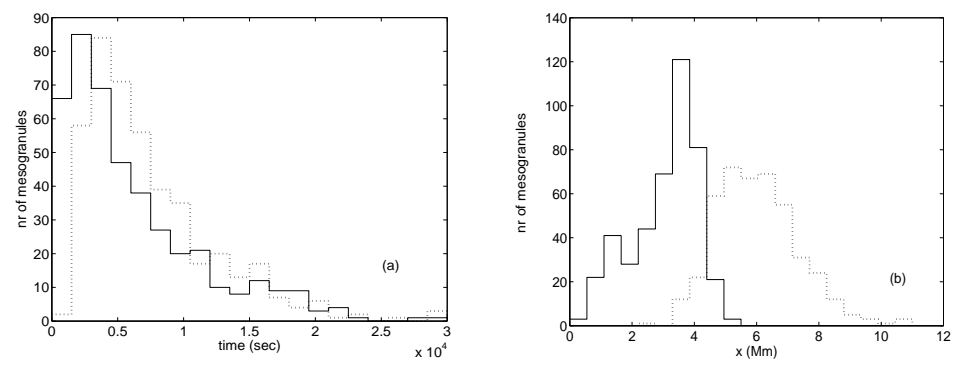

Figure 2. Mesogranule lifetime (a) and size (b) histograms extracted from the 1D model (see Fig. 1). Solid lines represent meso-dissolvers and dotted lines meso-fragmenters. The averaging time equals $t_{0}=50 \mathrm{~min}$.

lanes marked as thick lines for $t_{0}=50$ minutes. Similarly to granules, mesogranules can also be divided into dissolvers and fragmenters. Figure 2 shows mesocell characteristics for averaging time $t_{0}=50 \mathrm{~min}$.

We find that the mesogranule characteristics depend only weakly on the details of the granule cell interaction. A random motion model, in which intergranular lanes perform a random walk in the domain, produces mesogranulation with similar characteristics.

\section{Two-dimensional model}

Figure 3 shows a snapshot from 2D extension of the cell evolution model. The cells are now triangles and the cell evolution is achieved by moving the vertices towards one of the neighbors along the connection lane (cell side). By employing four different motion rules as well as four different splitting rules (including a random motion model) we measure how sensitive the resulting mesostructures are to the details of the cell interaction.

To be able to compare these results with observations or numerical simulations, we also employ procedures similar to those used in solar data analysis for mesogranulation detection. Apart from the cork method, which outlines the velocity divergence areas, one can directly calculate the divergence and extract the statistical properties of the divergence patches, i.e., mesogranules. The velocity field is obtained with a Local Correlation Tracking (LCT) algorithm. Both the mesolane and the divergence patch methods produce similar exponential histograms of cell areas and lifetimes. The statistical properties of mesogranules in the model show only little dependence on the cell interaction rules, showing them to be robust features of such cellular automata models. Figure 4 shows an example of the mesogranulation characteristics for the mesolane detection method.

\section{Conclusions}

We have shown that mesostructures, similarly defined as in observations, arise naturally in granulation-like cellular automata models, with no additional effects needed. Both the cork-equivalent as well as velocity divergence methods detect mesostructures in the mod- 


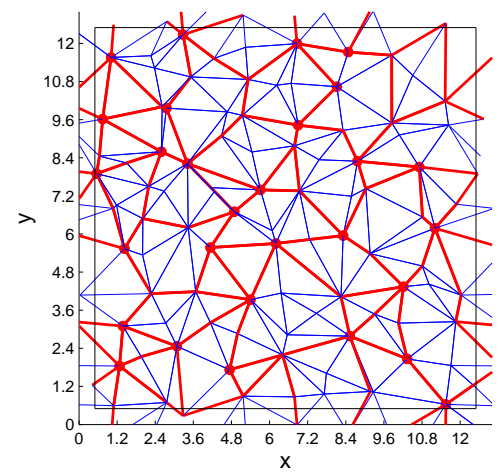

Figure 3. Example of the $2 \mathrm{D}$ extension of the granule evolution plot, with the mesogranular lanes marked as thicker light-grey lines, for $t_{0}=60 \mathrm{~min}$. Large dots are the mesovertices, i.e. vertices that are older than $t_{0}$.
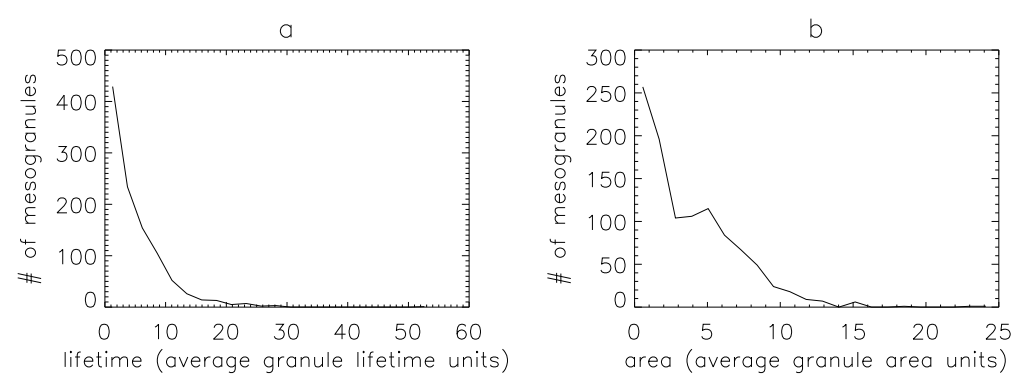

Figure 4. Example of the mesogranulation characteristics for the mesolane detection method for the 2D model (see Fig. 3); (a) is the lifetime histogram, (b) is the area histogram. Averaging time equals $t_{0}=60 \mathrm{~min}$.

els. Moreover, mesogranulation proves to be a robust feature of such models, only weakly depending on the detailed granule interaction rules.

\section{References}

Cattaneo, F., Lenz, D., \& Weiss, N. 2001, ApJ, 563, 91

Chou, D.-Y., Labonte, B. J., Braun, D. C., \& Duvall, T. L., Jr. 1991, ApJ, 372, 314

Lawrence, J. K., Cadavid, A. C., \& Ruzmaikin, A. 2001, Solar Phys., 202, 27

November, L. J.,Toomre, J., Gebbie, K. B., \& Simon, G. W. 1981, ApJ, 245, 123

Ploner, S. R. O., Solanki, S. K., \& Gadun, A. S. 2000, A\&A, 357, 1050

Rieutord, M., Roudier, T., Malherbe, J. M., \& Rincon, F. 2000 A\&A 357, 1063

Roudier, T., Lignières, F., Rieutord, M., Brandt, P. N., \& Malherbe, J .M. 2003, A\&A, 409, 299

Simon, G. W., Title, A. M., \& Weiss, N. O. 1991, ApJ, 375, 775

Straus, T. \& Bonaccini, D. 1997, A\&A, 324, 704

Straus, T., Deubner, F.-L., \& Fleck, B. 1992, A\&A, 256, 652S 


\title{
Sunspot models with bright rings
}

\author{
L. L. Kitchatinov ${ }^{1,2^{*}}$ and G. Rüdiger ${ }^{2}$ \\ ${ }^{1}$ Institute for Solar-Terrestrial Physics, Irkutsk, Russia \\ ${ }^{2}$ Astrophysikalisches Institut Potsdam, Germany \\ *Email: 1kitchatinov@aip.de
}

\begin{abstract}
A theoretical sunspot model is provided including magnetic suppression of the diffusivities and also a strong stratification of density and temperature. Heat diffusion alone with given magnetic field and zero mean flow only produces (after a very long relaxation time) dark spots without any bright ring. Models with full dynamics of both field and flow, however, provide rings and also the observed correlation of ring temperature excess and the spot size. The rings are formed as the result of heat transport by the resulting flow system and increased thermal diffusivity due to reduced magnetic quenching around spots.
\end{abstract}

\section{Introduction}

Measurements have shown the existence of bright rings around sunspots (Bonnet et al. 1978; Rast et al. 1999, 2001). The rings appear one spot radius beyond the spots and are reported to be about $10 \mathrm{~K}$ warmer than the photosphere. There is a clear trend that larger spots have brighter rings. We shall discuss in the following whether simple mean-field models of more or less flat sunspots do develop such rings or not.

\section{A simple model}

The model concerns a horizontal layer with top and bottom at depths $d_{\text {top }}$ and $d_{\text {bot }}$ below the photosphere. In our computations $d_{\text {top }}=1 \mathrm{Mm}$ is fixed, slightly beneath the typical depth of Wilson depression. The fluid is assumed to be a perfect gas. Density and temperature at the top $\left(\rho_{\text {top }}, T_{\text {top }}\right)$ and bottom $\left(\rho_{\text {bot }}, T_{\text {bot }}\right)$ boundaries are prescribed with the solar structure model by Stix \& Skaley (1990). The reference atmosphere is approximated by adiabatic profiles,

$$
T=T_{\text {top }}+\frac{g}{C_{\mathrm{p}}}(D-z), \quad \rho=\rho_{\text {top }}\left(\frac{T}{T_{\text {top }}}\right)^{\frac{1}{\gamma-1}} .
$$

Here the gravity is $g=2.74 \cdot 10^{4} \mathrm{~cm} \mathrm{~s}^{-2}, D=d_{\text {bot }}-d_{\text {top }}$ is the layer depth (here $15 \mathrm{Mm}$ ), $z$ is the vertical coordinate, $C_{\mathrm{p}}$ and $\gamma$ are specific heat and adiabaticity index defined by the condition that the bottom temperature and density are exactly reproduced, i.e.

$$
C_{\mathrm{p}}=\frac{g D}{T_{\text {bot }}-T_{\text {top }}}, \quad \gamma=1+\frac{\ln \left(T_{\text {bot }} / T_{\text {top }}\right)}{\ln \left(\rho_{\text {bot }} / \rho_{\text {top }}\right)} .
$$


The entropy equation with $S=C_{\mathrm{v}} \log \left(P / \rho^{\gamma}\right)$ is

$$
\rho T\left(\frac{\partial S}{\partial t}+\boldsymbol{u} \cdot \nabla S\right)=\boldsymbol{\nabla} \cdot(\rho T \chi \nabla S) .
$$

The sunspot darkness is usually explained in terms of convective heat transport suppressed by magnetic field. Accordingly, the eddy diffusivities in the model depend on the magnetic field (cf. Rüdiger \& Kitchatinov 2000). This dependence describes a steady decrease of the turbulent diffusivities with the magnetic field amplitude. Hence $\chi=\chi_{\mathrm{T}} \varphi(\beta)$, where $\chi_{\mathrm{T}}$ is nonmagnetic diffusivity and the quenching function

$$
\varphi(\beta)=\frac{3}{8 \beta^{2}}\left(\frac{\beta^{2}-1}{\beta^{2}+1}+\frac{\beta^{2}+1}{\beta} \arctan \beta\right)
$$

depends on the field strength normalized to the energy equipartition value, $\beta=B /\left(\sqrt{\mu_{0} \rho} u^{\prime}\right)$, $u^{\prime}$ is the rms turbulent velocity. Any anisotropy of the eddy diffusivities is neglected.

The depth profiles of the equipartition field, $B_{\mathrm{eq}}=\sqrt{\mu_{0} \rho} u^{\prime}$, and the (nonmagnetic) diffusivities are written in accordance with the mixing-length approximation, which yields

$$
\chi_{\mathrm{T}}(z)=\chi_{0}\left(\frac{T}{T_{\mathrm{top}}}\right)^{\frac{3 \gamma-4}{3(\gamma-1)}}, \quad B_{\mathrm{eq}}^{2}=B_{0}^{2}\left(\frac{T}{T_{\mathrm{top}}}\right)^{\frac{1}{3(\gamma-1)}},
$$

where $T$ has been defined in (1) while $\chi_{0}$ and $B_{0}$ are the thermal diffusivity and the equipartition field on the top boundary. A marginal value for thermal convection, $\chi_{0}=1.4 \cdot 10^{13} \mathrm{~cm}^{2} / \mathrm{s}$, was taken for the diffusivity, and $B_{0}=500$ Gauss. The density runs from $2.02 \times 10^{-6} \mathrm{~g} / \mathrm{cm}^{3}$ at the top to $1.8 \times 10^{-3} \mathrm{~g} / \mathrm{cm}^{3}$ at the bottom, and the temperature varies from $14,000 \mathrm{~K}$ to $120,000 \mathrm{~K}$.

The horizontal boundaries are stress-free and impenetrable. For the magnetic field a vacuum condition is used for the top while the field is assumed vertical on the bottom. Thermal conditions are the black-body radiation on the top and constant heat flux $\left(F_{0}=6.27 \cdot 10^{10} \mathrm{~g} \mathrm{~s}^{-3}\right)$ at the bottom. If wall boundaries are used we assume zero stress, zero normal velocity, and superconductor outside.
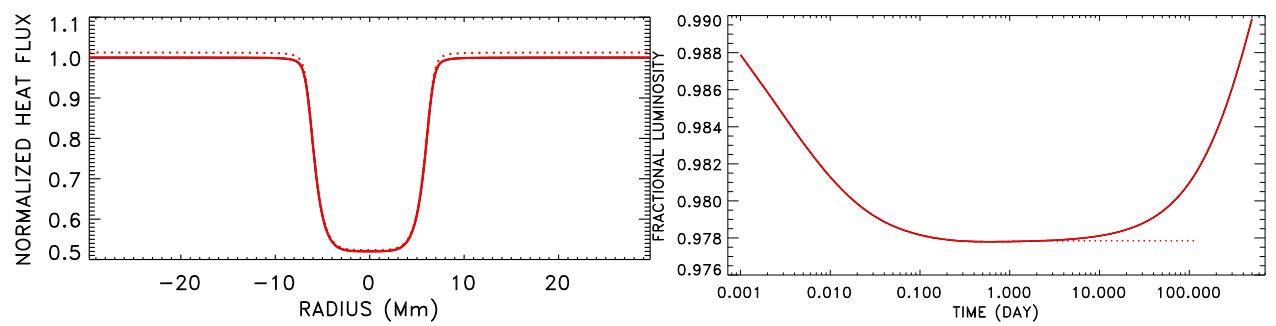

Figure 1. Left: Profiles of surface brightness normalized to $F_{0}$ after 1 day (solid) and after about 500 days (dotted) for vanishing heat flux across the wall boundary. Right: Time dependence of the normalized total irradiance at the top. The thermal conditions on the side wall are $F_{r}=0$ (solid) and $\partial T / \partial t=0$ (dotted).

A simplified model was used to probe the possibility of reproducing bright rings by heat transport alone. In this model the velocity is put to zero, the spot-like structure of the magnetic field is prescribed and stationary, and the diffusion equation for the entropy is solved. 
It is a rather flat spot with an aspect ratio $a=2$. The spot of this model has a magnetic flux of $3 \cdot 10^{21} \mathrm{Mx}$ and a depth of about $10 \mathrm{Mm}$.

The surface brightness at the initial state is uniform and equals $F_{0}$. The brightness of the region occupied by magnetic field decreases due to magnetic quenching of the thermal diffusivity. The normalized surface brightness approaches the profile shown by the solid line in Fig. 1 (left panel) already after several hours and varies slowly after this time. The profile does not show any bright ring.

The further evolution depends on the thermal conditions imposed on the wall boundary. The boundary condition (zero heat flux across the boundary) ensures that in the steady state the total irradiance from the surface equals the heat supply at the bottom $\left(\pi R^{2} F_{0}\right)$. In the steady solution the heat flux blocked by the spot can reemerge for flat spots as a bright ring (Eschrich \& Krause 1977). Figure 1 (right) shows that the luminosity does indeed recover after the initial reduction but it needs a very long time which is of the order of the KelvinHelmholtz time $\left(\tau_{\mathrm{KH}} \sim 10\right.$ yrs for our model).

The reason for this long time scale is the high heat capacity of the solar interior. The time passed before the rise in the surface irradiance can be seen is much longer than lifetimes of sunspots. Steady solutions of the heat transport equation are thus not relevant for sunspots. Also flatter spot models do not show any bright rings.

\section{A new MHD sunspot model}

For a more consistent MHD model for sunspots we work with the anelasticity condition, $\boldsymbol{\nabla} \cdot(\rho \boldsymbol{u})=0$, and the complete momentum equation together with the induction equation

$$
\frac{\partial \boldsymbol{B}}{\partial t}=\boldsymbol{\nabla} \times(\boldsymbol{u} \times \boldsymbol{B}-\eta \boldsymbol{\nabla} \times \boldsymbol{B}) .
$$
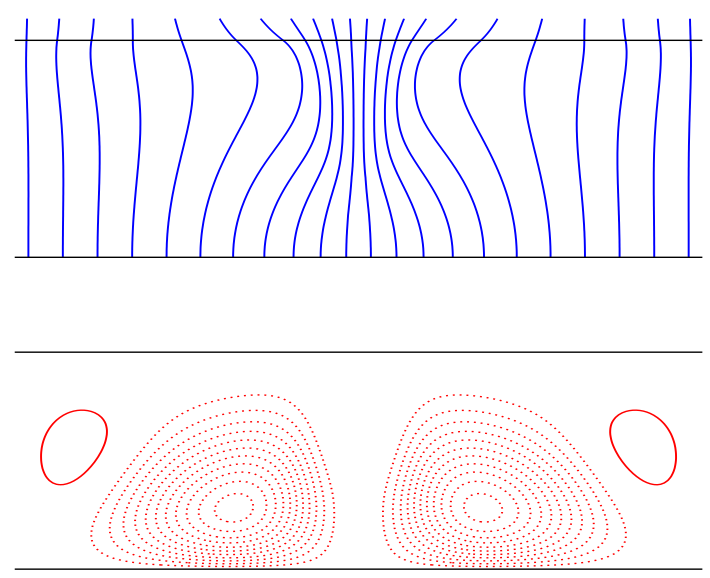

Figure 2. Field lines (top) and streamlines (bottom) after 3 days for an initial field of 600 Gauss. The dotted streamlines mean the flow convergent on the top and divergent on the bottom. The depth of the simulated spot is comparable to its diameter. The surface flow is convergent near the spot but divergent at larger distances. 
Amplitudes of the initial uniform field of several hundred Gauss are considered. Magnetic diffusivity and eddy viscosity are assumed to follow the same magnetic quenching expressions as in Eq. (4).

The vertical size of the simulated spots is comparable to their diameter. The resulting mean 'meridional circulation' is convergent on the top near the spots and it makes downdrafts beneath the spots in agreement with results of heliotomography (Fig. 2, bottom). The amplitude of the flow is about $800 \mathrm{~m} \mathrm{~s}^{-1}$. The flow direction becomes divergent at larger radial distances. The field strength in the spot is about 2700 Gauss. Brightness and field strength are almost uniform in the central parts of the spots but they change rapidly with radius beyond the 'umbra'.

The radial heat-flux profiles show bright rings around the spots. Our rings are somewhat brighter than the observed rings.

A comparison of Figs. 2 and Fig. 3 shows that the position of the ring maximum is at the radius where the horizontal surface flow changes its direction and an upwards directed subsurface flow appears. At the same radius the surface field strength has a minimum. Hence, the upward flow carries extra heat from beneath and the reduced field strength increases the diffusive heat flux due to reduced magnetic quenching of the thermal diffusivity (4). If the flow is switched off to probe its contribution to the bright rings they do not disappear. We conclude that both the flow and the reduced diffusivity quenching contribute to the resulting bright rings. The contribution by the circulation should basically be steady. Observations can help to decide between the two possible explanations for the rings by finding out whether the rings around big spots are decaying on a time scale of some days or not.

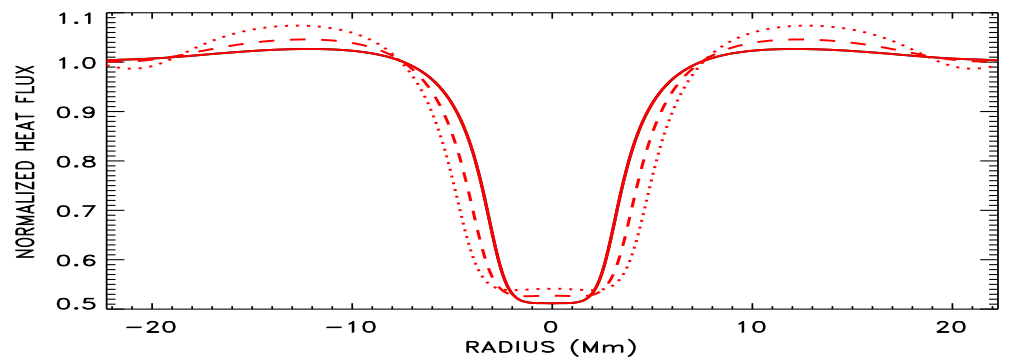

Figure 3. Surface brightness normalized to $F_{0}$ for the time of 3 days in the runs for initial fields of 400 (full line), 500 (dashed), and 600 Gauss (dotted). All runs show bright rings which are more pronounced for larger spots.

\section{References}

Bonnet, R. M., Lemaire, P., Vial, J. C., et al. 1978, ApJ, 221, 1032

Eschrich, K.-O. \& Krause, F. 1977, Astron. Nachr., 298, 1

Kitchatinov, L. L., Pipin, V. V., \& Rüdiger, G. 1994, Astron. Nachr., 315, 157

Rast, M. P., Fox, P. A., Lin, H., et al. 1999, Nature, 401, 678

Rast, M. P., Meisner, R. W., Lites, B. W., et al. 2001, ApJ, 557, 864

Rüdiger, G. \& Kitchatinov, L. L. 2000, Astron. Nachr., 321, 75

Stix, M. \& Skaley, D. 1990, A\&A, 232, 234 


\title{
Numerical modeling of MHD wave propagation in sunspots: a 3D case
}

\author{
V. Olshevsky ${ }^{1, *}$, E. Khomenko ${ }^{2,1}$, and M. Collados ${ }^{2}$ \\ ${ }^{1}$ Main Astronomical Observatory, NAS, Kyiv, Ukraine \\ ${ }^{2}$ Instituto de Astrofísica de Canarias, La Laguna, Tenerife, Spain \\ *Email:sya@mao.kiev.ua
}

\begin{abstract}
We present the first results of a 3D numerical modeling of linear MHD wave propagation in a realistic sunspot model. In our simulations, a piston located at the base of the photosphere generates waves with a certain period. The ratio between the acoustic and the Alfvén speed, $c_{S} / v_{A}$, decreases from much larger than one at the photosphere to much lower than one in the chromosphere in our simulation domain. Waves propagate through the region where $c_{S}=v_{A}$, where mode transformation is observed. At a somewhat higher region, where $c_{S} \ll v_{A}$, the fast (magnetic) mode reflects back to the photosphere due to the vertical and horizontal gradients of $v_{A}$. The slow (acoustic) mode propagates to the upper layers and increases its velocity amplitude. Unlike the 2D simulations, the Alfvén mode is also generated by the piston and experiences transformations at the $c_{S}=v_{A}$ layer. The behaviour of this mode requires further study.
\end{abstract}

\section{Introduction}

Wave phenomena in solar magnetic structures have recently become a subject of great scientific interest. The latest observational studies of sunspots by Centeno et al. (2006) suggest that the observed 3-min velocity oscillations in the umbral chromosphere are due to the propagation of a 3-minute perturbation from the underlying photosphere. As theoretically investigated by Cally (2005) and Schunker \& Cally (2006), wave mode coupling and transformation near the equipartition layer, where Alfvén and sound speeds coincide, are essential for the energy propagation to the chromosphere of sunspots. These phenomena were studied via numerical 2D modeling by Khomenko \& Collados (2006). They adopt a magnetostatic model of a sunspot in which linear waves propagate upwards and mode transformation occurs near the $c_{S}=v_{A}$ line. A complex behaviour of the waves is observed in higher regions. The fast (magnetic) mode reflects back to the photosphere, and only some part of the wave energy is transported upwards by the slow (acoustic) mode along the field lines. Although computations were made for 10 -sec period oscillations, these authors suggest that a similar behaviour is expected for oscillations with frequencies higher than acoustic cut-off.

In order to make such modeling more realistic we address in this work the same questions as Khomenko \& Collados (2006), but extend our computations to 3 dimensions and study waves with a somewhat higher period of 20 seconds. 


\section{Computational scheme}

We investigate linear waves propagating in a 3D magnetostatic (MHS) model of a sunspot. We consider the following ideal MHD equations:

$$
\begin{aligned}
& \frac{\partial \rho}{\partial t}+\boldsymbol{\nabla}(\rho \boldsymbol{V})=0, \\
& \frac{\partial \rho \boldsymbol{V}}{\partial t}+\boldsymbol{\nabla}\left(\rho \boldsymbol{V} \boldsymbol{V}+\left(P+\frac{B^{2}}{8 \pi}\right) \hat{I}-\frac{\boldsymbol{B} \boldsymbol{B}}{4 \pi}\right)=\rho \boldsymbol{g}, \\
& \frac{\partial}{\partial t}\left(\frac{1}{2} \rho V^{2}+\frac{B^{2}}{8 \pi}+\frac{P}{\gamma-1}\right)+\nabla\left(\frac{1}{2} \rho V^{2} \boldsymbol{V}+\frac{\gamma}{\gamma-1} P \boldsymbol{V}+\frac{1}{4 \pi} \boldsymbol{B} \times(\boldsymbol{V} \times \boldsymbol{B})\right)=\boldsymbol{V} \rho \boldsymbol{g}+\rho Q, \\
& \frac{\partial B}{\partial t}=\boldsymbol{\nabla} \times(\boldsymbol{V} \times \boldsymbol{B}),
\end{aligned}
$$

where all the symbols have their usual meaning and $\hat{I}$ is a unity tensor. We neglect energy losses, which are represented by the term $\rho Q$, which we set to zero and thus consider only adiabatic waves. We use the equation of state of the perfect gas. As a first approach all equations were linearized and solved using a fourth-order Runge-Kutta numerical scheme.

\section{Computational domain and boundary conditions}

For our 3D modeling, we have taken an axial-symmetric MHS model of a sunspot computed by Khomenko \& Collados (2006). A part of this cube of size $1.8 \times 1.8 \times 0.8 \mathrm{Mm}^{3}$ was chosen as a computational domain (see Fig. 1 in Khomenko \& Collados 2006). As in the latter work we apply the perfectly matched layer boundary conditions (Berenger 1994), though extended to $3 \mathrm{D}$, at all domain boundaries. These conditions exclude all reflections from the boundaries and absorb all incoming waves.

Waves were excited by a driver placed at the base of the umbral photosphere. The driver had a Gaussian shape in horizontal directions. It initiated periodical velocity perturbations. Two types of drivers were applied: horizontal and vertical. The horizontal driver initiates mostly a set of slow magneto-acoustic waves and Alfvén waves. The second, vertical driver, produces mainly the fast magneto-acoustic waves. The behaviour of different wave types as they are rising into the upper layers is described below.

\section{Results of the calculations}

The two magneto-acoustic modes (fast and slow) differ in their nature depending on the value of the $c_{S} / v_{A}$ ratio. In the photosphere, where $c_{S} / v_{A} \gg 1$, the fast mode is acoustic, while the slow mode is magnetic. In the high layers, where $c_{S} / v_{A} \ll 1$, the situation is the opposite. The magnetic waves, as well as the Alfvén waves can be distinguished by plotting the transversal velocity oscillations $(\boldsymbol{V} \perp \boldsymbol{B}$, columns of panels 1 and 3 in Fig. 1$)$ and the acoustic waves by plotting the longitudinal ones ( $\boldsymbol{V} \| \boldsymbol{B}$, columns of panels 2 and 4 in Fig. 1).

Unlike the 2D case, the Alfvén mode can be generated in simulations with a horizontal driver. Due to the inclination of the field lines, the driver does not generate pure modes, but rather a set of Alfvén waves, slow and fast magneto-acoustic waves, with the latter being 

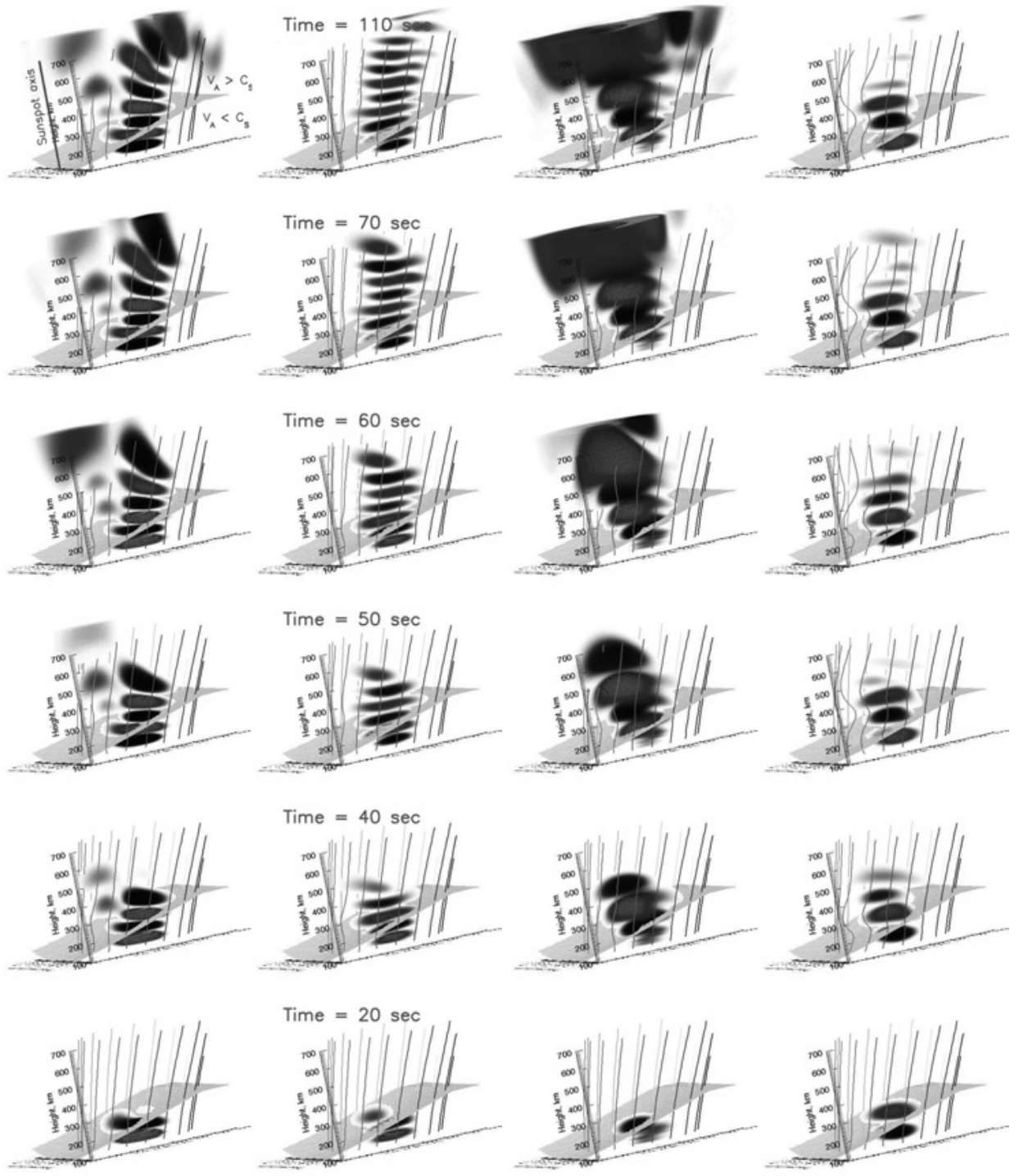

Figure 1. The four columns of panels show velocity oscillations produced by MHD waves. The two left sets correspond to the simulations with the horizontal driver, two right sets correspond to the simulations with the vertical driver. For each driver the left panels display transversal $(\boldsymbol{V} \perp \boldsymbol{B})$, while the right panels display longitudinal $(\boldsymbol{V} \| \boldsymbol{B})$ velocity components. The surface where $v_{a}=c_{S}$ is shown with a gray color. Oscillations of the magnetic field are multiplied by a certain factor to clearly show the variations of the field lines, shown as thin solid lines. Darker shades correspond to larger velocity perturbations, but the scale is arbitrary, since we consider a linear case. The sunspot axis is parallel to the $\mathrm{Z}$ axis and crosses the $\mathrm{Y}$ axis in the middle of the computational domain (see the top left panel). 
less important. It is seen in the two left columns of Fig. 1 that slow-to-slow mode transformation plays an important role and a large amount of the wave energy is transported into the upper chromosphere along the field lines by the slow wave. The Alfvén wave generated by the driver is not observed in the upper layers. Thus, we conclude that, possibly, it is transformed into the other wave types. A further analysis of the simulations is required, however, to understand the behaviour of this mode. In the upper layers, where the magnetic wave becomes fast, its propagation direction does not depend on $\boldsymbol{B}$ and it is reflected back to the photosphere. Note that there appear two fast waves: one that refracts towards the spot axis, and another one that refracts away from it.

In the simulations with a vertical driver (two right columns of Fig. 1) the fast (acoustic) mode generation is the most efficient. Near the $c_{S}=v_{A}$ layer the fast-to-fast mode transformation is very sizeable. Fast (magnetic) waves form a fountain-like structure due to refraction and finally reflect back to the photosphere.

From these calculations we can conclude that the slow-to-slow and fast-to-fast mode transformations are very effective at the $c_{S}=v_{A}$ layer. As a consequence, only if the slow magnetic mode is generated in the deep photosphere, wave energy transport to the chromosphere by this mode can be effective.

\section{Conclusions}

Different wave phenomena which were seen in 2D calculations and explained by Khomenko $\&$ Collados (2006) are also observed in our 3D modeling. They prove that only some part of the driver energy can reach the upper chromosphere. Only the slow wave can transport energy to the upper layers, since the fast mode is reflected back. The slow-to-slow and fastto-fast mode transformations seem to be more efficient in 3D than in 2D. Consequently, at least for short-period waves, the slow mode should be generated directly by the driver, to make the energy transport more efficient.

In the future, our 3D simulations can be used to reveal effects which are not accessible by 2D methods, such as generation and transformation of the Alfvén mode, effects of the twist of the flux tube, etc.

Acknowledgements. V.L.O. acknowledges financial support by the Deutsche Forschungsgemeinschaft for participation in the workshop. This research was partly funded by the Spanish Ministerio de Educación y Ciencia through project AYA2004-05792.

\section{References}

Berenger, J. P. 1994, J. Comp. Phys., 114, 185

Cally, P. S. 2005, MNRAS, 358, 353

Centeno, R., Collados, M., \& Trujillo Bueno, J. 2006, ApJ, 640, 1153

Khomenko, E. \& Collados, M. 2006, ApJ, 653, 740

Schunker, H. \& Cally, P. S. 2006, MNRAS, 372, 551 


\title{
Seismology of sunspot atmosphere: from chromospheric resonance to nonlinear antireflection
}

\author{
Y. D. Zhugzhda \\ IZMIRAN, Troitsk, Russia \\ Email: zhu@izmiran.ru
}

\begin{abstract}
The theory of 3-min oscillations in sunspot chromospheres is revised. Linear and nonlinear effects are taken into account. It is revealed that not only the chromospheric resonance is responsible for the 3-min oscillations in sunspots. The spectrum of oscillations in chromosphere and transition region appears due to the combined effects of chromospheric resonance, cut-off frequency of temperature minimum and nonlinear antireflection of the sunspot atmosphere.
\end{abstract}

\section{Introduction}

There are numerous observations of 3-min oscillations in chromosphere and transition region of sunspots. Oscillations are detected in optical (Centeno et al. 2006), UV (Brynildsen et al. 2004) and radio (Gelfreikh et al. 2006) ranges. The most important feature of 3-min oscillations is the occurrence of few peaks in their spectrum which correspond to the periods of 3 min and less. The explanation of the fine structure of 3-min spectrum of the chromospheric oscillations of sunspots was found by Zhugzhda \& Locans (1981). They were the first who did not consider sunspot oscillations as eigen-oscillations. The propagation of slow MHD waves through sunspot atmosphere was explored. The treatment of the wave propagation was based on the finding of Syrovatskii \& Zhugzhda (1968) who revealed that slow waves propagate in a stratified atmosphere along the field lines with sound speed if the magnetic field is sufficiently strong. The filter theory was used for exploration of empirical models of the sunspot atmosphere (Zhugzhda et al. 1983, 1984, 1985, 1987; Settele et al. 2001). A new approach to the seismology of sunspot atmosphere is proposed in the present paper.

We need to mention that the filter theory of sunspot oscillations is confused sometimes with the eigen theory because of a chromospheric resonance is mentioned for the explanation of the frequencies of transmission. But a chromospheric resonator works as an interference filter for slow waves since it is excited not from inside but from the outside by waves coming from the subphotospheric layers.

The structure of the sunspot atmosphere is not known well enough. This point becomes clear from the comparison of distinct empirical models of sunspot atmosphere. There are essential differences between empirical models of Staude (1981), Maltby et al. (1986) and Lites \& Skumanich (1982). For example the thickness of the temperature plateau in the chromosphere varies from $400 \mathrm{~km}$ for Staude's model to $800 \mathrm{~km}$ for the Maltby et al. model. 

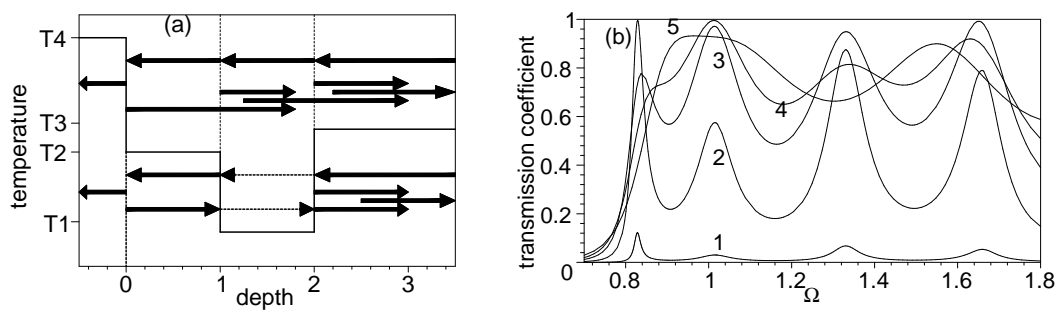

Figure 1. (a) Schemes of wave propagation through a four-layer atmosphere for waves of frequencies below cut-off frequency (bottom of the plot) and above cut-off (top). (b) The dependence of filter transmission on dimensionless frequency for various coefficients of amplitude reduction due to nonlinear dissipation. Curves $1-5$ correspond to the reduction coefficients $1,0.83,0.67,0.37,0.07$.

The thickness of the temperature minimum differs by more than a factor 2 for various models.

\section{Four-layer model of sunspot atmosphere}

To explore the filter properties of the sunspot atmosphere we consider a four-layer isothermal model of the sunspot atmosphere which is shown in Fig. 1a. Four isothermal layers of temperatures $T 1, T 2, T 3, T 4$ correspond to corona, temperature plateau of chromosphere, temperature minimum and photosphere-subphotosphere layers of a sunspot, respectively. This model permits to play with the parameters of the sunspot atmosphere which is not possible with the empiric models. Fig. 1a shows two scenarios of wave propagation through the sunspot atmosphere. The first scenario occurs for slow waves with the frequencies less than the cut-off frequency of the temperature minimum. In this case the waves incident upon the temperature minimum from below undergo strong reflection and penetrate through the temperature minimum to the chromosphere due to a tunnel effect considered for the first time for waves in the solar atmosphere by Zhugzhda (1972). After penetration to the chromosphere the waves are reflected from the corona and tunnelled back through the temperature minimum to the photosphere. The waves reflected by the temperature minimum and corona interfere in the photosphere. If they are out of phase destructive interference occurs and the reflection from the upper sunspot atmosphere drops. It happens at the frequency of the chromospheric resonance. Thus, the sunspot atmosphere works in this case as a Fabry-Perot interference filter for slow waves. This scenario is shown by arrows in the bottom of Fig. 1a. But there is the essential difference from the conventional interference filter due to strong dependence of wave reflection from the temperature minimum on frequency. As a result, the only one passband could appear for slow waves. As a rule, the second harmonic of chromospheric resonance has the frequency above the cut-off frequency of the temperature minimum which exludes resonance. If the temperature plateau is assumed to be so thick that the frequency of the second harmonic of a chromospheric resonator is below the cutoff frequency, the transmission of the passband for the first harmonic reduces so much that the filter does not work for this frequency. The filtering due to chromospheric resonance is effective only for the limited range of frequencies below the cut-off frequency since the Fabry-Perot interference filter for slow waves works only when reflections from the corona and the temperature minimum are of the same order. Only in this case waves are concealed 
almost completely due to destructive interference. No more than one harmonic of the chromospheric resonator can be in the range of frequencies which are suitable for an effective interference filter. It could happen that all of the harmonics of a chromospheric resonator are out of this range of frequencies. In this case an interference filter for slow waves has low transmission and a corresponding peak in the spectrum of 3-min oscillations is so small that it can be missed. Thus, the chromospheric resonance can not be responsible for the appearance of multiple peaks in the spectrum.

To understand the origin of the peaks in the spectrum we need to consider the second scenario of wave propagation when the frequency is above the cut-off frequency of the temperature minimum. The scheme of wave propagation is shown in the upper part of Fig. 1a. In this case there is no strong reflection of waves from the temperature minimum. If the spectrum of incident waves is decreasing fast with frequency the peak around the cut-off frequency has to appear. The origin of passbands above the cut-off frequency is related with the so-called antireflection effect which appears in a multilayered medium. This effect appears when the medium consists of few layers with more or less sharp boundaries between them. The interference of the waves reflected from the boundaries makes the media partly transparent for the waves of some specific frequencies. The temperature plateau and temperature minimum are the layers responsible for the appearance of multiple peaks in the spectrum of 3-min oscillations. The frequencies of these peaks are higher than the cut-off frequency.

A concern arises due to the evidence that 3-min oscillations are nonlinear in the chromosphere (Centeno et al. 2006) and the transition region (Brynildsen et al. 2004). But the interference responsible for the chromospheric interference filter and antireflection effect takes place in the temperature minimum and photosphere where the linear approximation works perfectly. Thus the nonlinearity of 3-min oscillations does not affect the interference process. But the nonlinear dissipation of waves has to reduce the amplitudes of waves coming from above to the temperature minimum. The observations of Brynildsen et al. (2004) show decreasing amplitudes in the transition region for temperatures above $10^{5} \mathrm{~K}$. To explore the effect of nonlinearity on filtering of slow waves by sunspot atmospheres it is enough to explore the consequences of amplitude reduction the waves traveling from upper layers to the temperature minimum. Since it is difficult to estimate a nonlinear dissipation, various coefficients of amplitude reduction of the waves reflected from the top of the atmosphere were used to calculate the transmission function. Fig. 1b shows just one example of the calculation of the transmission coefficient for the four-layer atmosphere as a function of the dimensionless frequency $\Omega$ normalized to the cut-off frequency. The curves $1-5$ on the plot of Fig. 1b show the transmission of the filter as a function of $\Omega$ for different values of the amplitude reduction coefficient, namely, $1,0.83,0.67,0.37,0.07$. The transmission of all filter passbands increases, as a rule. The transmission of the passband due to the chromospheric resonance $(\Omega=0.815)$ increases with decreasing amplitude of the reflected waves up to the amplitude reduction coefficient 0.83 . A further reduction of the waves reflected from the transition region leads to a decrease of the passband transmission and disappearance of the chromospheric resonance when the amplitude of reflected waves reduces by a factor of $10-15$. The transmission of the passband due to the cut-off frequency $(\Omega=1)$ increases as well with decreasing amplitude of the reflected wave. The transmission of passbands due to antireflection effect increases along with the decrease of the reflected wave amplitudes. Thus, we see that the nonlinear damping of the waves in chromosphere and transition region leads to a higher quality of filter passbands. 

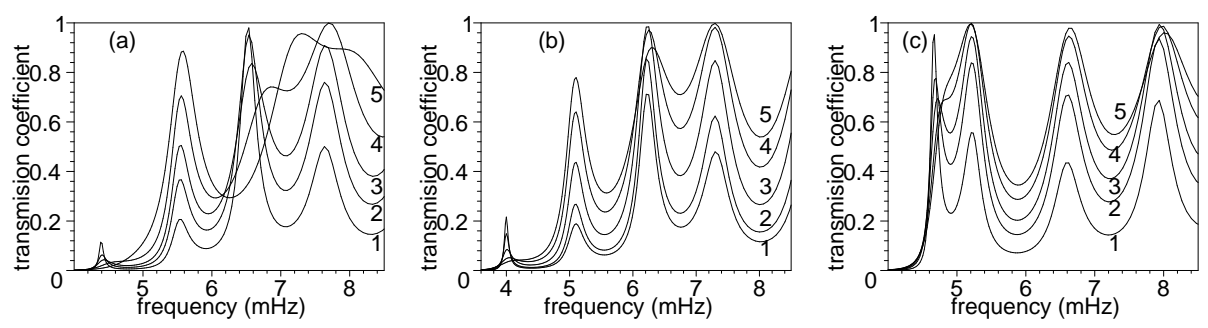

Figure 2. Transmission as a function of frequency for empirical models of (a) Lites \& Skumanich (1982), (b) Maltby et al. (1986) and (c) Staude (1981). Curves 1-5 correspond to reduction of reflected waves by factors $0.7,0.5,0.25,0.1,0.0$, respectively.

\section{Analysis of empirical models of sunspot atmosphere}

Figures $2 \mathrm{a}, \mathrm{b}$ and $\mathrm{c}$ show the transmission coefficient for the empirical models of Lites \& Skumanich (1982), Maltby et al. (1986) and Staude (1981). The passband due to the cutoff frequency appears almost at the same frequency for all three models since the sound velocities in the temperature minimums of these models are nearly the same. In contrast, the passband due to the chromospheric resonance occurs at different frequencies for different models. Besides, the transmission of this passband increases with the increase of its frequency. The maximum transmission occurs for Staude's model. The interval between antireflection passbands is different for the models of Lites \& Skumanich (1982), Maltby et al. (1986) and Staude (1981) since the thickness of temperature minimum and temperature plateau differs for these models. Thus, the frequencies of passbands of the 3-min spectrum provide information on the parameters of sunspot atmospheres. The curves $1-5$ in the plots of Fig. 2a,b,c show the dependence of passbands on the reduction of amplitude of reflected waves which has to occur due to nonlinear dissipation of waves. On the whole, the dissipation of waves in transition region and upper chromosphere causes an increase of passband transmission.

Acknowledgements. This research was supported by RFFI grant 06-02- 16359

\section{References}

Brynildsen, N., Maltby, P., Foley, C. R., Fredvik, T., \& Kjeldseth-Moe, O. 2004, Solar Phys., 221, 237 Centeno, R., Collados, M., \& Trujillo Bueno, J. 2006, ApJ, 640, 1153

Gelfreikh, G. B., Nagovytsyn, E., \& Nagovytsyna, Yu. 2006, Publ. Astron. Soc. Japan, 58, 29

Lites, B. W. \& Skumanich, A. 1982, ApJS, 49, 293

Maltby, P., Avrett, E., Carlsson, M., Kjeldseth-Moe, O., Kurucz, R., \& Loeser, R. 1986, ApJ, 306, 284

Settele, A., Staude, J., \& Zhugzhda, Y. D. 2001, Solar Phys., 202,282

Staude, J. 1981, A\&A, 100, 284

Syrovatsky, S. I. \& Zhugzhda, Y. D. 1968, Soviet Astronomy, 11, 945

Zhugzhda, Y. D. 1972, Solar Phys., 25, 329

Zhugzhda, Y. D. \& Locans, V. 1981, Sov. Astronomy Letters, 7, 25

Zhugzhda, Y., Locans, V., \& Staude, J. 1983, Solar Phys., 82, 369

Zhugzhda, Y., Staude, J., \& Locans, V. 1984, Solar Phys., 91, 219

Zhugzhda, Y., Locans, V., \& Staude, J. 1985, A\&A, 143, 201

Zhugzhda, Y., Locans, V., \& Staude, J. 1987, Astron. Nachr., 308, 257 


\title{
Ray tracing of ion-cyclotron waves in a coronal funnel
}

\author{
R. Mecheri" and E. Marsch \\ Max-Planck-Institut für Sonnensystemforschung, Katlenburg-Lindau, Germany \\ *Email: mecheri@ mps.mpg.de
}

\begin{abstract}
Remote observations of coronal holes have strongly implicated the kinetic interactions of ion-cyclotron waves with ions as a principal mechanism for plasma heating and acceleration of the fast solar wind. In order to study these waves, a linear perturbation analysis is used in the work frame of the collisionless multi-fluid model. We consider a non-uniform background plasma describing a funnel region and use the ray tracing equations to compute the ray path of the waves as well as the spatial variation of their properties.
\end{abstract}

\section{Introduction}

The ultraviolet spectroscopic observations made by SUMER and UVCS aboard SOHO indicated that heavy ions in the coronal holes are very hot with high temperature anisotropy (see, e.g., Kohl et al. 1997; Wilhelm et al. 1998). This result is a strong indication for heating by ion-cyclotron resonance (i.e., collisionless energy exchange between ions and wave fluctuations, see Stix 1992, Chap 10) involving ion-cyclotron waves that are presumably generated in the lower corona from small-scale reconnection events (Axford \& McKenzie 1995). Performing a Fourier plane wave analysis, ion-cyclotron waves are studied using the collisionless multi-fluid model. While neglecting the electron inertia, this model permits the consideration of ion-cyclotron wave effects that are absent from the one-fluid MHD model. Realistic models of density and temperature as well as a 2D funnel model describing the open magnetic field are used to define the background plasma. Considering the WKB approximation, we first solve locally the dispersion relation and then perform a non-local wave analysis using the ray-tracing theory, which allows to compute the ray path of the waves in the funnel as well as the spatial variation of their properties.

\section{Basic equations}

The cold collisionless fluid equations for a particle species $j$ are:

$$
\begin{gathered}
\frac{\partial n_{j}}{\partial t}+\nabla \cdot\left(n_{j} \mathbf{v}_{j}\right)=0 \\
m_{j} n_{j}\left(\frac{\partial \mathbf{v}_{j}}{\partial t}+\mathbf{v}_{j} \cdot \nabla \mathbf{v}_{j}\right)-q_{j} n_{j}\left(\mathbf{E}+\mathbf{v}_{j} \times \mathbf{B}\right)=0,
\end{gathered}
$$



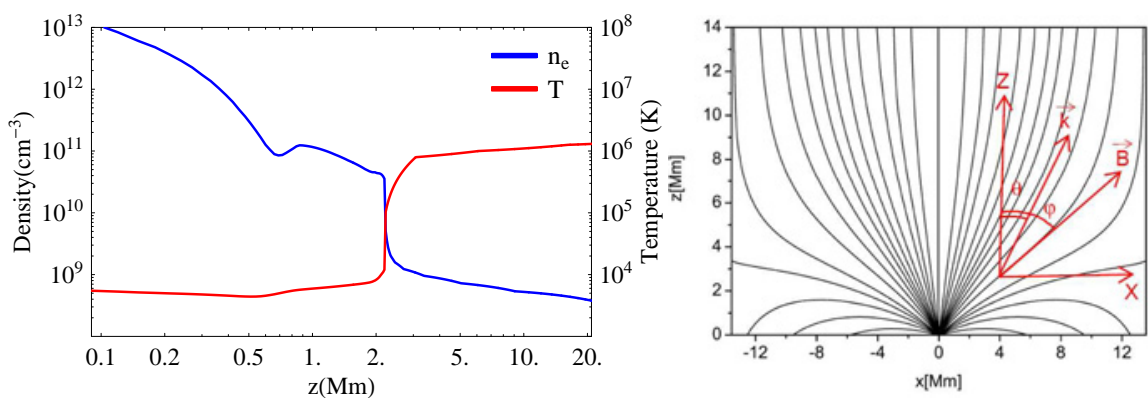

Figure 1. Left: Electronic density $\left(n_{e}\right)$ and temperature $(T)$ model-profiles of the chromosphere (Fontenla et al. 1993) and the lower corona (Gabriel 1976). Right: Funnel magnetic field geometry as obtained from a 2-D potential field model (Hackenberg et al. 2000).

where $m_{j}, n_{j}$ and $\mathbf{v}_{j}$ are respectively the mass, density and velocity of a species $j$. The electric field $\mathbf{E}$ and the magnetic field $\mathbf{B}$ are given by Faraday's law, i.e. $\nabla \times \mathbf{E}=-\partial \mathbf{B} / \partial t$. The background density, temperature and magnetic field are described in Fig. 1. Considering the quasi-neutrality and no ambient electric field, we perform a Fourier plane-wave analysis. The dispersion relation is obtained using

$$
D(\omega, \mathbf{k}, \mathbf{r})=\operatorname{Det}\left[\frac{c^{2}}{\omega^{2}} \mathbf{k} \times(\mathbf{k} \times \mathbf{E})+\boldsymbol{\varepsilon}(\omega, \mathbf{k}, \mathbf{r}) \cdot \mathbf{E}\right]=0
$$

where $c$ is the speed of light in vacuum, $\varepsilon$ is the dielectric tensor which is a function of the wave frequency $\omega$, the wave vector $\mathbf{k}$ and the large-scale position vector $\mathbf{r}$. The wave vector $\mathbf{k}$ lies in the $x-z$ plane, with $\mathbf{k}=k(\sin \theta, 0, \cos \theta)$.

The dispersion relation (3) is used to compute the Hamiltonian-type ray-tracing equations (Weinberg 1962):

$$
\frac{\mathrm{d} \mathbf{r}}{\mathrm{d} t}=-\frac{\partial D(\omega, \mathbf{k}, \mathbf{r}) / \partial \mathbf{k}}{\partial D(\omega, \mathbf{k}, \mathbf{r}) / \partial \omega}, \frac{\mathrm{d} \mathbf{k}}{\mathrm{d} t}=\frac{\partial D(\omega, \mathbf{k}, \mathbf{r}) / \partial \mathbf{r}}{\partial D(\omega, \mathbf{k}, \mathbf{r}) / \partial \omega}
$$

\section{Numerical results}

We consider a cold plasma made of electrons $\left(n_{e}\right)$, protons $\left(n_{p}\right)$ and alpha particles $\left(\mathrm{He}^{2+}\right.$, indicated by $\alpha$ ) with $n_{\alpha}=0.1 n_{p}$. In this case, the dispersion relation (3) is a quadratic polynomial of degree 6 , which means that 3 modes exist and each one is represented by an oppositely propagating ( $\omega>0$ and $\omega<0$ ) pair of waves. These modes, for which the dispersion curves are shown in the right panel of Fig. 2, are the ion-cyclotron modes 1 (IC1) and 2 (IC2) and the fast mode. For large k, the two IC modes reach a resonance regime at each one of the ion-cyclotron frequencies, i.e. $\omega=\Omega_{p}$ and $\omega=\Omega_{\alpha}=\Omega_{p} / 2$. The fast mode has a cut-off frequency $\omega_{c o} / \Omega_{p}=0.583$ and couples and mode converts with the IC1 mode at the so-called cross-over frequency $\omega_{c r} / \Omega_{p}=0.612$. The polar plots of the group velocity for $k V_{A p} / \Omega_{p}=0.2$ (Fig. 2), show that the IC2 mode is mainly anisotropic and cannot propagate perpendicularly to $\mathbf{B}_{0}$. Thus the corresponding energy mainly flows along the magnetic field lines, with maximum group velocity at parallel propagation. On the other 

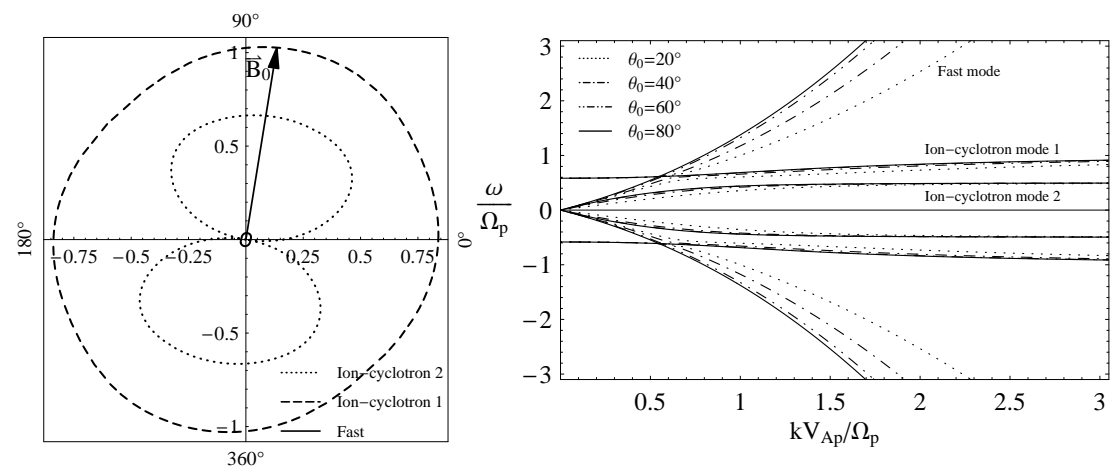

Figure 2. Right: Dispersion curves in the cold three-fluid (e-p- $\left.\mathrm{He}^{2+}\right)$ model at the location $(x=7.5$ $\mathrm{Mm}, \mathrm{z}=2.5 \mathrm{Mm}$ ) in the funnel with a $\mathbf{B}_{0}$-inclination angle $\varphi \approx 79^{\circ}$ and for different propagation angles $\theta\left(\Omega_{p}\right.$ and $\mathrm{V}_{A p}$ are the proton cyclotron frequency and Alfvén speed). Left: Polar plot of the group velocity as a function of $\theta$ and for $k V_{A p} / \Omega_{p}=0.2$.

hand, the IC1 mode and the fast mode propagate almost isotropically, and consequently the energy flow is fairly isotropic. These results are confirmed by the ray-tracing computation which clearly shows that the IC2 mode is well guided along the field lines, since the ray paths (direction of the group velocity) for various initial angles of propagation $\theta_{0}$ nicely follow the magnetic field lines (Fig. 3, p. 358). Indeed, the maximal angular deviation is $\psi \approx 6.5^{\circ}$ at $\mathrm{z}$ $\approx 4 \mathrm{Mm}$ and $\psi \approx 0^{\circ}$ (quasi-parallel propagation) in the upper part of the funnel, where the wave is quasi-electrostatic, i.e. $|\xi| \approx 1$, and has a nearly linear polarization, i.e. $|\varrho| \approx 0$. On the contrary to that behavior, the IC1 mode and the fast mode are unguided with a ray path having mainly a strait trajectory. The angular deviation $\psi$ between the ray path and $\mathbf{B}_{0}$ varies between $-60^{\circ}$ and $80^{\circ}$ for the IC1 mode and $-40^{\circ}$ and $60^{\circ}$ for the fast mode. The polarization is in general elliptical, right-handed $(\varrho>0)$ for the IC1 mode and left-handed $(\varrho<0)$ for the fast mode, except for $\theta=20^{\circ}$ in the upper part of the funnel $(\mathrm{z} \gtrsim 6 \mathrm{Mm})$ where it is mainly circular, right-handed $(\varrho=-1)$ for the IC1 mode and left-handed $(\varrho=-1)$ for the fast mode.

\section{References}

Axford, W. I. \& McKenzie, J. F. 1995, in Solar Wind Conference, 31

Fontenla, J. M., Avrett, E. H., \& Loeser, R. 1993, ApJ, 406, 319

Gabriel, A. H. 1976, Royal Society of London Philosophical Transactions Series A, 281, 339

Hackenberg, P., Marsch, E., \& Mann, G. 2000, A\&A, 360, 1139

Kohl, J. L., Noci, G., Antonucci, E., et al. 1997, Solar Phys., 175, 613

Stix, T. H. 1992, Waves in Plasmas (New York: American Institute of Physics, 1992)

Weinberg, S. 1962, Physical Review, 126, 1899

Wilhelm, K., Marsch, E., Dwivedi, B. N., et al. 1998, ApJ, 500, 1023 

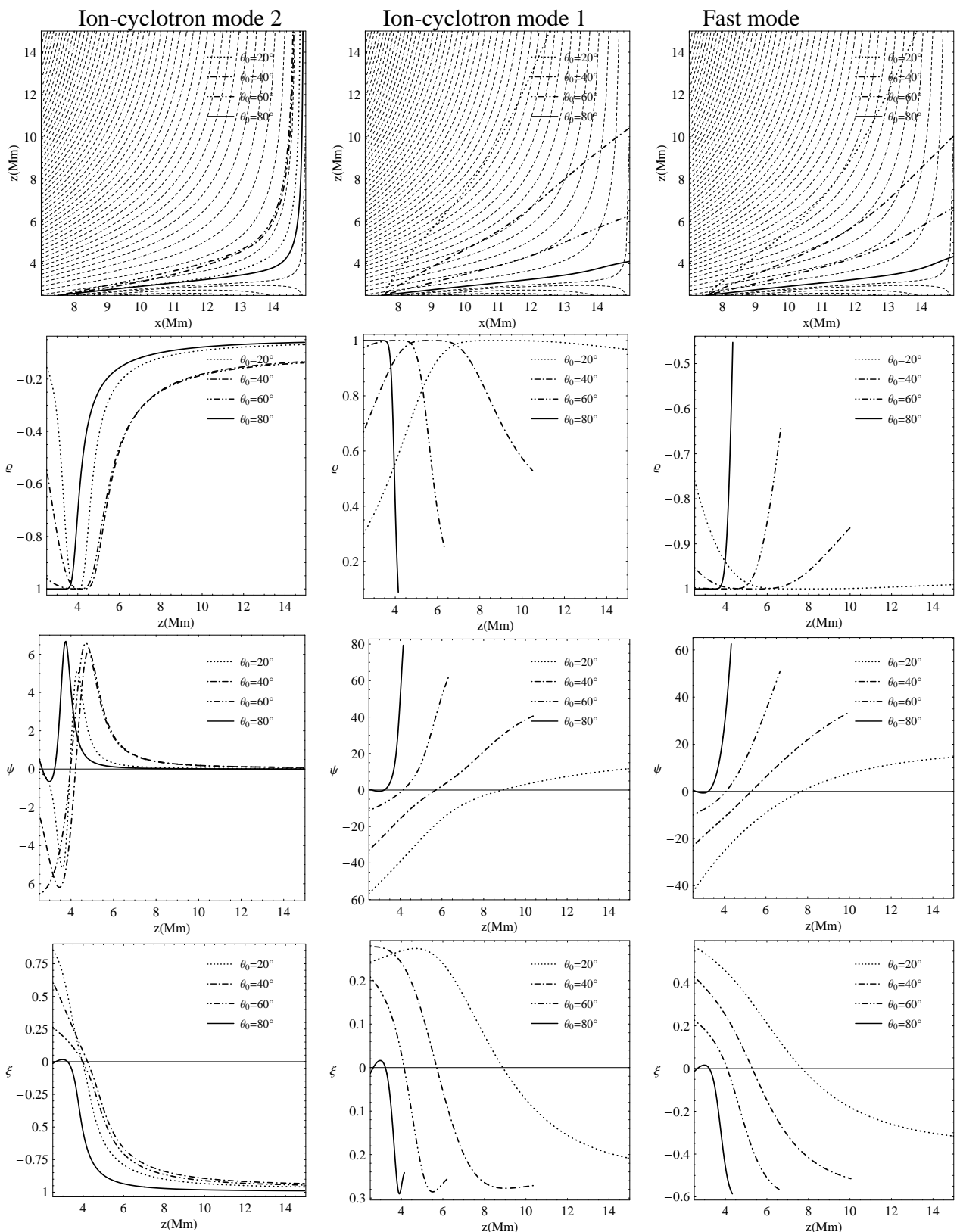

Figure 3. Ray paths of the waves and the spatial variation of their basic properties. The waves are launched at the location $\left(\mathrm{x}_{0}=7.5 \mathrm{Mm}, \mathrm{z}_{0}=2.5 \mathrm{Mm}\right)$ in the coronal funnel (with a $\mathbf{B}_{0}$-inclination angle $\varphi_{0} \approx 79^{\circ}$ ) with an initial normalized wave number $\mathrm{k}_{0}=0.2$ and different initial angles of propagation $\theta_{0}$. In the top panels, the dashed lines represent the funnel field lines. The wave properties are: $(\varrho)$ helicity (degree of circular polarization), $(\psi)$ angle between the direction of the group velocity and $\mathbf{B}_{0}$, $(\xi)$ the electrostatic part of the wave. 


\title{
Nanoflare model of emission line radiance distributions in active region coronae
}

\author{
H. Safari ${ }^{1,2, *}$, D. E. Innes ${ }^{2}$, S. K. Solanki ${ }^{2}$, and A. Pauluhn ${ }^{3}$ \\ ${ }^{1}$ Institute for Advanced Studies in Basic Sciences, Zanjan, Iran \\ ${ }^{2}$ Max-Planck-Institut für Sonnensystemforschung, Katlenburg-Lindau, Germany \\ ${ }^{3}$ Paul Scherrer Institut, Villigen, Switzerland \\ *Email: hsafary@iasbs.ac.ir
}

\begin{abstract}
Nanoflares are small impulsive bursts of energy that blend with and possibly make up much of the solar background emission. Determining their overall contribution is central to understanding the heating of the solar corona. Here, a simple nanoflare model based on three key parameters: the flare rate, the flare damping time, and the power-law slope of the flare energy frequency distribution has been used to simulate emission line radiances observed by SUMER in the corona above an active region. The three lines analysed, Fe xIX, Ca XIII, and $\mathrm{Si}$ III have very different formation temperatures, damping times and flare rates but all suggest a power-law slope greater than 2 . Thus it is possible that nanoflares provide a significant fraction of the flare energy input to active region coronae.
\end{abstract}

\section{Introduction}

Nanoflares were postulated first by Levine (1974) and later by Parker (1988) to be the source of the solar corona's background heat. The majority would be small fluctuations on the overall background and impossible to detect, so their energy has been estimated by extrapolating the energy frequency distribution of larger flares. The energy frequency distribution of larger flares tends to follow a power-law distribution

$$
\frac{d N}{d E} \sim E^{-\alpha}
$$

where $d N$ is the flare number in energy interval $d E$. The energy of small flares dominates if $\alpha>2$ (Hudson 1991). The standard method to evaluate $\alpha$ is to evaluate the energy of many flares in a series of observations and then plot their frequency in bins of energy $d E$. The majority of analyses based on this type of event counting deduce $\alpha \approx 1.7$, a value smaller than the critical 2 (Lin et al. 1984; Shimizu 1995; Aschwanden \& Parnell 2002). These results may, however, be misleading. For example, Parnell (2004) demonstrated that one can obtain $\alpha$ ranging from 1.5 to 2.6 for the same dataset using different but still reasonable sets of assumptions for the analyses.

Here we take an alternative approach and model UV radiances observed by SUMER in an active region corona, assuming that the radiance fluctuations and the nearly constant 'background' emission are caused by small-scale stochastic flaring (Pauluhn \& Solanki 2004, 2007). The model has successfully been applied to UV radiance fluctuations in the quiet 

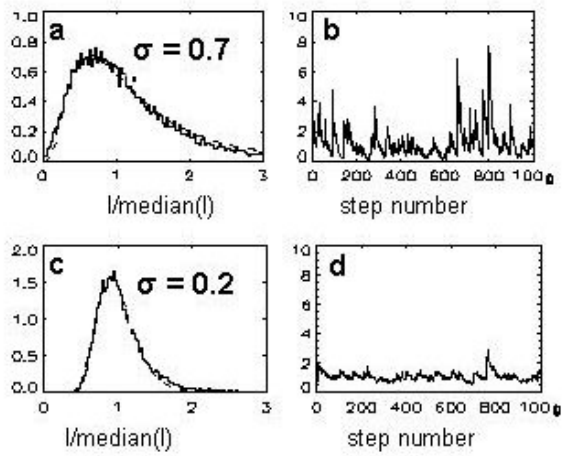

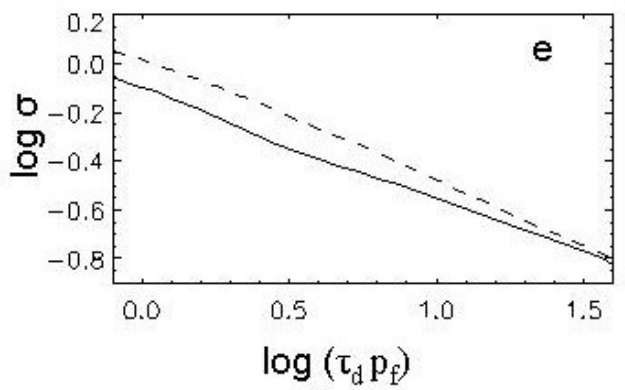

Figure 1. Simulation radiance distributions $(\mathbf{a}, \mathbf{c})$ for the light curves $(\mathbf{b}, \mathbf{d})$ on their right and (e) the dependence of shape parameter $\sigma$ on $\tau_{d} p_{f}$ for $\alpha=1.6$ (dashed) and $\alpha=2.4$ (solid).

Sun (Pauluhn \& Solanki 2007). The lines chosen, Fe xix (7 MK), Ca XIII (1 MK) and Si III (0.05 MK), cover two decades in formation temperature. In a previous paper, event counting in the Fe xIX dataset deduced an $\alpha \approx 1.8$ (Wang et al. 2006).

\section{The Model}

A detailed outline of the model and assumptions are given in Pauluhn \& Solanki (2007). Basically, flares with a power-law frequency distribution in radiance are assumed to erupt with a frequency, $p_{f}$, and then decay with a damping time, $\tau_{d}$. The radiance observed is the sum of all overlapping radiances.

For a large number of independent random flares, the radiance distribution is lognormal:

$$
\rho(x)=\exp \left(-\frac{(\log (x))^{2}}{2 \sigma^{2}}\right) /(\sigma x \sqrt{2 \pi}),
$$

where $\sigma$ is the shape parameter. Small $\sigma(<0.3)$ indicates a symmetric distribution due to high background emission caused by either a long damping time, $\tau_{d}$, or a high flare frequency, $p_{f}$. Examples are given in Fig. 1a-d. $\sigma$ is inversely proportional to $\sqrt{\tau_{d} p_{f}}$ (Pauluhn $\&$ Solanki 2007) with a slight $\alpha$ dependence as shown in Fig. 1e. The radiance distribution can be used to obtain the value $\tau_{d} p_{f}$ to within $\approx 20 \%$.

\subsection{Wavelet analysis}

Since our model is random no periods are expected and this is observed when analysing the model light curves. We notice however that the steepness of the wavelet global power spectrum at high frequencies depends inversely on the damping time, so can be used to determine $\tau_{d}$ to within $\approx 20 \%$. Global power spectra for different $\tau_{d}$ are shown in Fig. 3e with the global power spectra of the data overplotted. 


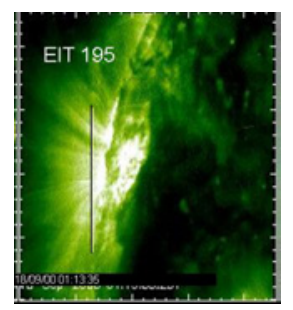

Figure 2. EIT $195 \AA$ image taken on 17 Sep 2000 of observed active region showing the position of the SUMER slit (black vertical line).

\section{Observations}

The active region corona was observed at a fixed position above the limb (Fig. 2) in the three lines, Fe XIX, Ca XIII and Si III by SUMER. Observations were made over periods of 14 hours with an exposure time of $1.5 \mathrm{~min}$. Therefore a typical period would consist of about 500 exposures. Observations were made over several days but for this analysis we look at data from the 14 hours when the active region was closest to the limb for the Fe XIX and Ca XIII. The Si III data were taken on the following day when a number of small surge-like events were seen above the limb. More details of the observational setup are given in Wang et al. (2006). The images in Fig. 3a, show the time series of radiance seen along the slit for the analysed periods.

For each line, radiance distribution functions were computed from the complete dataset and fitted with the lognormal function. Using the different $\sigma$ 's we are able to obtain $\tau_{d} p_{f}$ from the relationship plotted in Fig. 1e. Then using the wavelet analysis to get the $\tau_{d}$, we obtain the $p_{f}$ s. Given the $\tau_{d}$ and $p_{f}$, we run the flare simulations for various $\alpha$ 's. This gives light curves that can be compared with our SUMER observations. In Figs. 3b,c,d we show simulated and observed light curves. As $\alpha$ increases, the background becomes smoother and single flares become more prominent. A foolproof technique to quantify this effect is the next stage of our analysis. For the moment, we can say that the simulations with $\alpha=2.4$ look the most realistic. Those with $\alpha=1.6$ have too much background variation, whereas those with $\alpha=3$ do not have enough larger flares.

Acknowledgements. H. S. acknowledges warm hospitality and financial support during his research visit to the solar group, MPS, Katlenburg-Lindau.

\section{References}

Aschwanden, M. J. \& Parnell, C. E. 2002, ApJ, 572, 1048

Hudson, H. S. 1991, Solar Phys., 133, 357

Levine, R. H. 1974, ApJ, 190, 457

Lin, R. P., Schwartz, R. A., Kane, S. R., Pelling, R. M., \& Hurley, K. C. 1984, ApJ, 283, 421

Parker, E. N. 1988, ApJ, 330, 474

Parnell, C. E. 2004, in ESA SP-575: SOHO 15 Coronal Heating, 227

Pauluhn, A. \& Solanki, S. K. 2004, in ESA SP-575: SOHO 15 Coronal Heating, ed. R. W. Walsh, J. Ireland, D. Danesy, \& B. Fleck, 501 


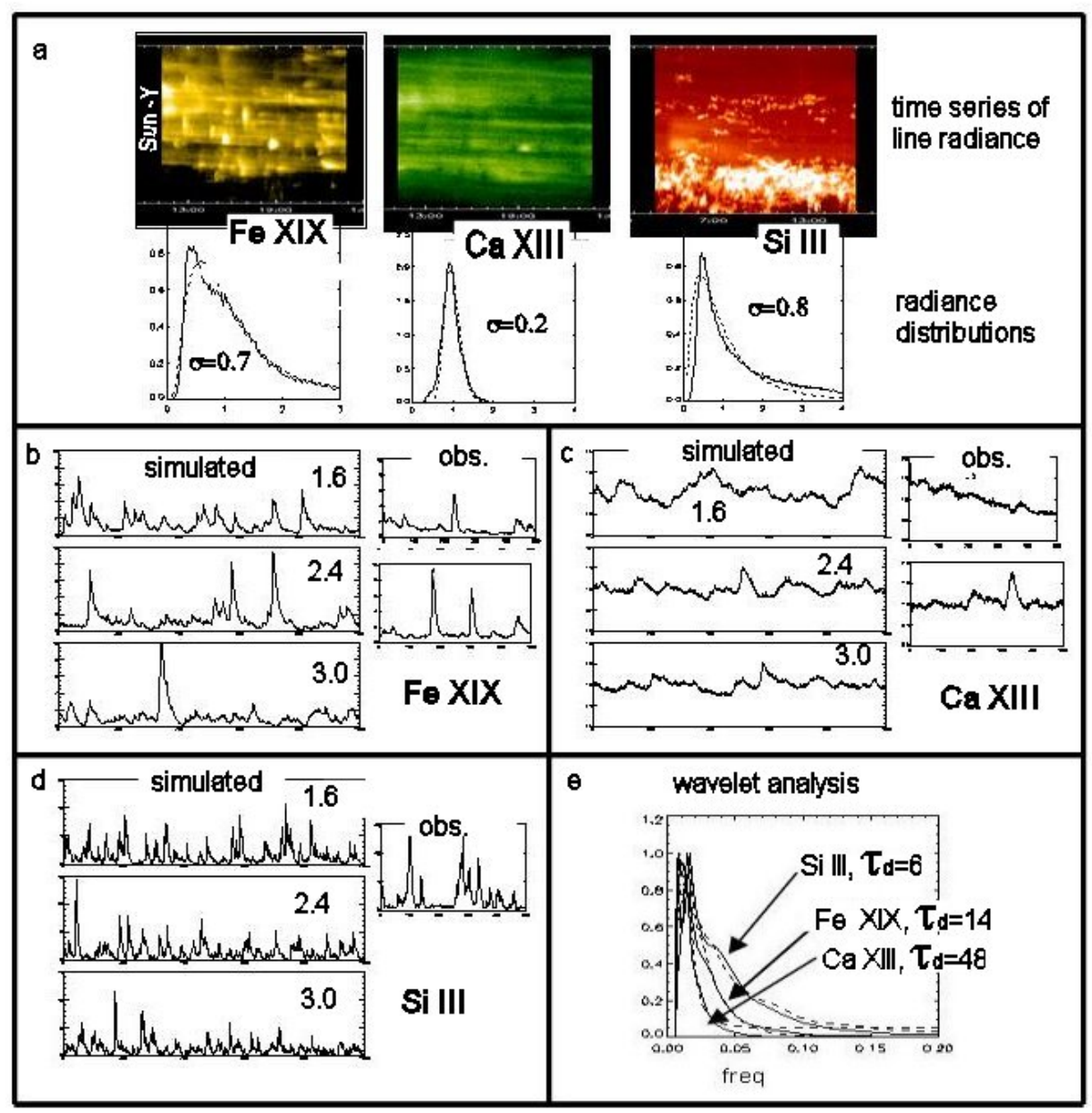

Figure 3. SUMER observations and modelling. (a) From left to right: Fe XIX, Ca XIII, Si III time series of line radiance along the spectrometer slit together with the corresponding radiance distributions of the data in the pictures above (solid) and the lognormal fit (dashed). (b) Fe XIX simulations with $\tau_{d}=14$, $p_{f}=0.1$ and $\alpha=1.6,2.4,3.0$ and observed light curves taken from two of the rows in a. (c) Ca xIII simulations with $\tau_{d}=48, p_{f}=0.6$ and $\alpha=1.6,2.4,3.0$ and observed light curves. (d) Si III simulations with $\tau_{d}=6, p_{f}=0.2$ and $\alpha=1.6,2.4,3.0$ and a typical observed light curve. (e) Wavelet global power spectra for light curves with different $\tau_{d}$ (solid) and for our data (dashed). The simulation light curves are the average over about fifty 500 step light curves. The data are the average of all rows along the slit of the 500 step block on 17-Sep (Fe XIX, Ca XIII) and on 18-Sep ( $\mathrm{Si}$ III).

Pauluhn, A. \& Solanki, S. K. 2007, A\&A, 463, 311

Shimizu, T. 1995, PASJ, 47, 251

Wang, T. J., Innes, D. E., \& Solanki, S. K. 2006, A\&A, 455, 1105 


\title{
Modeling large-scale coronal structures
}

\author{
P. Ruan*, T. Wiegelmann, and B. Inhester \\ Max-Planck-Institut für Sonnensystemforschung, Katlenburg-Lindau, Germany \\ *Email: ruan@mps.mpg.de
}

\begin{abstract}
Current measurements provide the photospheric magnetic field (e.g., from MDI on SOHO) and line-of-sight integrated 2D coronal images (e.g., EIT). Our aim is to use these observations to reconstruct the $3 \mathrm{D}$ structure of the solar corona. Here we do the reconstruction in two steps. We compute a global coronal magnetic field model with the help of a potential or linear force-free model. In a subsequent step we model the coronal plasma radiation with the help of scaling laws. Scaling laws which relate loop emissivities with plasma parameters will be tested with these models.
\end{abstract}

\section{Introduction}

To understand the physical processes in the solar corona (e.g. coronal mass ejections), it is important to get insights into the solar magnetic field which couples the solar interior, the photosphere and the corona. Unfortunately, because of low plasma density and high temperature in the corona, a direct measurement of the coronal magnetic field is extremely difficult. Therefore the extrapolation of the photospheric magnetic field into the solar corona is very important. Here we use the extrapolation results of the potential field model and linear force free model on global scales.

The scaling laws which relate loop emissivities with plasma parameters will be tested with these models. The distribution of the plasma and the shape of the loops in these models compared to what is seen in the EUV and white light images will reveal how well the magnetic field is reconstructed. We discuss how future measurements from different viewpoints with STEREO can be used to refine the model.

\section{Method of extrapolation}

We construct a field $B$ which satisfies

$$
\begin{aligned}
\nabla \times \mathbf{B} & =\alpha \mathbf{B} \\
\nabla \cdot \mathbf{B} & =0
\end{aligned}
$$

with a constant value of $\alpha$ ( $\alpha=0$ means potential field model). We also fit our extrapolation to surface observations.

A field which satisfies Eqs. (1) and (2) can be calculated from its poloidal and toroidal components (Morse \& Feschbach 1953)

$$
\mathbf{B}=\mathbf{P}(\phi)+\alpha \mathbf{T}(\phi)
$$




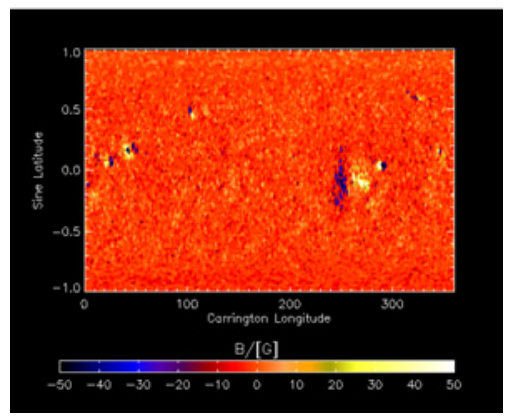

Figure 1. Surface data of magnetic field: MDI observation.
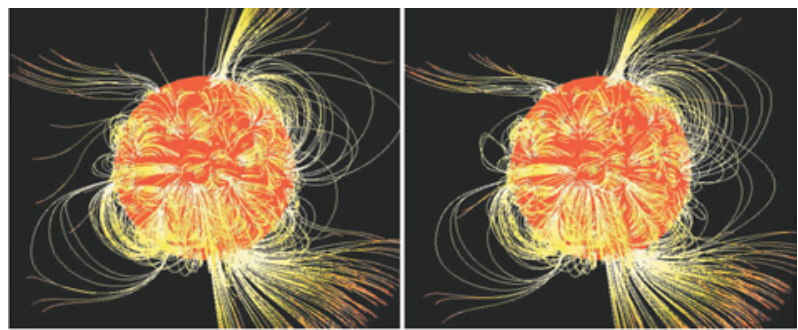

Figure 2. Extrapolation results: $\alpha=0$ for left panel and $\alpha=0.3$ for right panel.

$$
\begin{aligned}
& \mathbf{P}(\phi)=\nabla \times \nabla \times \mathbf{r} \phi \\
& \mathbf{T}(\phi)=\nabla \times \mathbf{r} \phi,
\end{aligned}
$$

where $\mathbf{r}$ is the radius vector in units of the solar radius $R_{\odot}$.

The potential $\phi$ satisfies a Helmholtz equation

$$
\Delta \phi=-\alpha^{2} \phi
$$

$\phi$ is fitted to surface measurements by a decomposition into spherical harmonics

$$
\phi=\sum_{n m} \frac{1}{(\alpha r)^{n+1}} P_{n}^{m}(\theta)\left(a_{n m} \cos p+b_{n m} \sin p\right) \text {. }
$$

The fit is achieved by minimizing the following expression at $r=1$ :

$$
\sum_{\theta p}[\mathbf{B}(1, \theta, p) \cdot \mathbf{e}-D(\theta, p)]^{2} \longrightarrow 0
$$

Here $\mathbf{e}$ is the known vector of the view direction and $D$ is the line-of-sight (LOS) field component obtained on the surface. From this we get the coefficients $a_{n m}$ and $b_{n m}$. 

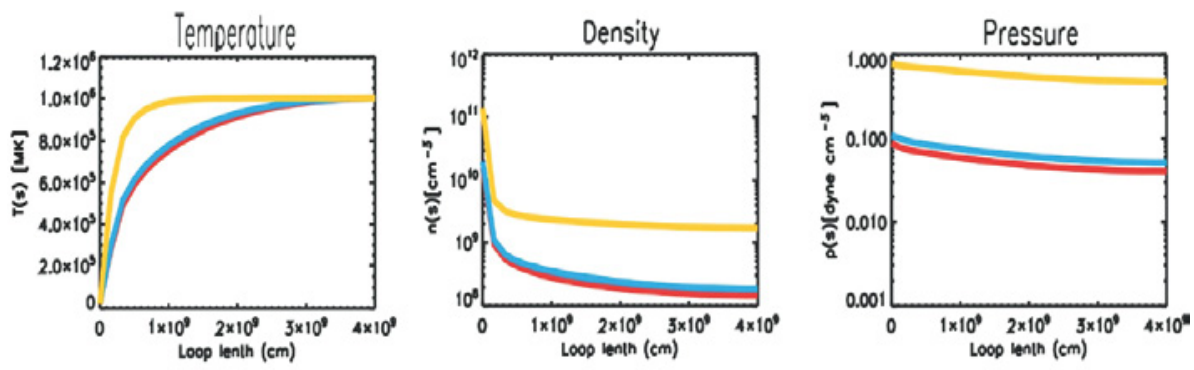

Figure 3. Influence of the heating scale height $S_{H}$ on loop properties. The values of $S_{H}$ (in Mm) are 4, 40 and 4000 for the top, middle and bottom curve, respectively.

\section{Measurements and results of extrapolation}

Figure 1 gives the MDI LOS magnetogram of the synoptic magnetic field from May 11, 1998 to June 7, 1998 at the photosphere. Figures 2 and 3 show the result of our linear force-free field extrapolation with different values of the free parameter $\alpha$. Careful inspection of these two panels shows that the extrapolation results are different.

\section{Scaling laws}

Because of the low plasma $\beta$ in the low corona, plasma can move only along magnetic field line. We write the energy equation along the field line as

$$
E_{H}-E_{R}-E_{C}=0
$$

where $E_{H}, E_{R}$ and $E_{C}$ are heating the term, the radiative loss term and the conductive term, respectively. Rosner et al. (1978) derived scaling laws to approximate the plasma parameter along the loops. These scaling laws have been refined by Aschwanden \& Schrijver (2002) who found the analytic expression

$$
E_{H}(s)=E_{0} \exp \left[-\left(s-s_{0}\right) / S_{H}\right],
$$

where $S_{H}$ is a heating scale height. $E_{R}$ and $E_{C}$ have no free parameters. With the heat input as in Eq. (10), they obtained the distribution of temperature, pressure and density as a function of heating scale height and loop length. Schrijver et al. (2004) extrapolated magnetic fields to the solar corona with a potential field model and filled the field lines with the plasma according to the above scaling laws. They also compared their simulation result with observations and reached the conclusion that the best heating flux densities $F_{H}$ depend on magnetic filed strength $B$ and loop length $L$ as: $F_{H}=c B^{1.0 \pm 0.3} / L^{1.0 \pm 0.5}$ (c is a constant).

Figure 3 shows the influence of $S_{H}$ on loop properties. We have combined their scaling laws with our magnetic field model. Figure 4 provides the temperature distribution in the solar corona as a cut through the longitude $40^{\circ}$. 


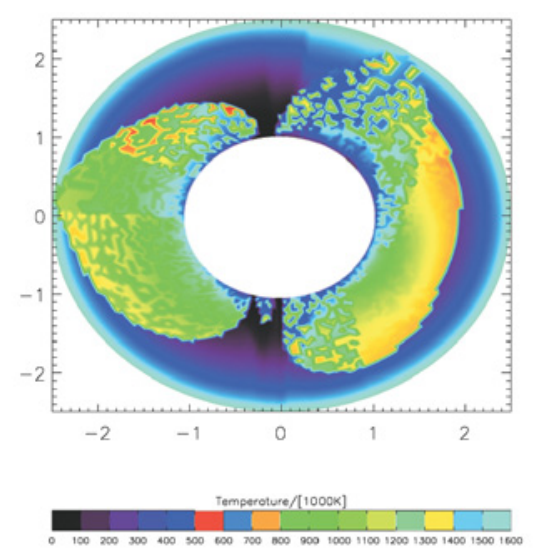

Figure 4. Temperature distribution in the solar corona, cut through longitude $40^{\circ}$.

\section{Discussion and outlook}

We extrapolate the magnetic field in the solar corona with the potential field model and the linear force-free model, and fill the loops with plasma according to the scaling laws. We cannot compare our temperature distribution with observations directly. In order to enable direct comparisons of our model with observations, we intend to calculate the plasma emissivities in the corona and integrate the LOS emissivities to produce artificial images. After that, we can compare our simulations and observations directly.

STEREO provides the opportunity to observe the Sun from two view directions. We will apply an advanced models, such as the MHS model (e.g., Neukirch 1995), to compute the coronal magnetic field and the plasma self-consistently. Our models contain free parameters, e.g., the optimal linear force-free parameter $\alpha$ and the details of the scaling laws. To optimize these parameters, we will compare our artificial images with the real images from STEREO. This allows us to find the optimal parameter set within our model approximation which fits the observations best.

Acknowledgements. We thank the SOHO/MDI research group to make their data available. The work of P. Ruan was supported by the International Max Planck Research School on Physical Processes in the Solar System and Beyond at the Universities of Braunschweig and Göttingen. The work of $T$. Wiegelmann was supported by DLR-grant 500c0501.

\section{References}

Aschwanden, M. J. \& Schrijver, C. J. 2002, ApJ, 142, 269

Morse, P. M. \& Feschbach, H. 1953, Methods of Theoretical Physics (International Series in Pure and Applied Physics), McGraw-Hill, New York

Neukirch, T. 1995, A\&A, 301, 628

Rosner, R., Tucker, W. H., \& Vaiana, G. S. 1978, ApJ, 220, 643

Schrijver, C. J., Sandman, A. W., Aschwanden, M. J., \& DeRosa, M. L. 2004, ApJ, 615, 512 


\title{
Magnetic flux transport and the lifetimes of spots on active cool stars
}

\author{
E. Işık*, M. Schüssler, and S. K. Solanki \\ Max-Planck-Institut für Sonnensystemforschung, Katlenburg-Lindau, Germany \\ *Email: ishik@mps.mpg.de
}

\begin{abstract}
We present results of numerical simulations of magnetic flux transport on the surfaces of cool stars with radii of $1 R_{\odot}$ and $3.3 R_{\odot}$. The effects of differential rotation and the tilt angle on the lifetimes of stellar bipolar magnetic regions are discussed. The existence of long-lasting polar spots can be explained by high-latitude persistent emergence of bipolar regions.
\end{abstract}

\section{Introduction}

It is evident from studies of Zeeman-Doppler imaging of rapidly rotating cool stars that starspots are magnetic structures. We have applied the magnetic flux transport model, which works reasonably for the transport of magnetic fields on the Sun (Wang et al. 1989; Dikpati \& Choudhuri 1995; van Ballegooijen et al. 1998; Mackay et al. 2002; Baumann et al. 2004) to understand the effects of large-scale surface flows on the lifetimes of starspots.

\section{The model}

The surface flux transport model for purely radial magnetic fields with horizontal and radial diffusion is used (Baumann et al. 2004, 2006). The horizontal and radial turbulent diffusivities are taken as $\eta_{h}=600 \mathrm{~km}^{2} \mathrm{~s}^{-1}$ and $\eta_{r}=100 \mathrm{~km}^{2} \mathrm{~s}^{-1}$, respectively. The observed differential rotation profiles of the Sun (Snodgrass 1983), AB Dor and HR 1099 (Donati et al. 2003) as well as the solar surface meridional flow profile (Hathaway 1996) are used. A starspot pair is represented by a bipolar magnetic region (hereafter BMR) emerged with a tilt angle $\alpha=0.5 \lambda_{0}$ between the bipole axis and the local latitudinal circle, where $\lambda_{0}$ is the emergence latitude. The area covered by the starspot is defined by the region with the field strength above a certain threshold level (for details, see Işık et al. 2007).

\section{The effects of large-scale flows on starspot lifetimes}

Figure 1 shows the evolution of three BMRs that emerged at different latitudes. The strongest rotational shear is at mid-latitudes. It leads to shorter lifetimes owing to a faster reduction of the characteristic length scales of the BMR, which leads to faster diffusion. Near the equator, local shear is less pronounced and this results in slightly longer lifetimes. At high latitudes, although the local shear rate is higher than in the equatorial regions, the lifetime is relatively 

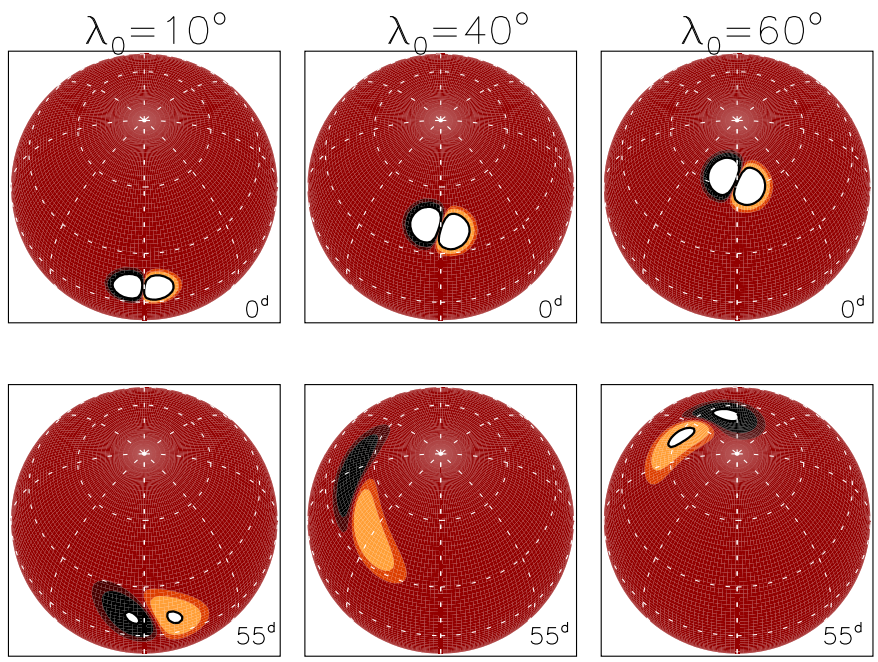

Figure 1. Evolution of BMRs with different emergence latitudes, with initial tilt angles $\alpha=0.5 \lambda_{0}$, and with solar surface shear (shown with respect to the rest frame of the equator). The top panels indicate the initial states, and the bottom panels show the states after 55 days. The field strength above a threshold level (14\%) is indicated by white regions enclosed by a thick black contour. Contours for $4 \%$ and $2 \%$ of the initial peak strength are filled with shades of gray for each polarity.
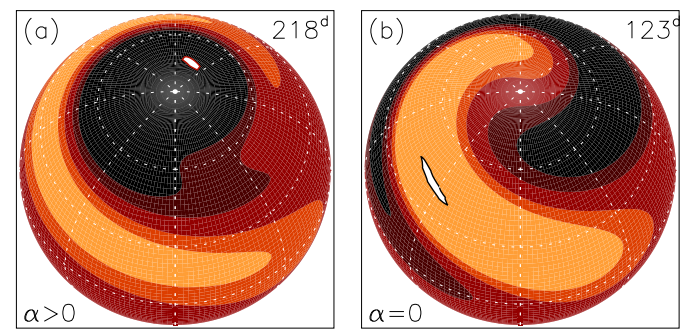

Figure 2. Two large BMRs with (a) and without (b) an initial tilt angle $\left(\alpha=0.5 \lambda_{0}\right)$, at times shortly before their peak strength falls below the threshold.

long. The reasons are, firstly, that the poleward meridional flow decelerates with increasing latitude, and secondly, that the meridional flow advects the BMR to regions with weaker shear. Both effects prolong the lifetimes of high-latitude starspots. A BMR covering $12 \%$ of the stellar surface, emerging at $\lambda_{0}=40^{\circ}$ with a tilt angle $\alpha=20^{\circ}$ may live as long as 7 months, because it develops into a roughly circular polar cap (Figure 2a). Polar caps require an initial tilt of the BMR (Figure 2b), because otherwise the enhanced flux cancellation leads to a relatively short lifetime.

In the case of (sub)giant stars, lifetimes increase as proportional to the square of the stellar radius. When a BMR, which covers about $0.8 \%$ of the stellar surface, emerges at $70^{\circ}$ latitude, it lives for about 2 years. Emergence of additional BMRs at mid- to high latitudes, can lead 
to the formation of polar spots which live for many years. For instance, random emergence of 6 BMRs, each covering $3 \%$ of the stellar surface, during 1.4 years, leads to a lifetime increase of an already existing polar cap from 3 years to 10 years (Iş1k et al. 2007).

\section{References}

Baumann, I., Schmitt, D., Schüssler, M., \& Solanki, S. K. 2004, A\&A, 426, 1075

Baumann, I., Schmitt, D., \& Schüssler, M. 2006, A\&A, 446, 307

Donati, J.-F., Collier-Cameron, \& Petit, P. 2003, MNRAS, 345, 1187

Dikpati, M. \& Choudhuri, A. R. 1995, Sol. Phys., 161, 9

Hathaway, D. H. 1996, ApJ, 460, 1027

Işık, E., Schüssler, M., \& Solanki, S. K. 2007, A\&A, in press

Mackay, D. H., Priest, E. R., \& Lockwood, M. 2002, Sol. Phys., 209, 287

Snodgrass, H. B. 1983, ApJ, 270, 288

van Ballegooijen, A. A., Cartledge, N. P., \& Priest, E. R. 1998, ApJ, 501, 866

Wang, Y.-M., Nash, A. G., \& Sheeley, N. R. 1989, ApJ, 347, 529 



\title{
Mass loss rates and wind ram pressures of cool stars
}

\author{
V. Holzwarth ${ }^{1,{ }^{*}}$ and M. Jardine ${ }^{2}$ \\ ${ }^{1}$ Max-Planck-Institut für Sonnensystemforschung, Katlenburg-Lindau, Germany \\ ${ }^{2}$ School of Physics and Astronomy, University of St Andrews, St Andrews, Scotland (UK) \\ *Email: holzwarth@mps.mpg.de
}

\begin{abstract}
The stellar mass loss rate is important for the rotational evolution of a star and for its interaction with the circumstellar environment. Based on empirical mass loss rates of cool stars, we develop a model for the increase of stellar wind ram pressures and mass fluxes with the stellar rotation rate. On the main-sequence, the predicted mass loss rates do not exceed about ten times the present solar value. We expect the erosion of planetary atmospheres and the detectability of magnetospheric radio emissions originating from extra-solar giant planets to be strongest during the pre-main sequence phase of the host star.
\end{abstract}

\section{Introduction}

The rotational evolution of cool stars and their interaction with the interstellar medium or with planetary companions depend on the stellar mass loss rate. Astrospheres carved out from the ambient interstellar medium by tenuous winds cause absorption features in the stellar Ly $\alpha$ emission line profile. By fitting observed and simulated absorption features, Wood et al. $(2002,2005)$ deduced mass loss rates of cool stars in the solar neighbourhood, which indicate a correlation between stellar mass loss rates and coronal activity levels. Their method is based on the assumption of thermally driven winds with a solar-like terminal velocity and disregards the influence of magnetic fields on the acceleration of outflowing plasma. By comparing self-consistently determined wind ram pressures with the empirical constraints, we investigate the impact of magnetic fields on the mass loss and on the rotational evolution of cool main-sequence stars (Holzwarth \& Jardine 2006).

\section{Model setup}

We consider a $1 \mathrm{M}_{\odot}$ main-sequence star with rotation rates $0.6 \leq \Omega / \Omega_{\odot} \leq 11.3$. Following the approach of Weber \& Davis (1967), we assume a split-monopole field geometry for the open magnetic field and that the entire stellar surface contributes to the mass loss. The properties of the polytropic wind are specified through its temperature, $T_{0}$, (particle) density, $n_{0}$, and radial magnetic field strength, $B_{0}$, at the reference level $r_{0}=1.1 \mathrm{R}_{\odot}$ close to the stellar surface (Holzwarth 2005). The dynamical impact of the stellar wind on the interstellar medium depends on the mass loss rate, $\dot{M}$, and the terminal wind velocity, $v_{\infty}$. In contrast to the wind ram pressure, which decreases far away from the star $\propto r^{-2}$, the ram force per solid 


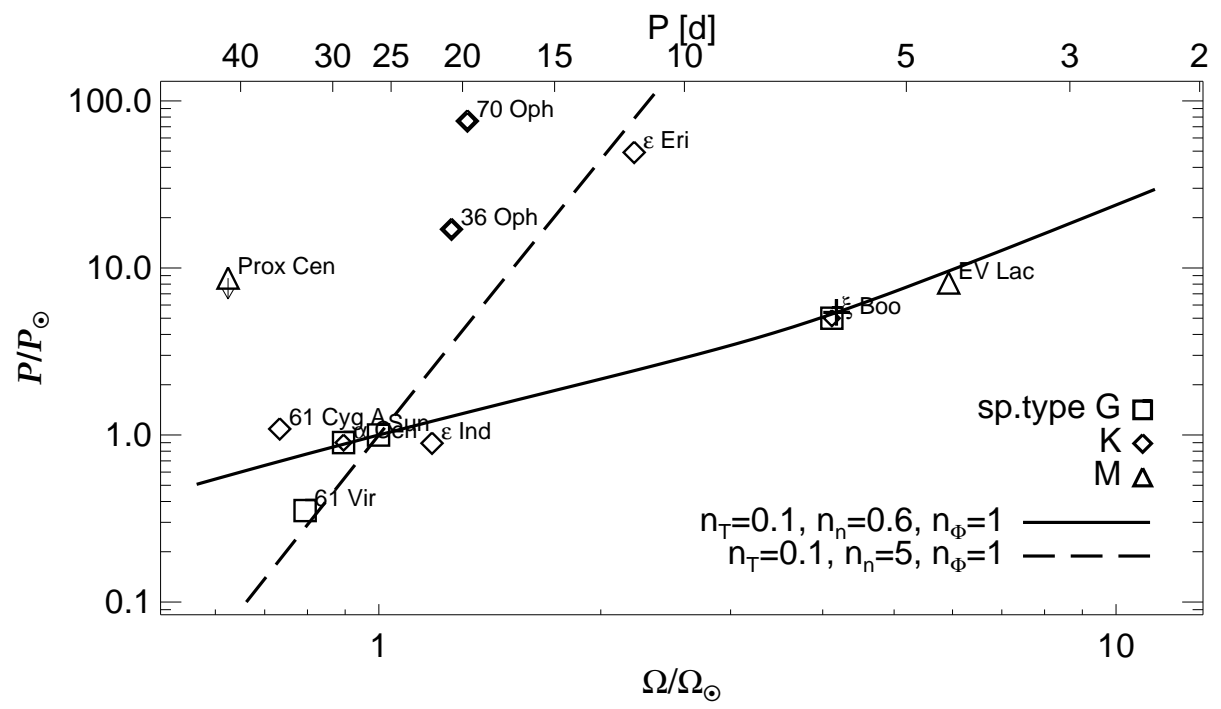

Figure 1. Comparison between empirical CWRP (symbols, indicating spectral type) and theoretical CWRP (curves) of main-sequence stars. The cross marks the transition from slow to fast magnetic rotators.

angle, $\dot{M} v_{\infty} /(4 \pi)$, is independent of the distance from the star. To compare our theoretical results with the empirical values of Wood et al. (2002, 2005), we use the ram force per stellar surface area, $\mathcal{P} \propto \dot{M} v_{\infty} / R^{2}$, as the principal quantity of our analysis and refer to it as the characteristic wind ram pressure (CWRP).

Observations indicate an increase of the coronal temperature, density, and activity level with the stellar rotation rate, $\Omega$. This aspect is taken into account by using rotation-dependent wind parameters:

$$
\frac{T_{0}}{T_{0, \odot}}=\left(\frac{\Omega}{\Omega_{\odot}}\right)^{n_{T}}, \quad \frac{n_{0}}{n_{0, \odot}}=\left(\frac{\Omega}{\Omega_{\odot}}\right)^{n_{n}}, \quad \frac{B_{0}}{B_{0, \odot}}=\left(\frac{\Omega}{\Omega_{\odot}}\right)^{n_{\Phi}}
$$

The power-law indices $n_{T}, n_{n}$ and $n_{\Phi}$ determine the rates of increase of the thermal and magnetic wind parameters. The reference parameters, $T_{0, \odot}=2.9 \cdot 10^{6} \mathrm{~K}, n_{0, \odot}=2.8 \cdot 10^{6} \mathrm{~cm}^{-3}$, and $B_{0, \odot}=3 \mathrm{G}$, are specified through the requirement that for the rotation rate $\Omega_{\odot}=2.8 \cdot 10^{-6} \mathrm{~s}^{-1}$ the model yields solar wind conditions as observed at earth orbit $\left(v_{r} \simeq 400 \mathrm{~km} / \mathrm{s}, n_{\mathrm{p}} \simeq\right.$ $\left.7 \mathrm{~cm}^{-3}, T \simeq 2 \cdot 10^{5} \mathrm{~K}, B \simeq 5 \cdot 10^{-5} \mathrm{G}\right)$. The polytropic index is $\Gamma=1.22$, and the mean molecular weight $\mu=0.5$.

\section{Wind ram pressures of cool stars}

Within the framework of our model, the observed high CWRPs of the moderately rotating targets $36 \mathrm{Oph}, 70 \mathrm{Oph}$, and $\epsilon$ Eri can only be explained in terms of very dense stellar winds (Fig. 1). Such a wind scenario is in contrast with recent X-ray observations, which indicate that the coronal densities of these targets are rather solar-like (Wood \& Linsky 2006). 

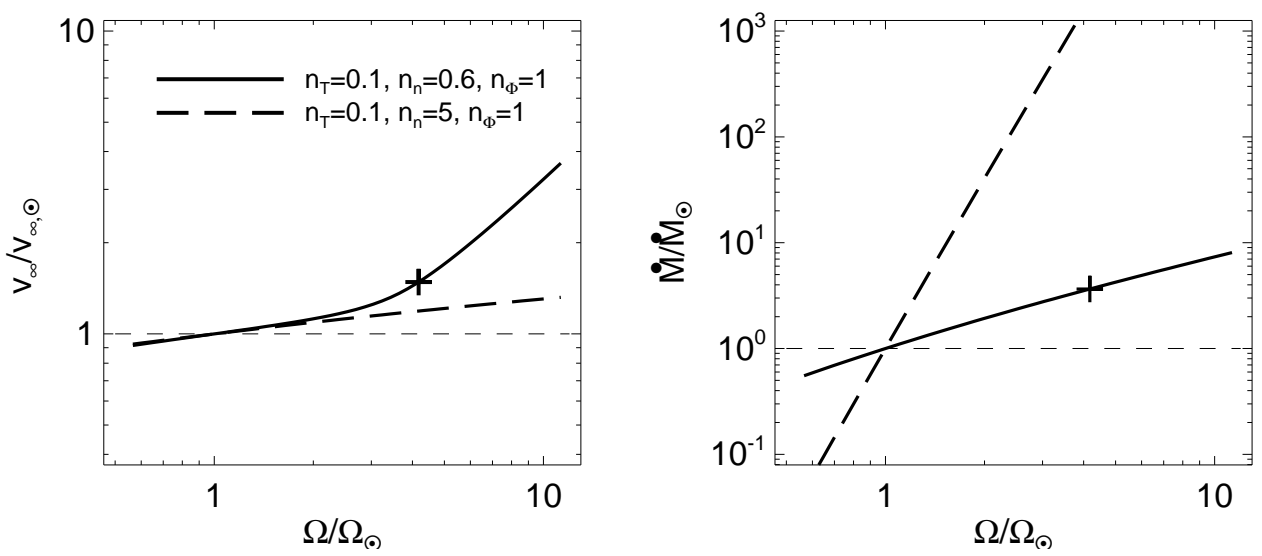

Figure 2. Terminal wind velocities (left) and mass fluxes (right). The cross marks the transition from slow to fast magnetic rotators.

The CWRPs of the more rapidly rotating targets $\xi$ Boo and EV Lac are consistent with wind scenarios which are based on a moderate increase of the thermal and magnetic wind parameters. The rates of increase applied here are in agreement with empirical values of previous investigations (e.g. Saar 2001; Ivanova \& Taam 2003). The character of the increase of both the mass loss rate and the terminal wind velocity depends on whether the star is a slow magnetic rotator with a thermally driven wind, or a fast magnetic rotator with high wind velocities caused by an efficient magneto-centrifugal driving (Fig. 2).

\section{Impact on stars and planets}

To analyse the impact of the two wind scenarios on the rotational evolution of a $1 \mathrm{M}_{\odot}$ star, we apply them to the stellar rotational evolution model described by Holzwarth \& Jardine (2005). The larger amount of angular momentum carried away by very dense winds hampers the pre-main sequence spin-up of stellar rotation (Fig. 3, upper panel), which is in discord with the observed existence of very rapidly rotating young stars. The predicted mass loss rates and wind ram pressures are highest during the pre-main sequence phase. Since the level of magnetospheric radio emission from planets increases with the kinetic and magnetic energy flux of the incident stellar wind (e.g. Grießmeier et al. 2005), we expect that the detectability of extra-solar giant planets is largest for young and rapidly rotating host stars.

\section{Conclusion}

The increase of the wind ram pressure of cool stars is characterised by the slow and rapidly rotating targets, and quantified through the rates of increase $n_{T} \simeq 0.1, n_{n} \simeq 0.6$, and $n_{B} \lesssim 1$; these values are consistent with previous investigations. The high CWRPs of the moderately rotating targets $36 \mathrm{Oph}, 70 \mathrm{Oph}$, and $\epsilon$ Eri are peculiar and imply significantly different wind properties; their role has to be clarified. The predicted mass loss rates of cool main-sequence 


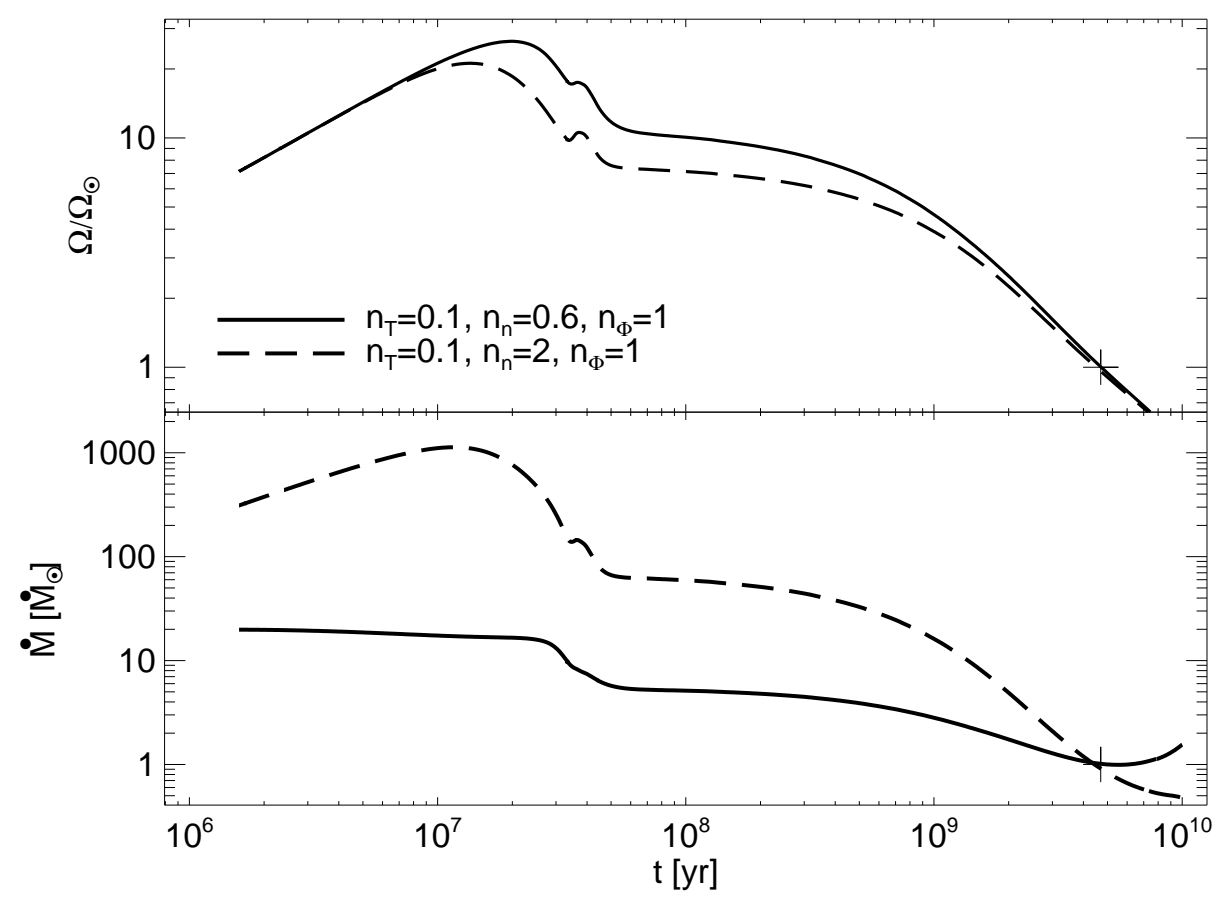

Figure 3. Evolution of the stellar surface rotation rate (upper panel) and mass loss rate (lower panel) of a $1 \mathrm{M}_{\odot}$ star subject to different wind scenarios. Crosses mark the current state of the Sun.

stars do not exceed about $10 \dot{M}_{\odot}$. The most promising targets for radio searches of extra-solar giant planets are predicted to be rapidly rotating pre-main sequence stars.

Acknowledgements. VH gratefully acknowledges support for this research through a PPARC standard grant and through a MPG fellowship.

\section{References}

Grießmeier, J-M., Motschmann, U., Mann, G., \& Rucker, H. O. 2005, A\&A, 437, 717

Holzwarth, V. 2005, A\&A, 440, 411

Holzwarth, V. \& Jardine, M. 2005, A\&A, 444, 661

Holzwarth, V. \& Jardine, M. 2006, A\&A, accepted, astro-ph/0611430

Ivanova, N. \& Taam, R. E. 2003, ApJ, 599, 516

Saar, S. H. 2001, in ASP Conf. Ser. 223: 11th Cambridge Workshop on Cool Stars, Stellar Systems and the Sun, ed. R. J. García López, R. Rebolo, \& M. R. Zapaterio Osorio, 292-299

Weber, E. J. \& Davis, L. J. 1967, ApJ, 148, 217

Wood, B. E. \& Linsky, J. L. 2006, ApJ, 643, 444

Wood, B. E., Müller, H., Zank, G. P., \& Linsky, J. L. 2002, ApJ, 574, 412

Wood, B. E., Müller, H.-R., Zank, G. P., Linsky, J. L., \& Redfield, S. 2005, ApJL, 628, L143 


\section{List of participants}

Conference secretaries:

N. Böker, M. Hüttenmeister, K. Wolters

Institut für Astrophysik

Friedrich-Hund-Platz 1

D-37077 Göttingen

sekr@astro.physik.uni-goettingen.de

Aznar Cuadrado, Regina

MPI für Sonnensystemforschung

Max-Planck-Str. 2

D-37191 Katlenburg-Lindau

Germany

aznar@mps.mpg.de

Balthasar, Horst

Astrophysikalisches Institut Potsdam

An der Sternwarte 16

D-14482 Potsdam

Germany

hbalthasar@aip.de

Beck, Christian

Kiepenheuer-Institut für Sonnenphysik

Schöneckstr. 6

D-79104 Freiburg

Germany

cbeck@kis.uni-freiburg.de

Bello González, Nazaret

Institut für Astrophysik

Friedrich-Hund-Platz 1

D-37077 Göttingen

Germany

nazaret@astro.physik.uni-goettingen.de
Berkefeld, Thomas

Kiepenheuer-Institut für Sonnenphysik

Schöneckstr. 6

D-79104 Freiburg

Germany

berke@kis.uni-freiburg.de

Berrilli, Francesco

Università di Roma Tor Vergata

V. le Ricerca Scientifica, 1

I-00133 Roma

Italy

berrilli@roma2.infn.it

Bilenko, Irina A.

Sternberg State Astronomical Institute

Universitetskii prospekt 13

RU-119992 Moscow

Russia

bilenko@sai.msu.ru

Blanco Rodríguez, Julián

Institut für Astrophysik

Friedrich-Hund-Platz 1

D-37077 Göttingen

Germany

blanco@astro.physik.uni-goettingen.de

Bloomfield, D. Shaun

MPI für Sonnensystemforschung

Max-Planck-Str. 2

D-37191 Katlenburg-Lindau

Germany

bloomfield@mps.mpg.de 
Bothmer, Volker

Institut für Astrophysik

Friedrich-Hund-Platz 1

D-37077 Göttingen

Germany

bothmer@astro.physik.uni-goettingen.de

Cameron, Robert

MPI für Sonnensystemforschung

Max-Planck-Str. 2

D-37191 Katlenburg-Lindau

Germany

cameron@mps.mpg.de

Carballo Bello, Julio Alberto

Institut für Astrophysik

Friedrich-Hund-Platz 1

D-37077

Göttingen

Germany

wackito7@yahoo.es

Carroll, Thorsten A.

Astrophysikalisches Institut Potsdam

An der Sternwarte 16

D-14482 Potsdam

Germany

tcarroll@aip.de

Centeno, Rebecca

Instituto de Astrofísica de Canarias

Vía Láctea s/n

E-38200 La Laguna/Tenerife

Spain

rce@ hao.ucar.edu

Ceppatelli, Guido

Fundacin Galileo Galilei - INAF

calle Alvarez de Abreu 70

E-38700 Sta. Cruz de La Palma/La Palma

Spain

ceppatel@tng.iac.es

Collados, Manuel

Instituto de Astrofísica de Canarias

Vía Láctea s/n

E-38200 La Laguna/Tenerife

Spain

mcv@iac.es
Danilovic, Sanja

MPI für Sonnensystemforschung

Max-Planck-Str. 2

D-37191 Katlenburg-Lindau

Germany

danilovic@mps.mpg.de

Deinzer, Willi

Institut für Astrophysik

Friedrich-Hund-Platz 1

D-37077 Göttingen

Germany

deinzer@astro.physik.uni-goettingen.de

Denker, Carsten J.

New Jersey Institute of Technology

Center for Solar-Terrestrial Research

323 Martin Luther King Blvd

Newark, NJ 07102

USA

cdenker@adm.njit.edu

Döhring, Thorsten

SCHOTT AG

Hattenbergstr. 10

D-55122 Mainz

Germany

thorsten.doehring@schott.com

Feller, Alex

Institut für Astronomie

ETH Zentrum, SEC

CH-8092 Zürich

Switzerland

afeller@astro.phys.ethz.ch

Feng, Li

MPI für Sonnensystemforschung

Max-Planck-Str. 2

D-37191 Katlenburg-Lindau

Germany

feng@mps.mpg.de

Fleck, Bernhard

ESA Space Science Department c/o NASA Goddard Space Flight Center Greenbelt, MD 20771

USA

bfleck@esa.nascom.nasa.gov 
Gandorfer, Achim

MPI für Sonnensystemforschung

Max-Planck-Str. 2

D-37191 Katlenburg-Lindau

Germany

gandorfer@mps.mpg.de

Giordano, Silvia

Universitá di Roma Tor Vergata

V. le Ricerca Scientifica, 1

I-00133 Roma

Italy

giordano@roma2.infn.it

Gisler, Daniel

Institut für Astronomie

ETH Zentrum, SEC E2

Scheuchzerstr. 7

CH-8092 Zürich

Switzerland

dgisler@astro.phys.ethz.ch

Gizon, Laurent

MPI für Sonnensystemforschung

Max-Planck-Str. 2

D-37191 Katlenburg-Lindau

Germany

gizon@mps.mpg.de

Gömöry, Peter

Astrophysikalisches Institut Potsdam

An der Sternwarte 16

D-14482 Potsdam

Germany

pgoemoery@aip.de

Haindl, Michal

Inst. of Information Theory and Automation

Pod vodarenskou vezi 4

CZ-182 08 Prague

Czech Republic

haindl@utia.cas.cz

Hirzberger, Johann

MPI für Sonnensystemforschung

Max-Planck-Str. 2

D-37191 Katlenburg-Lindau

Germany

hirzberger@mps.mpg.de
Holzwarth, Volkmar

MPI für Sonnensystemforschung

Max-Planck-Str. 2

D-37191 Katlenburg-Lindau

Germany

holzwarth@mps.mpg.de

Innes, Davina

MPI für Sonnensystemforschung

Max-Planck-Str. 2

D-37191 Katlenburg-Lindau

Germany

innes@mps.mpg.de

Işık, Emre

MPI für Sonnensystemforschung

Max-Planck-Str. 2

D-37191 Katlenburg-Lindau

Germany

ishik@mps.mpg.de

Jackiewicz, Jason

MPI für Sonnensystemforschung

Max-Planck-Str. 2

D-37191 Katlenburg-Lindau

Germany

jackiewicz@mps.mpg.de

Janssen, Katja

INAF, Osserv. Astrofisico di Arcetri

Largo Eenrico Fermi, 5

I-50125 Firenze

Italy

kjanssen@arcetri.astro.it

Jurčák, Jan

Astronomical Institute

Fricova 298

CZ-25165 Ondřejov

Czech Republic

jurcak@asu.cas.cz

Khomenko, Elena

Instituto de Astrofísica de Canarias

Vía Láctea s/n

E-38200 La Laguna/Tenerife

Spain

khomenko@iac.es 
Kitchatinov, Leonid L.

Astrophysikalisches Institut Potsdam

An der Sternwarte 16

D-14482 Potsdam

Germany

lkitchatinov@aip.de

Kneer, Franz

Institut für Astrophysik

Friedrich-Hund-Platz 1

D-37077 Göttingen

Germany

kneer@astro.physik.uni-goettingen.de

Kobel, Philippe

MPI für Sonnensystemforschung

Max-Planck-Str. 2

D-37191 Katlenburg-Lindau

Germany

kobel@mps.mpg.de

Kotrč, Pavel

Astronomical Institute

CZ-25165 Ondřejov

Czech Republic

pkotrc@asu.cas.cz

Koza, Julius

Sterrekundig Instituut

Universiteit Utrecht

P.O. Box 80000

NL-3508 TA Utrecht

The Netherlands

j.koza@astro.uu.nl

Lagg, Andreas

MPI für Sonnensystemforschung

Max-Planck-Str. 2

D-37191 Katlenburg-Lindau

Germany

lagg@mps.mpg.de

Löfdahl, Mats

Institute for Solar Physics

Alba Nova University Center

SE-10691 Stockholm

Sweden

mats@astro.su.se
Madjarska, Maria S.

MPI für Sonnensystemforschung

Max-Planck-Str. 2

D-37191 Katlenburg-Lindau

Germany

madjarska@mps.mpg.de

Marsch, Eckard

MPI für Sonnensystemforschung

Max-Planck-Str. 2

D-37191 Katlenburg-Lindau

Germany

marsch@mps.mpg.de

Martínez González, María Jesús

MPI für Sonnensystemforschung

Max-Planck-Str. 2

D-37191 Katlenburg-Lindau

Germany

martinez@mps.mpg.de

Matloch, Lucasz

MPI für Sonnensystemforschung

Max-Planck-Str. 2

D-37191 Katlenburg-Lindau

Germany

matloch@mps.mpg.de

Mecheri, Redouane

MPI für Sonnensystemforschung

Max-Planck-Str. 2

D-37191 Katlenburg-Lindau

Germany

mecheri@mps.mpg.de

Mikurda, Kasia

Kiepenheuer-Institut für Sonnenphysik

Schöneckstr. 6

D-79104 Freiburg

Germany

kasia@kis.uni-freiburg.de

Myagkova, Irina N.

Lomonosov Moscow State Univ. Skobeltsyn

Institute of Nuclear Physics

1-2 Leninskie Gory, GSP-2

RU-119992 Moscow

Russia

irina@srd.sinp.msu.ru 
Narayan, Gautam

Institute for Solar Physics

Alba Nova University Center

SE-10691 Stockholm

gautam@astro.su.se

Oklay, Nilda

Istanbul University

Department of Astronomy \& Space Sciences

University-Istanbul

Turkey

oklay@mps.mpg.de

Okunev, Oleg

Main Astronomical Observatory

of the Russian Academy of Sciences

Pulkovskoye chausee 65/1

RU-196140 St. Petersburg

Russia

olok@bk.ru

Olshevsky, Vyacheslav L.

Main Astronomical Observatory of the

National Academy of Sciences of Ukraine

27 Akademika Zabolotnoho St.

UA-03680 Kyiv

Ukraine

sya@mao.kiev.ua

Oreshina, Inna V.

Sternberg Astronomical Institute

Moscow State University

Universitetskii prospekt, 13

RU-119992 Moscow

Russia

ivo@sai.msu.ru

Pío Jiménez, Miguel Ángel

Institut für Astrophysik

Friedrich-Hund-Platz 1

D-37077 Göttingen

Germany

mapj@astro.physik.uni-goettingen.de
Puschmann, Klaus Gerhard

Institut für Astrophysik

Friedrich-Hund-Platz 1

D-37077 Göttingen

Germany

kgp@astro.physik.uni-goettingen.de

Reiners, Ansgar

Hamburger Sternwarte

Gojenbergsweg 112

D-21029 Hamburg-Bergedorf

Germany

areiners@hs.uni-hamburg.de

Reinsch, Klaus

Institut für Astrophysik

Friedrich-Hund-Platz 1

D-37077 Göttingen

Germany

reinsch@astro.physik.uni-goettingen.de

Riethmüller, Tino

MPI für Sonnensystemforschung

Max-Planck-Str. 2

D-37191 Katlenburg-Lindau

Germany

riethmueller@mps.mpg.de

Roth, Markus

MPI für Sonnensystemforschung

Max-Planck-Str. 2

D-37191 Katlenburg-Lindau

Germany

roth@mps.mpg.de

Ruan, Peng

MPI für Sonnensystemforschung

Max-Planck-Str. 2

D-37191 Katlenburg-Lindau

Germany

ruan@linmpi.mpg.de

Rüdiger, Günther

Astrophysikalisches Institut Potsdam

An der Sternwarte 16

D-14482 Potsdam

Germany

gruediger@aip.de 
Ruiz Cobo, Basilio

Instituto de Astrofísica de Canarias

Vía Láctea s/n

E-38200 La Laguna/Tenerife

Spain

brc@iac.es

Sailer, Markus

Institut für Astrophysik

Friedrich-Hund-Platz 1

D-37077 Göttingen

Germany

msailer@astro.physik.uni-goettingen.de

Sánchez-Andrade Nuño, Bruno

Institut für Astrophysik

Friedrich-Hund-Platz 1

D-37077 Göttingen

Germany

bruno@ astro.physik.uni-goettingen.de

Sasso, Clementina

MPI für Sonnensystemforschung

Max-Planck-Str. 2

D-37191 Katlenburg-Lindau

Germany

sasso@mps.mpg.de

Schlichenmaier, Rolf

Kiepenheuer-Institut für Sonnenphysik

Schöneckstr. 6

D-79104 Freiburg

Germany

schliche@kis.uni-freiburg.de

Schmitt, Dieter

MPI für Sonnensystemforschung

Max-Planck-Str. 2

D-37191 Katlenburg-Lindau

Germany

schmitt@mps.mpg.de

Schühle, Udo

MPI für Sonnensystemforschung

Max-Planck-Str. 2

D-37191 Katlenburg-Lindau

Germany

schuehle@mps.mpg.de
Schüssler, Manfred

MPI für Sonnensystemforschung

Max-Planck-Str. 2

D-37191 Katlenburg-Lindau

Germany

schuessler@mps.mpg.de

Schunker, Hannah

MPI für Sonnensystemforschung

Max-Planck-Str. 2

D-37191 Katlenburg-Lindau

Germany

schunker@mps.mpg.de

Shapiro, Alexander

Institut für Astronomie

ETH Zentrum, SEC

Scheuchzerstr. 7

CH-8092 Zürich

Switzerland

shapiro@astro.phys.ethz.ch

Šimberová, Stanislava

Astron. Inst. Academy of Sciences

CZ-25165 Ondřejov

Czech Republic

ssimbero@asu.cas.cz

Sobotka, Michal

Astron. Inst. Academy of Sciences

CZ-25165 Ondřejov

Czech Republic

msobotka@asu.cas.cz

Steiner, Oskar

Kiepenheuer-Institut für Sonnenphysik

Schöneckstr. 6

D-79104 Freiburg

Germany

steiner@kis.uni-freiburg.de

Steinhof, Wilfried

Institut für Astrophysik

Friedrich-Hund-Platz 1

D-37077 Göttingen

Germany

steinhof@astro.physik.uni-goettingen.de 
Strassmeier, Klaus G.

Astrophysikalisches Institut Potsdam

An der Sternwarte 16

D-14482 Potsdam

Germany

kstrassmeier@aip.de

Theissen, Armin

MPI für Sonnensystemforschung

Max-Planck-Str. 2

D-37191 Katlenburg-Lindau

Germany

theissen@mps.mpg.de

Tothova, Danica

MPI für Sonnensystemforschung

Max-Planck-Str. 2

D-37191 Katlenburg-Lindau

tothova@mps.mpg.de

Trujillo Bueno, Javier

Institut für Astrophysik

Friedrich-Hund-Platz 1

D-37077 Göttingen

Germany

jtb@iac.es

Volkmer, Reiner

Kiepenheuer-Institut für Sonnenphysik

Schöneckstr. 6

D-79104 Freiburg

Germany

volkmer@kis.uni-freiburg.de
Wiehr, Eberhard

Ewaldstr. 75

D-37075 Göttingen

Germany

Wittmann, Axel D.

Institut für Astrophysik

Friedrich-Hund-Platz 1

D-37077 Göttingen

Germany

wittmann@astro.physik.uni-goettingen.de

Yelles Chaouche, Lotfi

MPI für Sonnensystemforschung

Max-Planck-Str. 2

D-37191 Katlenburg-Lindau

Germany

yelles@mps.mpg.de

Zakharov, Vasily

MPI für Sonnensystemforschung

Max-Planck-Str. 2

D-37191 Katlenburg-Lindau

Germany

zakharov@mps.mpg.de

Zhugzhda, Yuzef D

IZMIRAN, Troitsk

RU 142190 Moscow Region

Russia

yzhugzhda@mail.ru 
